\title{
Proton Coupled Electron Transfer at Heavy Metal Sites
}

\author{
DISSERTATION
}

FOR THE AWARD OF THE DEGREE

“DOCTOR RERUM NATURALIUM"

OF THE GEORG-AUGUST-UNIVERSITÄT GÖTTINGEN

WITHIN THE DOCTORAL CHEMISTRY PROGRAM OF THE GEORG-AUGUST UNIVERSITY SCHOOL OF SCIENCE (GAUSS) IN THE GRADUATE SCHOOL BENCH

SUBMITTED BY

DANIEL DELONY, M. SC.

FROM CELLE, GERMANY

GÖTTINGEN, 2020 


\title{
Thesis Committee
}

\section{Prof. Dr. Sven Schneider}

Institut für Anorganische Chemie, Georg-August Universität Göttingen

\section{Prof. Dr. Franc Meyer}

Institut für Anorganische Chemie, Georg-August Universität Göttingen

Prof. Dr. Max. C. Holthausen

Institut für Anorganische und Analytische Chemie, Johann Wolfgang Goethe-Universität Frankfurt am Main

\section{Members of the Examination Board}

1. Reviewer

\author{
Prof. Dr. Sven Schneider
}

Institut für Anorganische Chemie, Georg-August Universität Göttingen

1. Reviewer

\section{Prof. Dr. Franc Meyer}

Institut für Anorganische Chemie, Georg-August Universität Göttingen

Prof. Dr. Max. C. Holthausen

Institut für Anorganische und Analytische Chemie, Goethe-Universität Frankfurt am Main

Further members

Prof. Dr. Ricardo Mata

of the

Institut für Physikalische Chemie, Georg-August Universität Göttingen

examination

Dr. Michael John

board

\author{
Institut für Organische und Biomolekulare Chemie, Georg-August Universität Göttingen \\ Dr. Christian Sindlinger \\ Institut für Anorganische Chemie, Georg-August Universität Göttingen
}


Für Katharina und Theo 



\section{Mein besonderer Dank gilt:}

meinem Betreuer Prof. Dr. Sven Schneider, für die interessanten wissenschaftlichen Fragestellungen und die große Freiheit mit der ich an die Themen herangehen durfte. Sven, dein ständiges Hinterfragen von Ergebnissen hat meinen Sinn für sauberes Arbeiten weiter geschärft.

Meinem Zweitbetreuer Prof. Dr. Franc Meyer, für die Übernahme der Zweitkorrektur und die anregenden Gespräche.

Prof. Dr. Max. C. Holthausen, für die unglaublich herzliche Aufnahme in deinen Arbeitskreis und die Übernahme der Drittkorrektur und natürlich für die erfolgreichen Kooperationen.

Dr. Martin Diefenbach, für das begeisterte Unterrichten der Quantenchemie. Ohne dich hätte ich in so kurzer Zeit niemals so viel lernen können.

Dem AK Holthausen, dafür dass ich mich selten so willkommen gefühlt habe wie bei euch.

My collaboration partners, for the successful collaborations and their scientific contribution to this thesis: Dr. Christian Würtele (X-Ray, many good conversations), Dr. Serhiy Demeshko (SQUID), Dr. Milan Orlita (magnetic IR), Dr. A. Claudia Stückl (EPR), Prof. Dr. Bas de Bruin (EPR) and Gannon Connor, Prof. Patrick L. Holland and Prof. James M. Mayer ( $\mathrm{NH}_{3}$ oxidation collaboration).

Dem AK Schneider, für die schöne und prägende Zeit in der Gruppe. Mein Dank gilt hier auch insbesondere Dr. Markus van Alten, Dr. Josh Abbenseth und Dr. Florian Wätjen für die gemeinsamen Projekte und Gespräche. Bei Isa, Markus, Josh und Sarah möchte ich mich des Weiteren für die Vernichtung zahlreicher Tonnen an Kaffeebohnen und die dabei verbrachte Zeit bedanken.

Meinen Korrekturlesern, Dr. Bastian Schluschaß, Richt van Alten und Jascha Lau. Vielen Dank für die vielen hilfreichen Anmerkungen und die gute Zeit während der Promotion.

Dem Graduiertenkolleg BENCh, für die Erweiterung meines Horizonts auf die Quantenchemie und die Ermöglichung meines Austausches in Frankfurt.

\section{Zuletzt möchte ich mich aus tiefstem Herzen bei meiner Familie bedanken:}

Meinen Eltern, dafür dass ihr immer an mich geglaubt habt und mich bereits in jungen Jahren bedingungslos unterstützt habt meinen Weg zu gehen. Ohne euch hätte ich nicht einmal Abitur machen können, da ein Kind aus einer Arbeiterfamilie ja „nichts auf dem Gymnasium zu suchen hat“....

Meiner Frau Katharina, dafür dass du seit mittlerweile sieben Jahren geduldig darauf wartet, dass ich mit meiner „Ausbildung“ abschließe. Vielen Dank auch für die jahrelange Unterstützung, Beratung und Ermöglichung eines Soziallebens. Mein Leben wurde durch dich bunter und vollkommener.

Meinem Sohn Theo, mein letzter Dank geht an dich. Du weißt es zwar nicht, aber du hast entscheidend dazu beigetragen, dass diese Arbeit schneller fertiggestellt wurde. Du hast mich zum einen zusätzlich motiviert fertig zu werden und warst zum anderen seit deiner Geburt das liebste Kind der Welt. Du hast mich nachts schlafen lassen und mir tagsüber den Freiraum gegeben zu schreiben. 


\section{Table of Contents}

Table of Contents

$\begin{array}{ll}\text { I. INTRODUCTION } & 6\end{array}$

1. Oxygen and Oxygenation chemistry 1

1.1 Oxygenation Chemistry in Nature $\quad 1$

$\begin{array}{lll}1.2 & \text { Terminal Oxo Complexes } & 3\end{array}$

2. Proton-Coupled Electron Transfer $\quad 8$

2.1 General Aspects in PCET Chemistry $\quad 8$

$\begin{array}{lll}2.2 & \text { PCET Thermochemistry } & 9\end{array}$

3. Computational Thermochemistry 12

3.1. Computational Methods $\quad 12$

3.2. Relativistic Effects on Thermochemistry 16

$\begin{array}{lr}\text { 4. Outline } & 18\end{array}$

$\begin{array}{llr}4.1 & \text { Outline of Chapter II } & 18\end{array}$

$\begin{array}{lll}4.2 & \text { Outline of Chapter III } & 19\end{array}$

$\begin{array}{llr}4.3 & \text { Outline of Chapter IV } & 20\end{array}$

II. A TERMINAL IRIDIUM OXO COMPLEX WITH A TRIPLET GROUND STATE 22

1. Synthesis of Terminal Iridium Hydroxo Complexes $\quad 23$

2. Synthesis and Characterisation of a Terminal Iridium Oxo Complex 27

3. Reactivity of the Terminal Iridium Oxo Complex 35

3.1. Oxygen Atom Transfer and Philicity Derivation 35

3.2. $\mathrm{BD}(\mathrm{F}) \mathrm{E}$ determination of the hydroxo/oxo couple 2/4 40

3.3. $\quad \mathrm{C}-\mathrm{H}$ bond Activation Chemistry of $4 \quad 44$

\begin{tabular}{l} 
4. Summary of Chapter II \\
\hline 0
\end{tabular}

$\begin{array}{lr}\text { III. EXCURSUSES } & 52\end{array}$

1. Excursus 1: Interconversion of Phosphinyl Radical and Phosphinidene Complexes by Proton Coupled Electron Transfer 
2. Excursus 2: Facile Conversion of Ammonia to a Nitride in a Rhenium System that Cleaves Dinitrogen 56

IV. ON THE EFFECT OF SPIN-ORBIT COUPLING IN SOLUTION THERMOCHEMISTRY

1. Characterisation of the rhenium(III/IV) amine complexes 23,25 and of rhenium(IV) amide 24

1.1 Characterisation of rhenium(III) amine complex 23

60

1.2 Characterisation of the Rhenium(IV) Amide Complex 24

63

1.3 Characterisation of cationic rhenium(IV) amine complex 25

64

2. Computational Rationalisation of the Spectroscopic and Magnetic Features 66

2.1. Computational Rationalisation of rhenium(III) amine complex 2366

2.2. Computational Rationalisation of rhenium(IV) amide complex 24

2.3. Computational Rationalisation of rhenium(IV) amine complex 25

3. (Free) Reaction Enthalpy Determination 75

3.1. Experimental (Free) Reaction Enthalpy Determination 75

$\begin{array}{lll}\text { 3.2. Computational (Free) Enthalpy Determination } & 78\end{array}$

$\begin{array}{ll}\text { 4. Summary of Chapter IV } & \mathbf{8 1}\end{array}$

V. CONCLUSION AND OUTLOOK 84

$\begin{array}{ll}\text { VI. EXPERIMENTAL DETAILS } & 90\end{array}$

1. Materials and Methods $\quad 91$

$\begin{array}{lll}1.1 & \text { General remarks } & 91\end{array}$

$\begin{array}{lll}1.2 & \text { Analytical Methods } & 92\end{array}$

2. Synthesis $\quad 95$

2.1. Improved synthesis for [(PNP)|rOH] $2 \quad 95$

2.2. Synthesis of $\left[\mathrm{Na}(\text { thf)(PNP)|rOH }]_{2} \mathbf{1} 96\right.$

2.3. Improved synthesis of [(PNP)IrOH][PF6] 3

2.4. Synthesis of [(PNP)IrO] $4 \quad 98$

2.5. Synthesis of $\left[(\mathrm{PNP}) \mid \mathrm{ICO}_{3}\right] \mathbf{8} \quad 99$

2.6. Synthesis of [(PNP)IrPMe 3 10 100

\begin{tabular}{lr} 
2.7. & Reactivity of 4 towards $\mathrm{H}_{2}$ \\
\hline
\end{tabular}

$\begin{array}{ll}\text { 2.8. } & \text { Reactivity of } 4 \text { towards CO } \\ & 101\end{array}$

2.9. Synthesis of [(PNP)IrO( $\left.\left.\mathrm{C}_{12} \mathrm{H}_{8} \mathrm{O}\right)\right]$ "Xanthenolate" $11 \quad 101$

2.10. Synthesis of [(PNP)IrO( $\left.\left.\mathrm{C}_{13} \mathrm{H}_{9}\right)\right]$ “Fluorenolate” 12

2.11. Reactivity of 4 towards benzaldehydes ( $\left.\mathrm{X}^{\text {para }}=\mathrm{H}, \mathrm{Me}, \mathrm{OMe}, \mathrm{F}\right) \quad 101$ 
2.12. General synthesis of $\left[(P N P) \mid \mathrm{rO}_{2} \mathrm{CPh}-\mathrm{X}\right]$ 14-X with $\left.X^{\text {para }}=\mathrm{F}, \mathrm{OMe}, \mathrm{Me}, \mathrm{H}\right)$

2.13. Synthesis of $[(\mathrm{PNP}) \operatorname{Ir}(\mathrm{H})(\mathrm{Cl})] \mathbf{1 5} \quad 104$

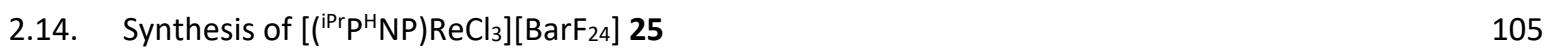

2.15. Deprotonation of [( $\left.\left(^{\mathrm{iPr}} \mathrm{P}^{\mathrm{H}} \mathrm{NP}\right) \mathrm{ReCl}_{3}\right]\left[\mathrm{BarF}_{24}\right] 25$

2.16. N-H/D exchange of $23 \quad 105$

2.17. Reevaluation of the 23/25 redox potential 106

$\begin{array}{lr}\text { 3. Isothermal Titration Calorimetry } & 107\end{array}$

$\begin{array}{llr}3.1 & B D(F) E \text { determination of } 4 & 107\end{array}$

$\begin{array}{llr}3.2 & \text { BDE determination of } 23 & 108\end{array}$

$\begin{array}{ll}\text { 3.2.1 Enthalpy determination of the PCET reaction in THF } & 108\end{array}$

3.2.2 Enthalpy determination of the PCET reaction in DCM 111

$\begin{array}{lll}3.3 \quad \mathrm{p} K_{\mathrm{A}} \text { value determination of } 25 & 114\end{array}$

$\begin{array}{lll}3.4 & \mathrm{p} K_{\mathrm{A}} \text { value determination of } \mathrm{Mes} * \mathrm{OH} & 116\end{array}$

$\begin{array}{lll}3.5 & \text { P-H BDE value determination of } 17 & 118\end{array}$

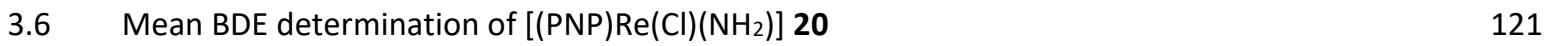

4. Computational Details $\quad 124$

$\begin{array}{lr}\text { 4.1. General Remarks } & 124\end{array}$

4.2. A Terminal Iridium Oxo with a Triplet Ground State 126

4.2.1. Molecular Geometries 126

$\begin{array}{ll}\text { 4.2.1. Spin State and Reaction Energetics } & 127\end{array}$

$\begin{array}{ll}\text { 4.2.2. Total Energies } & 128\end{array}$

$\begin{array}{ll}\text { 4.2.3. State-Averaged CASSCF computations } & 129\end{array}$

4.3. On the Spin-Orbit Coupling influence in solution thermochemistry 133

4.3.1. Molecular Geometries 133

4.3.1. State Averaged CASSCF Computations 134

4.3.2. CASSCF/NEVPT2 State Composition and QDPT Eigenvectors 136

$\begin{array}{lll}\text { 4.3.3. } & \text { Comparison of Magnetic Properties Theory vs. Experiment } & 137\end{array}$

$\begin{array}{lll}\text { 4.3.4. Spin State and Reactions Energetics } & 138\end{array}$

$\begin{array}{ll}\text { 4.3.5. Total Energies } & 141\end{array}$

4.4 Interconversion of Phosphinyl Radical and Phosphinidene Complexes by Proton Coupled Electron Transfer 
A.1. X-Ray Single-Crystal Analysis of $1 \quad 159$

A.2. X-Ray Single-Crystal Analysis of $3 \quad 161$

A.3. X-Ray Single-Crystal Analysis of $4 \quad 163$

A.4. X-Ray Single-Crystal Analysis of $8 \quad 165$

$\begin{array}{lll}\text { A.5. X-Ray Single-Crystal Analysis of } 10 & 167\end{array}$

A.6. X-Ray Single-Crystal Analysis of 14-H 169

A.7. X-Ray Single-Crystal Analysis of 14-F 171

A.8. X-Ray Single-Crystal Analysis of 14-OMe 173

A.9. X-Ray Single-Crystal Analysis of $11 \quad 175$

A.10. X-Ray Single-Crystal Analysis of $12 \quad 177$

A.11. X-Ray Single-Crystal Analysis of $15 \quad 179$

A.12. X-Ray Single-Crystal Analysis of $25 \quad 181$

$\begin{array}{lr}\text { B. List of Chemical Compounds } & 183\end{array}$

$\begin{array}{lr}\text { C. Independent PCET Model } & 184\end{array}$

C.1. Derivation of the fitting function $\quad 184$

$\begin{array}{lr}\text { C.2. Incooperation into Visual Basics } & 185\end{array}$

D. Cartesian Coordinates of Computed Structures $\quad 186$

$\begin{array}{lr}\text { E. List of abbreviations } & 206\end{array}$

$\begin{array}{lr}\text { F. Curriculum Vitae } & 208\end{array}$ 

Chapter I

I. Introduction 


\title{
1. Oxygen and Oxygenation chemistry
}

\author{
"Oxygen: An essential toxin"
}

I. C. Gunsalus, $1973^{[1]}$

\subsection{Oxygenation Chemistry in Nature}

Oxygen is the most abundant element in the Earth crust and after iron the most abundant element on Earth in general. ${ }^{[2]}$ The dioxygen essential to respiration was nevertheless not always part of the Earth atmosphere. Only since the first cyanobacteria started to produce dioxygen as a waste product of photosynthesis, the oxygen amount started to rise slowly to the present value of $21 \%$. On the way there, oxygen was responsible for the first mass extinction in Earth history, known as the great oxygenation event (GOE). ${ }^{[3]}$ This catastrophe can be seen as the first indication of the high reactivity of dioxygen. Life had to adapt to the new atmosphere and overcame it utilizing the former poison as an energy supply. An evolutionary stroke of genius, since the aerobic respiration had a significantly higher energy potential than former anaerobic processes, thus making the way free for higher life forms. ${ }^{[4]}$ The biological oxygen fixation takes place in the coordination sphere of base metals with either copper (hemocyanin) or iron (haemoglobin) in the centre of attraction for invertebrates and vertebrates, respectively. ${ }^{[5]}$ The latter has an iron(II)-high spin (hs) metal centre coordinated to a porphyrin ring in the active site of the protein (I), which loses its paramagnetic ground state upon coordination of $\mathrm{O}_{2}$ and yields a diamagnetic oxyhaemoglobin species (II) (Figure i1, a). ${ }^{[6]}$

a)

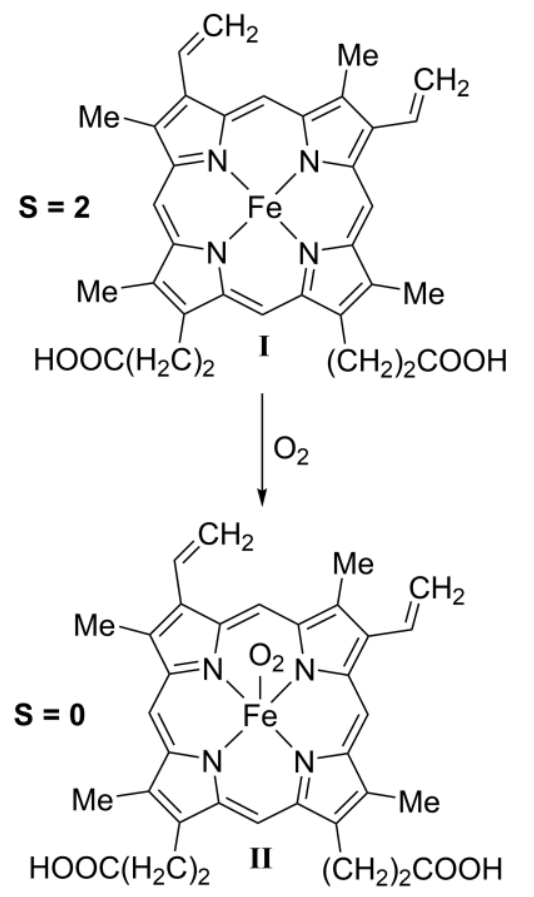

b)

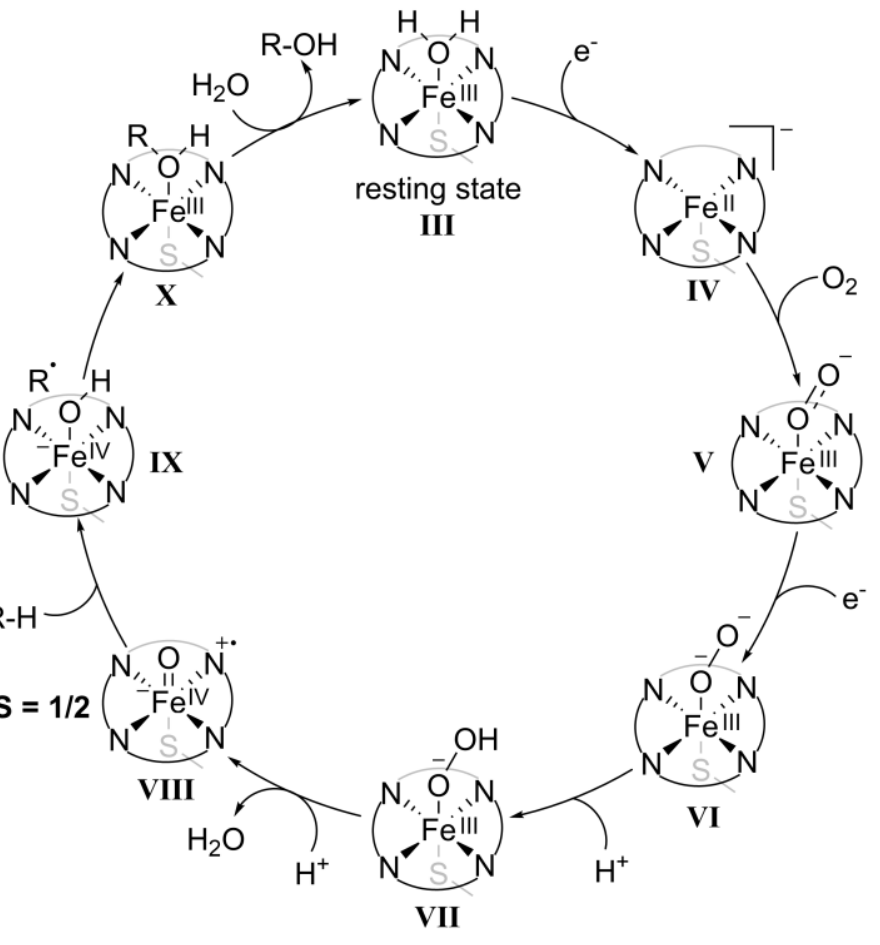

Figure i1: a) Chemical structure of the heme-b unit of haemoglobin and cytochrome P450 (top) and schematic end-on oxygen binding to haemoglobin (bottom). b) Catalytic oxygenation cycle of cytochrome P450.[1,7] 
This finding in 1936 started an intense and still ongoing discussion about the nature of the $\mathrm{Fe}-\mathrm{O}_{2}$ binding, including a low-spin (Is) Fe(II, Is)- $\mathrm{O}_{2}$ (singlet) interaction (Pauling model), an $\mathrm{Fe}(\mathrm{III}, \mathrm{Is})-\mathrm{O}_{2}{ }^{-}$interaction, in which the superoxo ligand is antiferromagnetically coupling to the iron centre (Weiss model) and an antiferromagnetically coupled intermediate-spin model (is) Fe(II, is)- $\mathrm{O}_{2}$ (triplet) (McClure, Harcourt, Goddard model). ${ }^{[8]}$ All these models agree in some aspects with the performed experiments and are among the first examples of oxygen metal binding interactions discussed in the literature. The same structural motif as for haemoglobin can be found in cytochrome proteins which are responsible for biological electron transfer and oxygenation chemistry. ${ }^{[9]}$ Here, the ubiquitous class of cytochrome P450 enzymes is of special interest, since these enzymes play a vital role in the metabolism of xenobiotics ${ }^{1}$ and in the synthesis of critical signal molecules. ${ }^{[1]}$ In contrast to haemoglobin, the iron is in the ferric iron(III) oxidation state (III). Dioxygen binds after reduction of III and is further activated by an electronproton-proton transfer cascade (ET-PT-PT) ${ }^{2}$ (IV-VII), yielding a terminal iron(IV) oxo complex (VIII) as the key intermediate for the oxygenation of unactivated hydrocarbons (Figure i1, b). ${ }^{[1,7]}$ The crucial reaction step of the catalytic cycle is the hydrogen atom transfer (HAT) from the hydrocarbon substrate to VIII followed by the radical rebound of the carbo-radical to $\mathbf{I X}$, which yields the desired alcohol product complex $\mathbf{X}{ }^{\left[{ }^{[10]}\right.}$ Such iron oxo moieties play a vital role for many biological enzyme families like cyclooxygenases and peroxidases and are proposed key intermediates in many different reaction types like (cis)-hydroxylation, oxidative ligand transfer, desaturation/cyclisation by HAT and electrophilic aromatic substitution. ${ }^{[11-13]}$ Another important field of biochemistry in which a terminal oxo species is discussed is the oxygen evolving complex (OEC) in the heart of photosystem(II) (PSII) (Figure i2). ${ }^{[14]}$

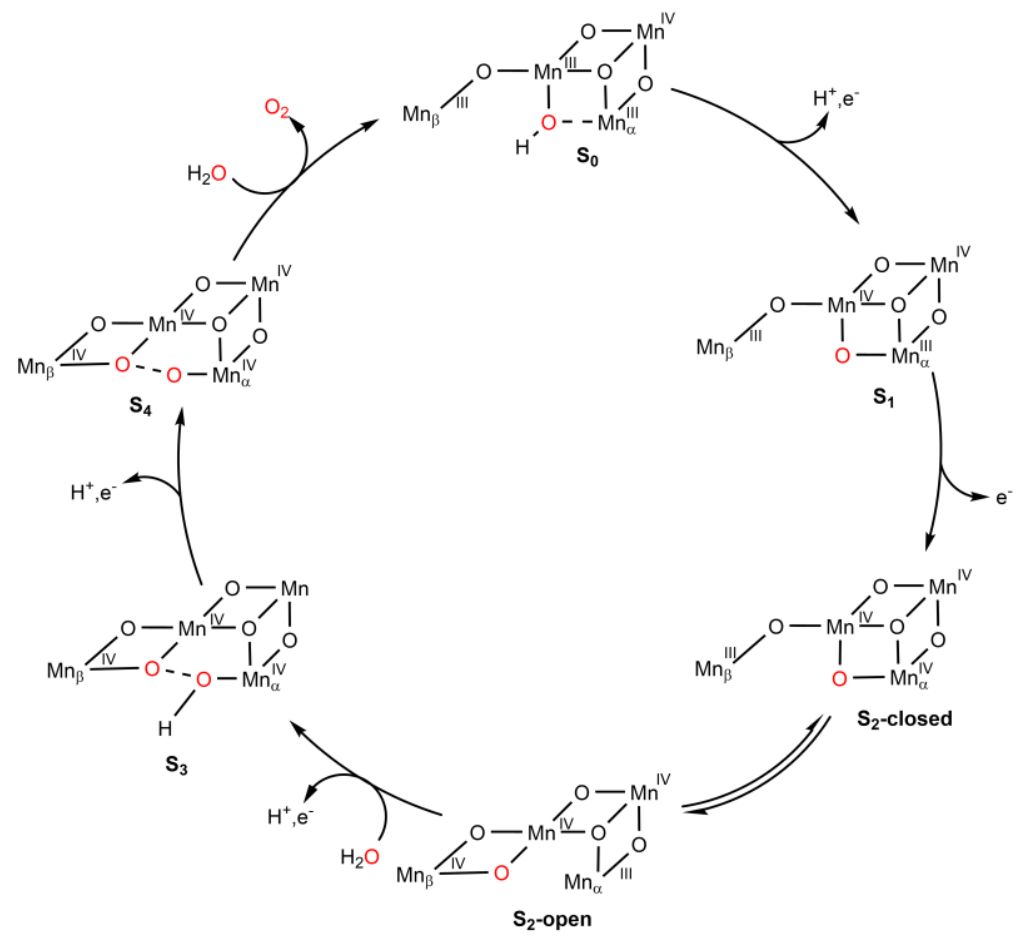

Figure i2: Schematic catalytic cycle of the oxygen evolving complex (OEC) according to Cox et al. ${ }^{[14]}$, structures of $\mathrm{S}_{0}$ and $\mathrm{S}_{1}$ in analogy to Pal et al. ${ }^{[15]}$

${ }^{1}$ Xenobiotics are compounds which are not naturally part of the organism.

2 For a detailed discussion of ETPT/HAT/PCET chemistry see Section 2. 
The manganese-calcium $\mathrm{Mn}_{4} \mathrm{O}_{5} \mathrm{Ca}$ cofactor in the active centre of PSII is activated by a proton-coupled electron transfer $(P C E T)^{3}$ step from the $S_{0}$ to the $S_{1}$ state, which is further oxidized to the $S_{2}$ state. Here, the cubic form of $\mathrm{S}_{2}$-closed is in equilibrium with an open form, $\mathrm{S}_{2}$-open, in which the $\mathrm{Mn}_{\alpha}$ now has a vacant coordination site to coordinate a second water molecule yielding $\mathrm{S}_{3}$ after PCET (Figure i2). For $\mathrm{S}_{3}$ an oxygen-oxygen interaction between $\mathrm{Mn}_{\alpha}-\mathrm{O}-\mathrm{-O}-\mathrm{Mn}_{\beta}$ is already predicted. The O-O bond is formed upon a last PCET step ( $\left.\mathrm{S}_{4}\right)$, leading to the rapid release of dioxygen upon coordination of a water molecule. ${ }^{[14,15]}$ These reaction types of transition metal oxo complexes, which nature performs with ease, are essential for the synthesis of fine chemicals from unactivated, fossil hydrocarbons as well as for future sustainable fuel cells and will be one of the main topics discussed in Chapter II of this thesis. ${ }^{[16,17]}$

\subsection{Terminal Oxo Complexes}

\section{"The Oxo Wall stands"}

\section{Craig L. Hill in correspondence with Harry B. Gray ${ }^{[18]}$}

Thousands of terminal transition metal oxo complexes are reported in the literature, but they are not evenly distributed over the d-block. ${ }^{[17]}$ Most transition metal oxo complexes are found for the early transition metals in high oxidation states bearing a low d-electron count. This finding was rationalized by Ballhausen and Gray in 1962 by the consideration of the molecular frontier orbitals $(\mathrm{MO})$ of the vanadyl oxo ion complex $\left[\left(\mathrm{H}_{2} \mathrm{O}\right)_{5} \mathrm{~V}(\mathrm{IV}) \mathrm{O}\right]^{2+} \mathbf{X I}$ (Figure i3). ${ }^{[19]} \mathbf{X I}$ is in tetragonal $\left(\mathrm{C}_{4 \mathrm{v}}\right)$ coordination with four equivalent water ligands in equatorial position and one water ligand and the oxo ligand in opposite axial positions. Considering the $3 \mathrm{~d}$, $4 \mathrm{~s}$ and $4 \mathrm{p}$ metal orbitals, a MO scheme with 17 orbitals arises which excellently reproduces the spectroscopic and magnetic data of $\mathbf{X I}{ }^{[19]}$ Additionally, a simplified MO scheme for near octahedral oxo complexes can be deducted from their elaborate approach which explains why only few examples of late transition metal oxo complexes exist (Figure i3, right). Together with the metal-oxo $\pi$-bonding interaction, a strong $\mathrm{M}-\mathrm{O}$ triple bond can be formed for low electron counts $d^{0-2}$, since only the non-bonding $d_{x y}$ will be populated. For higher $d$-electron counts the $\pi^{*}$-orbitals of the M-O unit will be populated and thus lead to a weakening of the M-O moiety. The vanadyl(IV) oxo complex XI exhibits with a $\mathrm{d}^{1}$-configuration a M-O triple bond, with one electron in the non-bonding $d_{x y}$, while the iron(IV) oxo VIII $\left(d^{4}\right)$ (see Figure i1) bears two electrons in the $\pi^{*}$-orbitals and therefore only exhibits a M-O double bond. This finding does not only explain the high reactivity of VIII, as discussed in Section 1.1, but also explains the spin state of the system. For the observed doublet ground state of VIII, a triplet configuration at the iron centre is needed that couples antiferromagnetically with the heme radical cation. This triplet configuration of the metal centre is predicted in the MO scheme due to the degeneracy of the $\pi^{*}$-orbitals.

The population of more than 5 d-electrons leads to a break down of the $\mathrm{M}-\mathrm{O} \pi$-bonding interaction, resulting in a basic oxo ligand which is e.g. not stable towards protonation. These findings led to the definition of the "Oxo-

\footnotetext{
${ }^{3}$ For a detailed discussion of PCET chemistry see Section 2.
} 
Wall": There is no stable terminal transition metal oxo complex in a tetragonal coordination sphere with d-electron counts higher than $d^{5}$. Since oxidation states are limited for the late transition metals, and very high oxidation states would favour the extrusion of $\mathrm{O}_{2}$ or the oxidation of the environment, the oxo wall is placed between group 8 and 9 of the periodic system. ${ }^{[20]}$

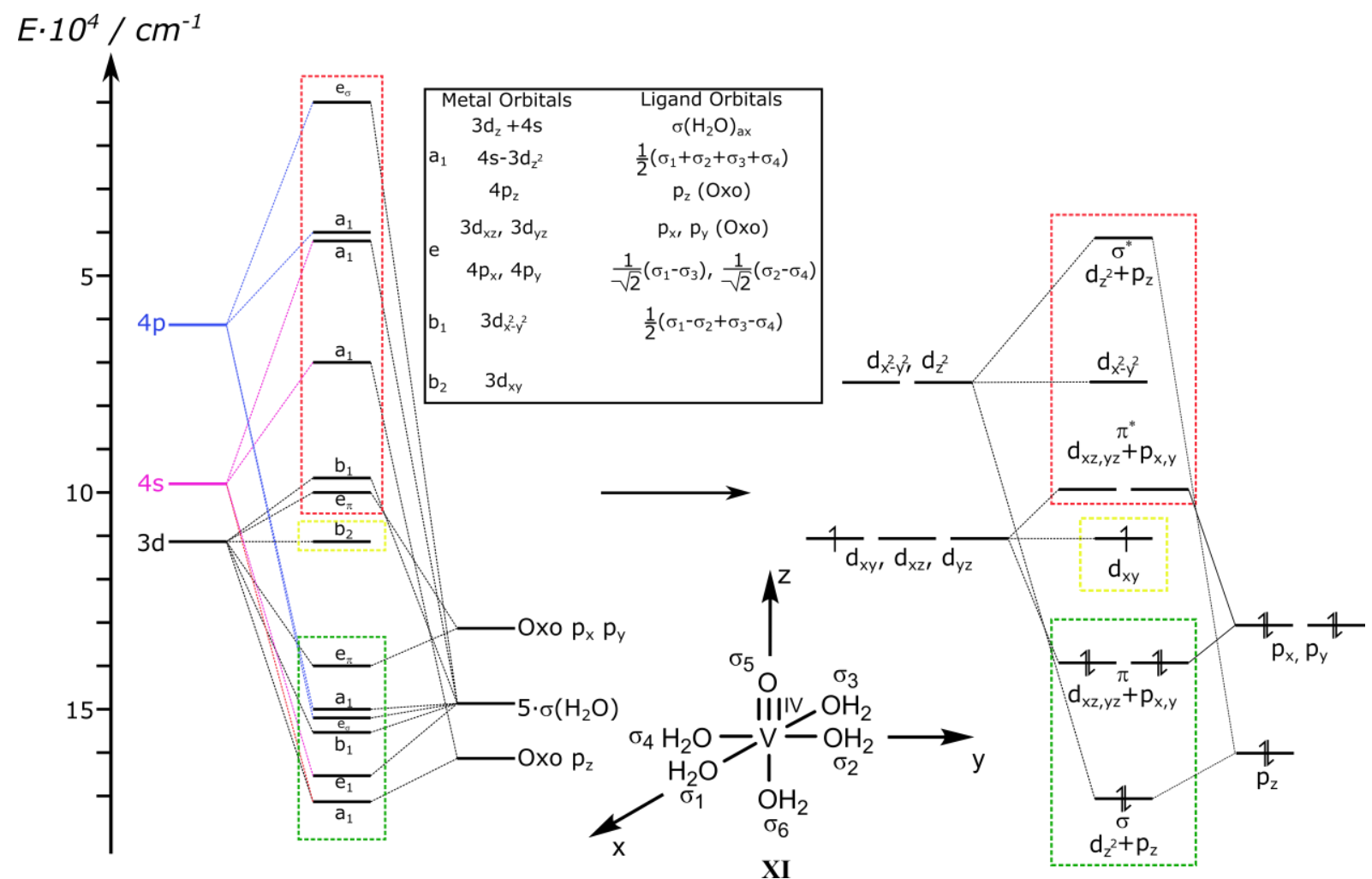

Figure i3: Full frontier orbital MO scheme of the vanadium(IV) oxo complex XI after Ballhausen and Gray with bonding orbitals (green) non-bonding orbital (yellow) and anti-bonding orbitals (red) (left) ${ }^{[19]}$ and simplified deducted $\mathrm{MO}$ scheme of a metal ion in near octahedral coordination sphere with an oxo ligand (right).

Late transition metals often favour lower coordination numbers than the (pseudo)octahedral coordination discussed above, but the lower coordination numbers change the d-orbital splitting in e.g. tetrahedral and squareplanar complexes and thus the starting point of the metal oxo bonding discussion changes. The $t_{2}$ orbital set in tetrahedral coordination interacts with the oxo ligand, forming the $\sigma$ - and $\pi$-bonding interactions, while the former e orbital set remains unchanged, thus forming two non-bonding orbitals (Figure i4, left). ${ }^{4} \mathrm{~A}$ similar situation arises for a square-planar oxo complex. Here, the $d_{z 2}$ and $d_{y z}$ orbitals do not interact with the oxo moiety and are therefore non-bonding orbitals. The only difference to the (pseudo)tetrahedral coordination is that the nonbonding orbitals are not degenerate as for the trigonal coordination (Figure i4, right). With the two non-bonding orbitals in (pseudo)tetrahedral and (pseudo)square-planar geometry, the formal oxo wall in these coordination environments is shifted from a $d^{5} / d^{6}$ to $a d^{7} / d^{8}$ configuration. The oxo wall in these coordination spheres is therefore formally between group 10 and 11, and up to date, there is no isolated transition oxo complex that violates the oxo wall. ${ }^{[18]}$

${ }^{4}$ Please note, that here the $z$-axis in tetrahedral coordination is ligand centred for clarity. 

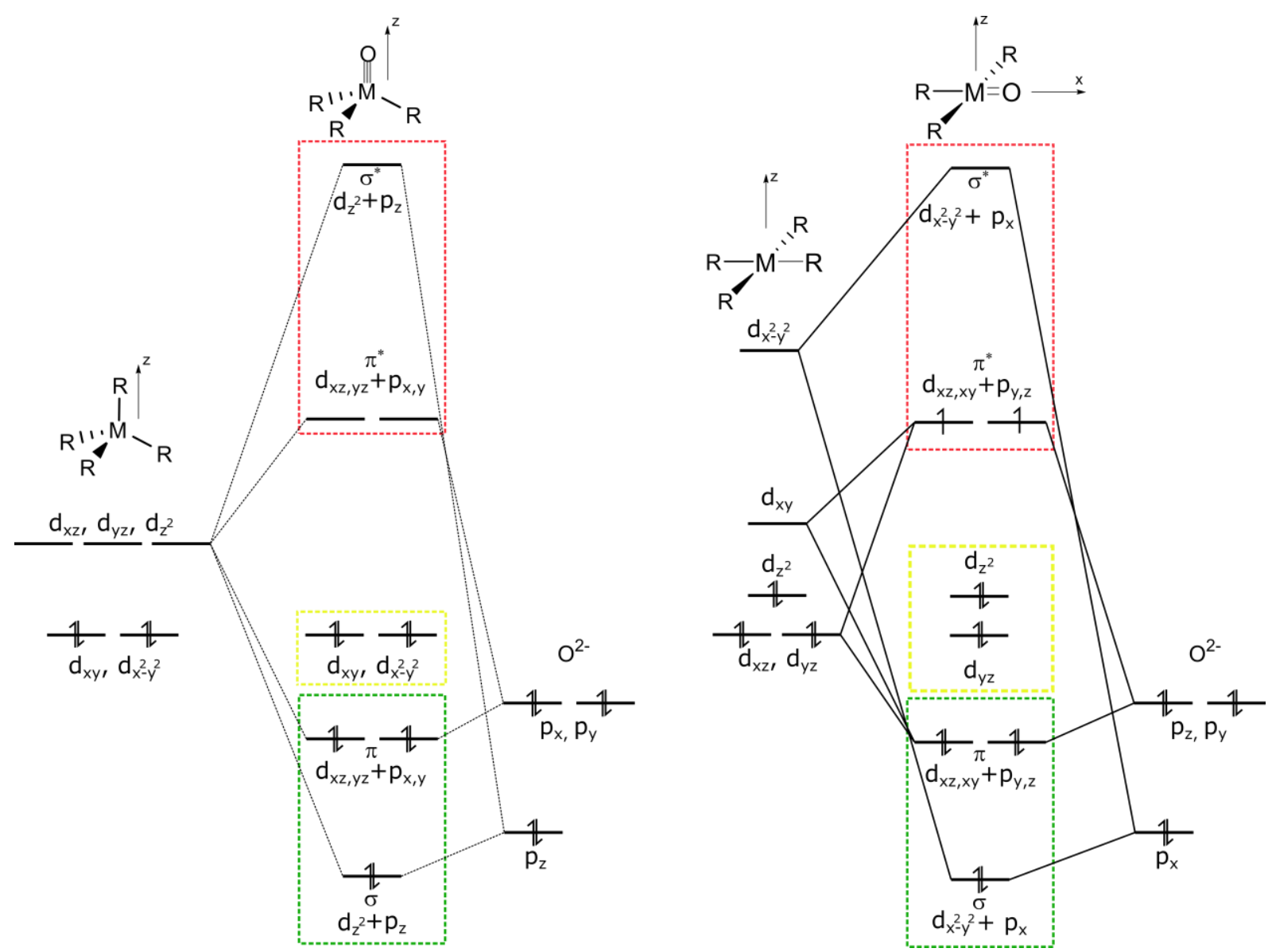

Figure i4: Schematic MO scheme for a terminal oxo complex in trigonal coordination sphere depicted exemplarily for a $\mathrm{d}^{4}$ metal ion (left). Schematic MO scheme for a terminal oxo complex in $C_{2 v}$ symmetry depicted exemplarily for a $\mathrm{d}^{6}$ metal ion (right)

The M-O $\pi^{*}$ population of late, electron rich, group 9 transition metals ( $\mathrm{Rh}, \mathrm{Ir}$ ) explains their prevalence in synthetic hydroxylation, water oxidation and related (isoelectronic) nitrene transfer chemistry. ${ }^{[21-26]}$ The isolation of the reactive late transition metal oxo/nitrene intermediates is therefore important for a detailed understanding of their reactivity. The few reported examples of this complex class in group 9 are dominated by pseudo(tetrahedral) geometries and terminal imido complexes are by far more common than terminal oxo complexes which most likely can be attributed to their higher steric protection from the N-R group (Figure i5). ${ }^{[27-33][34][35-37]}$ Only two terminal oxo complexes of group 9 are reported, both are closed-shell and in (pseudo)-tetrahedral coordination. The oxotrimesityliridium complex XXI by Wilkinson is in the iridium(V) oxidation state and thus exhibits a triple bond, while the thermolabile cobalt oxo complex XVII reported by Anderson is in the cobalt(III) oxidation state and thus exhibits double bond character. ${ }^{[33,36], 5}$ The lower bond order of XVII is reflected by its higher reactivity compared to XXI. While XXI is mostly inert and can only oxygenate phosphines, ${ }^{[38]}$ cobalt oxo XVII additionally is a strong hydrogen atom abstractor with an estimated bond dissociation free energy (BDFE) of $85 \mathrm{kcal} \mathrm{mol}^{-1}$.

\footnotetext{
${ }^{5}$ Anderson et al. discuss a triple bond in their paper even though the electron count [Co(III), $\left.d^{6}\right]$ formally forbids a triple bond in this geometry. Additionally, their own DFT computations have the Co-O $\sigma^{*}$-orbital as the HOMO thus reducing the bond order to two. For this reason, complex XVII is discussed to obtain double character, to stay consistent with the oxo-wall discussion above.
} 


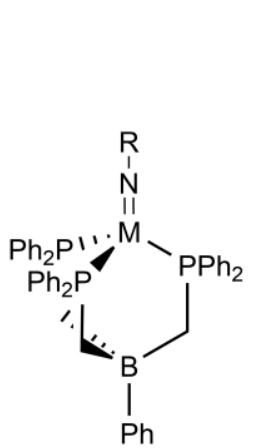

Co: Peters 2002 XII Rh: Tejel 2014 XIII

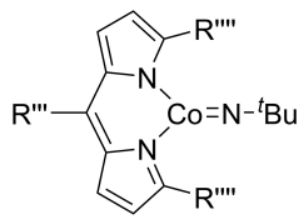

XIX

Betley 2012

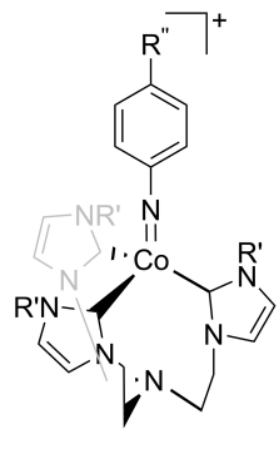

XIV

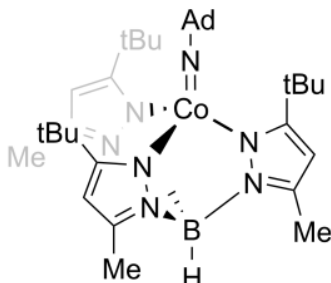

XV

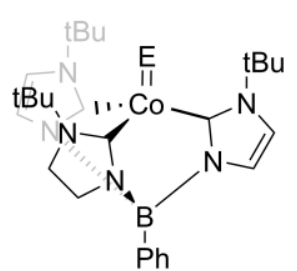<smiles></smiles>

$E=N^{t} B u$ Smith $2007 \mathbf{X V I}$

XVIII Theopold 2005

$E=$ O Anderson 2018 XVII Deng 2015<smiles>C[As](C)(C)=O</smiles>

XXI Wilkinson 1993

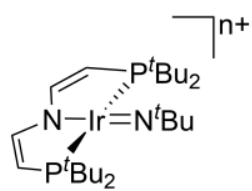

$\mathrm{n}=0$ (XXII), $\mathrm{n}=1$ (XXIII), $\mathrm{n}=2$ (XXIV) Schneider 2018

Figure i5: Literature reported isolated group 9 terminal imido and oxo complexes of cobalt ${ }^{[27-33]}$, rhodium ${ }^{[34]}$ and iridium ${ }^{[35-37]}$. $R(P e t e r s)=T$ Tol, $R(T e j e l)=A d, D i p p$, $\mathrm{C}_{6} \mathrm{~F}_{5} ; \mathrm{R}^{\prime}=\mathrm{H}, \mathrm{Me} ; \mathrm{R}^{\prime \prime}=\mathrm{OMe}, \mathrm{Me} ; \mathrm{R}^{\prime \prime \prime}=3,5$-bis(trifluormethyl)phenyl; $\mathrm{R}^{\prime \prime \prime \prime}=2,6$-bis(diphenylmethyl)-4-methylphenyl $\mathrm{R}^{\vee}={ }^{\mathrm{t}} \mathrm{Bu}, \mathrm{SiMe}_{2}{ }^{t} \mathrm{Bu}, 2,6$-dimethylphenyl, Dipp.

The reported group 9 oxo/imido complexes have a diamagnetic ground state, but catalytic nitrene and oxygenation transfer is often associated to proceed via radical pathways and therefore accessible open-shell states are discussed for the intermediates. ${ }^{[39,40]}$ The only exceptions here are the two-coordinate cobalt(II) imido complex XVIII of Deng et al. and the square-planar iridium(III/IV/V) imido redox series XXII-XXIV by Schneider et al (Figure i6). ${ }^{[31,37]}$ Iridium imide XXII represents an unprecedented iridium complex with a triplet ground state, which is strongly stabilised by spin-orbit coupling as shown by SQUID magnetometry and computational analysis. Substantial N-radical "imidyl" character of XXII with a $\left(\pi_{1}{ }^{*} / \pi_{2}{ }^{*}\right)^{2}$ configuration is predicted by computations, in perfect agreement with the MO scheme depicted in Figure i4, resulting in ambiphilic nitrene transfer reactivity with $\mathrm{CO}_{2}, \mathrm{PMe}_{3}$ and $\mathrm{H}_{2}$. On the other hand, the cationic imido species XXIII does not show reactivity with selected nucleo- and electrophiles, which is attributed to spin-delocalisation by spin-orbit coupling and is also in line with the formally higher Ir-N bond order. Reactivity studies of the dicationic complex XXIV were hampered by its thermally instability forming the cationic nitride complex XXVI and isobutene. ${ }^{[37]}$

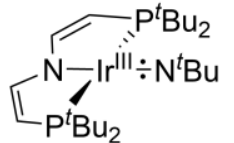

XXII

$S=1$

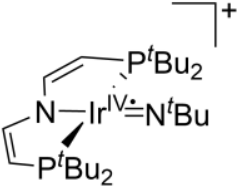

XXIII

$S=1 / 2$

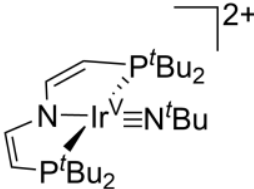

XXIV

$S=0$

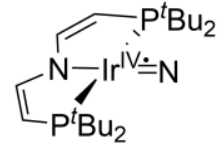

XXV

$S=1 / 2$

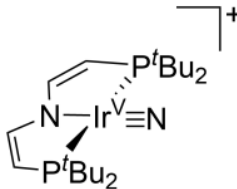

XXVI

$S=0$

Figure i6: Square-planar terminal imido and nitride complexes with their formal oxidation states reported by Schneider et al. ${ }^{[37,41]}$

Examples of terminal imido/oxo complexes beyond group 9 are even scarcer. ${ }^{[42-44][45][46]}$ Here, a series of nickel imides XXVII-XXIX by Hillhouse et al., a palladium imide XXX by Munz and co-workers, a platinum(IV) oxo XXXI by Milstein et al, and very recently a copper(I) supported open-shell nitrene complex XXXI by Betley and co-workers were reported (Figure i7). ${ }^{[42-47]}$ The platinum oxo complex XXXI of Milstein is a thermolabile 
compound which inserts the oxygen atom into the Pt-P bond when heated to room temperature. XXXI has diverse oxygenation chemistry with several substrates like carbon monoxide, phosphines, dihydrogen, hydrides and water. ${ }^{[47]}$ With an electron count of $d^{6}, \mathbf{X X X I}$ exhibits a Pt-O double bond but is significantly more reactive than the cobalt oxo XVII, thus highlighting the importance of the accessibility of the metal oxidation state on the stability/reactivity of the formed oxo complex. Very recently, a copper supported triplet nitrene complex XXXII was isolated by Betley and co-workers which shows diverse nitrene transfer chemistry.[46] Even though the short Cu-N bond distance $(1.75 \AA$ A) suggests multi-bonding character, XAS studies derived a copper(I) oxidation state which was further refined by SORCI-CAS ${ }^{6}$ computations. Here, the copper $(I)$-triplet $\left(\mathrm{d}^{10}\right)$ nitrene configuration dominates the ground state (58\%), while the copper(II) doublet imidyl configuration only sums up to $25 \%$. These results suggest, that XXXII can be considered as the only stable imido complex beyond the oxo wall, stabilized by the enormous steric bulk around the reactive centre. As discussed in this Section, isolated terminal oxo complexes beyond group 8 are rare complexes and especially only closed-shell species have been published until now. In Chapter II of this thesis the isolation and characterisation of a new terminal iridium oxo complex with a triplet ground state will be discussed.

\section{a) Group 10}

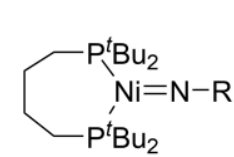

$\mathrm{R}=$ Dipp XXVII, 2001

$\mathrm{R}=\mathrm{dmp}$ XXVIII, 2010

Hillhouse<smiles>[R]n1ccnc1N=NO</smiles>

XXIX

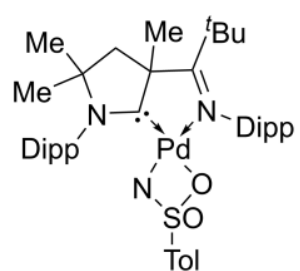

XXX

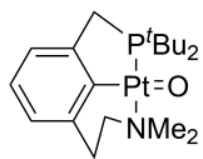

XXXI

Hillhouse 2011

Munz 2018

Milstein 2008

\section{b) Group 11}<smiles></smiles>

XXXII

Betley 2019

Figure i7: a) Literature reported terminal imido and oxo complexes of group 10 of nickel ${ }^{[43,44]}$, palladium ${ }^{[45]}$, platinum ${ }^{[47]}$;, $R^{\prime}=1,1,7,7$-tetraethyl-1,2,3,5,6,7hexahydro-3,3,5,5-tetramethyl-s-indacene; b) Only literature reported terminal imido complex of copper ${ }^{[46]} \cdot \mathrm{R}^{\prime \prime}=3,5$-bis(trifluormethyl)phenyl R"' $=\mathrm{O}^{\mathrm{t}} \mathrm{Bu},{ }^{\mathrm{t}} \mathrm{Bu}$.

${ }^{6}$ Spectroscopy-oriented con 


\title{
2. Proton-Coupled Electron Transfer
}

\section{"Among the most elementary and significant of all reactions is the transfer of a}

\author{
hydrogen atom..."
}

\section{Ahmed H. Zewail, Nobel Laureate 1999[48]}

\subsection{General Aspects in PCET Chemistry}

This Section will describe and define the terms proton-coupled electron transfer (PCET), hydrogen atom transfer (HAT) or stepwise proton-electron steps (PTET/ETPT), which are relevant to the chemistry of terminal oxo complexes. Transfer of electrons and protons is ubiquitous in bioenergetic processes in nature (e.g. Cytochrome P450, OER in photosynthesis, nitrogen fixation) ${ }^{[7,49,50]}$, synthesis (e.g. Hydroxylations, Hydroaminations, HaberBosch process ${ }^{[50,51]}$ and artificial energy conversions (e.g. fuel cells, combustion) ${ }^{[52]}$, just to name a few, and many excellent reviews ${ }^{[53,54]}$ and books ${ }^{[48]}$ have been published on the topic. Meyer et al. introduced the term Protoncoupled electron transfer (PCET) first in 1981 for concerted transfer of one proton and one electron, but the term is nowadays used more broadly for the general transfer of (multiple) protons and electrons even from different reaction sites. ${ }^{[55]}$ Many sub-terms have been introduced in the literature to differentiate between the various expressions and mechanisms of PCET chemistry (Figure i8, a).

a)

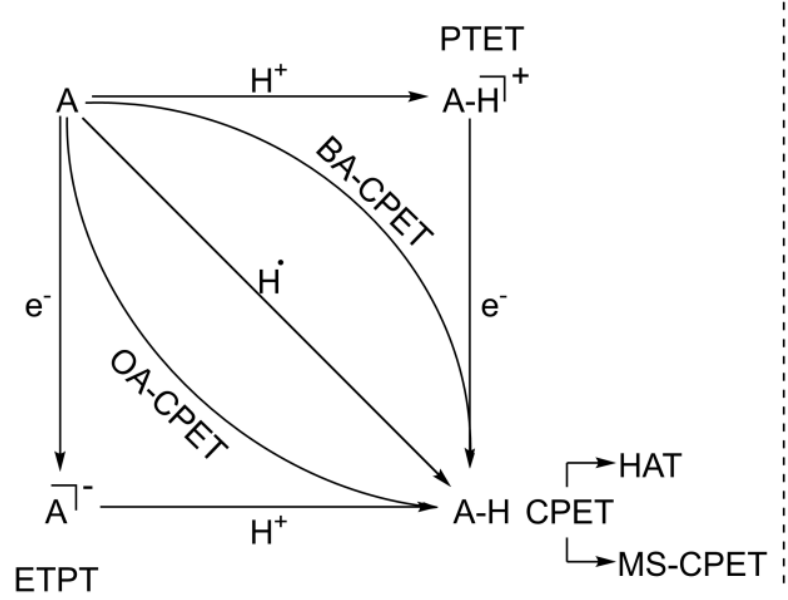

b) Thermodynamic Square-Scheme for a PCET

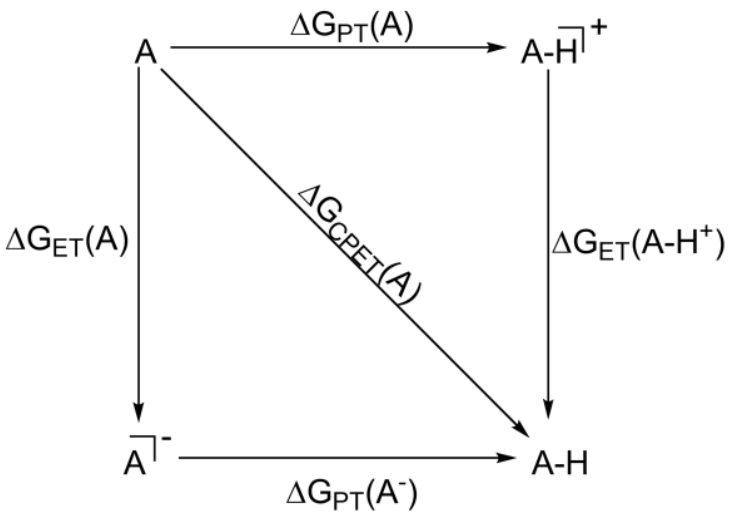

Figure i8: a) Acronyms for single-proton single-electron PCET transformations of an acceptor molecule A. PTET = Proton-transfer electron-transfer; CPET = Concerted proton-electron Transfer; ETPT = Electron-transfer proton-transfer; $\mathrm{BA}-\mathrm{CPET}^{7}=$ Basic asynchronous concerted proton-electron transfer; $\mathrm{OA}-\mathrm{CPET}^{-}$ Oxidative Asynchronous CPET; MS-CPET = Multiple-site CPET; HAT = Hydrogen Atom Transfer. b) Thermodynamic square-scheme for a PCET. $\Delta G_{\mathrm{ET}}=$ Free enthalpy of electron transfer, $\Delta G_{P T}=$ Free enthalpy of proton transfer, $\Delta G_{\text {CPET }}=$ Free enthalpy of concerted proton-electron transfer

The original definition of the PCET term by Meyer et al. is best described as a concerted proton-electron transfer (CPET) as first discussed by Savéant et al ${ }^{[56]}$, while reactions with subsequent proton/electron transfer are referred to as PTET or ETPT processes, respectively. ${ }^{[53]}$ For a CPET processes in which the formal hydrogen atom comes from multiple reaction sites (e.g. proton from the ligand, electron from the metal) the term multi-centre (MS)CPET is commonly used in the literature, while a process in which the hydrogen atom is transferred from one

${ }^{7}$ The abbreviations BA- and OA-CPET were introduced in this thesis for space reasons and are written out in their original publication by Anderson et al. 
reaction site is called hydrogen atom transfer (HAT). ${ }^{[53]}$ Recently the group of Anderson gave experimental proof for another nuance in PCET chemistry, an asynchronously concerted pathway, which is computationally predicted to proceed via lower activation barriers than the fully synchronous transfer (Figure i8, a, bend arrows). Here, the transition state is closer to either an oxidative (OA-CPET) or to a basic (BA-CPET) transfer character. ${ }^{[57,58]}$ The distinction between the different mechanistic models is often ambiguous. This can be visualised on the example of the crucial reaction step of cytochrome P450 (see Section 1.1, Figure i1). Here, the Fe(IV) oxo moiety reacts with an unactivated hydrocarbon in which the proton and the electron both arise from one $\mathrm{C}-\mathrm{H}$ bond rendering this reaction as a HAT, but on the acceptor site the proton binds to the oxygen and the electron quenches the radical cation in the porphyrin unit rendering it as a MS-CPET.

\subsection{PCET Thermochemistry}

\section{"Not radical character but driving force quantifies PCET chemistry"}

\section{Key message of James M. Mayer, 1998 ${ }^{[59]}$}

The (free) enthalpy of a formal hydrogen atom transfer can be split, following Hess' law, into the sum of enthalpies of proton transfer and subsequent electron transfer (Figure i8, b,eq.i1). ${ }^{[60]}$ The $p K_{\mathrm{A}}$ value of a substance is derived as the decadic logarithm of the proton transfer equilibrium constant with a reference base (eq.i2) ${ }^{[61]}$, thus the free enthalpy of proton transfer $\Delta G$ PT is derived for standard conditions via eq.i3. The free energy of electron transfer can be derived analogously from the difference of the redox potentials between two reactants following eq.i4. With these equations at hand, the reaction free enthalpy of a CPET step is obtained from eq.i5.

$$
\begin{aligned}
& \mathrm{A}^{\cdot}+\mathrm{D}-\mathrm{H} \longrightarrow \mathrm{A}-\mathrm{H}+\mathrm{D}^{\cdot} \\
& \Delta G_{\mathrm{CPET}}=\Delta G_{\mathrm{PT}}+\Delta G_{\mathrm{ET}} \quad \text { eq.i1 } \\
& \Delta \mathrm{p} K_{\mathrm{A}}=\log _{10} \Delta K_{\text {eq }} \quad \text { eq.i2 } \\
& \Delta G_{\mathrm{pT}}=\mathrm{RT} \ln K_{\text {eq }}=\mathrm{RT} \ln 10 \Delta \mathrm{p} K_{\mathrm{A}}=5.70 \Delta \mathrm{p} K_{\mathrm{A}} \quad \text { eq.i3 } \\
& \Delta G_{\mathrm{ET}}=\mathrm{ZF} \Delta E^{0}=96.5 \Delta E^{0} \quad \text { eq.i4 } \\
& \Delta G_{\text {CPET }}=5.70 \Delta \mathrm{p} K_{\mathrm{A}}+96.5 \Delta E^{0} \quad \text { eq. } 15 \\
& \mathrm{~A}-\mathrm{H} \longrightarrow \mathrm{A}^{\cdot}+\mathrm{H}^{\cdot} \\
& \operatorname{BDFE}(\mathrm{A}-\mathrm{H})=5.70 \mathrm{p} K_{\mathrm{A}}+96.5 E^{0}-96.5 E^{0}\left(\mathrm{H}^{+} / \mathrm{H}_{2} \text {, solv }\right)+\Delta G_{\text {solv }}^{0}\left(\mathrm{H}^{-}\right)+\Delta G_{\mathrm{f}}^{0}\left(\mathrm{H}^{\cdot}\right) \quad \text { eq.i6 } \\
& \rightarrow \operatorname{BDFE}(\mathrm{A}-\mathrm{H})=5.70 \mathrm{p} K_{\mathrm{A}}+96.5 E^{0}+\mathrm{C}_{\mathrm{G}} \quad \text { eq.i7 } \\
& C_{H}=C_{G}-T\left[S_{f}{ }^{0}\left(H^{\prime}\right)+\Delta S_{\text {solv }}{ }^{0}\left(H^{\cdot}\right)\right] \text {, for } \Delta S_{\text {solv }}{ }^{0}(A-H) \approx \Delta S_{\text {solv }}{ }^{0}\left(A^{\prime}\right) \quad \text { eq.i8 }
\end{aligned}
$$

Figure i9: Thermodynamic equations (in $\mathrm{kJ} \mathrm{mol}^{-1}$ ) based on the square scheme Figure i8,b for a CPET reaction between an acceptor molecule $\mathrm{A}$ and a donor molecule D-H (top) and derivation of the absolute bond dissociation (free) energy equation in analogy to Bordwell and Tilset. [62,63]

Absolute bond dissociation (free) energies $\mathrm{BD}(\mathrm{F}) \mathrm{E}^{\prime} \mathrm{S}$ are commonly used in PCET literature to simplify the comparison between different substrates. To convert the relative reaction free enthalpy of eq.i5 into a BDFE value, the enthalpy of the formally released "free hydrogen atom" must be considered. Here, three terms are important: 
1) The standard reduction potential of the $\mathrm{H}^{*} / \mathrm{H}^{+}$couple in the respective solvent ${ }^{8}$, 2) The free enthalpy of solvation $\Delta G_{\text {solv }}^{0}\left(H^{\prime}\right)$ and 3) The free enthalpy of formation $\Delta G_{f}^{0}\left(H^{\cdot}\right)$ of the hydrogen atom (eq.i6). ${ }^{[63]}$ The enthalpy of solvation for a hydrogen atom is commonly approximated by the solvation enthalpy of argon, while in older literature the solvation enthalpy of $\mathrm{H}_{2}$ was used. ${ }^{[64]}$ The three terms are commonly abbreviated in the literature with the solvent depended term $C_{G}$ yielding Bordwell's equation (eq.i7). ${ }^{[62]}$ Especially in organic literature, the enthalpic term bond dissociation energy ( $\mathrm{BDE}$ ) is commonly used. For the derivation of a $\mathrm{BDE}$ the $C_{\mathrm{G}}$ term has to be replaced by the term $C_{H}$, in which the entropic contribution of the hydrogen atom formation is subtracted from $C_{G}$ (eq.i8), under the assumption that the solvation entropy difference between $\mathrm{A}-\mathrm{H}$ and $\mathrm{A}$ ' is negligible. ${ }^{[65]}$ This assumption holds true for most organic PCET reagents, but cannot generally be applied to transition metal complexes, since a PCET from the latter may lead to electronic rearrangement and thus results in significant changes in the solvation entropy. ${ }^{[66]}$ Here, equilibration techniques and calorimetric measurements, as described in the main part of this thesis, enable the direct measurement of the CPET reaction enthalpy for transition metal complexes.

A long-standing discussion in PCET chemistry was the necessity of radical character for hydrogen atom transfer. Organic HAT transformations generally do involve radicals $(\mathrm{RO} ; \mathrm{Cl})$, while the oxidation of hydrocarbons with chromates or permanganates start from diamagnetic species. ${ }^{[59]}$ Radical intermediates are discussed in the literature to be part of the mechanism of permanganate oxidation reactions. ${ }^{[67,68]}$ First hints, that the radical character is not rendering the HAT reactivity of transition metal compounds gave the analysis of the rhenium(I) oxo [(bis-acetylene)Re(I)O] anion XXXIII by Mayer et al. XXXIII exhibits significant $\mathrm{Re}=\mathrm{O} \pi^{*}$-character and yields upon one-electron oxidation the rhenium(II) oxo XXXIV which indeed shows radical reactivity but not arising from the oxo ligand. Instead of the expected formation of an hydroxo ligand, the metal centre is the reactive site, yielding the dimerization of XXXV to XXXVI or, in low yields, hydrogen atom abstraction from tributyltin hydride, to the rhenium hydride complex XXXVI (Figure i10). ${ }^{[69]}$ This finding lead to the detailed kinetic investigation of the oxidation of hydrocarbons with closed-shell $\mathrm{CrO}_{2} \mathrm{Cl}_{2}$, in which the bimolecular attack of $\mathrm{CrO}_{2} \mathrm{Cl}_{2}$ was found to be the rate determining step. ${ }^{[70,71]}$ Furthermore, a positive linear correlation between the activation enthalpy and the reaction enthalpy is found, supporting the formation of carboradicals over carbocations. Additionally for substrate oxidation with closed-shell $\mathrm{MnO}_{4}{ }^{-}$, a linear free energy relationship between the bond strength and rate constant is found, which leads to the key message: Not radical character but the driving force of the reaction is rendering a proton-coupled electron transfer. ${ }^{[59]}$

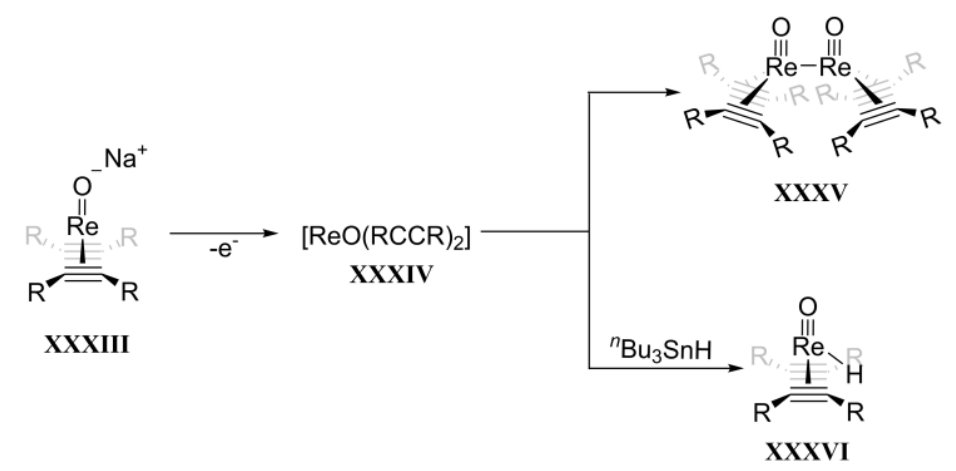

Figure i10: Radical reactivity of rhenium(II) oxo complex XXXV reported by Mayer et al.

8 Potentials referenced vs. the ferrocene/ferrocenium couple in organic media. 
The PCET chemistry of transition and f-block metals is generally of interest, since the weakest found ElementHydrogen bonds are found in this field. One important reagent here is samarium dioide. ${ }^{[72]}$ The oxophilic $\mathrm{Sm}^{2+}$ ion weakens the $\mathrm{O}-\mathrm{H}$ bonds of alcohols and water by up to $280 \mathrm{~kJ} \mathrm{~mol}^{-1}$ through coordination to the oxygen atom and thus enables the hydrogen atom transfer from the $\mathrm{O}-\mathrm{H}$ group to a substrate. ${ }^{[73,74]}$ Also simple transition metal hydrides $\left(\mathrm{BDFE}\left[\mathrm{CpCr}(\mathrm{CO})_{3}-\mathrm{H}\right]=240 \mathrm{~kJ} \mathrm{~mol}^{-1} ; \mathrm{BDFE}\left[\mathrm{CpFe}(\mathrm{CO})_{2} \mathrm{H}\right]=210 \mathrm{~kJ} \mathrm{~mol}^{-1}\right)$ are commonly used in catalysis and can posses BDFE values close to the limit $\left(\operatorname{BDFE}\left(\mathrm{H}_{2, \text { gas }}\right) / 2=203 \mathrm{~kJ} \mathrm{~mol}^{-1}\right)$ of thermodynamic favourable dihydrogen gas formation. ${ }^{[53]}$ Transition metal ion PCET chemistry is long known to be critically pH dependent, which was first rationalized by Pourbaix in 1945. ${ }^{[75], 9}$ Here, the chemistry of transition metal oxo species was of special interest, since these species were important in biochemical (see Section 1.1) and organic oxidation reactions. ${ }^{[76,77]}$ One of the best analysed examples in the field is the aquo-hydroxo-oxo complex series [cis(bpy) ${ }_{2}($ py $\left.) \mathrm{RuOH}_{\mathbf{x}}\right]^{\mathrm{y}, 10} \mathbf{X X X V I I}(\mathbf{x}, \mathbf{y})$ whose ET, PT, PCET, hydride transfer and hydrocarbon oxidation chemistry was investigated in detail (Figure i11, a). ${ }^{[53,78,79]}$

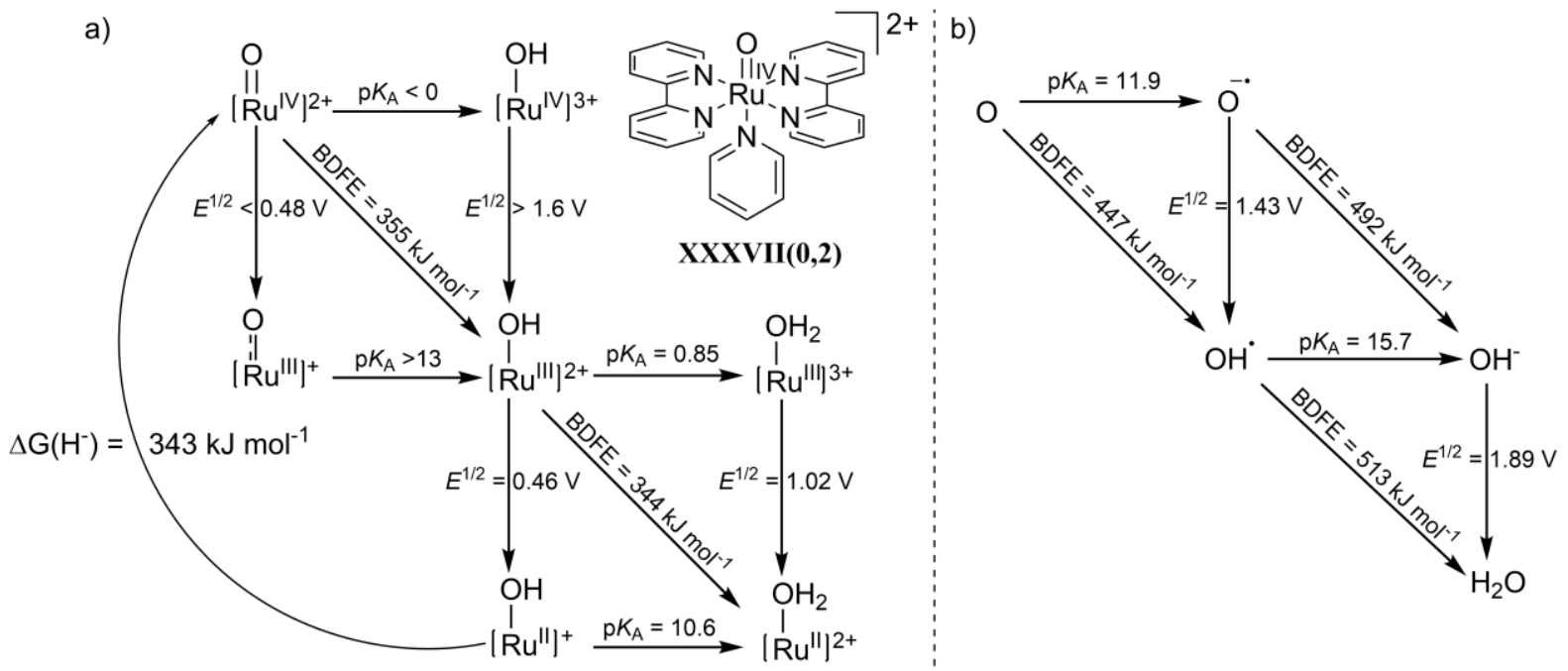

Figure i11: a) Thermodynamic data of the PCET chemistry of XXXVII in aqueous solution. Potentials are given vs. NHE. Values without an equal sign are upper limits. ${ }^{[53,78,79]}$ b) Thermodynamic data of the PCET chemistry of water. Potentials are given vs. NHE. ${ }^{[53,80]}$

The most interesting finding in these studies, expressed in the poorly defined $\mathrm{p} K_{\mathrm{A}}$ and $E^{1 / 2}$ values between XXXVII (0,2) and XXXVII(1,2), is the strong preference of XXXVII(0,2) to undergo a CPET step over a stepwise proton/electron transfer. The O-H BDFE of water strongly decreases by $160 \mathrm{~kJ} \mathrm{~mol}^{-1}$ (Figure i11, b) upon coordination to XXXVII, but still yields high BDFE values of $355 \mathrm{~kJ} \mathrm{~mol}^{-1}$ and $344 \mathrm{~kJ} \mathrm{~mol}^{-1}$ for $\mathbf{X X X V I I ( 0 , 2 )}$ and XXXVII(1,2), respectively. This rationalizes the high hydrocarbon oxidation capability of XXXVII and related systems. ${ }^{[81]}$ This thesis will in the following Chapters focus on the investigation of PCET chemistry of heavy metal complexes.

${ }^{9}$ In the preface of this book it is mentioned that the first version of the atlas is published in the dissertation of M. Pourbaix in 1945.

${ }^{10}$ bpy = bipyridine; py= pyridine 


\section{Computational Thermochemistry}

The main Chapters II and IV include the computational rationalisation of a spin-orbit coupling effect (SOC) on PCET solution thermochemistry. The following Section will therefore give a brief introduction to the performed computational methods and to the treatment of relativistic effects. Since this is no pure theoretical work, this Section is meant as a brief introduction for the interested reader and to find orientation in the performed methods and corresponding literature.

\subsection{Computational Methods}

\section{"I think I can safely say that nobody understands quantum mechanics"}

\section{Richard P. Feynman, $1964^{11}$}

Two main methods are frequently used for the computation of thermochemical data. Density functional theory (DFT) and wavefunction theory (WFT) based ab initio (from first principle) methods with the Hartree-Fock (HF) theory at the fundament. ${ }^{[82]}$ On the one hand, modern DFT methods are indispensable tools for the fast, cost efficient computation of ground- and excited-state structures and many more properties, but on the other hand they lack a systematically improvement towards the "real" state of the analysed system. ${ }^{[82-84], 12}$ The solution of the time-dependent Schrödinger equation (eq.i9) can yield the desired "real" properties of a molecule, but only for non-trivial systems like $\mathrm{H}_{2}{ }^{+}$etc. a strategy for its exact solution is known. ${ }^{[84]}$

$$
\begin{array}{cc}
\widehat{H} \Psi=E \Psi & \text { eq.i9 } \\
\widehat{H}=\widehat{T}+\widehat{V}_{N e}+\widehat{V}_{e e}{ }^{13} & \text { eq.i10 }
\end{array}
$$

A systematic approach towards the exact solution of eq.i9, and therefore towards a better understanding of the errors of the computations, can be achieved by ab initio techniques. ${ }^{[85]}$ The Hartree-Fock method is based on a mean-field approach, in which electron $n$ only experiences the mean electric field of the other $n-1$ electrons of the molecule. This non-interacting electrons approach thus only represents a rough estimate of the electronic situation in the molecule. ${ }^{[86,87]}$ Electrons as charged particles experience coulombic interactions with other electrons in the molecule and their movement must therefore be correlated. This can be systematically treated by post Hartree-Fock methods by introducing the correlation energy $E_{c}$ as the difference between the HF energy $E_{\mathrm{HF}}$ to the (unknown) exact energy $E_{\text {exact }}$ of the system (eq.i11). ${ }^{[87,88]}$ The correlation energy is, due to the variational principle, always a negative quantity and "stabilises" the system.

$$
E_{\mathrm{C}}=E_{\text {exact }}-E_{\mathrm{HF}}
$$

\footnotetext{
${ }^{11}$ Richard P. Feynman on his Messenger Lecture at Cornell University. I am deeply sorry for all the footnotes in this Section.

${ }^{12}$ For time reasons, DFT is not discussed in detail. An excellent book for a chemist's introduction to DFT by Koch and Holthausen can be found in the quotation.

${ }^{13}$ Electronic Hamiltonian $\widehat{T}=$ electron kinetic energy, $\widehat{V}_{N e}=$ potential nucleus/e- energy $\widehat{V}_{e e}=$ potential e-/e- energy.
} 
The correlation treatment is encountered by "opening" the unoccupied orbitals for the electrons through an excitation operator. A straightforward approach for the excitation treatment is called configuration interaction $(\mathrm{Cl})$ in which e.g. electron $i$ from orbital $\Phi_{i}$ is excited into the unoccupied orbital $\Phi_{a}$ for a single excitation, electrons i and j from the orbital $\Phi_{i}$ and $\Phi_{j}$ are excited into unoccupied $\Phi_{a}$ and $\Phi_{b}$ for a double excitation etc. The specific method is then named after the performed excitations (e.g. CISDT $=\mathrm{Cl}$ with single, double and triple excitations). Full configuration interaction $(\mathrm{FCl})$ is reached for $n$-fold excitations and here the only difference to the exact solution of the Schrödinger equation is due to the use of finite basis sets. ${ }^{[87], 14}$

$$
\Psi_{\mathrm{CI}}=\Phi_{0}+\sum_{i, a} C_{i}^{a} \Phi_{i}^{a}+\sum_{\substack{i<j \\ a<b}} C_{i j}^{a b} \Phi_{i j}^{a b}+\ldots
$$

Unfortunately, an $\mathrm{FCl}$ treatment of the correlation problem scales with the number of electrons $n$ of the system with basis functions $N$ to $N^{n}$, thus the computational costs for the description of a $5 \mathrm{~d}$ metal complex system with $n>100$ electrons are elusive. ${ }^{[87]}$ Therefore, the excitations need to be treated in a more cost-efficient manner. The modern "gold standard" of computational chemistry is the coupled-cluster (CC) approach with single, double and perturbative triple excitations $\operatorname{CCSD}(T) .{ }^{[89], 15}$ Here, the excitations are treated within an exponential operator $\widehat{T}$ (eq.i13), which is expanded by a Tailor series (eq.i14). The higher excitation amplitudes are now expressed as products of the single and double excitations (for CCSD), which is drastically reducing the computational costs by maintaining high predictive power. ${ }^{[87]}$

$$
\begin{gathered}
\Psi_{\mathrm{CC}}=\exp (\widehat{T}) \Psi_{\mathrm{HF}} \\
\exp (\widehat{T})=1+\widehat{T}+\frac{1}{2 !} \widehat{T} \hat{T}+\frac{1}{3 !} \hat{T} \hat{T} \widehat{T}+\ldots, \text { with } \widehat{T}=\widehat{T}_{1}+\widehat{T}_{2} \text { for CCSD }
\end{gathered}
$$

For comparison, the computational error of $\operatorname{CCSD}(\mathrm{T})$ for small molecules $\left(\mathrm{BH}, \mathrm{HF}, \mathrm{H}_{2} \mathrm{O}\right)$, compared to a $\mathrm{FCl}$ computation, is $~ 16$ times smaller than for a CISDT computation, while the formal excitation level is the same. ${ }^{[87]}$ Even though the computational demand of the $\operatorname{CCSD}(\mathrm{T})$ method is drastically reduced compared to $\mathrm{Cl}$ methods, it nevertheless scales with $N^{7} .{ }^{[00]}$ Therefore, the computation of medium-sized or larger transition metal complex systems is currently prohibitive, even on modern computer clusters. This issue can be addressed by several methods such as partitioning or localization schemes. ${ }^{[91-96]}$ An "our own n-layered integrated molecular orbital and molecular mechanics" (ONIOM) approach is partitioning the molecule in different "onion like" layers, in which the correlation problem is then treated on the inner layer at the high $\operatorname{CCSD}(T)$ level of theory, while the outer layer(s) are computed with cheaper methods e.g. DFT and Molecular Mechanics (MM) (Figure i12). ${ }^{[96-98], 16} \mathrm{~A}$ possible truncation of the haemoglobin molecule from Section 1.1 could therewith include the iron and its direct coordination environment in the inner layer (Figure i12, green), the porphyrin ring in the medium layer (Figure i12, orange) and the protein chain in the outer layer (Figure i12, red). The ONIOM3 (three layers) energy is then derived via eq.i15. ONIOM methods perform excellently in several benchmarks and are therefore a good method for the computation of accurate energies for metal complex systems. ${ }^{[96]}$

\footnotetext{
${ }^{14}$ The term basis set will be briefly introduced later.

${ }^{15}$ In conjunction with a complete basis set extrapolation

${ }^{16}$ Please note that the outer layers are composed of the inner layer plus additional parts, e.g. the intermediate layer of Figure i12 also includes the iron centre.
} 

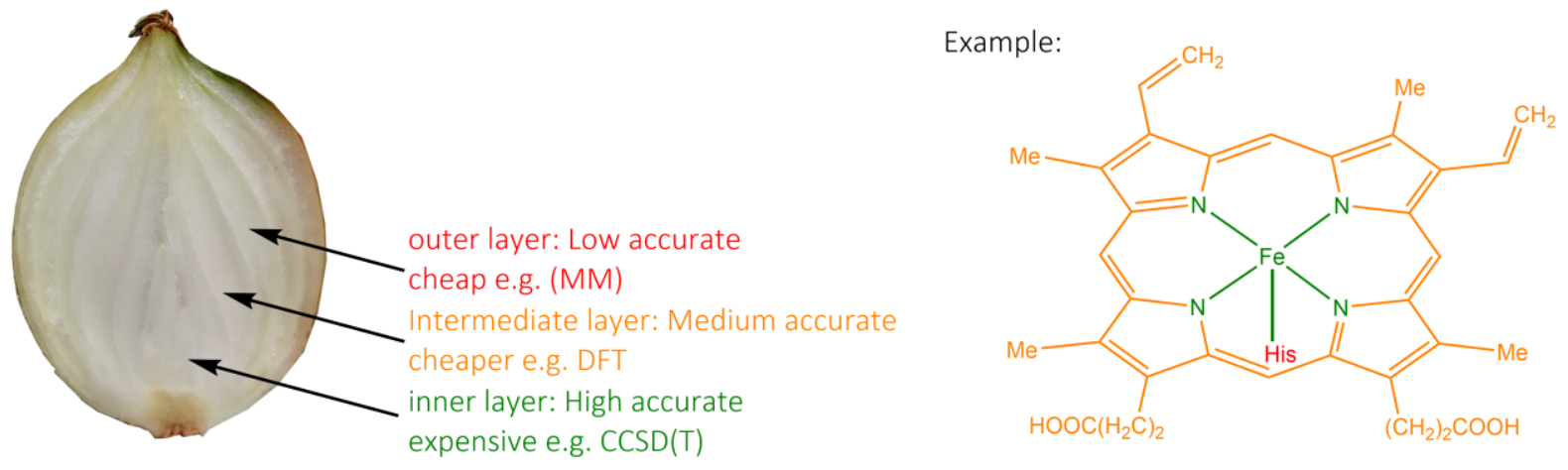

Figure i12: Illustration of the ONIOM approach based on Svenson et al. ${ }^{[98]}$

$$
E^{\text {ONIOM3 }}=E_{\text {Inner layer }}^{\text {High }}+E_{\text {Intermediate Layer }}^{\text {Medium }}-E_{\text {Inner Layer }}^{\text {Medium }}+E_{\text {Outer Layer }}^{\text {Low }}-E_{\text {Intermediate Layer }}^{\text {Low }}
$$

A second method, which does not rely on the truncation of the molecule into different layers but on the localisation of the correlation problem, is the domain-based local pairs of natural orbitals CCSD(T) (DLPNO$\operatorname{CCSD}(T)$ ) approach of the Neese group. ${ }^{[94]}$ Here, pairs of natural orbitals (PNOs) ${ }^{[99,100]}$ are constructed from a set of projected atomic orbitals (PAOs) which are assigned to a given electron pair specific local domain. This enables the localised treatment of the correlation problem or simply speaking: Electron $i$ on one end of the molecule is not correlated with electron $j$ on the opposite edge. The computational costs of the DLPNO method scale almost linearly with the system size, while $99.9 \%$ of the $\operatorname{CCSD}(\mathrm{T})$ correlation energy can be recovered, rendering DLPNO$\operatorname{CCSD}(T)$ as a very cost efficient and precise method for accurate thermochemistry. ${ }^{[95]}$

Besides the dynamical correlation mentioned above, the "static correlation" as a second contribution to the correlation energy must be mentioned. Static correlation describes the situation, in which the electronic ground state cannot be described with a single slater determinant e.g. the ground state of a molecule is not a singlet or a triplet but a combination of both. ${ }^{[87]}$ Multiconfigurational character can already be observed for small molecules of the type $\mathrm{XH}_{2}$. While for water $\left(\mathrm{X}=\mathrm{O}, \sigma=104.5^{\circ}\right)^{[101]}$ the $3 \mathrm{a}_{1}$ orbital lies well below the $1 \mathrm{~b}_{1}$ orbital (one Slater determinant is sufficient) the orbitals are close for methylene $\left(X=C, \sigma=134^{\circ}\right)^{[102]}$ and thus the singlet and triplet state are close in energy. ${ }^{[103,104]}$

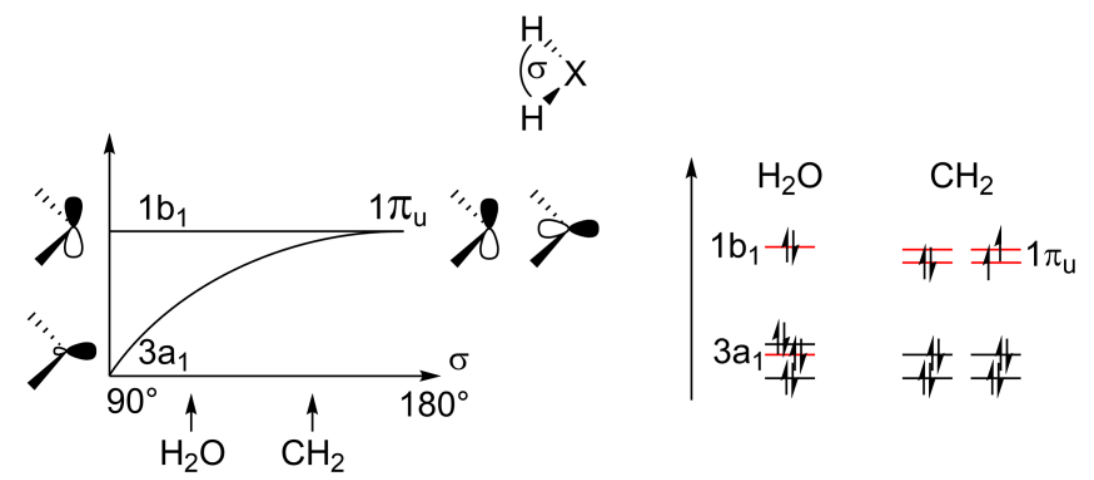

Figure i13: Walsh type diagram of the active orbitals for a $\mathrm{XH}_{2}$ molecule (left ${ }^{[103]}$ and simplified molecular orbital scheme for water and methylene deducted from the Walsh diagram. 1s electrons are omitted for clarity (right)

Multireference character is commonly addressed by a complete active space self consistent field (CASSCF) treatment. Here, the valence orbitals of a molecule are defined as active orbitals in which an $\mathrm{FCl}$ treatment is performed. ${ }^{[105-108]}$ Additionally, the MO coefficients of the active orbitals are optimised for a better description of the electronic,multireference ground state. On the example of Figure i13, the methylene molecule has two 
electrons in the two active orbitals leading to a 2,2 CAS expansion. Several methods are known for the (expensive) combination of static and dynamic correlation and an excellent review on this topic is written by Shepard et al. ${ }^{[109]}$ One particular method applicable to larger molecules is the $n$-electron valence perturbation theory of second order from the Cimiraglia group (NEVPT2). ${ }^{[10-112]}$ NEVPT2 has two main advantages compared to the also common complete active space perturbation theory of second order (CASPT2). ${ }^{[113,114]}$ NEVPT2 exhibits exact size consistency ${ }^{[115]}$ and lacks of intruder states. ${ }^{[116], 17}$

The predictive power of any computation is always determined by the methods level of theory and equally important by the applied basis set. An excellent book Chapter by Jensen et al. is written on this topic. ${ }^{[117]}$ A given basis set is a set of functions for the atomic orbitals $(\mathrm{AO})$ of the atoms in the molecule, which are then yielding the molecular orbitals (MO) in the computation as linear combinations (LCAO approach). ${ }^{18}$ The simplest basis set of single-zeta (SZ) type only has functions for the occupied AOs (e.g. s- and p-type functions for Li-Ar) and thus has no flexibility to describe bonding interactions. A double-zeta (DZ) type basis adds functions with one higher angular momentum than the occupied AOs (e.g. d-orbitals for p-block elements) etc. Additionally, polarisation and augmentation functions can be added to a given basis to further enhance its flexibility for chemical bonding. ${ }^{[117]}$ While general chemical trends can already be described on low level basis sets (e.g. double zeta), accurate thermochemistry needs computations close to the complete basis set (CBS) limit. The Karlsruhe basis sets denoted e.g. def2-TZVP (second generation-triple zeta valence + polarisation) have been shown to be close to the DFT CBS limit with rational computational costs and are thus frequently used in the literature. ${ }^{[118]}$ For ab initio methods the correlation consistent Dunning basis sets (cc-VnZ, correlation consistent-n-zeta valence) are of particular interest, since they are designed in "shells"19 in which each shell captures a similar amount of correlation energy, thus enabling an accurate extrapolation to the CBS. ${ }^{[119,120]}$ The extrapolations are however only most accurate when large basis sets $(n=Q, 5)$ are used. ${ }^{[121]}$ Here, explicitly correlated F12 extensions to the Dunning basis sets perform excellently and converge faster and closer to the CBS even when a double zeta basis is used. They rely on R12 methods (interelectronic distance) with non-linear terms and density fitting (RI) for faster convergence and are used in conjunction with explicitly correlated F12 coupled cluster expansions. ${ }^{[122-127]}$

For this reason, this thesis will feature the ONIOM approach mentioned above, including CCSD(T)-F12/VTZ computations, and the DLPNO-CCSD(T) method for the accurate computation of $5 \mathrm{~d}$ metal complex systems.

\footnotetext{
17 The cited papers show that CASPT2 is not generally fulfilling these criteria. For NEVPT2 the size consistency and the lack of intruder states are shown in the original publications.

18 Plane wave basis sets for surface chemistry are not discussed.

${ }^{19} \mathrm{~A}$ shell refers to the cardinal number of the basis set e.g. First shell no correlation (HF), $c c-V D Z=S e c o n d$ shell,$c c-V T Z=$ third shell etc.
} 


\title{
3.2. Relativistic Effects on Thermochemistry
}

\section{"I know that Einsteins theory of special relativity is correct because every weekend goes by twice as normal"}

\begin{abstract}
Anonymous
The special theory of relativity was introduced by Albert Einstein in his 1905 publication "Zur Elektrodynamik bewegter Körper" and is based on one fundamental assumption: Light is moving at a constant, universal speed in vacuum. ${ }^{[128]}$ Many important quantities can be deduced from this elemental assumption, but for chemists the most important consequence is the acceleration of the inner electrons of heavy elements, which leads to the relativistic contraction and stabilisation of the $s$ - and p-electrons, while the $d$ - and f-electrons are destabilised. ${ }^{[129]}$ Several basic phenomena are attributed to this scalar relativistic effect such as the yellow colour of gold, the "inertpair" effect in main group chemistry or the tendency for higher oxidation states of $5 \mathrm{~d}$ metals compared to their $4 d$ analogues. ${ }^{[130]}$ For valence electrons the relativistic effect scales with $\sim Z^{2}{ }^{[131]}$ A second important relativistic effect, which also scales with $\sim Z^{2}{ }^{[132]}$ is the coupling of the spin- and angular momentum of the electron, termed spin-orbit coupling (SOC). Even though SOC also scales with $\sim Z^{2}$ for valence orbitals, it already has a non negligible effects on the $\mathrm{F}+\mathrm{H}_{2}$ reaction cross section. ${ }^{[133]}$ Furthermore, the enhanced reactivity of $5 \mathrm{~d}$ metals in gas phase methane activation, compared to their $4 \mathrm{~d}$ analogous, can be explained by high relativistic stabilisation and significant SOC contributions on the formed carbene complexes. ${ }^{[134]}$ The scalar relativistic effects are routinely incorporated in general quantum chemistry, while SOC effects are mostly neglected, since they are assumed to be "quenched by the ligand field". ${ }^{135-139]}$ Contrary theoreto Kývala and Rulišek reported in 2008 on the computational prediction of $\mathrm{M}^{3+/ 2+}(\mathrm{M}=\mathrm{Ru}, \mathrm{Os})$ reduction potentials of twelve different complexes by multireference methods. Here, the inclusion of SOC was indispensable for the agreement with the experiment, resulting in an average potential shift of $-70 \mathrm{mV}$ and $-300 \mathrm{mV}$ for ruthenium and osmium, respectively. ${ }^{[140]} \mathrm{A}$ vivid review on relativistic effects in chemistry is given by Pyykkö. ${ }^{[141]}$

Relativistic effects are included in the time-dependent Schrödinger equation. An approach for its solution is the Dirac-Fock (DF) method, which uses a four-component Hamiltonian with time as a variable. For the majority of molecules with $>2$ atoms this approach is elusive, since the DF method has high computational costs and also suffers from negative energy solutions. ${ }^{[142]}$ Additionally, dynamic correlation is not included in the classic DF method resulting in a good description of relativistic effects on cost of the description of correlation effects. For this reason, several empirical approaches have been developed to add relativity to non-relativistic methods. For heavy metals the electrons are often divided into valence and core electrons (for correlated methods) in which the latter are then described with a scalar-relativistic electron core potentials (ECP), but also all-electron scalar relativistic basis sets (SARC) are known. ${ }^{[143,144]}$ ECPs are derived from a relativistic all electron treatment of the corresponding atom and have shown to sufficiently describe the electronic situation of heavy metals. Additionally, they remove a significant number of electrons from the explicit treatment in the computation and are therefore crucial for the computation of big to medium sized $5 \mathrm{~d}$ metal complex systems, e.g. the relativistic "Stuttgart" ECP on iridium ${ }^{[145]}$ removes 60 electrons from the explicit treatment. A comprehensive book Chapter on this topic is written by Dolg. ${ }^{[146]}$ One approach to reduce the four-component Hamiltonian of the time-dependent Schrödinger
\end{abstract}


equation to a two-component Hamiltonian is called zeroth order regular approximation (ZORA). ${ }^{[142,147-152], 20}$ ZORA is e.g. the recommended method of choice for relativistic DFT computations in the ADF program packages (also including a spin-orbit coupling ZORA method) and is as well implemented in the ORCA program package. ${ }^{[153,154]}$ SOC can also be introduced to a non-relativistic, correlated computation by applying a spin-orbit mean field (SOMF) operator, which is reproducing the full relativistic ${ }^{21} \mathrm{SOC}$ stabilisations of e.g. $\mathrm{Pd}, \mathrm{PdCl}$ and $\mathrm{Pd}_{2}^{+}$within a few wavenumbers. ${ }^{[155]}$ Further development of this methodology led to the inclusion of the SOMF operator into CASSCF. ${ }^{[156]}$ In the following chapters the SOC effect on $5 \mathrm{~d}$ metal pincer PCET chemistry will be analysed by CASSCFNEVPT2-QDPT computations, where QDPT is the quasi-degenerate perturbation theory. By QDPT a SOMF is applied on the CASSCF-NEVPT2 wavefunction for the explicit treatment of SOC.

${ }^{20}$ Two-component: Neglecting the positronic solutions and thus also the problems of the negative solutions. A book chapter by M. Barysz is given in the quotation. ${ }^{21}$ Full Breit-Pauli Operator 


\section{Outline}

\subsection{Outline of Chapter II}

The synthesis and characterisation of late transition metal oxo complexes is, as discussed in Chapter 1 , an active field of chemistry, with only two reported examples in group 9. ${ }^{[33,36]}$ Here, open-shell systems are of special interest, since they are frequently suggested as key intermediates in oxygenation chemistry and in related nitrene transfer catalysis. ${ }^{[39,40]}$ The group of Schneider recently presented the successful isolation of a terminal iridium imido complex series $\left[(P N P) \mid r N^{t} B u\right]^{n+}(n=0,1,2)$ XXII-XXIV featuring the first iridium complex (XXII) with a triplet ground state. ${ }^{[37]}$ This is rendering the PNP pincer platform as an ideal ligand for the stabilisation of an isoelectronic terminal iridium oxo complex. Additionally, the open-shell ground state of XXII hints towards an open-shell character of the desired oxo species (Figure i14).

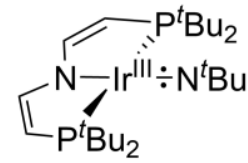

XXII

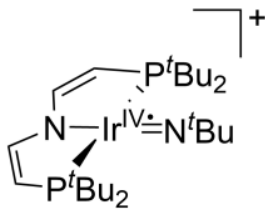

XXIII

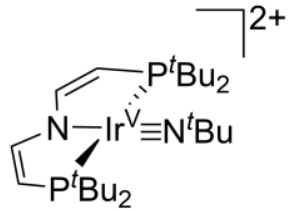

XXIV
$S=1$
$S=1 / 2$
$S=0$

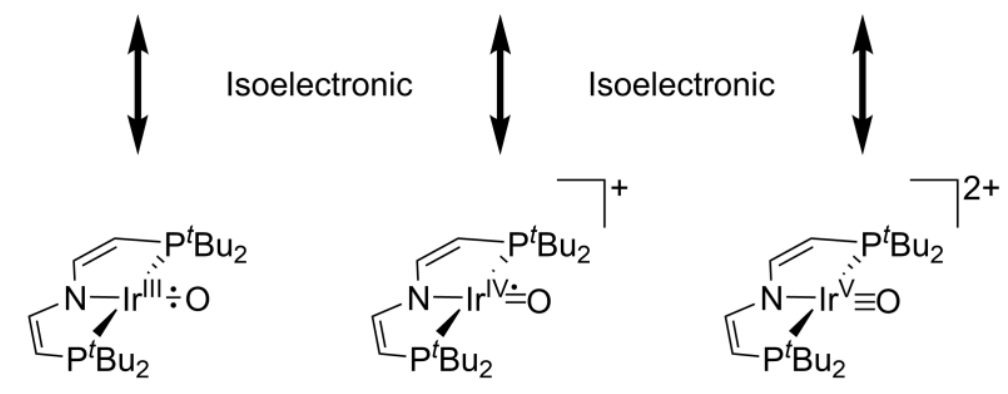

Figure i14: Potential isoelectronic terminal iridium oxo complexes to the terminal iridium imido complexes reported by Schneider et al.

In Section 1 of the upcoming Chapter, the synthesis and characterisation of an iridium(I,II,II) hydroxo complex redox series will be covered, including improved synthetic routes compared to the published ones in the Ph.D. thesis of Dr. Markus van Alten born Kinauer. In Section 2 the synthetic pathways towards the terminal iridium(III) oxo complex 4 and its full spectroscopic and magnetic characterisation will be discussed, including rationalisation by high level ab initio computations of the electronic structure of 4 and on the PCET thermochemistry of the $\mathrm{O}-\mathrm{H}$ bond cleavage from 2 to 4 . The reactivity of 4 will be analysed in Section 3, including the determination of its philicity by reaction with selected small molecules, the derivation of the O-H bond strength (BD(F)E) of 2 by isothermal titration calorimetry and the comparison to the theoretically predicted value. The Section ends with the $\mathrm{C}-\mathrm{H}$ bond oxygenation reactivity of $\mathbf{4}$, including thermal reactivity and reactivity under irradiation. At the end a summary of the Chapter can be found in Section 4. 


\subsection{Outline of Chapter III}

In the excurses Chapter III, results are discussed which arose from collaborations within our group with Dr. Josh Abbenseth (Section 1) and with the external collaboration partners from the Yale University Prof. James Mayer and Prof Patrick Holland and Gannon Connor as their Ph.D. student (Section 2).

Excursus 1: Interconversion of Phosphinyl Radical and Phosphinidene Complexes by Proton Coupled Electron Transfer discusses the calorimetric P-H BDE determination of a [PNP)Os(H)(PHMes*)] phosphinyl radical complex 17 which forms the phosphinidene [(PNP)Os(H)(PMes*)] complex 19 upon PCET. Additionally, the derivation of the $\mathrm{p} K_{\mathrm{A}}$ value of the cationic phosphide complex [(PNP)Os(H)(PHMes*)] ${ }^{+} 18$ will be reported(Figure i15).

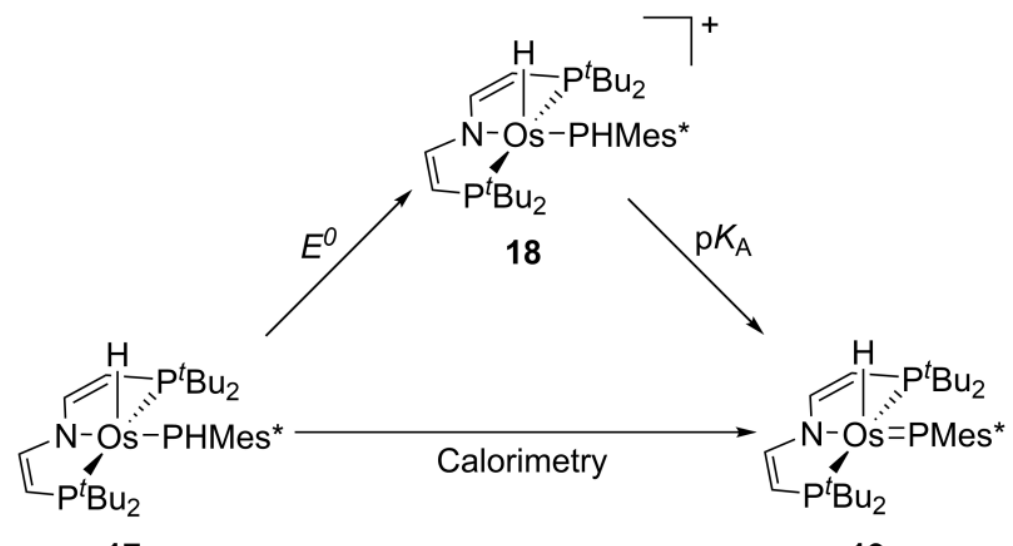

17

19

Figure i15: Thermodynamically examined PCET chemistry of the osmium phosphanyl radical/phosphinidene complex couple.

Excursus 2: is about the thermodynamic analysis of a rhenium(III) amide $\left[(\mathrm{PNP}) \operatorname{Re}(\mathrm{Cl})\left(\mathrm{NH}_{2}\right)\right]$, rhenium(V) nitride $[(\mathrm{PNP}) \operatorname{Re}(\mathrm{Cl})(\mathrm{N})]$, complex couple $20 / 22$ relevant for ammonia oxidation. Here, the $\mathrm{N}-\mathrm{H}$ BDE of the double PCET step is determined by isothermal titration calorimetry (Figure i16).

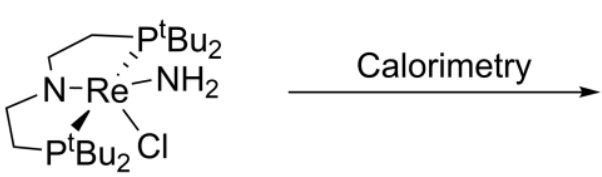

20

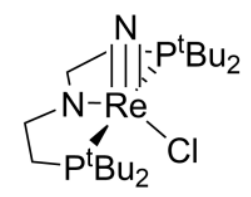

22

Figure i16: Investigation of the mean BDE of the double PCET step from 13 to 12 by isothermal titration calorimetry. 


\subsection{Outline of Chapter IV}

In Chapter II, the O-H bond of the terminal iridium(II) hydroxo/iridium(III) oxo couple 2/4 was found to be stabilised by $2.9 \mathrm{~kJ} \mathrm{~mol}^{-1}$ as a reason of spin-orbit coupling (SOC). ${ }^{[157]}$ The small SOC effect on the O-H BDE was attributed to the negligible spin-change at the heavy metal centre upon PCET, resulting in similar SOC stabilisations for 2 and $\mathbf{4}$, respectively. In Chapter IV the SOC effect on the PCET chemistry at heavy metal sites will be further analysed. Here, the rhenium(III) amine complex $\left[\left(\mathrm{P}^{\mathrm{H}} \mathrm{NP}\right) \operatorname{Re}(\mathrm{III}) \mathrm{Cl}_{3}\right] 23$, which has been shown to be a good a starting platform for the incorporation of $\mathrm{N}_{2}$ into benzonitrile, is chosen as a platform. 23 exhibits an extraordinary shift of its ${ }^{31} \mathrm{P}$ NMR signal to $\delta_{31 P}=-1526 \mathrm{ppm}$, which was assigned to high temperature independent paramagnetism (TIP) as a result of strong SOC. This finding is rendering this platform as an ideal candidate for the investigation of a high SOC effect on its PCET thermochemistry, especially since the corresponding product of HAT the rhenium(IV) amide [(PNP)Re(IV)Cl 3 ] 24 is as well accessible. ${ }^{[158]}$ In chapter IV the PCET process from 23 to 24 will be thermochemically analysed via direct calorimetric measurement or via oxidation to the rhenium(IV) amine complex $\left[\left(\mathrm{P}^{\mathrm{H}} \mathrm{NP}\right) \mathrm{Re}(\mathrm{IV}) \mathrm{Cl}_{3}\right]^{+}$ 25 and subsequent deprotonation (Figure i17). On the computational site, the reaction thermochemistry will be computed "spin-free" via the ONIOM and DLPNO method (see Chapter I Section 3.1), while SOC is introduced by CASSCF-NEVPT2-QDPT computations (see Chapter I Section 3.2).

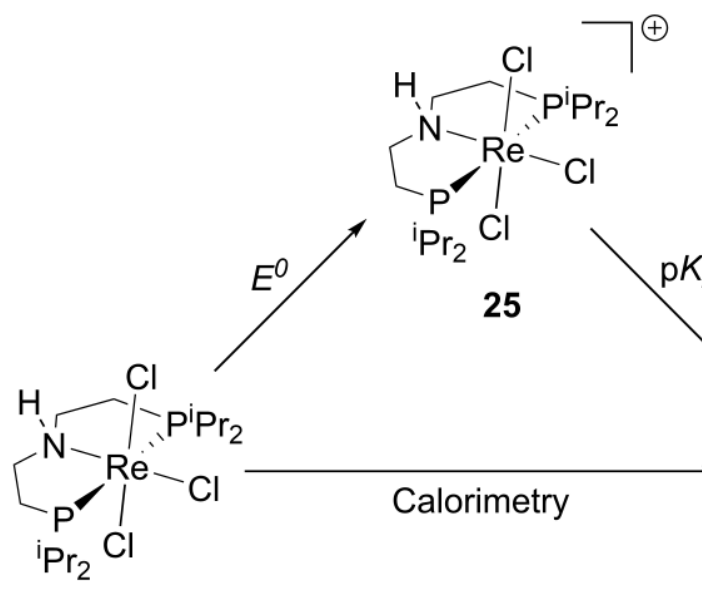

23

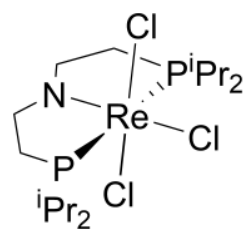

24

Figure i17: Examination scheme for the N-H BD(F)E of 15 by direct HAT (calorimetry) or oxidation and subsequent deprotonation (square scheme).

In Section 1, the structural, spectroscopic and magnetic properties of the rhenium(III) amine 23 (Section 1.1) and rhenium(IV) amide complex 24 (Section 1.2) are discussed, extended by the characterisation of the cationic rhenium(IV) amine complex $25\left[(\mathrm{PHNP}) \operatorname{Re}(\mathrm{IV})(\mathrm{Cl})_{3}\right]\left[\mathrm{BArF}_{24}\right]$ (Section 1.3).

In Section 2, the ab initio computational rationalization of the spectroscopic and magnetic features of the complexes 23 (Section 2.1), 24 (Section 2.2) and 24 (Section 2.3) are presented by means of complete active space self consistent field (CASSCF) computations extend by n-electron valence perturbation theory of second order (NEVPT2) followed by quasi-degenerate perturbation theory (QDPT) to include spin-orbit coupling effects. In Section 3, the (free) reaction enthalpy of the PCET reaction is measured experimentally (Section 3.1) and compared to the predicted value by the $a b$ initio techniques DLPNO and ONIOM(CCSD(T)-f12/DFT) (Section 3.2). At the end in Section 4, a summary of the Chapter can be found. 


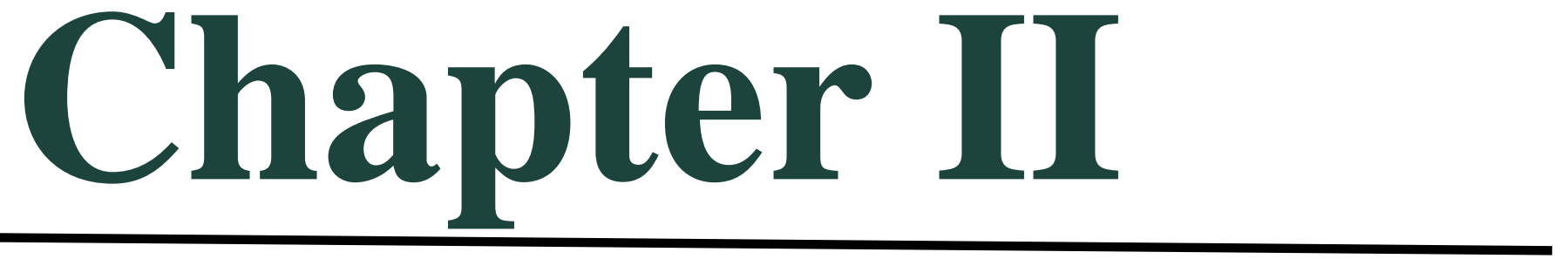

II. A Terminal Iridium Oxo Complex with a Triplet Ground State 


\section{Synthesis of Terminal Iridium Hydroxo Complexes}

Parts of this Chapter have been published in: D. Delony, M. Kinauer, M. Diefenbach, S. Demeshko, C. Würtele, M. C. Holthausen, S. Schneider, Angew. Chem. Int. Ed. 2019 58, 10971-10974. "A Terminal Iridium Oxo Complex with a Triplet Ground State. The initial synthesis of the neutral Ir(II) hydroxo complex 2 as well as the cationic Ir(III) hydroxo complex 3 and their analysation, as well as first attempts towards the iridium oxo complex were performed by Dr. Markus Van Alten born Van Alten born Kinauer and will be briefly discussed here.

Towards the synthesis of a terminal oxo complex of iridium a suitable precursor platform must be found. Possible pathways would be (1) Oxygen Atom Transfer (OAT) with reagents like pyridine-N-oxide or trimethylamine- $\mathrm{N}$-oxide with a low valent iridium platform or (2) Hydrogen Atom Transfer (HAT) from terminal hydroxo complexes. Both pathways are literature known. The only known terminal oxo complex of iridium by Wilkinson et al. ${ }^{[36]}$ (see Chapter I in Section 1.2) was synthesized by treatment of $\operatorname{Ir}(\mathrm{Mes})_{3}$ with trimethylamine-N-oxide, while for the isoelectronic terminal imido complexes, the HAT route was utilized for the synthesis of $[(P N P) \mid r N R]^{n+}\left(R={ }^{t} B u, P h\right.$, $\mathrm{n}=0,1,2) \mathbf{X X I I - X X I V} .^{[37,159]}$ Due to the structural similarity of the latter to the pincer platform utilized in this thesis, the HAT route is followed here. Therefore, the synthesis of terminal hydroxo complexes of the type (PNP)Ir$\mathrm{OH}$ is required as a starting platform towards the synthesis of a terminal oxo complex. Of course, HAT is not the only possible route to transform a terminal hydroxo complex into a terminal oxo complex. Depending on the metal oxidation state, also hydride transfer and deprotonation should be considered (Scheme 1).

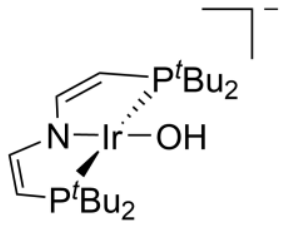

1

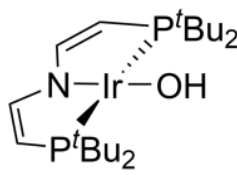

2

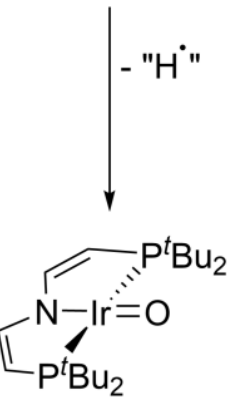

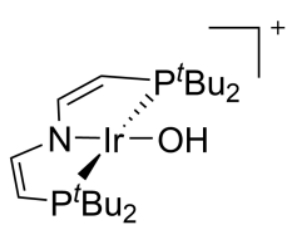

3

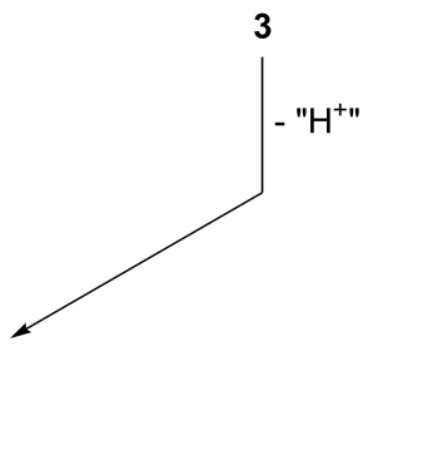

4

Scheme 1: Possible Pathways from terminal iridium hydroxo platforms bearing a PNP pincer ligand towards a terminal oxo. Hydride Transfer (left), Hydrogen Atom Transfer (HAT, middle) and deprotonation (right).

As a starting platform for this project, the well established square planar iridium chloro pincer complex [(PNP)Ir(Cl)] 5 was chosen, ${ }^{[160]}$ which has proven to be a versatile precursor for formally isoelectronic complexes to a terminal oxo, like terminal nitrides and imido complexes. ${ }^{[37,41]}$ Salt metathesis with $\mathrm{NaOH}$ in a mixture of THF and water (7:1) is yielding the desired $\operatorname{Ir}(I I)$ hydroxo complex $2[(\mathrm{PNP}) \operatorname{Ir}(\mathrm{OH})]$ in very good yields (>90\%) (Figure 1a). Only a careful exclusion of $\mathrm{O}_{2}$ in the synthesis leads to high yields, due to the inherently higher instability of 2 towards dioxygen compared to 5 . 
a)<smiles>Cl[Pb]1N2C=CN([Pb])[Pb]1C=C2</smiles>

5

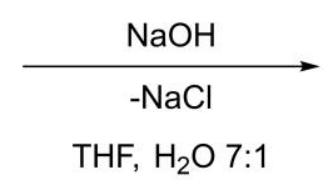

RT, 16 h

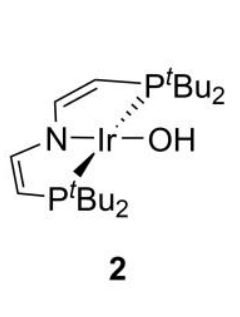

b)

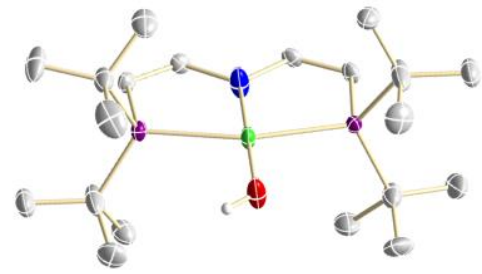

c)
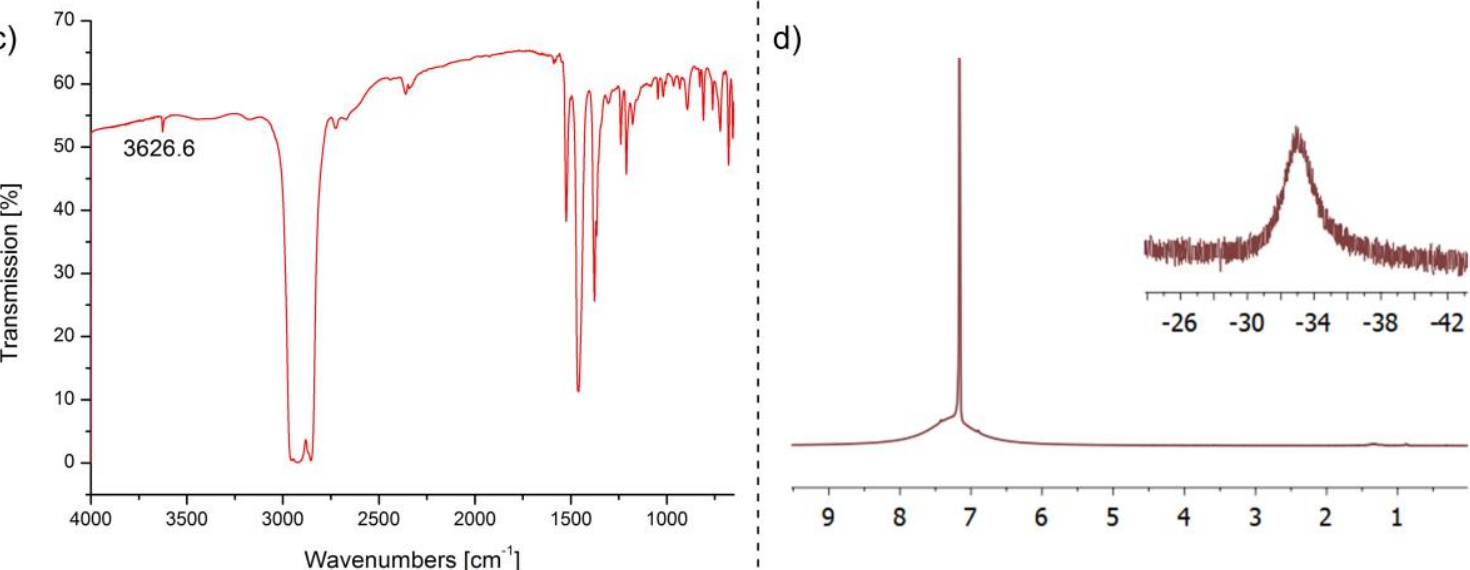

Figure 1: a) Improved synthetic route from 5 to 2 by salt metathesis with $\mathrm{NaOH}$. b) Molecular structure in the solid state of 2 obtained by $\mathrm{X}$-ray single crystal diffraction. The ORTEP plot shown with anisotropic displacement parameters at $50 \%$. All C-H hydrogen atoms as well as the disorder of the molecule are omitted

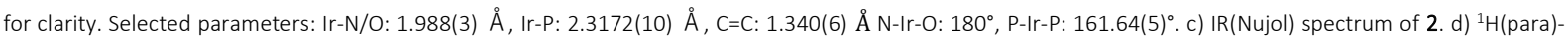
NMR spectrum of 2 in benzene at $25^{\circ} \mathrm{C}$.

Structural confirmation for 2 was obtained by single crystal X-ray diffraction, which shows the expected square planar coordination mode (angle sum around $\left.\mathrm{Ir}=360^{\circ}\right) .{ }^{22}$ The molecule is heavily disordered in the solid state with the oxygen and nitrogen atoms on opposite positions, thus the distinction between the Ir-N and Ir-O bond length is impossible $\left(d_{1 \mathrm{r}-\mathrm{N} / \mathrm{O}}=1.988 \AA\right.$ ) (Figure $\left.1 \mathrm{~b}\right)$. The $\mathrm{O}-\mathrm{H}$ stretch in the infrared spectrum of 2 appears as a weak transmission at $v_{\mathrm{O}-\mathrm{H}}=3627 \mathrm{~cm}^{-1}$ (Figure 1c) in line with other literature known square planar iridium hydroxo complexes like the complexes reported by Werner et al. ${ }^{[161,162]}\left[\operatorname{trans}-\mathrm{Ir}(\mathrm{OH})\left(\mathrm{C}_{2} \mathrm{H}_{4}\right)\left(\mathrm{P}^{i} \mathrm{Pr}_{3}\right)_{2}\right]\left(v_{\mathrm{O}-\mathrm{H}}=3652 \mathrm{~cm}^{-1}\right)$ and [trans- $\operatorname{Ir}(\mathrm{OH})(=\mathrm{C}=\mathrm{C}=\mathrm{CPh} 2)\left(\mathrm{P}^{i} \mathrm{Pr}_{3}\right)_{2}\left(V_{\mathrm{O}-\mathrm{H}}=3643 \mathrm{~cm}^{-1}\right)$ or the pincer complexes by Parvez et al. ${ }^{[163]}\left[\mathrm{PCP}^{R}\right) \mathrm{IrOH}^{23}$ $\left(v^{R=t B u_{\mathrm{O}-\mathrm{H}}}=3642 \mathrm{~cm}^{-1}, v^{R=i P r} \mathrm{O}-\mathrm{H}==3625 \mathrm{~cm}^{-1}\right)$ and Burger et al. ${ }^{[164]}[(\mathrm{PDI}) \mid \mathrm{rOH}]^{24}\left(v_{\mathrm{O}-\mathrm{H}}=3561 \mathrm{~cm}^{-1}\right)$. The hydroxO complex 2 has a paramagnetic ground state with broadened signals ( $\delta=7.2 \mathrm{ppm}$ and $\delta=-33 \mathrm{ppm})$ in the ${ }^{1} \mathrm{H}$ (para)-NMR spectrum (Figure $1 \mathrm{~d}$ ). It is noteworthy that 2 is therefore representing the only literature reported paramagnetic iridium hydroxo complex. The electrochemistry of 2 was investigated by cyclic voltammetry (CV) to analyse the accessibility of the anionic and cationic hydroxo complexes 1 and 3 . On first glance, both redox events $\left(E^{0}(\operatorname{Red})=-2.12 \mathrm{~V} ; E^{0}(\mathrm{Ox})=-0.37 \mathrm{~V}\right)$ seem to be reversible, which indicates that hydroxo complex 1 and 3 are both stable and isolatable (Figure 2). A more detailed analysis by the Randles-Sevcik equation (see Chapter III, Section 1.2.1) gives a reversible electrochemical process with fast electron transfer to freely diffusing ions (Figure 2a), but the analysation of the scan rate depended peak ratio (forward vs. backward peak) reveals for the

\footnotetext{
${ }^{22}$ Crystal grown by Dr. Markus van Alten, born Kinauer, crystal structure solved by Dr. Christian Würtele.

${ }^{23} \mathrm{PCP}=\operatorname{Bis}(2$-diisopropylphosphaneyl)phenyl)carbene

${ }^{24} \mathrm{PDI}=$ Pyridine-diimine
} 
reduction, a moderate decrease of the peak ratio at higher scan rates, indicating a quasi-reversible process with a slowly equilibrating chemical reaction after the electrochemical step (EC mechanism) (Figure 2c, left).

a)

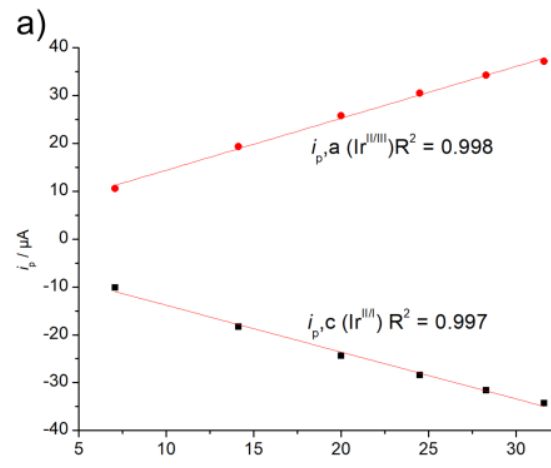

c)

\begin{tabular}{|c|c|c|}
\hline$v / \mathrm{mV} \mathrm{s}^{-1}$ & $\begin{array}{c}\text { Reduction } \\
\qquad i_{\mathrm{p}}, \mathrm{c} / i_{\mathrm{p}}, \mathrm{a}\end{array}$ & $\begin{array}{l}\text { Oxidation } \\
\qquad i_{\mathrm{p}, \mathrm{a} / i_{\mathrm{p}}, \mathrm{c}}\end{array}$ \\
\hline 50 & 0.96 & 1.0 \\
\hline 200 & 0.83 & 1.1 \\
\hline 400 & 0.80 & 1.1 \\
\hline 600 & 0.80 & 1.1 \\
\hline 800 & 0.77 & 1.1 \\
\hline 1000 & 0.78 & 1.1 \\
\hline
\end{tabular}

b)

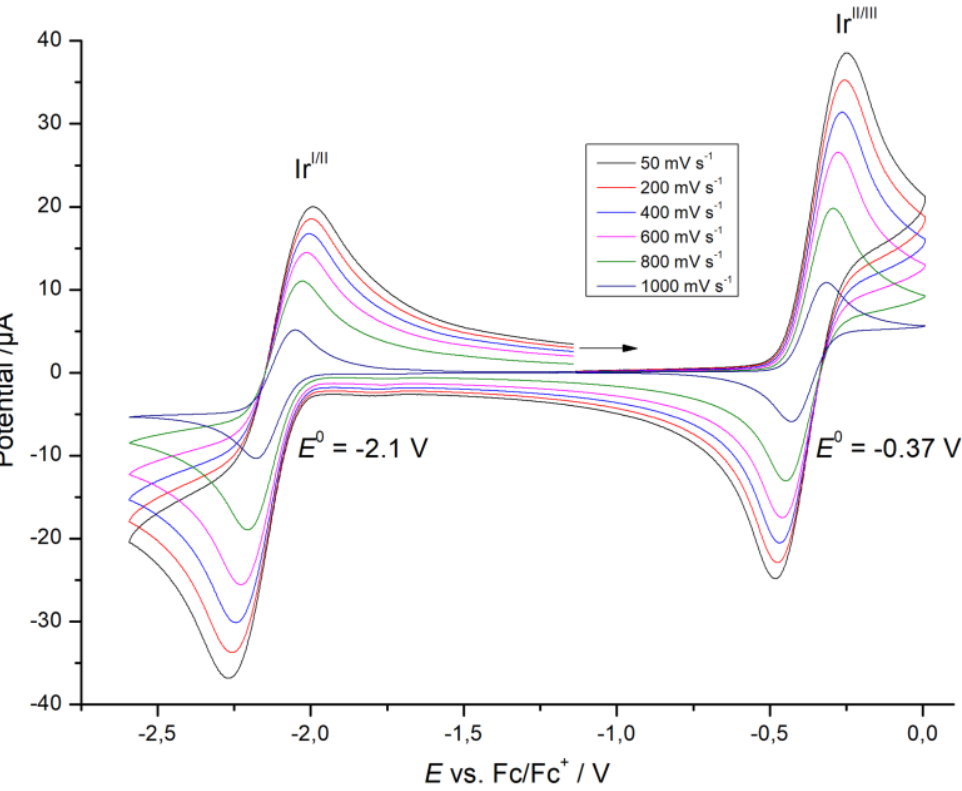

Figure 2: a) Linear regression of $i_{p}$ vs. $v^{1 / 2}\left(i_{p}, c\right.$ for the reduction iridium (II/I) couple and $i_{p}$, a for the iridium (II/III) couple) for the oxidation. b) Cyclic Voltammogram of $2(1 \mathrm{mM})$ at different scan rates in THF with $\mathrm{NBu}_{4} \mathrm{PF}_{6}(0.1 \mathrm{M})$ as the conducting salt. Shown is the first cycle. c) Scan rate dependent peak ratio of the forward and backward peak for the reduction as well as for the oxidation.

A possible explanation for the chemical irreversibility is the dimerization of the formed iridium(I) hydroxo species which is a literature known process. ${ }^{[161,165]}$ On the oxidative site the $i_{p}$ ratio is, at any scan rate close to unity and supports a reversible process. Indeed the oxidation of 2 with $\mathrm{AgPF}_{6}$ as the oxidizing agent in DCM yields selectively (isolated yield: 92\%) the desired cationic hydroxo species 3 [(PNP)Ir(OH)][PF6] (Figure 3a and d). The cationic hydroxo species could be confirmed by XRD analysis (Figure 3b). ${ }^{25}$ Again, the angle sum around iridium sums up to $360^{\circ}$, but no disorder of the molecule in the $\mathrm{N}-\mathrm{Ir}$-O plane is observed as for 2 . The Ir-O bond length is significantly shortened by $0.05 \AA$ to $d_{1 \mathrm{r}-\mathrm{o}}=1.935 \AA$, which indicates an increase in bond strength and represents the by far shortest Ir-OH bond reported in literature. ${ }^{26,[166-174]}$ Also, the $\mathrm{N}-\mathrm{Ir}-\mathrm{O}$ angle is slightly deviating from $180^{\circ}$ ( $\mathrm{N}-\mathrm{Ir}-\mathrm{O}=$ $177^{\circ}$ ) and hints towards a minor amount of $\pi$-backdonation from the pincer nitrogen, however it could as well arise from packing effects in the crystal. IR spectroscopy yields a decrease of the $\mathrm{O}-\mathrm{H}$ stretching frequency ( $\nu_{0}-\mathrm{H}=3577 \mathrm{~cm}^{-1}$ ) by 50 wavenumbers compared to 2 , which supports a higher degree of activation of the $\mathrm{O}-\mathrm{H}$ bond of 3 (Figure 3c). 3 exhibits a $C_{2 v}$ symmetric signal set with four signals in the ${ }^{1} \mathrm{H}-\mathrm{NMR}$ spectrum $(\delta=14.6$ $\mathrm{ppm}, \delta=7.1 \mathrm{ppm}, \delta=5.2 \mathrm{ppm}$ and $\delta=1.8 \mathrm{ppm})^{\text {and }}$ one signal for the pincer phosphorous atoms in the ${ }^{31} \mathrm{P}-\mathrm{NMR}$ spectrum ( $\delta=41.3 \mathrm{ppm}$ ). The $\mathrm{O}-\mathrm{H}$ group of 3 is remarkably deshielded with a proton shift to $\delta$ O-H $=14.6 \mathrm{ppm}$, which hints towards an acidification of the $\mathrm{O}-\mathrm{H}$ group (Figure $3 \mathrm{~d}$ ).

\footnotetext{
${ }^{25}$ Crystal grown by Dr. Markus Van Alten born Kinauer, crystal structure solved by Dr. Christian Würtele.

${ }^{26}$ Scifinder(C) and Google Scholar (C) search on „iridium hydroxide“ on 20.04 .2020 and additional tracing of cross references.
} 
a)

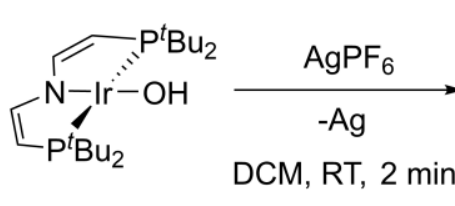

2

c)

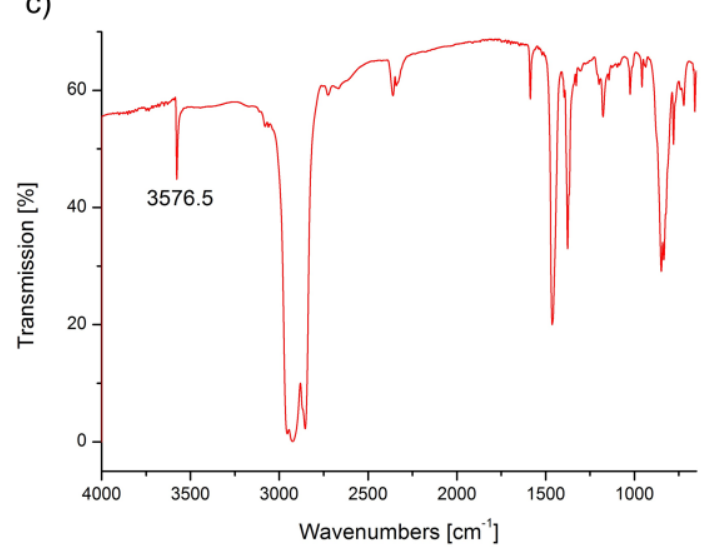

b)

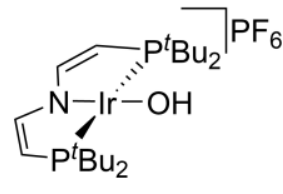

3
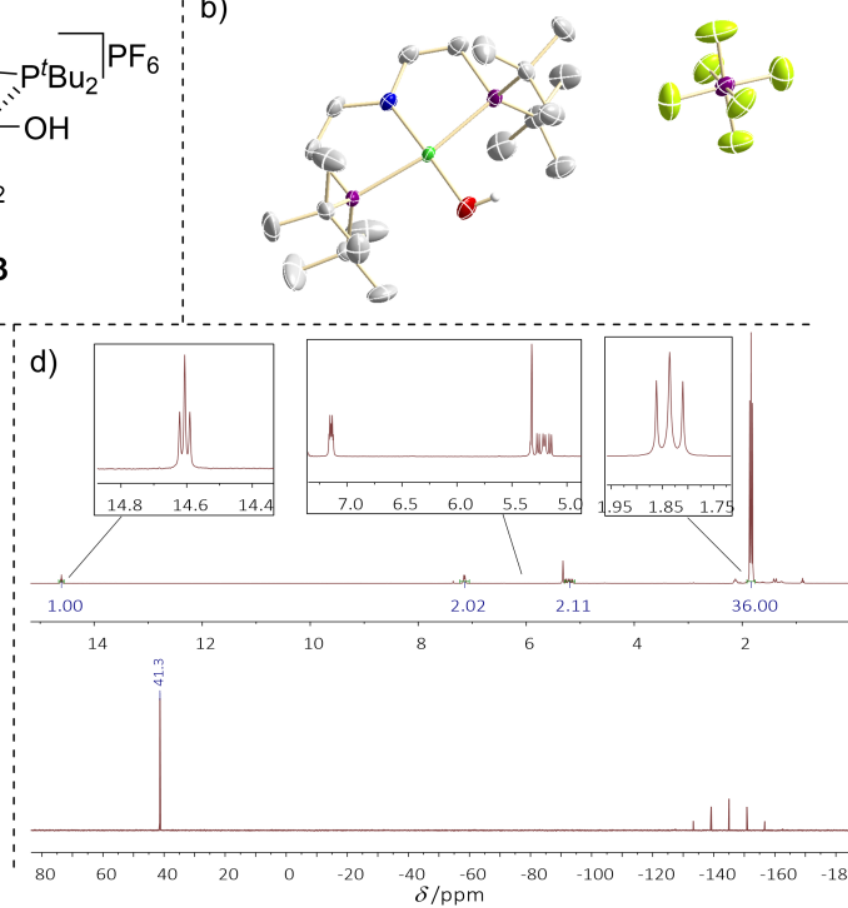

Figure 3: a) Improved synthetic route to the cationic hydroxo species 3 by oxidation of 2 with $\mathrm{AgPF}_{6}$. b) Molecular structure in the solid state of 3 obtained by $\mathrm{X}$ ray single crystal diffraction. The ORTEP plot shown with anisotropic displacement parameters at $50 \%$. All C-H hydrogen atoms and disorder omitted for clarity.

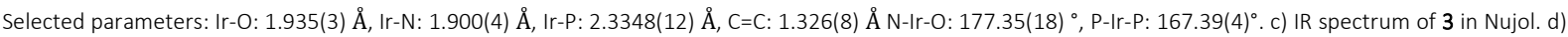
${ }^{1} \mathrm{H}-\mathrm{NMR}$ spectrum (top) and ${ }^{31} \mathrm{P}\left\{{ }^{1} \mathrm{H}\right\}$ NMR spectrum (bottom) of 3 in DCM- $d_{2}$ at $25^{\circ} \mathrm{C}$.

The reduction towards an anionic iridium(I) hydroxo complex can be achieved by treatment of 2 with $1 \mathrm{M} \mathrm{Na} / \mathrm{Hg}$ as the reducing agent in few THF, yielding an orange product (Figure 4a). The solution needs to be cooled immediately to $-40{ }^{\circ} \mathrm{C}$ because the product is temperature sensitive and decomposes to several species at prolonged times, at room temperature as well as in the solid state. The molecular structure of the reduction product was determined by XRD analysis. Complex 1 is not monomeric but a dimer of the iridium(I) hydroxo species $[(\mathrm{PNP}) \operatorname{Ir}(\mathrm{NaOH})(\mathrm{thf})]_{2}$ with bridging sodium atoms, which confirms the hypothesis regarding the chemically non-reversible behaviour in the cyclic voltammogram of 2 (Figure $4 \mathrm{~b}$ ). ${ }^{27}$ The metal centre is coordinated in a square-planar fashion with only weak distortion from the ideal $360^{\circ}$ (Angle sum around iridium $=358^{\circ}$ ) and the $\mathrm{Ir}$ $\mathrm{O}$ bond is drastically elongated to $d_{\mathrm{Ir}-\mathrm{O}}=2.134 \AA$ and therefore reflects the high electron count. The $\mathrm{O}-\mathrm{H}$ hydrogen atoms could be found in the electronic density map and isotopically refined, giving an $\mathrm{O}-\mathrm{H}$ bond length of $d_{\mathrm{O}-\mathrm{H}=}=$ $0.79 \AA$, which is already close to the $\mathrm{O}-\mathrm{H}$ bond length in pure $\mathrm{NaOH} \cdot \mathrm{H}_{2} \mathrm{O} d_{\mathrm{O}-\mathrm{H}}=0.74 \AA .{ }^{[175]} \mathrm{A} C_{2 v}$ symmetric signal set is found for 1 with four signals in the ${ }^{1} \mathrm{H}-\mathrm{NMR}$ spectrum $(\delta=6.89 \mathrm{ppm}, \delta=4.02 \mathrm{ppm}$ and $\delta=1.35 \mathrm{ppm}$, $\delta=-2.1 \mathrm{ppm})$ and one signal in the ${ }^{31} \mathrm{P}\left\{{ }^{1} \mathrm{H}\right\}-\mathrm{NMR}$ spectrum $(\delta=57.2 \mathrm{ppm})$. The dimeric nature of 1 could be observed as well in solution by the broadened NMR-signals, due to hindered rotation (Figure 4c). The O-H protons of 1 are shifted to $\delta_{\mathrm{O}-\mathrm{H}}=-2.1 \mathrm{ppm}$ which hints towards some hydridic character.

\footnotetext{
${ }^{27}$ Crystal structure solved by Dr. Christian Würtele. The same structure was previously reported by Dr. Markus van Alten born Kinauer.
} 
a)

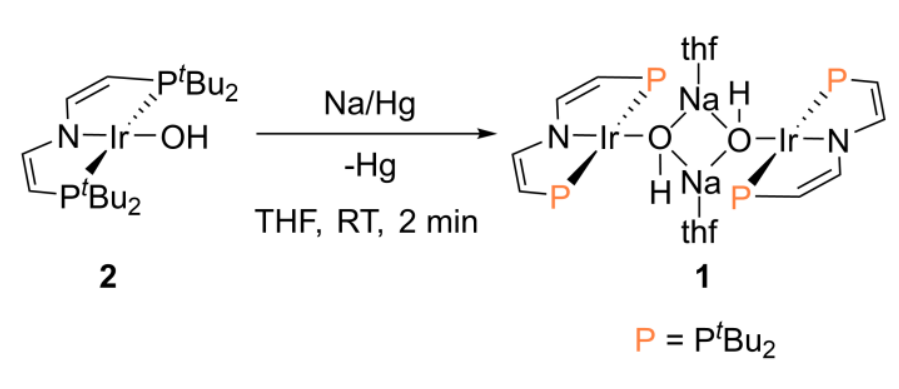

b)

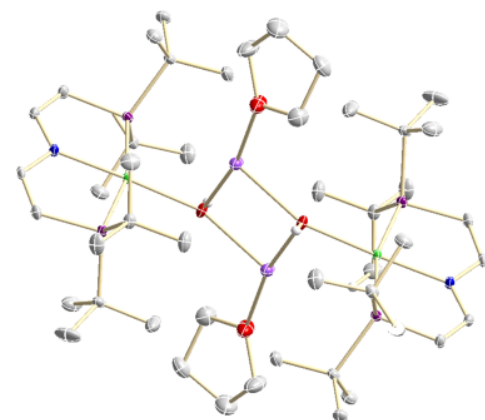

c)
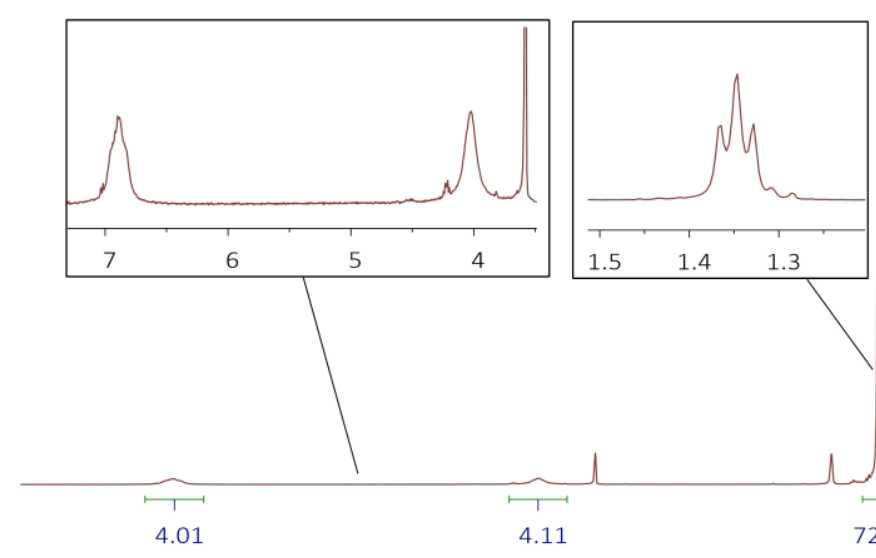

$1.5 \quad 1.4$

$\begin{array}{lllllllll}8.0 & 7.5 & 7.0 & 6.5 & 6.0 & 5.5 & 5.0 & 4.5 & 4.0\end{array}$

72.00

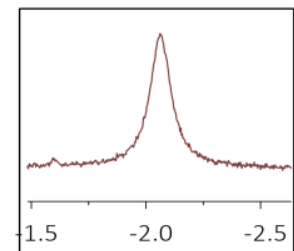

$-2.5$

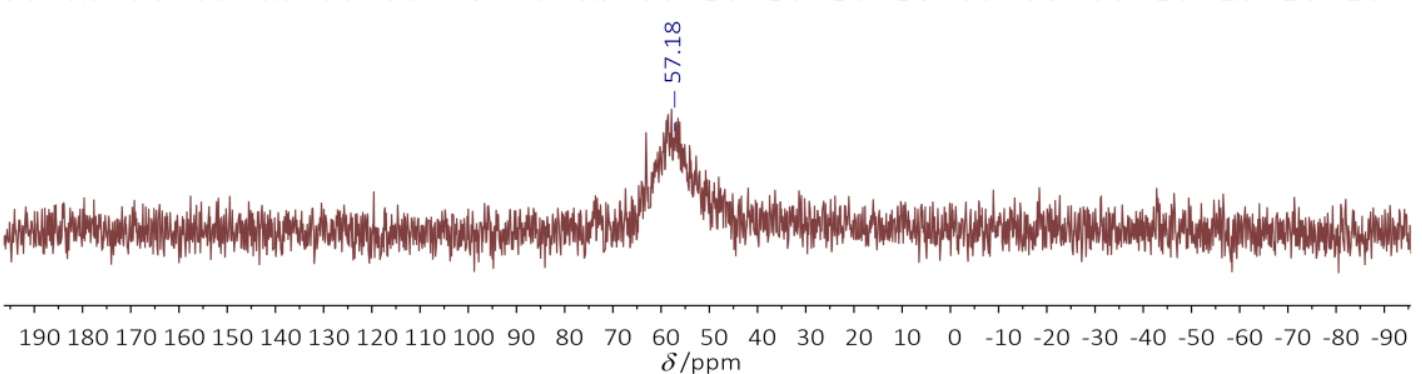

Figure 4: a) Synthetic route towards the iridium(I) hydroxo species 1 from 2 by reduction with sodium amalgam in THF. b) Molecular structure in the solid state of 1 obtained by X-ray single crystal diffraction. The ORTEP plot shown with anisotropic displacement parameters at $50 \%$. All C-H hydrogen atoms, disorder and a

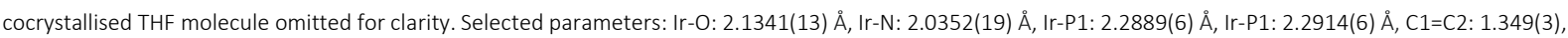
$\mathrm{C} 11=\mathrm{C} 12: 1.345(3), \mathrm{Na1}-01:$ 2.235(2), O1-Na1-O1\#: $91.60(7)^{\circ}, \mathrm{N}-\mathrm{Ir}-\mathrm{O}: 176.53(7)^{\circ}, \mathrm{P}-\mathrm{Ir}-\mathrm{P}: 163.26(2)^{\circ}$. c) ${ }^{1} \mathrm{H}-\mathrm{NMR}$ (top) and ${ }^{31} \mathrm{P}\left\{{ }^{1} \mathrm{H}\right\}-\mathrm{NMR}(\mathrm{bottom})$ of 1 in THF- $d_{8}$ at $25^{\circ} \mathrm{C}$.

\section{Synthesis and Characterisation of a Terminal Iridium Oxo Complex}

The inherent thermal instability of 1 prevents its use as a synthetic starting platform, therefore only the hydroxo complexes 2 and 3 were probed as precursors towards the terminal iridium oxo complex. Addition of $\mathrm{KO}^{t} \mathrm{Bu}$ in $\mathrm{THF}$ to 3 leads to a direct colour change from red-violet to purple. Washing with pentane and crystallisation from toluene leads to the isolation of the desired terminal oxo complex $4[(P N P) \operatorname{Ir}(O)]$ in moderate yields as confirmed by single crystal X-ray analysis (Figure $5, \mathrm{~b}$ ). It must be noted, that absolutely pure and $\mathrm{Na} / \mathrm{K}$ dried toluene must be used, since otherwise exclusively the hydroxo complex 2 will be obtained (Figure 5a). Even with sublimed $\mathrm{KO}^{t} \mathrm{Bu}$ the yield of 4 does not exceed $60 \%$ and green, pentane soluble side products are formed. A second route towards 4 is the HAT reaction from 2, therefore the strong hydrogen atom abstracting reagent 2,4,6-tris-tert-butylphenoxyl 
(Mes*O) was added to 2. Performing this reaction in polar solvents like THF will only lead to a 50:50 mixture of 4 and $\mathbf{2}$, indicating a very low driving force for the reaction, however in contrast to the deprotonation route from 3 no side products are observed. The equilibrium can be shifted exclusively to the product site by usage of an unpolar solvent like hexamethyldisiloxane (HMDSO) or pentane. Here, the additional driving force arises from the precipitation of 4 , while the starting materials as well as the second product 2,4,6-tris-tert-butylphenol $(\mathrm{Mes} * \mathrm{OH})$ are all soluble. Filtration of the reaction mixture, washing with pentane and crystallisation from toluene yields the terminal oxo complex 4 pure in high isolated yields (80\%) (Figure 5a).

a)

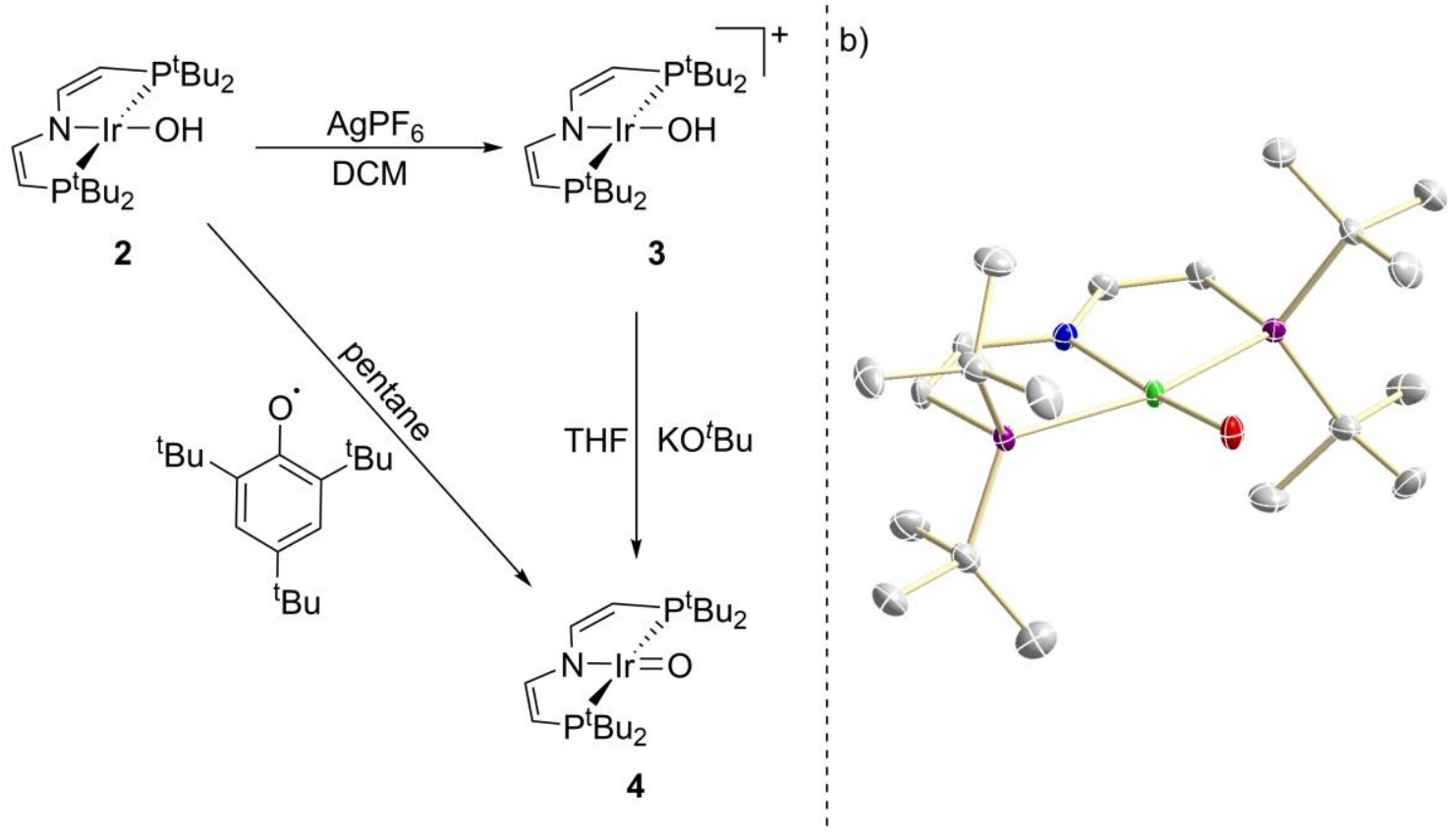

Figure 5: a) Synthetic routes towards 4 by oxidation of 2 with $\mathrm{AgPF}_{6}$ to 3 and subsequent deprotonation with KOtBu in THF or HAT from 2 with Mes*O in pentane. b) Molecular structure in the solid state of 4 obtained by X-ray single crystal diffraction. The ORTEP plot shown with anisotropic displacement parameters at $50 \%$.

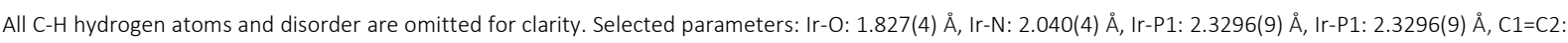
1.344(4), N-Ir-O: $173.6(6)^{\circ}$, P-Ir-P: $163.16(4)^{\circ}$.

XRD analysis of 4 reveals a square-planar coordinated iridium metal centre (angle sum around $\left.\operatorname{Ir}=360^{\circ}\right),{ }^{28}$ with a drastically reduced Ir-O bond length $\left(d_{\mathrm{lr}-\mathrm{O}}=1.827(4) \AA\right)$ compared to the hydroxo species 1-3, which indicates multibonding character within the Ir-O moiety (Figure 5b). This is also reflected by the N-Ir-O angle of $174^{\circ}$, which speaks for the avoidance of $\pi$-back donation from the pincer nitrogen. For the isoelectronic iridium imido complex XXII [(PNP) Ir(N $\left.\left.{ }^{t} B u\right)\right]$ a longer $\mid r-N$ bond length was reported $\left(d_{1 r-N}=1.868(2) \AA\right)$. This reflects the high steric shielding of the $\mathrm{N}^{-}{ }^{\mathrm{t}} \mathrm{Bu}$ group. ${ }^{[37]}$ The $\mathrm{r}-\mathrm{O}$ bond of 4 is significantly elongated $(\Delta=0.1 \AA$ ), compared to the only other reported iridium oxo complex by Wilkinson et a ${ }^{[36]} \mathbf{X X I}\left(d_{1 \mathrm{r}-\mathrm{O}}=1.73 \AA\right.$ ) (Figure 6a, left). This can be explained by a simple molecular orbital (MO) scheme of the Ir-O bonding for both species. The terminal oxo by Wilkinson is in trigonal coordination with a formal iridium $(\mathrm{V})$ oxidation state. The $\mathrm{d}_{z^{2}}$ orbital is forming the $\sigma$-bonding interaction between iridium and oxygen and the $d_{y z}$ and $d_{x z}$ orbitals the $\pi$-bonding interactions, while the $d_{x^{2}-y^{2}}$ and $d_{x y}$ orbitals stay non-bonding. With the six electrons from the formal $\mathrm{O}^{2-}$ ligand and the four electrons from the iridium $(V)$ metal centre only bonding and non-bonding orbitals are occupied, resulting in an Ir-O triple bond (Figure 6a, left). The terminal oxo 4 is in a square planar coordination mode with a formal iridium(III) metal centre. Here,

\footnotetext{
${ }^{28}$ Crystal structure solved by Dr. Christian Würtele.
} 
the $d_{x^{2}-y^{2}}$ is forming the $\sigma$-bonding interaction and the $d_{x z}$ and $d_{x y}$ orbitals the $\pi$-bonding interactions, while the $d_{z^{2}}$ and $d_{y z}$ orbitals remain non-bonding. The two additional electrons from the formal iridium(III) oxidation state result in the population of antibonding $\pi^{*}$ orbitals which reduces the bond order of 4 to an Ir-O double bond (Figure 6a, left).

a)

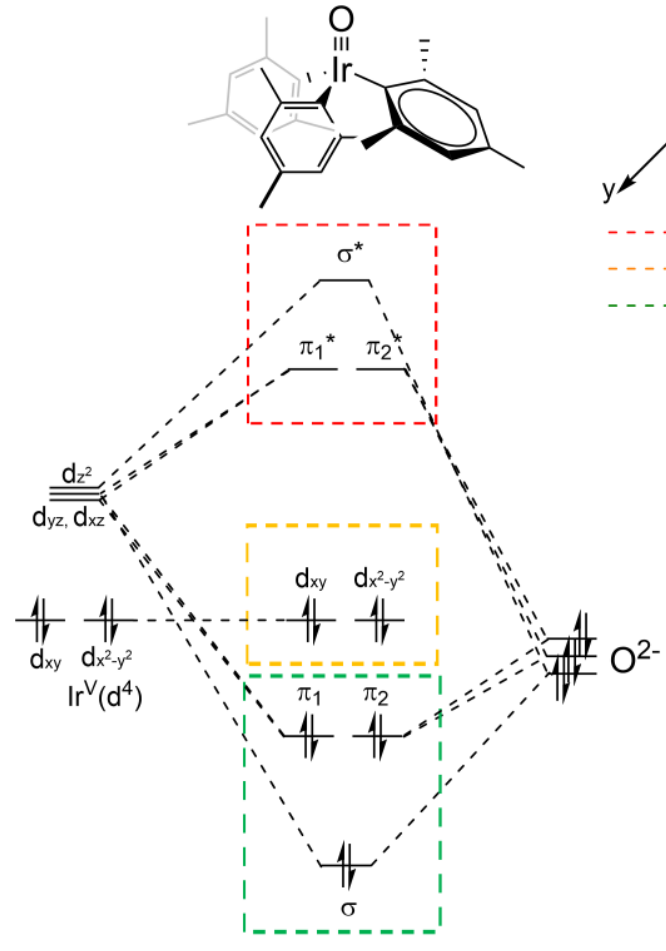

Bond Order: 3.0

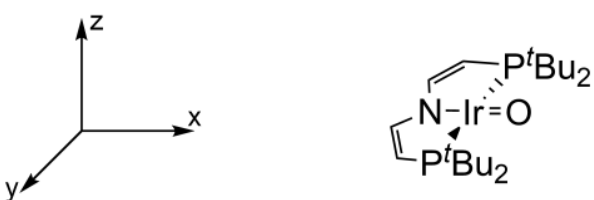

- - - - antibonding

- - non-bonding - bonding

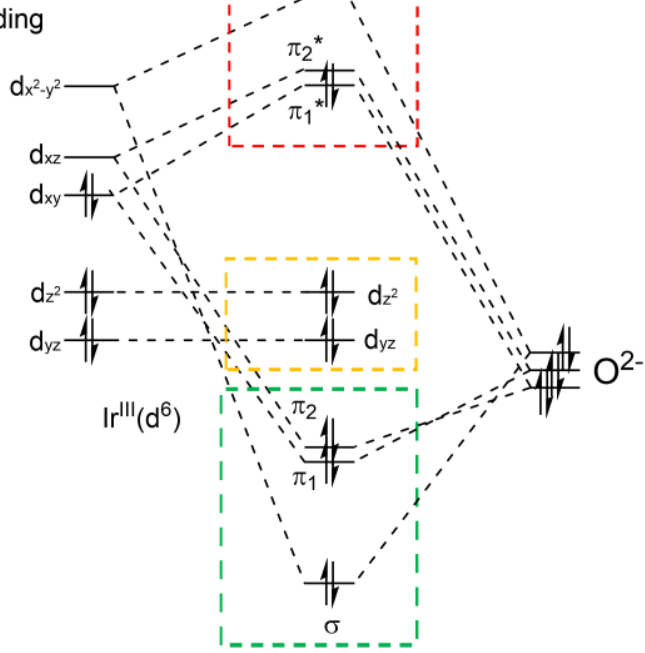

Bond Order: 2.0

b)
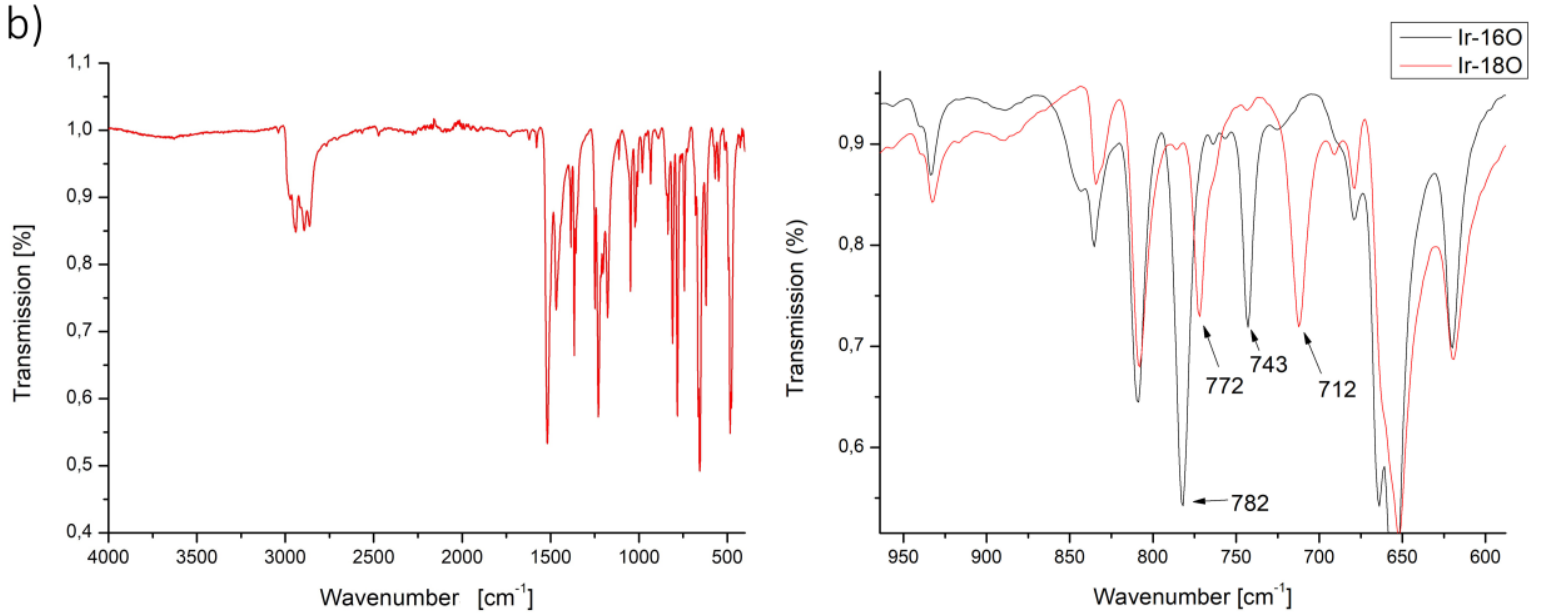

Figure 6: a) MO-scheme of the Ir-O bond for 4 (right) and the Wilkinson oxo complex XXI (left). b) IR(ATR) spectrum of 4 (left) and overlay of the IR(ATR) spectrum of 4 and its ${ }^{18} \mathrm{O}$ isotopologue.

The IR $\left(\mathrm{ATR}^{29}\right)$ of 4 is silent in the region of around $3500 \mathrm{~cm}^{-1}$ were $\mathrm{O}-\mathrm{H}$ stretches would be expected, which supports the formation of a terminal oxo complex (Figure 6a). For further bonding analysis the ${ }^{18} \mathrm{O}$-isotopologue of 4 was synthesised. The Ir-O stretching frequency was thereby assigned to $1 \mathrm{rr}-\mathrm{O}=743 \mathrm{~cm}^{-1}$, while the $\mid \mathrm{Ir}^{-18} \mathrm{O}$ stretching frequency of $v_{\mathrm{rr}-\mathrm{O}}=712 \mathrm{~cm}^{-1}$ is in good agreement to the expected value of the harmonic oscillator

${ }^{29} \mathrm{ATR}=$ Attenuated total reflection 
approximation $\left(v_{\mathrm{rr}-\mathrm{O}}(\mathrm{HO})=701 \mathrm{~cm}^{-1}\right)$. The other visible deviations in the ${ }^{18} \mathrm{O}$-isotopologue spectrum at e.g. $v=$ $772 \mathrm{~cm}^{-1}$ can tentatively be assigned to Fermi resonances in the ${ }^{16} \mathrm{O}$ isotopologue which cancel upon labelling (Figure $6 b$, right). A comparison of the $\mid r-O$ frequencies of 4 and $\mathbf{X X I}\left(v_{\mathrm{Ir}-\mathrm{O}}=802 \mathrm{~cm}^{-1}\right)^{[36]}$ supports the stronger bond for the latter, confirming the picture of a double bond for 4 and a triple bond for XXI.

The electrochemistry of 4 was analysed by cyclic voltammetry, revealing two redox events at $E_{1 / 2}=-1.95 \mathrm{~V}$ and $E_{1 / 2}=-0.13 \mathrm{~V}$ vs. $\mathrm{Fc} / \mathrm{Fc}^{+}$(Figure $\left.7 \mathrm{a}\right)^{30}$. The reductive wave is quasi-reversible, which can be rationalized by the higher electron count of the formal anionic oxo species 4 - and thus less stabilizing $\pi$-bonding character within the Ir-O unit. In analogy to the MO-scheme of Figure 6a the Ir-O bond of the anionic terminal oxo 4- is further activated (the bond order drops to 1.5) and thus the complex is destabilized. The oxidative event is at a very mild potential for a formal Ir III/Ir' oxidation ${ }^{[176]}$ reflecting the high covalency in the Ir-O unit and therefore the low validity of the concept of oxidation numbers for very covalent complexes in general.

a)

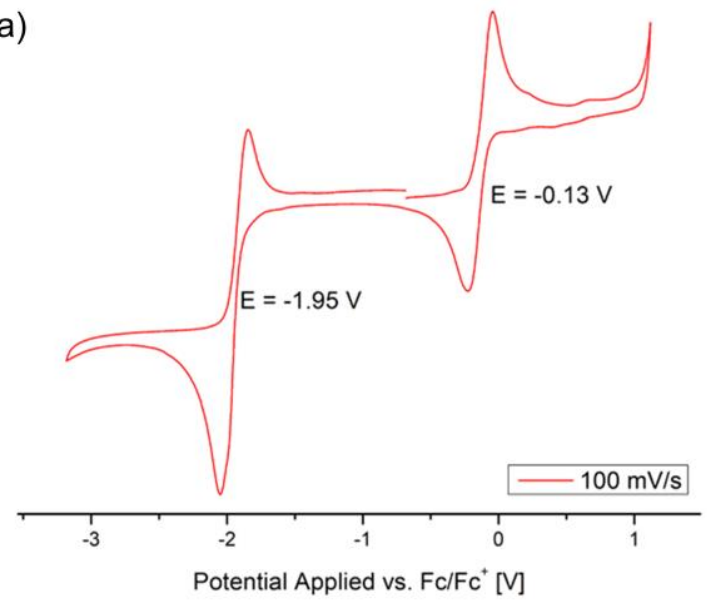

c)

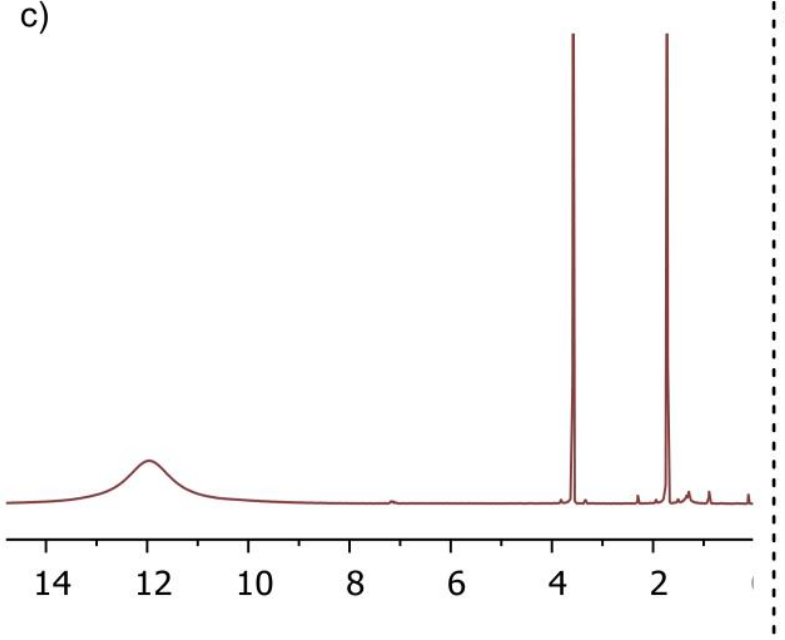

ib)

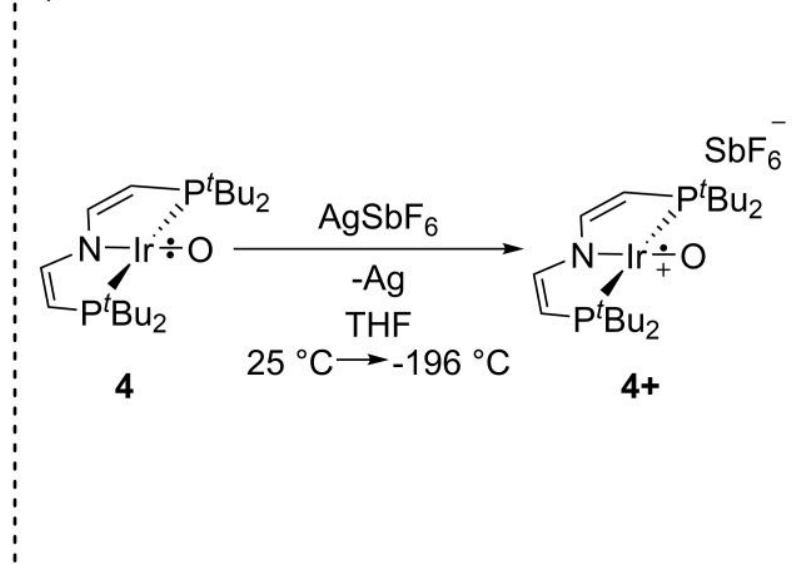

d)

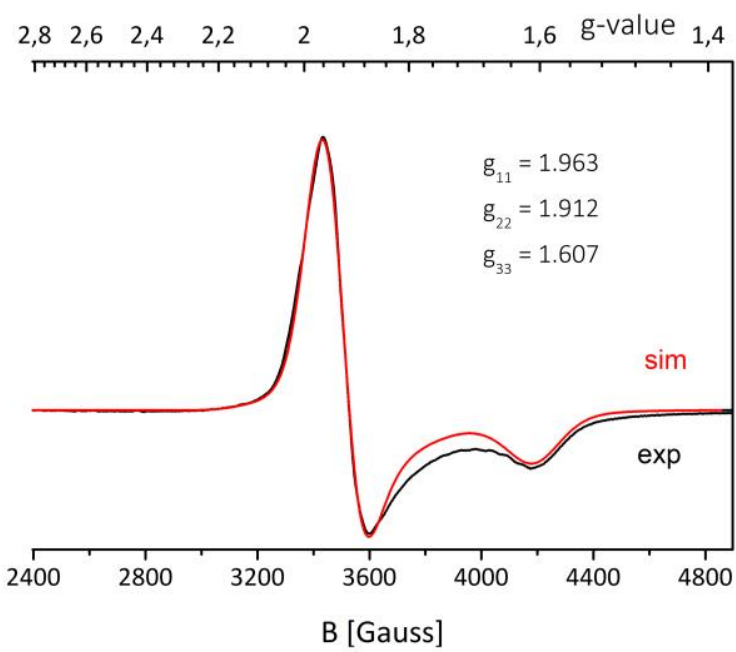

Figure 7: a) Cyclic Voltammogram of $4(1.0 \mathrm{mM})$ in THF with $0.1 \mathrm{M} \mathrm{NBu}_{4} \mathrm{PF}_{6}$. Shown is the $2^{\text {nd }}$ cycle. b) Oxidation of 4 with $\mathrm{AgSbF} 6$ in THF at room temperature and trapping of the formed species $4+$ with liquid nitrogen. c) ${ }^{1} \mathrm{H}$ (para)-NMR spectrum of the reaction after filtration. d) X-Band EPR spectrum at $143 \mathrm{~K}$ obtained by oxidation of 4 with $\mathrm{AgSbF}_{6}$ in THF. ${ }^{31}$

${ }^{30}$ Cyclic voltammogram recorded by Niklas Welker.

${ }^{31}$ Simulation of the EPR spectrum by Prof. Dr. Bas de Bruin University of Amsterdam. 
The electrochemistry of $\mathbf{4}$ seems very promising for the synthesis of a cationic terminal oxo, thus the chemical oxidation with $\mathrm{AgSbF}_{6}$ in $\mathrm{PhCl}$ was probed (Figure $7 \mathrm{~b}$ ). An instant colour change from the reddish purple of 4 to a blue violet is observed. Keeping this solution at ambient temperature will lead to the decomposition of the species, in which the cationic hydroxo 3 could be observed as the main decomposition product. Directly cooling with liquid nitrogen after the reaction enables the spectroscopic characterisation of $4+[(P N P) \operatorname{Ir}(O)]\left[S_{b F}\right]$. The ${ }^{1} \mathrm{H}($ para) $-\mathrm{NMR}$ of 4+ speaks for a $\mathrm{C}_{2 v}$ symmetric complex with only one main signal at $\delta=11.9 \mathrm{ppm}$, which indicates that $4+$ is also a terminal oxo complex, since a decomposition pathway by insertion into the metal phosphorous bond, as observed for the platinum oxo by Milstein, ${ }^{[47]}$ would reduce the product symmetry to $C_{1}$ (Figure 7c). Transient 4+ was further analysed by EPR spectroscopy where an almost axial spectrum with a very low $g$-anisotropy for a $5 \mathrm{~d}$ metal compound $\left(g_{11}=1.96, g_{22}=1.91\right.$ and $\left.g_{33}=1.61\right)$ can be observed. ${ }^{32}$ A comparison to the formally isoelectronic iridium(IV) nitride complex $\mathbf{X X V}[(\mathrm{PNP}) \operatorname{Ir}(\mathrm{N})]$ and the iridium(IV) imido complex XXIII $\left[(\mathrm{PNP}) \operatorname{Ir}(\mathrm{NtBu}]^{+}\right.$highlights the special electronic structure of $4+.^{[37,41]}$ For $\mathbf{X X V}\left(g_{11}=1.86, g_{22}=1.58, g_{33}=1.32\right)$ and XXIII $\left(g_{11}=1.71, g_{22}=1.63, g_{33}=1.33\right)$ the $g$-anisotropy is much more distinct and the values are less deviating from the value of the free electron $g_{e}=2.002 .{ }^{33}$ This supportsa ligand centred oxyl radical character for 4+. Further insight into the electronic structure of 4+ was obtained by computational analysis on the PBEOD3/def2TZVP level of theory. ${ }^{34}$ The cationic oxo $4+$ is computed to be in square planar coordination geometry (angle around iridium $=360^{\circ}$ ) with a significantly shortened $\mid r-O$ bond $\left(d_{1 r-0}=1.75 \AA\right)$ compared to the crystal structure of 4 and additionally a lower $\mathrm{N}-\mathrm{Ir}-\mathrm{O}$ angle $\left(169.7^{\circ}\right)$. This nicely reflects the trend of rising bond order and thus shorter Ir-O bond length due to less electrons in antibonding $\pi^{*}$-orbitals. Additionally, the rare oxyl radical character of $4+$ is confirmed by the Mulliken spin density analysis (O: $75 \%$ ).

a)

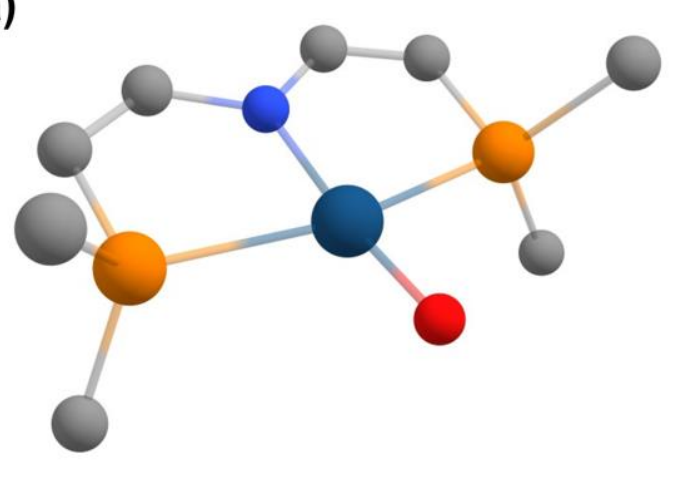

b)

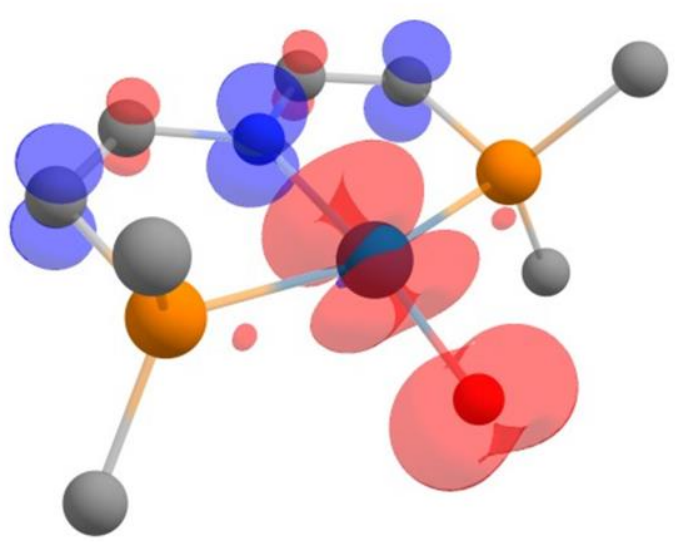

Figure 8: a) Structure of $4+$ on the PBEO-D3/def2TZVP level of theory. b) Mulliken spin density plot at isolevel $0.0075 \mathrm{a}_{0}{ }^{-3}$ computed at the PBEO-D3/def2TZVP level of theory. Ir: $42 \%$ O: $75 \% \mathrm{~N}:-18 \%$

\footnotetext{
32 The fitting of the EPR spectrum was performed by Prof. Dr. Bas de Bruin, University of Amsterdam.

${ }^{33}$ https://physics.nist.gov/cgi-bin/cuu/Value?gem/search_for=all free electron g factor

${ }^{34}$ For more details see Section 4.2 of Chapter VI.
} 
The three sharp and strongly paramagnetically shifted signals in the ${ }^{1} \mathrm{H}\left\{\right.$ para\}-NMR spectrum of $4\left(\delta_{\mathrm{t} \mathrm{Bu}}=+21 \mathrm{ppm}\right.$, $\delta_{2 \times \mathrm{CH}}=-62 \mathrm{ppm}, \delta_{2 \times \mathrm{CH}}=-76 \mathrm{ppm}$ ) support a $\mathrm{C}_{2 \mathrm{v}}$ symmetric structure on the NMR timescale (Figure 9a). The signals are almost temperature invariant over a wide range $\left(-75\right.$ to $\left.+65{ }^{\circ} \mathrm{C}\right)$ besides the third signal $\left(\delta_{2 \times \mathrm{CH}}=-76 \mathrm{ppm}\right)$, which exhibits approximately Curie dependence above $\sim 250 \mathrm{~K}$, which indicates accessible thermally excited states (Figure 9b). Such spectroscopic behaviour was also found for the isoelectronic [(PNP)|r=N ${ }^{t}$ Bu] complex XXII and was rationalized by ab initio computations. Imido complex XXII has a triplet ground state which is strongly stabilised by spin-orbit coupling (SOC). This causes a non-magnetic ground state that mixes due to temperature independent paramagnetism (TIP) with the open-shell excited states. ${ }^{[37]}$ This explains the sharp signals NMR signals and their paramagnetic shift in contrast to the broadened signals expected for a classic, paramagnetic compound.
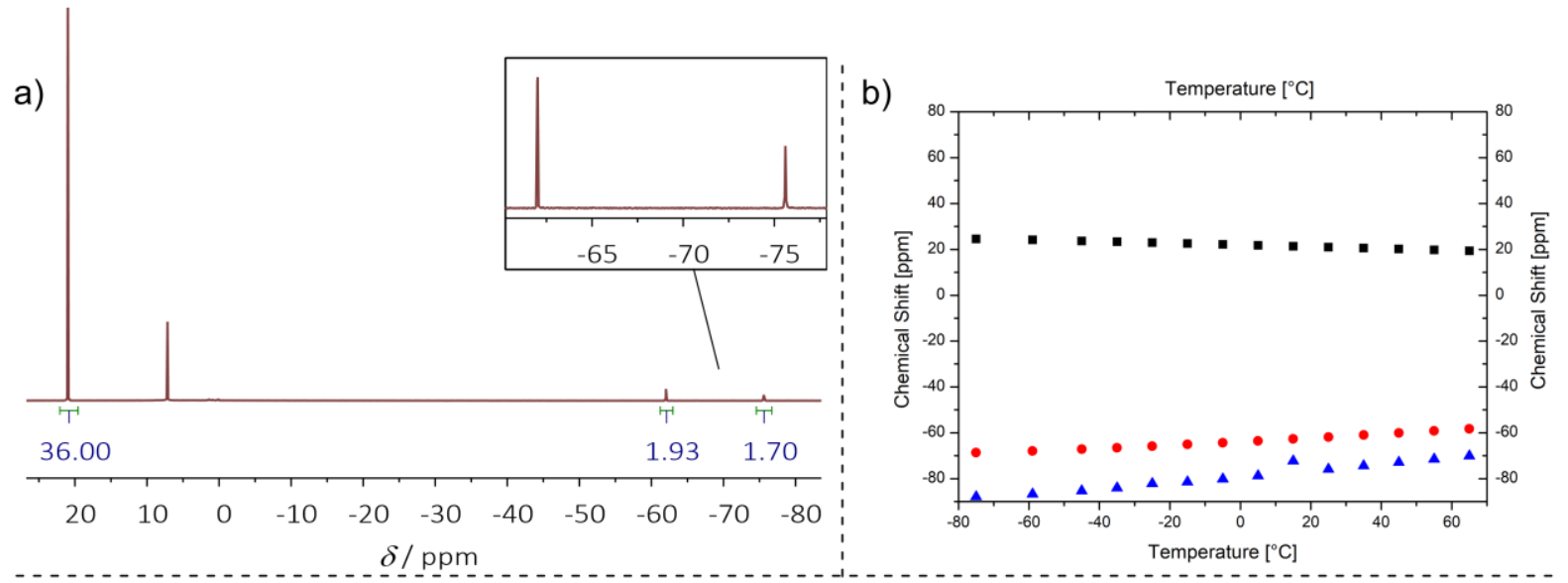

c)

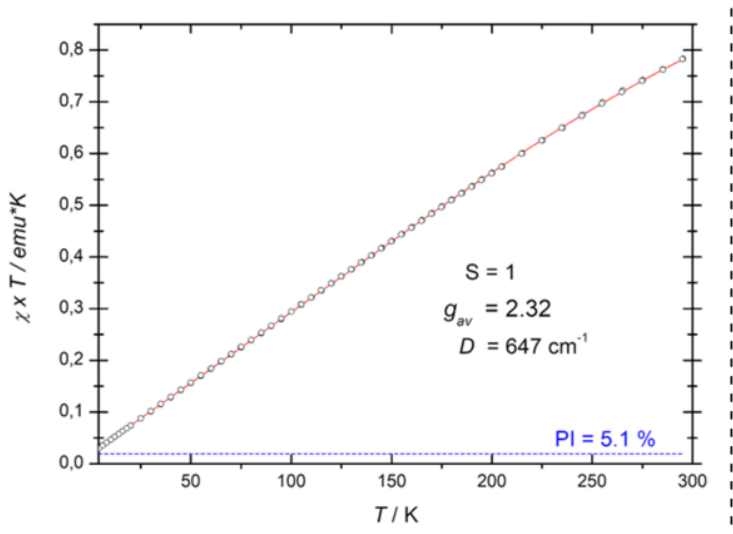

d)

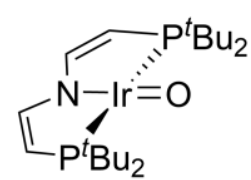

${ }^{1} \mathrm{~A}$

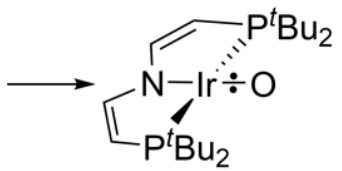

${ }^{3} \mathrm{~A}$

Figure 9: a) ${ }^{1} \mathrm{H}$ \{para\}-NMR of 4 in $\mathrm{C}_{6} \mathrm{D}_{6}$ at $25^{\circ} \mathrm{C}$. b) VT-NMR of 4 from $-75^{\circ} \mathrm{C}$ to $+65^{\circ} \mathrm{C}$. c) Temperature dependent magnetic susceptibility measurement by SQUID magnetometry. d) Revision of the Lewis-structure of 4 reflecting the triplet ground state.

Accordingly, the $\chi_{\mathrm{M}} T$ vs. T curve of a powder sample of 4, obtained by SQUID magnetometry features temperature independent paramagnetism up to around $250 \mathrm{~K}$ and shows slight bending of the curve at higher temperatures which arises from the partial population of excited states (Figure 9c). ${ }^{35}$ The data can be fitted with a spin Hamiltonian for a triplet state $\left(S=1, g_{\mathrm{av}}=2.32\right)$ with a large zero-field splitting $\left(D=647 \mathrm{~cm}^{-1}\right)$ which is $181 \mathrm{~cm}^{-1}$ higher compared to imido complex XXII. This can be attributed within a simple ligand-field picture to reduced covalent bonding of 4 in the $\operatorname{Ir}=E(E=E R, O)$ moiety, which leads to a smaller relativistic nephelauxetic effect for 4

${ }^{35}$ SQUID magnetometric measurements performed by Dr. Serhiy Demeshko. 
and therefore to a higher effective spin-orbit coupling parameter $\zeta_{\text {eff. }}{ }^{36}$ With these spectroscopic and magnetic data at hand, the Lewis-structure of $\mathbf{4}$ should be revised in analogy to Figure $9 \mathrm{~d}$ as a biradical species.

The spectroscopic and magnetic description of $\mathbf{4}$ is corroborated by high level ( $a b$ initio) computations. ${ }^{37}$ The structures are optimized by DFT on the B3LYP(V)-(D3)/def2TZVP level of theory and reproduce the short Ir-O bond $d^{\mathrm{DFT}}{ }_{\mathrm{Ir}-\mathrm{O}}=1.80 \AA$ and the near linearity of the $\mathrm{N}-\mathrm{Ir}-\mathrm{O}$ moiety $\left(179.0^{\circ}\right)$ for the ${ }^{3} \mathrm{~A}^{\prime \prime}$ state (Figure 10a). In contrast, the lowest singlet state $\left({ }^{1} \mathrm{~A}^{\prime}\right)$ of 4 exhibits a high deviation from linearity $\left(157.0^{\circ}\right)$ as also found computationally for the isoelectronic platinum(IV) pincer (PCN) oxo complex XXXI by Milstein et al. (Triplet state: C-Pt-O = $175.4^{\circ} ;$ Singlet state: $\left.\mathrm{C}-\mathrm{Pt}-\mathrm{O}=138.2^{\circ}\right) \cdot{ }^{[47,177]}$ The adiabatic triplet-singlet gap of 4 is significantly higher with $\Delta \mathrm{E}(\mathrm{T} / \mathrm{S})=41 \mathrm{~kJ} \mathrm{~mol}^{-1}$ compared to the computational value of XXXI which vanishes on high level ab initio computations resulting in virtually isoenergetic states of XXXI. ${ }^{[177]}$

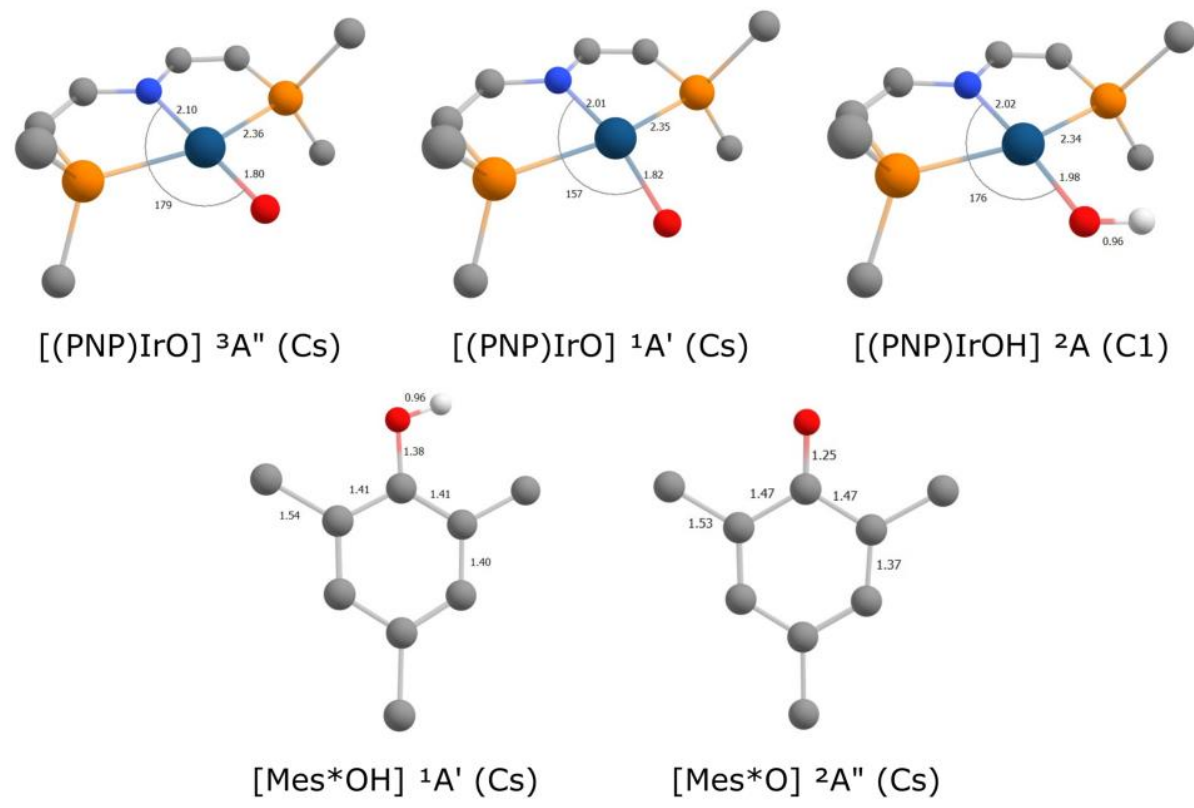

Figure 10: a) Computed structures for $4\left({ }^{3} \mathrm{~A}^{\prime \prime},{ }^{1} \mathrm{~A}^{\prime}\right), 2\left({ }^{2} \mathrm{~A}\right)$ as well as for Mes*OH and Mes*O on the B3LYP(V)-D3/def2TZVP level of theory. Selected parameters: $4\left({ }^{3} \mathrm{~A}^{\prime \prime}\right)$ : Ir-O: 1.80 , Ir-N: $2.10 \AA$, Ir-P: $2.36 \AA$, N-Ir-O: $179^{\circ} ; 4\left({ }^{1} \mathrm{~A}^{\prime}\right)$ : "): Ir-O: $1.82 \AA$, Ir-N: $2.01 \AA$, Ir-P: $2.35 \AA$, N-Ir-O: $157^{\circ} ; 2\left({ }^{2} A^{\prime \prime}\right):$ Ir-O: $1.98 \AA$, Ir-N: $2.02 \AA$, Ir-P: $2.34 \AA$,

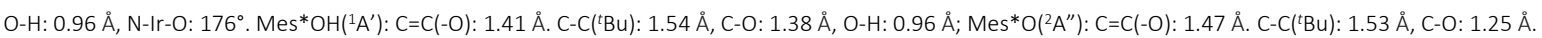

For a more detailed insight into the electronic structure and the influence of spin-orbit coupling (SOC) in 4 and 2 , state averaged multireference computations on the CASSCF/NEVPT2 level of theory, extended by quasidegenerate perturbation theory (QDPT), were performed. The active spaces were constructed to reflect the bonding situation around the iridium metal centre. In addition to the $5 \mathrm{~d}$ orbitals of iridium the $\sigma-$ and $\pi$-bonding interaction of the oxo moiety, the $\sigma$-bonding interaction of the phosphorous atoms and the $\pi$-bonding interaction of the pincer-nitrogen atom were considered leading to a $(16,10)$ CAS extension for 4 and to a $(17,11)$ CAS extension for 2 , where the $\mathrm{O}-\mathrm{H} \sigma$-bonding interaction is additionally considered. In analogy to the DFT computations, the CASSCF wavefunction also predicts a triplet ground state for $4 \Delta E_{\mathrm{T} / \mathrm{s}}=52 \mathrm{~kJ} \mathrm{~mol}^{-1}$ with a Ir-O

\footnotetext{
${ }^{36}$ The spin-Hamiltonian formally loses its physical meaning in cases of strong spin-orbit coupling, making "spin" a bad quantum number. It is, nevertheless, easier to capture. For further information please see reference ${ }^{[258]}$.

37 The computations of this chapter, except for the computations for $4+$, were performed by Dr. Martin Diefenbach. A comprehensive discussion of the computations performed for this Section can be also found in Section 4.2 of chapter VI.
} 
$\left(\pi_{1}{ }^{*} \pi_{2}{ }^{*}\right)^{2}$ configuration and a doublet ground state $\Delta E(D / Q)=388 \mathrm{~kJ} \mathrm{~mol}^{-1}$ for 2 (Figure 11 ). Upon treatment of spin-orbit coupling (SOC) by QDPT the triplet ground state of 4 is strongly stabilised $\left(\triangle E(S O C)=-23.5 \mathrm{~kJ} \mathrm{~mol}^{-1}\right)$ and splits into its $m_{s}=0, \pm 1$ microstates, resulting in an isolated "non-magnetic" ground state.

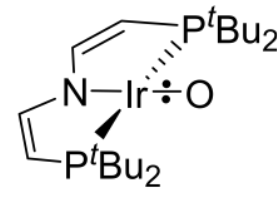

4

$$
\overbrace{}^{E_{\mathrm{rel}} / \mathrm{C}}
$$

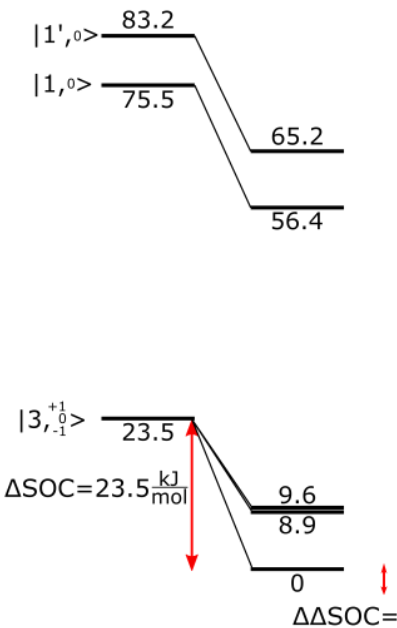

$\Delta \triangle \mathrm{SOC}=2.9 \frac{\mathrm{kJ}}{\mathrm{mol}}$

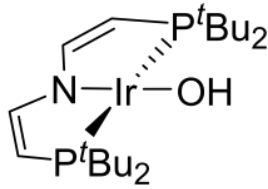

2

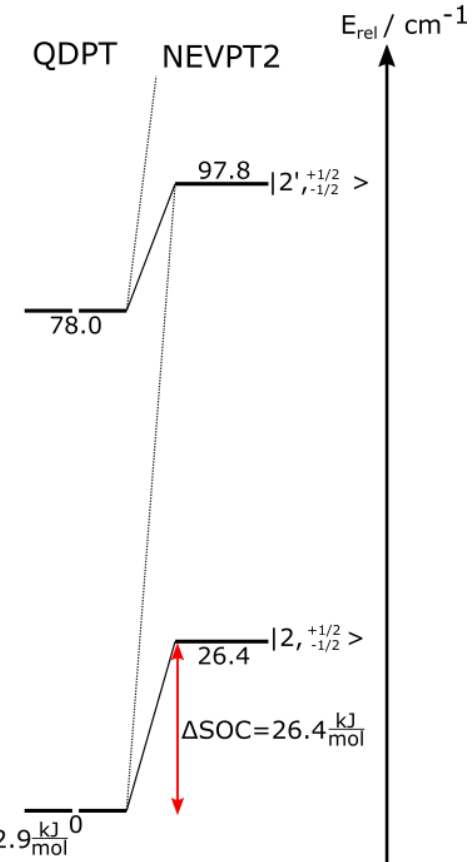

Figure 11: CASSCF state correlation on the NEVPT2 level of theory and with spin-orbit coupling (SOC) treatment by QDPT for 4 (16,10) (left) and 2 (17,11) (right) based on state averaged CASSCF wavefunctions over all roots which arise from excitations within the $5 \mathrm{~d}$ shell of the metal centre 4 ( 50 singlet, 45 triplet, and 5 quintet states), 2 (40 doublet, 10 quartet states)

The zero-field splitting to the excited states was computed to DQDPT $=775 \mathrm{~cm}^{-1}$, which very nicely reflects the experimental value of $647 \mathrm{~cm}^{-1}$. The same picture was derived for the imido complex XXII, even though the computed and experimentally found zero-field splitting was significantly lower (DQDPT $\left.=450 \mathrm{~cm}^{-1}\right) \cdot{ }^{[37]}$ Conclusively, imido complex XXII and oxo complex 4 have both a spin-orbit stabilized triplet ground state, but $\mathbf{4}$ experiences a higher SOC stabilisation due to the reduced covalency of the $\operatorname{Ir}-E(E=N / O)$ unit. The doublet ground state of 2 has contributions from the first excited doublet root and is even exhibiting a higher stabilisation by SOC than 4 $\left(\Delta E(\mathrm{SOC})=-26.4 \mathrm{~kJ} \mathrm{~mol}^{-1}\right)$ resulting in a differential SOC effect of $-2.9 \mathrm{kJmol}^{-1}$ which formally describes a strengthening of the $\mathrm{O}-\mathrm{H}$ bond by SOC (Figure 11). 


\section{Reactivity of the Terminal Iridium Oxo Complex}

\subsection{Oxygen Atom Transfer and Philicity Derivation}

With the terminal oxo complex 4 at hand, its reactivity towards oxygen atom transfer (OAT), including the derivation of its philicity, was probed with selected reagents. Hydrogenolysis of 4 with 1 bar $\mathrm{H}_{2}$ at ambient temperature in benzene leads to a direct colour change from purple to green (Figure 12a). 4 is already after 5 min fully converted as seen by the immediate measurement of ${ }^{1} \mathrm{H}-\mathrm{NMR}$ after the addition of dihydrogen (Figure $12 \mathrm{~b}$, top). A broad signal at $\delta=7 \mathrm{ppm}$ indicates the formation of 2 and of a second, $\mathrm{C}_{2 v}$ symmetric product, with broadened signals $\left(\delta_{2 \times \mathrm{CH}}=7.16 \mathrm{ppm}, \delta_{2 \times \mathrm{CH}}=4.49 \mathrm{ppm}, \delta_{4 \times \mathrm{C}\left(\mathrm{CH}_{3}\right)_{3}}=1.32 \mathrm{ppm}\right)$ accompanied by the formation of $\mathrm{H}_{2} \mathrm{O}$ at $\delta \sim 0$ ppm. 2 is fully converted after one hour reaction time and yields a new orange product (Figure 12b, bottom).

a)

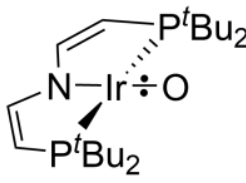

4

b)

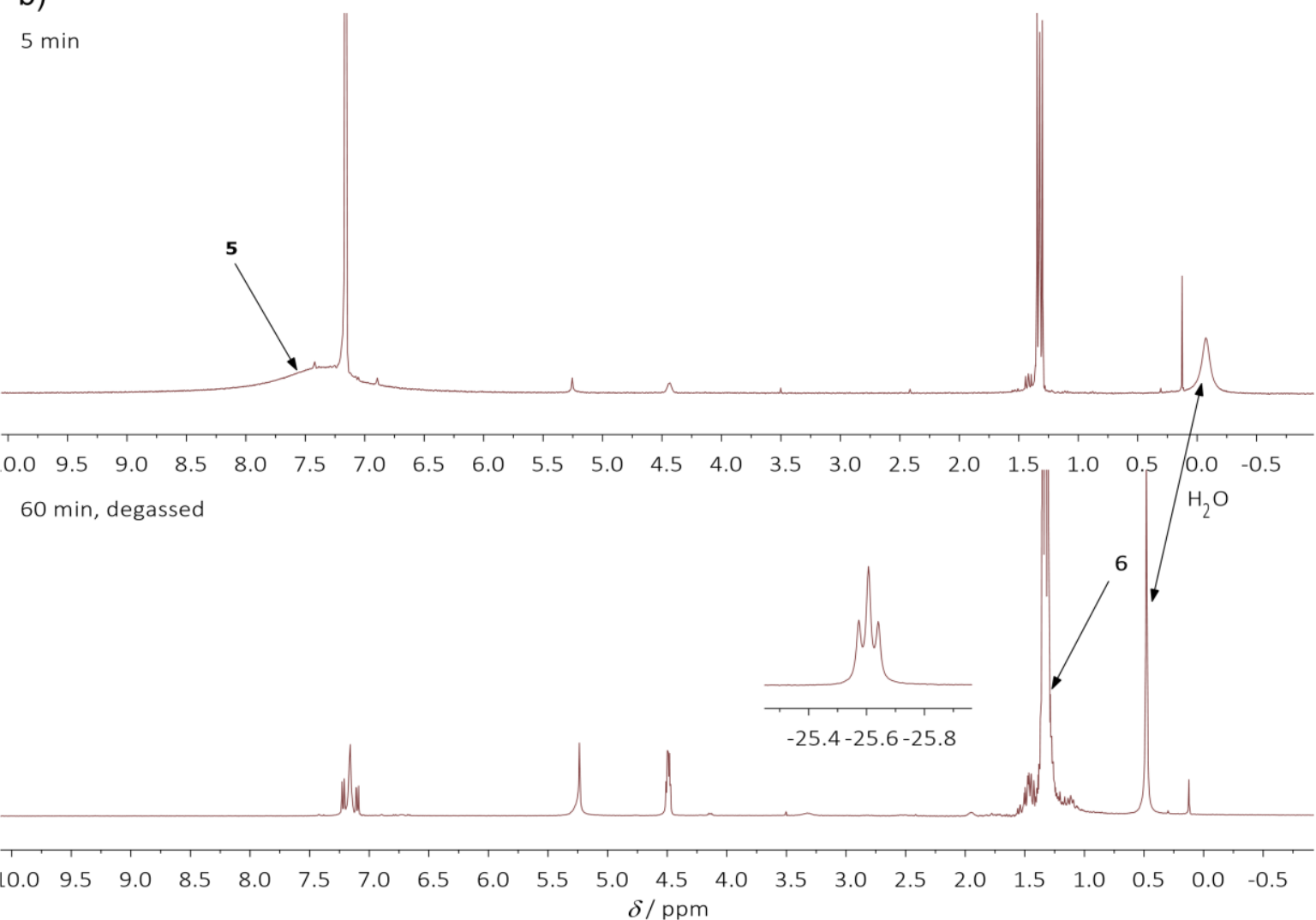

Figure 12: a) Hydrogenolysis of 4 with 1 bar $\mathrm{H}_{2}$ at ambient temperature in benzene to 6 and water. b) ${ }^{1} \mathrm{H}-\mathrm{NMR}$ of the reaction mixture under 1 bar of $\mathrm{H}_{2}$ gas 5 min after the addition. c) ${ }^{1} \mathrm{H}$ (para)-NMR of the reaction mixture $1 \mathrm{~h}$ after the addition after degassing and addition of $\mathrm{Ar}$ 
The signals of the new product are broadened in the presence of $\mathrm{H}_{2}$ which speaks for chemical exchange. The $\mathrm{H}_{2} \mathrm{O}$ signal shifts (concentration depended) to $\delta \sim 0.4 \mathrm{ppm}$ after freeze-pump-thaw degassing the mixture, which resembles the literature known shift in $\mathrm{C}_{6} \mathrm{D}_{6}$ (Figure $\left.12, \mathrm{c}\right) .{ }^{[178]}$ Additionally a new signal at $\delta=-26 \mathrm{ppm}$ can be observed, integrating to two protons, thus the NMR signals can be assigned to the dihydride complex 6 $\left[(\mathrm{PNP}) \operatorname{Ir}(\mathrm{H})_{2}\right]$ previously reported by Schneider et al. ${ }^{[37]}$ Here, hydrogenolysis of XXII also led to the formation of 6 and ${ }^{t} \mathrm{BuNH} 2$ as the second product and again reflects the chemical relationship between 4 and XXII.

a)

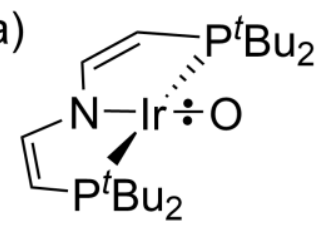

4

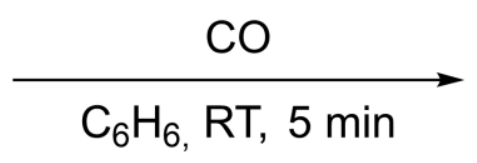

$\mathrm{C}_{6} \mathrm{H}_{6}, \mathrm{RT}, 5 \mathrm{~min}$

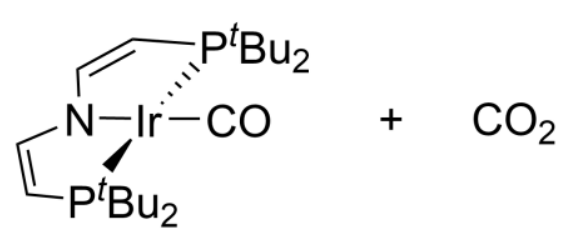

7

b)

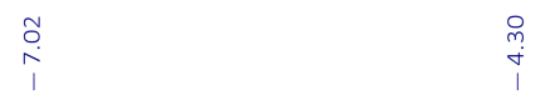

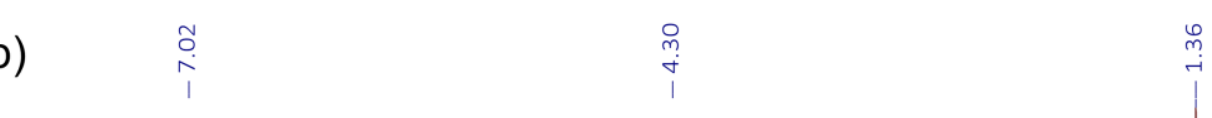
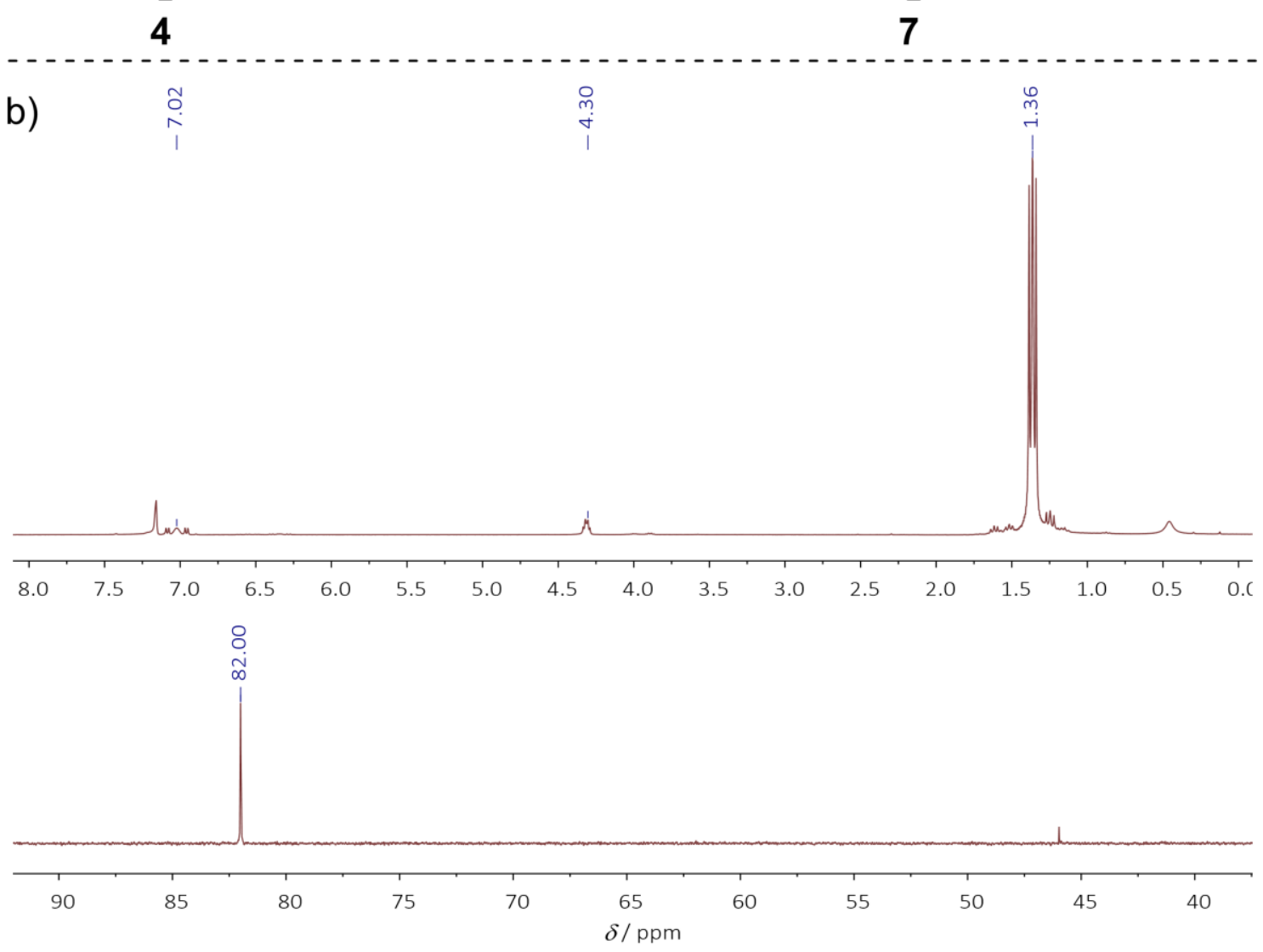

Figure 13: a) Carbonylation of 4 in benzene at ambient temperature yielding 7 and $\mathrm{CO}_{2}$. b) ${ }^{1} \mathrm{H}$-NMR spectrum of the reaction mixture. c) ${ }^{31}$-NMR spectrum of the reaction mixture. d) Headspace analysis plot of the reaction mixture.

The reaction of 4 with CO gas (1 bar) in benzene at ambient temperature gives an immediate colour change to pale yellow (Figure 13a). The ${ }^{1} \mathrm{H}-\mathrm{NMR}$ spectrum of the reaction mixture shows the formation of one, $\mathrm{C}_{2 \mathrm{v}}$ symmetric, main product with three distinct signals in the spectrum ( $\delta_{2 \times \mathrm{CH}}=7.02 \mathrm{ppm}, \delta_{2 \times \mathrm{CH}}=4.30 \mathrm{ppm}, \delta_{4 \times \mathrm{C}(\mathrm{CH}) 3}=1.36 \mathrm{ppm}$ ) and one signal in the ${ }^{31} \mathrm{P}-\mathrm{NMR}$ spectrum ( $\left.\delta=82 \mathrm{ppm}\right)$ which confirms the synthesis of the previously reported [(PNP)!rCO] carbonyl complex 7 (Figure 13b). ${ }^{[179]}$ Thus, in analogy to Milstein's platinum oxo XXXI $[(\mathrm{PCN}) \mathrm{Pt}(\mathrm{O})][\mathrm{BF} 4]^{38}$, the second product must be $\mathrm{CO}_{2}$, which could be confirmed by headspace analysis. ${ }^{[47], 39}$ The

${ }^{38} \mathrm{PCN}=\mathrm{C}_{6} \mathrm{H}_{3}\left[\mathrm{CH}_{2} \mathrm{P}(\mathrm{t}-\mathrm{Bu})_{2}\right]\left(\mathrm{CH}_{2}\right)_{2} \mathrm{~N}\left(\mathrm{CH}_{3}\right)_{2}$

${ }^{39}$ Head space analysis performed by M.Sc. Marcus Thater 
reaction of $\mathrm{CO}_{2}$ with iridium hydroxo- and imido complexes forming the (hydrogen)carbonate analogous is literature reported and speaks for a nucleophilic oxo moiety. ${ }^{[35,37,168,172]}$ In the ${ }^{31}$ P-NMR spectrum of the carbonylation reaction of 4 , also a second species at $\delta=46 \mathrm{ppm}$ can be found, which might arise from the reaction of 4 with the formed $\mathrm{CO}_{2}$.

To test the hypothesis, that the side product in the carbonylation reaction of 4 arises from reactivity with the second product $\mathrm{CO}_{2}, 4$ was directly reacted with carbon dioxide. The addition of $\mathrm{CO}_{2}$ to a degassed THF solution of 4 at ambient temperature leads to an instant colour change to deep red (Figure 14a). The ${ }^{1} \mathrm{H}-\mathrm{NMR}$ spectrum of the reaction exhibits no paramagnetic species but three distinct signals $\left(\delta_{2 \times \mathrm{CH}}=6.40 \mathrm{ppm}, \delta_{2 \times \mathrm{CH}}=5.45 \mathrm{ppm}\right.$, $\left.\delta_{4 \times C\left(\mathrm{CH}_{3}\right)_{3}}=1.47 \mathrm{ppm}\right)$ which belong to one species by integration. The ${ }^{31} \mathrm{P}-\mathrm{NMR}$ spectrum exhibits one signal at $\delta=46 \mathrm{ppm}$, which is identical to the second product in the carbonylation of 4 (Figure 14b and c) and thus supports the aforementioned hypothesis. A potential candidate for the quarteric carbonyl C atom is found at $\delta=174 \mathrm{ppm}$ in the ${ }^{13} \mathrm{C}-\mathrm{NMR}$ spectrum, which exhibits no coupling to the phosphorous atoms (Figure $14 \mathrm{~d}$ ).

a)

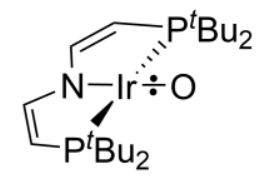

4

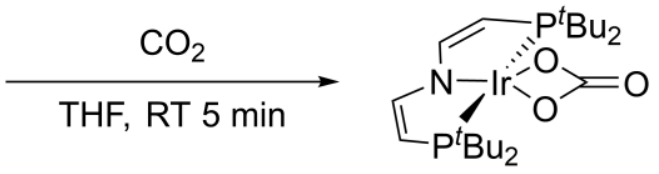

8

b)

c)

d)

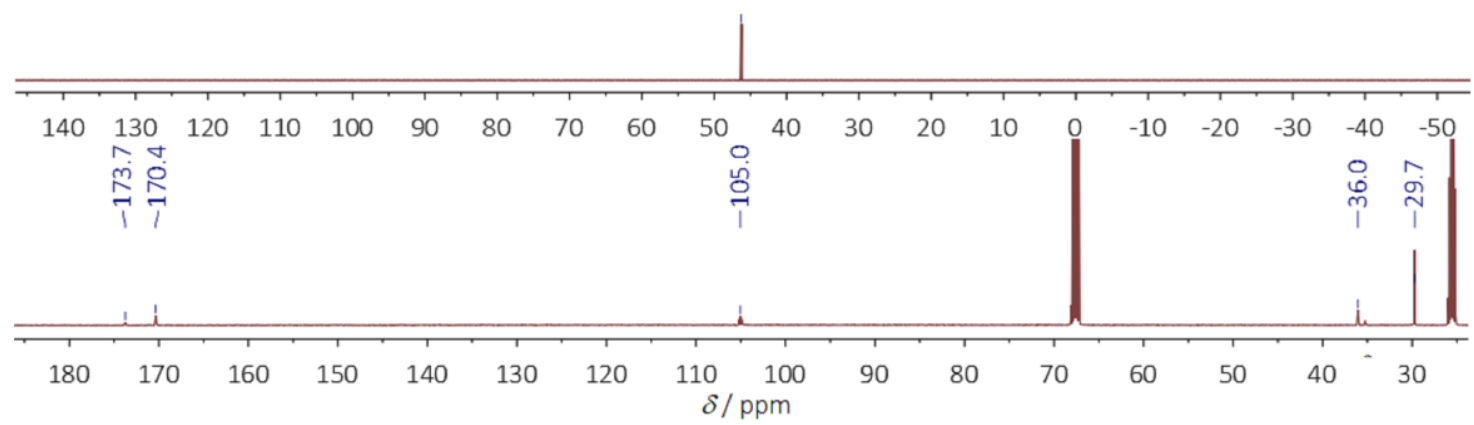

Figure 14: a) Synthesis scheme for the reaction of 4 with $\mathrm{CO}_{2}$ in THF at ambient temperature yielding the carbonate complex 5 . b) ${ }^{1} \mathrm{H}-\mathrm{NMR}$ spectrum of 5 in THF$d_{8}$ at ambient temperature. Signals marked with ${ }^{*}$ arise from $n$-pentane. c) ${ }^{31}$ P-NMR spectrum of 8 in THF- $d_{8}$ at ambient temperature. $\left.d\right){ }^{13} C$-NMR spectrum of 5 at ambient temperature. Signals marked with * arise from $n$-pentane.

The IR(ATR) spectrum exhibits three distinct sharp signals in the "carbonyl region" at $v=1663 \mathrm{~cm}^{-1}, 1625 \mathrm{~cm}^{-1}$ and $1553 \mathrm{~cm}^{-1}$, which as well supports the formation of a carbonate complex (Figure 15a). Crystallisation from few toluene at $-40{ }^{\circ} \mathrm{C}$ yields crystals suitable for XRD analysis and finally confirms the successful isolation of the 
iridium(III) carbonate complex [(PNP)Ir(CO 3$)] 8 .^{40}$ The metal centre in 8 is in square planar coordination (angle sum around $\left.\operatorname{Ir}=360^{\circ}\right)^{41}$ with two oxygen atoms of the carbonate unit coordinated to the metal centre at angles of $\mathrm{N}$ $\mid \mathrm{r}-\mathrm{O}_{1}=151^{\circ}$ and $\mathrm{N}-\mathrm{Ir}_{\mathrm{r}-\mathrm{O}_{2}}=146^{\circ}$. The Ir-O bonds are elongated compared to $4\left(d_{\mathrm{Ir}-01}=2.06 \AA\right.$ and $\left.d_{\mathrm{Ir}-02}=2.07 \AA\right)$ resembling their single bond character. The carbonyl atom has two different sets of $\mathrm{C}-\mathrm{O}$ bonds. The $\mathrm{C}-\mathrm{O}$ bond to the uncoordinated oxygen is significantly shortened $\left(d_{\mathrm{c}-03}=1.224(4) \AA\right)$ which indicates double bond character, while the bonds to the coordinating oxygen atoms are elongated ( $d_{\mathrm{c}-01}=1.32 \AA$ and $d_{\mathrm{c}-02}=1.31 \AA$ ) compared to "free" carbonate (Figure 15, b). ${ }^{[180]}$

a)

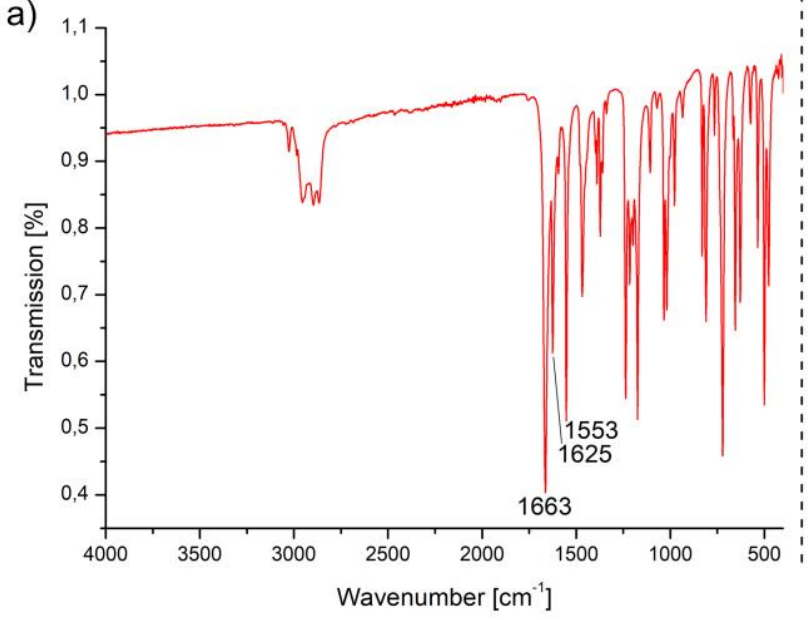

b)

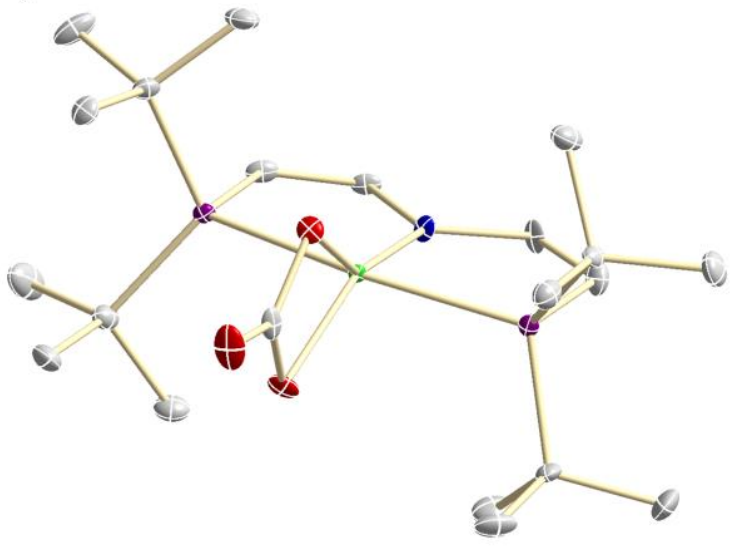

Figure 15: a) IR(ATR) spectrum of $\mathbf{8}$ b) Molecular structure in the solid state of $\mathbf{8}$ obtained by X-ray single crystal diffraction. The ORTEP plot shown with anisotropic displacement parameters at $50 \%$. All C-H hydrogen atoms, disorder and a cocrystallised toluene molecule omitted for clarity. Selected parameters: Ir- ${ }_{1}: 2.061(2)$ $\AA$, Ir-O $\mathrm{O}_{2}: 2.068(2) \AA \mathrm{Ir}-\mathrm{N}: 1.928(3) \AA$, Ir-P1: $2.3374(8) \AA$, Ir-P2: 2.3383(8) $\AA, \mathrm{C} 1=\mathrm{C} 2: 1.326(4), \mathrm{O}_{1}-\mathrm{C}_{21}: 1.317(4), \mathrm{O}_{2}-\mathrm{C}_{21}: 1.313(4), \mathrm{O}_{3}-\mathrm{C}_{21}: 1.224(4), \mathrm{N}-\mathrm{Ir}-\mathrm{O}_{1}: 151.47(10)^{\circ}$, $\mathrm{N}-\mathrm{Ir}-\mathrm{O}_{2}: 146.35(10)^{\circ}, \mathrm{P}-\mathrm{Ir}-\mathrm{P}: 163.75(3)^{\circ} \mathrm{N}-\mathrm{Ir}_{\mathrm{r}} \mathrm{C}_{21}: 177.38(12)^{\circ}$

The philicity of 4 was further analysed with trimethyl phosphine as an ambiphilic reagent. ${ }^{[37,181,182]}$ While the addition of 1 eq. $\mathrm{PMe}_{3}$ to a suspension of 4 in pentane at ambient temperature only results in partial conversion, the addition of 2 eq. leads to full conversion of the starting materials accompanied by the formation of a new complex and OPMe ${ }_{3}^{[183]}$ as determined by ${ }^{31} \mathrm{P}-\mathrm{NMR}$ spectroscopy (Figure 16a and c). ${ }^{42}$ Besides the main signal in the ${ }^{31} \mathrm{P}-\mathrm{NMR}$ at $\delta=61.5 \mathrm{ppm}$, a second signal at $\delta=61.3 \mathrm{ppm}$ can be observed, which is assigned to the hydrido phenyl complex $9[(\mathrm{PNP}) \operatorname{Ir}(\mathrm{H})(\mathrm{Ph})]$, this indicates an oxidative addition of the NMR solvent to an intermediately formed iridium(I) species. ${ }^{[179]}$ The NMR solvent was changed to cyclohexane- $d_{12}$ in which side reactivity only played a very minor role. The OPМез can be removed by washing with hexamethyldisiloxane (HMDSO) and crystallisation at $-40{ }^{\circ} \mathrm{C}$ yields material pure enough for further analysis. In the ${ }^{31} \mathrm{P}-\mathrm{NMR}$ spectrum, two sets of signals were found at $\delta_{2 \mathrm{P}}=61.75 \mathrm{ppm}, \delta_{\mathrm{P}}=-52.6 \mathrm{ppm}$ which integrate in a 2:1 ratio and exhibit P-P coupling $\left(J_{\mathrm{P}-\mathrm{P}}=16.9 \mathrm{~Hz}\right)$ and thus support the coordination of a second equivalent $\mathrm{PMe}_{3}$ to the iridium(I) complex intermediate, which is left after the oxygenation of the first equivalent of $\mathrm{PMe}_{3}$ (Figure 16e). ${ }^{43}$

\footnotetext{
${ }^{40}$ Picking and measurement of the crystal by Dr. Sebastian Forrest, solving of the crystal structure by Dr. Christian Würtele.

${ }^{41}$ Angle sum computed with the carbonyl $\mathrm{C}$ atom as the $4^{\text {th }}$ substituent.

42 The solvent is changed to the more unreactive pentane, compared to the published route, to encounter the reactivity of 10 the reaction time could drastically be reduced.

${ }^{43}$ Please note, that for a reliable integration of ${ }^{31} \mathrm{P}-\mathrm{NMR}$ spectra the $T_{1}$ time needs to be adjusted, which was not done here. Therefore, the integrated values can only be estimates.
} 
In analogy, the ${ }^{1} \mathrm{H}-\mathrm{NMR}$ spectrum exhibits a $\mathrm{C}_{2 \mathrm{v}}$ symmetric species with four sets of signals $\left(\delta_{2 \times \mathrm{CH}}=7.00 \mathrm{ppm}\right.$, $\delta_{2 \times \mathrm{CH}}=4.19 \mathrm{ppm}, \delta_{\mathrm{P}\left(\mathrm{CH}_{3}\right)_{3}}=1.82 \mathrm{ppm}$ and $\left.\delta_{4 \times C\left(\mathrm{CH}_{3}\right)_{3}}=1.31 \mathrm{ppm}\right)$ which integrate to 2:2:9:36. This further supports the coordination of $\mathrm{PMe}_{3}$ (Figure $16 \mathrm{~d}$ ). ${ }^{44}$ XRD analysis finally confirms the coordination of $\mathrm{PMe}_{3}$ to the formed iridium(I) fragment (Figure 16b). The iridium(I) PMe 3 complex [(PNP)Ir(PMe3)] 10 is in square planar coordination environment (angle sum around iridium $=360.0^{\circ}$ ) with one $\mathrm{PMe}_{3}$ ligand coordinating. The Ir- $\mathrm{N}$ bond length is, due to the trans-effect of the strongly $\sigma$-donating phosphine, elongated compared to the other complexes reported in this thesis. From this reactivity the philicity of $\mathbf{4}$ can be attributed to be of nucleo- to ambiphilic character.

a)

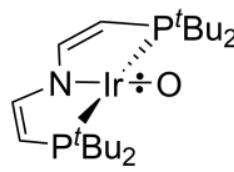

4

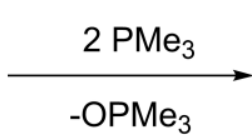

pentane, RT, $1 \mathrm{~h}$

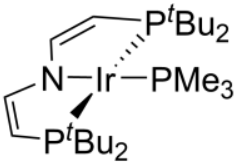

10 b)

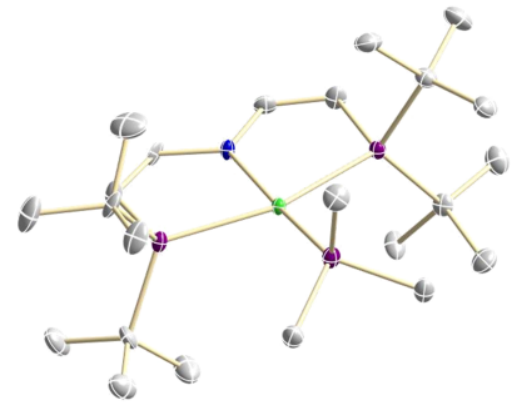

c)
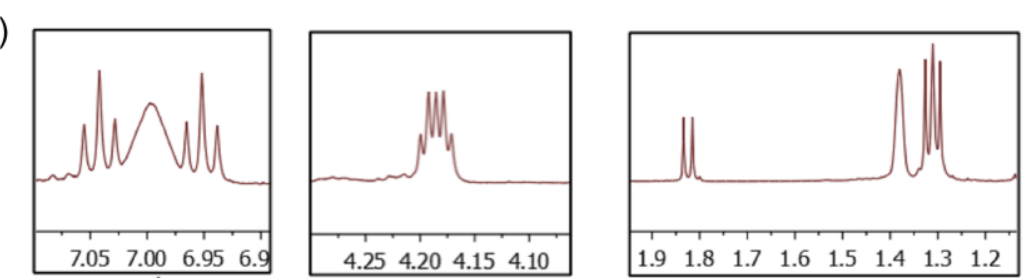

$\begin{array}{llllllllllll}1.9 & 1.8 & 1.7 & 1.6 & 1.5 & 1.4 & 1.3 & 1.2\end{array}$

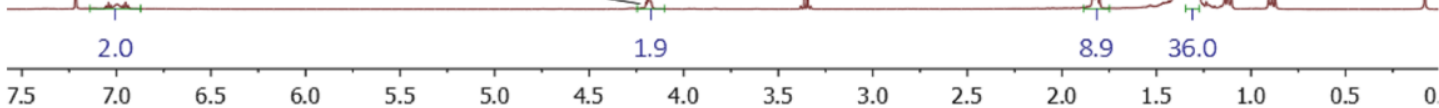

d)

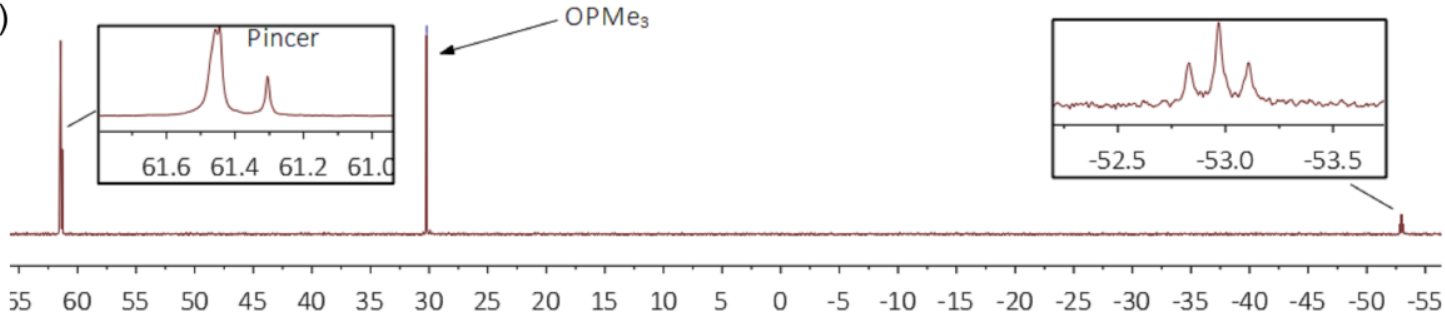

e)

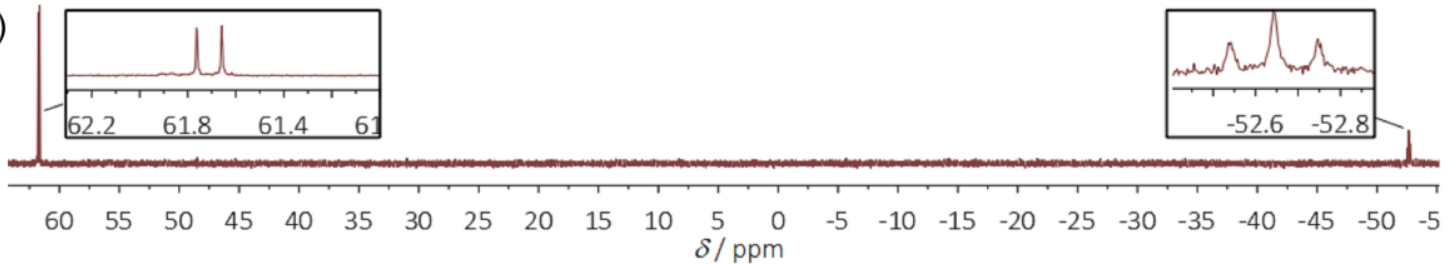

Figure 16: a) Synthetic scheme for the reaction of 4 with 2 eq. $\mathrm{PMe}_{3}$ to 10 and $\mathrm{OPMe}_{3}$ in THF at ambient temperature. b) Molecular structure in the solid state of 10 obtained by X-ray single crystal diffraction. The ORTEP plot shown with anisotropic displacement parameters at $50 \%$. All C-H hydrogen atoms are omitted for

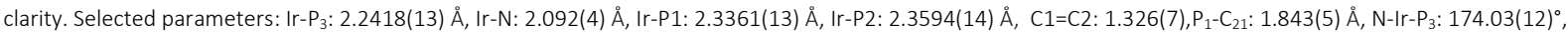
P-Ir-P: $160.37(4)^{\circ}$. c) ${ }^{31}$ P-NMR spectrum of the reaction mixture in $\mathrm{C}_{6} \mathrm{D}_{6}$ (reaction also carried out in $\mathrm{C}_{6} \mathrm{D}_{6}$ ). d) ${ }^{1} \mathrm{H}-\mathrm{NMR}$ spectrum of the 10 in cyclohexane- $\mathrm{d}_{12}$ at ambient temperature. e) ${ }^{31}$ P-NMR spectrum of 10 in cyclohexane- $d_{12}$. Signals marked with an asterix belong to pentane.

${ }^{44}$ This special solvent was chosen to partially overcome the thermal instability of 10 . 


\section{2. $B D(F) E$ determination of the hydroxo/oxo couple $2 / 4$}

An important measure for the ability of terminal oxo complexes to activate and oxygenate $\mathrm{C}-\mathrm{H}$ bonds, is the bond dissociation (free) energy $(\mathrm{BD}(\mathrm{F}) \mathrm{E})$ of the formed $\mathrm{O}-\mathrm{H}$ bond upon hydrogen atom transfer. Thus the $\mathrm{O}-\mathrm{H}$ bond strength of the hydroxo/oxo couple $2 / 4$ in THF was determined by isothermal titration calorimetry (ITC). ${ }^{45}$

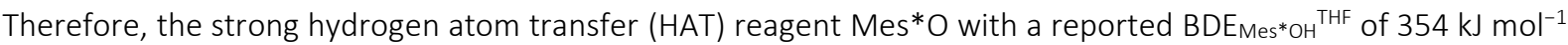
was chosen as a titrant to 2 (Figure 18a). ${ }^{[184]}$

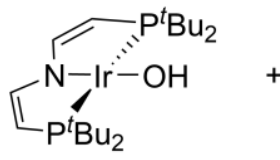<smiles>CC(C)(C)c1cc(Br)c(O)c(Br)c1</smiles>

${ }^{t} \mathrm{Bu}$

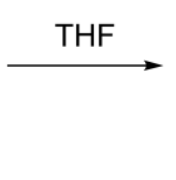<smiles>Oc1c(Br)cc(Br)cc1Br</smiles>

${ }^{\mathrm{t}} \mathrm{Bu}$

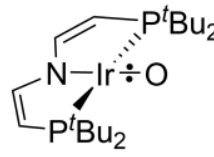

4

Scheme 2: Reaction analysed by isothermal titration calorimetry

The reaction is only slightly exothermic with a maximum heat rate of $16 \mu \mathrm{J} \mathrm{s}^{-1}$ and has no s-shaped character of the titration curve, which implies an equilibrium constant close to unity (Figure 18b). The background corrected integrated thermogram is fitted with an independent PCET model (for more information of this model see Section $\mathrm{C}$ in Chapter 7) and yields indeed a low reaction enthalpy of $\Delta H_{r}=-3.8 \mathrm{~kJ} \mathrm{~mol}^{-1}$ and an even slightly endergonic equilibrium constant $K=0.993$. Appealingly, the stoichiometry factor $n=0.998$ is very close to the ideal value of unity for an $A+B \rightarrow C+D$ reaction and indicates the clean conversion of 2 to 4 without severe weighting errors or impurities of the compounds (Figure 18c and d). The low driving force is not unexpected, since in Section 2 the synthetic hydrogen atom transfer (HAT) from 2 with Mes*O in THF also does not lead to full conversion but runs into an equilibrium. The driving force for the synthesis of 4 was here enhanced by an unpolar solvent to enable precipitation of 4 . With the literature known BDE value of Mes* $\mathrm{O}$ an experimental value for the $\mathrm{O}-\mathrm{H}$ bond of 2 can

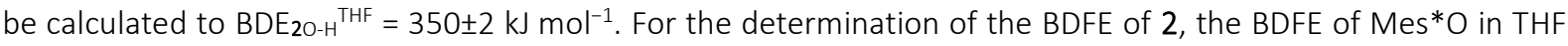
had to be determined first via a solvent transfer (MeCN $\rightarrow$ THF) utilizing the Abrahams solvation model as described by Mayer et al. (eq. 1).[53,185,186],46

\begin{tabular}{|c|c|c|c|c|}
\hline a) & $\alpha$ & $\beta$ & 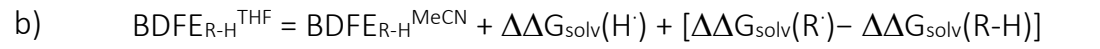 & eq. 1 \\
\hline THF & 0 & 0.51 & {$\left[\Delta \Delta G_{\text {solv }}\left(R^{\prime}\right)-\Delta \Delta G_{\text {solv }}(R-H)\right]=-41.92\left[\alpha_{\text {Mes* }}{ }^{\circ} \beta_{\text {MeCN}}-\alpha_{\text {Mes* }}{ }^{*} \beta_{\text {THF }}\right]=0.63 \mathrm{~kJ} \mathrm{~mol}^{-1}$} & eq. 2 \\
\hline MeCN & 0 & 0.22 & & \\
\hline Mes*OH & 0.22 & --- & BDFE $_{R-H}{ }^{T H F}=(323+1.03+0.63) \mathrm{kJ} \mathrm{mol}^{-1}=325 \mathrm{~kJ} \mathrm{~mol}^{-1}$ & \\
\hline
\end{tabular}
$\mathrm{MeCN}\left(\mathrm{BDFE}_{\mathrm{Mes}^{*} \mathrm{OH}} \mathrm{MeCN}^{\mathrm{M}}=323 \mathrm{~kJ} \mathrm{~mol}^{-1}\right)$ respectively. ${ }^{[53]} \Delta \Delta \mathrm{G}_{\text {solv }}(\mathrm{H})$ differential free energy of solvation between MeCN and THF of a hydrogen atom $\Delta \mathrm{G}_{\mathrm{THF}}{ }^{298.15 \mathrm{~K}}\left(\mathrm{H}_{2}\right)$

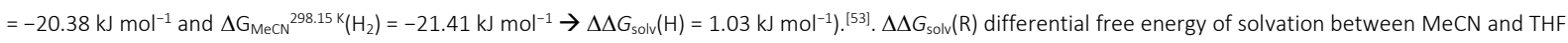
of the residual radical. $\Delta \Delta G_{\text {solv }}(R-H)$, the differential free energy of solvation between MeCN and THF of the HAT reagent. eq. 2: Calculation of the differential free energy of solvation between the solvents with the Abrahams parameters.

The free energy of solvation of the hydrogen atom was approximated by the free energy of solvation of $\mathrm{H}_{2}$ and the solvation difference of the residual radical Mes* $\mathrm{O}$ and $\mathrm{Mes} * \mathrm{OH}$ were obtained with the Abrahams parameters in eq. 2. The BDFE for Mes* OH in THF is calculated to $325 \mathrm{~kJ} \mathrm{~mol}^{-1}$ and therewith the BDFE of 2 is determined with

\footnotetext{
${ }^{45} \mathrm{~A}$ comprehensive description of the experiment can be found in Section 3.1 of chapter VI.

${ }^{46}$ Please note, that after this work a new reference value for Mes*O was published: $B D F E=311 \mathrm{~kJ} \mathrm{~mol}^{-1}{ }^{[.259]}$ Based on this value the $\mathrm{BDE}$ of Mes*O can be calculated (Approximating $\left.T S^{0}(H) \approx T S^{0}\left(H_{2}\right)=27 \mathrm{~kJ} \mathrm{~mol}^{-1}\right)^{[260]}$ to $B D E=338 \mathrm{~kJ} \mathrm{~mol}^{-1}$. Therefore, the values of 2 change to BDFE $=311 \mathrm{~kJ} \mathrm{~mol}-1 / B D E=334 \mathrm{~kJ} \mathrm{~mol}^{-1}$
} 
the experimental value of $\Delta G_{r}=0$ to $325 \pm 6 \mathrm{~kJ} \mathrm{~mol}^{-1}$. Additionally, via a square scheme approach, the $p K_{\mathrm{A}}$ value of the cationic hydroxo complex 3 can be calculated with the reported ${ }^{[187]} \mathrm{CH}_{\mathrm{H}}$ value of $66 \mathrm{kcal} \mathrm{mol}^{-1}$ in $\mathrm{THF}^{\mathrm{f}}$ to $\mathrm{p} K_{\mathrm{A}}^{30-\mathrm{H}}=19$.

a)

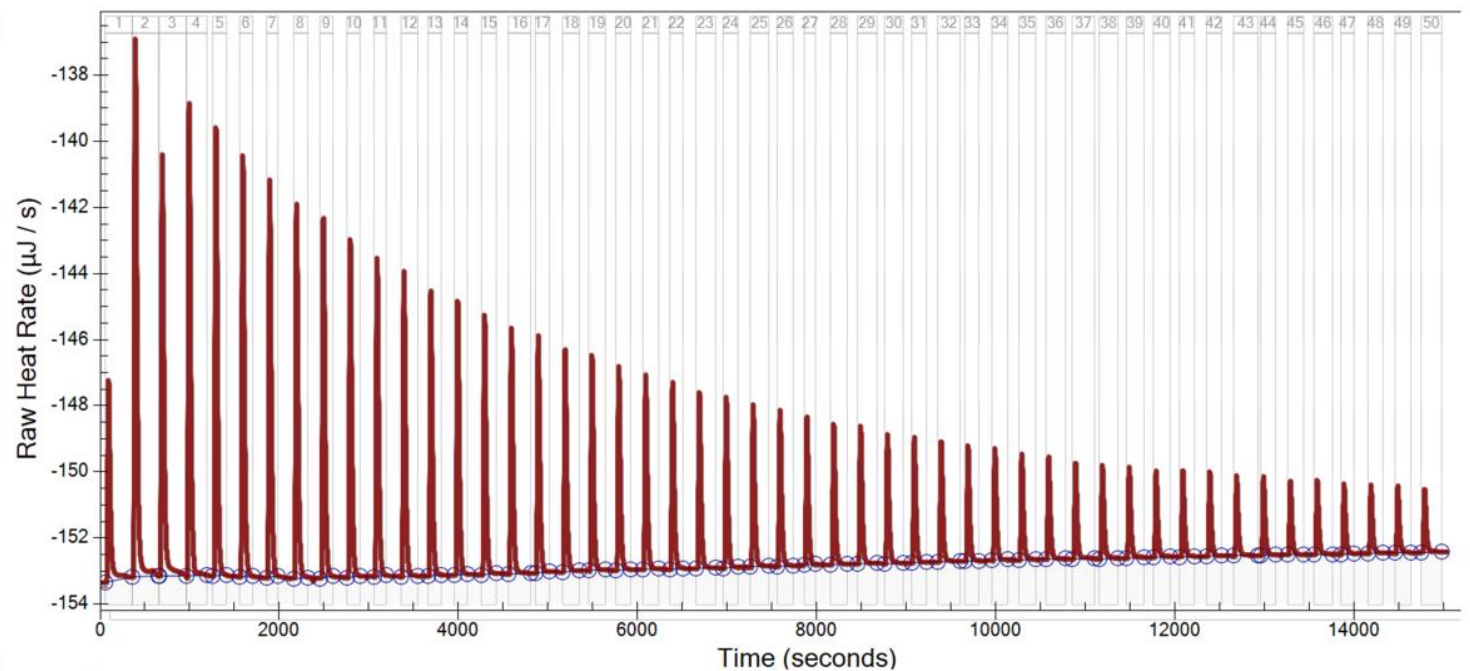

b)

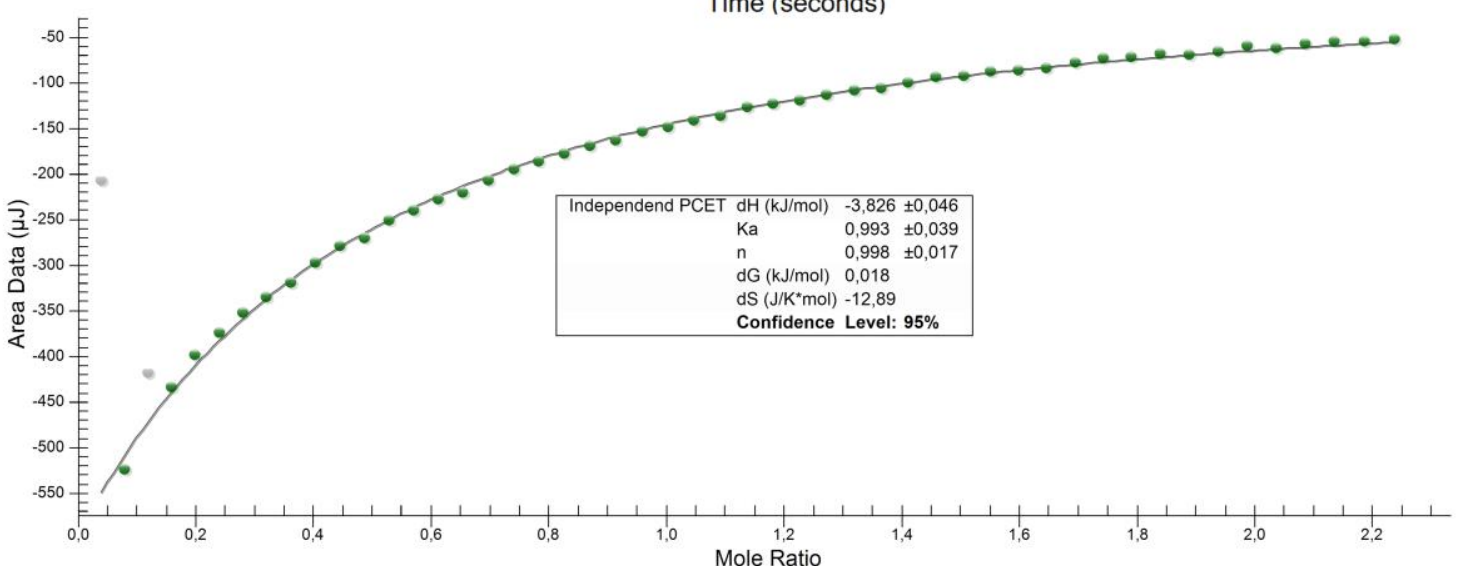

c)

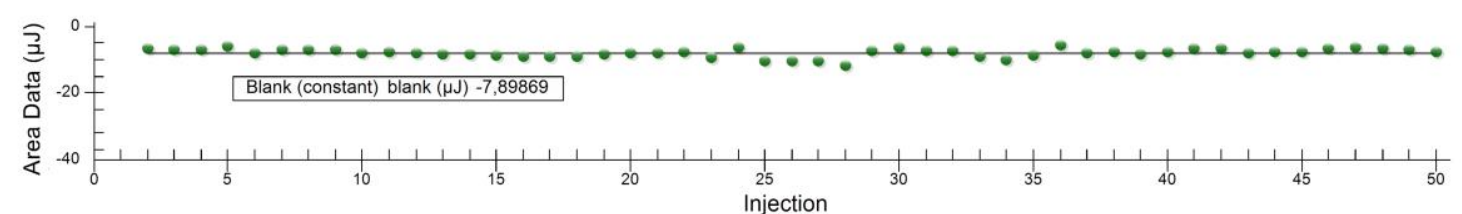

Figure 18: a) Raw heat curve of the titration of $2(2.0 \mathrm{mM}, 950 \mu \mathrm{L})$ with Mes* $(15.3 \mathrm{mM}, 250 \mu \mathrm{L})$ in THF at $25^{\circ} \mathrm{C}$ with fifty $5 \mu \mathrm{L}$ injection steps. b) Background corrected integrated thermogram fitted with an independent PCET model. c) Background of the titration, the first point was out of range and it not shown here.

The PCET reaction was additionally analysed by computational means with the high level ab initio techniques ONIOM(CCSD(T)-F12b/DFT $\left.{ }^{47}\right)$ and DLPNO, ${ }^{[95,97,98], 48}$ where the ONIOM energy is computed via eq. 3.

$$
E_{O N I O M}=E(\text { Truncation })_{C C S D(T)-F 12 b}-E(\text { Truncation })_{D F T}+E(\text { Fullsystem })_{D F T}
$$

Truncation: Referring to truncated model systems; Fullsystem: Referring to the untruncated systems.

Two different truncation levels were probed for the ONIOM approach, "H-truncation" where hydrogen atoms replace the tert-butyl groups on the complexes 2 and 4 and Mes*OH and Mes*O, respectively and "Me-

${ }^{47}$ DFT: B3LYP(V)-D3/def2TZVP + Solvation Correction (SMD:THF) on the converged structures.

${ }^{48}$ Computations performed by Dr. Martin Diefenbach. A comprehensive computational analysis of the thermochemistry can be found in Section 4.2 of chapter VI. 
truncation" where methyl groups replace tert-butyl groups. The ab initio methods also assign the triplet electromer of 4 as the ground state, but with a smaller triplet/singlet gap is smaller $(\Delta E(\mathrm{~T} / \mathrm{S}))^{a b}$ initio $=$ $37-41 \mathrm{~kJ} \mathrm{~mol}^{-1}$ ) compared to the value of the DFT computation $\left.(\Delta E(\mathrm{~T} / \mathrm{S}))^{\mathrm{DFT}}=74 \mathrm{~kJ} \mathrm{~mol}^{-1}\right)$. The DFT level of theory places the simple HAT reaction (Figure 19i and Table 1) of the complexes 4 and 2, forming a free hydrogen atom, at $322 \mathrm{~kJ} \mathrm{~mol}^{-1}$, whereas the ab initio techniques converge at much higher values. The DLPNO approach predicts for this reaction step a BDE of $349 \mathrm{~kJ} \mathrm{~mol}^{-1}$, while all ONIOM approaches converge at $339 \mathrm{~kJ} \mathrm{~mol}^{-1}$. The computation of the Mes*OH/Mes*O couple (Figure 19ii) is resulting in a BDE of $308 \mathrm{~kJ} \mathrm{~mol}^{-1}$ on the DFT level and a BDE of $335 \mathrm{~kJ} \mathrm{~mol}^{-1}$ at the DLPNO level of theory, while the results are more differing between the ONIOM approaches (Table 1). Here, the Mes* $\mathrm{O}-\mathrm{H}$ bond is predicted to be stronger, the higher the level of theory. While the $\mathrm{H}$ truncation on double-zeta basis is resulting in an BDE of $326 \mathrm{~kJ} \mathrm{~mol}^{-1}$ the Me-truncation triple-zeta basis computation results in a BDE of $331 \mathrm{~kJ} \mathrm{~mol}^{-1}$. All computational approaches predict an endergonic reaction enthalpy for reaction iii), while experimentally a slightly exothermic reaction was observed (Table 1). A possible explanation is the higher uncertainty on the Mes*OH/Mes*O couple for all computational methods, while the computation of the "pure" BDE of the $2 / 4$ couple (reaction i) yields comparable values to the experimental BDE of $350 \mathrm{~kJ} \mathrm{~mol}^{-1}$.

i)

ii)

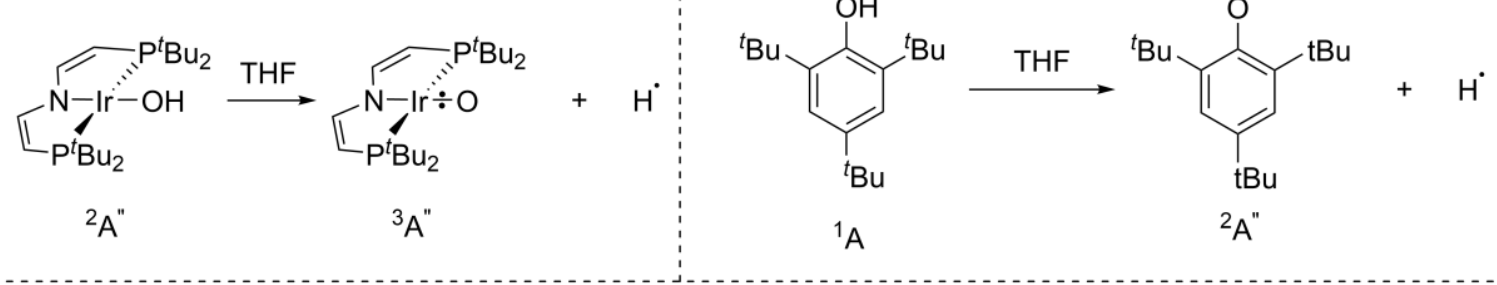

ii)

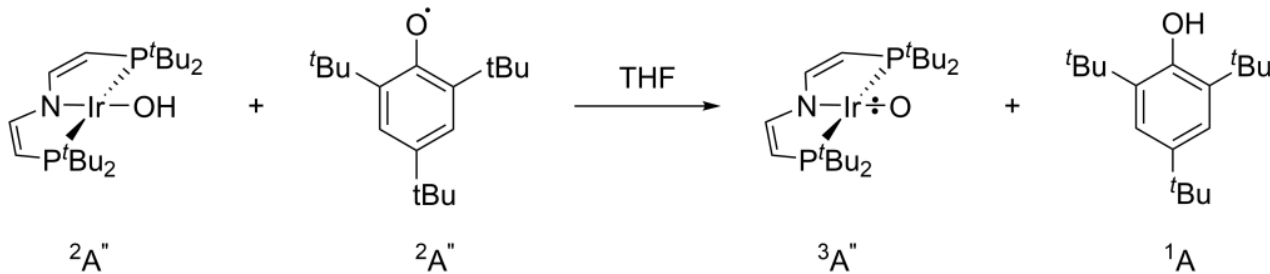

Figure 19: i) HAT reaction between 2 and $\mathbf{4}$ forming a free hydrogen atom. ii) HAT reaction between Mes*OH and Mes*O forming a free hydrogen atom. iii) PCET reaction between 2 and $\mathrm{Mes}^{*} \mathrm{O}$ forming iroxo and $\mathrm{Mes}^{*} \mathrm{OH}$.

Table 1: Summary table of the thermochemical computations a) All structures computed at the DFT(B3LYP(V)-D3/def2TZVP) level of theory values given in $\mathrm{kJ} \mathrm{mol}{ }^{-1}$. b) Triplet/Singlet gap on different levels of theory, Excitation energy at $0 \mathrm{~K}$ from the ${ }^{3} \mathrm{~A}^{\prime \prime}\left(\mathrm{C}_{\mathrm{s}}\right)$ to the lowest energy ${ }^{1} \mathrm{~A}^{\prime \prime}\left(\mathrm{C}_{\mathrm{s}}\right)$ electromer. c) Reaction enthalpy at $298 \mathrm{~K}$ of reaction i) including solvation in THF. d) Reaction enthalpy at $298 \mathrm{~K}$ of reaction i) including solvation in THF and spin-orbit coupling derived by CASSCFNEVPT2/QDPT $\Delta \Delta E_{\text {SOC }}=-2.9 \mathrm{~kJ} \mathrm{~mol}^{-1}$. e) Reaction enthalpy at $298 \mathrm{~K}$ of reaction ii) including solvation in THF. f) Reaction enthalpy at $298 \mathrm{~K}$ of reaction iii) including solvation in THF. g) Reaction enthalpy at $298 \mathrm{~K}$ of reaction iii) including solvation in THF and spin-orbit coupling derived by CASSCF-NEVPT2/QDPT $\Delta \Delta E_{\text {SOC }}=-2.9 \mathrm{~kJ}$

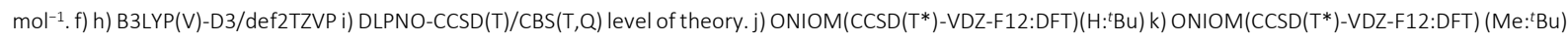
I) ONIOM(CCSD(T*)-VTZ-F12:DFT)(H:tBu) m) ONIOM(CCSD(T*)-VTZ-F12:DFT) (Me: $\left.{ }^{t} \mathrm{Bu}\right)$.

\begin{tabular}{|c|c|c|c|c|c|c|}
\hline Method $^{\text {a) }} / \mathrm{kJ} \mathrm{mol}^{-1}$ & $\Delta E(\mathrm{~T} / \mathrm{S})^{\mathrm{b})}$ & $\Delta H_{\text {solv }}{ }^{\mathrm{c})}$ i) & $\left.\Delta H_{\text {solv }}(\mathrm{SOC})^{\mathrm{d})} \mathrm{i}\right)$ & $\Delta H_{\text {solv }}{ }^{\mathrm{e})} \mathrm{ii}$ & $\Delta H_{\text {solv }}{ }^{\text {f) }}$ iii) & $\left.\Delta H_{\text {solv }}(\mathrm{SOC})^{\mathrm{g})} \mathrm{iii}\right)$ \\
\hline $\mathrm{DFT}^{\mathrm{h})}$ & 74.0 & 321.8 & 324.7 & 308.3 & 16.4 & 13.5 \\
\hline DLPNOi) & 41.1 & 349.1 & 352.1 & 335.1 & 17.0 & 14.1 \\
\hline ONIOM(H:VDZ-F12b)j) & 36.7 & 338.6 & 341.5 & 326.4 & 15.1 & 12.2 \\
\hline ONIOM(Me:VDZ-F12b)k) & 36.9 & 339.0 & 342.2 & 327.6 & 14.5 & 11.6 \\
\hline ONIOM(H:VTZ-F12b)!) & 39.9 & 339.3 & 341.9 & 329.2 & 12.7 & 9.8 \\
\hline ONIOM(Me:VTZ-F12b) & 40.5 & 339.4 & 342.3 & 330.6 & 11.7 & 8.8 \\
\hline
\end{tabular}


The spin-orbit coupling effect determined by multi-reference treatment of 2 and 4 in Section 2 (Figure 11, $\Delta \Delta E_{\text {soc }}$ $=-2.9 \mathrm{~kJ} \mathrm{~mol}^{-1}$ ) is almost closing the gap between experiment and theory. The rather small value of the SOC effect was however unexpected. In a simple picture, 2 with its doublet ground state, only has one electron on the heavy nucleus which experiences spin-orbit coupling, while $\mathbf{4}$ is in a triplet ground state with two unpaired electrons which can be influenced by SOC. In this simple picture, 4 should be more stabilized by SOC than 2 . A possible explanation for the small SOC effect comes from the computed spin-density plots of 2 and $\mathbf{4}$ on the DFT and NBONPA level of theory (Figure 20). While the SOMOs of 4 predominantly exhibit Ir-O $\pi^{*}$-character, which results in an equal spin-density distribution over iridium and oxygen, the spin-density of 2 is mainly focused on the metal centre. The experimental and computational analysis of $\mathbf{4}$ is therewith representing an unprecedented iridium oxo biradical with strong oxyl radical character. The overall spin density at the iridium metal centre of 4 therefore does no change significantly compared to 2 upon PCET, which results in similar SOC stabilisations and thus no substantial overall SOC effect on the thermochemistry. This is a contrary situation as the quenching of the SOC effect by the ligand field commonly proposed in the literature. ${ }^{[134,136-138]}$

a)

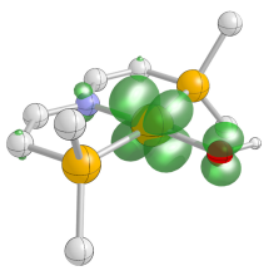

2

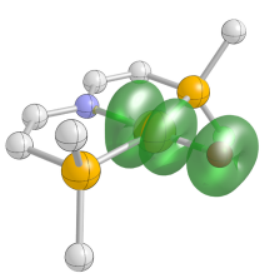

4 b)

\begin{tabular}{|c|cc|c|c|}
\hline \multirow{2}{*}{ Spin Density } & \multicolumn{2}{|c|}{$\mathbf{2}$} & \multicolumn{2}{c|}{$\mathbf{4}$} \\
\cline { 2 - 5 } & Ir & O & Ir & O \\
\hline Mulliken & 0.77 & 0.13 & 0.95 & 0.88 \\
\hline NBO & 0.72 & 0.16 & 0.89 & 0.94 \\
\hline
\end{tabular}

Figure 20: a) B3LYP(V)-D3/def2TZVP spin-density plots for the triplet ground state of $\mathbf{4}\left({ }^{3} \mathrm{~A}^{\prime \prime}\right)$ (top) and the doublet ground state of $2\left({ }^{2} \mathrm{~A}\right.$ ) $)($ bottom) shown at isovalue $0.0075 \mathrm{a}_{0}^{-3}$. b) Mulliken and NBO/NPA analysis of the spin density of 2 and 4 .

With all these thermodynamic parameters at hand, a full square scheme for all discussed oxo species can be formulated (Figure 21). The $p K_{A}$ value of 19 of 3 is much more acidic than for $2\left(p K_{A}=46\right)$ but still in the medium range in THF, thus 4 is stabilized as a weak base. The irreversible reduction in the cyclic voltammogram of 4 can be easily explained by the super base character of the formed anionic oxo 4- which might even be capable of deprotonating the solvent THF itself. The $\mathrm{O}-\mathrm{H}$ bond strength rises within the hydroxo redox series. The anionic hydroxo complex 1 has the lowest BDE with $336 \mathrm{~kJ} \mathrm{~mol}^{-1}$, therefore the anionic oxo complex 4- will favour deprotonation over hydrogen atom abstraction. The BDE of neutral oxo complex 4 lies directly in the middle and is with a BDE value of $350 \mathrm{~kJ} \mathrm{~mol}^{-1}$ strong enough for the activation of weak $\mathrm{C}-\mathrm{H}$ bonds but is not sufficient for the cleavage of the strong $\mathrm{C}-\mathrm{H}$ bonds of most solvents. The same picture arises for the $\mathrm{p} K_{\mathrm{A}}$ value of 4 and thus explains why 4 is stable in the first place. The cationic hydroxo complex 3 is bearing the strongest $\mathrm{O}-\mathrm{H}$ bond with $372 \mathrm{~kJ} \mathrm{~mol}^{-1}$. A comparison of the spin densities at the oxygen atom of 4 (0: $\left.95 \%\right)$ and $4+(0: 75 \%)$ reveals, that the more reactive cationic oxo 4+ has less radical character, which supports the statement of James Mayer that "Not radical character but bond strength is defining radical reactivity". ${ }^{25]}$ 


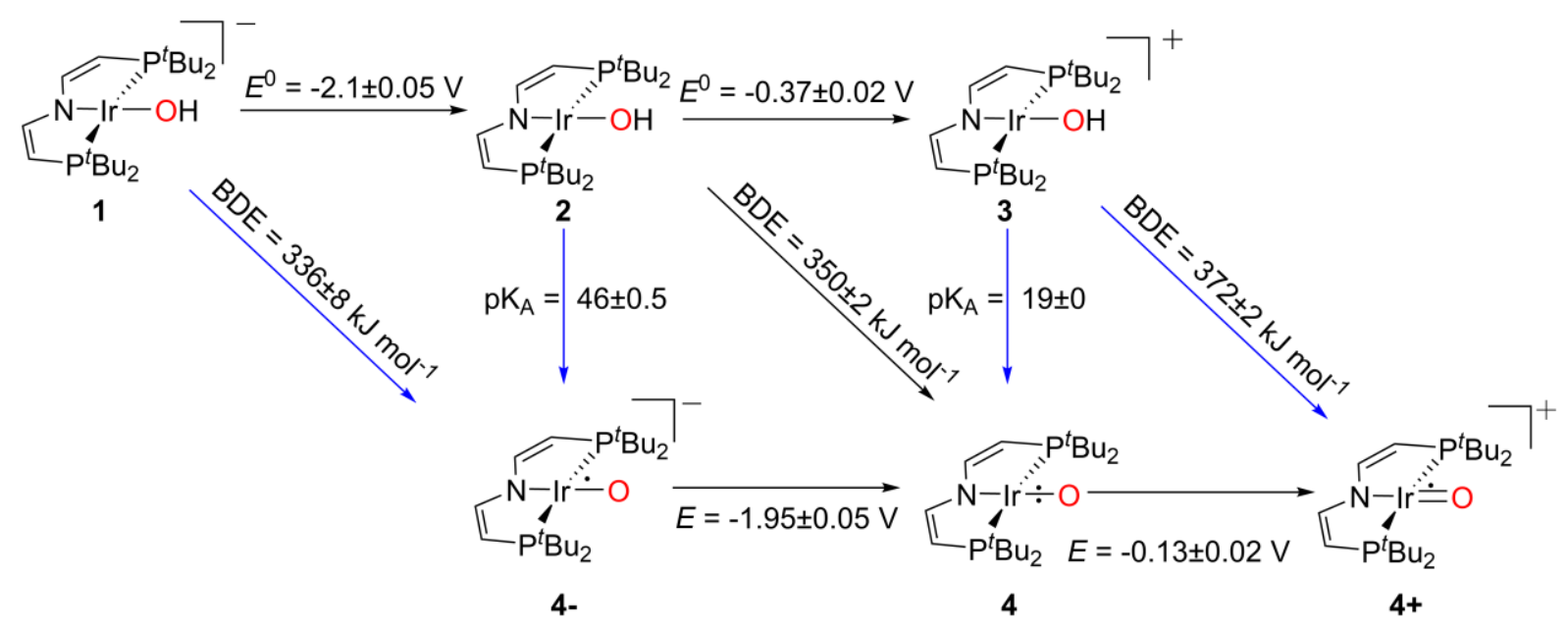

Figure 21: Thermodynamic parameters of $4-, 4$ and $4+$ with a square scheme approach: Blue $=$ calculated values via $B D E^{T H F}=23.06 E^{0}+1 \cdot 37 p K_{A}+66^{[187]}$. The errors for the cyclic voltammograms are taken from upper limits standard errors of the technique. For the irreversible processes the error is assumed to be higher. Please note, that for reliable data only electrochemically reversible signals should be considered, thus the values shown here give a qualitative picture.

\section{3. $\mathrm{C}-\mathrm{H}$ bond Activation Chemistry of 4}

With the $\mathrm{O}-\mathrm{H}$ bond strength of 2 at hand $\left(\mathrm{BDE}=350 \mathrm{~kJ} \mathrm{~mol}^{-1}, \mathrm{BDFE}=325 \mathrm{~kJ} \mathrm{~mol}^{-1}\right)$, the $\mathrm{C}-\mathrm{H}$ bond activation capability of 4 is analysed. $\mathrm{C}-\mathrm{H}$ bonds belong to the strongest bonds in nature with BDE values up to $558 \mathrm{~kJ} \mathrm{~mol}^{-1}$ ( $\mathrm{HC}=\mathrm{C}-\mathrm{H}$, gas), $472 \mathrm{~kJ} \mathrm{~mol}^{-1},\left(\mathrm{C}_{6} \mathrm{H}_{6}\right.$, gas) or $439 \mathrm{~kJ} \mathrm{~mol}^{-1}\left(\mathrm{CH}_{4}\right.$, gas), and can surpass elemental hydrogen gas (436 kJ $\left.\mathrm{mol}^{-1}\right) \cdot{ }^{[53]}$ The $\mathrm{C}-\mathrm{H}$ bond strength decreases when the formed carboradicals can be stabilized by delocalisation over larger $\pi$-systems or when aromaticity can be reached upon HAT (Figure 22).

i)<smiles>[CH](c1ccccc1)c1ccccc1</smiles>

$\mathrm{BDE}=352 \mathrm{~kJ} \mathrm{~mol}^{-1}$ ii)<smiles>c1ccc(C(c2ccccc2)c2ccccc2)cc1</smiles>

$\mathrm{BDE}=349 \mathrm{~kJ} \mathrm{~mol}^{-1}$ iii)<smiles>C1=CCC=C1</smiles>

iv)<smiles>c1ccc2c(c1)Cc1ccccc1-2</smiles>

$\mathrm{BDE}=345 \mathrm{~kJ} \mathrm{~mol}^{-1}$ v)<smiles>C1=Cc2ccccc2C1</smiles>
DMSO

DMSO

vi)<smiles>c1ccc2c(c1)Cc1ccccc1C2</smiles>

$\mathrm{BDE}=337 \mathrm{~kJ} \mathrm{~mol}^{-1}$ vii)<smiles>c1ccc2c(c1)Cc1ccccc1O2</smiles>

$\mathrm{BDE}=326 \mathrm{~kJ} \mathrm{~mol}^{-1} \quad \mathrm{BDE}=318 \mathrm{~kJ} \mathrm{~mol}^{-1}$

\section{DMSO}

viii)

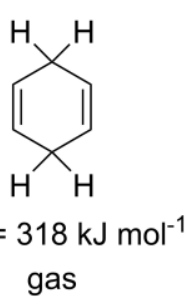

Figure 22: Selection of $\mathrm{C}-\mathrm{H}$ bond strength in the range of 4 sorted by BDE. The weak $\mathrm{C}-\mathrm{H}$ bonds are depicted for clarity. For 1,4-cyclohexadiene (viii) no value in DMSO was found. ${ }^{[53]}$

The selective oxygenation of $\mathrm{C}-\mathrm{H}$ bonds is a fundamental process in nature and an active field in chemical research, thus the most desired reactivity of 4 would be the formation of C-O bonds after the initial HAT reaction. ${ }^{[188,189]}$ For HAT reagents with two active HAT sites like cyclohexadiene or dihydroanthracene (Figure 22vi and viii, respectively) the driving force towards the aromatic products benzene and anthracene will most likely by higher than towards the formation of an alkoxide complex (Figure 23a). On the other hand, reagents with only one active hydrogen atom can form an intermediate carboradical which can further react with a second equivalent of 4 to form a C-O bond (Figure 23b). 
a)

2

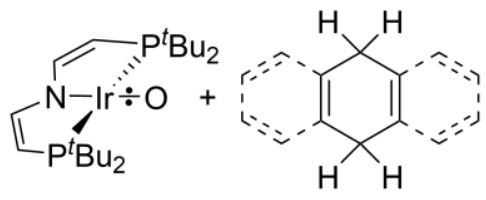

b)

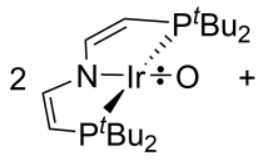

)

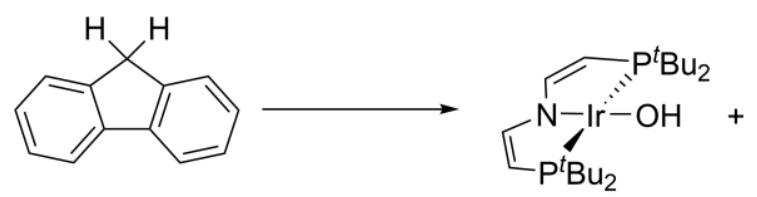

2

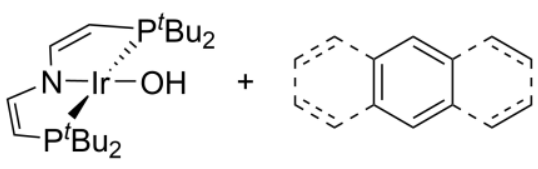

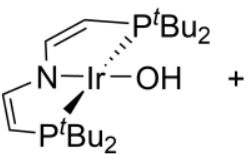<smiles></smiles>

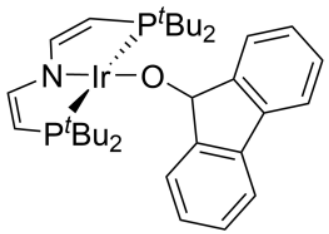

Figure 23: a) Expected reactivity of $\mathbf{4}$ with HAT donor bearing two active hydrogen atoms forming only 2 and the aromatic by-product. b) Expected reactivity of 4 with single HAT donor reagents with the example of fluorene.

Therefore, single HAT reagents were reacted with 4. Stirring a solution of xanthene (Figure 22vii) and two equivalents of 4 in THF overnight at $60{ }^{\circ} \mathrm{C}$ leads to a colour change to green blue (Figure 24, a). The ${ }^{1} \mathrm{H}-\mathrm{NMR}$ (para) spectrum shows an aromatic, diamagnetic species and two paramagnetic species with main peaks at $\delta=8.2 \mathrm{ppm}$, $\delta=7.2 \mathrm{ppm}$ and $\delta=6.2 \mathrm{ppm}$. Here, the signal at $\delta=7.2 \mathrm{ppm}$ can be assigned to hydroxo complex 2 (Figure 24b). Column chromatography over silanized silica enables the separation of both complexes but the organic, diamagnetic compound in the aromatic region of the spectrum cannot be separated on this way (Figure 24c). LIFDI-mass spectrometry gives one single complex product signal at $\mathrm{m} / \mathrm{z}=746.2$ hinting towards the desired $\mathrm{C}-\mathrm{O}$ bond formation towards an [(PNP)Ir(II)(xanthenolate)] complex 11 (Calculated: $\mathrm{m} / \mathrm{z}=746.3$ ) (Figure 24d). Structural proof of the C-O bond formation was obtained by XRD analysis (Figure 24e). The xanthenolate complex 11 is in a square-planar coordination environment (angle sum around Ir $=360^{\circ}$ ), but the $\mathrm{N}-\mathrm{Ir}-\mathrm{O}$ and O-Ir-P1\# angles deviate from an ideal square-planar coordination $\left(167^{\circ}\right.$ and $111^{\circ}$, respectively). The elongated $\mathrm{Ir}-\mathrm{O}$ bond of $d_{\mathrm{r}-\mathrm{O}}=$ $1.98 \AA$ and the Ir-O-C angle of $130^{\circ}$ indicates no multibonding character in the Ir-O unit or in the xanthene fragment. A closer look at the ${ }^{1} \mathrm{H}$-NMR spectra reveals two main tert-butyl peaks for 11 suggests a $\mathrm{C}_{\mathrm{s}}$ symmetric structure in solution. The integration of both peaks ( $\delta=8.2 \mathrm{ppm}$ and $\delta=6.2 \mathrm{ppm}$ ) of 11 combined does however not reach the integral of the main peak of 2 which speaks for a non-selective reaction. Here, the organic side product helps to identify the second reaction pathway and gives inside into the mechanism of the reaction. The signal at $\delta=4.0 \mathrm{ppm}$ in the ${ }^{1} \mathrm{H}-\mathrm{NMR}$ spectrum can be assigned to the $\mathrm{C}_{\text {sp3 }}-\mathrm{H}$ proton of dimeric xanthene, integrating $4: 2$ with the aromatic protons, instead of 2:2 for monomeric xanthene. ${ }^{[190]}$ Therefore, the first step in the xanthene activation is the hydrogen atom abstraction forming one molecule of 2 and an intermediate xanthenyl radical. At this point the reaction pathways divide to 1) C-O bond formation between a second equivalent of 4 and the xanthenyl radical and 2) Dimerization of two xanthenyl radicals towards bixanthene. Independent synthetic routes towards 11, like ligand substitution to the chlorine complex 5 or the amide complex [(PNP)|rNH$\left.H_{2}\right] 13$ with free xanthenol under basic conditions unfortunately failed by now, which hinders the full characterisation of 11 . 
a)

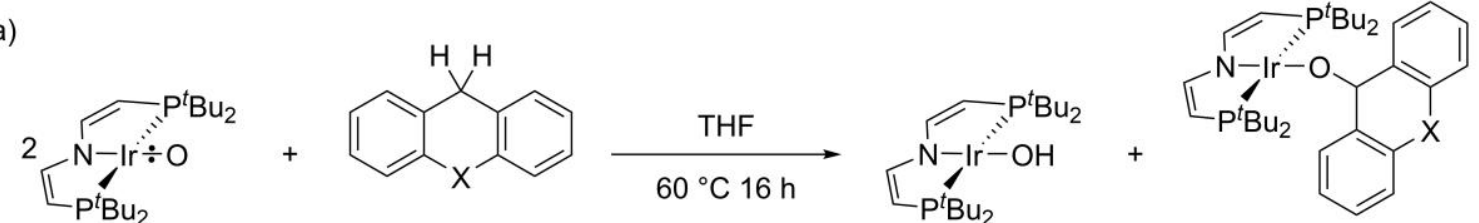

4 $\mathrm{X}=$ none $/ \mathrm{O}$

2

$X=O(11)$, none (12)

b)

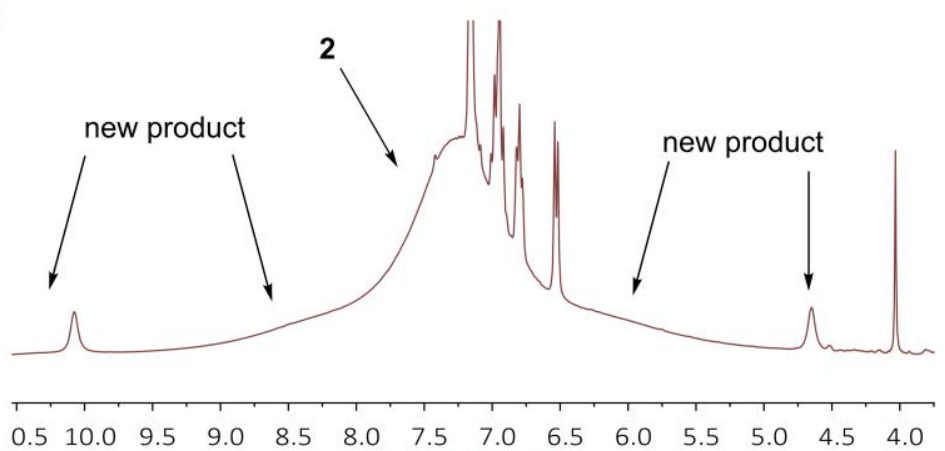

c)

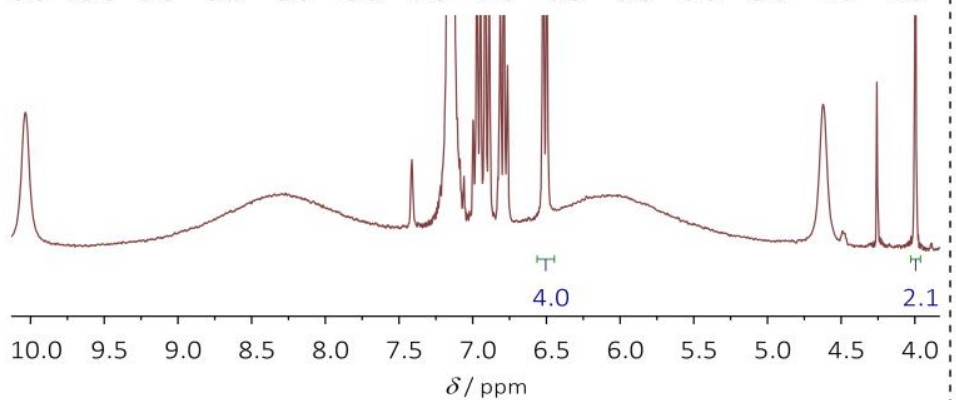

d)

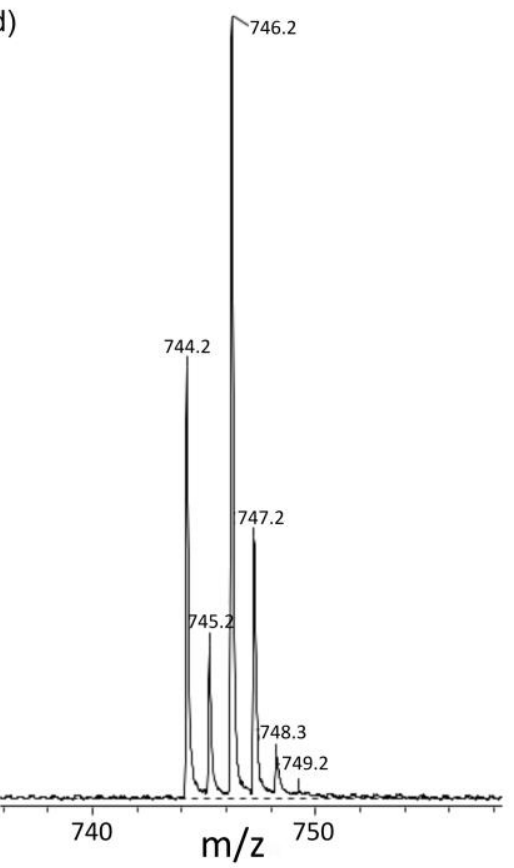

e)

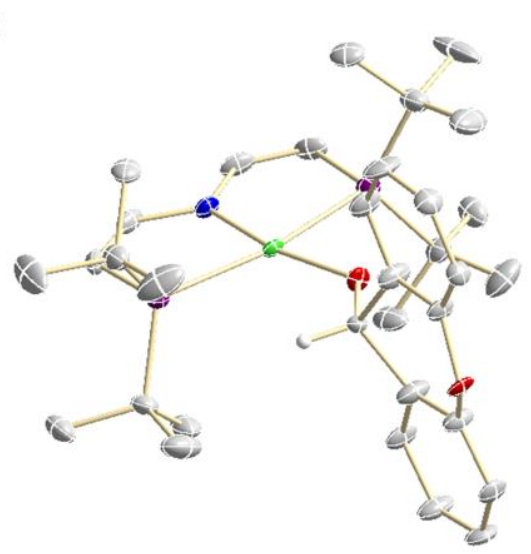

f)

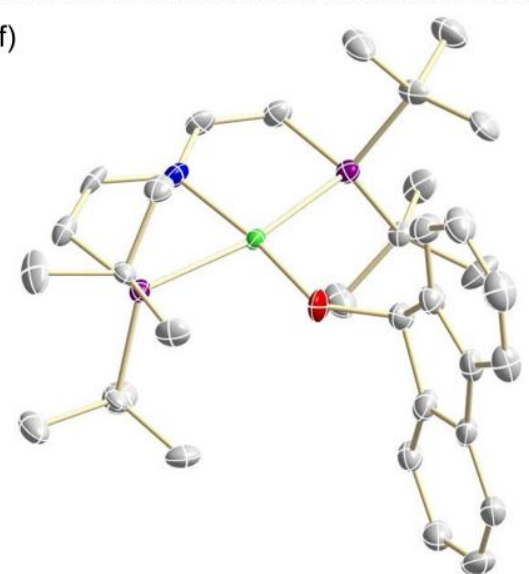

Figure 24: a) Reaction of 4 in THF at $60^{\circ} \mathrm{C}$ for $16 \mathrm{~h}$ with xanthene $(\mathrm{X}=\mathrm{O})$ or fluorene ( $\mathrm{X}=$ none) forming 2 and 11 or 12 , respectively. b) ${ }^{1} \mathrm{H}($ para)-NMR spectrum of the reaction mixture for xanthene in $\mathrm{C}_{6} \mathrm{D}_{6}$ at $25^{\circ} \mathrm{C}$. c) Pentane phase of the reaction mixture after column chromatography over silanized silica for xanthene. $d$ ) LIFDI-mass spectrometry results of 12, creation parameters: Average(MS[1] Time 0.66..0.72). e+f) Molecular structure in the solid state obtained by XRD analysis of $11(\mathrm{e})$ and 12 (f), respectively. The ORTEP plots are shown with anisotropic displacement parameters at $50 \%$. All C-H hydrogen atoms and disorder of the molecule omitted for clarity. Selected parameters 11: Ir- $\mathrm{O}_{2}: 1.983(5) \AA$, Ir- $\mathrm{N}: 1.988(4) \AA$, Ir-P1: 2.3327(9) $\AA, \mathrm{C} 1=\mathrm{C} 2: 1.327(6) \AA, \mathrm{O}_{2}-\mathrm{C}_{23}: 1.385(8) \AA, \mathrm{N}-\mathrm{Ir}-\mathrm{O}_{2}$ :

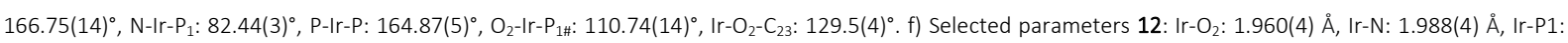

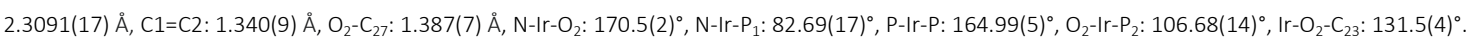

The same picture arises, when 4 is reacted with fluorene (Figure 22iv) with the corresponding [(PNP)Ir(II)(fluorenolate)] complex 12 as the main product, besides other diamagnetic species. ${ }^{49}$.The solid state structure of 12 resembles the structure of 11 (Figure 24f) but with a smaller angle distortion of the N-Ir-O and $\mathrm{P}_{2-}$

${ }^{49}$ One signal can be assigned to the dihydride complex 6, while the other one is commonly observed with wet reagents which speaks for an impurity of fluorene. 
Ir-O, which can be ascribed to the lower steric demand of the fluorenolate ligand. It should be noted that the reaction of 4 with xanthene also proceeds slowly at room temperature, while heating to $60{ }^{\circ} \mathrm{C}$ is mandatory for the reaction with fluorene, which reflects the decreasing driving force $(\mathrm{BD}(\mathrm{F}) \mathrm{E}$ difference) of the two compounds compared to 2 .

Benzaldehydes are an ideal platform to test for the concurrence reaction between HAT abstraction of the aldehyde $\mathrm{C}-\mathrm{H}$ proton vs. a nucleophilic attack at the carbonyl atom, since they feature a relatively weak and sterically unprotected $\mathrm{C}-\mathrm{H}$ bond $\left(\mathrm{BDE}\left(\mathrm{PhC}(\mathrm{O})-\mathrm{H} \sim 365 \mathrm{~kJ} \mathrm{~mol}^{-1}\right)^{[191]}\right.$ and an electrophilic carbonyl group in close proximity. Thermal reactivity could not be observed, but the filter less irradiation with a mercury arc lamp of 4 in benzene with 10-fold excess of the benzaldehyde is yielding two paramagnetic products (Figure 25b).

a)

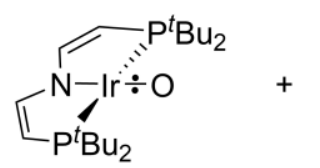<smiles>[X]c1ccc(C=O)cc1</smiles>

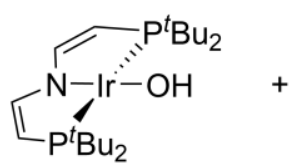<smiles></smiles>

4 $\mathrm{X}=\mathrm{F}, \mathrm{H}, \mathrm{Me}, \mathrm{OMe}$

14-X = F, H, Me, OMe

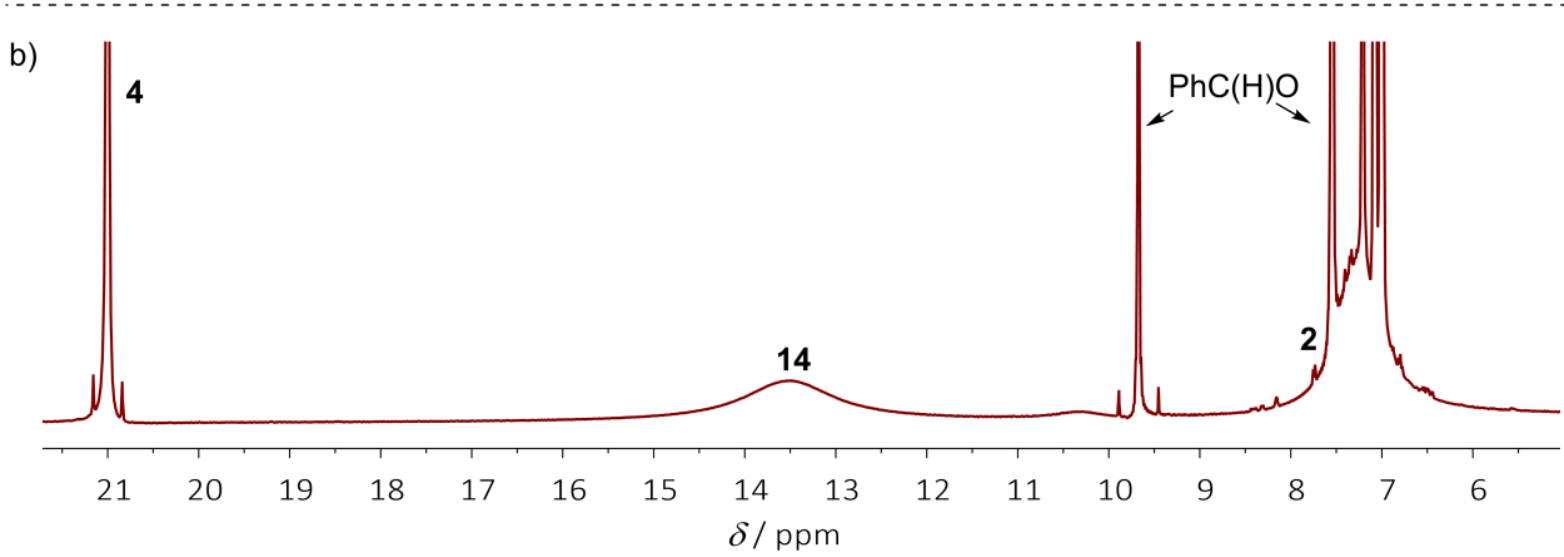

Figure 25: a) Irradiation of 4 with a mercury arc lamp (no filter) in benzene with different benzaldehydes (10 eq.) present (X=F, H, Me, OMe) forming 2 and the corresponding benzoate complex. 9-x. b) ${ }^{1} \mathrm{H}$-para NMR spectrum after 30 min irradiation of 4 with benzaldehyde $\mathrm{X}=\mathrm{H}$ after $30 \mathrm{~min}$.

From the characteristic peak at $\delta=7.2 \mathrm{ppm}$ in the ${ }^{1} \mathrm{H}-\mathrm{NMR}$ the formation of hydroxo complex 2 can be concluded, while besides 2 a second paramagnetic product with signals at $(\delta \sim 13 \mathrm{ppm}, \delta=4.9 \mathrm{ppm}, \delta=2.8 \mathrm{ppm}, \delta=-4.9$ ppm and $\delta=-5.7 \mathrm{ppm}$ ) can be observed, suggesting the coordination of benzaldehyde to 4 . A rational guess for benzaldehyde activation is the HAT transfer of the substrate to one molecule 4, forming 2 , followed by the oxygenation of the benzaldehyde residue with a second equivalent of 4 , which yields the corresponding [(PNP)Ir $\left.\left(\mathrm{O}_{2} \mathrm{CPh}-\mathrm{X}\right)\right]$ complex 14-x, analogous to the reaction with xanthene and fluorene. To test this hypothesis an independent synthetic route towards 14-x was probed. Reaction of the corresponding benzoic acid with the iridium amido complex 13, yields one paramagnetic product with the exact same NMR signature as observed in the irradiation experiment, confirming the oxygenation of benzaldehydes by 4 under irradiative conditions (Scheme 3). 


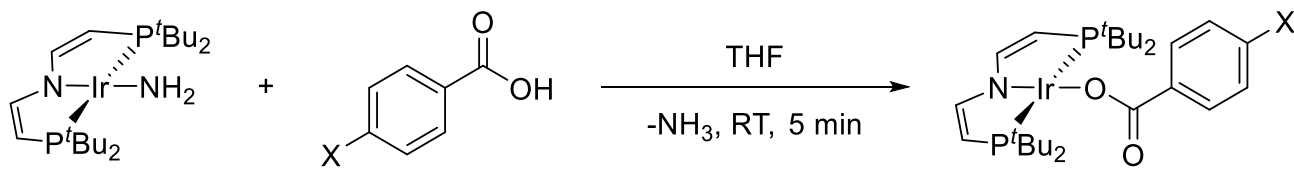

13

Scheme 3: Independent synthetic route towards $9-\mathbf{x}$ by reaction of 13 with the corresponding benzoic acid in THF at ambient temperature.

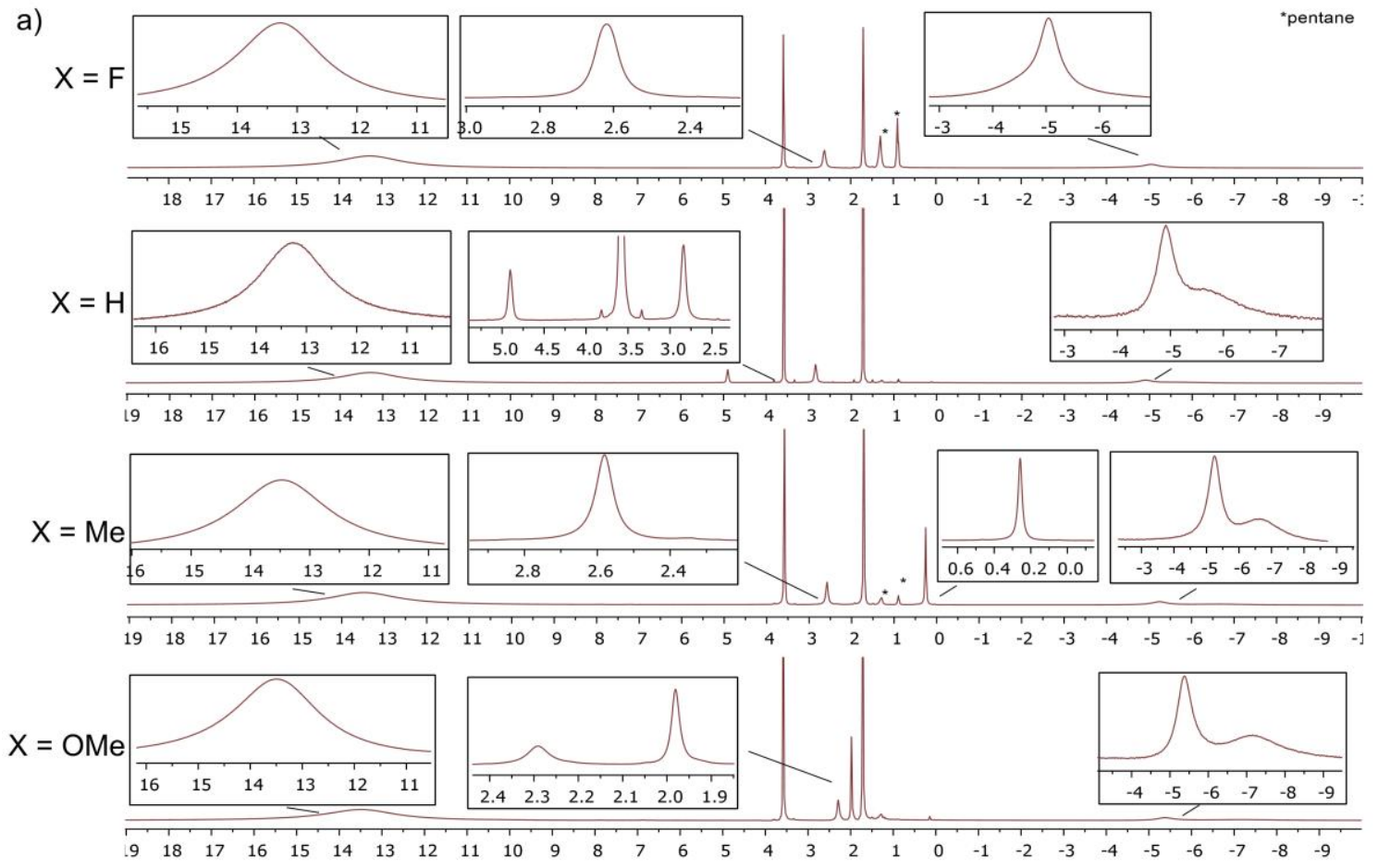

b)

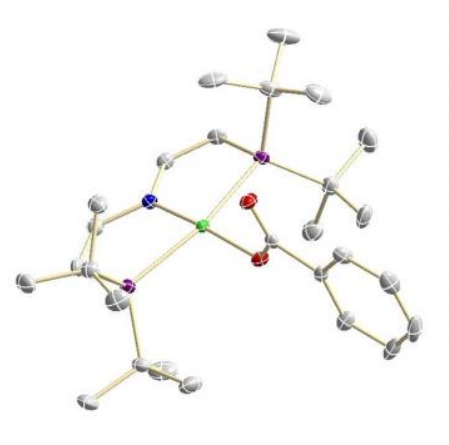

c)

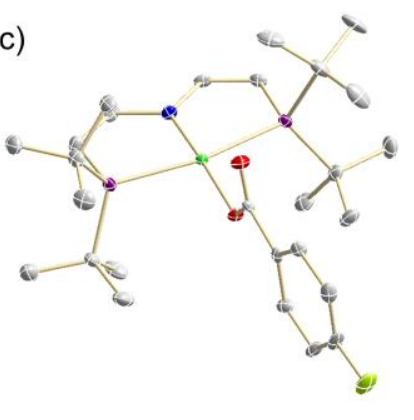

d)

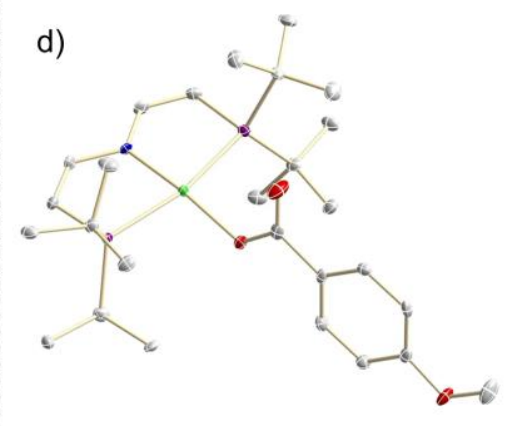

Figure 26: a) ${ }^{1} \mathrm{H}$ (para)-NMR spectra of the benzoate complexes 14-x with ( $\mathrm{X}=\mathrm{F}, \mathrm{H}, \mathrm{Me}$, OMe. Molecular structure in the solid state of b) 14- $\left.\mathrm{H}, \mathrm{C}\right)$ 14- $\mathrm{F}$ and d) $14-$ OMe obtained by X-ray single crystal diffraction. The ORTEP plot shown with anisotropic displacement parameters at $50 \%$. All C-H hydrogen atoms are omitted for clarity. For b) Two molecules crystalized in the asymmetric unit only one is shown for clarity. Selected parameters: Ir-O $\mathrm{O}_{1}: 2.061(2) \AA$, Ir- $\mathrm{N}: 1.960(3) \AA$, Ir-P1:

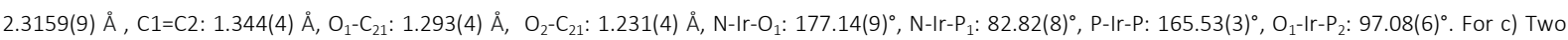
molecules crystalized in the asymmetric unit only one is shown for clarity. Selected parameters: Ir- $\mathrm{O}_{1}:$ : 2.048(2) $\AA$, Ir- $\mathrm{N}: 1.963(3) \AA$, Ir-P1: $2.3149(10) \AA, C 1=C 2$ : $1.340(5) \AA, O_{1}-C_{21}: 1.288(4) \AA, O_{2}-C_{21}: 1.226(4), N-I r-O_{1}: 174.24(12)^{\circ}, N-I r-P_{1}: 82.84(9)^{\circ}$, P-Ir-P: $166.43(3)^{\circ}, O_{1}-I_{r}-P_{2}: 95.75(7)^{\circ}$. For c) Selected parameters: Ir-O ${ }_{1}$ :

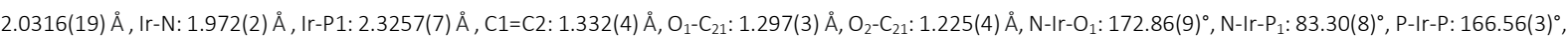
$\mathrm{O}_{1}-\operatorname{Ir}-\mathrm{P}_{2}: 94.82(6)^{\circ}$.

The ${ }^{1} \mathrm{H}-\mathrm{NMR}$ signals of the benzoate complexes $14-\mathrm{H}, 9-\mathrm{F}, 14-\mathrm{Me}$ and $14-\mathrm{OMe}$ are paramagnetically shifted with the main peak, corresponding to the tert-butyl groups at $\delta \sim 13 \mathrm{ppm}$. Further, the signal at $\delta=4.9 \mathrm{ppm}(\mathrm{X}=\mathrm{H})$ is not present for $\mathrm{X}=\mathrm{F}$, therefore this signal can be assigned to the para- $\mathrm{H}$ in the phenyl ring, while the new signals at $\delta=0.3 \mathrm{ppm}(\mathrm{X}=\mathrm{Me})$ and $\delta=2.0 \mathrm{ppm}(\mathrm{X}=\mathrm{OMe})$ can be assigned to the methyl groups in this compounds. The 
assignment of the other signals is more uncertain. It can be seen, that the signal at $\delta \sim 2.5 \mathrm{ppm}$ is shifting less within the complex series and thus suggests that it is not close to the rest $X$ and therefore most likely a pincer backbone signal, while the two signals at $\delta \sim-5.0 \mathrm{ppm}$ exhibit a higher shift with different rests $\mathrm{X}$ and thus can be assigned to the ortho and meta phenyl protons (Figure 26a). Final structural proof is obtained by single XRD analysis $\left(X=H^{50}, F, O M e^{51}\right.$ ) (Figure 26b). All benzoate complexes are in square-planar coordination environment (angle sum around iridium $=360^{\circ}$ ). The $\mathrm{Ir}$-O bond is elongated compared to 4 to $d_{\mathrm{Ir}-\mathrm{O}} \geq 2.0 \AA$, which indicates no multi-bond character in the Ir-O unit, while the uncoordinated oxygen atom of the aldehyde bears an shorter bond to the carbonyl centre $d_{\mathrm{c} 21-02} \leq 1.23 \AA$ than the coordinated one $d_{\mathrm{c} 21-01} \leq 1.29 \AA$, which suggests double bond character in the carbonyl group. A Hammett analysis of the irradiation reaction is performed to get insight into the reaction mechanism. ${ }^{52}$ The analysis reveals a clear positive slope of the linear regression, and supports either a $\mathrm{C}-\mathrm{H}$ bond weakening of the excited state benzaldehyde and thus fast HAT from the aldehyde to 4 , forming 2 , in the first step or a nucleophilic attack of the excited state 4 at the carbonyl centre. Since the reactivity of 4 has been previously shown to exhibit nucleo- to ambiphilic character, the latter explanation is more convenient.

a)
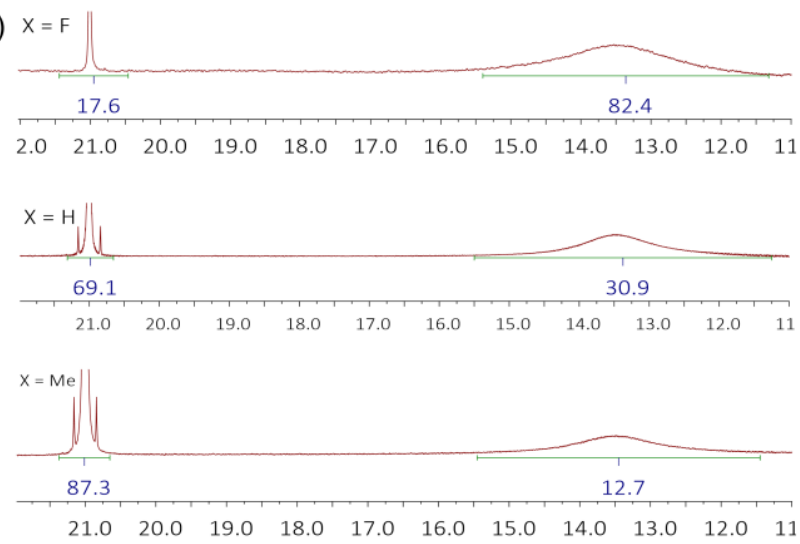

$\mathrm{x}=\mathrm{OMe}$

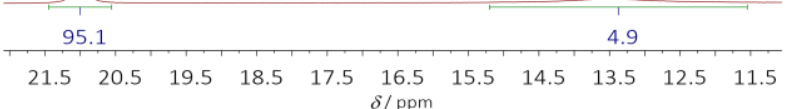

b)

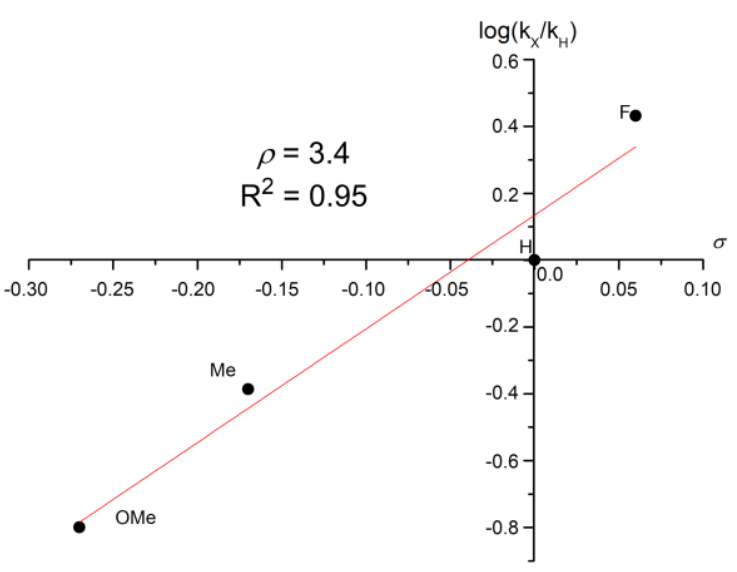

Figure 27: Irradiation of 4 in benzene solution at ambient temperature. ${ }^{53} \mathrm{~b}$ ) ${ }^{1} \mathrm{H}($ para)-NMR spectra of the irradiation of 4 in benzene. c) Hammett analysis of the irradiation experiment, with the Hammett/Taft parameters for the para position of the substituents. ${ }^{[192]}$

\footnotetext{
${ }^{50}$ Crystals grown by Niklas Welker and measured by Dr. Christian Würtele.

${ }^{51}$ Crystals grown by Matthieu Haake and measured by Dr. Matthias Otte.

${ }^{52}$ See Section 2.11 in chapter VI for experimental details.

${ }^{53}$ Experiment performed by Niklas Welker.
} 


\section{Summary of Chapter II}

In summary the isolation and full characterisation of an open-shell terminal iridium oxo complex was presented. Spectroscopic and crystallographic characterisation led to the assignment of an iridium oxygen double bond in contrast to the triple bond character of the only other terminal iridium oxo complex by XXI Wilkinson et al. SQUID magnetometry revealed a thermally well separated $\left(650 \mathrm{~cm}^{-1}\right)$ triplet ground state with large TIP from SOC. The zero-field splitting is $200 \mathrm{~cm}^{-1}$ higher than for the isoelectronic terminal imido complex XXII, which can be rationalised by a smaller relativistic nephelauxetic effect of 4 due to less covalent $\mid r-E(E=N / O)$ bonding, which results in a higher effective SOC parameter $\zeta_{\text {eff }}$ for 4 . Multi-reference computations reproduce this picture and predict a triplet ground state with a high zero-field splitting $\left(750 \mathrm{~cm}^{-1}\right)$. Additionally, a small but non-negligible SOC effect on the PCET thermochemistry of hydroxo/oxo couple $2 / 4$ is predicted. The oxo complex 4 is therefore best described as a rare biradical species with substantial oxyl radical character as confirmed by Mulliken spin density and NBO/NPA analysis. 4 exhibits a versatile nucleo- to ambiphilic oxygenation chemistry as shown by reaction with $\mathrm{PMe}_{3}, \mathrm{CO}, \mathrm{CO}_{2}, \mathrm{H}_{2}$ and benzaldehydes. The calorimetrically determined $\mathrm{O}-\mathrm{H} \mathrm{BD}(\mathrm{F}) \mathrm{E}$ of the hydroxo/oxo complex couple $2 / 4\left(350 \mathrm{~kJ} \mathrm{~mol}^{-1},\left(325 \mathrm{~kJ} \mathrm{~mol}^{-1}\right)\right)$ is in line with the predicted value from ab initio computations $\left(\mathrm{BDE}^{\mathrm{ab} \text { initio+SOC}}=343 \mathrm{~kJ} \mathrm{~mol}^{-1}\right)$ and is in the thermodynamic region of activated $\mathrm{C}-\mathrm{H}$ bonds. Indeed, by reaction with xanthene $\left(B D E=326 \mathrm{~kJ} \mathrm{~mol}^{-1}\right)$ and fluorene $\left(B D E=343 \mathrm{~kJ} \mathrm{~mol}^{-1}\right)$ the $\mathrm{C}-\mathrm{H}$ oxygenation products are obtained.

The transient cationic iridium(IV) oxo species $4+$ is accessible at very mild oxidation potentials $\left(E_{1 / 2}=-0.13 \mathrm{~V}\right.$ vs. $\left.\mathrm{Fc} / \mathrm{Fc}^{+}\right)$, which reflects the high covalent iridium-oxygen bonding and therewith the low validity of oxidation numbers is such systems. The almost axial X-band EPR spectrum of $\mathbf{4 +}$ is further supporting a ligand centred oxyl radical which is backed up by DFT analysis. The Mulliken spin density of 4+ (0: 75\%) is mainly oxygen centred, speaking for a rare oxyl radical complex, but with a lower oxygen spin density as for 4 (O: 88\%). A comparison of the $\mathrm{O}-\mathrm{H}$ BD(F)E of neutral oxo $4\left(\mathrm{BDE}=350 \mathrm{~kJ} \mathrm{~mol}^{-1}\right)$ with its cationic counterpart $4+\left(\mathrm{BDE}=372 \mathrm{~kJ} \mathrm{~mol}^{-1}\right)$ supports the key message of James Mayer, that not radical character is rendering PCET chemistry, but the driving force of the reaction. 


\section{Chapter III}

III. Excursuses 


\section{Excursus 1: Interconversion of Phosphinyl Radical and Phosphinidene Complexes by Proton Coupled Electron Transfer}

Parts of this Chapter are published in: J. Abbenseth, D. Delony, M. C. Neben, Christian Würtele, B. de Bruin, S. Schneider, Angew. Chemie. Int. Ed. 201958 6338. "Interconversion of Phosphinyl Radical and Phosphinidene Complexes by Proton Coupled Electron Transfer". The synthetic work presented here was performed under the supervision of Dr. Josh Abbenseth by Marc. C. Neben.

Phosphorous compounds like phosphinyl radicals $\left(\mathrm{PR}_{2}\right)$ are key intermediates in $\mathrm{P}-\mathrm{C}$ bond formations, due to their electronically unsaturated nature. ${ }^{[193-197]}$ Catalytic P-R bond formation processes remain nevertheless scarce, while the analogue nitrene transfer reactions are commonly catalytically utilized in organic synthesis. ${ }^{[24,40,198-200]} \mathrm{A}$ possible explanation is the lack of suitable oxidizing transfer reagents for PR moieties. Here, primary phosphines present attractive precursors from a thermochemical point of view, since their $\mathrm{P}-\mathrm{H}$ bonds are inherently weaker compared to their $\mathrm{N}-\mathrm{H}$ analogues (Scheme 4a) ${ }^{[184]}$. Additionally, the coordination of the free phosphine to a metal catalyst site might further weaken the P-H bonds, as observed for water and ammonia. [201,202]

a)

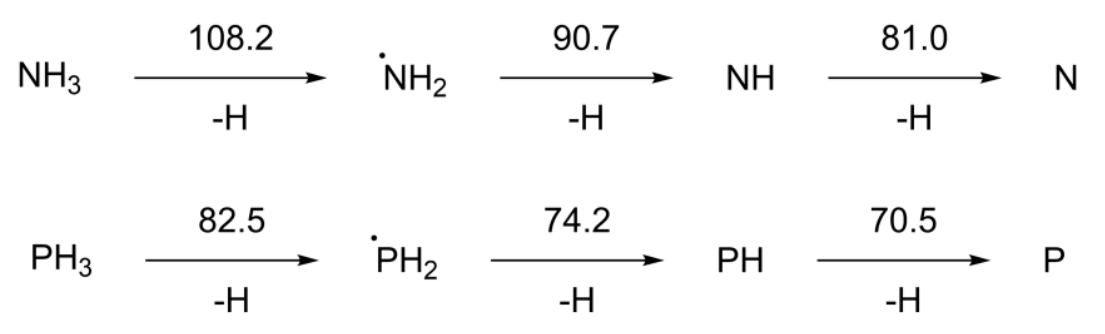

b)

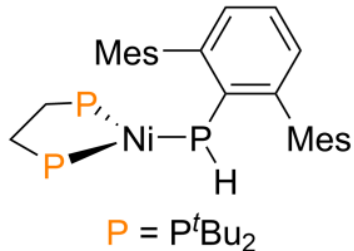

XXXVIII

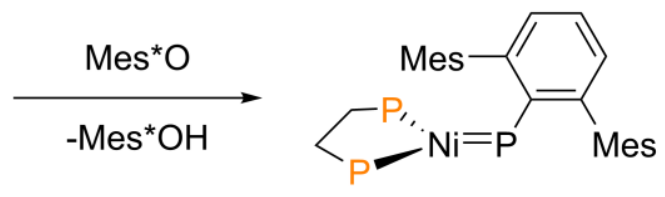

XXXIX

Scheme 4: a) N-H and P-H bond dissociation energies (BDEs) in the gas phase in kcal mol ${ }^{-1} \cdot{ }^{[184]}$ b) Synthesis of a nickel phosphinidene complex by PCET reported by Hillhouse et al. (Mes = 2,4,6-trimethyl-phenyl, Mes* $=2,4,6$-tris-tert-butyl-phenyl). ${ }^{[203]}$

While N-H bond homolysis from amide ligands is an active field of chemistry, P-H bond homolysis was only reported by Hillhouse et al. from the nickel platform XXXVIII (Scheme 4b). ${ }^{[203]}$ In this light the [Os(H)(PHMes*)(PNP)] phosphinyl radical complex 17 was synthesized by oxidative addition of $\mathrm{PH}_{2} \mathrm{Mes}^{*}$ to the previously reported square-planar [Os(Cl)(PNP)] complex 16, followed by subsequent reduction with cobaltocene in benzene. ${ }^{[204]}$ Insight into the PCET chemistry of the thermally instable phosphinyl radical complex 17 was obtained by a square scheme approach and by direct HAT (Scheme 5). Oxidation of 17 using the silver "crossing" salt $\left(\mathrm{Ag}\left[\mathrm{Al}\left(\mathrm{OC}\left(\mathrm{CF}_{3}\right)\right)_{4}\right]\right)$ at $-35^{\circ} \mathrm{C}$ gave access to the thermally instable, cationic osmium(IV) phosphide complex 18. Cyclic Voltammetry gave a reversible oxidative wave $\left(E_{1 / 2}=-0.88 \mathrm{~V}\right.$ vs. $\left.\mathrm{FeCp}_{2}{ }^{+/ 0}\right)$ which indicates that the chemical decomposition is not happening within the time frame of $\mathrm{CV}$. 


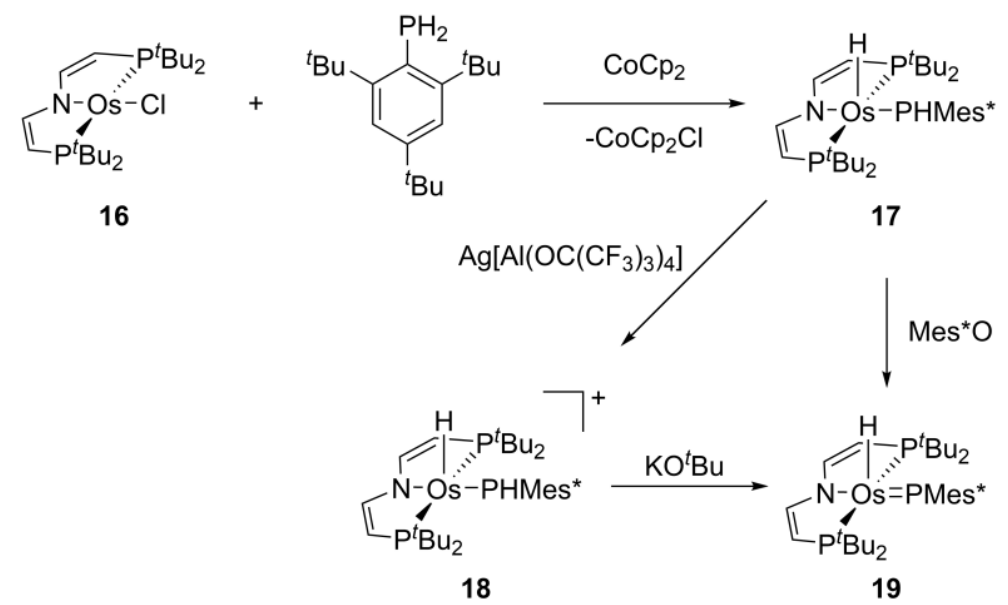

Scheme 5: Synthesis of the phosphinyl radical complex 17 by oxidative addition of PH2Mes* to 16 and subsequent reduction with CoCp $\mathrm{p}_{2}$ in benzene and PCET chemistry of 10 by oxidation with silver "crossing" salt and subsequent deprotonation with KOtBu or HAT with Mes*O.

Deprotonation of 18 with $\mathrm{KO} \mathrm{B}^{\mathrm{B} u}$ at $-80^{\circ} \mathrm{C}$ yields quantitatively a purple product, which could be assigned to the phosphinidene complex 19 with ${ }^{31}$ P-NMR by its characteristic signal at $\delta_{\mathrm{P}}=825 \mathrm{ppm}$ for the PMes* group. The thermal instability of 18 nevertheless hampered the determination of a reliable $p K_{A}$ value. Thus, the direct determination of the $\mathrm{P}-\mathrm{H}$ bond strength of 17 by HAT was focused. Reaction with Mes* $\mathrm{O}$ gave the clean formation of 17 , which supports this reaction as suitable for further analysis by isothermal titration calorimetry. The BDE determination was carried out at $10{ }^{\circ} \mathrm{C}$ to supress the thermal decomposition of both 17 and 19 , respectively (Figure 28a).

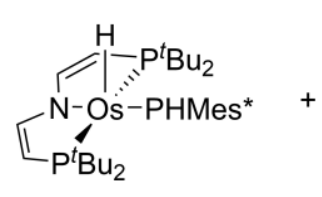

17<smiles>CC(C)(C)c1cc(Br)c([O])c(C(C)(C)C)c1</smiles>

${ }^{t} \mathrm{Bu}$

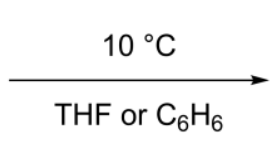

HF or $\mathrm{C}_{6} \mathrm{H}_{6}$

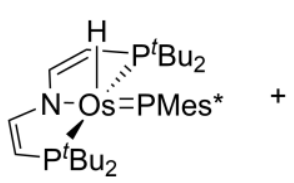

19<smiles>CC(C)(C)c1cc(Br)c(O)c(Br)c1</smiles>

${ }^{t} \mathrm{Bu}$

Scheme 6: PCET reaction of 17 with Mes*O forming 19 and Mes*OH analysed by Isothermal Titration Calorimetry in THF and benzene at $10^{\circ} \mathrm{C}$.

The ITC was recorded in THF and benzene and reveals a very exothermic reaction with $\Delta H(T H F)=-69 \mathrm{~kJ} \mathrm{~mol}^{-1}$ and $\Delta H\left(\mathrm{C}_{6} \mathrm{H}_{6}\right)=-71 \mathrm{~kJ} \mathrm{~mol}^{-1}$ leading to a P-H BDE of $67 \mathrm{kcal} \mathrm{mol}^{-1}$ in THF and of $65.5 \mathrm{kcal} \mathrm{mol}^{-1}$ in benzene (Figure 28c and $d$ ). A reliable determination of the free bond dissociation energy (BDFE) in both solvents was hampered by the thermal instability of 19 . Here, the thermal decomposition led to increased tailing of the peaks around the equivalence point, thus a meaningful fit of the titration curve is hampered (Figure 28b). The effect of P-H bond weakening upon coordination mentioned above is supported by computational analysis by means of DFT. ${ }^{54}$ Here, the free HPMes*/PMes* couple exhibits a $6 \mathrm{kcal} \mathrm{mol}^{-1}$ higher BDE compared to the coordinated fragment, supporting the general assumption that primary phosphines are suitable precursors for PR transfer reactivity. With the P-H BDE value and oxidation potential of 17 in THF at hand, the calculation of the pKA value of 18 by a thermochemical square-scheme is possible (Figure 28e). With the Bordwell equation ${ }^{[205]} \mathrm{BDE}=1.37 \mathrm{p} K_{\mathrm{A}}+23.06 E^{0}$ $+\mathrm{C}_{\mathrm{H}}$, with $\mathrm{C}_{\mathrm{H}}(\mathrm{THF})=66 \mathrm{kcal} \mathrm{mol}^{-1}[187]$, the value can be calculated to $\mathrm{p} K_{\mathrm{A}}(18, \mathrm{THF})=16$.

\footnotetext{
${ }^{54}$ The computations discussed here are not presented in the publication. They are nevertheless on a comparable level of theory (PBE-D3/defSVP but without the RI-J approximation). A detailed discussion of the computations can be found in chapter VI Section 4.4
} 
a)

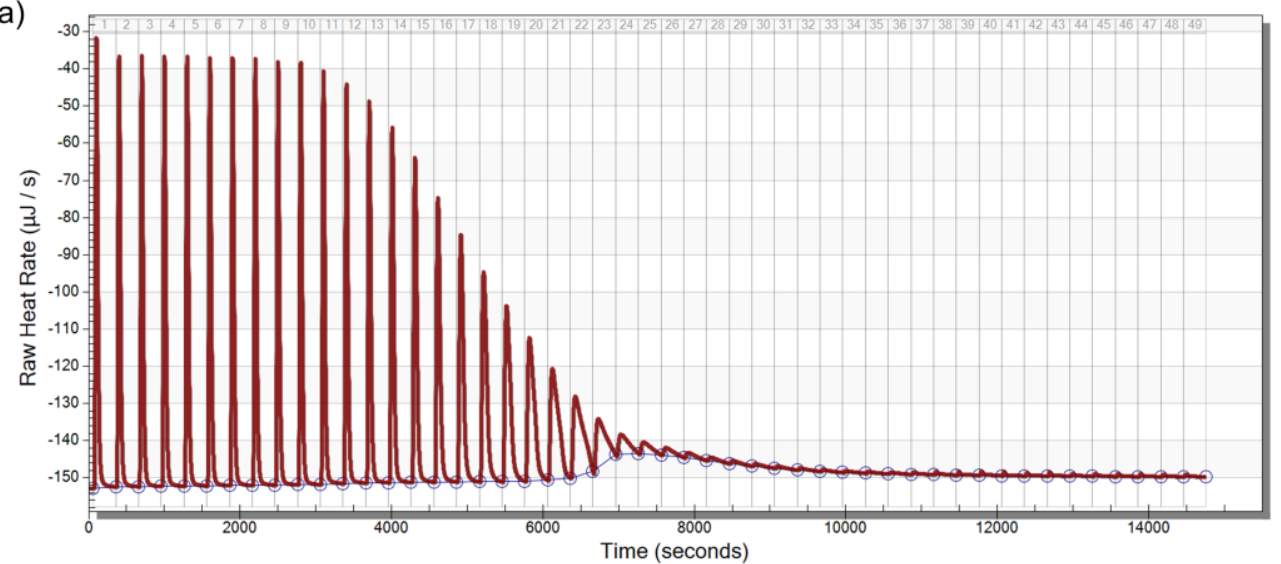

b)

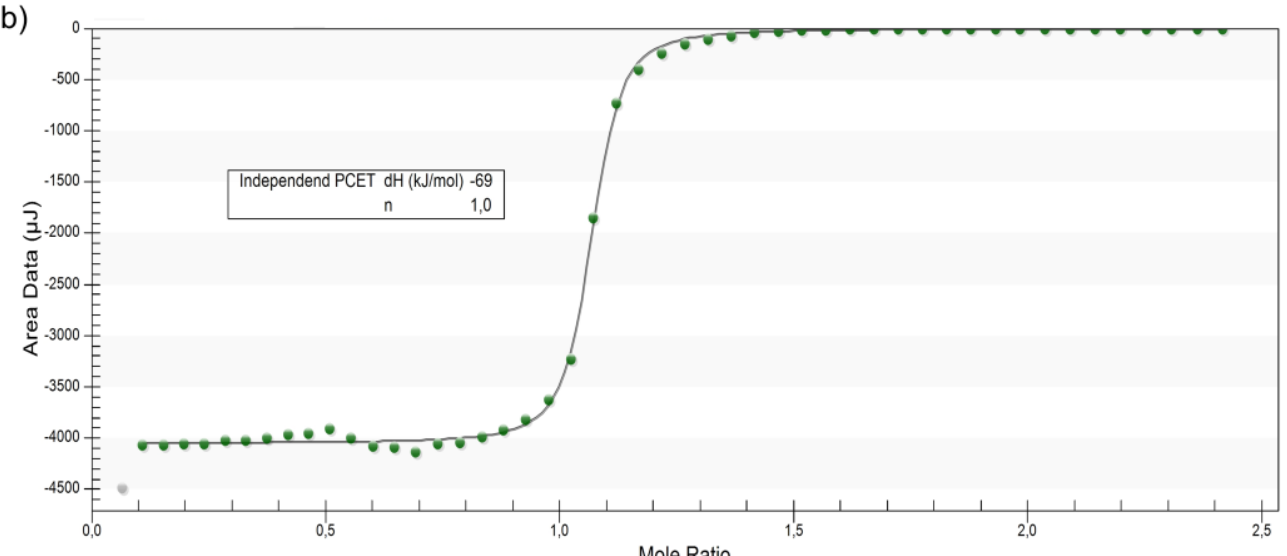

c)

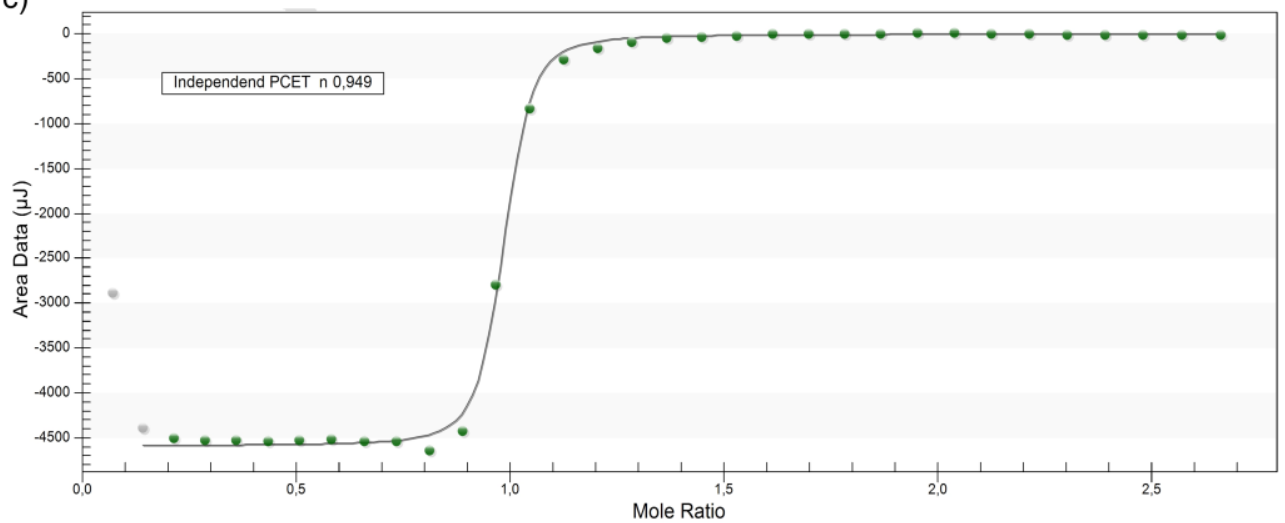

d)

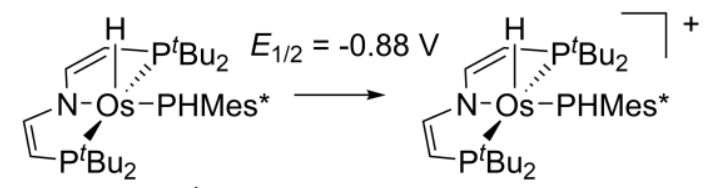

17

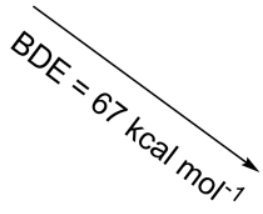

18

$\mathrm{p} K_{\mathrm{A}}=16$

$\stackrel{\mathrm{H}}{-} \mathrm{P}^{t} \mathrm{Bu}_{2}$

$\mathbb{L}_{\mathrm{P}^{t} \mathrm{Bu}_{2}}^{\mathrm{N}-\mathrm{Os}=\mathrm{PMes}^{*}}$

19

Figure 28: a) Representative thermogram of the titration curve in THF with peak tailing around the equivalence point. b) Representative integrated thermogram in benzene fitted with an independent PCET model (see Chapter 7, Section C). c) Representative integrated thermogram in THF fitted with an independent PCET model. d) Thermochemical square scheme for the $p K_{\mathrm{A}}$ determination of 18 


\title{
2. Excursus 2: Facile Conversion of Ammonia to a Nitride in a Rhenium System that Cleaves Dinitrogen
}

\author{
The manuscript of this Chapter is currently in progress in collaboration with Prof. Holland and Prof. Mayer (Yale \\ University). G. Connor, D. Delony, B. Q. Mercado, J. B. Curley S. Schneider., J. M. Mayer, P. L. Holland "Working \\ title: Facile Conversion of Ammonia to a Nitride in a Rhenium System that Cleaves Dinitrogen". The synthetic work \\ presented here was performed by Gannon Connor.
}

The interconversion of atmospheric dinitrogen to $\mathrm{NH}_{3}$ is one of the most important syntheses developed in the $20^{\text {th }}$ century, since the so obtained fertilizers are responsible for around $40 \%$ of our food production and thus supply the growing world population. ${ }^{[206]}$ The microscopic reverse reaction from $\mathrm{NH}_{3}$ to $\mathrm{N}_{2}$ is equally of high research interest, since the enormous energy density of ammonia $\left(\omega_{\mathrm{NH} 3}=22.5 \mathrm{MJ} / \mathrm{kg}\right)$ renders it as a candidate for carbon-free fuels. ${ }^{[207]}$ For the realisation of the potential of such an ammonia-based fuel cell, the underlying principles and chemical reaction steps must be investigated in detail. Here, the analysation of the single PCET steps from ammonia to a nitride ligand, which finally couples to $N_{2}$, is of special interest on the search for potential bottle necks in the interconversion. A promising system for this reaction is a complex which already has shown to be active in $\mathrm{N}_{2}$ fixation and cleavage. Here, rhenium pincer complexes have recently gained attention. ${ }^{[158,208,209]}$ The rhenium(V) nitride complex $[(P N P) \operatorname{Re}(V)(C l)(N)] 22$ is capable of nitrogen incorporation into nitriles, but was shown to be unreactive towards organic hydrogen atom transfer reagents or $\mathrm{H}_{2}$. Thus, the PCET thermochemistry of the microscopic reverse reaction from the rhenium(III) amide complex [(PNP)Re(III)(CI) $\left.\left(\mathrm{NH}_{2}\right)\right] 20$ towards the nitride complex 22 will be analysed, to gain insight into this bottle neck (Scheme 7).

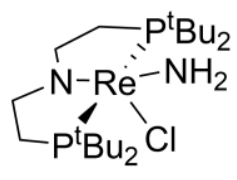

20

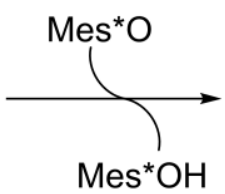

$\mathrm{Mes}{ }^{*} \mathrm{OH}$

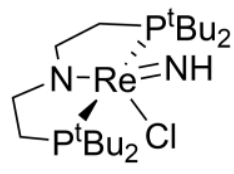

21

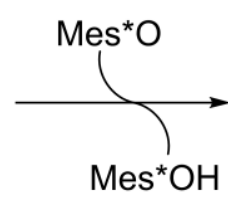

Mes* $\mathrm{OH}$

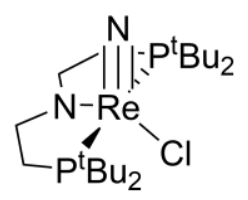

22

Scheme 7: Analysed PCET reaction in this excursus of 20 to 22 with intermediate imido complex 21

The mean N-H BDE of the 20/22 couple is determined by isothermal titration calorimetry by titration of 20 with Mes* $\left(\mathrm{BDE}_{-\mathrm{H}}\left(\mathrm{Mes}{ }^{*} \mathrm{OH}\right)=354 \mathrm{kJmol}^{-1}\right)$ in $\mathrm{THF}^{.55}$ The heat of reaction does not change after the addition of one equivalent Mes*O (Figure 29a), which is reproduced by NMR spectroscopy, in which the intermediately formed terminal imido complex [(PNP) Re(IV)(Cl)(NH) 21 cannot be observed. Since the single PCET steps are not separable calorimetrically, only the determination of a mean $\mathrm{N}-\mathrm{H}$ BDE is possible. Exactly after the addition of the second equivalent of $\mathrm{Mes}^{*} \mathrm{O}$, the reaction stops with a step slope. This indicates that the (double) PCET step is irreversible (Figure 29a). The data is fitted with the independent PCET model (see Section C), to obtain the stoichiometry of the reaction, while the reaction enthalpy is determined by the mean heat of reaction until the equivalence point (Figure 29b). To benchmark the so obtained data, the experiment is independently performed three times, resulting in a weighted average reaction enthalpy of $\Delta H^{\text {Double PCET }}=-213 \pm 2 \mathrm{~kJ} \mathrm{~mol}^{-1}$. Therefore, for each single PCET

\footnotetext{
${ }^{55} \mathrm{~A}$ detailed discussion of the performed experiments can be found in Section 3.6 of chapter VI.
} 
step an average of $\Delta H^{\text {Single PCET }}=-107 \pm 1 \mathrm{~kJ} \mathrm{~mol}^{-1}$ is released, which results in a mean binding energy of $\mathrm{BDE}^{20}=$ $59.2 \pm 0.25 \mathrm{kcal} \mathrm{mol}^{-1}$. This remarkable weak BDE also explains why the microscopic reverse reaction from 22 towards 20 does not take place, since most of the standard hydrogen atom donors possess stronger bonds than 20.

a)
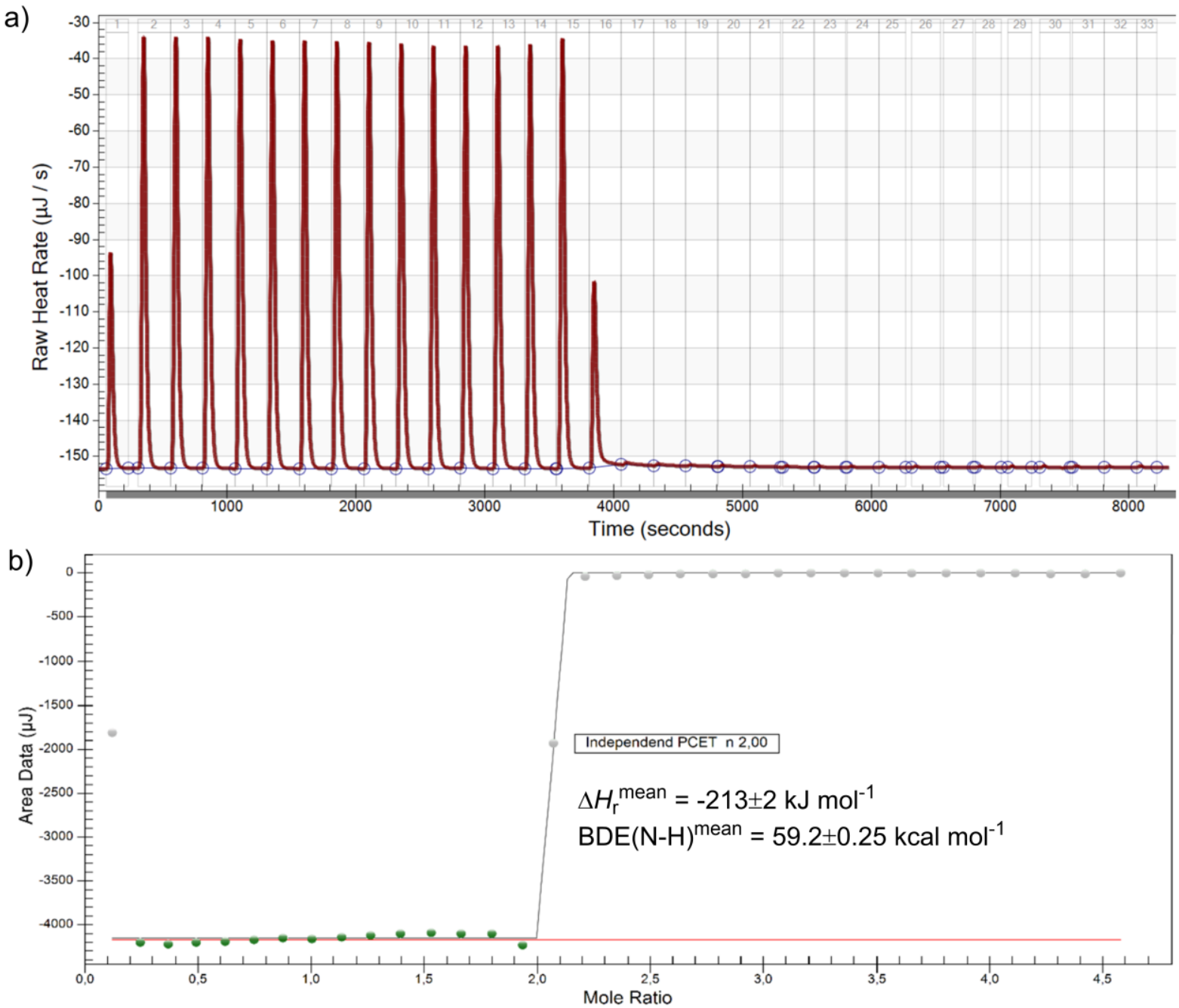

Figure 29: a) Representative thermogram of the titration of 20 with 22 with Mes*O in THF at 298 K. b) Integrated heat curve of the titration of 20 with 22 with Mes*O in THF at $298 \mathrm{~K}$ and results of the titrations. 
Chapter IV

IV. On the Effect of Spin-Orbit Coupling in Solution Thermochemistry 
For parts of the results of this Chapter a manuscript is in progress: D. Delony, F. Wätjen, M. Diefenbach, S. Demeshko, M. Orlita, M. C. Holthausen, S. Schneider "Evaluating the Effect of Spin-Orbit Coupling on the Thermochemistry of Proton Coupled Electron Transfer" The synthesis and characterisation of the rhenium(III) amine complex 23 and the rhenium(IV) amide complex 24 was described by Dr. F. Wätjen born Schendzielorz in F. Schendzielorz, M. Finger, J. Abbenseth, C Würtele, V. Krewald, S. Schneider, Angew. Chem. Int. Ed. $201958,830$. This Section will resume these results and extend the characterisation by further magnetic and spectroscopic data.

The vital problem of the small SOC effect (see Chapter II Section 3.2) of the terminal iridium hydroxo/oxo couple 2 and $\mathbf{4}$ was the nearly unchanged metal centred spin at the "spin-orbit coupling centre" iridium (see page 43), thus both complexes 2 and 4 were highly stabilised by SOC which led to the near cancellation of the differential SOC effect $(\triangle \Delta S O C)$. Thus, for a high $\Delta \Delta S O C$, a significant spin change at the metal centre upon reaction must take place. In a recent work from our group on benzonitrile formation from $\mathrm{N}_{2}$ the rhenium(III) amine complex $23^{56}$ exhibited an extraordinary shift of the ${ }^{31} \mathrm{P}-\mathrm{NMR}$ signal to $\delta=-1536 \mathrm{ppm}$, which was assigned to high temperature independent paramagnetism (TIP) as a result of strong SOC. ${ }^{[158]}$ Additionally, the corresponding rhenium(IV) amide complex 23 was well accessible by HAT from 23, rendering this reaction a promising candidate to investigate the influence of SOC on the PCET reaction (Scheme 8).

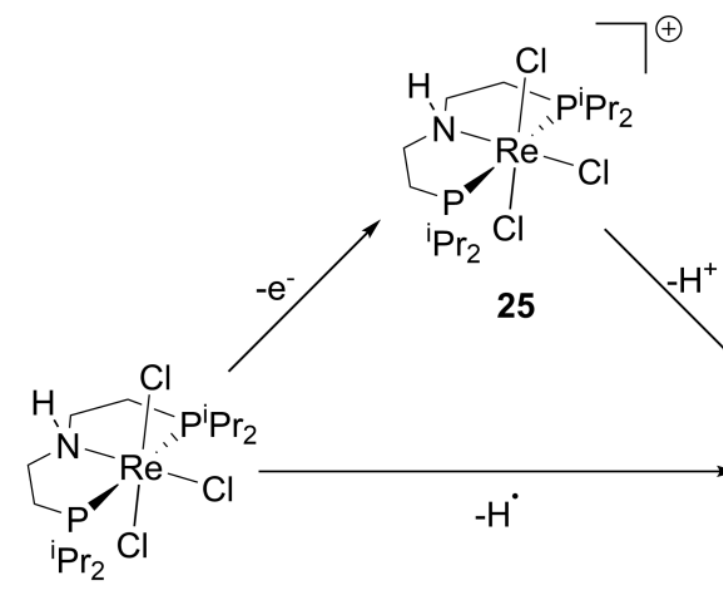

23

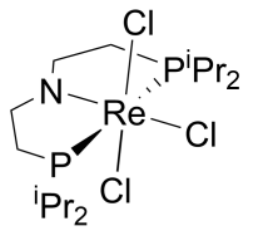

24

Scheme 9: Square scheme for the PCET reaction of 23 to 24 by direct HAT (bottom) or via oxidation to 25 and subsequent deprotonation (top).

In the following Chapter the rhenium(III) amine 23, the rhenium(IV) amide 24 and the cationic rhenium(IV) amine 25 will be characterized by spectroscopic and magnetic means and their properties will be rationalized by ab initio computations. The SOC effect on the PCET thermochemistry will then be analysed as the difference of the $a b$ initio computed reaction (free) enthalpy (with and without SOC) to the experimentally determined value.

\footnotetext{
${ }^{56}$ Please note: The pincer ligands referred to in this chapter differ from the ligand in chapter II. Here [PHNP] stands for Bis(di-isopropylphosphinoethyl)amine: $\overbrace{{ }_{2} \mathrm{PIP}}^{\mathrm{H}} \overbrace{\mathrm{PPr}_{2}}^{\mathrm{H}}$
} 


\section{Characterisation of the Rhenium(III/IV) Amine Complexes 23, 25 and of Rhenium(IV) Amide 24}

This Section will be about the characterisation of the three rhenium complexes 23, 24 and 25. To present a full picture, also the experimental data obtained by Dr. Florian Wätjen born Schendzielorz published in the publication mentioned above are presented. ${ }^{[158]}$

\subsection{Characterisation of Rhenium(III) Amine Complex 23}

The rhenium(III) amine complex 23 is coordinated meridionally by the pincer ligand with near $C_{s}$ symmetry as confirmed by XRD analysis (Figure 31a). ${ }^{[158]} 23$ possesses, due to the rapid electronic relaxation of Re ${ }^{\text {III }}$, ${ }^{[210]}$ sharp but extraordinary paramagnetically shifted NMR signals $\left(\delta\left({ }^{1} \mathrm{H}\right)=156 \mathrm{ppm}\right.$ to $\left.-11 \mathrm{ppm} ; \delta\left({ }^{31} \mathrm{P}\right)=-1526 \mathrm{ppm}\right)$, which were assigned to strong temperature independent paramagnetism (TIP) as a result of strong spin-orbit coupling on the ground state of 23 . This is a typical phenomenon for heavy $d^{4}$ metal ions like rhenium(III) or osmium(IV). ${ }^{[158,211]}$ For a closer insight into the nature of the paramagnetic behaviour of 23 , variable temperature NMR measurements between $190 \mathrm{~K}$ and $310 \mathrm{~K}$ were performed (Figure 30). The $\mathrm{N}-\mathrm{H}$ proton experiences the strongest, temperature dependent shift of $10 \mathrm{ppm}$ over the whole temperature range (Figure 30, top left), while the other signals only experience very minor $(<2 \mathrm{ppm}$ ) or no shifts (Figure 30, top right, bottom). This can be rationalised by solvation effects on the $\mathrm{N}-\mathrm{H}$ bond. All NMR signals exhibit a linear $\delta \propto T$ (non-Curie type) behaviour, which supports TIP arising from admixture of thermally not populated excited state as the origin of the NMR shifts. ${ }^{[212-216]}$ Further insight into the magnetic nature of 23 was obtained by SQUID magnetometry (Figure 31, b). The magnetic moment in the solid state at room temperature of $23 \mu_{\text {eff }}=1.60 \mu_{\mathrm{B}}$ is in the typical range for an open-shell, octahedrally coordinated rhenium(III) complex but significantly lower than the expected spin-only value for a triplet ground state $\left(\mu_{\mathrm{eff}}=2.83 \mu_{\mathrm{B}}\right){ }^{[211,215,217]}$ The linear non-Curie magnetism of 23 from the VT-NMR is as well reflected by the SQUID data, where in a temperature range from 2 to $300 \mathrm{~K}$ a complete linear curve of $X_{M} T$ vs. $T$ could be observed. This is in contrast to the magnetic data of oxo complex 4 (c.f. Figure 30, b black dots, Figure 9), which showed a bend SQUID curve at higher temperatures. ${ }^{57}$ The magnetic data can be fitted with two different models, which are both based on a spin-Hamiltonian picture (Figure 31, b red line). 1) A singlet ground state with strong mixing to open-shell excited states, which results in an extremely high TIP value of $1063.4 \cdot 10^{-6}$ $\mathrm{cm}^{3} \mathrm{~mol}^{-1}$ or 2) A triplet ground state with a very high zero-field splitting parameter of $D \geq 1302 \mathrm{~cm}^{-1}$, which is twice the zero-field splitting of terminal oxo $4\left(650 \mathrm{~cm}^{-1}\right)$ (Figure 31, b). Both models are only approximations for the electronic structure of 23 , since the spin-Hamiltonian is losing its physical meaning, when large SOC contributions lead to the splitting into spin-orbit states. The spectroscopic and magnetic data of 23 therefore support the picture of a non-magnetic $(J=0)$ ground state, thermally well separated $\left(>>\mathrm{k}_{\mathrm{B}} T\right)$ by SOC from the excited states. ${ }^{[218]}$

\footnotetext{
57 SQUID measurement and fitting by Dr. Serhiy Demeshko sample preparation by Dr. Florian Wätjen.
} 


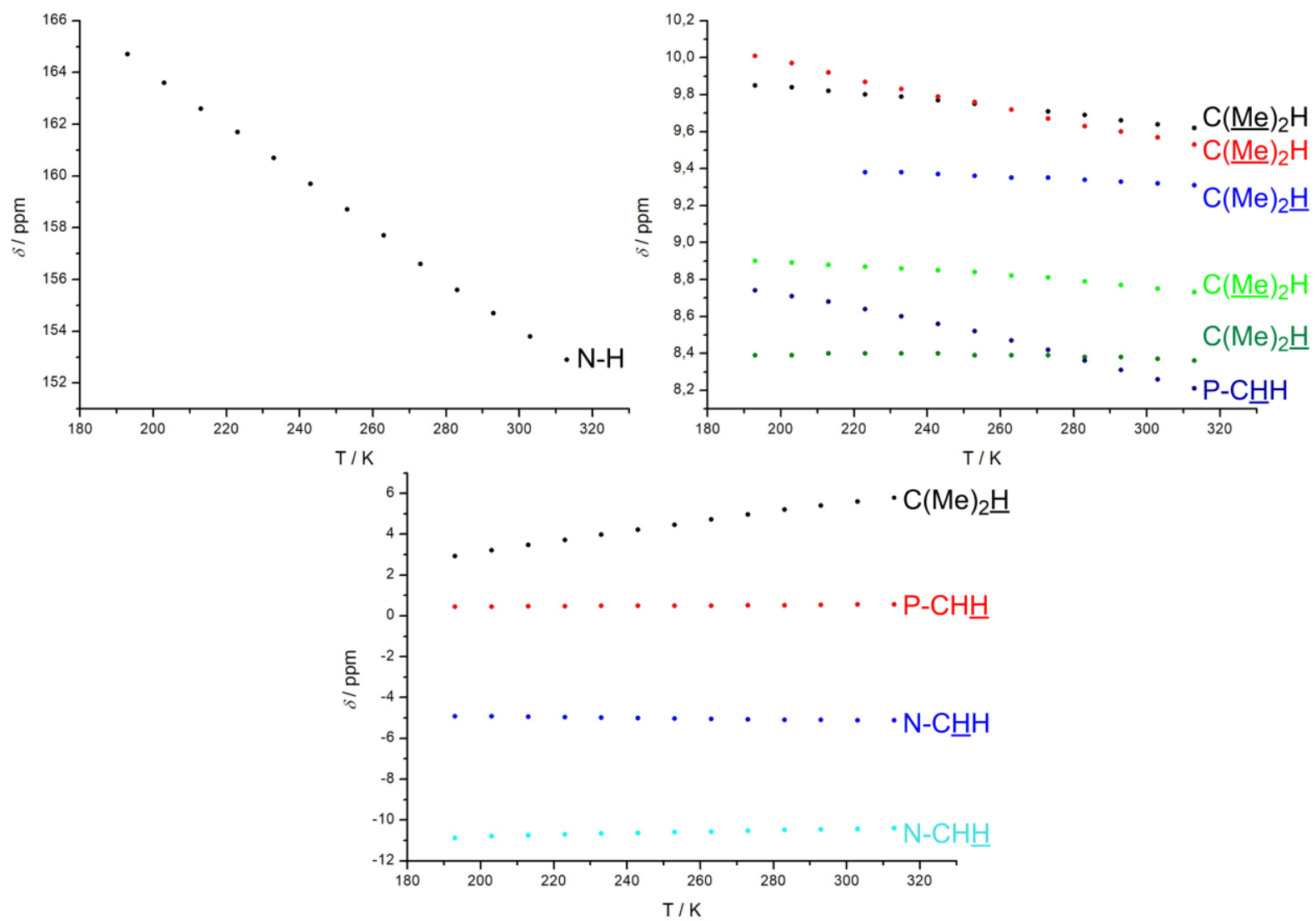

Figure 30: ${ }^{1} \mathrm{H}-\mathrm{VT}$-NMR plots recorded from $193 \mathrm{~K}$ to $313 \mathrm{~K}$ in THF of 23 . For each data point an independent lock and shim were performed after at least 10 min equilibration time at the given temperature.

a)

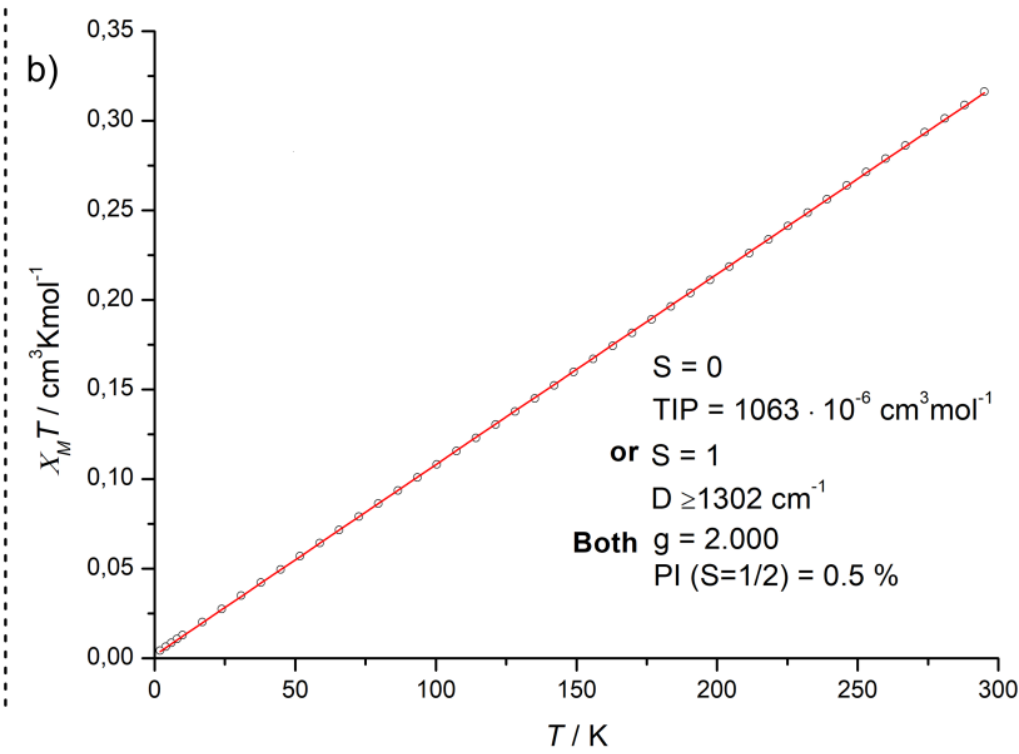

Figure 31: a) Solid state structure of 23 obtained by single crystal X-ray diffraction. All C-H protons neglected for clarity. Selected parameters: Re-N: $2.158(6) \AA$,

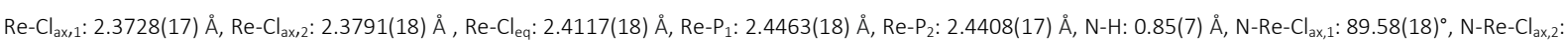

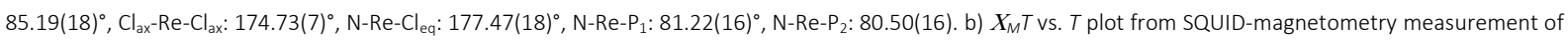
solid 15 from $2 \mathrm{~K}$ to $300 \mathrm{~K}$ (dots measured data, red line Spin-Hamiltonian fit) for a detailed description of the fitting procedure see Section 1.2 .10 in Chapter VI. 
The mid-IR spectrum of 23 exhibits three signals beyond the fingerprint region. Besides the $\mathrm{C}-\mathrm{H}$ vibrations at around $2900 \mathrm{~cm}^{-1}$, one weak but sharp band at $3183 \mathrm{~cm}^{-1}$ and one intense, broad transition at $3510 \mathrm{~cm}^{-1}$ are found. Here, the weak band at $3183 \mathrm{~cm}^{-1}$ can be assigned to the $\mathrm{N}-\mathrm{H}$ stretch by a deuteration experiment $\left(v_{\mathrm{N}-\mathrm{D}}=\right.$ $2363 \mathrm{~cm}^{-1}$ ) (Figure 32a). ${ }^{58}$ In previous publications, broad transitions in the mid-IR spectral range of rhenium phosphine complexes were tentatively assigned to arise from ligand field transitions, but their electronic nature was never proven spectroscopically. ${ }^{[219,220]}$ In contrast to a vibrational transition, electronic transitions to $\left|M_{\jmath}\right|>0$ states should change in an applied magnetic fields. A magnetic field dependent IR measurement of the band at $3510 \mathrm{~cm}^{-1}$ would therefore enable the definite assignment to an electronic transition. In collaboration with Dr. Milan Orlita ${ }^{59}$ mid-IR spectra at differing magnetic fields from 0-11 T were recorded. The normalized transmission $T_{\mathrm{B}} / T_{0}$ shows a rising signal intensity at increasing magnetic field only for the transition at $3510 \mathrm{~cm}^{-1}$, which confirms unequivocally an electronic transition in the mid-IR range (Figure 51c). The further analysation of the rhenium(III) amine complex 23 therefore supports the description of a complex with high SOC stabilisation of the ground state and encourages the further investigation on the SOC effect on the PCET reaction (Scheme 9).

a)

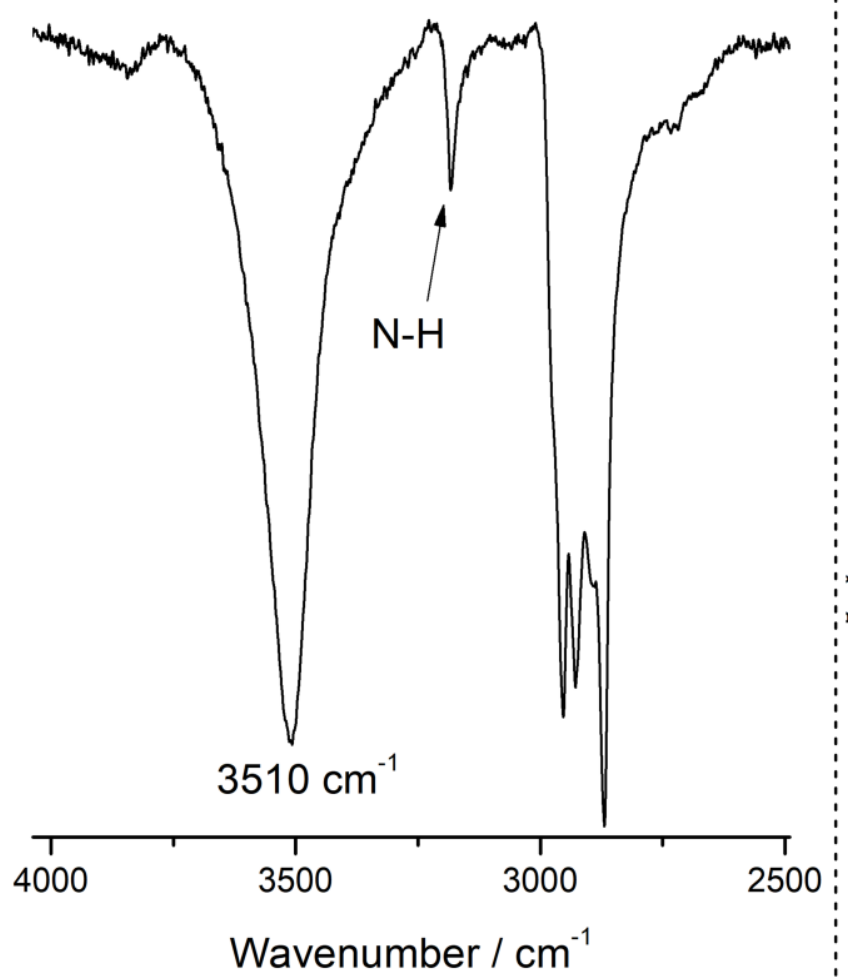

ib)

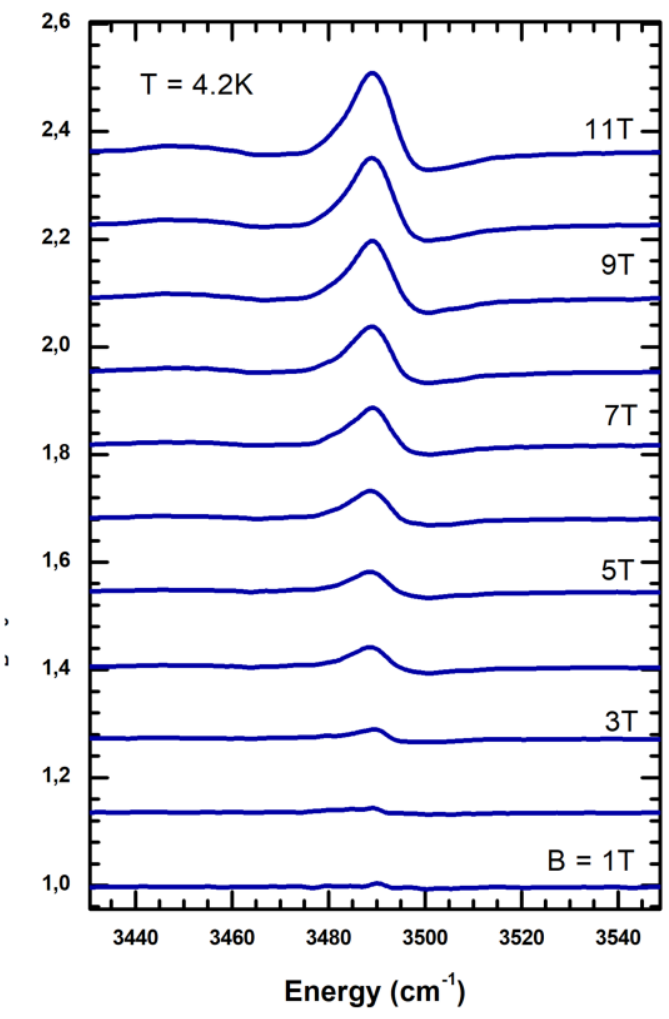

Figure 32: a) Section of the mid-IR(ATR) region above the fingerprint region of 23. b) Magnetic field dependent IR(KBr) data of 23 showing the transmission spectrum with applied field $\left(T_{\mathrm{B}}\right)$ divided by the zero-field spectrum $\left(T_{0}\right)$.

\footnotetext{
58 Spectroscopy and deuteration experiment (see Section 2.16 of Chapter VI) performed by Dr. Florian Wätjen.

59 Laboratoire National des Champs Magnetiques Intenses.
} 


\subsection{Characterisation of the Rhenium(IV) Amide Complex 24}

Next, the product of the PCET reaction, the rhenium(IV) amide 24, is further analysed. By addition of 1 eq. Mes*O to 23 in THF 24 can be selectively synthesized. 24 also adopts a meridional coordination with the pincer ligand as confirmed by XRD analysis (Figure 33b). ${ }^{[158]}$ While the solid state structure is slightly deviating from any symmetry,

24 adopts $C_{2 v}$ symmetry in solution, as shown by the two signals for the methyl groups in the NMR spectrum (Figure 33a). ${ }^{60}$

a)

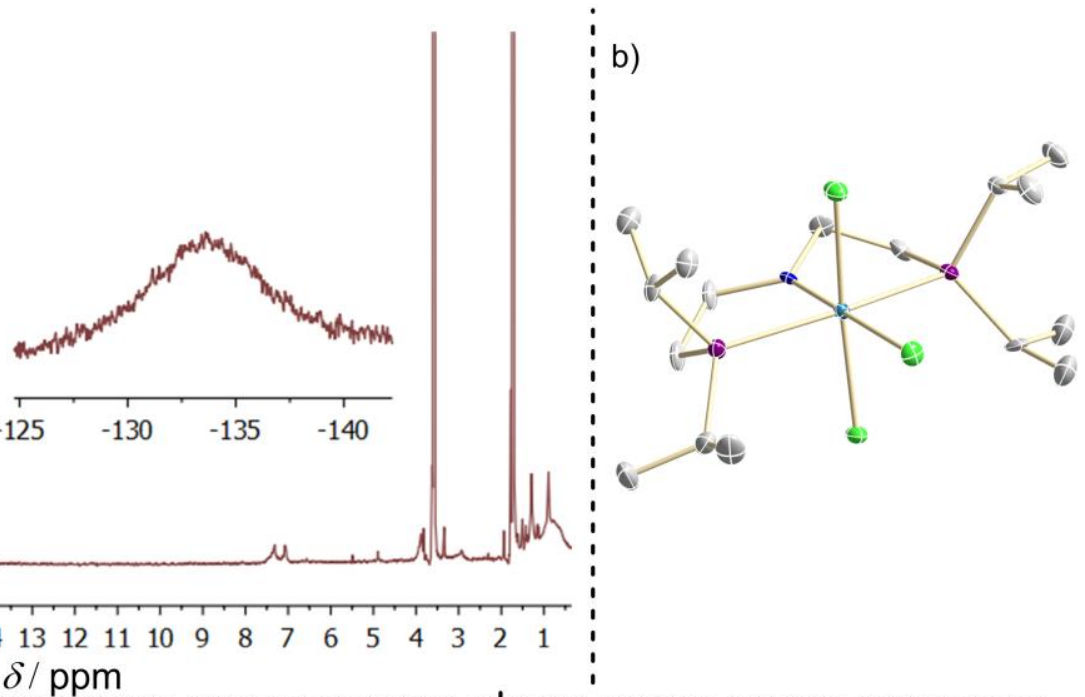

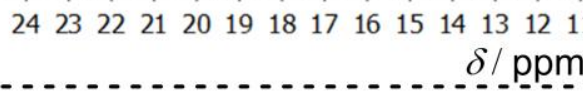

c)

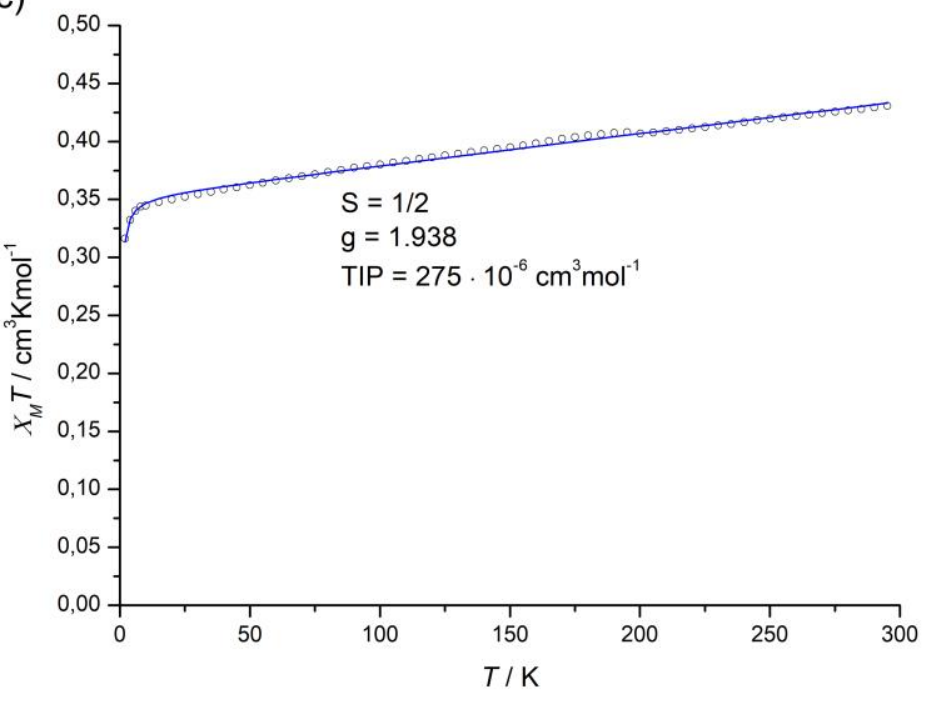

id)

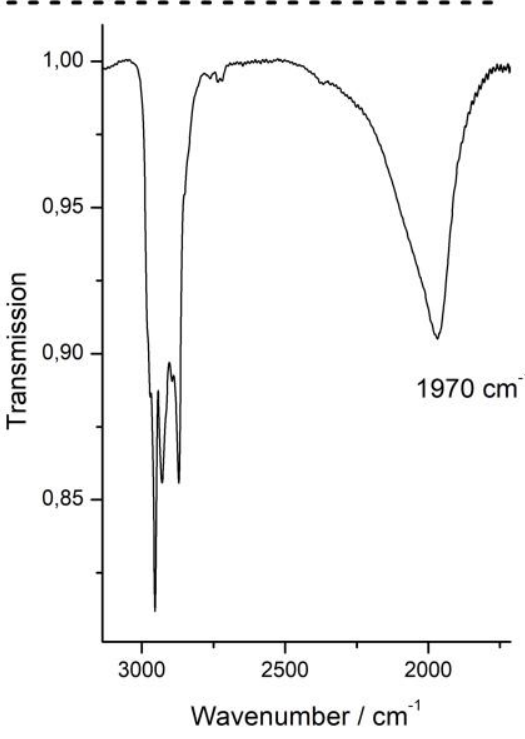

Figure 33: a) $1 \mathrm{H}-\mathrm{NMR}$ (para) of 24 in THF-d 8 at r.t. b) Structure of 24 in the solid state obtained by single crystal X-ray diffraction. Selected bond parameters: Re-N:

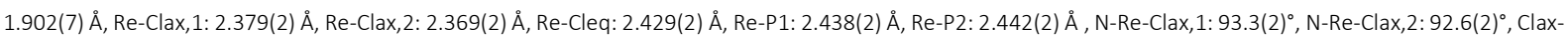
Re-Clax: $174.01(8)^{\circ}, \mathrm{N}-$ Re-Cleq: $178.7(2)^{\circ}, \mathrm{N}-\mathrm{Re}-\mathrm{P} 1: 81.9(2)^{\circ}, \mathrm{N}-\mathrm{Re}-\mathrm{P} 2: 82.4(2)^{\circ}$. c) $X_{\mathrm{M}} \mathrm{T}$ vs. T plot from SQUID-magnetometry measurement of solid 24 from $2 \mathrm{~K}$ to $300 \mathrm{~K}$ (dots measured data, red line Spin-Hamiltonian fit) for a detailed description of the fitting procedure see Section 1.2.10 in Chapter VI. d) Section of the midIR(ATR) region above the fingerprint region of 24 .

60 Improved synthesis using one equivalent of Mes*O in THF. The Re(III) imide impurities at around 10 ppm reported in the publication are gone. Additionally, the "lost" signal is found at $-134 \mathrm{ppm}$ 
In comparison to 23 the signals are still paramagnetically shifted but broader, reflecting the formally $1 \mathrm{e}^{-}$oxidized metal centre resulting in an odd electron count. The magnetic properties of 23 were analysed by SQUID magnetometry (Figure 33c). ${ }^{61}$ The obtained data (black dots) can be fitted within a spin-Hamiltonian picture (blue line) to a doublet ground state. The magnetic moment in the solid state at room temperature of $24\left(\mu_{\mathrm{eff}}=1.86 \mu_{\mathrm{B}}\right)$, is higher than the expected spin-only value for a doublet ground state ( $\mu_{\mathrm{eff}}=1.73 \mu_{\mathrm{B}}$ ), which speaks for a SOC induced admixture of excited quartet states to the ground state. This is described by the pronounced TIP value of $275 \cdot 10^{-6} \mathrm{~cm}^{3} \mathrm{~mol}^{-1}$. The low-spin ground state of 24 can be rationalized to strong $\mathrm{p}$-donation of the amide ligand, which rises one of the two MOs with $b$ character in energy. The mid-IR spectrum of 24 features a broad band at $1968 \mathrm{~cm}^{-1}$ comparable to the electronic band of 23 (Figure 33d). The presented data support 24 to exhibit a doublet ground state with significant, but lower, SOC stabilisation than 23 (cf. $\operatorname{TIP}(23)=1063.4 \cdot 10^{-6}, \operatorname{TIP}(24)=$ $\left.275 \cdot 10^{-6} \mathrm{~cm}^{3} \mathrm{~mol}^{-1}\right)$, which anticipates a high differential SOC effect on the PCET reaction.

\subsection{Characterisation of Cationic rhenium(IV) Amine Complex 25}

For the measurement of the PCET free reaction enthalpy by a square-scheme formalism (see Scheme 9), the cationic rhenium(IV) amine species 25 must be synthesised and characterised. Oxidation of 23 with ferrocenium tetrakis[3,5-bis(trifluoromethyl)phenyl]borate $\left(\mathrm{BArF}_{24}\right)$ in dichloromethane leads to a direct colour change to deep red (Figure 34 ,a). Crystallisation from benzene yields material suitable for single crystal XRD analysis and confirms the successful synthesis of the cationic rhenium(IV) amine complex $\left[\left(\mathrm{P}^{\mathrm{H} N P}\right) \operatorname{Re}(\mathrm{Cl})_{3}\right][\mathrm{BArF} 24] 25$ (Figure 34b). The nitrogen atom is in distorted tetrahedral coordination (angle sum around nitrogen $339^{\circ}$ ) which supports an amine species and the ligand backbone as well as directly coordinated ligands are close to $C_{s}$ symmetry in the solid-state structure, while the isopropyl groups do not show any symmetry in the solid state. The Re-Cl bonds are drastically shortened by up to $0.13 \AA$ compared to 23 , which can be rationalized by less $\pi$-backbonding to the chlorine ligands, while all angles to the ligands do not significantly differ.

a)

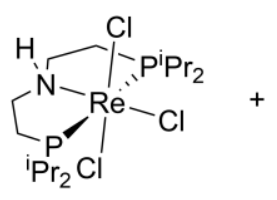

23
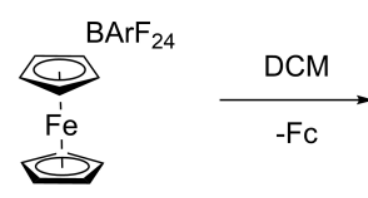

$-\mathrm{Fc}$

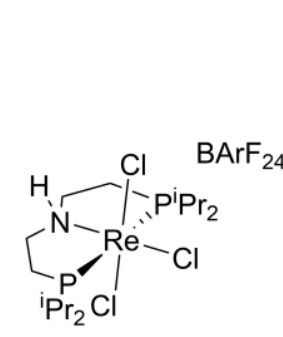

25

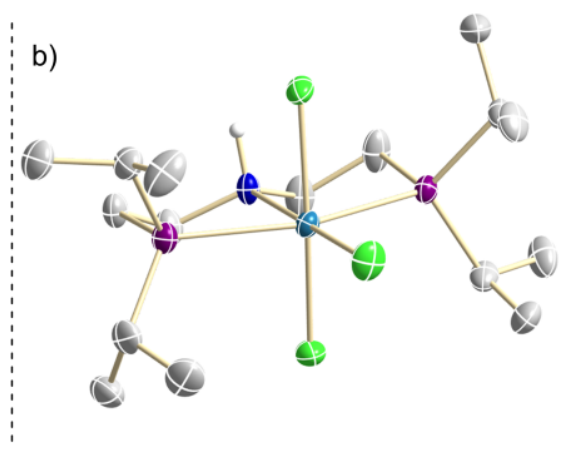

Figure 34: a) Reaction scheme for the synthesis of 25 by oxidation of 23 with ferrocenium BArF $_{24}$ in DCM at ambient temperature. b) Structure of 25 in the solid state obtained by single crystal X-ray diffraction. Cocrystallized benzene and the counter ion $\mathrm{BArF}_{24}$ are omitted for clarity. Selected bond parameters: Re-N:

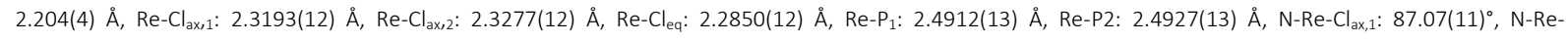
$\mathrm{Cl}_{\mathrm{ax}, 2}: 86.45(11)^{\circ}, \mathrm{Cl}_{\mathrm{ax}}-\mathrm{Re}-\mathrm{Cl}_{\mathrm{ax}}: 173.42(5)^{\circ}, \mathrm{N}-\mathrm{Re}-\mathrm{Cl}_{\mathrm{eq}}: 179.50(11)^{\circ}, \mathrm{N}-\mathrm{Re}-\mathrm{P}_{1}: 80.07(10)^{\circ}, \mathrm{N}-\mathrm{Re}-\mathrm{P}_{2}:$ : 79.09(10). 
The signals in the ${ }^{1} \mathrm{H}-\mathrm{NMR}$ spectrum are broadened compared to 23 , but significantly sharper than for 24 and give a $C_{s}$ symmetric signal set (Figure 35c). The signals for the methyl groups can be found around 30 ppm, while the backbone- and tertiary $\mathrm{C}-\mathrm{H}$ protons are shifted over a wide range from $15 \mathrm{ppm}$ to $-105 \mathrm{ppm}$. The $\mathrm{N}-\mathrm{H}$ proton signal overlaps with the two isopropyl group signals at $30.5 \mathrm{ppm}$ as indicated by the integral of 13 . The effective magnetic moment of 25 was determined by the Evans method in THF- $d_{8}$ to $\mu_{\mathrm{eff}}=2.9 \mu_{\mathrm{B}}$ and is therefore significantly over the expected spin-only value for a doublet ground state $\left(\mu_{\mathrm{eff}}=1.73 \mu_{\mathrm{B}}\right)$. Since, its electron count must be odd, the best rationalisation is a quartet ground state for 25. In the infrared spectrum of 25 a weak signal at $3095 \mathrm{~cm}^{-1}$ is found, which can be tentatively assigned to the $\mathrm{N}-\mathrm{H}$ vibration but in contrast to the complexes 23 and 24 no broad electronic transition over the whole range from $500-6500 \mathrm{~cm}^{-1}$ was found for 25 . The $\mathrm{N}-\mathrm{H}$ vibration is $88 \mathrm{~cm}^{-1}$ lower in energy compared to neutral 23, which supports a higher degree of activation of the $\mathrm{N}-\mathrm{H}$ bond for 25 (Figure 35d). With the electronic, spectroscopic and magnetic data of the complexes 23-25 at hand, the next step is their rationalisation by ab initio computations.

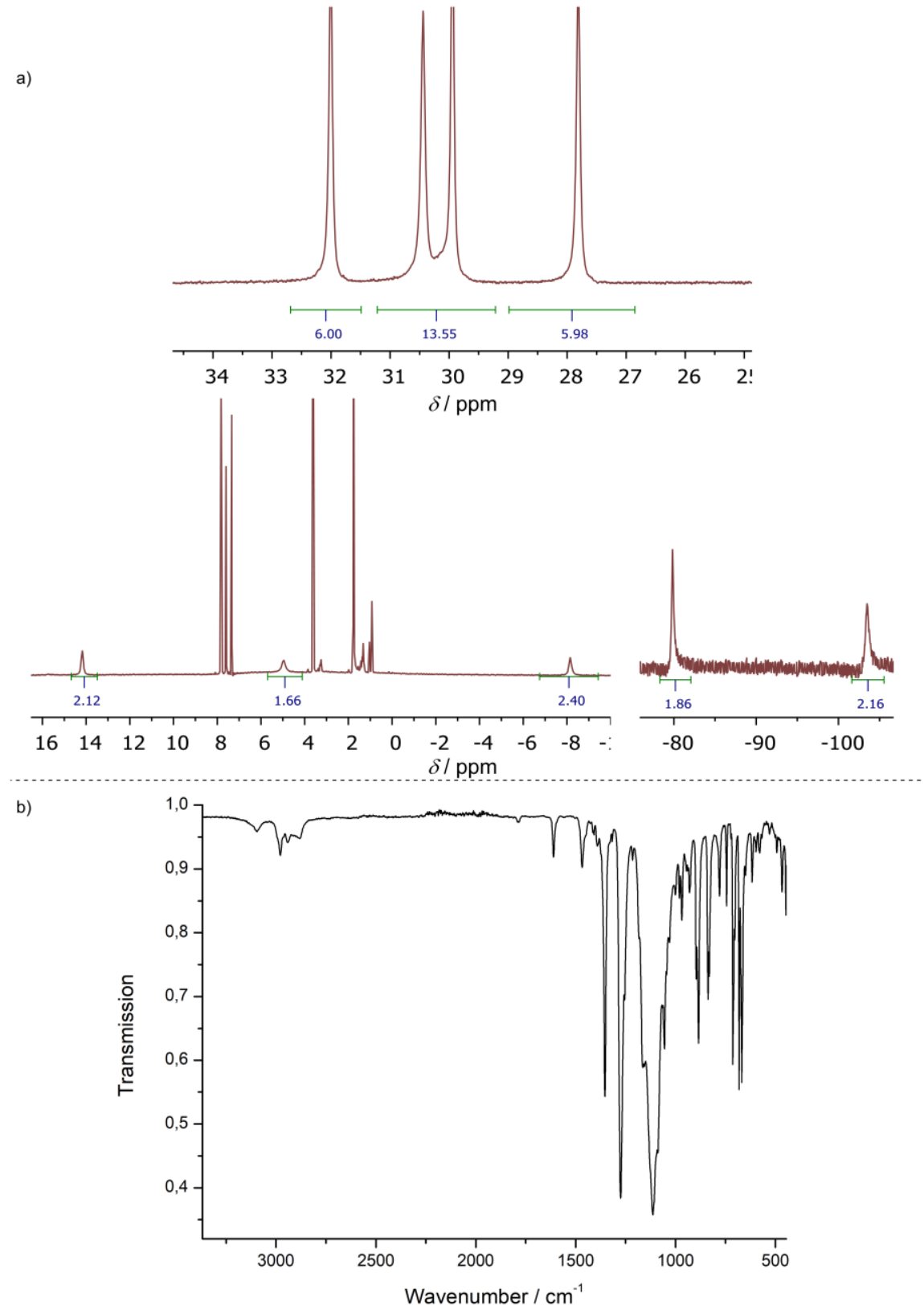

Figure 35: a) ${ }^{1} \mathrm{H}-\mathrm{NMR}$ (para) spectrum of 25 in THF- $\mathrm{d}_{8}$ at ambient temperature. c) IR (ATR) spectrum of 25. 


\section{Computational Rationalisation of the Spectroscopic and Magnetic}

\section{Features}

For the rationalisation of the spectroscopic and magnetic features of the complexes 23-25, reported in Section 1 , state averaged CASSCF calculations were performed. The computations are corrected by the NEVPT2 ansatz to include dynamic correlation effects and are further refined by spin-orbit coupling contributions by quasidegenerate perturbation theory (QDPT). ${ }^{62}$

\subsection{Computational Rationalisation of Rhenium(III) Amine Complex 23}

DFT analysis of 23 on the PBE0-D3/def2TZVP level of theory places the triplet state below the singlet by $\Delta E_{T / S}=$ $38.7 \mathrm{~kJ} \cdot \mathrm{mol}^{-1}$ and therefore confirms the electronic ground state picture which evolved from the spectroscopic and magnetic characterisation (Figure 36a). The calculated structure for the triplet ground state converged in $C_{s}$ symmetry and is in good agreement with the found structure by XRD analysis, while the singlet structure gives higher deviations, especially for the equatorial chlorine bond length. The Mulliken and NBO/NPA spin densities support mainly metal centred radical character (Mulliken: 1.85; NBO/NPA:63 1.61) and give reason to expect a high SOC contribution if excited states are accessible. (Figure 36b)

a)

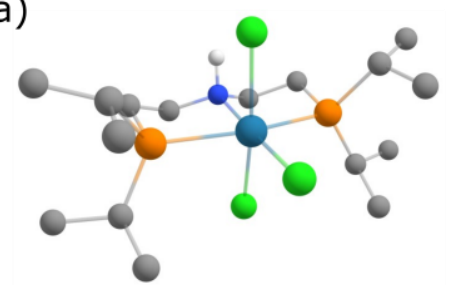

$23{ }^{3} A^{\prime \prime}$

\begin{tabular}{c|ccc}
$\AA$ & 23 XRD analysis & 23 DFT $\left({ }^{3} \mathrm{~A}^{\prime \prime}\right)$ & 23 DFT $\left({ }^{1} \mathrm{~A}^{\prime}\right)$ \\
\hline $\mathrm{Re}-\mathrm{N}$ & 2.16 & 2.20 & 2.20 \\
$\mathrm{Re}-\left.\mathrm{Cl}\right|_{\text {eq }}$ & 2.41 & 2.39 & 2.32 \\
$\mathrm{Re}-\mathrm{Cl} \mathrm{eq}$ & 2.38 & 2.36 & 2.37 \\
$\operatorname{Re}-\mathrm{P}$ & 2.44 & 2.42 & 2.38
\end{tabular}

b)

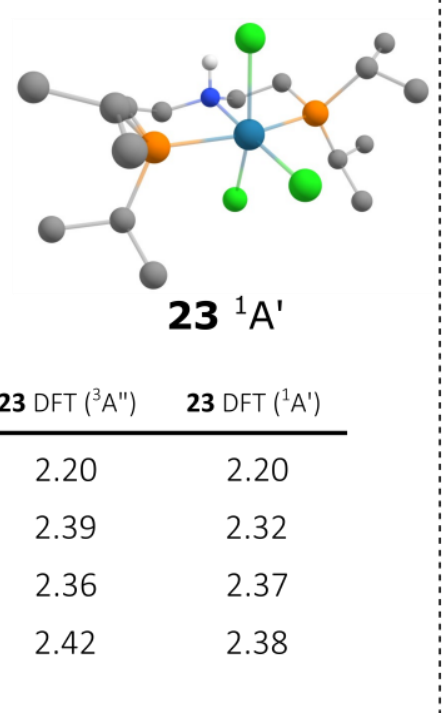

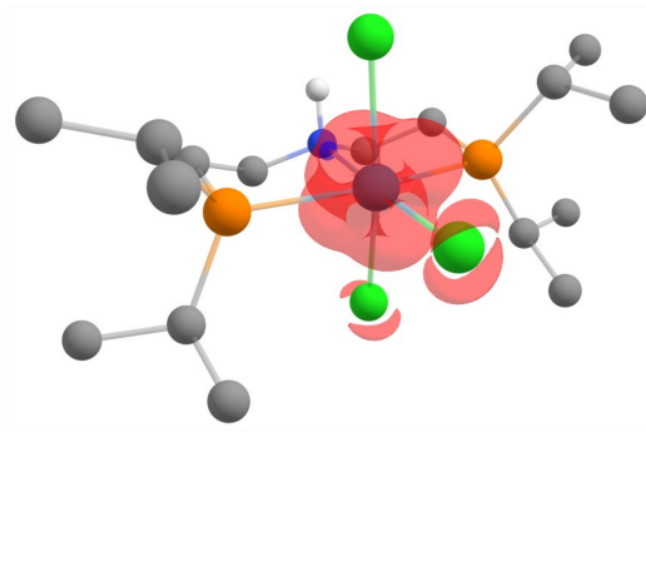

Figure 36: a) $C_{s}$ symmetric computed structures for 23 (Triplet ${ }^{~} \mathrm{~A}^{\prime \prime}$ and Singlet ${ }^{1} \mathrm{~A}^{\prime}$ ) on the PBE0-D3/def2TZVP level of theory and comparison of selected bond length to the experimental found structure by XRD analysis. b) DFT spin-density plot at isovalue $0.0075 \mathrm{a}_{0}{ }^{-3}$ for the ${ }^{3} \mathrm{~A}^{\prime \prime}$ ground state of 23 showing a mainly metal centred spin-density.

For the CASSCF computations two different active spaces were probed. A small CAS space, only including the $5 \mathrm{~d}$ orbitals of the rhenium metal centre (4x5) (Figure 37, left) and a bigger CAS space, additionally including the rhenium bonding interactions with the ligand environment resulting in a $(14,10)$ CAS extension (Figure 37 , right).

${ }^{62}$ For a detailed discussion of the performed computations in this Section see Section 4.3.1 in chapter VI.

${ }^{63}$ NBO/NPA: Natural Bond Orbital/Natural Population Analysis 


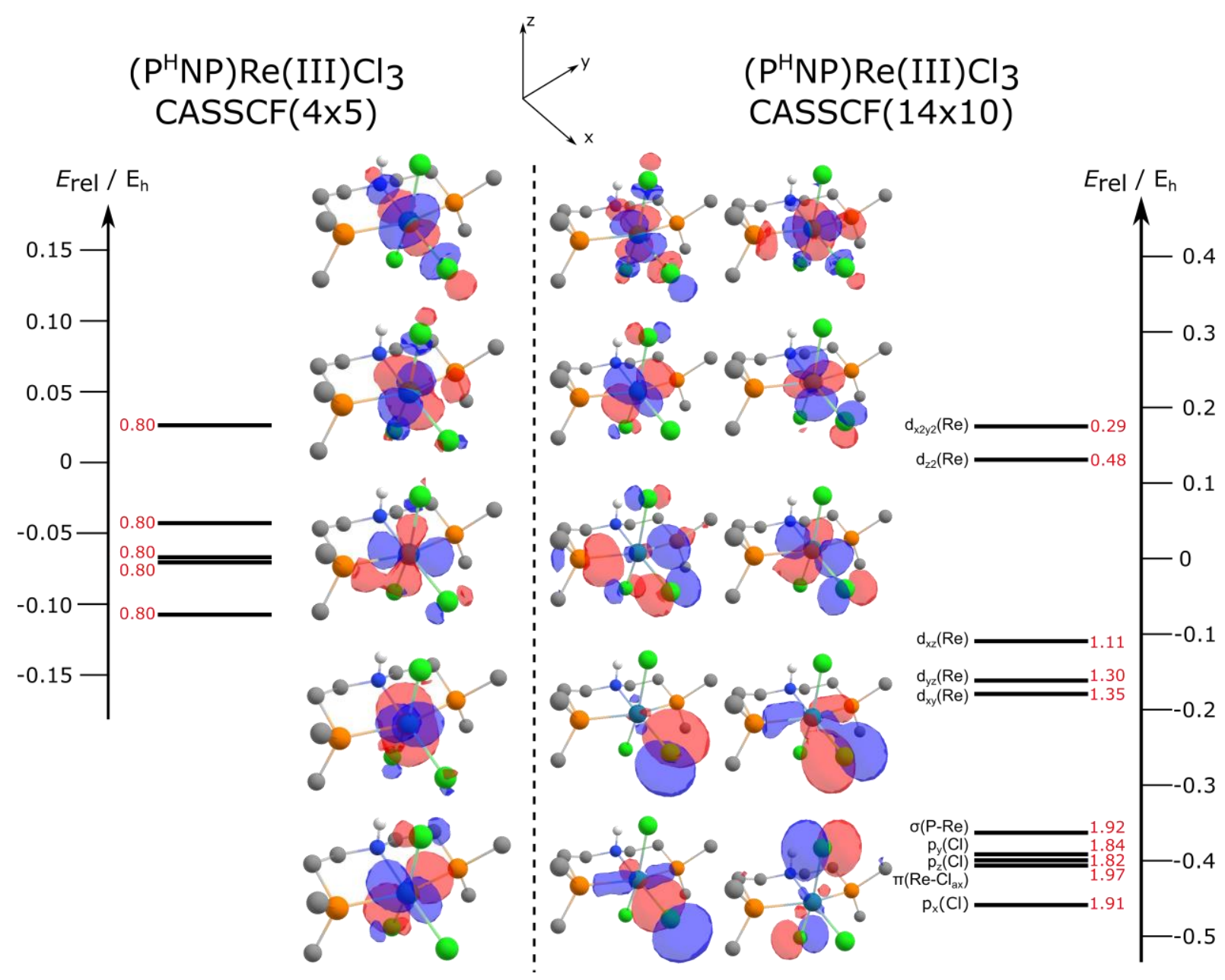

Figure 37: CASSCF orbitals of 23 for the small $(4,5)$ CAS space with only d orbitals encountered (left) and CASSCF orbitals for the large $(14,10)$ CAS space with the d-orbitals and the ligand bonding interactions encountered (right) orbital order shown from bottom left to top right.

The CASSCF/NEVPT2 wavefunctions place the triplet electromer over the singlet by $58.6 \mathrm{~kJ} \mathrm{~mol}^{-1}$ and $39.6 \mathrm{~kJ} \mathrm{~mol}^{-1}$ for the small and big CAS space, respectively and thus reproduce the electronic ground state picture obtained by DFT (Figure 38). The ground state of 23 in the big CAS space on the NEVPT2 level is dominated by the $\left[\left(d_{x y}{ }^{2}\right)\left(d_{y z}{ }^{1}\right)\left(d_{x z}{ }^{1}\right),(68 \%)\right]$ configuration and the first excited state $\left[\left(d_{x y}{ }^{1}\right)\left(d_{y z}{ }^{2}\right)\left(d_{x z}{ }^{1}\right),(68 \%)\right]$ is only $5 \mathrm{~cm}^{-1}$ higher in energy, which results in an effective twofold degenerate $\left({ }^{3} E\right)$ ground state. These two states are separate by over $1000 \mathrm{~cm}^{-1}$ in the small CAS space. Treatment of spin-orbit coupling by means of QDPT leads to extensive mixing of the triplet states of 23 , which results in a high SOC stabilisation of -44.9 and $-46.4 \mathrm{~kJ} \mathrm{~mol}^{-1}$ for the small and big CAS space, respectively. Delightfully, for both CAS spaces bright transitions from the ground state to the $4^{\text {th }} / 5^{\text {th }}$ excited state are predicted. While the small CAS space places these transitions at $4400 \mathrm{~cm}^{-1}$, the big CAS space predicts them at $3900 \mathrm{~cm}^{-1}$. 
( $\left.\mathrm{P}^{\mathrm{H}} \mathrm{NP}\right) \mathrm{Re}(\mathrm{III}) \mathrm{Cl}_{3}$

CASSCF $(4 \times 5)$

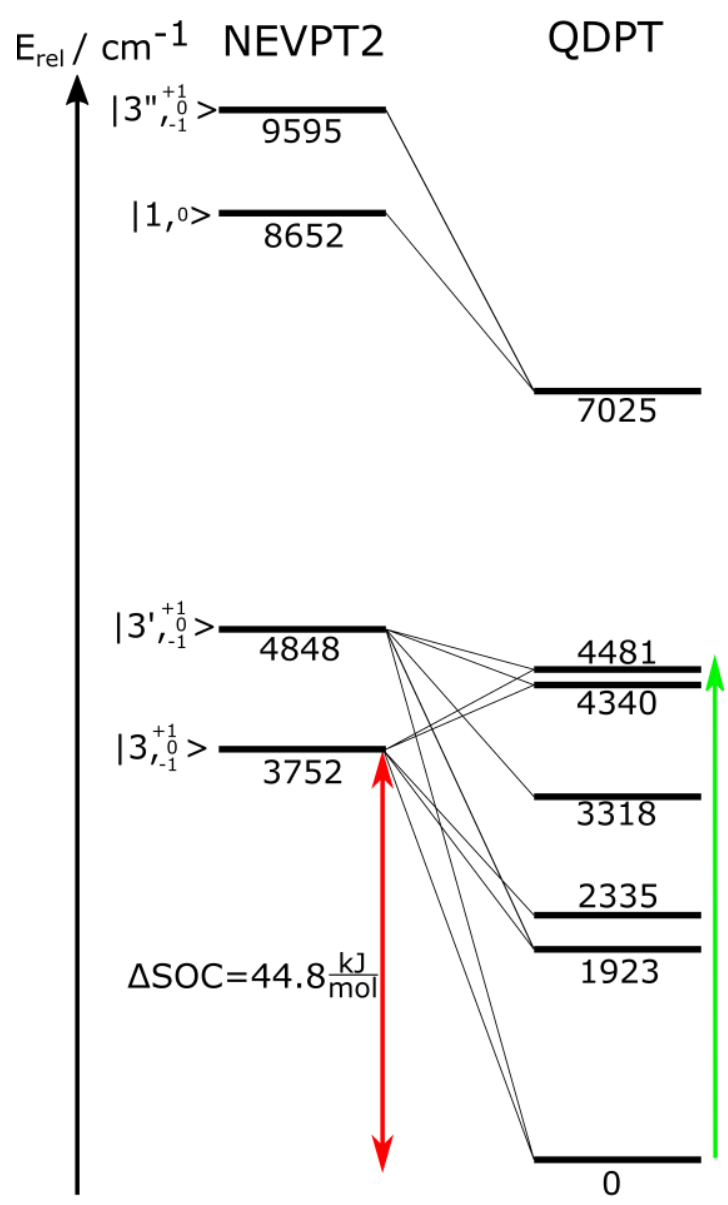

$\left(\mathrm{P}^{\mathrm{H}} \mathrm{NP}\right) \mathrm{ReCl}_{3}$

CASSCF $(14 \times 10)$

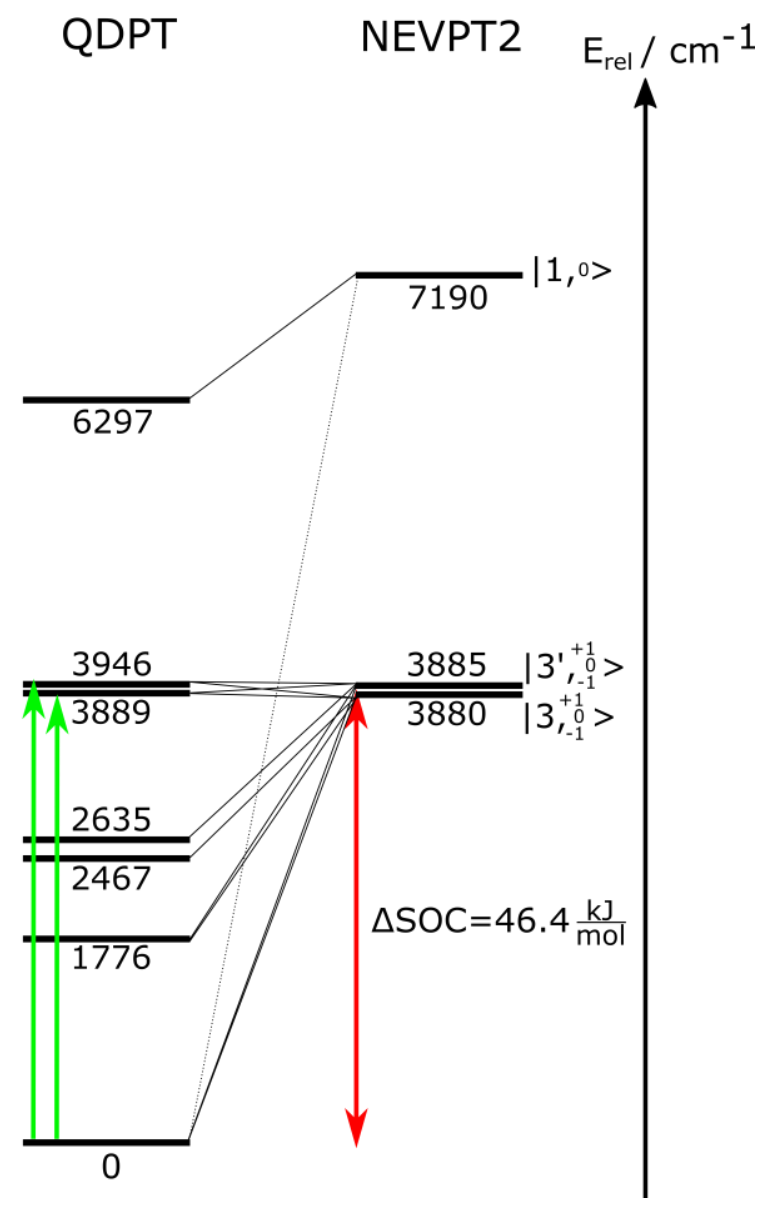

Figure 38: State correlation diagrams for the small $(4,5)$ and big $(14,10)$ complete active spaces from NEVPT2 calculations with (QDPT) and without $($ NEVPT2) spinorbit coupling for 23.

A comparison between the predicted values to the observed transition in the midIR spectrum of 23 at $3500 \mathrm{~cm}^{-1}$ (Figure 32) gives a significantly higher agreement of the experiment with the big CAS space than with the small CAS space. For this reason, the big $(14,10)$ CAS space is analysed in more detail. The lowest eigenstate of 23 on the QDPT level is equally composed of the two "degenerate" triplet states and also the first singlet is mixed in with a minor amount $\left.\left(|3\rangle\left(m_{s}= \pm 1\right)(40 \%) ;\left|3^{\prime}>\left(m_{s}= \pm 1\right)(40 \%) ;\right| 1\right\rangle(6 \%)\right)$. The computed temperature dependent magnetisation of 23 (Figure 39, a yellow) is in line with the experimentally found data by SQUID magnetometry and only underestimates to a minor degree the experimentally found values (Figure 39, b). To benchmark the CASSCF wavefunction further, also the UV-Vis and NIR spectra were recorded. 23 shows one intense charge transfer transition at $409 \mathrm{~nm}\left(24400 \mathrm{~cm}^{-1}\right)$ and two weak d-d transitions at $523 \mathrm{~nm}\left(19100 \mathrm{~cm}^{-1}\right)$ and $800 \mathrm{~nm}$ $\left(12500 \mathrm{~cm}^{-1}\right)$ respectively, while in the NIR region three small transitions at $1000 \mathrm{~nm}\left(10000 \mathrm{~cm}^{-1}\right), 1500 \mathrm{~nm}$ $\left(6700 \mathrm{~cm}^{-1}\right)$ and $1750 \mathrm{~nm}\left(5700 \mathrm{~cm}^{-1}\right)$ are observed (Figure $39 \mathrm{c}$ black and red). ${ }^{64}$

The computed spectrum on the CASSCF-NEVPT2/QDPT level of theory (Figure 39c magenta) reproduces the experimental spectrum, even though some differences can be observed. The intense charge transfer transition at

${ }^{64}$ The spectra are shown with a linear energy scale $\left(\right.$ in $\mathrm{cm}^{-1}$ ) for a better comparability tot he computed spectra. 
$409 \mathrm{~nm}$ in the experimental spectrum is computed to $358 \mathrm{~nm}$ on the ab initio level of theory. This quite high deviation from the experiment can be explained by solvent interactions with the $\mathrm{N}-\mathrm{H}$ proton, since charge transfer bands are commonly observed to be highly solvent dependent. ${ }^{[221]}$ TD-DFT computations were performed to test the this hypothesis (Figure 39, b). Without solvent correction by SMD, a transition at $404 \mathrm{~nm}$ is predicted (Figure 39b black), while the band shifts upon treatment with SMD(DCM) to $424 \mathrm{~nm}$ (Figure 39b red), which confirms the charge transfer character. The transitions in the NIR spectrum of 23 are all well represented, even though blue shifted. Here again, solvation effects as well as vibrational coupling in the NIR region are possible explanations for the deviations from the experimental spectra. Both effects are neglected within the CASSCF computations. All together, the ab initio model satisfactorily reproduces the experimental found spectroscopic and magnetic features of 23 .
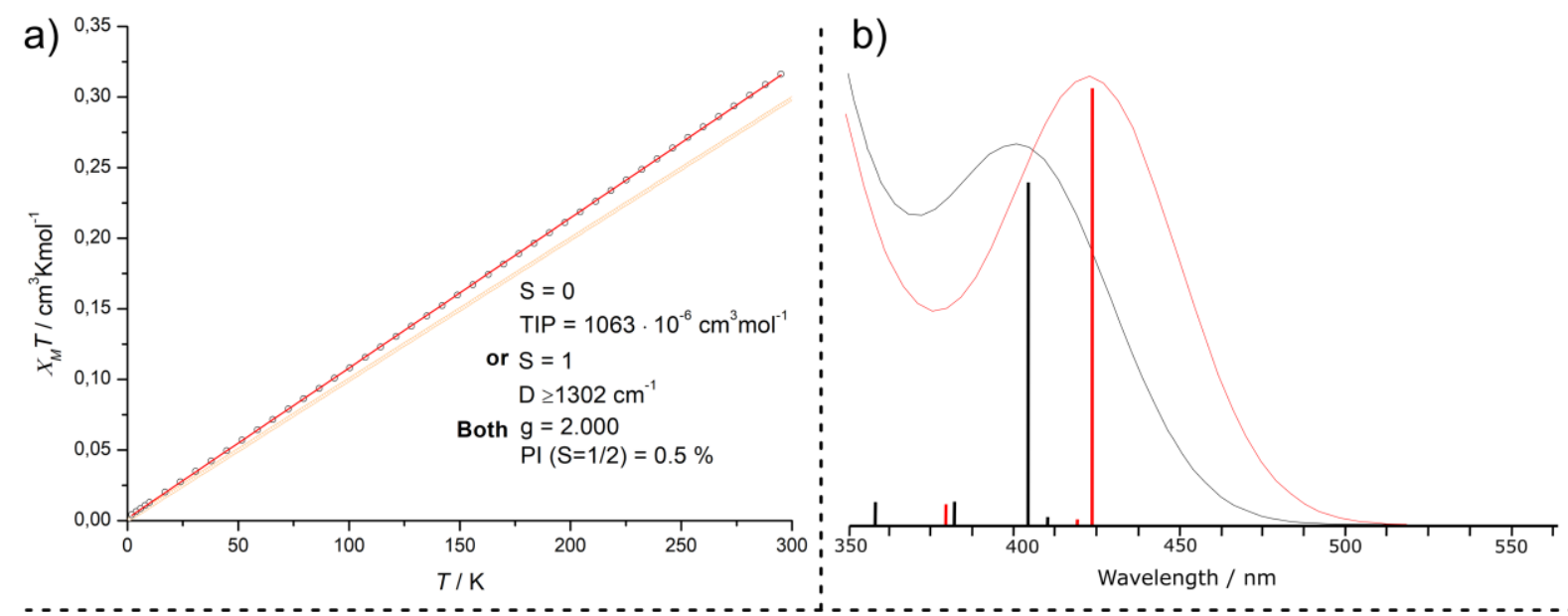

c)

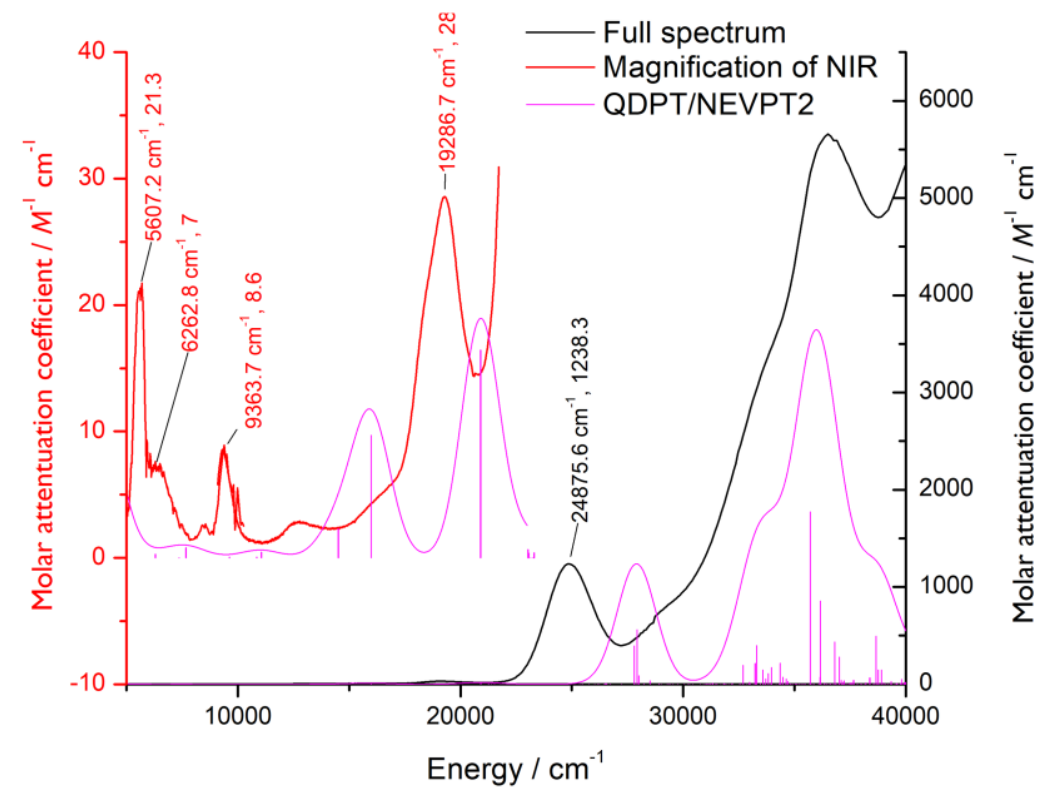

Figure 39: a) Comparison of the computed magnetisation for 23 on the $(14,10)$ CASSCF/NEVPT2-QDPT level of theory (orange) vs. the found magnetisation by SQUID magnetometry (black+red fit). b) Comparison of the TD-DFT(50 states) data of 23 with and without solvent correction by SMD for dichloromethane. c) Computed UV/Vis and NIR spectra of 23 on the $(14,10)$ CASSCF/NEVPT2-QDPT level of theory (pink) compared to the experimental spectra (black and red). 


\subsection{Computational Rationalisation of Rhenium(IV) Amide Complex 24}

Next, the electronic structure of the rhenium(IV) amide was analysed. DFT analysis on the PBE0-D3/def2TZVP level of theory confirms the doublet ground state $\left(\Delta \mathrm{ED}_{\mathrm{D}} \mathrm{Q}=15.3 \mathrm{~kJ} \cdot \mathrm{mol}^{-1}\right)$ of 24 , which reproduces the electronic ground state picture from the spectroscopic and magnetic characterisation (Figure 40a). The calculated structure for the doublet ground state converged in $C_{s}$ symmetry and is in very good agreement to the found structure by XRD analysis, while the quartet structure exhibits higher deviations especially for the Re-N bond length. The Mulliken and NBO/NPA analysis yield again mainly metal centred spin densities (Mulliken: 0.96; NBO/NPA: 0.84) and give reason to expect a high, but smaller SOC contribution than for 23 (Figure 40, b).

a)

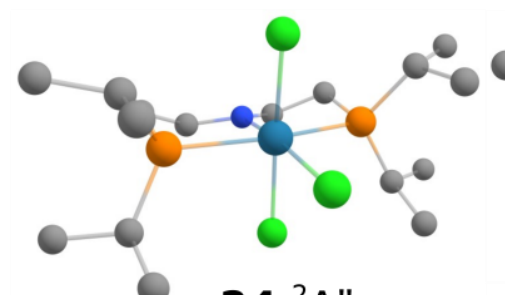

$24{ }^{2} \mathrm{~A} "$

\begin{tabular}{c|ccc}
$\AA$ & 24 XRD analysis & 24 DFT $\left({ }^{2} \mathrm{~A}^{\prime \prime}\right)$ & $\mathbf{2 4}$ DFT $\left({ }^{4} \mathrm{~A}^{\prime}\right)$ \\
\hline $\mathrm{Re}-\mathrm{N}$ & 1.90 & 1.92 & 2.05 \\
$\mathrm{Re}-\mathrm{Cl}$ & 2.43 & 2.43 & 2.36 \\
$\mathrm{Re}-\mathrm{Cl}$ & 2.37 & 2.32 & 2.36 \\
$\mathrm{Re}-\mathrm{P}$ & 2.45 & 2.43 & 2.49
\end{tabular}

$24{ }^{4} A^{\prime}$ b)
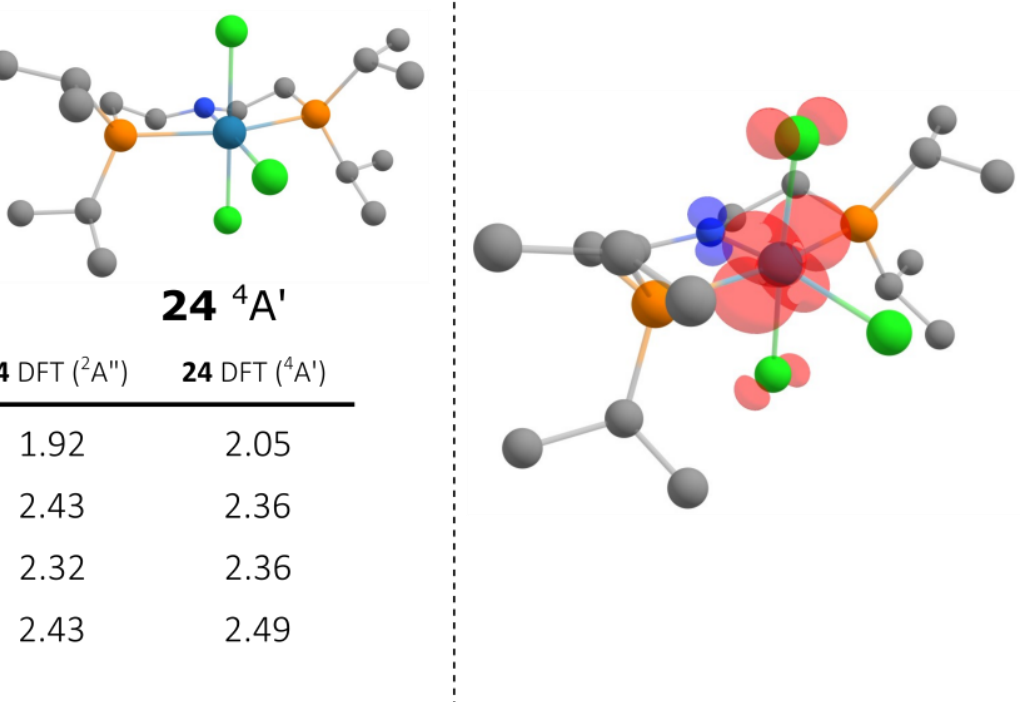

Figure 40: a) $C_{s}$ symmetric computed structures for 24 (Doublet ${ }^{2} A^{\prime \prime}$ and Quartet ${ }^{4} A^{\prime}$ ) on the PBEO-D3/def2TZVP level of theory and comparison of selected bond length to the experimental found structure by XRD analysis. b) DFT spin-density plot at isovalue $0.0075 \mathrm{a}_{0}^{-3}$ for the ${ }^{2} \mathrm{~A}^{\prime \prime}$ ground state of 24 .

A more detailed analysis of the electronic structure was again reached by CASSCF-NEVPT2 analysis. The same CAS spaces as for 23 were chosen with the difference, that for the big CAS space the additional $N$-Re $\pi$-bond was considered, leading to a $(3,5)$ and $(15,11)$ CAS extension, respectively (Figure 41 , left and right). The CASSCFNEVPT2 wavefunctions place the doublet electromer over the singlet by $60.4 \mathrm{~kJ} \mathrm{~mol}^{-1}$ for the small CAS space and by $43.3 \mathrm{~kJ} \mathrm{~mol}^{-1}$ for the big CAS space and thus reproduce the electronic ground state picture obtained by DFT (Figure 41, bottom). The ground state is dominated by the $\left(d_{x y}{ }^{2}\right)\left(d_{y z}{ }^{1}\right)$ configuration $(82 \%)$ and experiences strong mixing with the first excited doublet state $\left(\left|2>\left(m_{s} \pm 1 / 2\right)(54 \%) ;\right| 2^{\prime}>\left(m_{s} \pm 1 / 2\right)(35 \%)\right)$ upon treatment of spin-orbit coupling by means of QDPT, which leads to a stabilisation of $-22.3 \mathrm{~kJ} \mathrm{~mol}^{-1}$ and $-18.5 \mathrm{~kJ} \mathrm{~mol}^{-1}$ for the small and big CAS space, respectively (Figure 41, bottom). Delightfully, the SOC stabilisation of 24 is therefore $28 \mathrm{~kJ} \mathrm{~mol}^{-1}$ lower in energy compared to the SOC stabilisation of 23, thus a high SOC effect on the PCET thermochemistry is expected. Additionally, also a transition from the ground state to the $1^{\text {st }} / 2^{\text {nd }}$ excited state of 23 with non-negligible oscillator strength is found in both CAS spaces. Again, the bigger $(15,11)$ CAS space performs significantly better with a predicted transition at $2200 \mathrm{~cm}^{-1}$, compared to the found transition at $2000 \mathrm{~cm}^{-1}$, while the small $(3,5)$ CAS places the same transition at $2600 \mathrm{~cm}^{-1}$. 


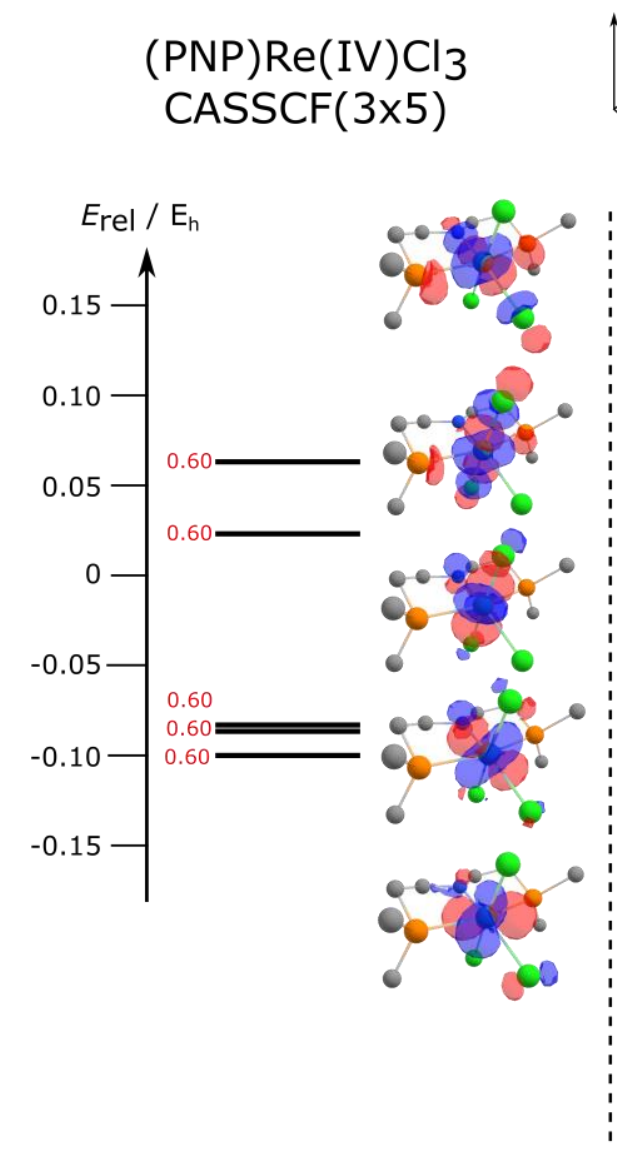

(PNP)Re(IV)Cl

CASSCF $(3 \times 5)$

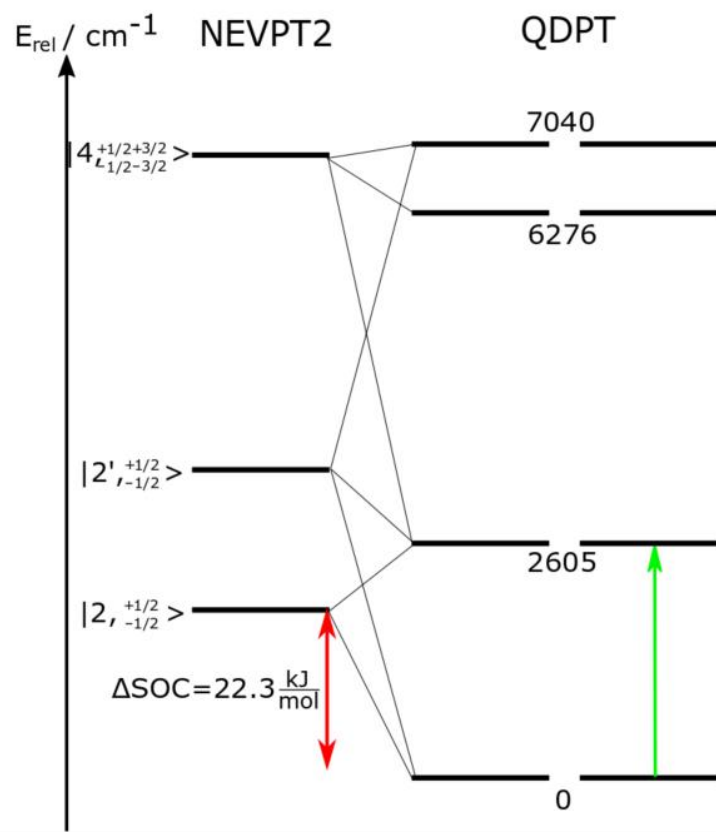

(PNP) $\mathrm{Re}(\mathrm{IV}) \mathrm{Cl}_{3}$

CASSCF $(15 \times 11)$

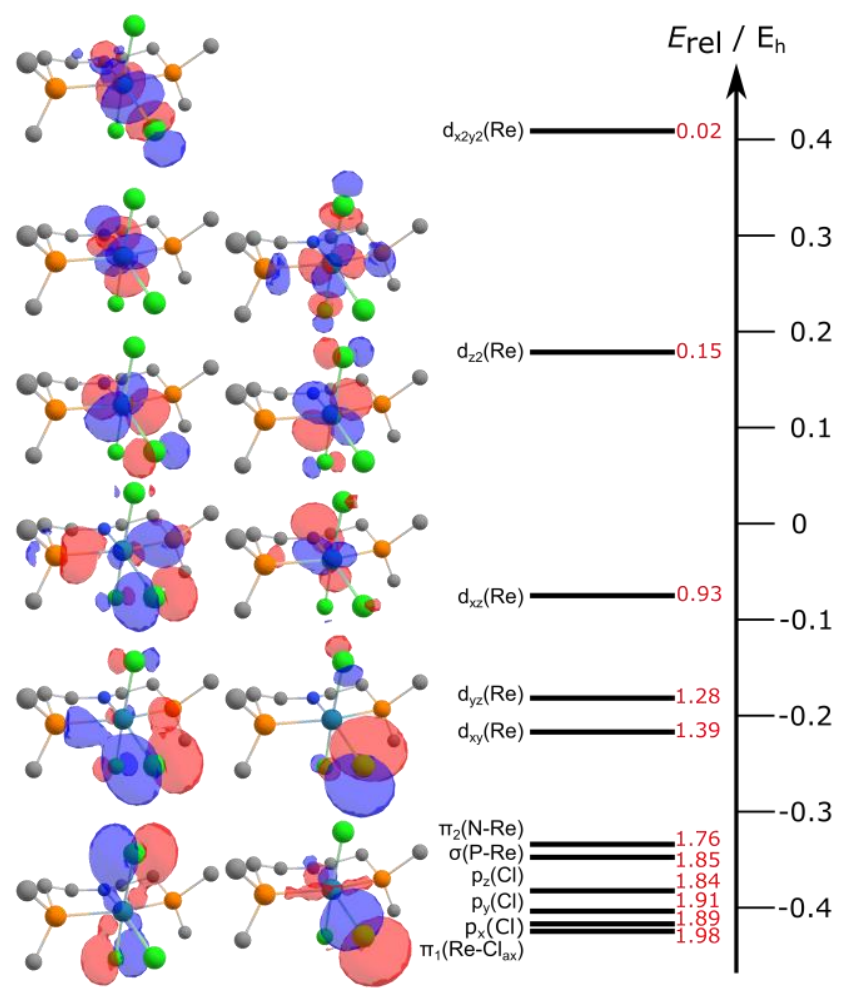

(PNP) $\operatorname{Re}(\mathrm{IV}) \mathrm{Cl}_{3}$

CASSCF $(15 \times 11)$

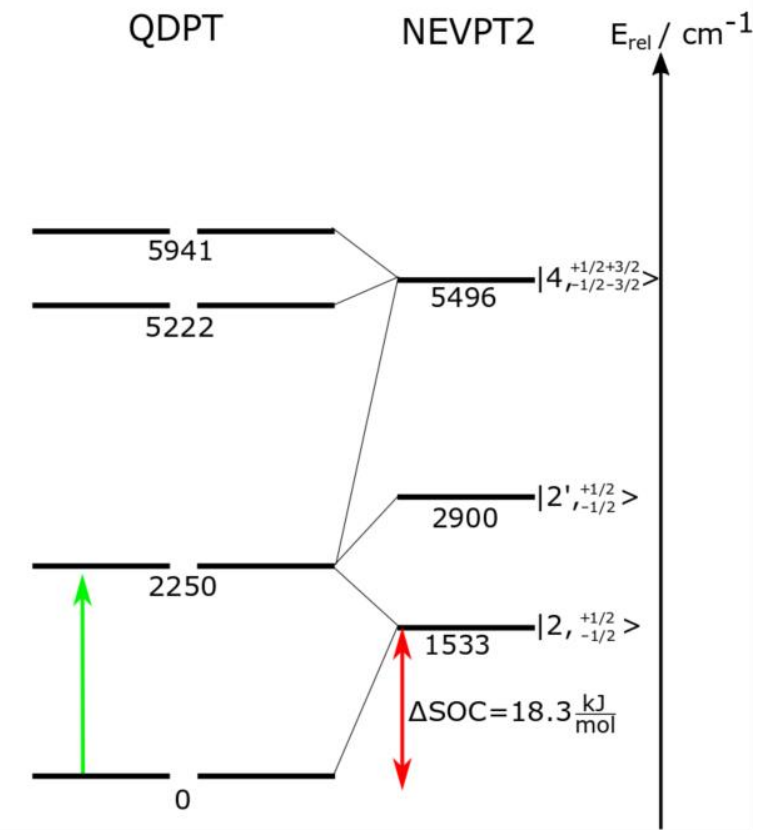

Figure 41: Top) CASSCF orbitals of the small $(3,5)$ CAS space (left) CASSCF orbitals of the big $(15,11)$ CAS space (right) for 24 , orbitals shown from lowest in energy left bottom to top. bottom) State correlation diagrams for the big $(15,11)$ (left) and small $(3,5)$ (right) complete active spaces from NEVPT2 calculations with $($ QDPT) and without spin-orbit coupling for 24 
The computed temperature dependent magnetisation of 24 (Figure 42a cyan) reproduces the experimentally found data by SQUID magnetometry and the computed TIP is only underestimated to a minor degree compared to the experimentally found values (cf. Figure 42, cyan/blue). The CASSCF wavefunction is further benchmarked by a comparison of the computed (Figure 42c magenta) to the experimentally recorded UV-Vis and NIR spectra of 24 (Figure 42c red and black). The UV-Vis spectrum of 24 has an intense transition at $290 \mathrm{~nm}\left(34440 \mathrm{~cm}^{-1}\right)$, a dominant charge transfer band at $520 \mathrm{~nm}\left(19200 \mathrm{~cm}^{-1}\right)$ with a low intensity edge around $600 \mathrm{~nm}\left(16700 \mathrm{~cm}^{-1}\right)$, which can be assigned to a $d$ - $d$ transition, while in the NIR region only one very broad signal at $1600 \mathrm{~nm}\left(6250 \mathrm{~cm}^{-1}\right)$ can be found (Figure 42). The intense transition at $290 \mathrm{~nm}$ is very well reproduced to $286 \mathrm{~nm}$, while the computed oscillator strength does not match at all the observed intensity. The chosen CAS space might be to small to describe the high energy of this transition, since not all MOs of the axial chlorine atoms are treated in the computation and thus their contribution to the transition is neglected. The intense transition at $520 \mathrm{~nm}$ is reproduced nicely by the computation to $533 \mathrm{~nm}$.
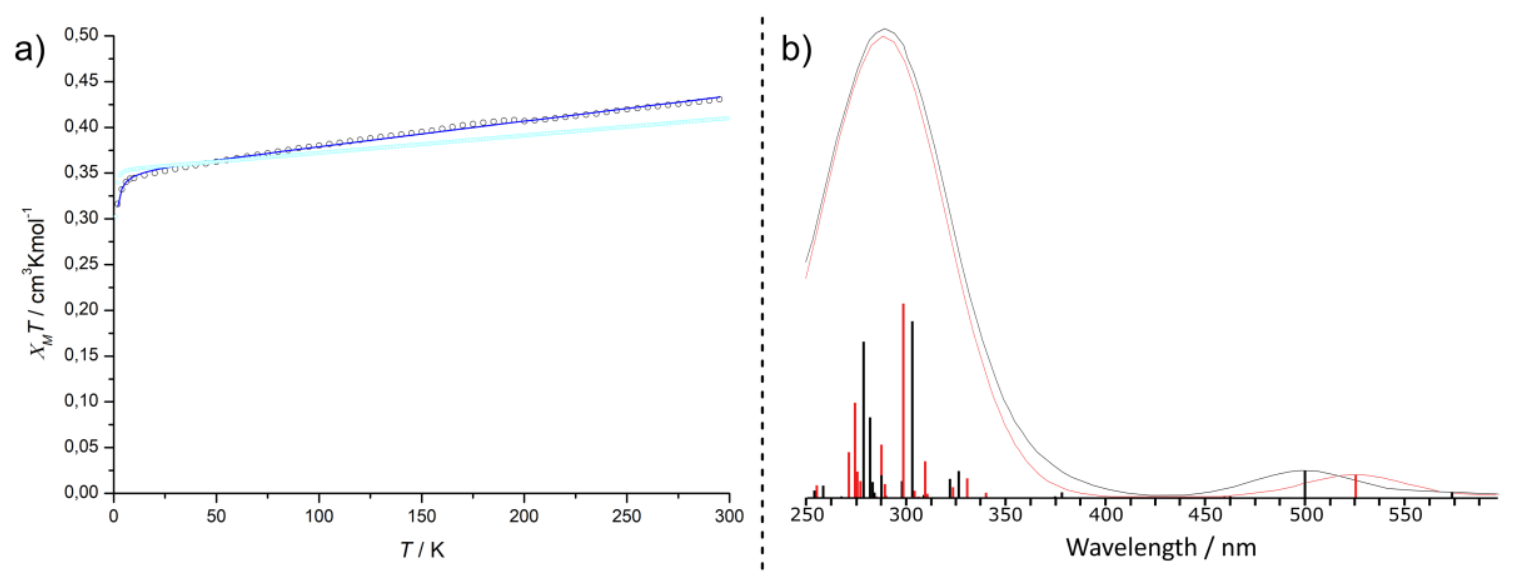

c)

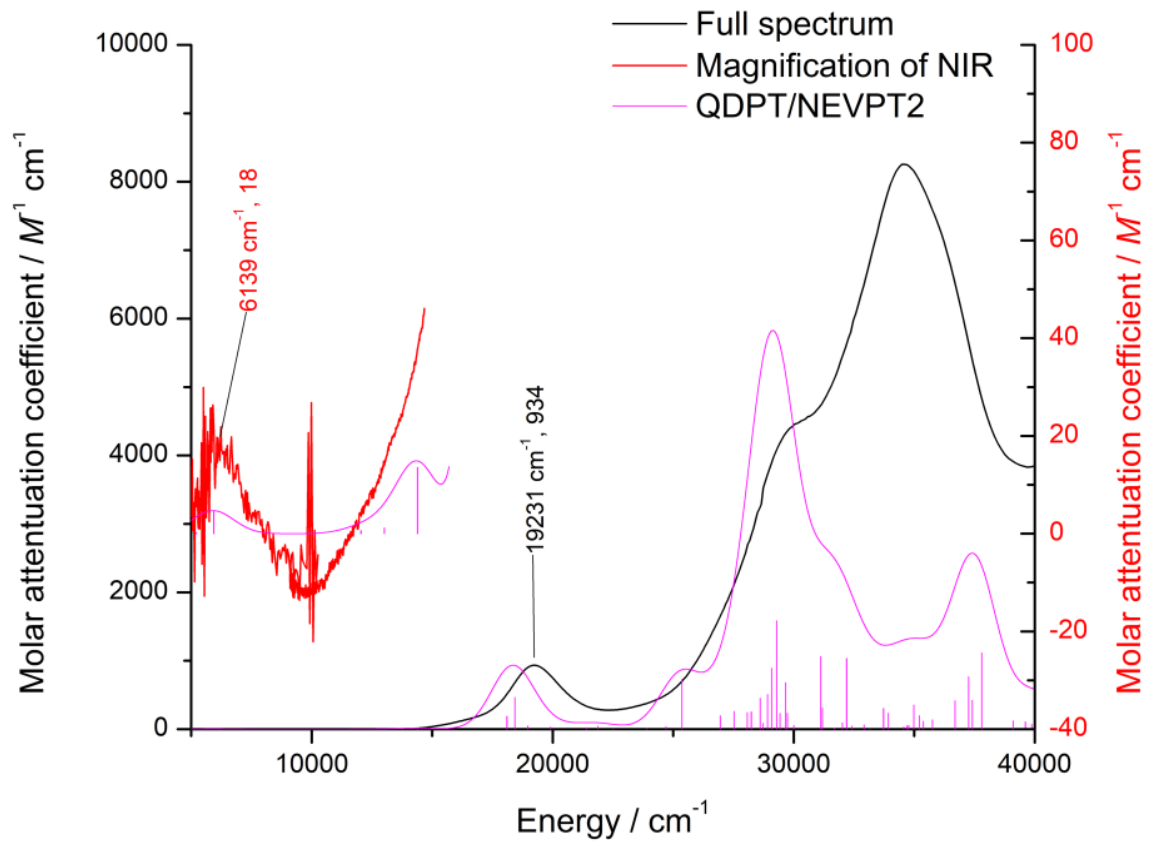

Figure 42: a) Comparison of the computed magnetisation for 24 on the $(15,11)$ CASSCF/NEVPT2-QDPT level of theory (cyan) vs. the found magnetisation by SQUID magnetometry (black+blue fit). b) Comparison of the TD-DFT(50 states) data of 24 with and without solvent correction by SMD for dichloromethane. c) Computed UV/Vis and NIR spectra of 24 on the (15,11) CASSCF/NEVPT2-QDPT level of theory (pink) compared to the experimental spectra (black and red). c) Computed UV/Vis and NIR spectra of 24 on the $(15,11)$ CASSCF/NEVPT2-QDPT level of theory (pink) compared to the experimental spectra (black and red). 
The charge transfer character of this band could be confirmed again by TD-DFT computations by the difference between the computed spectra with and without SMD correction. (Figure 42b). The better agreement on this band compared to 23 could be explained by less solvent interactions due to the abstracted N-H proton for 24 . In the end, the NIR region is as well, excellently reproduced by the computations. All together, the ab initio computation is satisfactorily reproducing the experimental found spectroscopic and magnetic features of 24 and additionally predicts an enormous differential spin-orbit coupling effect between 23 and 24 of $\Delta \Delta S O C=28 \mathrm{~kJ} \mathrm{~mol}^{-1}$.

\subsection{Computational Rationalisation of Rhenium(IV) Amine Complex 25}

At the end the electronic structure of the cationic rhenium(IV) amine 25 is computationally rationalized. DFT analysis on the PBEO-D3/def2TZVP level of theory confirms the predicted quartet ground $\mathrm{state}\left(\triangle \mathrm{E}_{\mathrm{D} / \mathrm{Q}}=\right.$ $73 \mathrm{~kJ} \mathrm{~mol}^{-1}$ ). The calculated structure for the quartet ground state converged in $\mathrm{C}_{\mathrm{s}}$ symmetry and is in very good agreement with the found structure by XRD analysis, while the doublet structure gives higher deviations especially for the Re-P bond length (Figure 44, a). The Mulliken spin density is mainly metal centred, with small contributions of the coordinated chlorine atoms ( $\mathrm{Re}: 2.56 ; \mathrm{Cl}_{\text {eq }} 0.21 ; 2 \mathrm{Clax}$ : 0.25 ) and gives reason to expect a high SOC contribution (Figure 44b). A more detailed analysis of the electronic structure was again obtained by CASSCFNEVPT2 analysis. For comparability, the same CAS space as for 23 and 24 was chosen resulting in a $(13,10)$ expansion (Figure 44, left). The CASSCF-NEVPT2 wavefunction places the quartet electromer over the doublet by $93 \mathrm{~kJ} \mathrm{~mol}^{-1}$ and thus reproduces the electronic ground state picture derived by DFT (Figure 44, right). The ground state is dominated by the $\left(\mathrm{d}_{x y}{ }^{1}\right)\left(\mathrm{d}_{\mathrm{yz}}{ }^{1}\right)\left(\mathrm{d}_{\mathrm{xz}}{ }^{1}\right)$ configuration and stabilizes upon treatment of spin-orbit coupling by means of QDPT by only $-11.6 \mathrm{~kJ} \mathrm{~mol}^{-1}$. The lowest eigenstates experience neglectable mixing with the excited states, explaining the low spin-orbit stabilisation, even though the spin density is metal centred. For this reason, also no IR active electronic transition was found, since the first excited states are insignificantly stabilized by SOC and thus located in the NIR region.

a)

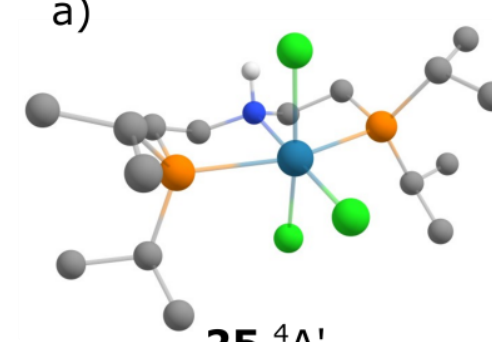

$25{ }^{4} A^{\prime}$

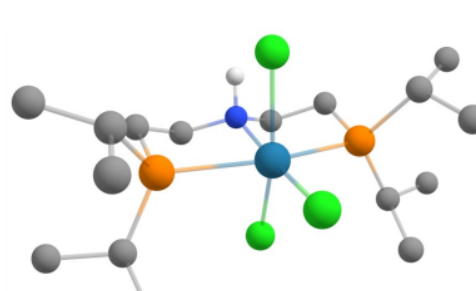

$25^{2} A^{\prime \prime}$

\begin{tabular}{c|ccc}
$\AA$ & $\mathbf{2 5}$ XRD analysis & $\mathbf{2 5}$ DFT $\left({ }^{4} \mathrm{~A}^{\prime}\right)$ & $\mathbf{2 5}$ DFT $\left({ }^{2} \mathrm{~A}^{\prime \prime}\right)$ \\
\hline $\mathrm{Re}-\mathrm{N}$ & 2.20 & 2.22 & 2.23 \\
$\mathrm{Re}-\mathrm{Cl} \mathrm{eq}$ & 2.29 & 2.28 & 2.24 \\
$\mathrm{Re}_{\mathrm{Cl}}$ & 2.33 & 2.33 & 2.32 \\
$\mathrm{Re}-\mathrm{P}$ & 2.49 & 2.50 & 2.46
\end{tabular}

b)

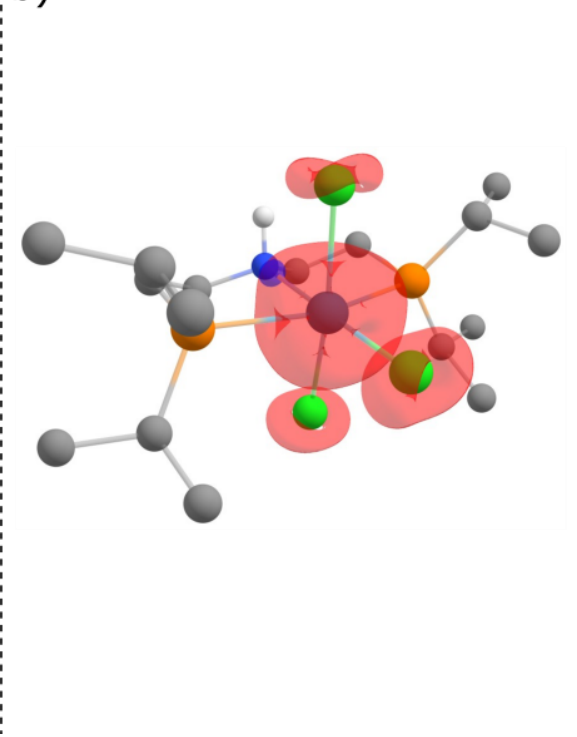

Figure 43: a) $C_{5}$ symmetric computed structures for 25 (Doublet ${ }^{2} \mathrm{~A}^{\prime \prime}$ and Quartet ${ }^{4} \mathrm{~A}^{\prime}$ ) on the PBE0-D3/def2TZVP level of theory and comparison of selected bond length to the experimental found structure by XRD analysis. b) DFT spin-density plot at isovalue $0.0075 \mathrm{a}_{0}{ }^{-3}$ for the ${ }^{4} \mathrm{~A}^{\prime}$ ground state of 25 . 


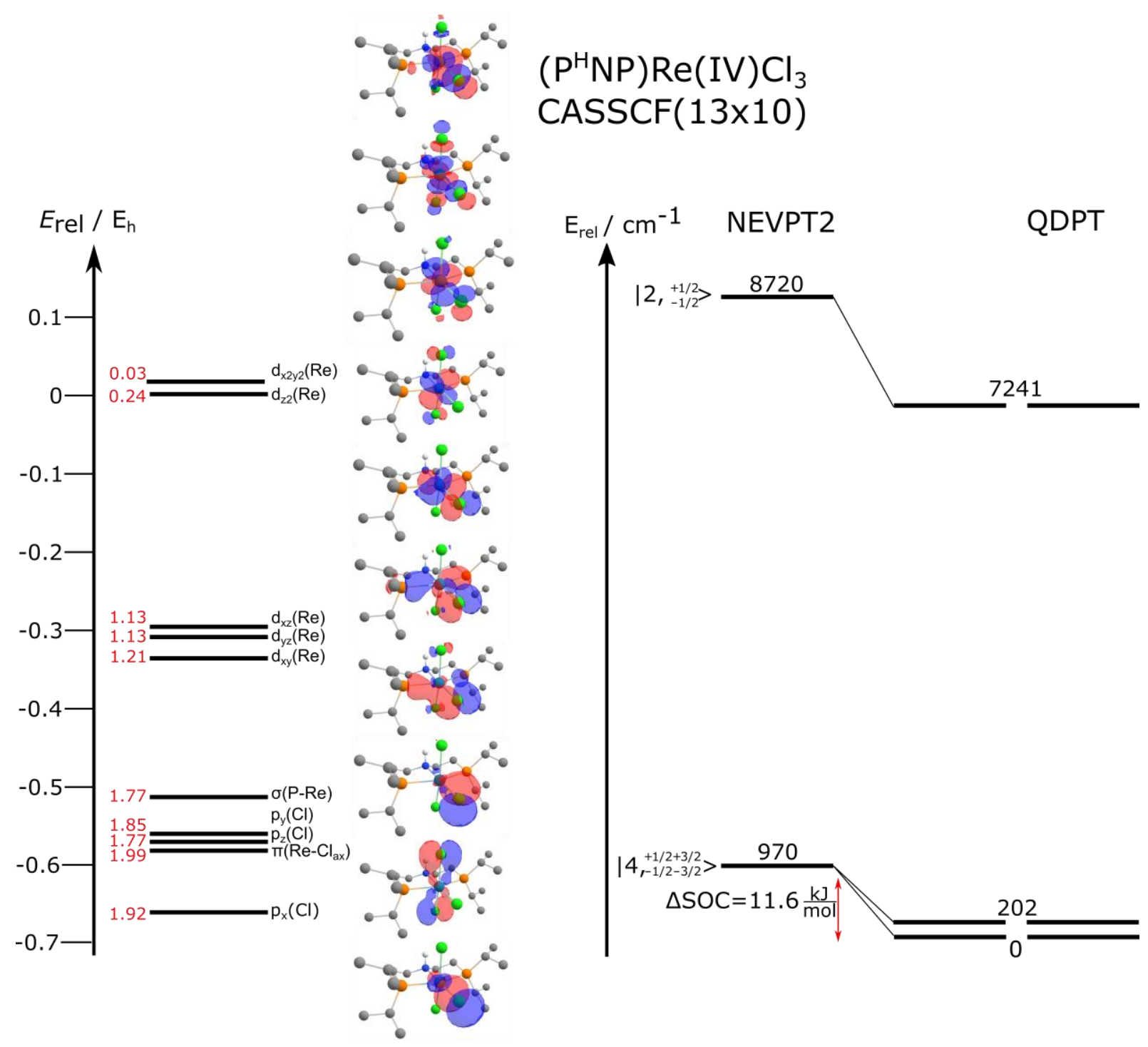

Figure 44: Left: CASSCF orbital scheme of 25 Right: State correlation diagrams of the $(13,10)$ complete active space from NEVPT2 calculations with (QDPT) and without spin-orbit coupling for 25 . 


\section{Reaction (Free) Enthalpy Determination}

\subsection{Experimental (Free) Reaction Enthalpy Determination}

Next, the reaction (free) enthalpy of the PCET reaction of 23 forming 24 is measured as a benchmark by isothermal titration calorimetry (ITC) ${ }^{65}$ for comparison of the spin-free computational results with the experiment. Therefore, the strong hydrogen atom acceptor Mes*O was titrated in small steps with 23 in THF and DCM (Scheme 10).

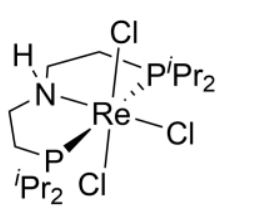

23<smiles>CC(C)(C)c1cc(Br)c(O)c(Br)c1</smiles>

${ }^{t} \mathrm{Bu}$

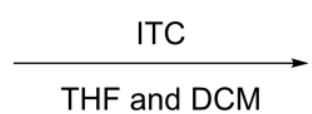

THF and DCM

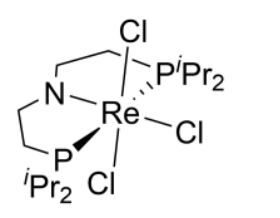

24<smiles>CC(C)(C)c1cc(Br)c(O)c(Br)c1</smiles>

${ }^{t} \mathrm{Bu}$

Scheme 10: Reaction of 23 with Mes* ${ }^{*}$ in THF or DCM at $25^{\circ} \mathrm{C}$ analysed by isothermal titration calorimetry.

The obtained data was integrated over time and fitted with an independent PCET model (Figure 45). ${ }^{66}$ On the one hand, the measured enthalpies all scatter within a narrow window of less than $1 \mathrm{~kJ} \mathrm{~mol}^{-1}$ and $0.1 \mathrm{~kJ} \mathrm{~mol}^{-1}$ for THF and DCM, respectively and are thus very precisely determined, while the free enthalpy of the reaction on the other hand could not be derived by ITC. Here, the to steep slope around the equivalence point hampered a meaningful fit of the titration curve. The reaction enthalpy is significantly changing between the two chosen solvents. While in THF $-40 \mathrm{~kJ} \mathrm{~mol}^{-1}$ are released (Figure $45 \mathrm{a}$ ), the enthalpy sums up to $-51 \mathrm{~kJ} \mathrm{~mol}^{-1}$ in DCM (Figure $45 \mathrm{~b}$ ). The high solvent dependency can be tentatively attributed to the higher tendency of THF to form hydrogen bonds with the $\mathrm{N}-\mathrm{H}$ and $\mathrm{O}-\mathrm{H}$ protons of 23 and $\mathrm{Mes} * \mathrm{OH}$. Since the $\mathrm{N}-\mathrm{H}$ proton of 23 is sterically less shielded than the O-H proton of Mes* OH, 23 could be higher stabilized by solvation in THF than in DCM, resulting in a lower reaction enthalpy. Since the free reaction enthalpy could not be determined by ITC, a square scheme approach was probed to access the Gibbs energy of reaction on a different way (Figure 46a). In the literature, the Bordwell equation (Figure 46b $\left.l^{e f t}\right)^{[60]}$ is used to determine the binding free dissociation energy (BDFE), where the $C_{G}$ value of this equation is a correction to capture the free enthalpy of reaction of the formally formed hydrogen atom (including e.g. solvation and the potential of the $\mathrm{H}^{+} / \mathrm{H}$ couple in the respective solvent). Since the solvation of the hydrogen atom can only be estimated, the obtained BDFE may have a systematical error. This is of no importance, if relative values in the respective solvents are compared, but hinders the absolute free reaction enthalpy determination as obtained from the computational site. ${ }^{67}$ This problem can be surpassed by the determination of the square scheme parameters for both reagents 23 and $\mathrm{Mes} * \mathrm{OH}$ and rearranging the equation to a form, in which $\mathrm{C}_{\mathrm{G}}$ cancels out (Figure 46b).

\footnotetext{
${ }^{65} \mathrm{~A}$ comprehensive description and analysis of the performed ITC experiments, including detailed measurement conditions and all titration plots can be found in Section 3 of chapter VI.

${ }^{66}$ The details of the independent PCET model can be found in Section C of chapter 7.

${ }^{67}$ For more details about PCET thermochemistry see Section 2.2 of chapter I.
} 
a)

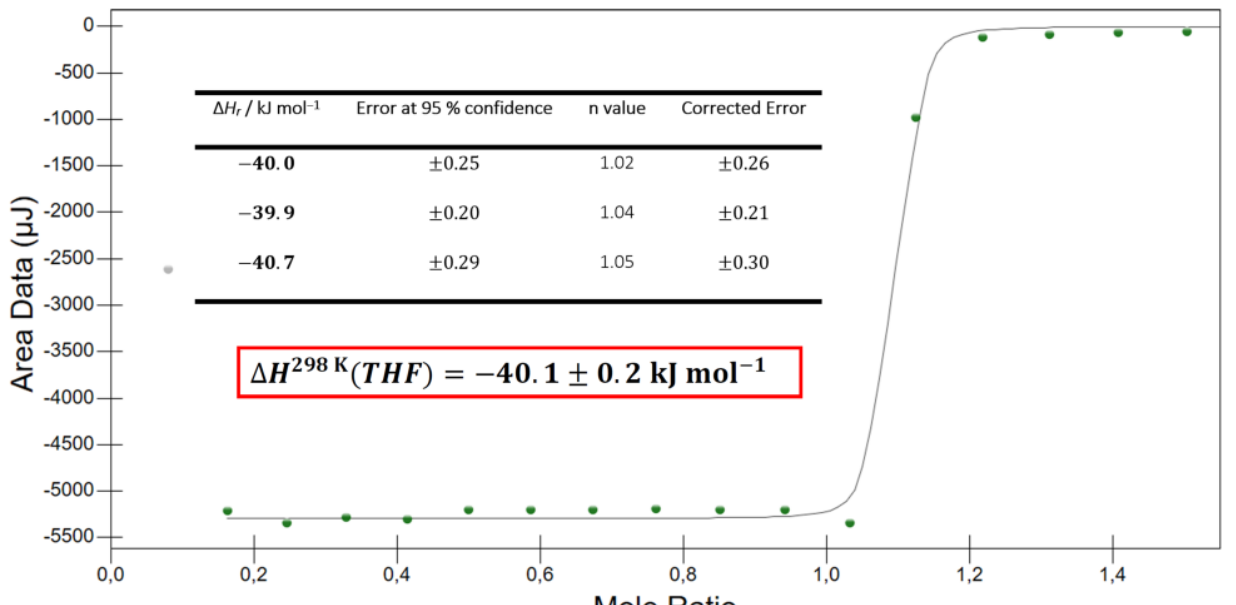

Mole Ratio

b)

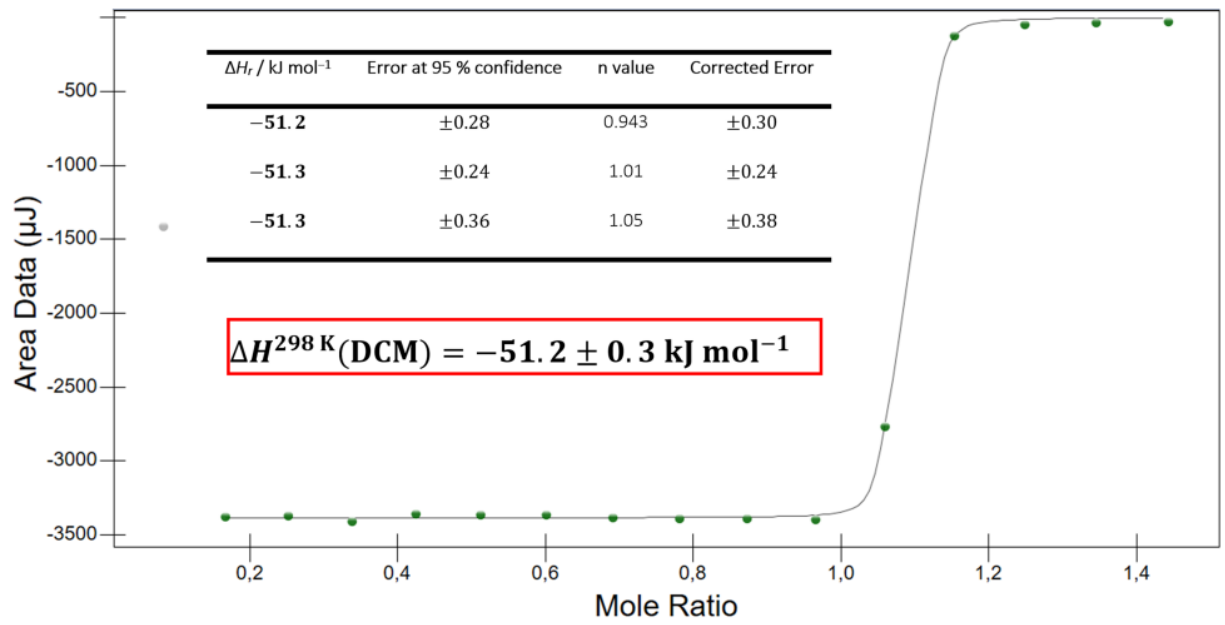

Figure 45: a) Representative titration plot and results table of three independent runs of the PCET reaction (Scheme 10) in THF. c) Representative titration plot and results table of three independent runs of the PCET reaction (Scheme 10) DCM.

a)

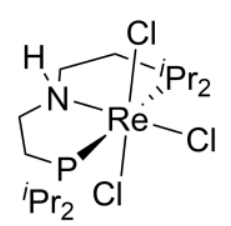

23<smiles>CC(C)(C)c1cc(Br)cc(C(C)(C)C)c1[O]</smiles>

$E^{o x}(15 / 17)$

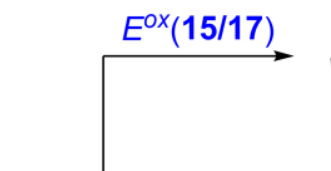

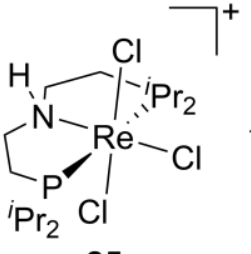

$\mathrm{p} K_{\mathrm{A}}(17)$

$\mathrm{Pr}_{2} \mathrm{Cl}$<smiles>C1CCC1</smiles>

$\Delta G(\mathrm{PT})$

$\stackrel{\Delta G_{\mathrm{r}}}{\longrightarrow} \quad 23$ $\mathrm{Pr}_{2} \mathrm{Cl}$
23
+<smiles>CC(C)(C)c1cc(Br)cc(C(Br)(Br)Br)c1O</smiles>

b)

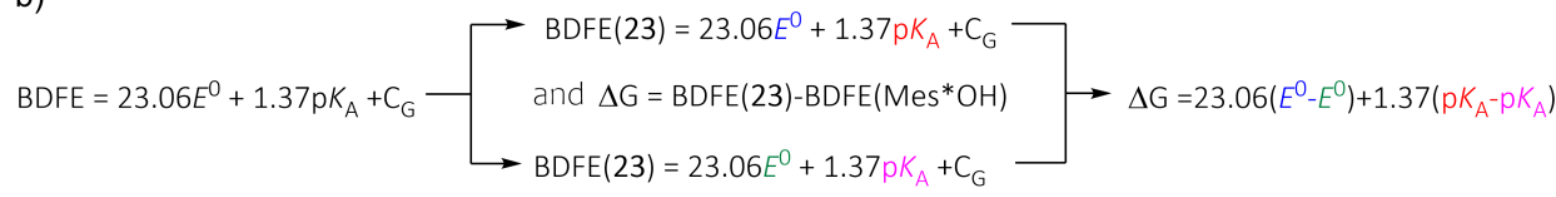

Figure 46: a) Approach for the determination of the free reaction enthalpy of the PCET reaction between 23 and Mes*O b) Rearrangement of the Bordwell equation for the cancelation of $C_{G}$. 
Therefore, the electrochemical potentials of the $23 / 25$ oxidation and $\mathrm{Mes}^{*} \mathrm{O} / \mathrm{Mes}^{*} \mathrm{O}^{-}$reduction as well as the $\mathrm{p} K_{\mathrm{A}}$ values of 25 and $\mathrm{Mes}^{*} \mathrm{OH}$ in THF must be determined. The Mes* $\mathrm{O}^{\mathrm{Mes}}{ }^{*} \mathrm{O}^{-}$electrochemical couple was reported previously ${ }^{[184]}$ to $E^{O}\left(\mathrm{Mes}^{*} \mathrm{O} / \mathrm{Mes}^{*} \mathrm{O}^{-}\right)=-0.96 \mathrm{~V}$ vs. $\mathrm{Fc} / \mathrm{Fc}^{+}$. The oxidation potential of 23 had to be reevaluated to $E^{0}(23 / 25)=-0.29 \mathrm{~V}$ vs. $F c / F c^{+}$in contrast to the published value of $E^{0}(23 / 25)=-0.24 \mathrm{~V} \mathrm{vs}$. Fc/Fc $\mathrm{Fhich}^{+}$wuffered from internal reference issues. ${ }^{[158], 68}$
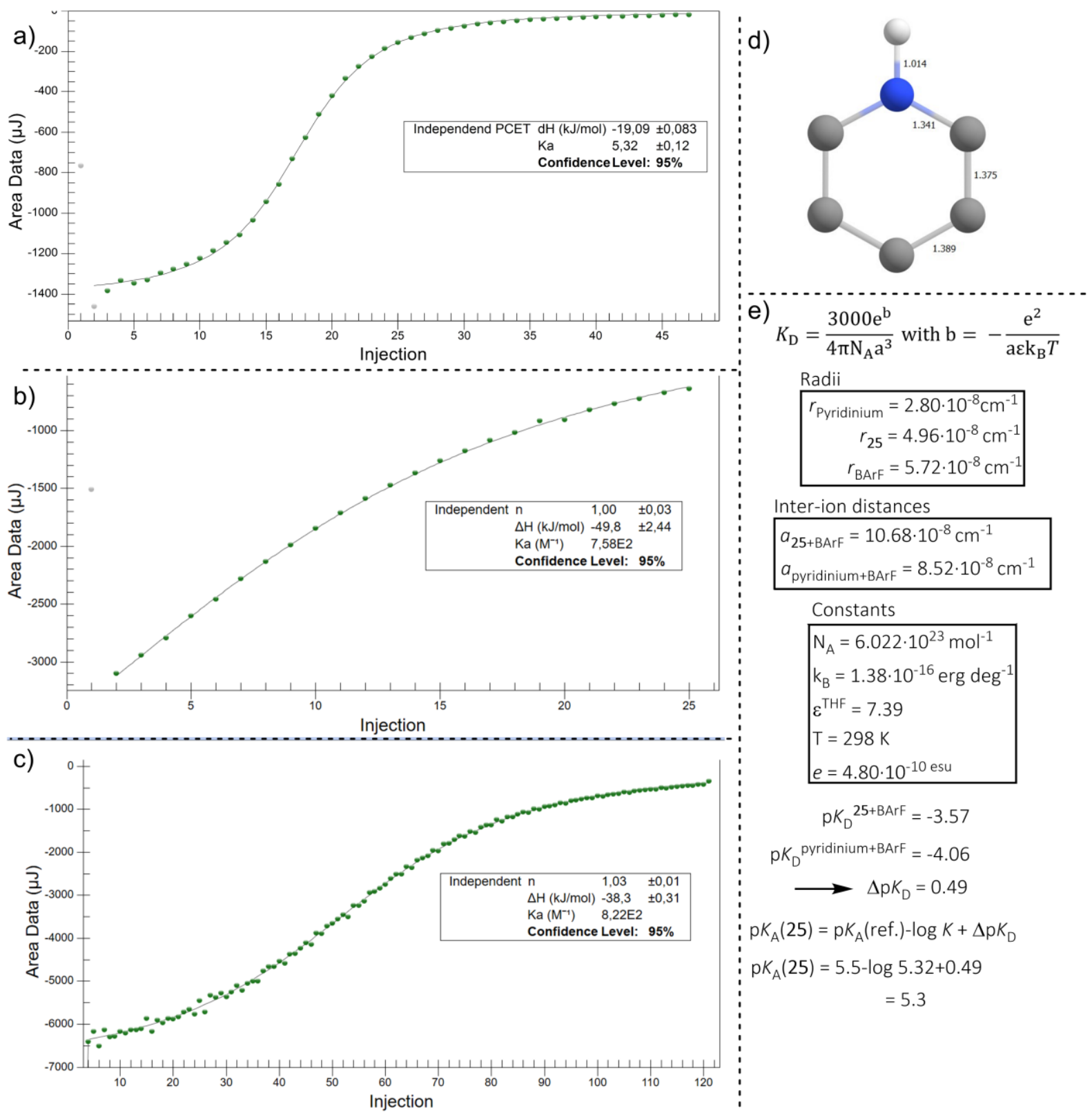

Figure 47: a) Representative titration plot and results table of the titration of 25 with pyridine in THF at $25^{\circ} \mathrm{C}$. b) Titration plot of the titration of Mes* ${ }^{*} \mathrm{OH}$ with $\mathrm{P}_{2}$ Et base in THF at $25^{\circ} \mathrm{C}$ at low concentration. c) Titration plot and results table of the titration of Mes* OH with $\mathrm{P}_{2}$-Et base in THF at $25^{\circ} \mathrm{C}$ at high concentration. $\mathrm{d}$ ) Converged $\mathrm{C}_{\mathrm{s}}$ symmetric structure of a pyridinium ion on the PBEO-D3/def2TZVP level of theory. All C-H protons are omitted for clarity. e) Fuoss equation (top) and the values for the calculation of the $\Delta \mathrm{p} K_{\mathrm{D}}$ value as well as the overlapping indicator equation (bottom) for the calculation of the $\mathrm{p} K_{\mathrm{A}}$ value.

The $\mathrm{p} K_{\mathrm{A}}$ values of 25 and Mes*OH were derived by isothermal titration calorimetry. Pyridine as a base $\left(\mathrm{p} K_{\mathrm{A}}^{\mathrm{THF}}=\right.$ $5.5)^{[222]}$ is sufficient to deprotonate 25 and the titration of 25 vs. pyridine gives an ideal, s-shaped titration curve, sufficient for the determination of an equilibrium constant $K=5.32$ (Figure 47a). To obtain the p $K_{\mathrm{A}}$ value of a

${ }^{68}$ See Chapter VI Section 2.17 
substance in THF, ion-pairing must be considered, which can be done by an electrostatic model based on work by Fuoss (Figure 47e). ${ }^{[223]}$ Since no crystal structure of an uncoordinated pyridinium-ion is known in the literature, its radius is estimated based on a structure computed on the PBEO-D3/def2TZVP level of theory (Figure 47d), ${ }^{69}$ while the radii of 25 and the $\mathrm{BArF}_{24}$ counterion are derived on basis of the found crystal structure (see Section 1.3). With this data the acidity of 25 is calculated to $p K_{A}{ }^{\mathrm{THF}}(25)=5.3$ (Figure $47 \mathrm{e}$ ).

The situation is more complicated for the $\mathrm{p} K_{\mathrm{A}}$ value derivation of $\mathrm{Mes} * \mathrm{OH}$. A diluted titration of $\mathrm{Mes} * \mathrm{OH}$ with the strong phosphazene base $\mathrm{P}_{2}-\mathrm{Et}^{70}\left(\mathrm{pK}_{\mathrm{A}}^{\mathrm{THF}}=25.3\right)$ gives a titration curve with a shallow slope (Figure $\left.47, \mathrm{~b}\right)$. This speaks either for a reaction with low driving force with dissociating ions or for an association reaction, in which the formed $\mathrm{Mes}^{*} \mathrm{O}^{-} / \mathrm{HP}_{2}-\mathrm{Et}^{+}$couple is not dissociating. To distinguish between these two situations, the titration was repeated with a ten-fold higher concentration, since only the association reaction is concentration dependent, while the titration curve would not change for a reaction with low driving force. At higher concentrations, the shape of the titration curve changes drastically to a s-shaped character, proofing the association mechanism (Figure 47c). Therefore, the titration curve has to be fitted by an independent model based on an association reaction. ${ }^{[224]}$ The so obtained fit of the titration curve already includes the ion-pairing correction and makes an additional Fuoss correction obsolete. With the equilibrium constant of $K=806$ the acidity of Mes*OH is calculated to $p K_{\mathrm{A}}{ }^{\mathrm{THF}}\left(\mathrm{Mes}{ }^{*} \mathrm{OH}\right)=22.4$, which finally enables the calculation of the free reaction enthalpy of the PCET reaction to $\Delta G_{\mathrm{r}}^{\mathrm{THF}}=-33 \pm 8 \mathrm{~kJ} \mathrm{~mol}^{-1} \cdot{ }^{71}$

\subsection{Computational (Free) Enthalpy Determination}

At last, the reaction (free) enthalpy of the PCET reaction (Scheme 10) is computed based on two different $a b$ initio models. A hybrid ONIOM(CCSD(T)-F12b/PBE0) approach including CCSD(T)-F12b single point computations on truncated model systems and a localized DLPNO-CCSD $(T)^{72}$ approach with single points on the full systems, extrapolated to the CBS from the triple and quadruple zeta basis (see Chapter I Section 3.1). The ab initio computations are performed based on structures obtained DFT level of theory (PBEO-D3/def2TZVP). Additionally, also the (free) enthalpic contributions for standard conditions (298 K/1 atm) are obtained on the DFT level of theory. $^{73}$

Two different truncation levels were probed to test the size consistency of the ONIOM approach. 1) Hydrogen truncation, where hydrogen atoms substitute the isopropyl and tert-butyl groups of $23 / 24$ and of Mes* $\mathrm{O} / \mathrm{Mes}^{*} \mathrm{OH}$, respectively and 2) Methyl truncation, where methyl groups are used for the substitution. Additionally, a single point $\operatorname{CCSD}(\mathrm{T})$ calculation without truncation was computed for the Mes*O/Mes*OH couple. Table 2 summarizes the obtained ab initio benchmarks for the PCET reaction (free) enthalpy with (SOC) and

\footnotetext{
${ }^{69} \mathrm{xyz}$ data of the converged structure can be found in Section D of chapter 7. The collision diameter was derived with Chemcraft V.1.8, www.chemcraftprog.com. ${ }^{70} \mathrm{P}_{2}$-Et $=$ Tetramethyl(tris(dimethylamino)phosphoranylidene)phosphorictriamid-Et-imin, CAS = 165535-45-5.

${ }^{71}$ The error of $\Delta G$ is estimated (Fuoss correction, $\mathrm{p} K_{\mathrm{A}}$ reference values have no reported errors, cumulative error of four independent experiments) to $8 \mathrm{~kJ}^{\mathrm{mol}} \mathrm{l}^{-1}$ 72 DLPNO computations performed by Dr. Martin Diefenbach.

${ }^{73} \mathrm{~A}$ comprehensive discussion of the performed computations can be found in Section 4.3.4 of chapter VI.
} 
without (spin-free) spin-orbit coupling $\left(\Delta \Delta \mathrm{SOC}=28 \mathrm{~kJ} \mathrm{~mol}{ }^{-1}\right) \cdot{ }^{74}$ Truncation of the tert-butyl groups has a very minor effect $\left(\Delta \Delta E=3 \mathrm{~kJ} \mathrm{~mol}^{-1}\right)$ on the total energy of the Mes*O/Mes*OH couple (Table 2, 1-3) as well as on the 23/24 couple $\left(\Delta \Delta E=0.5 \mathrm{~kJ} \mathrm{~mol}^{-1}\right)$ (Table 2, 1 and 4), which proofs the validity of the ONIOM approach for this reaction. A comparison between the VDZ-F12b and VTZ-F12b basis sets results in a higher deviation of $\Delta \Delta E=5$ $7 \mathrm{~kJ} \mathrm{~mol}^{-1}$ than for the different truncation levels, but still supports that a sufficient amount of electronic correlation is captured already with the double-zeta basis (Table 2, 1/7,2/8). The DLPNO-CCSD(To) computations do not differ between the correlation consistent Dunning basis sets and the Ahlrich basis sets (Table 2, 9 and 10), thus the more expensive $T_{1}$ triples, were probed with the Ahlrich basis set (Table 2,11). All together, the $a b$ initio models place the reaction (free) enthalpy at $\Delta H / G_{r}=-74$ to $-82 \mathrm{~kJ} \mathrm{~mol}^{-1}$ without including spin-orbit coupling, but with SOC the picture drastically changes. Here, the (free) reaction enthalpy is computed to $\Delta H / G_{r}=-46$ to $-54 \mathrm{~kJ} \mathrm{~mol}^{-1}$, which is in very good agreement to the experimentally found values of $\Delta H / G_{r}^{T H F}=-40.1 \pm 0.2 /-33 \pm 8 \mathrm{~kJ} \mathrm{~mol}^{-1}$ and $\Delta H^{\mathrm{DCM}}=-51.2 \pm 0.3 \mathrm{~kJ} \mathrm{~mol}^{-1}$. The small difference between experiment and theory can tentatively be accounted to solvation effects (THF/DCM) and to the methodical error of the performed computations (basis set limit, truncations, symmetry, active orbitals). These results finally proof the spin-orbit coupling effect on the thermochemistry of a PCET reaction.

${ }^{74}$ SOC value from the CASSCF/NEVPT2-QDPT computations, see Section 2. 
Table 2: Ab initio computations summary table for the PCET reaction (see Figure 45, a), the (relative) highest levels of theory are marked bold. [a] All ab initio data are based on structures optimized on PBEO-D3/def2TZVP level of theory. ONIOM energies computed with: $E^{O N I O M}=E_{M o d e l}^{C C S D(T)}-E_{M o d e l}^{D F T}+E_{F u l l s y s t e m}^{D F T}$ Results shown for the triplet ground state of 23 and the doublet ground state of 24 [b] (Free) Enthalpic contribution based on the DFT results on PBEO-(D3)/def2TZVP level of theory. [c] Spin-orbit contribution based on CASSCF/NEVPT2-QDPT results for 15 (14x10) and 16 (15x11). [d] CCSD(T)-f12/VDZ computations based on hydrogen truncated model systems. [e] CCSD(T)-f12/VDZ computations based on hydrogen truncated model systems for 23 and 24 and methyl truncation on the Mes*OH/Mes*O couple. [f] CCSD(T)-f12/VDZ computations based on hydrogen truncated model systems for 23 and 24 and no truncation on the Mes*OH/Mes*O couple. [g] CCSD(T)-f12/VDZ computations based on methyl truncated model systems for 23 and 24 and hydrogen truncated model systems for the Mes*OH/Mes*O couple. [h] CCSD(T)-f12/VDZ computations based on methyl truncated model systems. [i] CCSD(T)-f12/VDZ computations based on methyl truncated model systems for 23 and 24 no truncation on the Mes*OH/Mes*O couple. [j] CCSD(T)-f12/VTZ computations on hydrogen truncated model systems. [k] CCSD(T)-f12/VTZ computations on hydrogen truncated model systems of 23 and 24 and methyl truncated model systems of the Mes*OH/Mes*O [l] DLPNO with CCSD(TO) using Dunning basis sets extrapolated to the CBS limit from the triples and quadruples results. [m] DLPNO with CCSD(TO) using Ahlrichs basis sets extrapolated to the CBS limit from the triples and quadruples results [n] DLPNO with CCSD(T1) using Ahlrichs basis sets extrapolated to the CBS limit from the triples and quadruples results.

\begin{tabular}{|c|c|c|c|}
\hline & Method $^{[a]}$ & $\Delta H / G_{r}^{\text {spin-free }[b]} / \mathrm{kJ} \mathrm{mol}^{-1}$ & $\Delta H / G_{\mathrm{r}}^{\mathrm{SOC}[\mathrm{c}]} / \mathrm{kJ} \mathrm{mol}^{-1}$ \\
\hline 1 & ONIOM $\left(\mathrm{H}::^{i} \mathrm{Pr} /{ }^{t} \mathrm{Bu}, \mathrm{VDZ}\right)^{[\mathrm{d}]}$ & $-79.5 /-80.0$ & $-51.4 /-51.9$ \\
\hline 2 & ONIOM (H:iPr, Me: $\left.{ }^{t} B u, V D Z\right)^{[e]}$ & $-82.5 /-83.0$ & $-54.4 /-54.9$ \\
\hline 3 & ONIOM (H: Pr $\left.^{t} \mathrm{Bu}:{ }^{t} \mathrm{Bu}, \mathrm{VDZ}\right)^{[\mathrm{f}]}$ & $-81.4 /-81.8$ & $-53.3 /-53.7$ \\
\hline 4 & ONIOM (Me: $\left.\mathrm{Pr}, \mathrm{H}:{ }^{\mathrm{t}} \mathrm{Bu}, \mathrm{VDZ}\right)^{[\mathrm{g}]}$ & $-80.0 /-80.4$ & $-51.8 /-52.4$ \\
\hline 5 & ONIOM (Me: $\left.:^{i} \mathrm{Pr} /{ }^{t} \mathrm{Bu}, \mathrm{VDZ}\right)^{[\mathrm{h}]}$ & $-83.0 /-83.4$ & $-54.9 /-55.3$ \\
\hline 6 & ONIOM (Me: $\left.{ }^{i} \mathrm{Pr},{ }^{t} \mathrm{Bu}:{ }^{t} \mathrm{Bu}, \mathrm{VDZ}\right)^{[\mathrm{i}]}$ & $-81.8 /-82.3$ & $-53.7 /-54.2$ \\
\hline 7 & ONIOM $\left(\mathrm{H}::^{i} \mathrm{Pr} /{ }^{\mathrm{t}} \mathrm{Bu}, \mathrm{VTZ}\right)^{[\mathrm{jJ}]}$ & $-72.4 /-72.8$ & $-44.3 /-44.7$ \\
\hline 8 & ONIOM (H:'Pr, Me:'Bu, VTZ) ${ }^{[k]}$ & $-77.1 /-77.6$ & $-49.0 /-49.5$ \\
\hline 9 & $\operatorname{DLPNO}\left(\mathrm{CCSD}\left(\mathrm{T}_{0}\right) / \mathrm{cc}-\mathrm{pV}(\mathrm{T}, \mathrm{Q}) \mathrm{Z}(\mathrm{PP})\right)^{[]]}$ & $-69.4 /-69.8$ & $-41.3 /-41.7$ \\
\hline 10 & $\operatorname{DLPNO}\left(\operatorname{CCSD}\left(\mathrm{T}_{0}\right) / \operatorname{def} 2-\mathrm{p}(\mathrm{T}, \mathrm{Q}) Z \mathrm{ZVPP}\right)^{[\mathrm{m}]}$ & $-69.1 /-69.5$ & $-41.0 /-41.4$ \\
\hline 11 & $\operatorname{DLPNO}\left(C C S D\left(T_{1}\right) / \operatorname{def} 2-p(T, Q) Z V P P\right)^{[n]}$ & $-74.2 /-74.7$ & $-46.1 /-46.6$ \\
\hline
\end{tabular}




\section{Summary of Chapter IV}

In summary, in this Chapter the effect of spin-orbit coupling on the thermochemistry of a PCET reaction was analysed in detail. For this purpose, the rhenium(III) amine complex 23 was chosen which has shown to be greatly influenced by SOC in a previous publication. The thorough investigation of the spectroscopic and magnetic data of 23 led to the assignment of a triplet ground state, which is thermally well separated $\left(>1300 \mathrm{~cm}^{-1}\right)$ from excited states as a reason of extensive SOC. The SOC splits the triplet root into its microstates, resulting in an effective non-magnetic $J=0$ ground state. The temperature independent mixing with magnetic excited states explains the sharp but paramagnetically shifted ${ }^{1} \mathrm{H}-\mathrm{NMR}$ spectra of 23 as well as the linear $X_{\mathrm{M}} T$ vs. $T$ slope over the whole temperature range. Additionally, a broad and intense transition in the midIR region at $3500 \mathrm{~cm}^{-1}$ was observed with differing intensity upon applying a magnetic field. Therefore, a midIR transition of a transition metal complex could be unequivocally assigned to be of electronic nature. The PCET counterpart, the rhenium(IV) amide complex 24, exhibits a doublet ground state which is also significantly influence by TIP even though only with a third of the magnitude of 23. The effective magnetic moment at room temperature $\mu_{\text {eff }}=1.83 \mu_{\mathrm{B}}$ of 24 is in line with the expected spin-only value of $\mu_{\mathrm{eff}}^{\mathrm{sO}}=1.73 \mu_{\mathrm{B}}$ of a doublet ground state, but already hints for the mixing of excited states with the ground state. The midIR spectrum of 24 is featuring like 23 a broad, intense band at $1970 \mathrm{~cm}^{-1}$ which is tentatively assigned as well to be of electronic nature. For the determination of the thermodynamic PCET characterisation, the cationic rhenium(IV) amine complex 25 was successfully synthesised and characterised. The effective magnetic moment at room temperature in solution of $25 \mu_{\mathrm{eff}}=2.9 \mu_{\mathrm{B}}$ is well beyond the expected spinonly value for a doublet ground state, suggesting a quartet ground state for 25 , but no broad transition in the midIR as for 23 and 24 was observed.

DFT analysis on the PBE0-D3/def2TZVP level of theory confirmed the experimental ground state picture and excellently reproduced the found structures by XRD analysis. Additionally, for all three complexes mainly metal centred radical character is predicted by Mulliken and NBO/NPA analysis, thus giving reason to expect high SOC contributions, especially for the amine complexes 23 and 25 . The electronic structure was analysed in more detail by ab initio CASSCF-NEVPT2-QDPT computations. For 23 an effective ${ }^{3} E$ like ground state on the spin-free NEVPT2 level is computed, which strongly splits upon SOC into its microstates. This results in a huge SOC stabilisation of $\triangle \mathrm{SOC}=-46.4 \mathrm{~kJ} \mathrm{~mol}^{-1}$. The ab initio wavefunction was further benchmarked with SQUID, UV/Vis and (N)IR spectra of 23 and is in excellent agreement with the experiment. Here, especially the midIR transition is very well predicted to $3900 \mathrm{~cm}^{-1}$ (cf. $3500 \mathrm{~cm}^{-1}$ experimentally). The ab initio wavefunction nicely reproduces the experimental benchmarks of the rhenium(IV) amide complex 24 as well and predicts the midIR transition to $2200 \mathrm{~cm}^{-1}$ (cf. $2000 \mathrm{~cm}^{-1}$ experimentally). The computed SOC stabilisation of $24\left(-18.5 \mathrm{~kJ} \mathrm{~mol}^{-1}\right)$ is almost three times lower than for 23 and therewith follows an enormous SOC effect on the PCET thermochemistry of $\Delta \Delta \mathrm{SOC}=28 \mathrm{~kJ} \mathrm{~mol}^{-1}$. For the cationic amine 25 a very high doublet/quartet gap of $93 \mathrm{~kJ} \mathrm{~mol}^{-1}$ was computed, resulting in a decreased mixing by SOC $\left(\triangle \mathrm{SOC}=-11.6 \mathrm{~kJ} \mathrm{~mol}^{-1}\right)$ even though the spin density at the metal centre was the highest of the series.

The enthalpy of the PCET reaction was measured by isothermal titration calorimetry (ITC) and gives a $\Delta H_{r}=$ $-40.1 \pm 0.2 \mathrm{~kJ} \mathrm{~mol}^{-1}$ in THF and $\Delta H_{r}=-51.2 \pm 0.3 \mathrm{~kJ} \mathrm{~mol}^{-1}$ in DCM, while the free reaction enthalpy in THF was calculated by a square scheme approach via the determination of the oxidation potentials of $23\left(E^{0}=-0.29 \mathrm{~V}, \mathrm{vs}\right.$. 
$\left.\mathrm{Fc} / \mathrm{Fc}^{+}\right), \mathrm{Mes}^{*} \mathrm{O}^{-}\left(E^{O}=-0.96 \mathrm{~V}\right.$ vs. $\left.\mathrm{Fc} / \mathrm{Fc}^{+}\right)$and the $\mathrm{p} K_{\mathrm{A}}$ values of $25\left(\mathrm{p} K_{\mathrm{a}}=5.3\right)$ and $\mathrm{Mes} * \mathrm{OH}\left(\mathrm{p} K_{\mathrm{A}}=22.4\right)$ to $\Delta G_{r}=-33 \pm 8 \mathrm{~kJ} \mathrm{~mol}^{-1}$. Here, the high solvent dependency was attributed to the higher tendency of THF to form hydrogen bonds.

The spin-free $a b$ initio techniques ONIOM and DLPNO place the reaction (free) enthalpy of the PCET reaction at $\Delta H / G_{r}^{a b}$ initio $=-74$ to $-82 \mathrm{~kJ} \mathrm{~mol}^{-1}$ while the consideration of the SOC effect corrects the predicted value to $\Delta H / G_{r}^{a b}$ initio+SOC $=-46$ to $-54 \mathrm{~kJ} \mathrm{~mol}^{-1}$, which is in perfect agreement to the experimentally found values. The SOC effect $\left(\Delta \Delta S O C=28 \mathrm{~kJ} \mathrm{~mol}^{-1}\right)$ is in the same order of magnitude as the computed SOC effect on reduction potentials of osmium(II/III) (300 mV, $\left.28 \mathrm{~kJ} \mathrm{~mol}^{-1}\right)$ reported by Kývala and Rulišek. ${ }^{[140]}$ These results corroborate the fundamental importance a differential SOC effect can have in heavy metal chemistry. A $\Delta \Delta S O C$ effect especially needs to be considered when a spin change at the metal centre upon reaction takes place and excited states close to the ground state are available for mixing (23/24 vs. 25 ). 
Chapter V

V. Conclusion and Outlook 
This dissertation helped in the deeper understanding of two research topics:

\section{1) Electronic structure and reactivity of late, electron rich terminal oxo complexes}

\section{2) Proton coupled-electron transfer thermochemistry at heavy metal sites with a special emphasis on the effect of spin-orbit coupling.}

The synthesis and characterisation of a series of terminal iridium hydroxo complexes $[(\mathrm{PNP}) \operatorname{Ir}(\mathrm{OH})](1,2,3)$, including the first open-shell iridium hydroxo complex 2, enabled the targeted synthesis of a terminal iridium(III) oxo complex 4 by either deprotonation of the cationic hydroxo complex 3 or direct HAT from neutral hydroxo complex 2 . The iridium-oxygen bonding of 4 was investigated by XRD analysis and IR spectroscopy, including ${ }^{18} \mathrm{O}$ isotopic labelling, and revealed an elongated $(0.1 \AA)$ and weakened $\left(60 \mathrm{~cm}^{-1}\right.$ red shifted) Ir-O bond, compared to the Ir-O triple bond of Wilkinson's iridium oxo complex XXI, thus 4 was assigned to exhibit an Ir-O double bond. Variable temperature NMR spectroscopy and SQUID magnetometry revealed an open-shell ground state of 4 with a linear $X_{M} T$ vs. $T$ slope. A triplet ground state with a very high zero-field splitting (ZFS) of $D=647 \mathrm{~cm}^{-1}$ was assigned to 4 by a spin-Hamiltonian fit of the SQUID data. The ZFS is almost $200 \mathrm{~cm}^{-1}$ higher than for the isoelectronic terminal imido complex XXII. This is in line with a less covalent Ir-E (E=N/O) bonding interaction, which leads to a smaller relativistic nephelauxetic effect for 4 and therefore to a higher effective spin-orbit coupling parameter $\zeta$ eff. The magnetic and spectroscopic data of 4 led to the assignment of an unprecedented oxo biradical species. Electrochemical analysis of 4 revealed a reductive event at $E=-1.95 \mathrm{~V}$ and a remarkably low potential for the oxidation at $E=-0.13 \mathrm{~V}$ vs. $\mathrm{Fc} / \mathrm{Fc}^{+}$for a formally iridium(III/IV) couple, again confirming the covalent bonding within the Ir-O unit and thus making oxidation states a bad descriptor for such complexes. The oxidation of $\mathbf{4}$ yields the thermally instable 4+ complex, which was analysed in situ by NMR and EPR spectroscopy to exhibit $\mathrm{C}_{2 v}$ symmetry. The very low $g$-anisotropy speaks for a ligand centred radical, which is supported by DFT computations with a Mulliken spin density at the oxygen atom of $75 \%$.

The electronic structure of $\mathbf{2}$ and $\mathbf{4}$ was further analysed by high level $a b$ initio techniques. The CASSCF-NEVPT2QDPT computations support the triplet ground state of 4 which is heavily split by spin-orbit coupling into its microstates. Here, the computed zero-field of $750 \mathrm{~cm}^{-1}$ was in very good agreement to the experiment. A second noteworthy outcome of the CASSCF computations was the small, but non negligible differential SOC effect, which formally strengthens the $\mathrm{O}-\mathrm{H}$ bond of 2 by $2.9 \mathrm{~kJ} \mathrm{~mol}^{-1}$. The bond dissociation (free) energy (BD(F)E) of 2 was examined by isothermal titration calorimetry revealing a bond strength, in the regime of activated $\mathrm{C}-\mathrm{H}$ bonds. Here, $a b$ initio computations on the $\operatorname{CCSD}(\mathrm{T})-\mathrm{F} 12 \mathrm{~b}$ and DLPNO-CBS level of theory excellently support the experimentally found BDE values. With all these thermodynamic parameters at hand, a full square scheme for all discussed oxo species was formulated. With a BDE value of $350 \mathrm{~kJ} \mathrm{~mol}^{-1} 4$ can activate weak $\mathrm{C}-\mathrm{H}$ bonds but not the strong $\mathrm{C}-\mathrm{H}$ bonds of most solvents. The same picture arises for the $\mathrm{p} K_{\mathrm{A}}$ value of 4 , which is in the medium range of $\mathrm{p} K_{\mathrm{A}}$ values in THF. The cationic hydroxo complex 3 is bearing the strongest $\mathrm{O}-\mathrm{H}$ bond with $372 \mathrm{~kJ} \mathrm{~mol}^{-1}$. A comparison of the computed spin densities at the oxygen atom of $4(0: 95 \%)$ and $4+(0: 75 \%)$ reveals that the transient 4+ has less radical character but is more reactive which supports the statement of James Mayer that:

"Not radical character but bond strength is defining radical reactivity" [25] 
At last, the versatile reactivity of 4 was investigated within this thesis. Carbon monoxide could be oxidized to $\mathrm{CO}_{2}$, which was confirmed by head space analysis. $\mathrm{CO}_{2}$ could also serve as a reagent forming the iridium(III) carbonate complex 8 and thus revealing nucleophilic reactivity of 4.8 was also found to be a site product in the CO oxidation and therefore represents a thermodynamically more favoured product compared to the CO oxidation. With hydrogenolysis the iridium(III) dihydride complex 6 and water are formed, while the ambiphilic test reagent $\mathrm{PMe}_{3}$ can be oxygenated to $\mathrm{OPMe}_{3}$. The $\mathrm{C}-\mathrm{H}$ bond activation capability of 4 was tested with the reagent's xanthene and fluorene in which in both cases a thermal reaction towards the C-O bond formation product (11/12) was found. While the reaction with xanthene also proceeds slowly at room temperature, the reaction must be heated to $60^{\circ} \mathrm{C}$ for fluorene, which nicely reflects the decreasing BDE difference of the substrate to 4 . At last the irradiative oxygenation of benzaldehydes was reported and the formed benzoate complexes (14-x, X = F, H, Me, OMe) were independently characterized. A Hammett analysis revealed a positive slope of 3.7 for different benzaldehyde parasubstituents, thus supporting the nucleophilic reactivity of 4 .

Future work is this field should focus on the deeper understanding of the $\mathrm{C}-\mathrm{H}$ bond activation reactivity of 4 . Here, isotopic labelling experiments would give valuable insight into the rate determining step of the C-O bond formation and a detailed kinetic investigation could further help to distinguish between a pure concerted CPET or more asynchronous pathways (Figure 48A). The oxidation potential of the hydroxo complex 2 has shown to be very mild $\left(E^{0}=-0.37 \mathrm{~V} \mathrm{vs} . \mathrm{Fc} / \mathrm{Fc}^{+}\right)$and the $\mathrm{p} K_{\mathrm{A}}$ value of the corresponding cationic hydroxo complex 3 is as well accessible with comparably weak bases $\left(p K_{A}{ }^{T H F}=19\right)$ thus a potential catalytic hydroxylation process can be proposed. Here, 2 is electrochemically oxidized and subsequently deprotonated by a base, yielding the terminal oxo complex 4 . This step is followed by the C-H oxygenation, yielding one equivalent of $\mathbf{2}$ and of the alkoxide product (analogous to $12 / 11$ ). Since 2 is synthesised in a THF/water mixture, water is the ideal reagent for the substitution of the alkoxide recovering 2 and the free alcohol (Figure 48B). In the end a further investigation by XRD analysis of the cationic oxo species 4+ would help in the understanding of late transition metal multi-bonding interactions. Here, trapping experiments at very low temperatures in inert solvents as fluorobenzene could lead to the successful isolation of 4+. A comparison of the reactivity of the cationic oxo $4+$ with neutral 4 could additionally give insight into general philicity trends of terminal oxo complexes. 4 exhibited nucleo- to ambiphilic reactivity but for cationic oxo 4+ electrophilic reactivity is expected due to the cationic charge. Here, olefin epoxidation or water oxidation would be promising starting points for the reactivity investigation of 4+ (Figure 48C). A second research field is the synthesis and characterisation of the terminal oxo complex of the lighter homologue rhodium $[(P N P) \operatorname{Rh}(O)] 4^{\mathrm{Rh}}$. Preliminary computations on the PBE0-D3/def2TZVP level of theory (see Section D of Chapter 7 for xyz data) predict a $21 \mathrm{~kJ} \mathrm{~mol}^{-1}$ higher $\mathrm{O}-\mathrm{H}$ bond strength of the rhodium hydroxo $2^{\mathrm{Rh}} / \mathrm{vs}$. 2 which is close to aliphatic $\mathrm{C}-\mathrm{H}$ bonds (cf. $\mathrm{BDE}(\mathrm{THF})=92 \mathrm{kcal} \mathrm{mol}^{-1}, \mathrm{BDE}\left(2^{\mathrm{Rh}}\right)=89 \mathrm{kcal} \mathrm{mol}^{-1}$ ) (Figure 48D). Even if the isolation of the rhodium oxo $4^{\mathrm{Rh}}$ would be hampered by its high reactivity, a similar approach like in Figure $48 \mathrm{~B}$ could enable the catalytic oxygenation of a broader substrate scope with $4^{\text {Rh }}$ compared to 4 . 


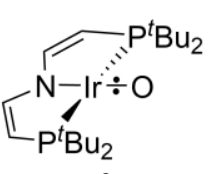<smiles>[2H]C1([2H])c2ccccc2Oc2ccccc2C1c1ccccc1Oc1ccccc1</smiles>

KIE

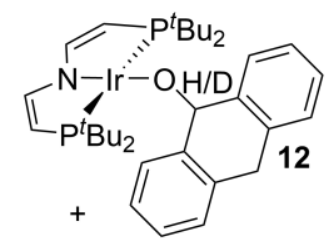


B $2 \mathrm{BaseH}^{+} \mathrm{N}^{\mathrm{t}} \mathrm{Bu}_{2}$

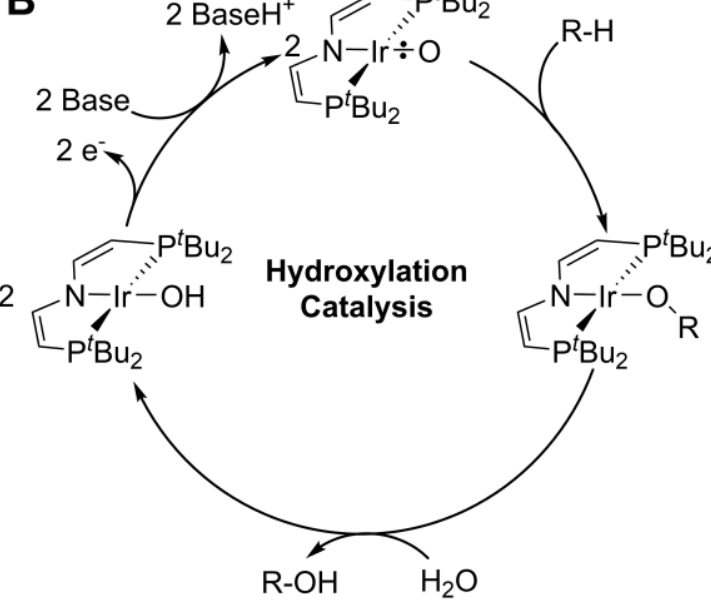

\section{C}<smiles></smiles>

Electrophilic Reactivity?

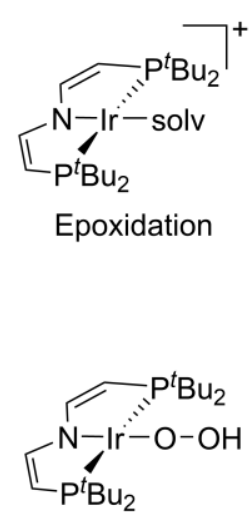

Water Oxidation
D

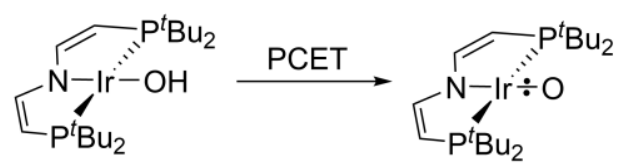

2

$\uparrow \quad$ Isoelectronic

$\downarrow$

$\mathbb{P}_{\mathrm{P}^{t} \mathrm{Bu}_{2}}^{\mathrm{N}-\mathrm{Rh}-\mathrm{OH}}$

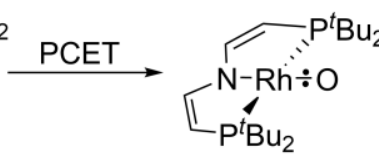

$2^{\text {Rh }}$

$4^{\mathrm{Rh}}$

$\mathrm{BDE}^{\mathrm{DFT}}\left(2^{\mathrm{Rh}}\right)=21 \mathrm{~kJ} \mathrm{~mol}^{-1}>\mathrm{BDE}^{\mathrm{DFT}}(\mathbf{2})$

Figure 48: A) Kinetic isotope experiment for the mechanistic investigation of the C-H oxygenation reactivity. B) Possible catalytic hydroxylation reactivity of 2. C) Proposed electrophilic reactivity of $4+$. D) Isoelectronic relationship of $2^{\mathrm{Rh}} / 2$ and $\mathbf{4}^{\mathrm{Rh}} / \mathbf{4}$ and predicted BDE difference on the PBEO-D3/def2TZVP level of theory.

The small but non-negligible SOC effect on the thermochemistry of the 2/4 couple inspired for the detailed and general investigation of SOC effects in PCET solution thermochemistry. For this purpose, the rhenium(III) amine complex 23 was chosen which has shown to be greatly influence by SOC in a previous publication. ${ }^{[158]}$ Detailed analysation of the spectroscopic and magnetic features led to the assignment of a triplet $J=0$ ground state with pronounced TIP. 23 exhibits an intense, broad peak at $3510 \mathrm{~cm}^{-1}$ in the mid-infrared spectrum, which arises from an electronic transition as assigned by magnetic field dependent IR spectroscopy. A similar picture arises for the rhenium(IV) amide complex 24. The magnetic data obtained by SQUID magnetometry gives a doublet ground state with a significant, but three times lower, contribution of temperature independent paramagnetism. The midIR spectrum of 24 is as well featuring a broad, intense band at $1970 \mathrm{~cm}^{-1}$ which can be tentatively assigned as well to an electronic transition. A new cationic rhenium(IV) amine complex 25 was synthesised by oxidation of 23 with $\mathrm{FcBArF}_{24}$ and fully characterized. The effective magnetic moment at room temperature of 25 led to the assignment of a quartet ground state, but no comparable IR band as for 23 and 24 was found for 25 .

DFT analysis on the PBE0-D3/def2TZVP level of theory confirmed the experimental ground state pictures and excellently reproduced the found structures by XRD analysis. Additionally, for all three complexes mainly metal centred radical character is predicted by Mulliken and NBO/NPA analysis, thus giving reason to expect high SOC 
contributions, especially for the amine complexes 23 and 25 . The electronic structure was analysed in more detail by ab inito CASSCF-NEVPT2-QDPT computations. For 23 a ${ }^{3} E$ like ground state on the spin-free NEVPT2 level is computed, which strongly splits upon SOC into its microstates, resulting in a huge SOC stabilisation of $\triangle \mathrm{SOC}=$ $-46.4 \mathrm{~kJ} \mathrm{~mol}^{-1}$. The ab initio wavefunction was further benchmarked with SQUID, UV/Vis and NIR spectra of 23 and gave a good agreement with the experiment. Here, especially the midIR transition is very well predicted to $3900 \mathrm{~cm}^{-1}$ (cf. $3500 \mathrm{~cm}^{-1}$ experimentally). For amide complex 24 the ab initio wavefunction i very well reproduces the experimental benchmarks as well and predicts the midIR transition to $2200 \mathrm{~cm}^{-1}$ (cf. $1970 \mathrm{~cm}^{-1}$ experimentally). The computed SOC stabilisation of 24 of $-18.5 \mathrm{~kJ} \mathrm{~mol}^{-1}$ is three times lower than for 23 and therewith predicts an enormous SOC effect on the PCET thermochemistry of $\Delta \Delta S O C=28 \mathrm{~kJ} \mathrm{~mol}^{-1}$. For the cationic amine 25 a very high doublet/quartet gap of $93 \mathrm{~kJ} \mathrm{~mol}^{-1}$ was computed, which results in a decreased mixing by $\mathrm{SOC}\left(\triangle \mathrm{SOC}=-11.6 \mathrm{~kJ} \mathrm{~mol}^{-1}\right)$ even though the spin density at the metal centre was the highest of the series.

The enthalpy of the PCET reaction was measured by isothermal titration calorimetry (ITC) and gives an enthalpy of $\Delta H_{r}=-40.1 \pm 0.2 \mathrm{~kJ} \mathrm{~mol}^{-1}$ in THF and $\Delta H_{r}=-51.2 \pm 0.3 \mathrm{~kJ} \mathrm{~mol}^{-1}$ in DCM, while the free reaction enthalpy in THF was calculated by a square scheme approach via the determination of the oxidation potentials of $23\left(E^{0}=-0.29 \mathrm{~V}\right.$, vs. $\left.\mathrm{Fc} / \mathrm{Fc}^{+}\right), \mathrm{Mes}^{*} \mathrm{O}^{-}\left(E^{0}=-0.96 \mathrm{~V}\right.$ vs. $\left.\mathrm{Fc} / \mathrm{Fc}^{+}\right)$and the $\mathrm{p} K_{\mathrm{A}}$ values of $25\left(\mathrm{p} K_{\mathrm{a}}=5.3\right)$ and $\mathrm{Mes} * \mathrm{OH}\left(\mathrm{p} K_{\mathrm{A}}=22.4\right)$ to $\Delta G_{\mathrm{r}}=-33 \pm 8 \mathrm{~kJ} \mathrm{~mol}^{-1}$.

The spin-free $a b$ initio techniques ONIOM and DLPNO place the reaction (free) enthalpy of the PCET reaction at $\Delta H / G_{\mathrm{r}}{ }^{a b}$ initio $=-74$ to $-82 \mathrm{~kJ} \mathrm{~mol}^{-1}$, while the consideration of the $\mathrm{SOC}$ effect corrects the predicted value to $\Delta H / G_{r}$ ab initio+SOC $=-46$ to $-54 \mathrm{~kJ} \mathrm{~mol}^{-1}$, which is in perfect agreement to the experimentally found values. These results corroborate the fundamental importance differential SOC effects can have in heavy metal chemistry. By consideration of the different SOC stabilisation of $23 / 24$ vs. 25 and their spin-densities the following conclusion can be drawn:

SOC has an important effect on heavy metal PCET thermochemistry when a metal centred spin change upon reaction takes place and excited open-shell states close to the ground state are available for mixing.

Future work on this topic should focus on general trends of thermochemical SOC contributions. Here, especially the evaluation of the rhenium tri-halogen series $\left[(\mathrm{PNP}) \operatorname{Re}(\mathrm{III})(\mathrm{Cl})_{3}\right] 23,\left[(\mathrm{PNP}) \operatorname{Re}(\mathrm{III})(\mathrm{Br})_{3}\right] 23^{\mathrm{Br}}$ and $\left[(\mathrm{PNP}) \operatorname{Re}(\mathrm{III})(\mathrm{I})_{3}\right]$ $23^{\prime}$ could bring valuable insight (Figure 49A). A second field of interest is the theoretical maximum of SOC effects which can be observed in solution thermochemistry. The analysed SOC effect in this thesis was reduced by the still high SOC stabilisation of the rhenium(IV) amide complex 24. 24 had a highly metal centred spin density (Re: Mulliken: 0.96; NBO/NPA: 0.84) and a relatively close excited doublet state ( $\left.\Delta E=16 \mathrm{~kJ} \mathrm{~mol}^{-1}, \mathrm{NEVPT2}\right)$ which resulted in the high SOC stabilisation of $-18.5 \mathrm{~kJ} \mathrm{~mol}^{-1}$ which overall reduced the differential SOC effect. The analysation of open-shell/closed-shell reactions could therefore lead to a significantly higher differential SOC effect. Here, HAT from closed-shell metal hydrides like the iridium(III) hydrid-chloride complex $15^{75}$, are interesting starting platforms. 15 exhibits a diamagnetic ground state and HAT yields the paramagnetic iridium(II) chloride complex 5, which may have significant contributions from SOC (Figure 49B).

\footnotetext{
${ }^{75}$ Complex 15 is fully characterised but not discussed within the main chapters of this thesis. For characterisation and XRD analysis see chapter VI Section 2.13 and
} chapter 7 Section A.11. 
A

Trends of SOC effects

Higher $\Delta \Delta S O C$ due to heavier elements

OR lower $\Delta \triangle S O C$ due to more covalent bonding?

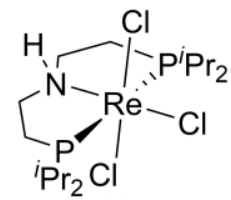

23

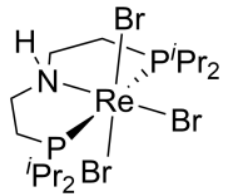
$23^{\mathrm{Br}}$

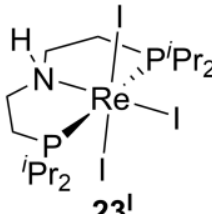
$23^{1}$

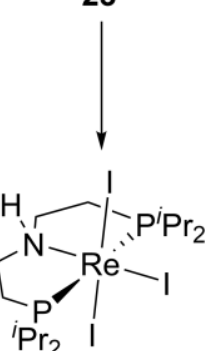

$24^{1}$

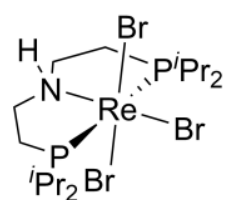

$24^{\mathrm{Br}}$

\section{B Maximisation of SOC effects}

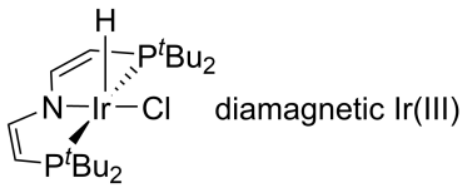

15

PCET

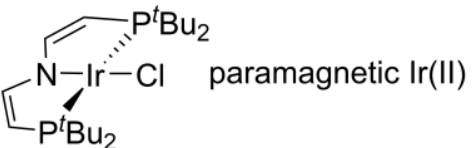

5

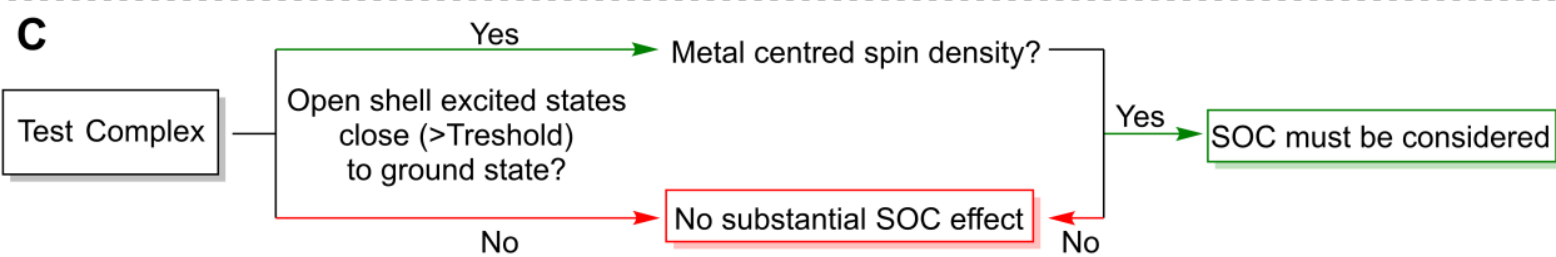

Figure 49: A) Trend analysis of the differential thermodynamic SOC effect on a PCET reaction within a halogen complex series (Cl, Br, I). B) Maximisation of the SOC effect by interconversion of a closed-shell metal hydride to an open-shell complex. C) Basic flow scheme for the theoretical prediction if a SOC effect must be explicitly encountered in a complex system.

This thesis has emphasised the importance of metal centred spin-density and accessible open-shell states for a high SOC effect. Therefore, the development of a theoretical procedure for the prediction, if a SOC effect must be considered computationally in the accurate thermochemical description of a reaction, is feasible. Such a procedure should include the computation of 1) At least the ground state/first excited state gap to test for accessible states for SOC mixing 2) The metal centred spin density. With a decent benchmark set at hand, thresholds could be defined which indicate for an unknown complex system, if SOC must be explicitly treated for its thermochemical description (Figure 49C). 
Chapter VI

VI. Experimental Details 


\section{Materials and Methods}

\subsection{General remarks}

All synthetic procedures were performed under an inert atmosphere of Ar (5.0 Linde gas) unless otherwise noted, using standard Schlenk and glovebox techniques. $\mathrm{CO}_{2}$ is purified by passing through $\mathrm{P}_{4} \mathrm{O}_{10}$, DRIERITE and cooling to $-40^{\circ} \mathrm{C} . \mathrm{H}_{2}$ was purified from water impurities by cooling with liquid nitrogen prior to use. All glassware was cleaned by a $\mathrm{KOH} /$ iso-propanol bath followed by an $\mathrm{HCl}$ bath and finished by rinsing with demineralized water. Unless otherwise stated, all glassware was heated to $120^{\circ} \mathrm{C}$ prior to use. Reactions in a small scale ( $\left.\sim \mathrm{mg}\right)$ were usually performed in J-Young NMR tubes. All solvents were purchased in HPLC quality from MERCK. Benzene, dichloromethane, ether, Pentane, tetrahydrofuran and Toluene, were pre-dried by an MBRAUN SOLVENT PURIFICATION SYSTEM. All solvents except dichloromethane and acetonitrile were dried additionally by stirring over $\mathrm{Na} / \mathrm{K}$ alloy for several days and subsequent trap-to-trap condensation. Dichloromethane and acetonitrile were additionally dried by stirring over $\mathrm{CaH}_{2}$ with subsequent trap-to-trap condensation. Deuterated solvents were obtained from EURISO TOP GmbH and were purified by the same procedures as their non-deuterated analogues.

List of literature procedures:

The iridium(II) chloride complex 5 was synthesised after Meiners et al. ${ }^{[160]}$, the iridium(II) amide complex 13 was synthesised after Scheibel et al. ${ }^{[225]}$, the phenoxy radical Mes*O was synthesised after Manner et al. ${ }^{[226]}$ The pincer ligands $\mathrm{P}^{\mathrm{H}} \mathrm{NP}(\mathrm{tBu}, \mathrm{iPr})$ were synthesised by our lab technician Kai Sebastian Kopp after Meiners et al. ${ }^{[227]}$ FcBArF can be obtained by ion exchange between $\mathrm{NaBArF}_{24}$ and $\mathrm{FCPF}_{6}$ (Dissolve in $\mathrm{DCM}$, remove solvent in vaccuo, extract with ether).

List of bought chemicals:

\begin{tabular}{|c|c|c|c|}
\hline $\mathrm{NaOH}$ & Sigma-Aldrich & $\mathrm{PhC}(\mathrm{H}) \mathrm{O}$ & Sigma-Aldrich \\
\hline $\mathrm{AgPF}_{6}$ & Sigma-Aldrich & $p-\mathrm{F}-\mathrm{PhC}(\mathrm{H}) \mathrm{O}$ & Sigma-Aldrich \\
\hline $\mathrm{FCPF}_{6}$ & Sigma-Aldrich & $p$-Me-PhC(H)O & Sigma-Aldrich (Merck) \\
\hline Mes*OH & Sigma-Aldrich & $p$-OMe-PhC(H)O & Sigma-Aldrich \\
\hline $\mathrm{AgSbF}_{6}$ & Sigma-Aldrich & $\mathrm{PhCO}_{2} \mathrm{H}$ & Sigma-Aldrich \\
\hline $\mathrm{H}_{2}$ & Linde & $p-\mathrm{F}-\mathrm{PhCO}_{2} \mathrm{H}$ & Sigma-Aldrich \\
\hline $\mathrm{CO}$ & AirLiquid & $p-\mathrm{Me}-\mathrm{PhCO}_{2} \mathrm{H}$ & Sigma-Aldrich \\
\hline $\mathrm{CO}_{2}$ & Linde & $p$-OMe- $\mathrm{PhCO}_{2} \mathrm{H}$ & Sigma-Aldrich \\
\hline $\mathrm{PMe}_{3}$ & Sigma-Aldrich & $\mathrm{KO}^{t} \mathrm{Bu}$ & Sigma-Aldrich \\
\hline Xanthene & Sigma-Aldrich & Pyridine & VWR \\
\hline $\mathrm{H}_{2}^{18} \mathrm{O}$ & Sigma-Aldrich & $\mathrm{P}_{2}$-Phosphazene base & Santa Cruz Biotechnology \\
\hline Fluorene & Sigma-Aldrich & & \\
\hline
\end{tabular}




\subsection{Analytical Methods}

\subsubsection{Cyclic Voltammetry}

Electrochemical data was recorded with a METROHM PGSTAT101 using the NOVA 2.0/2.1 software. The cyclo voltammograms were recorded in a $0.1 \mathrm{M} \mathrm{N}^{n} \mathrm{Bu}_{4} \mathrm{PF}_{6}$ solution within a UV/Vis cell equipped with a glassy carbon $(\varnothing=1.6 \mathrm{~mm})$ working electrode, a Pt wire as counter electrode and an Ag wire as pseudo-reference electrode. The data was finally internally referenced vs. the $\mathrm{Fc} / \mathrm{Fc}^{+}$couple. The internal resistance was compensated by standard procedures within the Nova 2.0 software.

To test for reversibility of redox events of freely diffusing-non adsorbed analytes, the scan rate dependence of the peak currents were analysed according to the Randles-Sevcik equation: ${ }^{[228]}$

$$
i_{p}=0.446 n F A C^{0} \sqrt{\frac{n F \vartheta D_{0}}{R T}}
$$

With $i_{\mathrm{p}}=$ Peak current, $F=$ Faraday's constant ${ }^{[229]}, A=$ Electrode surface area $/ \mathrm{cm}^{2}, C^{0}=$ Bulk concentration of the analyte $/ \mathrm{mol} \mathrm{cm}^{-3}, \mathrm{n}=\mathrm{Number}$ of transferred electrons, $v=$ scan rate $/ \mathrm{Vs}^{-1}, D_{0}=$ Diffusion coefficient of the oxidized species $/ \mathrm{cm}^{2} \mathrm{~s}^{-1}$

\subsubsection{Elemental Analysis}

Elemental analysis data were obtained from the ANALYTICAL LABORATORIES at the Georg-August University Göttingen using an ELEMENTAR VARIO EL 3 analyser.

\subsubsection{EPR spectroscopy}

X-band EPR spectra were recorded on a Bruker ELRXSYS E 500 spectrometer with an ER $4131 \mathrm{VT}$ temperature control device for liquid $\mathrm{N}_{2}(\mathrm{MW}=9.42 \mathrm{GHz}$ ) and an ER $4112 \mathrm{HV}$ for liquid $\mathrm{He}(\mathrm{MW}=9.39 \mathrm{GHz}$ ) cooled measurements. The spectra were simulated by Prof. Bas de Bruin University of Amsterdam.

\subsubsection{Head space Analysis}

Head space analysis was performed with a SHIMADZU-GC-2014 equipped with a TCD detector and a SHINCARBON ST80/100 Silico column.

\subsubsection{Irradiation Experiments}

Photolysis experiments were performed using a $150 \mathrm{~W} \mathrm{Hg}(\mathrm{Xe})$ arc lamp with a lamp housing and arc lamp power supply from LOT QUANTUM DESIGN GmbH. A water bath was used to ensure a constant temperature.

\subsubsection{Mass spectrometry}

LIFDI mass spectra were measured on a LINDEN CMS and ESI mass spectra on a BRUKER HCT ultra ESI-MS device by the "Zentrale Massenabteilung" at the Georg-August Universität Göttingen. 


\subsubsection{NMR spectroscopy}

All NMR data was recorded on machines from Bruker (AVANCE III 300, AVANCE III 400 and referenced to residual solvent signals (cyclohexane- $\mathrm{d}_{12}: \delta^{1 \mathrm{H}}=1.38 \mathrm{ppm},{ }^{[230]} \mathrm{THF}-\mathrm{d}_{8}: \delta^{1 \mathrm{H}}=3.58 \mathrm{ppm}^{[230]}, \delta^{13 \mathrm{C}}=67.6 \mathrm{ppm}^{[230]} ; \mathrm{C}_{6} \mathrm{D}_{6}: \delta^{1 \mathrm{H}}=$

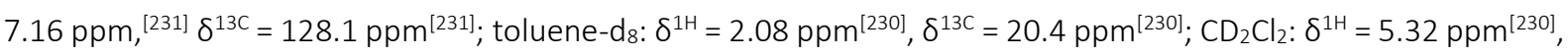
$\left.\delta^{13 C}=54.0 \mathrm{ppm}\right){ }^{[230]}{ }^{31} \mathrm{P}-\mathrm{NMR}$ chemical shifts are reported relative to the shift of Phosphoric acid $(\delta=0.00 \mathrm{ppm})$. For paramagnetic compounds ${ }^{1} \mathrm{H}-\mathrm{NMR}$ pulse sequences with reduced relaxation times were used $\left({ }^{1} \mathrm{H}\right.$-para). Signal multiplicity is denoted as broad (br), singlet (s), doublet (d), triplet(t), virtual triplet (vt) etc. For complex spin systems with higher order coupling effects multiplet $(m)$ is used.

\subsubsection{IR spectroscopy}

IR spectra of pure compounds were recorded at a BRUKER Alpha FT-IR spectrometer with a Platinum ATR module inside a glovebox. For IR measurements in the magnetic field, the spectra were recorded in a KBR pellet using a commercial Fourier transform spectrometer (VERTEX 80v) coupled to a superconducting coil with magnetic fields up to $11 \mathrm{~T}$. To measure transmittance, the radiation of the globar was delivered from the spectrometer using lightpipe optics to the sample inside the magnet and detected by a silicon composite bolometer (IRLABS) placed below the sample. Sample and bolometer were surrounded by a helium exchange gas and cooled down to $4.2 \mathrm{~K}$. Measurements in NUJOL were carried out with a Thermo Scientific Nicolet iZ10 FT/IR spectrometer at room temperature.

\subsubsection{Isothermal Titration Calorimetry}

Isothermal Titration Calorimetry was performed in a NANOITC device by TA INSTRUMENTS equipped with a $24 \mathrm{~K}$ gold cell and a sample volume of $1 \mathrm{~mL}$ operated in overfill mode. Measurement by ITCRun Version 3.4.6.0, TA INSTRUMENTS 2017 and evaluation by the NANOANALYZE software package Version 3.7.5, TA INSTRUMENTS 2015.

\subsubsection{SQUID magnetometry}

Temperature-dependent magnetic susceptibility measurements were performed with a QUANTUM-DESIGN MPMSXL-5 SQUID magnetometer equipped with a $5 \mathrm{~T}$ magnet. The temperature range was from $2 \mathrm{~K}$ to $295 \mathrm{~K}$ at a magnetic field of $0.5 \mathrm{~T}$. The powdered sample was contained in a Teflon bucket and fixed in a non-magnetic sample holder. The data is corrected for the diamagnetic contribution of the Teflon bucket by $M_{\text {dia }}($ bucket $)=X g \cdot m \cdot H$. with an experimentally determined gram susceptibility of the bucket. Experimental data were modelled with the JulX program ${ }^{[232]}$ for $S=0, S=1 / 2$ and $S=1$. Temperature Independent Paramagnetism (TIP) was modeled by $X_{\exp }=$ $(1-P I) \cdot X_{\text {sample }}+P I \cdot X_{P I}+$ TIP. For Zeeman and Zero-Field splitting the isotropic spin Hamiltonian $\widehat{H}=g \mu_{B} \vec{B} \vec{S}+$ $D\left(\hat{S}_{Z}^{2}-\frac{1}{3} S(S+1)\right)$ was used. 


\subsubsection{UV/Vis spectroscopy}

UV/Vis spectra were recorded in a cuvette equipped with a J-YOUNG cap at an AGILENT CARY 300 spectrometer using the SCAN software. Background measurements of the blank solvent were performed in an identical constructed cuvette and subtracted.

\subsubsection{X-ray-diffractometry}

Suitable single crystals for X-ray structure determination were selected from the mother liquor under an inert gas atmosphere and transferred into protective perfluoro polyether oil on a microscope slide. The selected and mounted crystals were transferred to the cold gas stream on the diffractometer. The diffraction data were obtained at $100 \mathrm{~K}$ on a Bruker D8 three-circle diffractometer, equipped with a PHOTON 100 CMOS detector and an INCOATEC microfocus source with Quazar mirror optics (Mo-K $\alpha$ radiation, $\lambda=0.71073 \AA$ ). The data obtained were integrated with SAINT and a semi-empirical absorption correction from equivalents with SADABS was applied. The structure was solved and refined using the Bruker SHELX 2016 software package. ${ }^{[233-236]}$ All nonhydrogen atoms were refined with anisotropic displacement parameters. All C-H hydrogen atoms were refined isotropically on calculated positions by using a riding model with their Uiso values constrained to 1.5 Ueq of their pivot atoms for terminal $\mathrm{sp}^{3}$ carbon atoms and 1.2 times for all other atoms.

\subsubsection{Evans Method}

Magnetic moments in solution were determined by NMR spectroscopy using the Evans' method as modified by Sur and co-workers and corrected for diamagnetic contribution. ${ }^{[237,238]}$

$$
X_{P}=X_{0}+\frac{3000 \Delta v}{4 \pi v_{0} c M}
$$




\section{Synthesis}

\subsection{Improved synthesis for $[(\mathrm{PNP}) \mid \mathrm{rOH}] 2$}

Degassed water $(4 \mathrm{~mL})$ is added to a suspension of 5 (200 mg, $354 \mu \mathrm{mol}, 1.00$ eq.) and NaOH powder (424 mg, $10.6 \mathrm{mmol}, 30$ eq.) in THF (14 mL). The suspension is vigorously stirred at $25^{\circ} \mathrm{C}$ for $16 \mathrm{~h}$. The solvent is removed in vacuo and the residue extracted with pentane and evaporated in vacuo. Washing the residue with cold pentane yields 5 as a blue greenish micro crystalline solid (176 mg, $322 \mu \mathrm{mol}, 91 \%$ ).

Elemental Analysis: Anal. Calc. for $\mathrm{C}_{20} \mathrm{H}_{41} \mathrm{IrNOP}_{2}$ (565.72): C, 42.46; H, 7.31; N, 2.48.

Found: C, 42.40; H, 7.52; N, 2.41

$\operatorname{NMR}\left(\mathrm{C}_{6} \mathrm{D}_{6}, 25^{\circ} \mathrm{C}\right):$

${ }^{1} \mathrm{H}(300 \mathrm{MHz}) \delta(\mathrm{ppm})=7.1\left(\mathrm{brs}, 36 \mathrm{H},\left(\mathrm{P}^{t} \mathrm{Bu} \mathrm{u}_{2}\right)_{2}\right),-32$ (brs, 2H, $(\mathrm{CH})_{2},-107$ (brs, $\left.2 \mathrm{H},(\mathrm{CH})_{2}\right)$.

LIFDI-MS:

Calculated: 566.23. Found: 566.2.

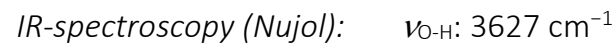

$C V\left(T H F, 25^{\circ} \mathrm{C}\right): \quad E_{1 / 2}($ red $)=-2.11 \mathrm{~V} ; E_{1 / 2}(\mathrm{ox})=-0.37 \mathrm{~V}$ 


\subsection{Synthesis of $[\mathrm{Na} \text { (thf)(PNP)|rOH }]_{2} 1$}

2 (25.0 mg, $44.2 \mu \mathrm{mol}, 1.00$ eq.) is dissolved in THF ( $5 \mathrm{~mL})$ and sodium amalgam (1.0 M, $900 \mathrm{mg}, 44.2 \mu \mathrm{mol}, 1.5$ eq.) is added and shaken for $5 \mathrm{~min}$. The amalgam is removed, and the solution is cooled to $-40^{\circ} \mathrm{C}$ yielding the product as a temperature sensitive orange, crystalline material.

$\operatorname{NMR}\left(\mathrm{THF}_{-} \mathrm{d}_{8}, 25^{\circ} \mathrm{C}\right)$ :

${ }^{1} \mathrm{H}-\mathrm{NMR}(300 \mathrm{MHz}) \delta(\mathrm{ppm})=6.89(\mathrm{~m}, 4 \mathrm{H}, 4 \times \mathrm{CH}), 4.02(\mathrm{~m}, 4 \mathrm{H}$, $4 \times C H), 1.35\left(v t, 72 \mathrm{H},\left(\mathrm{P}^{t} B u_{2}\right)_{2}\right),-2.06(\mathrm{~m}, 2 \mathrm{H}, \mathrm{OH})$.

31P-NMR: 57 ppm (broad). 


\subsection{Improved synthesis of $[(\mathrm{PNP}) \mid \mathrm{rOH}][\mathrm{PF} 6] 3$}

$\mathrm{AgPF}_{6}(22 \mathrm{mg}, 87.0 \mu \mathrm{mol}, 0.98 \mathrm{eq}$.) in dichloromethane $(2 \mathrm{~mL})$ is slowly added to a stirred solution of $2(50 \mathrm{mg}$, $88.4 \mu \mathrm{mol}, 1.0$ eq.) in dichloromethane ( $5 \mathrm{~mL}$ ) at room temperature. The solution is stirred for additional $2 \mathrm{~min}$ and filtered. The crude product precipitates upon addition of pentane. The residue is washed with pentane $(2 \times$ $2 \mathrm{~mL})$, extracted with dichloromethane $(5 \times 0.5 \mathrm{~mL})$ and the product is obtained as dark violet crystals after concentrating the solution and crystallization via gas diffusion layering with pentane (57.8 mg, $81.3 \mu \mathrm{mol}, 92 \%$ ).

Elemental Analysis:

$\operatorname{NMR}\left(\mathrm{CD}_{2} \mathrm{Cl}_{2}, 25^{\circ} \mathrm{C}\right)$ :

IR-spectroscopy (Nujol):
Anal. Calc. for $\mathrm{C}_{20} \mathrm{H}_{41} \mathrm{~F}_{6} \mathrm{IrNOP} 3$ (710.68): C, 33.80; H, 5.82; N, 1.97. Found: $\mathrm{C}, 33.82 ; \mathrm{H}, 6.11 ; \mathrm{N}, 1.95$

${ }^{1} \mathrm{H}\left\{{ }^{31} \mathrm{P}\right\}(300 \mathrm{MHz}) \delta(\mathrm{ppm})=14.60(\mathrm{~s}, 1 \mathrm{H}, \mathrm{OH}), 7.15(\mathrm{~d}, J=6.4 \mathrm{~Hz}, 1 \mathrm{H}$, $\mathrm{NCH}), 5.22(\mathrm{~d}, J=6.4 \mathrm{~Hz}, 1 \mathrm{H}, \mathrm{PCH}), 1.84\left(\mathrm{~s}, 36 \mathrm{H},\left(\mathrm{P}^{t} B u_{2}\right)_{2}\right)$.

$\left.{ }^{31} \mathrm{P}\left\{{ }^{1} \mathrm{H}\right\}(121.4 \mathrm{MHz}): \delta(\mathrm{ppm})=41.3\left(\mathrm{~s}, \mathrm{P}^{\mathrm{t}} \mathrm{Bu}_{2}\right)_{2}\right),-145$ (hept. ${ }^{1} \mathrm{JPF}_{\mathrm{PF}}=$ $\left.710.7 \mathrm{~Hz}, P \mathrm{~F}_{6}\right)$.

${ }^{13} \mathrm{C}\left\{{ }^{1} \mathrm{H}\right\}(75.4 \mathrm{MHz}): \delta(\mathrm{ppm})=170(2 \mathrm{C}, \mathrm{PCH}), 147.9(2 \mathrm{C}, \mathrm{NCH}), 37.3$ $\left(4 \mathrm{C}, \mathrm{PC}\left(\mathrm{CH}_{3}\right)_{3}\right), 32.1\left(12 \mathrm{C}, \mathrm{PC}\left(\mathrm{CH}_{3}\right)_{3}\right)$.

Vо-н: $3577 \mathrm{~cm}^{-1}$ 


\subsection{Synthesis of [(PNP)IrO] 4}

Route A: A solution of Mes*O (234 mg, $895 \mu \mathrm{mol}, 10.1$ eq.) in HMDSO (3 mL) is added to a stirred solution of 2 (50.0 mg, $88.3 \mu \mathrm{mol}, 1.00$ eq.) in $\mathrm{HMDSO}(3 \mathrm{~mL})$ at $25^{\circ} \mathrm{C}$. The solution is filtered and the remaining solid washed with pentane $(2 \times 0.5 \mathrm{~mL})$. Extraction with toluene $(3 \times 0.5 \mathrm{~mL})$ and crystallization at $-40{ }^{\circ} \mathrm{C}$ yields the product as purple crystals (14.2 mg, $70.7 \mu \mathrm{mol}, 80 \%)$.

Route $\mathrm{B}: \mathrm{KO} \mathrm{Bu}^{\mathrm{B}}(1.5 \mathrm{mg}, 13 \mu \mathrm{mol}, 1.0$ eq.) is added to a solution of 3 (9.4 mg, $13 \mu \mathrm{mol}, 1.0$ eq.) in THF (1 mL) at room temperature. The solution is stirred for additional 10 minutes and the solvent evaporated. The residue is washed with pentane $(3 \times 0.5 \mathrm{~mL})$. Crystallization from toluene at $-40{ }^{\circ} \mathrm{C}$ yields the product $(4.4 \mathrm{mg}, 7.8 \mu \mathrm{mol}$, $59 \%)$.

Preparation of the ${ }^{18} \mathrm{O}$ isotopolouge: Sodium $(7.5 \mathrm{mg})$ is added to $\mathrm{H}_{2}{ }^{18} \mathrm{O}(250 \mu \mathrm{L})$ at $4{ }^{\circ} \mathrm{C}$ and the produced $\mathrm{H}_{2}$ gas is removed by three freeze-pump-thaw cycles. A THF solution $(4 \mathrm{~mL})$ of $5(50 \mathrm{mg}, 85.6 \mu \mathrm{mol})$ is added and vigorously stirred for $5 \mathrm{~d}$. The solvents are removed in vacuo and the residue extracted with pentane. Without further purification a solution of Mes*O (234 mg, $895 \mu \mathrm{mol}, 10.5$ eq.) in pentane $(3 \mathrm{~mL})$ is added and the reaction mixture stirred for $5 \mathrm{~min}$. The solution is filtered off and the precipitate washed with pentane $(3 \times 0.5 \mathrm{~mL})$. The residue is extracted with toluene $(5 \times 0.5 \mathrm{~mL})$, evaporated to dryness and washed with pentane $(3 \times 0.5 \mathrm{~mL})$. Removing the solvents in vaccuo yielded material suitable for further analysis.

Elemental Analysis:

$\operatorname{NMR}\left(C_{6} D_{6}, 25^{\circ} \mathrm{C}\right)$

LIFDI-MS:

IR-ATR:

$\mathrm{CV}\left(\mathrm{THF}, 25^{\circ} \mathrm{C}\right)$ :

SQUID magnetometry:
Anal. Calc. for $\mathrm{C}_{20} \mathrm{H}_{40} \mathrm{IrNOP}_{2}$ (564.71): C, 42.54; H, 7.14; N, 2.48 .

Found: C, 42.94; H, 7.19; N, 2.47.

${ }^{1} \mathrm{H}(400 \mathrm{MHz}) \delta(\mathrm{ppm})=20.88(\mathrm{~s}, 36 \mathrm{H}$,

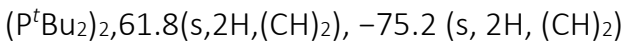

${ }^{31} \mathrm{P}\left\{{ }^{1} \mathrm{H}\right\}(162 \mathrm{MHz})$ : no signal from -2000 and $+2000 \mathrm{ppm}$

Calculated: 565.22 . Found: 565.2

Mr-160: $743 \mathrm{~cm}^{-1} ; \operatorname{Mr}-180: 712 \mathrm{~cm}^{-1}$

$E_{1 / 2}(\mathrm{red})=-1.95 \mathrm{~V}: E_{1 / 2}(\mathrm{ox})=-0.13 \mathrm{~V}$

$g=2.318, D=647 \mathrm{~cm}^{-1}, \mathrm{Pl}=5.1 \%$ 


\subsection{Synthesis of $\left[(\mathrm{PNP}) \mid \mathrm{rCO} 3 \mathrm{rCO}_{3}\right] 8$}

A solution of 4 ( $25 \mathrm{mg}, 44 \mu \mathrm{mol}, 1.0$ eq.) in THF ( $3 \mathrm{~mL})$ is freeze-pump-thaw degassed and thawed to room temperature under an atmosphere of $\mathrm{CO}_{2}$. All volatiles are removed in vacuo and the residue is washed with pentane $(2 \times 0.5 \mathrm{~mL})$ and extracted with toluene $(3 \times 0.5 \mathrm{~mL})$. Crystallization at $-40^{\circ} \mathrm{C}$ yields 8 as red brown needles (19 mg, $31 \mu \mathrm{mol}, 71 \%$ ).

Elemental Analysis:

$\operatorname{NMR}\left(T H F-d_{8}, 25^{\circ} \mathrm{C}\right)$ :

LIFDI-MS:

IR-spectroscopy (ATR):
Anal. Calc. for $\mathrm{C}_{21} \mathrm{H}_{40} \mathrm{IrNO}_{3} \mathrm{P}_{2}$ (608.72): C, 41.44; $\mathrm{H}, 6.62 ; \mathrm{N}, 2.30$.

Found: C, 41.44; H, 6.02; N, 2.30 .

${ }^{1} \mathrm{H}(500 \mathrm{MHz}) \delta(\mathrm{ppm})=6.40(\mathrm{~m}, 2 \mathrm{H}, 2 \mathrm{xCH}), 5.46(\mathrm{~m}$,

$2 \mathrm{H}, 2 \times \mathrm{CH}), 1.48\left(\mathrm{~m}, 36 \mathrm{H}, 4 \times \mathrm{C}(\mathrm{CH})_{3}\right)$.

$\left.{ }^{31} \mathrm{P}\left\{{ }^{1} \mathrm{H}\right\}(202 \mathrm{MHz}): \delta(\mathrm{ppm})=46.2\left(\mathrm{~s}, \mathrm{P}^{\mathrm{t}} \mathrm{Bu}_{2}\right){ }_{2}\right)$.

${ }^{13} \mathrm{C}\left\{{ }^{1} \mathrm{H}\right\}(75.4 \mathrm{MHz}): \delta(\mathrm{ppm})=173.35\left(\mathrm{~s}, \mathrm{CO}_{3}\right), 170(\mathrm{~m}, 2 \mathrm{C}, 2 \mathrm{xCH}), 105(\mathrm{~m}, 2 \mathrm{C}$, $2 \times C H), 36.1\left(m, 4 \mathrm{C}, 4 \times C\left(\mathrm{CH}_{3}\right)\right), 29.7\left(\mathrm{~m}, 12 \mathrm{C}, 4 \times \mathrm{C}\left(\mathrm{CH}_{3}\right)\right)$.

Calculated: 551.23. Found 551.2.

$1663 \mathrm{~cm}^{-1}, 1625 \mathrm{~cm}^{-1}, 1553 \mathrm{~cm}^{-1}$ 


\subsection{Synthesis of [(PNP)IrPMe $] 10$}

$\mathrm{PMe}_{3}\left(1.8 \mu \mathrm{L}, 18 \mu \mathrm{mol}, 2.0 \mathrm{eq}\right.$.) is added to a solution of $4\left(5.0 \mathrm{mg}, 8.9 \mu \mathrm{mol}, 1.0\right.$ eq.) in cyclohexane-d $\mathrm{d}_{12}(0.4 \mathrm{~mL})$. The solution is stirred for $2 \mathrm{~d}$. Quantitative precipitation of OPMeз is revealed by filtration. 10 , can be isolated by removal of the solvent in vacuo and crystallization from $\mathrm{HMDSO}-40{ }^{\circ} \mathrm{C}$ as a yellow crystalline solid, which was characterized by NMR spectroscopy. Any attempts to achieve elemental analysis data from the crystalline material failed, which is attributed to thermal instability.

NMR (Cyclohexane- $\left.d_{12}, 25^{\circ} \mathrm{C}\right)$ :

${ }^{1} \mathrm{H}-\mathrm{NMR}(300 \mathrm{MHz}) \delta(\mathrm{ppm})=7.00(\mathrm{~m}, 2 \mathrm{H}, 2 \times \mathrm{CH}), 4.19(\mathrm{~m}$,

$2 \mathrm{H}, 2 \times \mathrm{CH}), 1.82\left(\mathrm{~d}, \mathrm{~J}_{\mathrm{HP}}=7.4 \mathrm{~Hz}\right), 9 \mathrm{H}, \mathrm{P}\left(\mathrm{CH}_{3}\right)_{3}, 1.31\left(\mathrm{~m}, 36 \mathrm{H}, 4 \times \mathrm{C}\left(\mathrm{CH}_{3}\right)\right.$.

${ }^{31} \mathrm{P}-\mathrm{NMR}(121 \mathrm{MHz}) \delta(\mathrm{ppm})=61.7\left(\mathrm{~d}, 2 \mathrm{P},{ }^{2} \mathrm{JPP}_{\mathrm{PP}}=16.9 \mathrm{~Hz}, 2 \times \mathrm{P}^{\mathrm{t} B \mathrm{Bu}_{2}}\right)$, $-52.6\left(\mathrm{t}, 1 \mathrm{P},{ }^{2} \mathrm{JPp}_{\mathrm{pP}}=16.9 \mathrm{~Hz}, \mathrm{PMe}_{3}\right)$.

${ }^{13} \mathrm{C}-\mathrm{NMR}(100.6 \mathrm{MHz}) \delta(\mathrm{ppm})=162(\mathrm{~m}, 2 \mathrm{C}, 2 \times \mathrm{CH}), 86.5(\mathrm{~m}, 2 \mathrm{C}$, $2 \times C H), 36.7\left(m, 4 \mathrm{C}, 4 \times C(\mathrm{CH})_{3}\right), 31.2\left(\mathrm{~m}, 12 \mathrm{C}, 4 \times \mathrm{C}(\mathrm{CH})_{3}\right), 29.4(\mathrm{~m}, 3 \mathrm{C}$, $\left.\mathrm{P}\left(\mathrm{CH}_{3}\right)_{3}\right)$.

LIFDI-MS:

Calculated: 625.27. Found 625.3 


\subsection{Reactivity of $\mathbf{4}$ towards $\mathrm{H}_{2}$}

A degassed solution of $4(5.0 \mathrm{mg}, 8.9 \mu \mathrm{mol})$ in $\mathrm{C}_{6} \mathrm{D}_{6}(0.4 \mathrm{~mL})$ is set under an atmosphere of $\mathrm{H}_{2}(1$ bar) and shaken vigorously. Monitoring of the reaction by ${ }^{1} \mathrm{H}-\mathrm{NMR}(400 \mathrm{MHz})$ gives formation of $\left[(\mathrm{PNP}) \operatorname{Ir}(\mathrm{H})_{2}\right] 6^{[37]}$ and water after $5 \mathrm{~min}$ and full conversion to 6 and water after $30 \mathrm{~min}$.

\subsection{Reactivity of $\mathbf{4}$ towards $\mathrm{CO}$}

A solution of $4(5.0 \mathrm{mg}, 8.9 \mu \mathrm{mol})$ in $\mathrm{C}_{6} \mathrm{D}_{6}(0.4 \mathrm{~mL})$ is degassed and stirred under $\mathrm{CO}(1 \mathrm{bar})$ for 5 min resulting in near quantitative formation of [(PNP)IrCO] 7 by NMR spectroscopy. ${ }^{[179]}$ Gas-chromatographic headspace analysis confirms the release of $\mathrm{CO}_{2}$.

\subsection{Synthesis of $\left[(\mathrm{PNP}) \mid \mathrm{rO}\left(\mathrm{C}_{12} \mathrm{H}_{8} \mathrm{O}\right)\right]$ "Xanthenolate" 11}

To a solution of 4 ( $25 \mathrm{mg}, 44 \mu \mathrm{mol}, 2.0$ eq.) in THF (10 mL) a solution of xanthene ( $4.0 \mathrm{mg}, 22 \mu \mathrm{mol}, 1.0$ eq.) in THF $(10 \mathrm{~mL})$ was added. The solution was heated to $60^{\circ} \mathrm{C}$ for $24 \mathrm{~h}$ and the solvent was removed in vaccuo. The residue is solved in pentane and purified with column chromatography over silianized silica. Removal of the solvent in vaccuo yields the product as blue-green crystals contaminated with xanthene-dimer.

\subsection{Synthesis of $\left[(\mathrm{PNP}) \mid \mathrm{rO}\left(\mathrm{C}_{13} \mathrm{H}_{9}\right)\right]$ "Fluorenolate" 12}

To a solution of 4 (15 mg, $27 \mu \mathrm{mol}, 2.0$ eq.) in THF (10 mL) a solution of fluorene $(2.2 \mathrm{mg}, 13 \mu \mathrm{mol}, 1.0$ eq. $)$ in THF $(10 \mathrm{~mL})$ was added. The solution was heated to $60^{\circ} \mathrm{C}$ for $24 \mathrm{~h}$ and the solvent was removed in vaccuo. The residue is solved in pentane and purified with column chromatography over silianized silica. Removal of the solvent in vaccuo yields the product as blue-green crystals contaminated with fluorene-dimer.

LIFDI-MS:

Caluclated: 746.3; Found: 746.2

\subsection{Reactivity of 4 towards benzaldehydes ( $\left.\mathrm{X}^{\mathrm{para}}=\mathrm{H}, \mathrm{Me}, \mathrm{OMe}, \mathrm{F}\right)$}

To a solution of $4:\left(5.0 \mathrm{mg}, 8.9 \mu \mathrm{mol}, 1.0\right.$ eq.) in benzene- $\mathrm{d}_{6}(0.5 \mathrm{~mL})$ the corresponding benzaldehyde was added in 10-fold excess. For the irradiation NMR tubes with borosilicat were used. After irradiating for 30 min without a filter the ${ }^{1} \mathrm{H}$-para spectra were recorded. 
2.12. General synthesis of $\left[(P N P) \mid \mathrm{rO}_{2} \mathrm{CPh}-\mathrm{X}\right] 14-\mathrm{X}$ with $\left.\mathrm{X}^{\text {para }}=\mathrm{F}, \mathrm{OMe}, \mathrm{Me}, \mathrm{H}\right)$

To a solution of $13(25.0 \mathrm{mg}, 44.3 \mu \mathrm{mol}, 1.00 \mathrm{eq}$.) in pentane a solution of the corresponding benzaldehyde (1.00 eq.) in THF is added. The solution is stirred for $5 \mathrm{~min}$ and the solvents removed in vaccuo. The residue is taken up in few pentane and the solution is crystalized at $-40^{\circ} \mathrm{C}$ yielding turquoise crystals.

14-H:

Elemental Analysis:

$\operatorname{NMR}\left(T H F-d_{8}, 25^{\circ} \mathrm{C}\right)$ :

LIFDI-MS:

14-OMe:

Elemental Analysis:

$\operatorname{NMR}\left(T H F-d_{8}, 25^{\circ} \mathrm{C}\right):$

LIFDI-MS:
Anal. Calc. for $\mathrm{C}_{21} \mathrm{H}_{40} \mathrm{IrNO}_{3} \mathrm{P}_{2}$ (670.26): C, 48.42; $\mathrm{H}, 6.77 ; \mathrm{N}, 2.09$.

Found: C, 48.38; H, 6.49; N, 2.11.

${ }^{1} \mathrm{H}$-para $(300 \mathrm{MHz}) \delta(\mathrm{ppm})=13.31$ (brs, 36H, 4xC(CH)3), 4.90 (brs, $1 \mathrm{H}, \mathrm{p}-\mathrm{Ar}-$ $\mathrm{CH}), 2.83$ (brs, 2H), -4.92 (brs, 2H), -5.52 (brs, 2H), -125 (vbrs, 2H).

Calculated: 670.3. Found 670.2.

Anal. Calc. for $\mathrm{C}_{21} \mathrm{H}_{40} \mathrm{IrNO}_{3} \mathrm{P}_{2}$ (700.27): C, 47.56; H, 6.84; N, 2.04 .

Found: C, 48.05; H, 6.77; N, 2.00.

${ }^{1} \mathrm{H}$-para $(300 \mathrm{MHz}) \delta(\mathrm{ppm})=13.43(\mathrm{brs}, 36 \mathrm{H}, 4 \times \mathrm{C}(\mathrm{CH}) 3), 2.29(\mathrm{brs}, 2 \mathrm{H}), 1.98$ (brs, 3H, O-CH3), -5.37 (brs, 2H), -7.14 (brs, 2H), -135 (vbrs, 2H).

Calculated: 700.27. Found 700.3. 
14-Me:

Elemental Analysis:

$\operatorname{NMR}\left(T H F-d_{8}, 25^{\circ} \mathrm{C}\right):$

LIFDI-MS:

$\operatorname{NMR}\left(T H F-d_{8}, 25^{\circ} \mathrm{C}\right):$

LIFDI-MS:
Anal. Calc. for $\mathrm{C}_{21} \mathrm{H}_{40} \mathrm{IrNO}_{3} \mathrm{P}_{2}$ (684.27): C, 49.18; H, 6.93; N, 2.04 .

Found: C, 48.69; H, 6.50; N, 1.92.

${ }^{1} \mathrm{H}$-para $(300 \mathrm{MHz}) \delta(\mathrm{ppm})=13.43\left(\mathrm{brs}, 36 \mathrm{H}, 4 \times \mathrm{C}(\mathrm{CH})_{3}\right), 2.58(\mathrm{brs}, 2 \mathrm{H}), 0.26$ (brs, 3H, $\left.p-\mathrm{CH}_{3}\right),-5.26$ (brs, 2H), -6.62 (brs, 2H), -128 (vbrs, 2H).

Calculated: 684.3. Found 684.3.

Anal. Calc. for $\mathrm{C}_{21} \mathrm{H}_{40} \mathrm{IrNO} \mathrm{P}_{3} \mathrm{P}_{2}$ (688.25): C, 47.15; H, 6.45; N, 2.04 .

Found: C, 46.74; H, 6.08; N, 2.01.

${ }^{1} \mathrm{H}$-para $(300 \mathrm{MHz}) \delta(\mathrm{ppm})=13.30\left(\mathrm{brs}, 36 \mathrm{H}, 4 \times \mathrm{C}(\mathrm{CH})_{3}\right), 2.62(\mathrm{brs}, 2 \mathrm{H}),-5.05$ overlaid (2xbrs, 2x2H), -134 (vbrs, $2 \mathrm{H})$.

Calculated: 688.2. Found 688.2. 


\subsection{Synthesis of $[(P N P) \operatorname{Ir}(H)(C l)] 15$}

To a solution of 5 (33.0 mg, $56.5 \mu \mathrm{mol}, 1.00$ eq.) in pentane $(20 \mathrm{~mL}) n$-BuLi (1.6 M (hexane), $50 \mu \mathrm{L}, 80 \mu \mathrm{mol}, 1.4$ eq.) is added under stirring. When the green colour of 5 has vanished to a pale yellow benzoic acid (15.0 mg, $123 \mu \mathrm{mol}$, 2.17 eq.) in THF ( $1 \mathrm{~mL})$ is added under stirring. The solvents are removed in vaccuo and the solution is extracted with pentane $(3 \times 1 \mathrm{~mL})$. Concentration of the solution in vaccuo and crystallization at $-40{ }^{\circ} \mathrm{C}$ yields the product as deep pink crystals (25.3 mg, $43.2 \mu \mathrm{mol}, 77 \%$ ).

Elemental Analysis:

$\operatorname{NMR}\left(\right.$ THF- $\left.d_{8}, 25^{\circ} \mathrm{C}\right):$

LIFDI-MS:
Anal. Calc. for $\mathrm{C}_{20} \mathrm{H}_{41} \mathrm{rNNP}_{2}$ (585.17): C, 41.05; H, 7.06; N, 2.39.

Found: C, 40.98; H, 7.02; N, 2.31 .

$\left.{ }^{1} \mathrm{H}-\mathrm{NMR}(300 \mathrm{MHz}) \delta(\mathrm{ppm})=1.31(\mathrm{~m}, 36 \mathrm{H}, 4 \times \mathrm{C}(\mathrm{CH}) 3), 4.35(\mathrm{~m}, 2 \mathrm{H}), 2 \mathrm{xCH}\right)$, $7.05(\mathrm{~m}, 2 \mathrm{H}, 2 \mathrm{CCH}),-47.3\left(\mathrm{t}, 1 \mathrm{H},{ }^{2} J_{P-H}=11.5 \mathrm{~Hz}, \mathrm{Ir}-H\right)$.

${ }^{31} \mathrm{P}\left\{{ }^{1} \mathrm{H}\right\}-N M R(121 \mathrm{MHZ}) \delta(\mathrm{ppm})=62.8 \mathrm{ppm}$

Calculated: 585.2. Found: 585.2. 


\subsection{Synthesis of $\left[\left({ }^{\left(\mathrm{Pr}^{\mathrm{H}}\right.}{ }^{\mathrm{N} N}\right) \mathrm{ReCl}_{3}\right]\left[\mathrm{BarF}_{24}\right] 25$}

To a solution of $23(21.8 \mathrm{mg}, 41.8 \mu \mathrm{mol}, 1.00$ eq.) in DCM $(5 \mathrm{~mL})$ a solution of Fc[BArF 24$](36.9 \mathrm{mg}, 40.3 \mu \mathrm{mol}$, 0.96 eq.) $(5 \mathrm{~mL})$ is added under stirring and the reaction is stirred for $5 \mathrm{~min}$. The solvent is removed, and the residue washed with pentane $(5 \times 2 \mathrm{~mL})$ and extracted with ether until the ether phase remained colourless. Removal of the solvent yields the product as a red solid (43.2 mg, $33.4 \mu \mathrm{mol}, 81 \%$ ). Crystals suitable for XRD analysis can be obtained by crystallisation at $-40^{\circ} \mathrm{C}$ from ether.

Elemental Analysis:

Anal. Calc. for $\mathrm{C}_{20} \mathrm{H}_{40} \mathrm{IrNOP} 2$ (564.71): C, 39.46; H, 3.38; N, 0.96

Found: C, 40.02; H, 3.33; N, 0.93 .

$\operatorname{NMR}\left(T H F-d_{8}, 25^{\circ} \mathrm{C}\right):$

IR-ATR:

Evans method:

$\mu_{\mathrm{eff}}=2.9 \mu \mathrm{B}$

$v_{\mathrm{N}-\mathrm{H}}=3095 \mathrm{~cm}^{-1}$

\author{
${ }^{1} \mathrm{H}$-para $(400 \mathrm{MHz}) \delta(\mathrm{ppm})=32.0\left(\mathrm{~s}, 6 \mathrm{H}, 2 \times\left(\mathrm{PC}(\mathrm{H})\left(\mathrm{CH}_{3}\right)\right), 30.4(\mathrm{~s}, 6 \mathrm{H}\right.$, \\ 2x(PC(H) $\left.\left(\mathrm{CH}_{3}\right)\right), 29.9\left(\mathrm{~s}, 6 \mathrm{H}, 2 x\left(\mathrm{PC}(\mathrm{H})\left(\mathrm{CH}_{3}\right)\right), 27.8\left(\mathrm{~s}, 6 \mathrm{H}, 2 \times\left(\mathrm{PC}(\mathrm{H})\left(\mathrm{CH}_{3}\right)\right)\right.\right.$, \\ $14.2(\mathrm{~s}, 2 \mathrm{H}), 4.9(\mathrm{~s}, 2 \mathrm{H}),-8.2(\mathrm{~s}, 2 \mathrm{H}),-79.8(\mathrm{~s}, 2 \mathrm{H}),-103.4(\mathrm{~s}, 2 \mathrm{H})$.
}

\subsection{Deprotonation of $\left[\left({ }^{\left(\mathrm{Pr}^{\mathrm{H}}\right.}{ }^{\mathrm{H} P P}\right) \mathrm{ReCl}_{3}\right]\left[\mathrm{BarF}_{24}\right] 25$}

${ }^{1} \mathrm{H}$ (para)- NMR is measured after the addition of pyridine $(0.5 \mu \mathrm{L} \mathrm{mg}, 6 \mu \mathrm{mol}, 0.6$ eq.) to a solution of 25 (13.6 mg, $9.31 \mu \mathrm{mol}, 1.00$ eq.) in THF-d8 $(0.5 \mathrm{~mL})$ leads to clean conversion to 24.

\subsection{N-H/D exchange of 23}

Experiment performed by Dr. F. Wätjen. $23(6.0 \mathrm{mg}, 10.0 \mu \mathrm{mol}, 1.0 \mathrm{eq})$ is suspended in a 4:1 DCM/D ${ }_{2} \mathrm{O}$ mixture $(0.5 \mathrm{~mL})$ and stirred overnight. Afterwards the solvent is removed in vacuo and the spectroscopically clean product [(PDNP)ReCl 3 (23- $d$ ) is measured in $\mathrm{CD}_{2} \mathrm{Cl}_{2}$. The NMR spectra show largely identical signals to those of 1 . The signal corresponding to the $\mathrm{NH}$ proton is almost gone ( $2 \%$ remaining) and the signals at $\delta_{1 \mathrm{H}}=-5.10$ and $-10.44 \mathrm{ppm}$ exhibit different coupling patterns.

$\operatorname{NMR}\left(\mathrm{CD}_{2} \mathrm{Cl}_{2}, 25^{\circ} \mathrm{C}\right)$ :

${ }^{2} \mathrm{H}-\mathrm{NMR}(46.1 \mathrm{MHz}) \delta(\mathrm{ppm})=152.6(\mathrm{~s}, \mathrm{ND})$.

ATR-IR:

$v_{N-D}=2362.9\left(v_{N D}\right)$ and $3506.4(b r$, electronic absorption) 


\subsection{Reevaluation of the $23 / 25$ redox potential}

Cyclic voltammetry performed by Dr. F. Wätjen. The spectrum is now measured vs. acetylferrocene $\left(E^{\mathrm{ox}}(\mathrm{THF})=\right.$ $0.24 \mathrm{~V}$, unpublished data Richt van Alten).

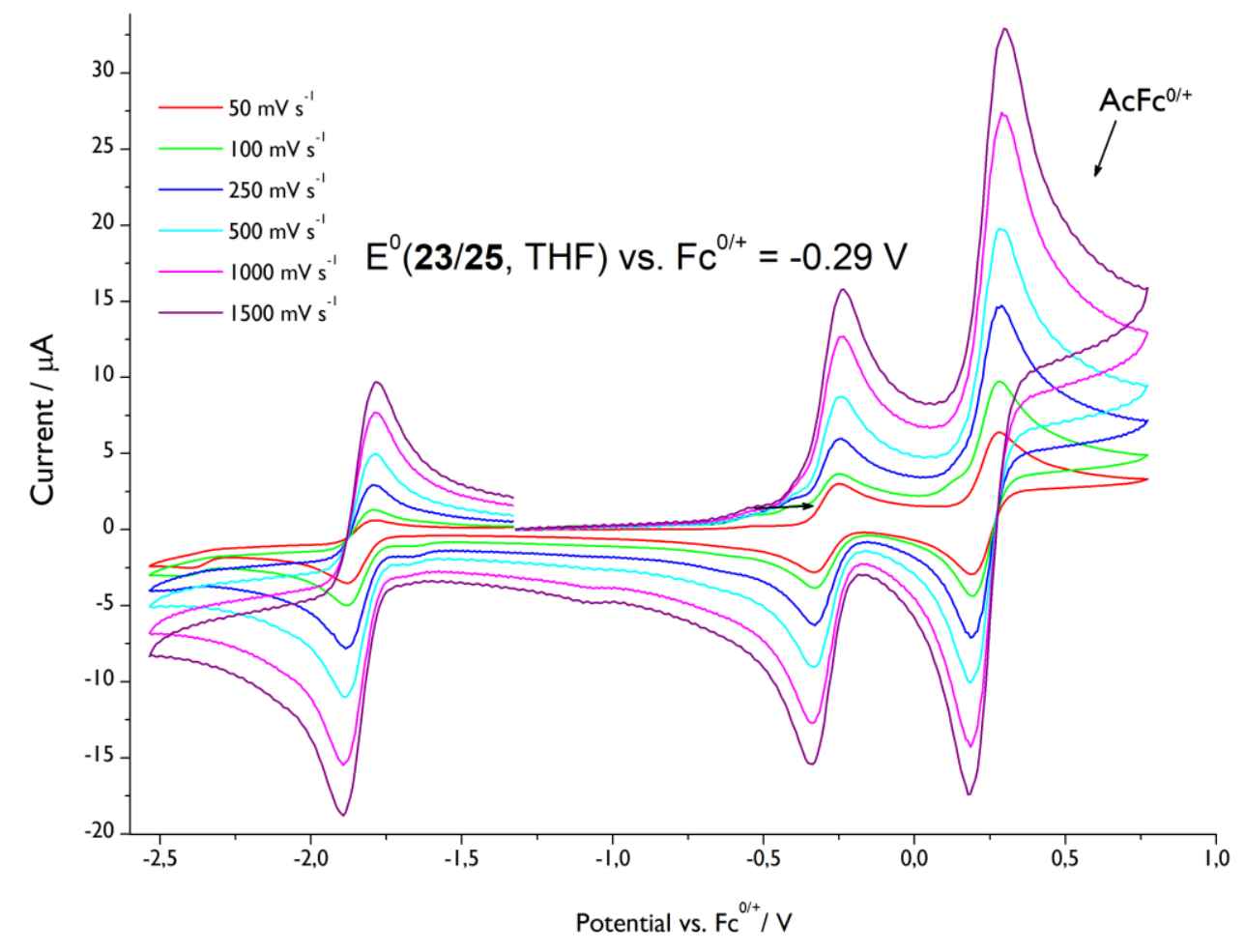

Figure 50: Cyclic voltammogram of 23 in THF with $\mathrm{AcFc}^{0 /+}$ present. 


\section{Isothermal Titration Calorimetry}

\section{1 $\mathrm{BD}(\mathrm{F})$ E determination of 4}

\section{Measurement Conditions}

The $\mathrm{O}-\mathrm{H}$ bond dissociation (free) energy $\mathrm{BD}(\mathrm{F}) \mathrm{E}$ of 2 is determined by reaction with the Mes*O $\left(\mathrm{BDE}_{\mathrm{-H}}(\mathrm{Mes} * \mathrm{OH})=354 \mathrm{kJmol}^{-1}\right)$ as a titrant with the experimental conditions shown in Table 3 .

Table 3: ITC measurement conditions for the BD(F)E determination of 2.

\begin{tabular}{c|c}
\hline Concentration and volume of 2 & $2.0 \mathrm{mM} / 950 \mu \mathrm{L}$ \\
Concentration and volume of Mes*O & $15.3 \mathrm{mM} / 250 \mu \mathrm{L}$ \\
Injection Steps/Injected Volume & $50 @ 5 \mu \mathrm{L}$ \\
Waiting time between two additions & $300 \mathrm{~s}$ \\
Stirring rate & $350 \mathrm{rpm}$ \\
Equilibration Time befor experiment start & $1229 \mathrm{~s}$ \\
\hline
\end{tabular}

\section{Titration}

A representative thermogram, integrated titration curve and measurement of the heat of dissolution of Mes*-O in THF are shown below. For each integrated titration step a constant value of $7.9 \mu \mathrm{J}$ is subtracted from the heat of reaction to correct for the heat of dissolution. The first titration data point is generally neglected due to a dilution effect within pre-experiment equilibration. The plot of the integrated heat curve is fitted with an independent model in analogy to Brandt's et al. but adjusted for a PCET process (see C, appendix). ${ }^{[224]}$

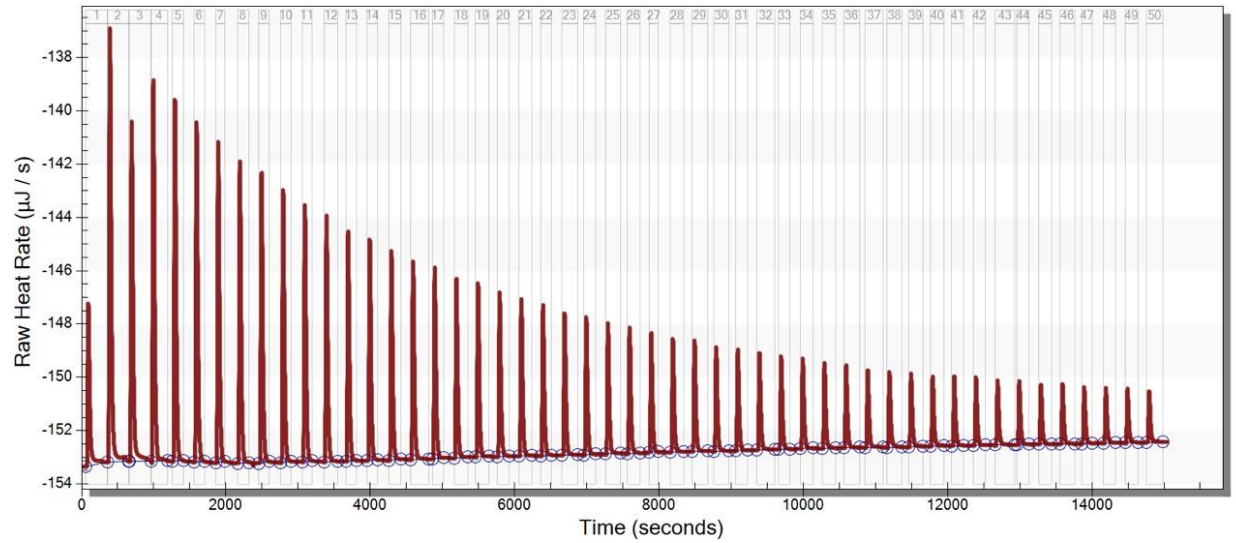

Figure 51: Heat vs. time thermogram of the titration of 2 vs. Mes* $\mathrm{O}$ in THF at $25^{\circ} \mathrm{C}$.

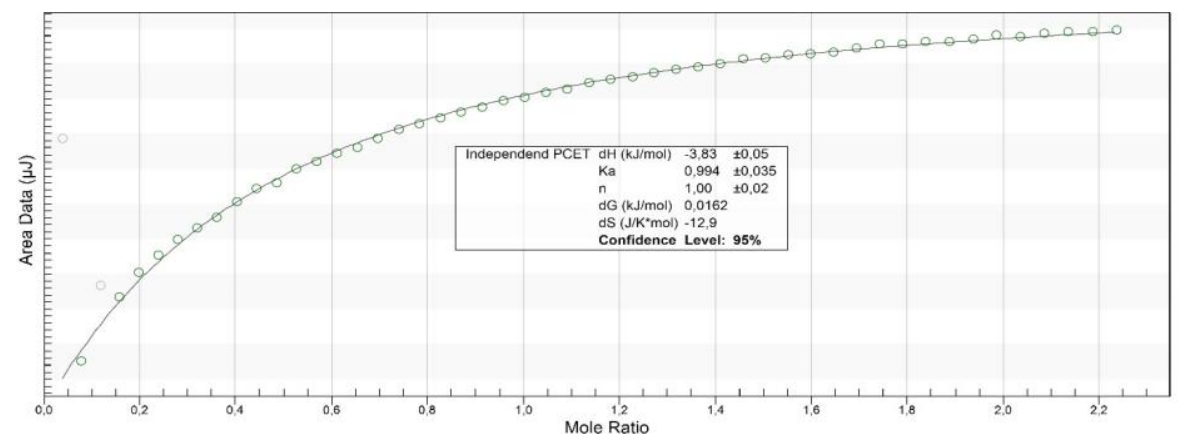

Figure 52: Integrated heat curve of the titration of $2 \mathrm{vs}$. Mes*O in THF at $25^{\circ} \mathrm{C}$. Shaded points were not included in the fitting process. 


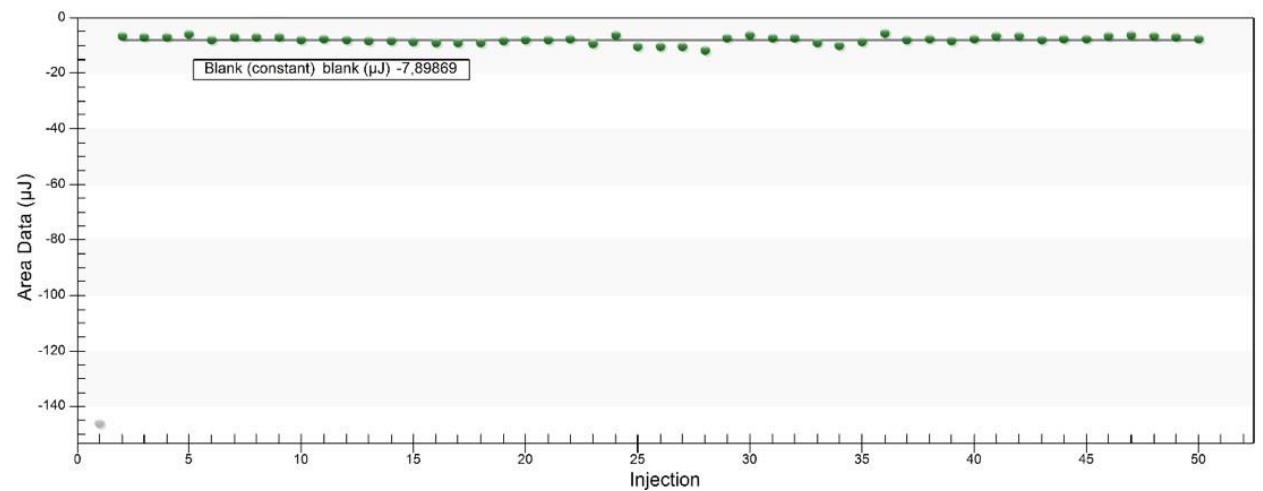

Figure 53: Heat of dissolution of Mes*-O in THF at $25^{\circ} \mathrm{C}$.

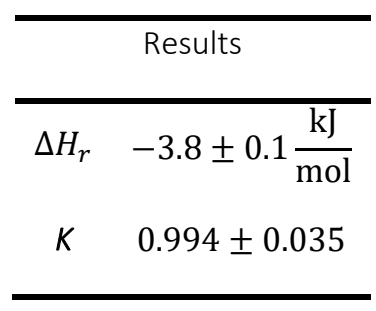

\subsection{BDE determination of 23}

\subsubsection{Enthalpy determination of the PCET reaction in THF}

\section{Measurement conditions}

The heat of reaction of the $23 / 24$ complex couple in THF was determined by titration with the Mes*-O. The experimental conditions were the following (Table 4).

Table 4: ITC measurement conditions

\begin{tabular}{ccc}
\hline & First Run/Second Run & Third Run \\
\hline Concentration and volume of $23[\mathrm{mM} / \mu \mathrm{L}]$ & $1.69 / 950$ & $1.69 / 950$ \\
Concentration and volume of Mes*-O $[\mathrm{mM} / \mu \mathrm{L}]$ & $13.3 / 250$ & $12.9 / 250$ \\
Injection Steps/Injected Volume $[\mu \mathrm{L}]$ & $25 @ 10$ & $250 * 10$ \\
Waiting time between two additions $[\mathrm{s}]$ & 350 & $200^{*}$ \\
Stirring rate $[\mathrm{rpm}]$ & $1200 / 1200$ & 350 \\
Equilibration time before start of experiment[s] & 1174 \\
\hline
\end{tabular}

*The waiting time for injection 13 was raised to $600 \mathrm{~s}$ to encounter slower kinetics around the equivalence point

\section{Titration}

The following figures show the thermograms and integrated titration curves of $\left(\mathrm{P}^{\mathrm{H} N P}\right) \mathrm{ReCl}{ }_{3} 23$ vs. Mes*-O in THF at 298 K. Greyed out points are not taken into account in the fitting process. The first point is generally neglected due to the dilution effect within the pre-experiment equilibration time. An Independent PCET model determined the heat of reaction and the stoichiometry. 

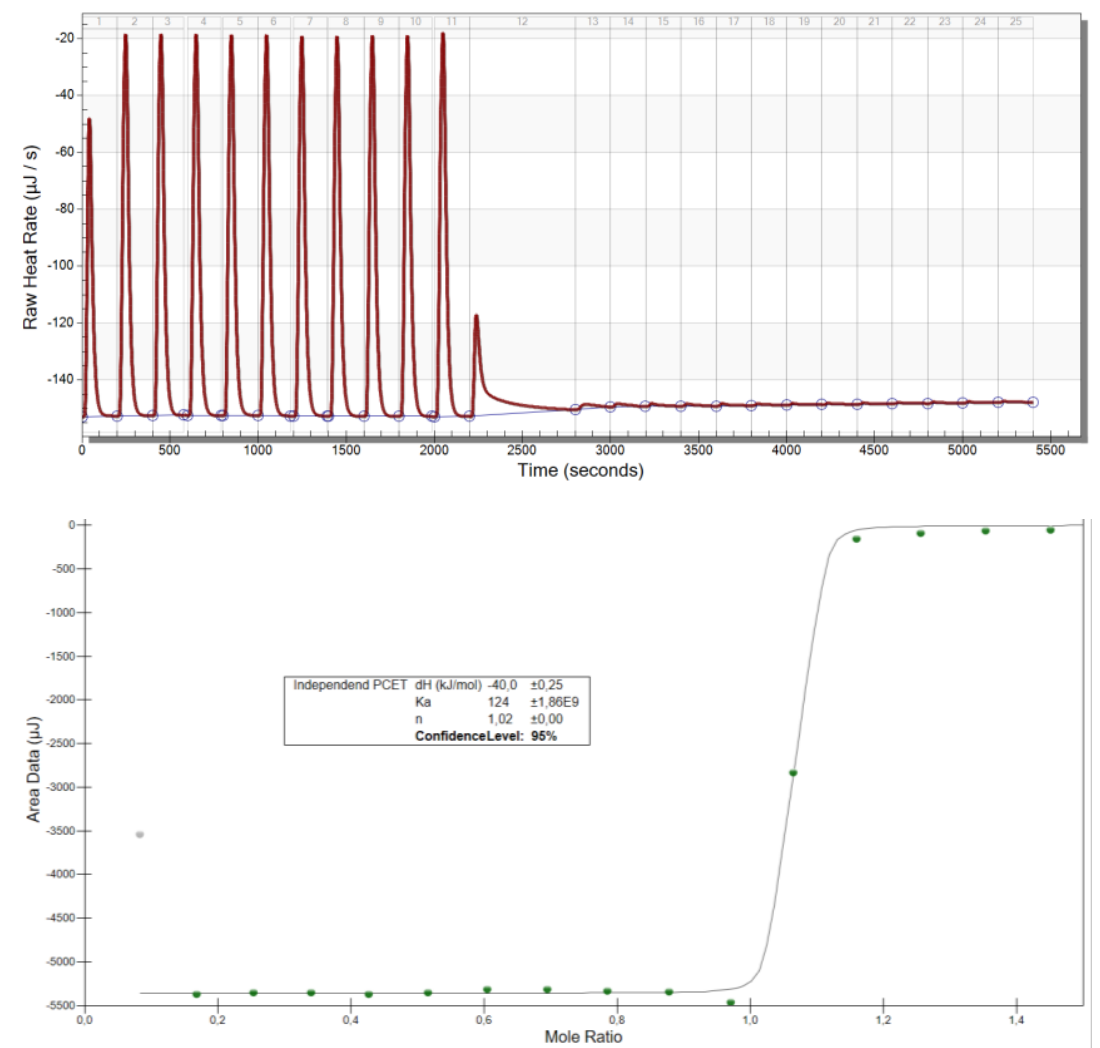

Figure 54: Thermogram (top) and integrated heat curve (bottom) of the first titration of $23 \mathrm{vs.} \mathrm{Mes*-O} \mathrm{at} 298 \mathrm{~K}, \mathrm{THF}$.
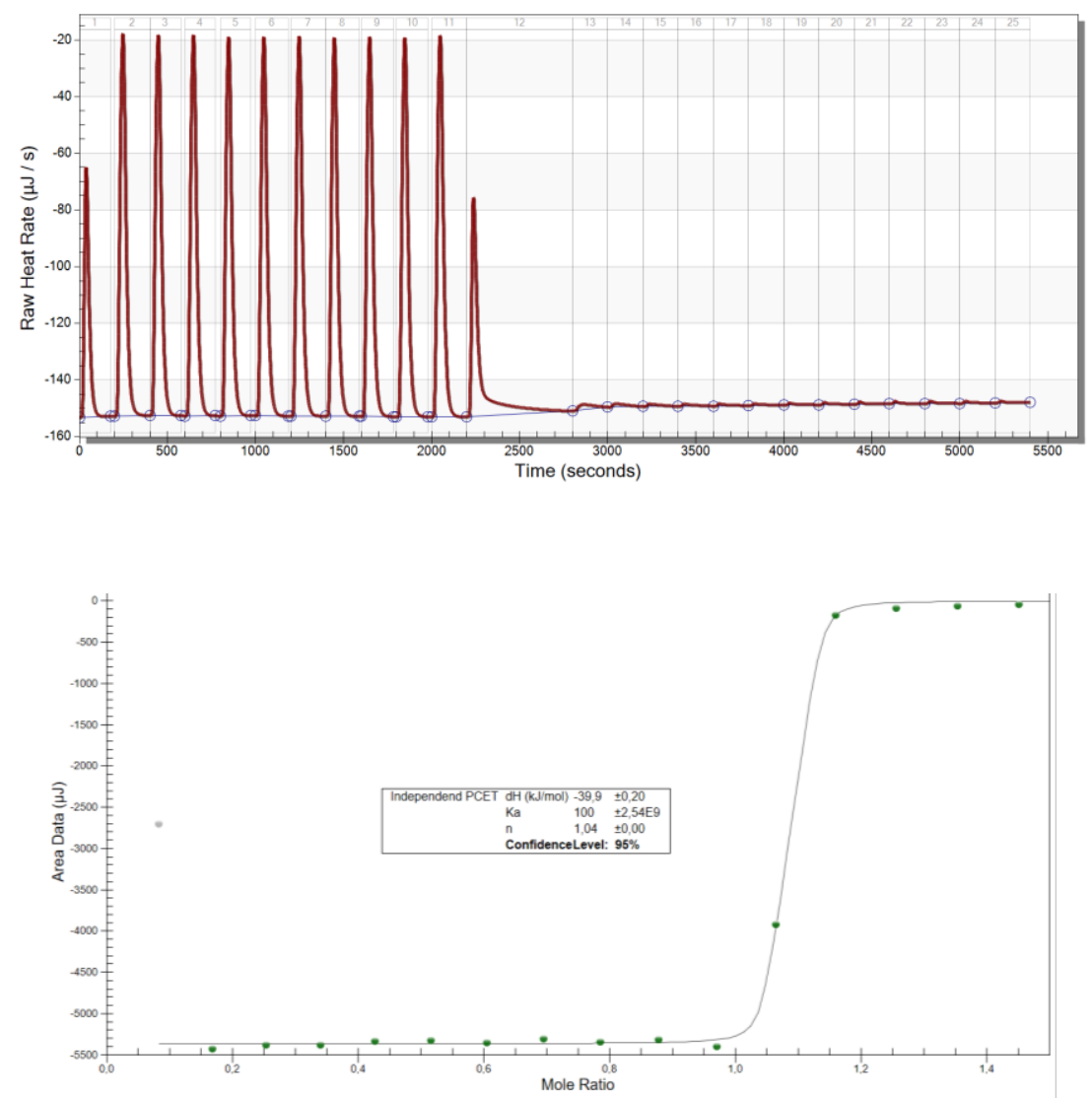

Figure 55: Thermogram (top) and integrated heat curve(bottom) of the second titration of 23 vs. Mes*-O at 298 K, THF. 

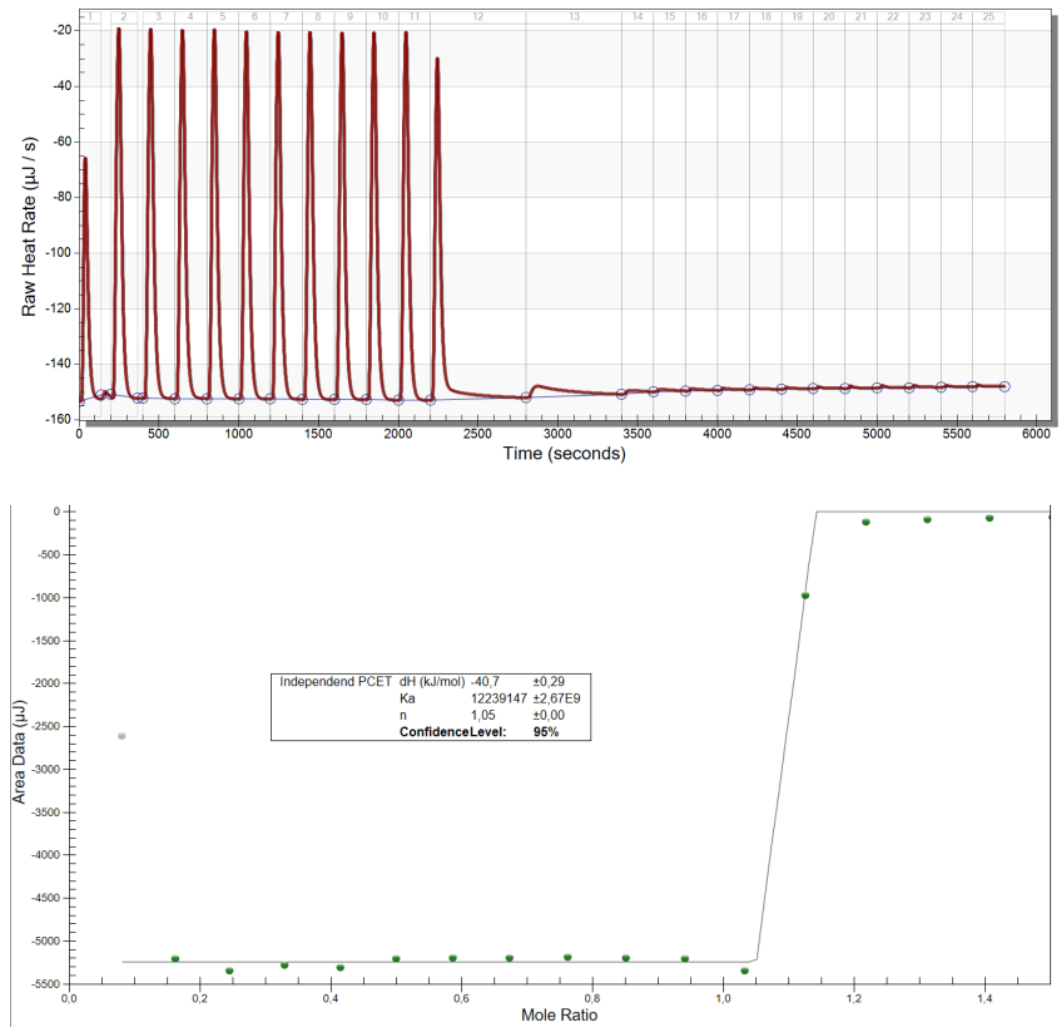

Figure 56: Thermogram (top) and integrated heat curve (bottom) of the third titration of 23 vs. Mes*-O at $298 \mathrm{~K}$, THF.

\section{Evaluation and error discussion}

Table 5: Summarized Results of the reaction enthalpy determination in THF.

\begin{tabular}{cccc}
\hline$\Delta H_{r} / \mathrm{kJ} \mathrm{mol}^{-1}$ & Error at $95 \%$ confidence & $\mathrm{n}$ value & Corrected Error \\
\hline-40.0 & \pm 0.25 & 1.02 & \pm 0.26 \\
-39.9 & \pm 0.20 & 1.04 & \pm 0.21 \\
-40.7 & \pm 0.29 & 1.05 & \pm 0.30 \\
\hline
\end{tabular}

The weighted average enthalpy of the reaction sums up to:

$$
\Delta H^{298 \mathrm{~K}}(T H F)=-40.1 \pm 0.2 \mathrm{~kJ} \mathrm{~mol}^{-1}
$$




\subsubsection{Enthalpy determination of the PCET reaction in DCM}

\section{Measurement Conditions}

The heat of reaction of the $23 / 24$ complex couple in DCM was determined by titration with the (Mes*-O). The experimental conditions where the following (Table 6).

Table 6: ITC measurement details

\begin{tabular}{ccc}
\hline & First Run/Second Run & Third Run \\
\hline Concentration and volume of $23[\mathrm{mM} / \mu \mathrm{L}]$ & $0.836 / 950$ & $1.69 / 950$ \\
Concentration and volume of Mes*-O $[\mathrm{mM} / \mu \mathrm{L}]$ & $6.54 / 250$ & $12.9 / 250$ \\
Injection Steps/Injected Volume $[\mu \mathrm{L}]$ & $14 / 16 @ 10$ & $16 @ 10$ \\
Waiting time between two additions $[\mathrm{s}]$ & $200^{[\mathrm{a}]}$ & $200^{*}$ \\
Stirring rate $[\mathrm{rpm}]$ & 350 & 350 \\
Equilibration time before start of experiment $[\mathrm{s}]$ & 1200 & 1200
\end{tabular}

[a] Following the decelerating kinetics around the equilibrium point the injection time was raised from injection 8 on by $50 \mathrm{~s}$ each up to $400 \mathrm{~s}$ at injection 11 , injection 12: $250 \mathrm{~s}$. [b] same as [a] but injection 12: 400s, injection $13250 \mathrm{~s}$.

\section{Titration}

The following figures show the thermograms and integrated titration curves of 23 vs. Mes*-O in DCM at $298 \mathrm{~K}$. Greyed out points are not considered in the fitting process. The first point is generally neglected due to the dilution effect within the pre-experiment equilibration time. An Independent PCET model determined the heat of reaction and the stoichiometry.
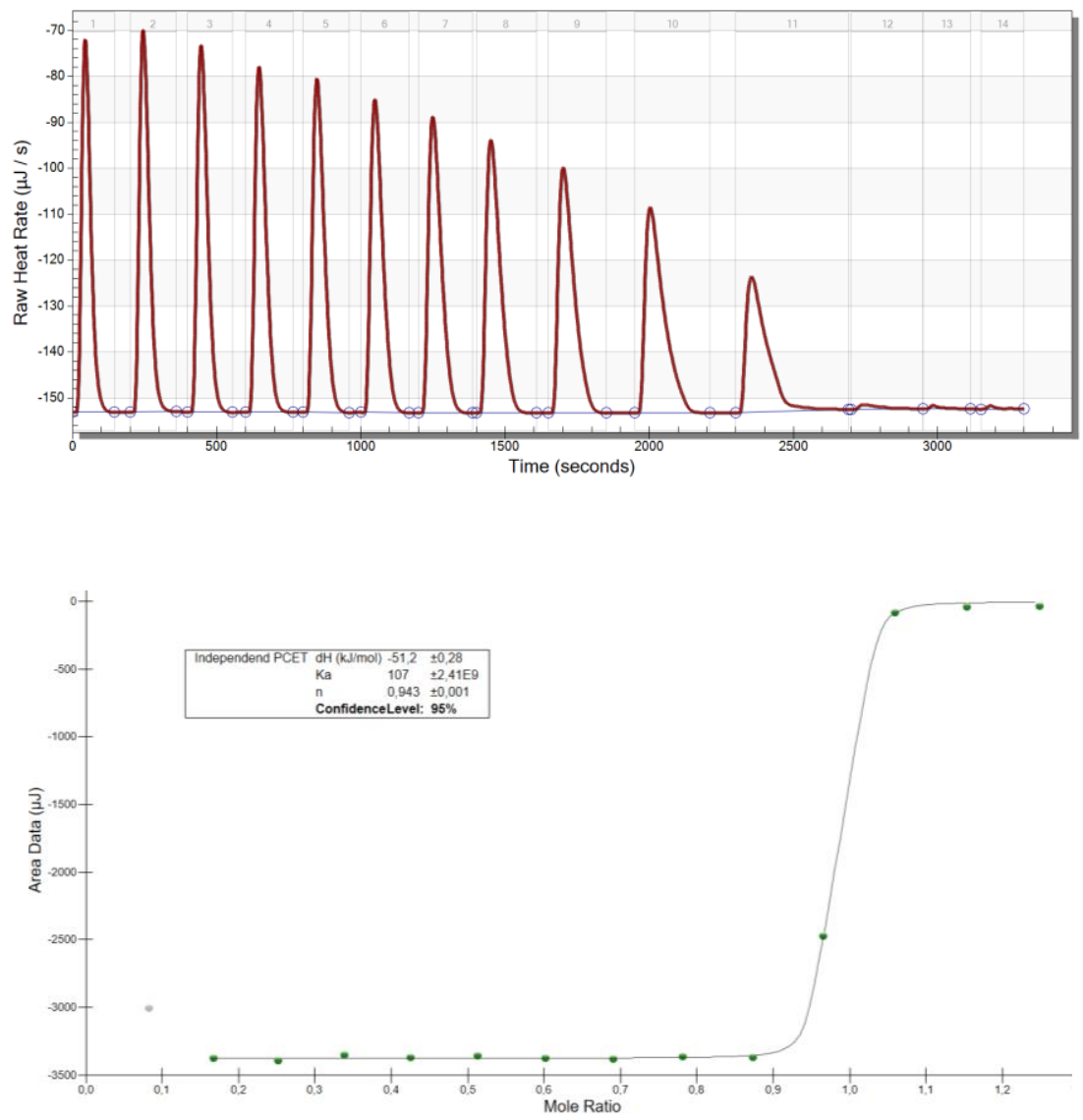

Figure 57: Thermogram (top) and integrated heat curve(bottom) of the first titration of 23 vs. Mes*-O at $298 \mathrm{~K}$, DCM. 

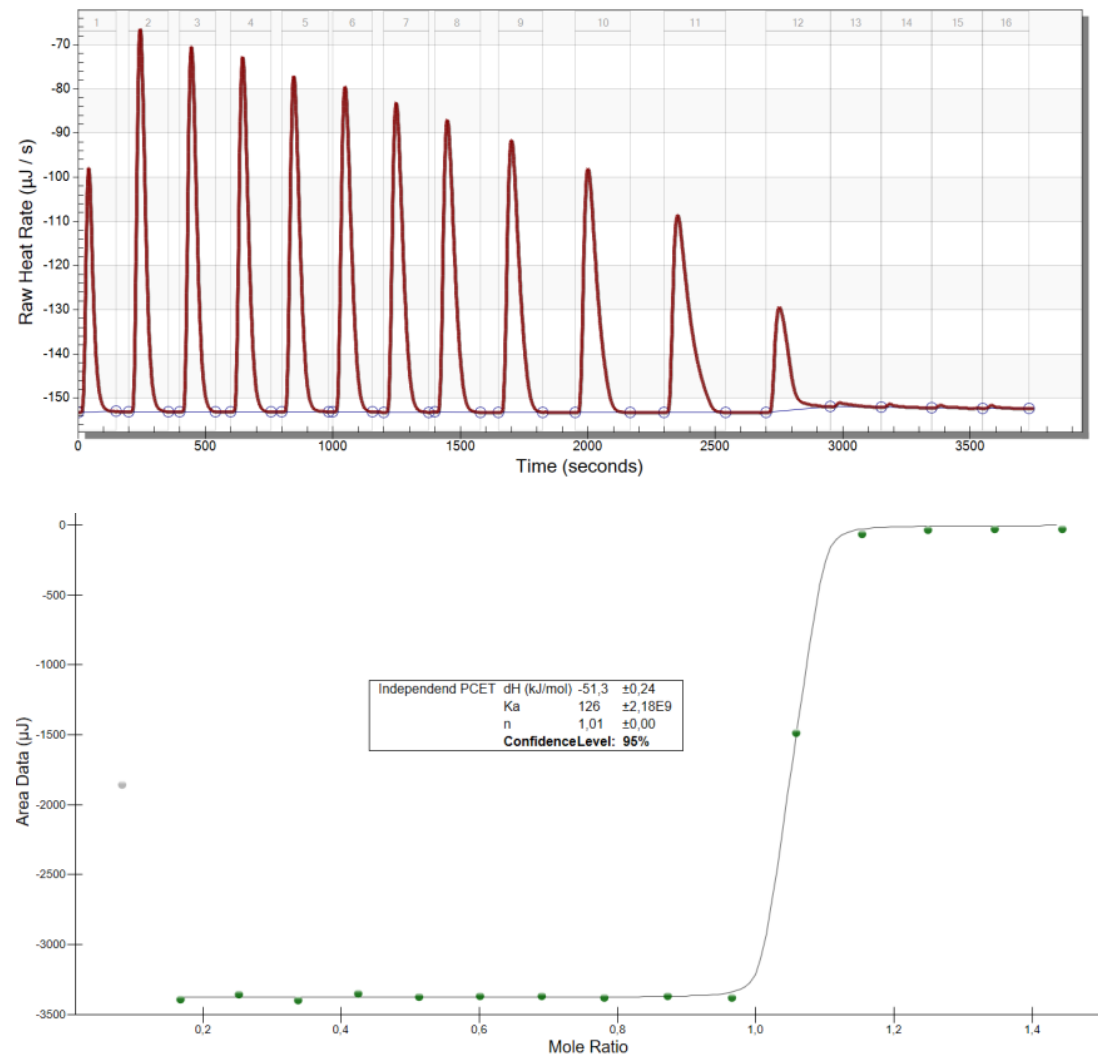

Figure 58: Thermogram (top) and integrated heat curve(bottom) of the second titration of 23 vs. Mes*-O at 298 K, DCM.
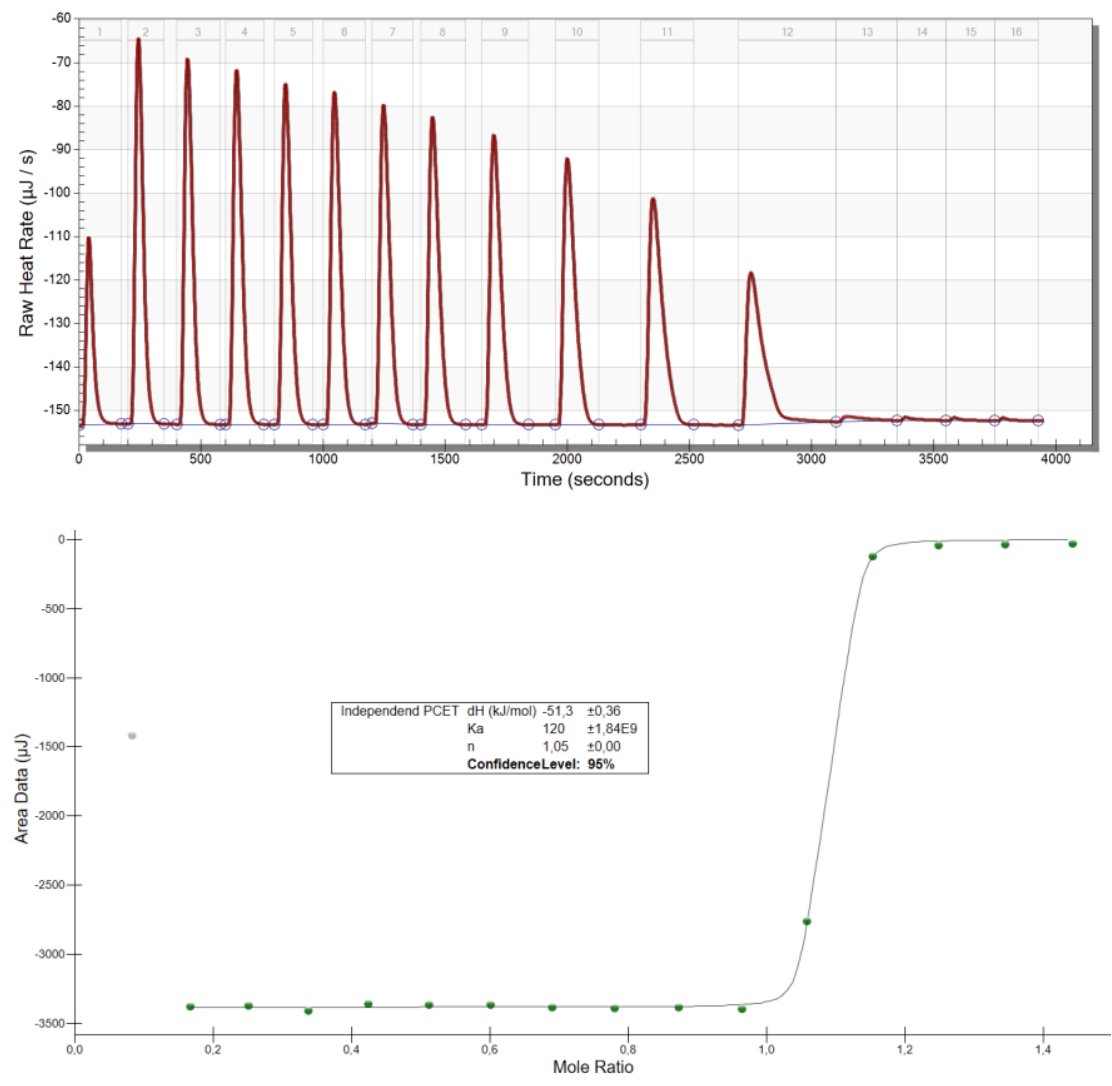

Figure 59: Thermogram (top) and integrated heat curve(bottom) of the third titration of 23 vs. Mes*-O at $298 \mathrm{~K}, \mathrm{DCM}$. 


\section{Evaluation and error discussion}

The extremely large error on the equilibrium constant within the fitting process indicates, that the data is only sufficient for $\Delta H$ determination. Table 7 summarizes the results of the three independent runs.

Table 7: Summarized results of the reaction enthalpy determination in DCM

\begin{tabular}{cccc}
\hline$\Delta H_{r} / \mathrm{kJ} \mathrm{mol}^{-1}$ & Error at $95 \%$ confidence & $\mathrm{n}$ value & Corrected Error \\
\hline-51.2 & \pm 0.28 & 0.943 & \pm 0.30 \\
-51.3 & \pm 0.24 & 1.01 & \pm 0.24 \\
-51.3 & \pm 0.36 & 1.05 & \pm 0.38 \\
\hline
\end{tabular}

The weighted average enthalpy of the reaction sums up to:

$$
\Delta H^{298 \mathrm{~K}}(\mathrm{DCM})=-51.2 \pm 0.3 \mathrm{~kJ} \mathrm{~mol}^{-1}
$$

\section{Thermodynamic Summary}

Table 8: Summarized results on the reaction enthalpy in THF and DCM.

\begin{tabular}{ccc}
\hline Solvent & THF & DCM \\
\hline$\Delta H / \mathrm{kJ} \mathrm{mol}^{-1}$ & $-40.1 \pm 0.2$ & $-51.2 \pm 0.3$
\end{tabular}




\section{3 $p K_{\mathrm{A}}$ value determination of 25}

\section{Measurement Conditions}

The $p K_{\mathrm{A}}$ value of 25 was determined by titration with pyridine as a base $\left(p K_{\mathrm{A}}{ }^{\mathrm{THF}}=5.5\right) .{ }^{[222]}$ The experimental conditions where the following (Table 6).

Table 9: ITC measurement details

\begin{tabular}{ccc}
\hline & First Run & Second Run \\
\hline Concentration and volume of $25[\mathrm{mM} / \mu \mathrm{L}]$ & $1.4 / 950$ & $1.44 / 950$ \\
Concentration and volume of pyridine $[\mathrm{mM} / \mu \mathrm{L}]$ & $12.4 / 250$ & $12.4 / 250$ \\
Injection Steps/Injected Volume $[\mu \mathrm{L}]$ & $47 @ 5$ & $33 @ 5$ \\
Waiting time between two additions $[\mathrm{s}]$ & 300 & 300 \\
Stirring rate $[\mathrm{rpm}]$ & 350 & 350 \\
Equilibration time before start of experiment[s] & 2040 & 1305 \\
\hline
\end{tabular}

\section{Titration}

The following figures show the thermograms and integrated titration curves of 25 vs. pyridine in THF at $298 \mathrm{~K}$. Greyed out points are not considered in the fitting process. The first point is generally neglected due to the dilution effect within the pre-experiment equilibration time. An Independent PCET model was fitted to the obtained data. $10 \mu \mathrm{J}$ per injection are subtracted to correct for the heat of dissolution.
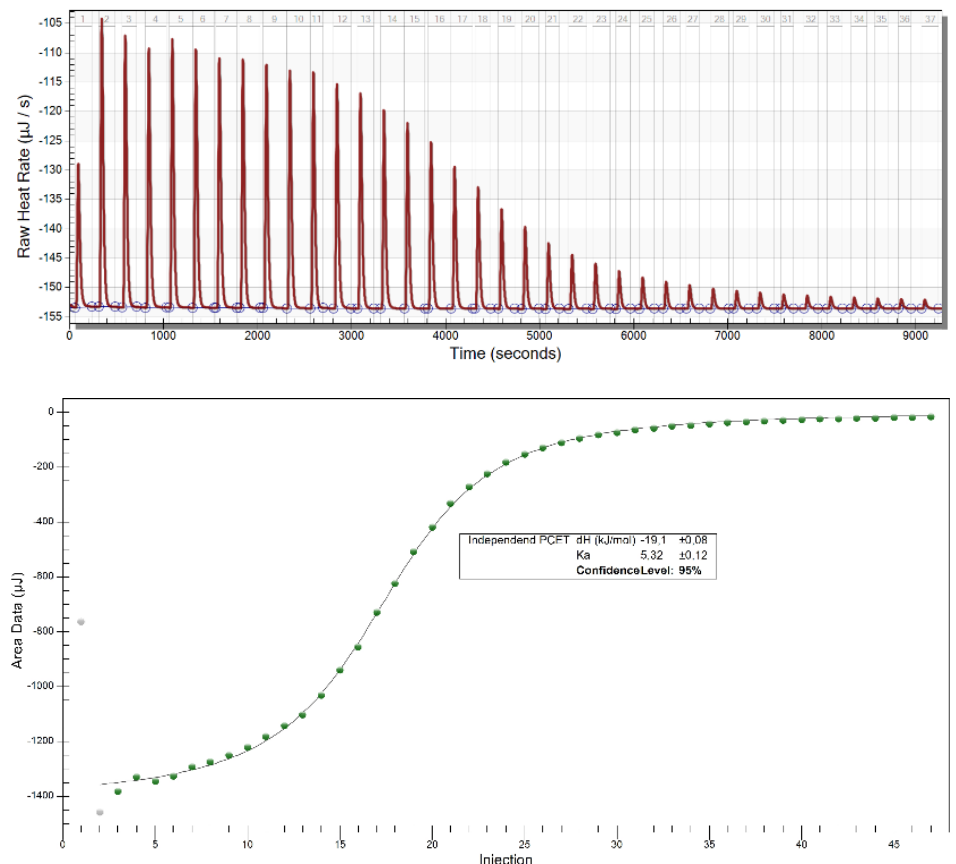

Figure 60: Thermogram (top) and integrated heat curve(bottom) of the first titration of 25 vs. pyridine at $298 \mathrm{~K}, \mathrm{THF}$. 

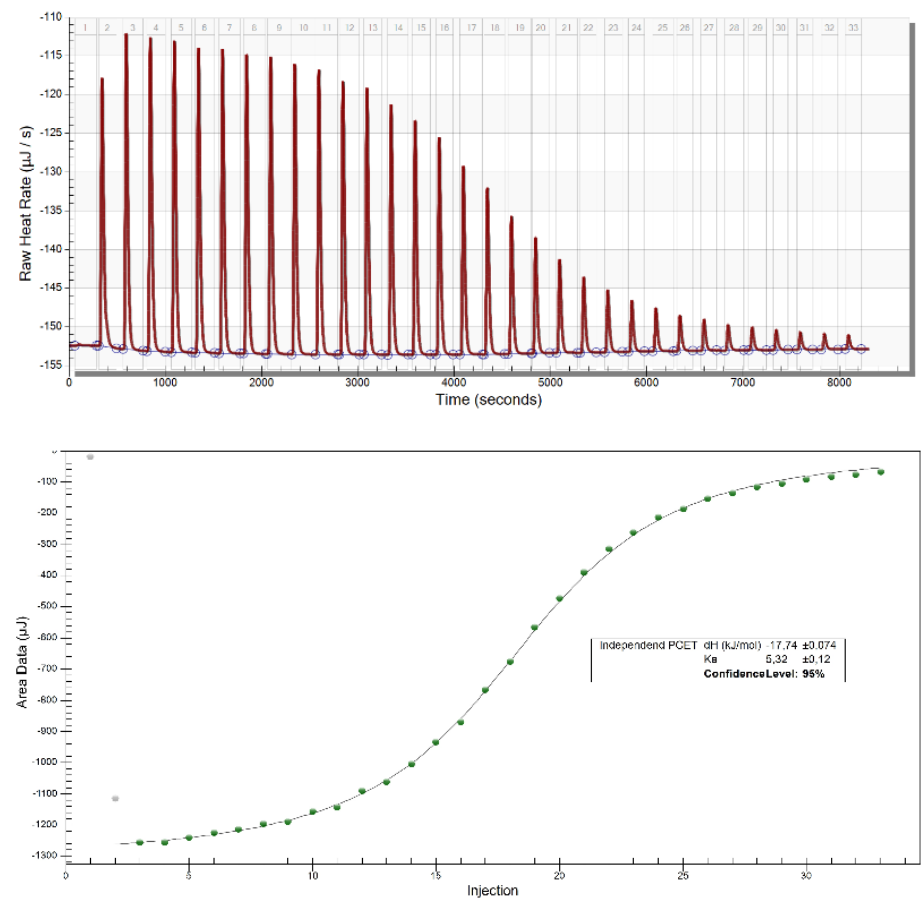

Figure 61: Thermogram (top) and integrated heat curve(bottom) of the second titration of 25 vs. pyridine at $298 \mathrm{~K}$, THF.

\section{Evaluation and error discussion}

Table 10: Results of the $\mathrm{p} K_{\mathrm{A}}$ titration of 25 with pyridine.

\begin{tabular}{ccc}
\hline Run & $K$ & $95 \%$ Confidence Error \\
\hline 1 & 5.32 & 0.12 \\
2 & 5.32 & 0.12 \\
Mean equilibrium constant & $5.32 \pm 0.12$ & \\
\hline
\end{tabular}

The titration curves have s-shaped character and are sufficient for the derivation of the equilibrium resulting in a value of $K=5.3 \pm 0.1$. Therefore, together with the Fuoss correction (see Section 3 of Chapter II) the acidity of 25 is calculated to $\mathrm{p} K_{\mathrm{A}}(25)=5.3$. 


\section{4 $\mathrm{p} K_{\mathrm{A}}$ value determination of $\mathrm{Mes}{ }^{*} \mathrm{OH}$}

\section{Measurement Conditions}

The $\mathrm{p} K_{\mathrm{A}}$ value of $\mathrm{Mes} * \mathrm{OH}$ was determined by titration with $\mathrm{P}_{2}$-Et as a base $\left(\mathrm{p} K_{\mathrm{A}}{ }^{\mathrm{THF}}=25.3\right){ }^{[222]}$ The experimental conditions where the following (Table 6).

Table 11: ITC measurement details

\begin{tabular}{ccc}
\hline & First Run & Second and third Run \\
\hline Concentration and volume of $\mathrm{Mes}{ }^{*} \mathrm{OH}[\mathrm{mM} / \mu \mathrm{L}]$ & $1.57 / 950$ & $12.6 / 950$ \\
Concentration and volume of P2-Et $\mathrm{mM} / \mu \mathrm{L}]$ & $12.5 / 250$ & $93.1 / 250$ \\
Injection Steps/Injected Volume $[\mu \mathrm{L}]$ & $25 @ 10$ & $125 @ 2$ \\
Waiting time between two additions $[\mathrm{s}]$ & 250 & 300 \\
Stirring rate $[\mathrm{rpm}]$ & 350 & 350 \\
Equilibration time before start of experiment[s] & 1200 & $827 / 1800$ \\
\hline
\end{tabular}

\section{Titration}

The following figures show the thermograms and integrated titration curves of Mes*OH vs. P2-Et in THF at $298 \mathrm{~K}$. Greyed out points are not considered in the fitting process. The first point(s) is generally neglected due to the dilution effect within the pre-experiment equilibration time. An Independent model was fitted to the obtained data. $10 \mu \mathrm{J}$ and $20 \mu \mathrm{J}$ per injection were subtracted to correct for the heat of dissolution for the diluted and concentrated titrations, respectively.
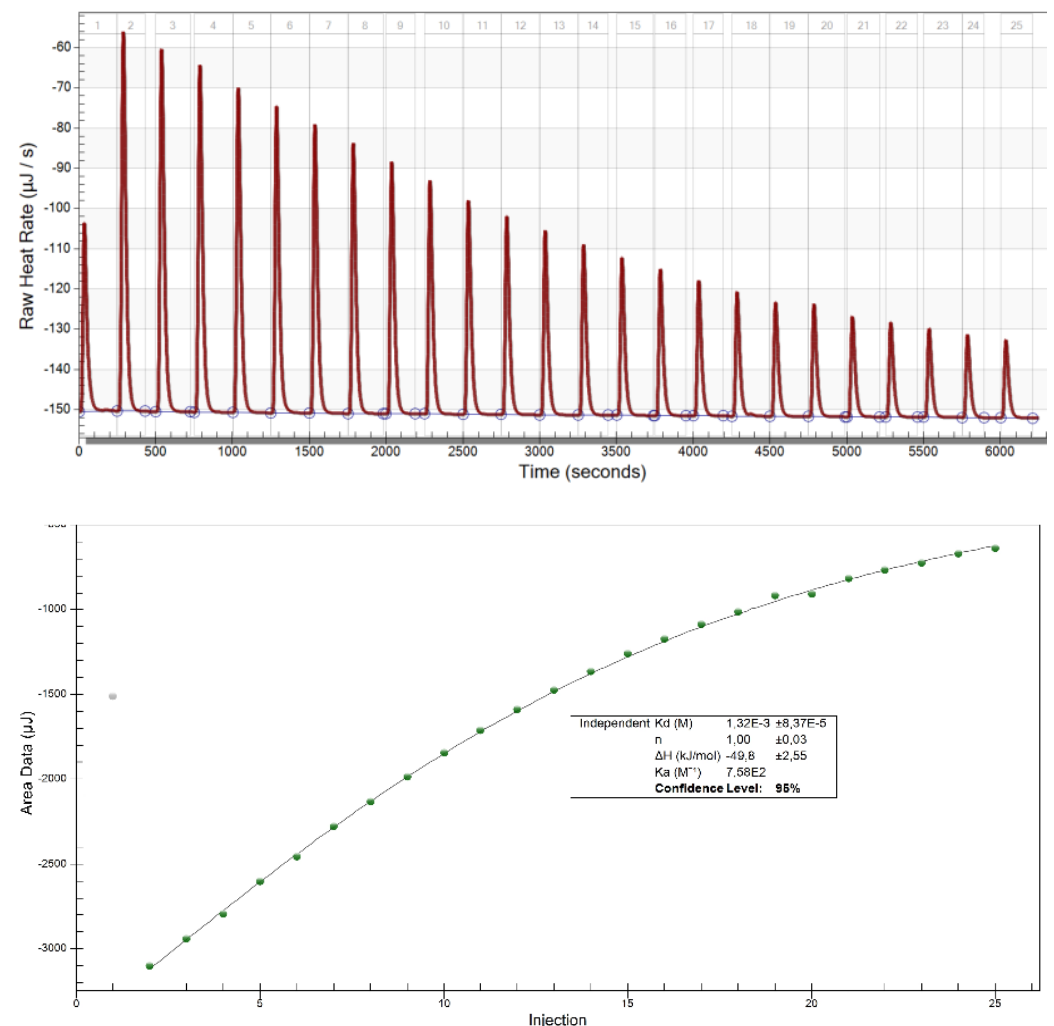

Figure 62: Thermogram (top) and integrated heat curve(bottom) of the first(diluted) titration of Mes*OH vs. P2-Et at 298 K, THF. 

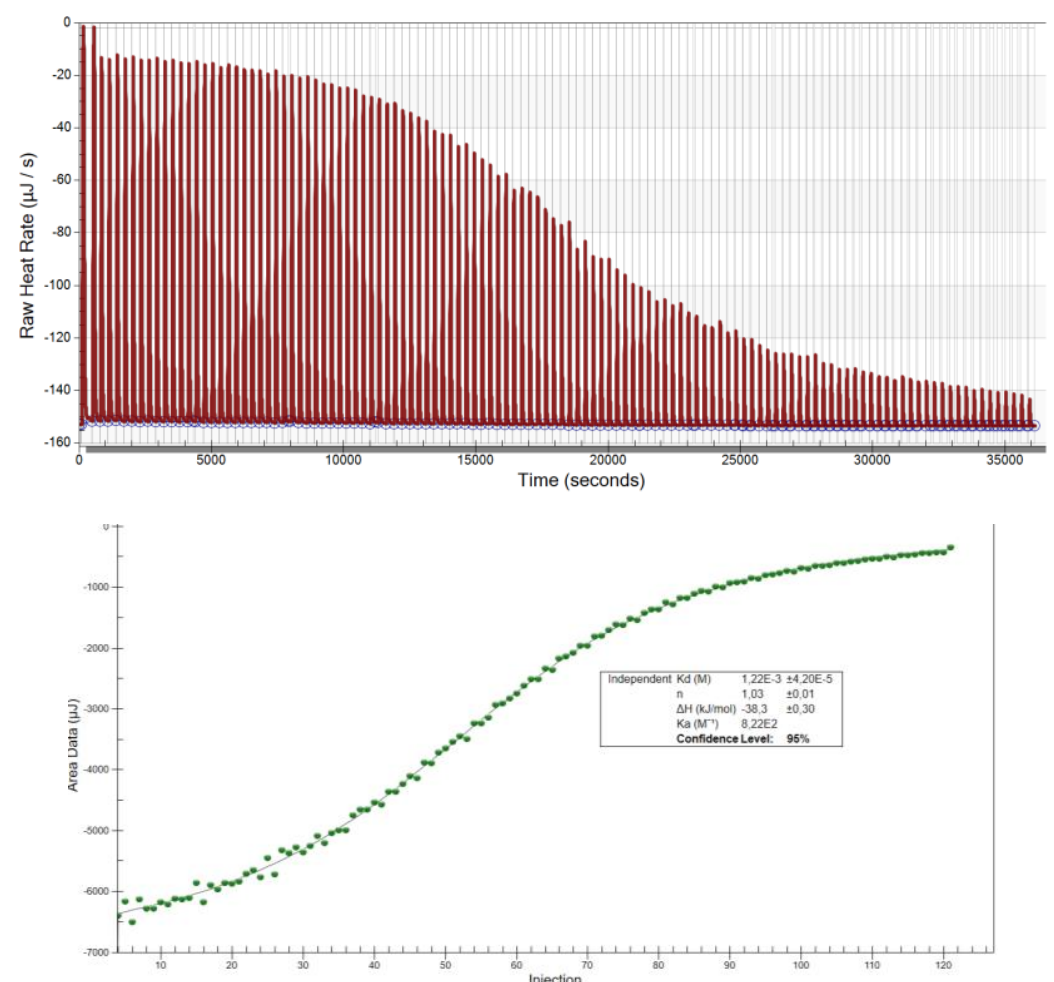

Figure 63: Thermogram (top) and integrated heat curve(bottom) of the second titration of Mes*OH vs. $\mathrm{P}_{2}$-Et at $298 \mathrm{~K}$, THF.
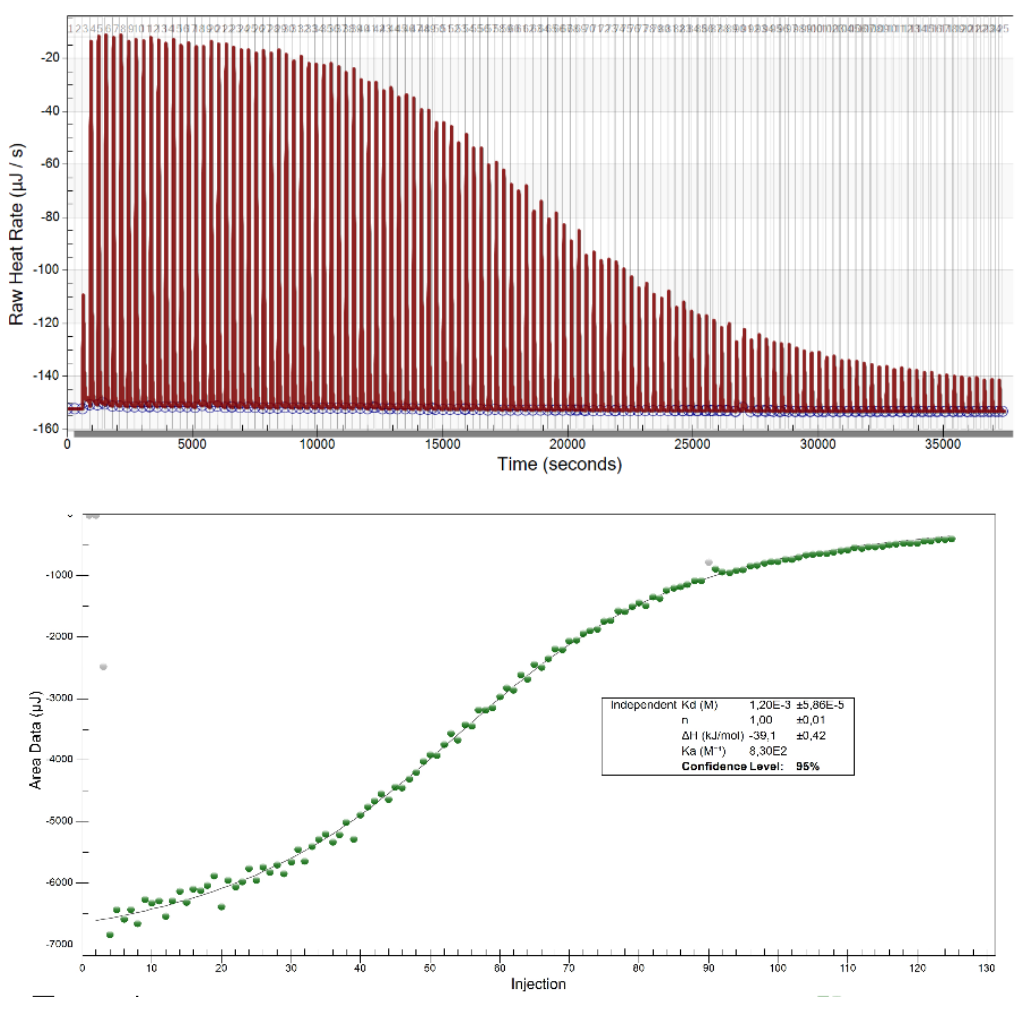

Figure 64: Thermogram (top) and integrated heat curve(bottom) of the third titration of Mes*OH vs. $\mathrm{P}_{2}$-Et at $298 \mathrm{~K}$, THF. 


\section{Evaluation and error discussion}

Due to the concentration dependence of the titration curve shape, an independent model for an association reaction had to be used in the fitting process. The results of the first titration are included in the final results but weighted only half, due to the lower amount of data points.

Table 12: Results table for the titration of Mes* $\mathrm{OH}$ with $\mathrm{P}_{2}$-Et base in THF at $298 \mathrm{~K}$.

\begin{tabular}{llcl}
\hline$K_{\mathrm{D}} / \mathrm{mol} \mathrm{L}^{-1}$ & Error at 95\% confidence & $\mathrm{n}$ value & Corrected Error \\
\hline $1.32 \cdot 10^{-3}$ & $\pm 0.08 \cdot 10^{-3}$ & 1.00 & $\pm 0.08 \cdot 10^{-3}$ \\
$1.22 \cdot 10^{-3}$ & $\pm 0.04 \cdot 10^{-3}$ & 1.03 & $\pm 0.04 \cdot 10^{-3}$ \\
$1.20 \cdot 10^{-3}$ & $\pm 0.06 \cdot 10^{-3}$ & 1.00 & $\pm 0.06 \cdot 10^{-3}$ \\
\hline
\end{tabular}

The weighted average enthalpy of the reaction sums up to $K_{D}=1.24 \pm 0.06 \cdot 10^{-3} \mathrm{M}^{-1}$, which gives a weighted averaged association constant as $K_{A}=806 \pm 39 \mathrm{M}$. Since this fit already includes the ion-pairing interaction, the $\mathrm{p} K_{\mathrm{A}}$ value is directly obtained by $p K_{A}($ Mes $* H)=25.3-\log (806 \pm 39)=22.4 \pm 0$.

\subsection{P-H BDE value determination of 17}

\section{Measurement Conditions}

The BDE of 17 was determined by isothermal titration calorimetry by titration with Mes*O $(\mathrm{BDE}(\mathrm{O}-\mathrm{H}) \mathrm{Mes} * \mathrm{OH}: \mathrm{a})$ $\left.\mathrm{C}_{6} \mathrm{H}_{6}=81.6 \mathrm{kcalmol}^{-1} ; \mathrm{THF}=84.5 \mathrm{kcalmol}^{-1}\right)^{[53,184]}$ was used as titrant. Titrations were carried out at $10{ }^{\circ} \mathrm{C}$ to suppress the decay of 17 and corrected vs. the addition of Mes*O to the solvent in the sample cell (18 $\mu \mathrm{J}$. addition). Further experimental conditions are summarized in Table 13 and Table 14.

Table 13: Experimental details of the BDE determination of 17 in benzene.

\begin{tabular}{cc}
\hline Concentration and volume of $17 / \mathrm{mM}$ and $\mu \mathrm{L}$ & $1.02 / 950$ \\
Concentration and volume of Mes*O / $\mathrm{mM}$ and $\mu \mathrm{L}$ & $9.18 \mathrm{mM} / 250 \mu \mathrm{L}$ \\
Injection Steps/Injected Volume / $\mu \mathrm{L}$ & $33 @ 7.5$ \\
Waiting time between two additions / $\mathrm{s}$ & $350-650$ \\
Stirring rate $/ \mathrm{rpm}$ & 350 \\
Equilibration time before experiment start / s & 1514 \\
\hline
\end{tabular}

*The peaks for the BDE determination had a waiting time of $350 \mathrm{~s}$.

Table 14: Experimental details of the BDE determination of 17 in THF.

\begin{tabular}{cc}
\hline Concentration and volume of $17 / \mathrm{mM}$ and $\mu \mathrm{L}$ & $1.4 / 950$ \\
Concentration and volume of Mes*O / $\mathrm{mM}$ and $\mu \mathrm{L}$ & $11.7 \mathrm{mM} / 250 \mu \mathrm{L}$ \\
Injection Steps/Injected Volume / $\mu \mathrm{L}$ & $49 @ 5$ \\
Waiting time between two additions / s & 300 \\
Stirring rate /rpm & 350 \\
Equilibration time before experiment start / s & 1671 \\
\hline
\end{tabular}




\section{Titration}

The first injection(s) of each titration were generally neglected due to diffusion of reactant solution into the cell during the equilibration of the instrument. Around the addition of one equivalent of $\mathrm{Mes}^{*} \mathrm{O}$, the peaks show increased tailing, which is tentatively attributed to follow-up reactivity of 17 . The P-H BDE was therefore determined at an early titration stage from the average of the heat of injections 3-6 for benzene and 2-5 for THF, respectively, while reliable data BDFE determination was not available. A representative titration is shown in Figure 65 for benzene and in Figure 66 for THF, respectively. The integrated results are summarized in Table 15 and Table 16
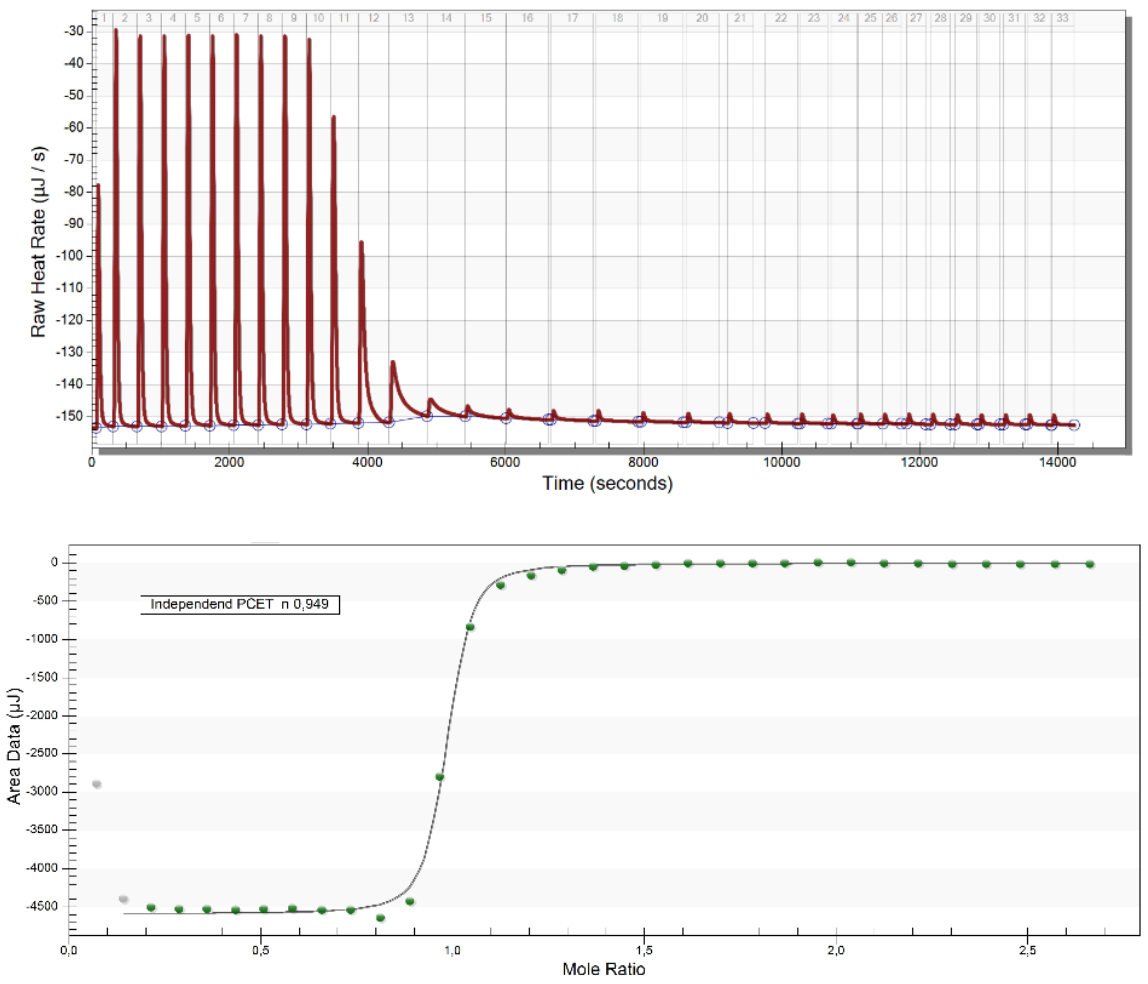

Figure 65: Thermogram of the titration of 17 with Mes* $\mathrm{O}$ at $283 \mathrm{~K}$ in benzene (top) and integrated titration curve of the titration (bottom).

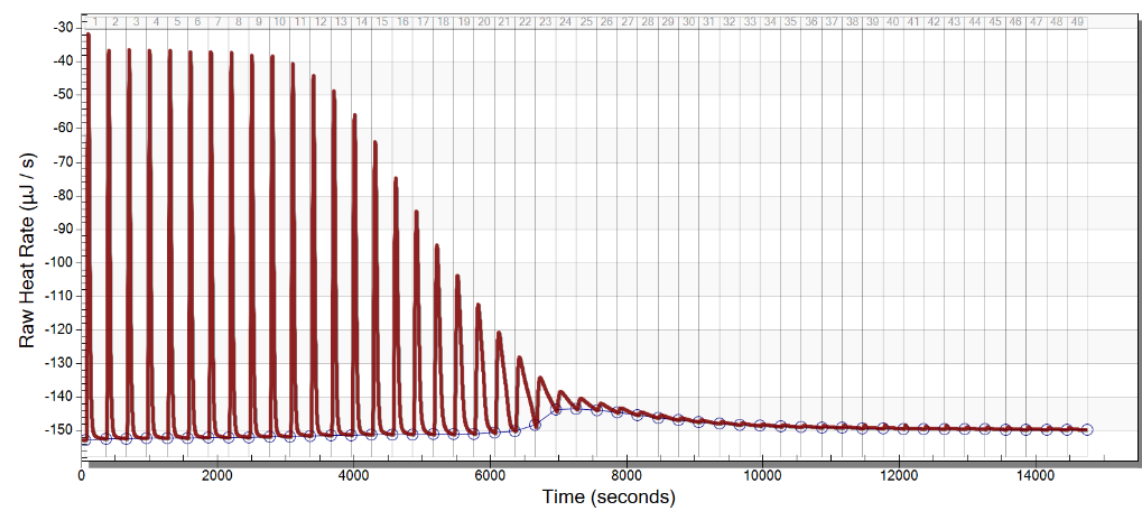




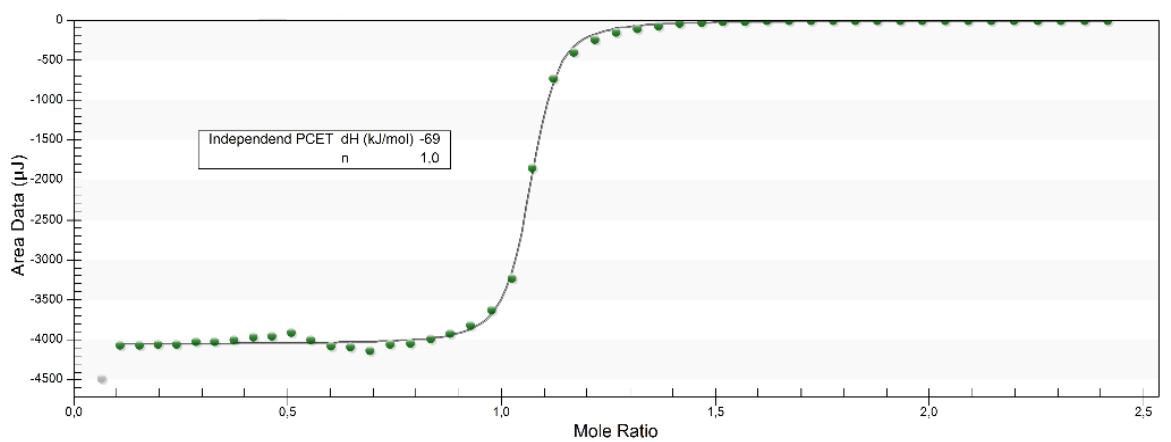

Figure 66: Thermogram of the titration of 17 with Mes* $\mathrm{O}$ at $283 \mathrm{~K}$ in THF (top) and integrated titration curve of the titration (bottom).

\section{Results and Error Discussion}

Table 15: ITC results of the BDE determination of 17 in benzene.

\begin{tabular}{cccc}
\hline Injection & Heat of injection of the $1^{\text {st }}$ run $/ \mu \mathrm{J}$ & Heat of injection of the $2^{\text {nd }}$ run $/ \mu J$ & Added moles per injection $/ \mathrm{nmol}$ \\
\hline 3 & -4682 & -4678 & 68.2 \\
4 & -4708 & -4678 & 68.2 \\
5 & -4703 & -4709 & 68.2 \\
6 & -4716 & -4711 & 68.2 \\
Mean & $-4702 \pm 20$ & $-4694 \pm 17$ & \\
Weighted Mean & $-4697 \pm 13$ & & \\
& & \\
Reaction Enthalpy $/ \mathrm{KJmol}^{-1}$ & $65.1 \pm 0.9^{*}$
\end{tabular}

*A cumulative error of $5 \%$ for stock solution preparation and weighting errors was estimated from the fitted $n$-value $(0.95)$ and multiplied with the weighted mean error.

Table 16: ITC results of the BDE determination of 17 in THF.

\begin{tabular}{ccc}
\hline Injection & Heat of injection of the $1^{\text {st }}$ run $/ \mu$ & Added moles per injection $/ \mathrm{nmol}$ \\
\hline 2 & -4069 & 56.9 \\
3 & -4066 & 56.9 \\
4 & -4050 & 56.9 \\
5 & -4049 & 56.9 \\
Mean & $-4059 \pm 10$ & \\
\hline Reaction Enthalpy $/ \mathrm{kJ} \mathrm{mol}^{-1}$ & & \\
P-H BDE(THF) kcal mol & & \\
& & -71 \\
\hline
\end{tabular}




\subsection{Mean BDE determination of $\left[(\mathrm{PNP}) \operatorname{Re}(\mathrm{Cl})\left(\mathrm{NH}_{2}\right)\right] 20$}

\section{Measurement Conditions}

The mean N-H BDE of the 20/22 couple is determined by titration with Mes* $\left(\mathrm{BDE}_{\mathrm{O}-\mathrm{H}}(\mathrm{Mes} * \mathrm{OH})=354 \mathrm{~kJ} \mathrm{~mol}^{-1}\right)$ with the experimental conditions shown below.

Table 17: Measurement details of the mean N-H BDE determination of 20.

\begin{tabular}{c|cc}
\hline & First Run & Second/Third Run \\
Concentration and volume of $20[\mathrm{mM} / \mu \mathrm{L}]$ & $0.341 / 950$ & $0.341 / 950$ \\
Concentration and volume of Mes*O $[\mathrm{mM} / \mu \mathrm{L}]$ & $5.28 / 250$ & $5.05 / 180$ \\
Injection Steps/Injected Volume $[\mu \mathrm{L}]$ & $33 @ 7.5$ & $24 @ 7.5$ \\
Waiting time between two additions $[\mathrm{s}]$ & 250 & 250 \\
Stirring rate $[\mathrm{rpm}]$ & 350 & 350 \\
\hline Equilibration time before start of experiment $[\mathrm{s}]$ & 1885 & 1453 and 1525 \\
\hline
\end{tabular}

\section{Titration}

The following figures show the thermograms and integrated titration curves of 20 with Mes*-O in THF. Greyed out points are not considered in the fitting process. The first point is generally neglected due to the dilution effect within the pre-experiment equilibration time. The mean heat value was determined for the titration steps before the equivalence point. The stoichiometry was determined by an Independent PCET model. (see C).
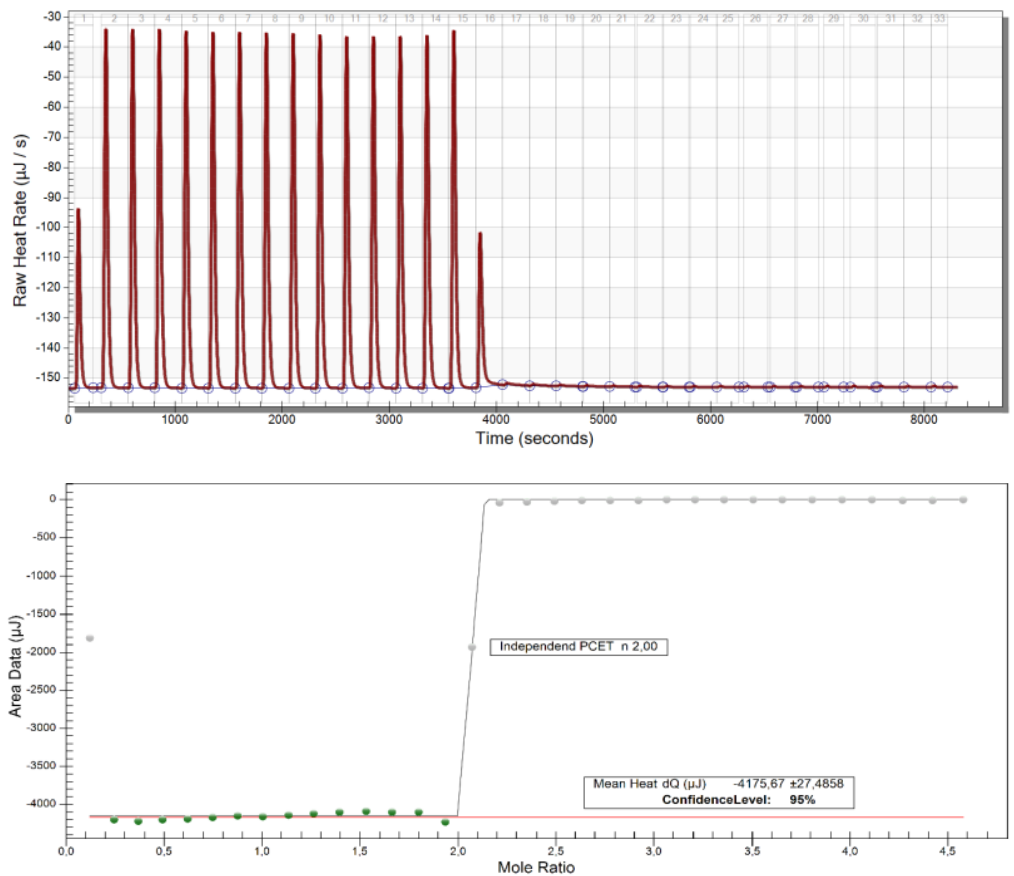

Figure 67: Thermogram of the first titration of 20 vs. Mes*O (4 eq.) 33@7.5 $\mu \mathrm{L}, 250$ s waiting time in THF@298 K (top) and integrated heat curve of the first titration of 20 vs. Mes*O (4 eq.) 33@7.5 $\mu \mathrm{L}, 250$ swaiting time in THF@298 K (bottom). 

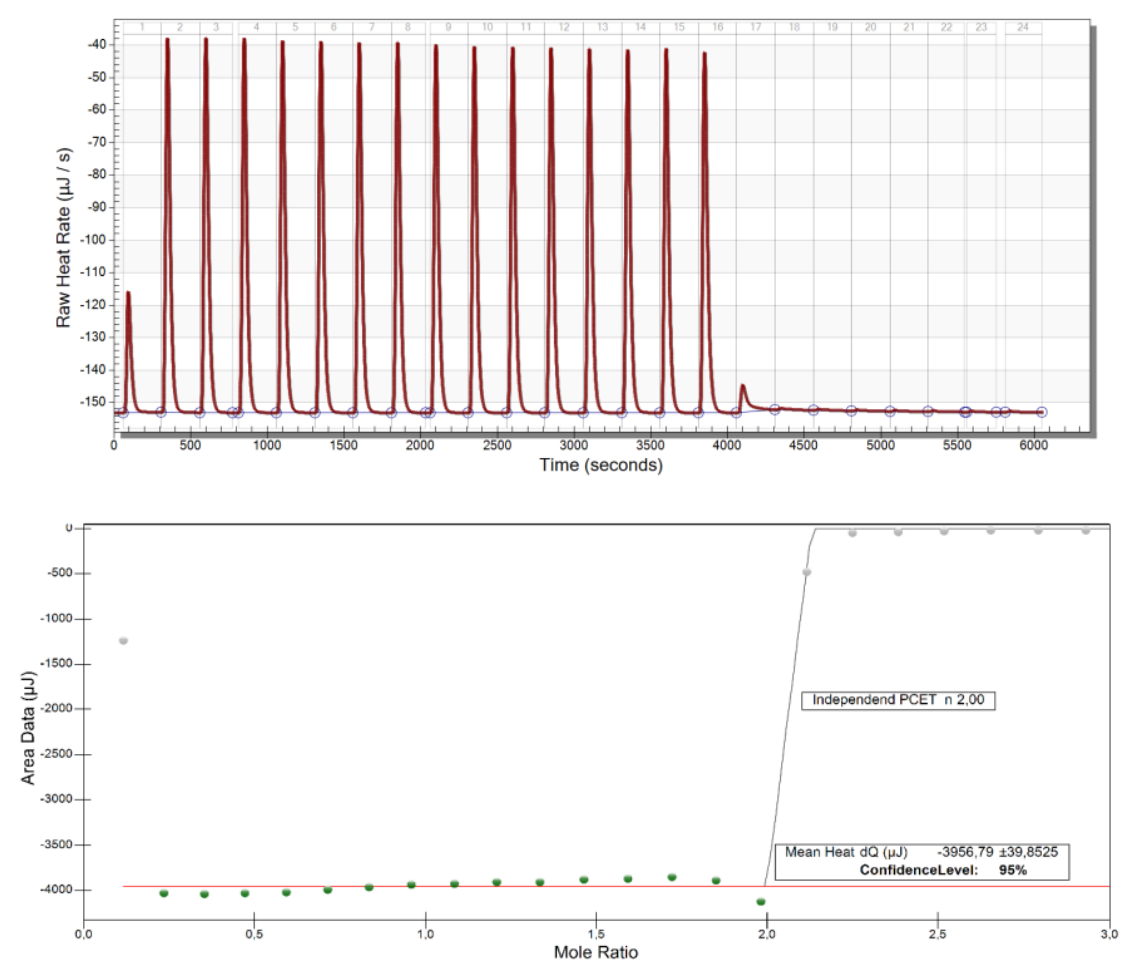

Figure 68: Thermogram of the second titration of 20 vs. Mes*O (4 eq.) 33@7.5 $\mu \mathrm{L}, 250$ s waiting time in THF@298 K (top) and integrated heat curve of the second titration of 20 vs. Mes*O (4 eq.) 33@7.5 $\mu$ L, 250 s waiting time in THF@298 K. (bottom).
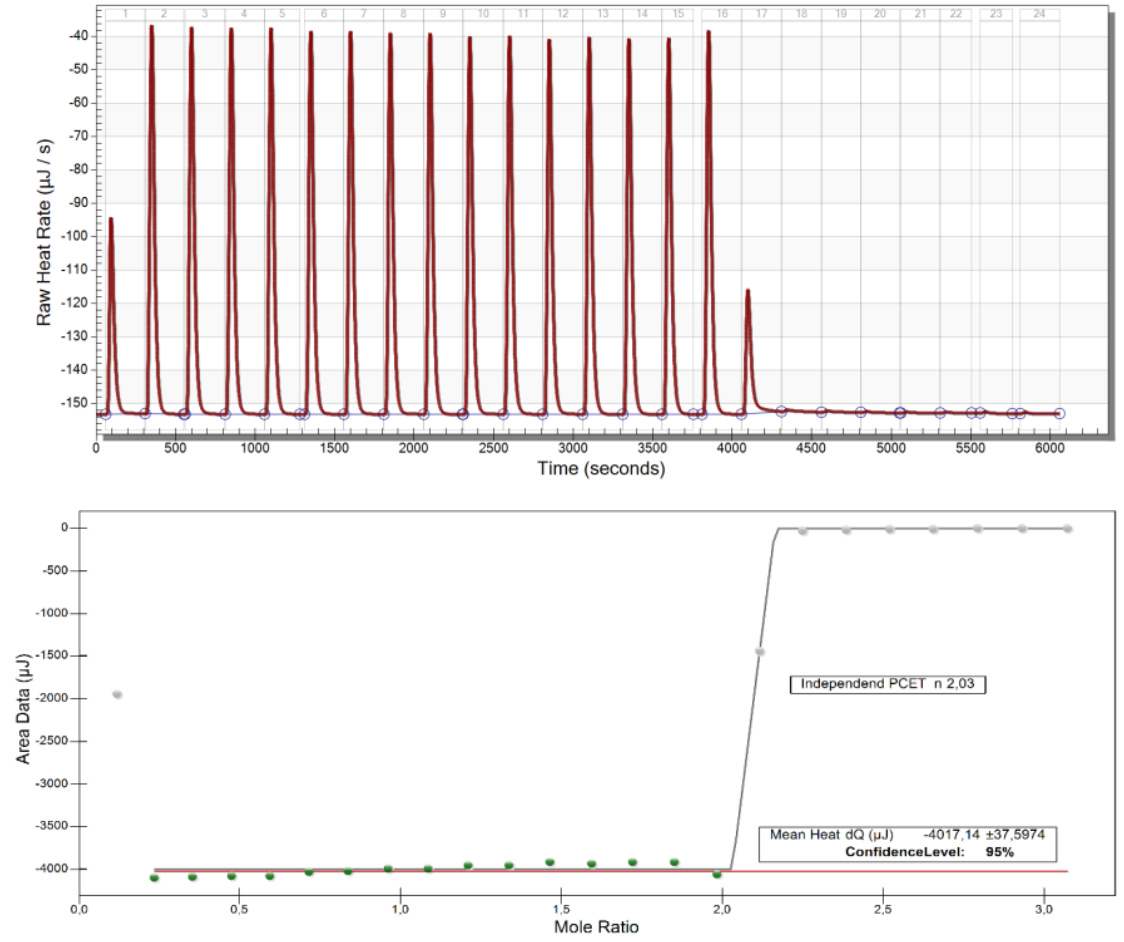

Figure 69: Thermogram of the third titration of 20 vs. Mes*O (4 eq.) 33@7.5 $\mu \mathrm{L}, 250$ s waiting time in THF@298 K (top) and integrated heat curve of the third titration of 20 vs. Mes*O (4 eq.) 33@7.5 $\mu \mathrm{L}, 250$ s waiting time in THF@298 K (bottom). 
Results and Error Discussion

\begin{tabular}{ccc}
\hline & Results & \\
Mean Heat $[\mu]]$ & Consumed moles per injection $[\mathrm{nmol}]$ & Enthalpy $[\mathrm{kJ} / \mathrm{mol}]$ \\
\hline$-4176 \pm 27$ & 19.62 & $-213 \pm 1$ \\
$-3957 \pm 40$ & 18.76 & $-211 \pm 2$ \\
$-4017 \pm 39^{*}$ & 18.76 & $-214 \pm 2$ \\
& Weighted mean enthalpy $=-213 \pm 2 \frac{\mathrm{kJ}}{\mathrm{mol}}$ & \\
& & \\
\hline
\end{tabular}

*The error for the third measurement is raised to scale for the deviation from the ideal $n$ value of $n=2.00$.

The enthalpy for the double PCET reaction is $-50.9 \mathrm{kcal} \mathrm{mol}^{-1}$. The mean Re-N(H)-H BDE is calculated to:

$$
\operatorname{BDE}_{[\mathrm{Re}] \mathrm{N}(\mathrm{H})-\mathrm{H}}^{\mathrm{THF}}=\mathrm{BDE}_{\mathrm{Mes} * 0-\mathrm{H}}^{\mathrm{THF}}-\frac{\Delta H}{2}
$$

$$
\mathrm{BDE}_{[\mathrm{Re}] \mathrm{N}(\mathrm{H})-\mathrm{H}}^{\mathrm{THF}}=\left(84.6-\frac{50.9}{2}\right) \frac{\mathrm{kcal}}{\mathrm{mol}}=59.2 \pm 0.2 \frac{\mathrm{kcal}}{\mathrm{mol}}
$$




\section{Computational Details}

\subsection{General Remarks}

All computations were performed on the Goethe CRC computation cluster in Frankfurt am Main. Optimizations of molecular geometries and analyses of the Hessian matrices were carried out with the Gaussian program ${ }^{[239]}$ under $^{2}$ gas-phase conditions utilizing the dispersion-corrected ${ }^{[240]}$ B3LYP(V)-D3 functional ${ }^{[241]}$ (iridium complexes and related structures) and the PBEO-D3 functional ${ }^{[242]}$ (rhenium complexes and related structures) the def2TZVPP118] orbital basis set including the quasi-relativistic 60-electron pseudopotentials (ECP60MWB) for iridium and rhenium $^{[243]}$ Thermal contributions to enthalpies were obtained from these results for $298.15 \mathrm{~K}$ and 1 atm. The effect of solvents was estimated via an implicit polarized continuum solvent model (SMD, ${ }^{[244]}$ solvent: tetrahydrofuran, dichloromethane) by computation of the single-point energy difference between gas-phase and solvent-based total energies. Improved relative energies were computed in single-point calculations on the full molecular systems with the Orca program ${ }^{[245,246]}$ using the domain-based local-pair natural orbital coupled-cluster approximation $^{[95]}$ employing tight accuracy settings (TightPNO, without full LMP2 guess also in the closed-shell calculations to ensure identical results for the RHF and UHF limits in Orca DLPNO/TightPNO computations) with single and double excitations and perturbative triples. For iridium the correlation-consistent triple- and quadruplezeta valence basis sets ${ }^{[119,120,247]}$ which include the relativistic pseudopotential (ECP6OMDF) on Ir ${ }^{[145]}$ were used in conjunction with the corresponding correlation fitting basis sets ${ }^{[248]}$ and extrapolated to the complete basis set (CBS) limit, DLPNO-CCSD $(T) / C B S(T, Q)$, as implemented in Orca, i.e. Eref(CBS) = Eref(Q) + (Eref(Q) - Eref(T) /exp $(\alpha$ $(\sqrt{ } 4-\sqrt{ } 3))-1) ; E \operatorname{corr}(C B S)=3 \beta E \operatorname{Ecorr}(T)-4 \beta E \operatorname{corr}(Q) 3 \beta-4 \beta$, where $\alpha=5.46$ and $\beta=3.05$. For the rhenium computations the correlation-consistent triple- and quadruple-zeta valence basis sets ${ }^{[119,120,247]}$ which include the relativistic pseudopotential (ECP60MDF) on $\mathrm{Re}^{[145]}$ were used in conjunction with the corresponding correlation fitting basis sets ${ }^{[248]}$ and extrapolated to the complete basis set (CBS) limit. Additionally the Ahlrich basis sets def2$(T, Q) Z V P P$ were used with the normal T0 triples as well as with the improved T1 triples ${ }^{[94]}$ and extrapolated to the CBS limit DLPNO-CCSD(TO/T1)/CBS(T,Q), as implemented in Orca, i.e. $\operatorname{Eref}(C B S)=\operatorname{Eref}(Q)+(\operatorname{Eref}(Q)-\operatorname{Eref}(T) / \exp (\alpha$ $(\sqrt{ } 4-\sqrt{ } 3))-1) ; \operatorname{Ecorr}(C B S)=3 \beta \operatorname{Ecorr}(T)-4 \beta E \operatorname{corr}(Q) 3 \beta-4 \beta$, where $\alpha=5.46$ and $\beta=3.05$ for cc-pVnZ and $\alpha=$ 7.88 and $\beta=2.97$ for the def2 basis set., respectively. Further, a composite ONIOM-like ${ }^{[97,98]}$ approach was employed to combine the DFT results of the full molecular systems with the highly accurate explicitly correlated F12B ansatz ${ }^{[249]}$ for truncated model systems, in which all ${ }^{t} \mathrm{Bu} /{ }^{\mathrm{P}} \mathrm{Pr}$ groups in each molecular system were either replaced by methyl groups (Me-truncated) or by hydrogen atoms (H-truncated). The CCSD(T*)-F12 ${ }^{[250]}$ variant was chosen as implemented in Molpro, ${ }^{[251]}$ where the perturbative triples contributions, denoted $\left(\mathrm{T}^{*}\right)$, are improved towards the complete basis set limit via F12-scaling ${ }^{[37]}$ according to the scale factor $E_{\text {corr }}(\mathrm{MP} 2$ F12)/Ecorr(MP2). The one particle space was described with the valence double- and triple-zeta orbital basis sets (OBS) cc-pV\{D,T\}Z-F12 for the non-metal atoms and aug-cc-pV\{D,T\}Z-PP[145] for iridium and rhenium, in conjunction with the corresponding triple-zeta auxiliary fit basis sets ${ }^{[252-255]}$ (also with the double-zeta OBSs), i.e., the JKfit sets for the Fock and exchange integrals, the MP2fit sets for density fitting and the OptRI/JKfit sets for construction of the complementary auxiliary basis set (OptRI for non-metal atoms, JKfit for iridium). Me- or H-truncated model

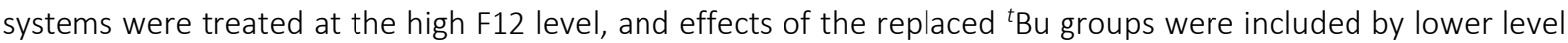


DFT calculations, ONIOM(F12b:DFT). To ensure a closest possible structural match to the fully optimized geometry of the real system, in the ONIOM approach the truncated model systems were constructed in constrained geometries at the DFT level. Thus, only the $\mathrm{C}-\mathrm{H}$ or $\mathrm{P}-\mathrm{H}$ bond lengths of the newly added hydrogen atoms (which replace the corresponding methyl or ${ }^{t} \mathrm{Bu} /{ }^{\prime} \operatorname{Pr}$ fragments of the ${ }^{t} \mathrm{Bu} /{ }^{\prime} \operatorname{Pr}$ groups) were allowed to relax, i.e., all angles and dihedrals are kept fixed and all remaining coordinates are unaltered.

Spin-orbit eigenstates were calculated at the DFT ground state geometries within the ORCA program ${ }^{[245,246]}$ from state-averaged CASSCF/NEVPT2 ${ }^{[110-112]}$ calculations followed by a quasi-degenerate perturbation theory treatment via a full spin-orbit mean field (SOMF) operator. ${ }^{[155]}$ CASSCF wavefunctions were optimized employing the ZORA approximation ${ }^{[256]}$ along with the ZORA-def2TZVP basis sets, ${ }^{[143]}$ which include the segmented all-electron relativistically contracted SARC-ZORA-TZVPP basis set for iridium and rhenium. The RIJK algorithm for fitting of the Coulomb and exchange integrals was used in conjunction with the def2/JK auxiliary basis sets. ${ }^{[257]}$ The active space of 4 comprises the five Ir-based $5 d$ orbitals, the $p-\sigma$ and the two $p-\pi$ orbitals of oxygen, and two occupied pincerligand based orbitals, giving rise to a $\operatorname{CAS}(16,10)$ expansion; the active space of 2 includes the bonding $\mathrm{p}-\sigma(\mathrm{O})-\mathrm{H}$ and additionally the antibonding $\mathrm{p}-\sigma^{*}(\mathrm{O})-\mathrm{H}$ orbitals, thus leading to a $\mathrm{CAS}(17,11)$ expansion. For the oxo-species, the CASSCF orbitals were optimized by the average of 5 quintet, 45 triplet and 50 singlet roots arising from the formal $d^{6}$ configuration of the iridium(III) centre. The formal $d^{7}$ iridium(II) hydroxo species gives rise to an average of 10 quartet and 40 doublet roots accordingly. Magnetic data where obtained by setting the ORCA keywords "MagneticField" and "dosusceptibility" to true. For the rhenium complexes two different actives spaces were probed. For the small active space only the $5 \mathrm{~d}$ orbitals of the complexes were analysed leading to a $\operatorname{CAS}(3 \times 5)$ expansion for the amido complex and to a $\mathrm{CAS}(4 \times 5)$ expansion for the amine complex. For both CAS expansions, the CASSCF orbitals for the amine species were optimized by the average of 5 quintet, 45 triplet and 50 singlet roots arising from the formal $d^{4}$ configuration of the rhenium(III) centre. The formal $d^{3}$ rhenium(IV) amido species gives rise to an average of 10 quartet and 40 doublet roots respectively. The big active space in the rhenium amido complex comprises the five Re-based $5 \mathrm{~d}$ orbitals, three p-orbitals of the axial chlorine, two occupied pincerligand/equatorial chlorines orbitals, and a filled Re-N $\pi$-based orbital, giving rise to a CAS $(15,11)$ expansion; the amine-species is compromised of the same orbital set, but without filled Re-N $\pi$-based orbital, thus leading to a CAS $(14,10)$ expansion. The final energies are obtained from NEVPT2 calculations, and the energies that enter the infinite-order SOC QDPT treatment via the SOMF operator are thus corrected to second order. 


\subsection{A Terminal Iridium Oxo with a Triplet Ground State}

\subsubsection{Molecular Geometries}

Figure 70 shows selected parameters of the optimized geometries for the full molecular systems. The $C_{s}$ symmetric iridium-oxo 4 exhibits a square-planar environment in the open-shell ${ }^{3} \mathrm{~A}^{\prime \prime}$ ground state and a slightly distorted structure in the less favourable closed-shell ${ }^{1} \mathrm{~A}^{\prime}$ state. The iridium-hydroxo 2 features a doublet electronic configuration in a square-planar environment. Compared to the triplet oxo species, the $\mathrm{Ir}-\mathrm{O}$ bond length increases by $0.18 \AA$ in the hydroxo complex and the $\mathrm{Ir}-\mathrm{N}$ contact shortens by $0.08 \AA$; the $\mathrm{O}-\mathrm{H}$ bond lies within the squareplane of the complex. Both, the 2,4,6-tri-tert-butyl-phenol (Mes* $\mathrm{OH})$ and the corresponding phenoxy-radical (Mes*O) also exhibit Cs symmetry, concomitant with a difference in C-O bond lengths of $0.12 \AA$. The xyz. data of all structures can be found in the appendix Section D.

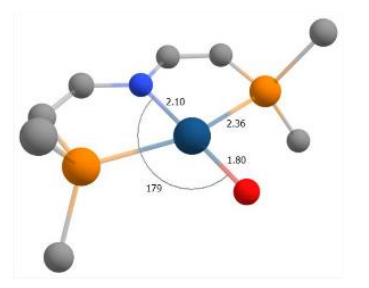

$[(P N P) \operatorname{IrO}]^{3} \mathrm{~A} "(\mathrm{Cs})$

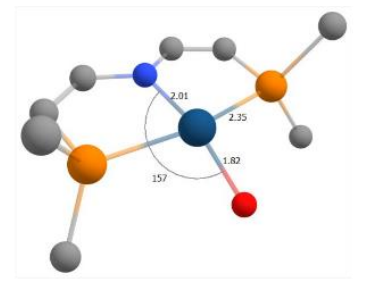

$[(P N P) I r O]^{1} A^{\prime}(C s)$

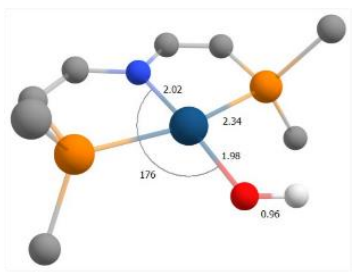

$[(\mathrm{PNP}) \mathrm{IrOH}]^{2} \mathrm{~A}(\mathrm{C} 1)$
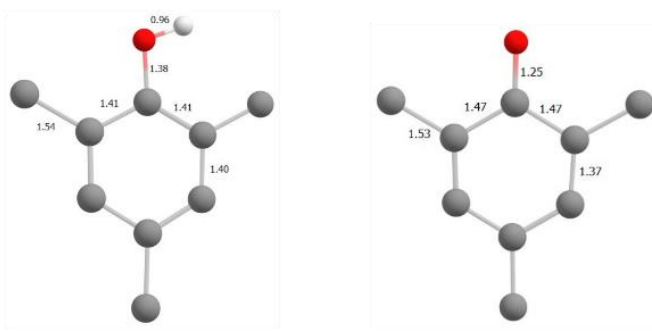

$\left[\mathrm{Mes}^{*} \mathrm{OH}\right]{ }^{1} \mathrm{~A}^{\prime}(\mathrm{Cs})$

$\left[\right.$ Mes*O ${ }^{2} \mathrm{~A}^{\prime \prime}(\mathrm{Cs})$

Figure 70: DFT-optimized molecular geometries (B3LYP(V)-D3/def2TZVP) for the triplet ground-state and the first singlet isomer of oxo species (PNP)IrO, and for the doublet ground-state of hydroxo-species (PNP)IrOH (top), as well as for the 2,4,6-tri-tert-butylphenol/phenoxy (Mes*OH/Mes*O) couple (bottom). All tBu groups and carbon-bonded hydrogen atoms are omitted for clarity. 


\subsubsection{Spin State and Reaction Energetics}

The relative spin-state and reaction energies were computed with DFT and coupled cluster methods. The results are collected in Table 24. The triplet-singlet gap $\Delta E(T / S)$ of 4 complex is computed following reaction 4.1.

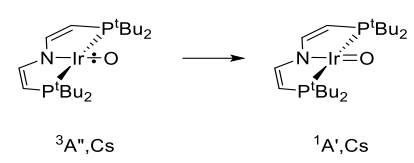

The half reaction energetics of the $4 / 2$ couple was computed for reaction 4.2 .

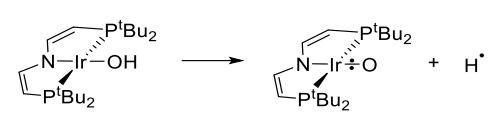

The half reaction energetics of the Mes* OH/Mes*O couple was computed for reaction 4.3.<smiles>CC(C)(C)c1cc(Br)cc(C(C)(C)C)c1O</smiles>

The half reaction energetics of the PCET reaction was computed for reaction 4.4 .

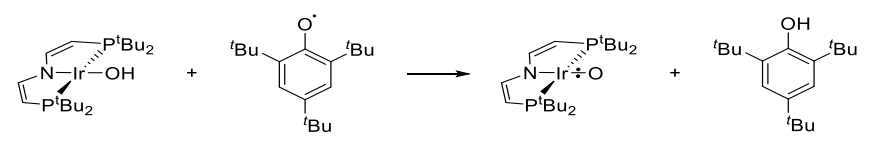

All computational approaches predict a triplet ground state for 4 . The hybrid functional places the singlet electromer at $\Delta E(T / S)=74 \mathrm{~kJ} \mathrm{~mol}^{-1}$ above the triplet, while coupled cluster expansions converge at a value of 40$41 \mathrm{~kJ} \mathrm{~mol}^{-1}$. Reaction (4.2) is strongly endothermic with to $\Delta_{\mathrm{r}} \mathrm{H}_{\mathrm{solv}}{ }^{\mathrm{SOC}}(4.2)=325 \mathrm{~kJ} \mathrm{~mol}^{-1}$ at the DFT level and $352 \mathrm{~kJ} \mathrm{~mol}^{-1}$ at the DLPNO-CCSD(T) level, while at the explicitly correlated coupled cluster ONIOM(F12:DFT) level it converges to $342 \mathrm{~kJ} \mathrm{~mol}^{-1}$. The enthalpy for reaction (3) amounts to $-311 \mathrm{~kJ} \mathrm{~mol}^{-1}$ at the DFT level and $-338 \mathrm{~kJ} \mathrm{~mol}^{-1}$ at the DLPNO-CCSD(T) level, and it reaches -331 to $-334 \mathrm{~kJ} \mathrm{~mol}^{-1}$ at the ONIOM(F12:DFT) approach, thus introducing a slightly larger uncertainty than the (PNP)IrO-H bond formation reaction (2). In summary, the PCET reaction (4) is computed endothermic with a reaction enthalpy of $14 \mathrm{~kJ} \mathrm{~mol}^{-1}$ at the DFT and at the DLPNO$\operatorname{CCSD}(\mathrm{T})$ levels of theory, and between 12 and $9 \mathrm{~kJ}$ mol ONIOM(F12b:DFT) approach. 


\subsubsection{Total Energies}

Table 18: Total energies $E_{\text {tot }}$ in Hartree for the full ( $\left.{ }^{(} \mathrm{Bu}\right)$ complexes 4 and 2 as well as the Mes* OH/Mes*O couple, and the corresponding Me- and $\mathrm{H}$ - truncated model systems, computed at the B3Lyp(V)-D3/def2TZVP level of theory.

\begin{tabular}{|c|c|c|c|c|c|c|}
\hline \multirow{3}{*}{ Species } & \multirow{3}{*}{ State } & \multicolumn{5}{|c|}{ B3LYP(V)-D3/def2TZVP } \\
\hline & & \multicolumn{3}{|c|}{${ }^{t} \mathrm{Bu}$} & \multirow{2}{*}{$\frac{\mathrm{Me}}{E_{\text {tot }}}$} & \multirow{2}{*}{$\frac{\mathrm{H}}{E_{\text {tot }}}$} \\
\hline & & Etot & $\Delta E_{\text {tot }}$ & $\Delta H_{\text {solv }}$ & & \\
\hline $\mathrm{H}$ & ${ }^{2} S$ & -0.498763 & 0.000416 & 0.002360 & & \\
\hline 4 & ${ }^{3} \mathrm{~A}^{\prime \prime}$ & -1703.034516 & -0.020352 & 0.602065 & -1231.404966 & -1074.147082 \\
\hline 4 & ${ }^{1} A^{\prime}$ & -1703.006333 & -0.023075 & 0.601908 & -1231.375861 & -1074.118083 \\
\hline 2 & ${ }^{2} \mathrm{~A}$ & -1703.665929 & -0.019095 & 0.613674 & -1232.037457 & -1074.782449 \\
\hline Mes*OH & ${ }^{1} A^{\prime}$ & -779.033070 & -0.012793 & 0.464384 & -425.325965 & -307.412571 \\
\hline Mes*O & ${ }^{2} A^{\prime \prime}$ & -778.405765 & -0.012633 & 0.451440 & -424.694213 & -306.770251 \\
\hline
\end{tabular}

Table 19: Total energies $E_{\text {tot }}$ in Hartree for the Me- and H-truncated model systems of the oxo/hydroxo complexes iroxo and 2 as well as of the Mes*OH/Mes*O couple with $\operatorname{CCSD}\left(T^{*}\right)-\mathrm{F} 12 / \mathrm{VnZ}(\mathrm{n}=\mathrm{D}, \mathrm{T})$.

\begin{tabular}{|c|c|c|c|c|c|}
\hline \multirow[b]{2}{*}{ Species } & \multirow[b]{2}{*}{ State } & \multicolumn{2}{|c|}{$\operatorname{CCSD}\left(T^{*}\right)-\mathrm{F} 12 @ \mathrm{Me}$} & \multicolumn{2}{|c|}{$\operatorname{CCSD}\left(T^{*}\right)-\mathrm{F} 12 @ \mathrm{H}$} \\
\hline & & VDZ & VTZ & VDZ & VTZ \\
\hline $\mathrm{H}$ & $2 S$ & -0.499811 & -0.499946 & -0.499811 & -0.499946 \\
\hline 4 & $3 A^{\prime \prime}$ & -1229.759513 & -1229.842592 & -1072.701371 & -1072.765121 \\
\hline 4 & $1 A^{\prime}$ & -1229.744546 & -1229.826262 & -1072.686589 & -1072.749105 \\
\hline 2 & $2 \mathrm{~A}$ & -1230.399599 & -1230.482968 & -1073.344184 & -1073.408341 \\
\hline Mes*OH & $1 A^{\prime}$ & -424.784878 & -424.837218 & -307.022741 & -307.061313 \\
\hline Mes*o & $2 A^{\prime \prime}$ & -424.144118 & -424.195793 & -306.372488 & -306.410402 \\
\hline
\end{tabular}

Table 20: Total Energies $E_{\text {tot }}$ in Hartree on the ONIOM(CCSD(T*)-F12-F12/VnZ:B3IYP(V)-D3/def2TZVP level of theory.

\begin{tabular}{|c|c|c|c|c|c|}
\hline \multirow[b]{2}{*}{ Species } & \multicolumn{5}{|c|}{ ONIOM(CCSD(T*)-F12/VnZ:B3LYP(V)-D3/def2TZVP[a] } \\
\hline & State & $\mathrm{F} 12 / \mathrm{VDZ}\left(\mathrm{Me}::^{t} \mathrm{Bu}\right)$ & $\mathrm{F} 12 / \mathrm{VTZ}\left(\mathrm{Me}:{ }^{t} \mathrm{Bu}\right)$ & $\mathrm{F} 12 / \mathrm{VDZ}\left(\mathrm{H}::^{\mathrm{t}} \mathrm{Bu}\right)$ & $\mathrm{F} 12 / \mathrm{VTZ}\left(\mathrm{H}:{ }^{t} \mathrm{Bu}\right)$ \\
\hline 4 & $3 A^{\prime \prime}$ & -1701.389063 & -1701.472142 & -1701.588805 & -1701.652555 \\
\hline 4 & $1 A^{\prime}$ & -1701.375018 & -1701.456734 & -1701.574839 & -1701.637355 \\
\hline 2 & $2 \mathrm{~A}$ & -1702.028071 & -1702.111440 & -1702.227664 & -1702.291821 \\
\hline Mes*OH & $1 A^{\prime}$ & -778.491983 & -778.544323 & -778.643240 & -778.681812 \\
\hline Mes*o & $2 A^{\prime \prime}$ & -777.855670 & -777.907345 & -778.008002 & -778.045916 \\
\hline
\end{tabular}

[a]Total energy according to e.g., $E_{\text {tot }}^{H, \text { iroxo }}(F 12 / V T Z: D F T)=E_{\text {tot }}^{\mathrm{H} \text {,iroxo }}(F 12 / V T Z)-E_{\text {tot }}^{\text {H,iroxo }}+E_{\text {tot }}^{\text {iroxo }}$ 


\begin{tabular}{ccccc}
\hline & & & DLPNO-CCSD(T) \\
Species & State & CC-pVTZ & cC-pVQZ & CBS(T,Q) \\
\hline $\mathrm{H}$ & ${ }^{2} \mathrm{~S}$ & -0.499810 & -0.499946 & -0.499986 \\
\hline 4 & ${ }^{3} \mathrm{~A}^{\prime \prime}$ & -1700.712855 & -1701.099834 & -1701.346959 \\
4 & ${ }^{1} \mathrm{~A}^{\prime}$ & -1700.695123 & -1701.083273 & -1701.331292 \\
2 & ${ }^{2} \mathrm{~A}$ & 1701.350845 & -1701.740892 & -1701.990023 \\
Mes*OH & ${ }^{1} \mathrm{~A}^{\prime}$ & -777.817247 & -778.044800 & -778.187044 \\
Mes*O & ${ }^{2} \mathrm{~A}^{\prime \prime}$ & -777.180946 & -777.406958 & -777.548300 \\
\hline
\end{tabular}

\subsubsection{State-Averaged CASSCF computations}

The CASSCF MO correlation diagram Figure 71 shows the relative energies and average occupation numbers of the active orbitals in the oxo and hydroxo species. The $\pi_{y}(\mathrm{Ir}-0)$ orbital of the oxo complex splits into the bonding and antibonding $\sigma(\mathrm{O}-\mathrm{H}) \mathrm{MOs}$ of the hydroxo species and a smaller splitting occurs for the dxy (Ir) orbital. Spinorbit coupling from QDPT treatment of the CASSCF/NEVPT2 wavefunctions has only a very minor effect of less than $3 \mathrm{kJmol}^{-1}\left(\Delta \Delta E_{\mathrm{soc}}=-254 \mathrm{~cm}^{-1}=-2.9 \mathrm{kJmol}^{-1}\right.$, Figure 72$)$, which is of comparable magnitude as the estimate for solvation effects in tetrahydrofuran $\left(\Delta \Delta E_{\text {solv }}=2.2 \mathrm{kJmol}^{-1}\right)$. Note that the computed zero-field splitting parameter $D=775 \mathrm{~cm}^{-1}$ for the triplet iridium-oxo complex is in good agreement with the experimentally determined value of $647 \mathrm{~cm}^{-1}$. Also note that the vertical excitation energy at the triplet geometry of $\Delta E(T / S)=$ $4349 \mathrm{~cm}^{-1}=52.0 \mathrm{~kJ} \mathrm{~mol}^{-1}$ computed at the NEVPT2/CAS $(16,10)$ level is in nice agreement with an upper bound to the (state-specifically relaxed) ONIOM(F12-VnZ/DFT) in Table 20. The state energies of the CAS and NEVPT2 wavefunctions are collected in Table 22 and Table 23. 


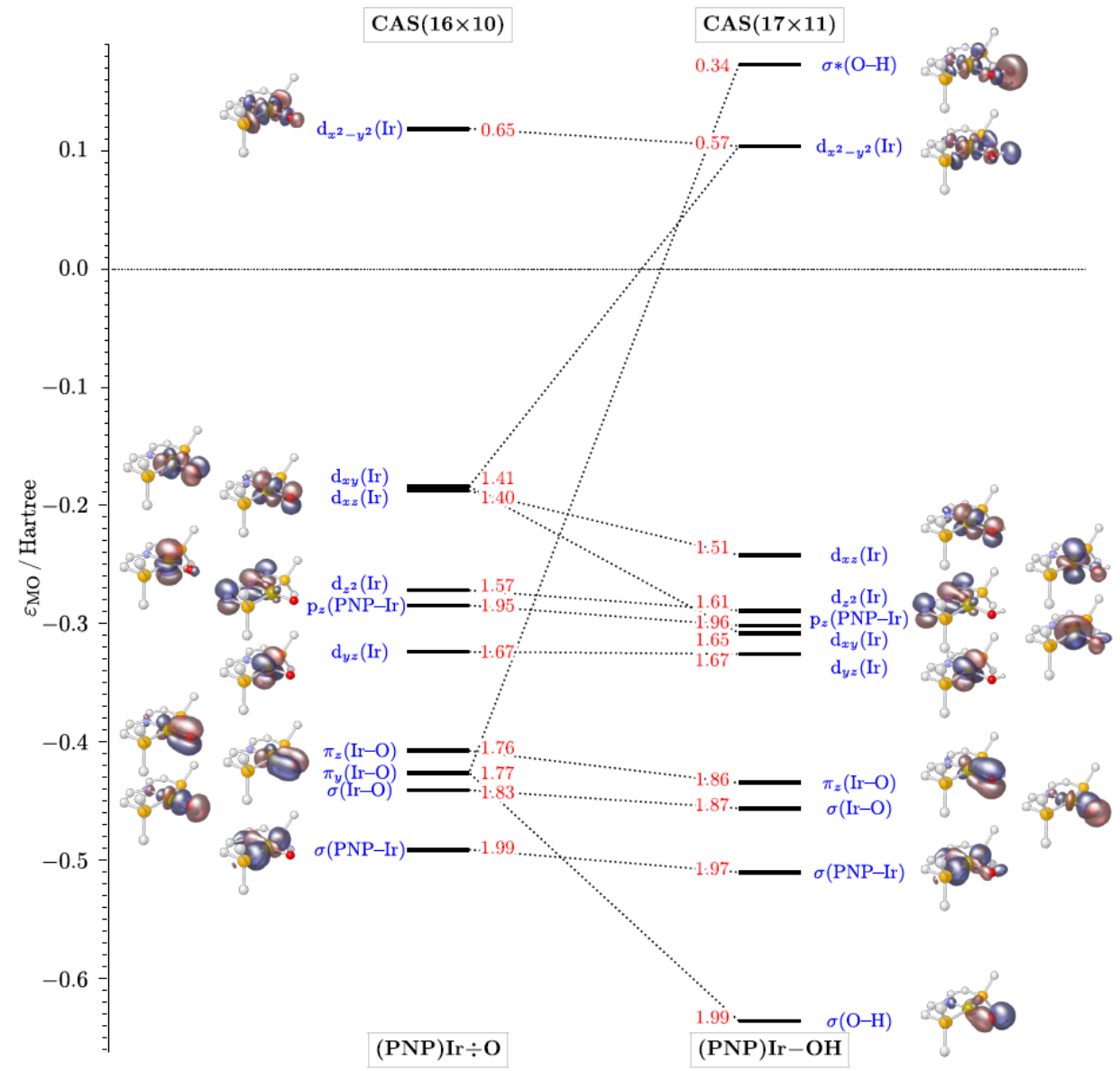

Figure 71: MO correlation diagram from CASSCF wavefunctions state-averaged over all roots which arise from excitations within the $5 \mathrm{~d}$ shell of the metal centre. MOs are computed for the iridium oxo species in a CAS $(16 \times 10)$ expansion averaged over 50 singlet, 45 triplet, and 5 quintet states, and for the iridium hydroxo species in a CAS $(17 \times 11)$ expansion averaged over 40 doublet and 10 quartet states. MO isosurfaces plotted at an isovalue of $0.05 \mathrm{a}_{0}{ }^{-3 / 2}$.

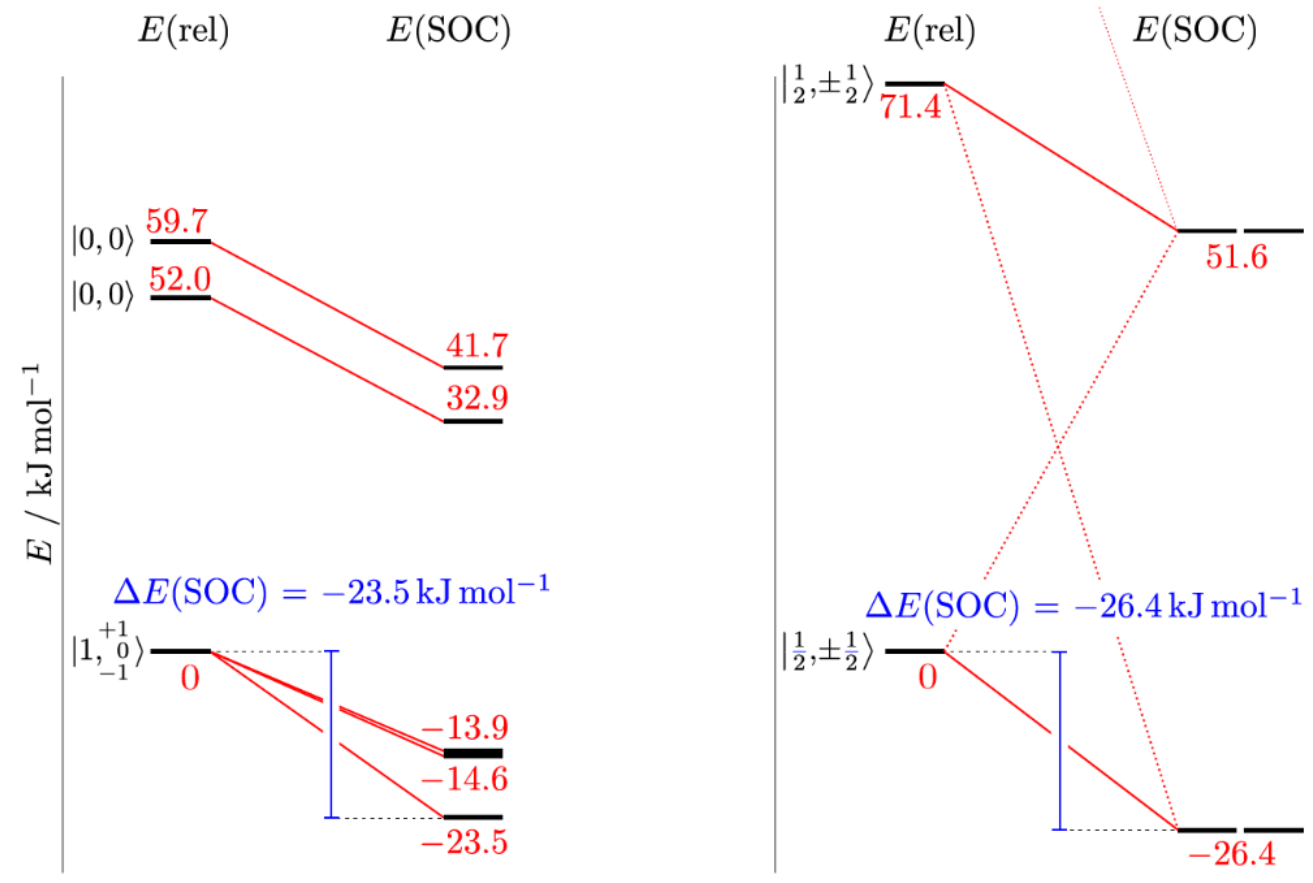


Table 22: State energies of the first ten states per multiplicity sorted by CAS energy. $\triangle E$ state and spin-orbit eigenvalues $\triangle E S O C$ in $\mathrm{cm}^{-1}$ from NEVPT2/SA$\operatorname{CASSCF}(16,10) /$ def2TZVP(ZORA) calculations for 4

\begin{tabular}{|c|c|c|c|c|}
\hline \multirow[b]{2}{*}{ root } & \multirow[b]{2}{*}{ mult } & \multicolumn{2}{|c|}{$\Delta E_{\text {state }}{ }^{[\mathrm{a}]}$} & \multirow{2}{*}{$\begin{array}{c}\Delta E_{\text {state }}{ }^{[\mathrm{b}]} \\
\text { NEVPT2/QDPT }\end{array}$} \\
\hline & & $\operatorname{CAS}(16 \times 10)$ & NEVPT2 & \\
\hline 0 & 3 & 0.0 & 0.0 & 0.0 \\
\hline 0 & 1 & 3499.4 & 4348.7 & 745.8 \\
\hline 1 & 1 & 3731.1 & 4992.6 & 804.0 \\
\hline 1 & 3 & 8529.4 & 11629.4 & 4715.0 \\
\hline 2 & 1 & 9330.0 & 9065.6 & 5452.5 \\
\hline 3 & 1 & 10044.6 & 13439.0 & 10644.8 \\
\hline 2 & 3 & 11270.1 & 14515.1 & 10867.0 \\
\hline 4 & 1 & 14142.9 & 17839.3 & 11938.8 \\
\hline 3 & 3 & 15412.1 & 18248.2 & 12337.3 \\
\hline 4 & 3 & 16899.5 & 19730.2 & 13213.9 \\
\hline
\end{tabular}

[a] Lowest root in $E_{\mathrm{h}} E_{\mathrm{CAS}}(16 \times 10)=-20321.802195 ; E_{\mathrm{NEVPT} 2}(16 \times 10)=-20326.823387$ [b] Lowest SOC eigenvalue in $E_{\mathrm{h}} E_{\mathrm{NEVPT} 2 / \mathrm{QDPT}}=-20326.832335$. Energy Stabilisation $-1964.1 \mathrm{~cm}^{-1}$.

Table 23: State energies of the first ten states per multiplicity sorted by CAS energy. $\triangle E$ state and spin-orbit eigenvalues $\triangle E_{S O C}$ in $\mathrm{cm}^{-1}$ from NEVPT2/SA$\operatorname{CASSCF}(16,10) /$ def2TZVP(ZORA) calculations for 2

\begin{tabular}{|c|c|c|c|c|}
\hline \multirow[b]{2}{*}{ root } & \multirow[b]{2}{*}{ mult } & \multicolumn{2}{|c|}{$\Delta E_{\text {state }}{ }^{[a]}$} & \multirow{2}{*}{$\begin{array}{c}\Delta E_{\text {state }}[\mathrm{b}] \\
\text { NEVPT2/QDPT }\end{array}$} \\
\hline & & $\operatorname{CAS}(16 \times 10)$ & NEVPT2 & \\
\hline 0 & 2 & 0.0 & 0.0 & 0.0 \\
\hline 1 & 2 & 4161.5 & 5967.9 & 0.0 \\
\hline 2 & 2 & 10451.3 & 11909.9 & 6523.3 \\
\hline 3 & 2 & 12301.7 & 11685.4 & 6523.3 \\
\hline 0 & 4 & 30639.0 & 32424.9 & 12853.6 \\
\hline 1 & 4 & 31271.1 & 33268.2 & 12853.6 \\
\hline 2 & 4 & 33384.9 & 35550.9 & 15989.8 \\
\hline 4 & 2 & 34739.2 & 32053.9 & 15989.8 \\
\hline 3 & 4 & 36655.2 & 40278.6 & 26863.0 \\
\hline 5 & 2 & 38385.0 & 38144.9 & 26863.0 \\
\hline
\end{tabular}

[a] Lowest root in $E_{\mathrm{h}} \mathrm{E}_{\mathrm{CAS}}(17 \times 11)=-20322.357149 ; E_{\mathrm{NEVPT} 2}(17 \times 11)=-20327.419124[\mathrm{~b}]$ Lowest SOC eigenvalue in $E_{\mathrm{h}} E_{\mathrm{NEVPT} 2} / \mathrm{QDPT}=-20327.429190$. Energy stabilisation $-2209.2 \mathrm{~cm}^{-1}$.

Overall, the computational approaches presented in Table 24 produce a consistent energetic picture, and, moreover, all ab initio coupled cluster methods show good agreement. The DLPNO-CCSD(T)/CBS(T,Q) and the ONIOM(F12:DFT) approaches thus operate at a comparable level in the current context. In particular the explicitly correlated ONIOM(F12:DFT) approaches are on a par, independent of basis set size and of truncation model. 
Table 24: Relative energies in $\mathrm{kJ} \mathrm{mol}^{-1}$ for the triplet-singlet gap (4.1), $\Delta \mathrm{E}(\mathrm{T} / \mathrm{S})$, in 4 complex and for the enthalpies $\Delta_{\mathrm{r}} \mathrm{H}^{298}$ of the O-H bond formation reaction

(4.2), of the $\mathrm{O}-\mathrm{H}$ bond homolysis reaction (4.3), and of the corresponding proton-coupled electron transfer (PCET) reaction (4.4) for the conversion of 2 towards the 4 .

\begin{tabular}{|c|c|c|c|c|}
\hline Method $^{[a]}$ & $\Delta E(T / S)^{[b]}$ & & $\overline{\Delta_{r} H_{\text {solv }}^{\text {SOC }[c]}}$ & \\
\hline & Reaction $(4.1)^{[b]}$ & Reaction $(4.2)^{[d]}$ & Reaction $(4.3)^{[\mathrm{e}]}$ & Reaction $(4.4)^{[f]}$ \\
\hline$\overline{D F T^{[g]}}$ & 74.0 & 324.7 & -311.2 & 13.5 \\
\hline DLPNO-CCSD $(T)^{[\mathrm{h}]}$ & 41.1 & 352.1 & -338.0 & 14.1 \\
\hline ONIOM(F12/VDZ:DFT)(H:Bu $)^{[i]}$ & 36.7 & 341.5 & -329.3 & 12.2 \\
\hline ONIOM(F12/VTZ:DFT)(H: Buu $)^{[j]}$ & 39.9 & 342.2 & -330.7 & 11.6 \\
\hline ONIOM(F12/VDZ:DFT)(Me: $\left.{ }^{t} \mathrm{Bu}\right)^{[\mathrm{k}]}$ & 36.9 & 341.9 & -332.1 & 9.8 \\
\hline ONIOM(F12/VTZ:DFT)(Me: $\left.:^{t} \mathrm{Bu}\right)^{[(])}$ & 40.5 & 342.3 & -333.5 & 8.8 \\
\hline$\Delta \Delta_{r} E_{\text {solv }}^{[\mathrm{m}]}$ & & -2.2 & -1.5 & -3.7 \\
\hline$\Delta \Delta_{r} E_{S O C}^{[n]}$ & & 2.9 & & \\
\hline
\end{tabular}

[a] All molecular geometries and thermal contributions to energies computed at B3LYP(V)-D3/def2TZVP level. [b] Excitation energy at $0 \mathrm{~K}$ from ${ }^{3} \mathrm{~A}^{\prime \prime}(\mathrm{Cs})$ triplet ground-state of 4 to the lowest-energy ${ }^{1} A^{\prime}(C s)$ singlet. [c] Reaction enthalpies computed at $298 \mathrm{~K}$, corrected for solvent effects in THF $\Delta \Delta_{\mathrm{r}} E($ solv) and for spin-orbit coupling contributions $\Delta \Delta_{\mathrm{r}} \mathrm{E}(\mathrm{SOC})$. [d] Reaction enthalpy for reaction (4.2) computed at $298 \mathrm{~K}$, corrected for solvent effects in $\mathrm{THF} \Delta \Delta \mathrm{rE}(\mathrm{solv})=-2.21 \mathrm{kJmol}{ }^{-1}$ and for spin-orbit coupling contributions $\Delta \Delta_{\mathrm{r}} \mathrm{E}(\mathrm{SOC})=254 \mathrm{~cm}^{-1}=2.93 \mathrm{kJmol}^{-1}$. [e] Reaction enthalpy for reaction (4.3) computed at $298 \mathrm{~K}$ and corrected for solvent effects in thf, $\Delta \Delta_{\mathrm{r}} \mathrm{E}(\mathrm{solv})=1.51 \mathrm{kJmol}^{-1}$. [f] Reaction enthalpy for reaction (4.4) computed at $298 \mathrm{~K}$, corrected for solvent effects in thf $\Delta \Delta_{\mathrm{r}} \mathrm{E}(\mathrm{solv})=3.72 \mathrm{~kJ}$ mol-1 and for spin-orbit coupling contributions $\Delta \Delta_{\mathrm{r}} \mathrm{E}(\mathrm{SOC})=-254 \mathrm{~cm}^{-1}=-2.93 \mathrm{~kJ} \mathrm{~mol}^{-1}$. [g] B3LYP(V)-D3/def2TZVP level. [h] DLPNO-CCSD(T)/CBS(T,Q) // B3LYP(V)D3/def2TZVP level. [i] ONIOM(CCSD(T*)-F12/VDZ: B3LYP(V)-D3/def2TZVP) level for H-truncated vs. full system. [j] ONIOM(CCSD(T*)-F12/VTZ: B3LYP(V)D3/def2TZVP) level for H-truncated vs. full system. [k] ONIOM(CCSD(T*)-F12/VDZ: B3LYP(V)-D3/def2TZVP) level for Me-truncated vs. full system. [l] ONIOM(CCSD(T*)-F12/VTZ: B3LYP(V)-D3/def2TZVP) level for Me-truncated vs. full system. [m] QDPT/NEVPT2 level.

As an extension, the cationic oxo species 4+ was computed on the PBE0-D3/def2TZVP level of theory. A doublet ground state is predicted for $4+$ with a doublet/quartet gap of $\Delta E(D / Q)=46 \mathrm{~kJ} \mathrm{~mol}^{-1}$. With a reduced $\mathrm{Ir}-\mathrm{O}$ bond length $\left(\mathrm{d}_{\mathrm{r}-\mathrm{o}}=1.75 \AA\right.$ ) of $0.05 \AA$ the doublet structure is nicely resembling the formally higher bond order due to less $\pi^{*}$ population (Figure 73). The computed energies are summarised in Table 25 while the xyz data can be found in Section D of Chapter 7.

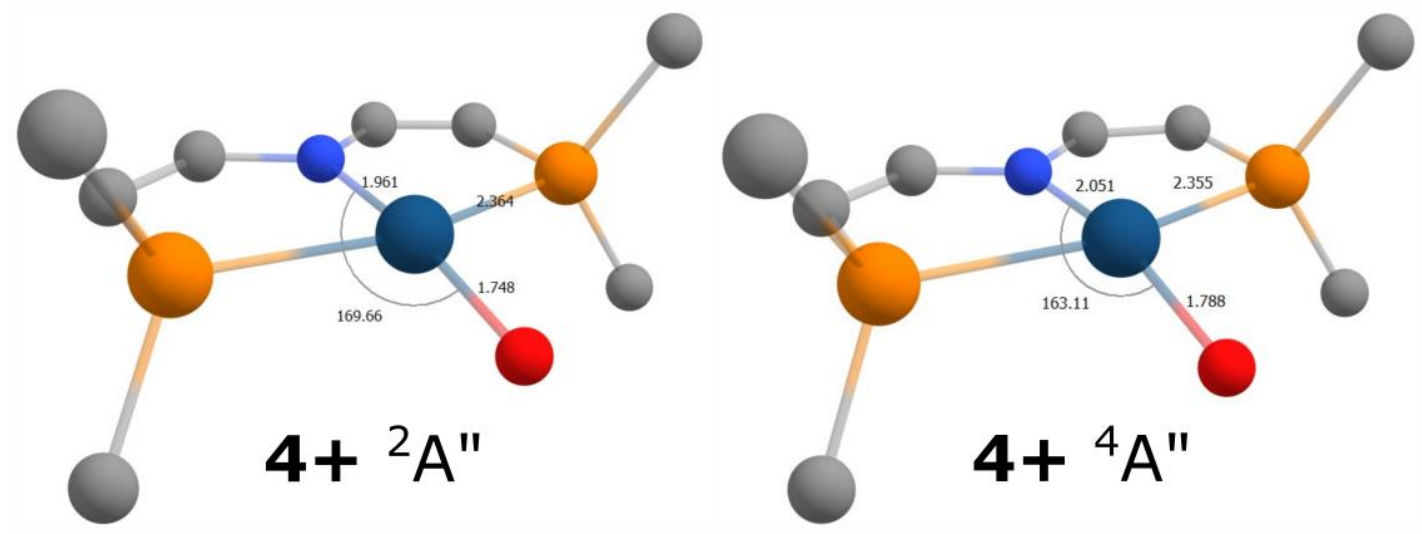

Figure 73: Computed structures of 4+ on the PBE0-D3/def2TZVP level of theory. Methyl groups are omitted for clarity.

Table 25: Relative energies of the DFT results for $4+$

\begin{tabular}{|c|c|c|c|c|c|c|}
\hline \multirow[b]{2}{*}{ Species } & \multirow[b]{2}{*}{ State } & \multicolumn{4}{|c|}{ PBEO-D3/def2TZVP } & \multirow[b]{2}{*}{$\Delta G / \mathrm{kJ} \mathrm{mol}^{-1}$} \\
\hline & & $E$ & $H(298)$ & $G(298)$ & $\Delta E / \mathrm{kJ} \mathrm{mol}^{-1}$ & \\
\hline $4+$ & $2 A^{\prime \prime}(C s)$ & -1702.181177 & 0.604576 & 0.507619 & 0.00 & 0.00 \\
\hline $4+$ & $4 \mathrm{~A}^{\prime \prime}(\mathrm{CS})$ & -1702.163178 & 0.603585 & 0.505842 & 47.25 & 42.59 \\
\hline
\end{tabular}




\subsection{On the Spin-Orbit Coupling influence in solution thermochemistry}

\subsubsection{Molecular Geometries}

Figure 74 shows selected parameters of the optimized geometries of the full molecular systems. All species converged in $C_{s}$ symmetry. The triplet ground state of 23 exhibits a distorted octahedral coordination sphere with the axial chlorine atoms significantly deviating from the ideal $90^{\circ}$ angle. The singlet isomer shows even higher deviation from ideal octahedral geometry. The doublet ground state of 24 is also octahedrally distorted but here the equatorial chlorine is tilted out of the N-Re-P plane, while the quartet isomer shows only minor deviations from an ideal octahedral coordination. Compared to the amine complex 23 the Re-N bond is significantly shortened by $0.28 \AA$ due to the $\pi$-bonding interaction. Also, the Mes* $\mathrm{O} / \mathrm{Mes}{ }^{*} \mathrm{OH}$ couple converged in $C_{s}$ symmetry. The C-O difference of the Mes*O radical is shortened by $0.12 \AA$ respectively. The xyz data of all structures can be found in the appendix Section D.

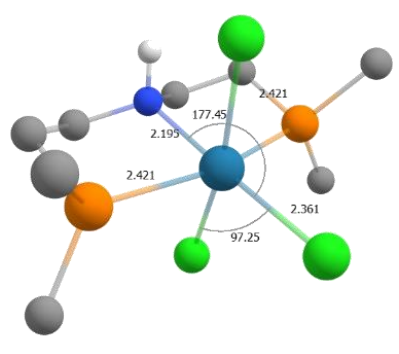

$\left(\mathrm{P}^{\mathrm{H}} \mathrm{NP}\right) \mathrm{ReCl}_{3}{ }^{3} \mathrm{~A} "(\mathrm{Cs})$
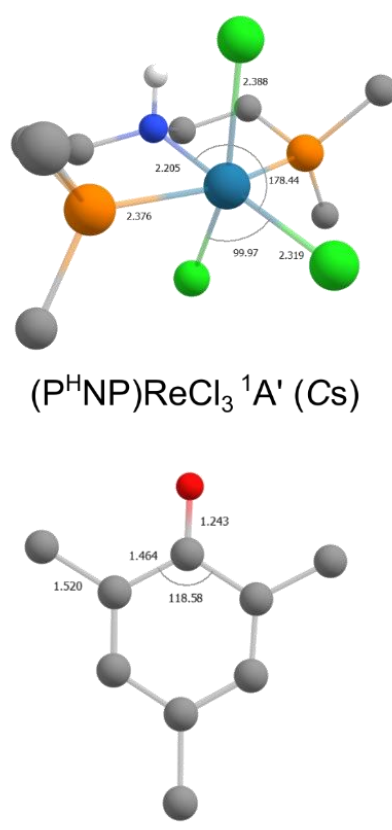

Mes*O ${ }^{2} A "(C s)$

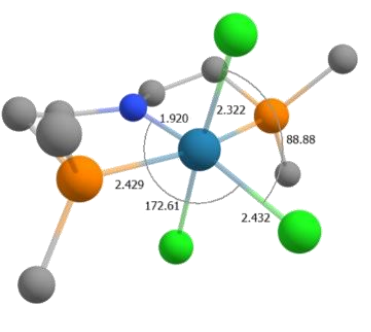

$(\mathrm{PNP}) \mathrm{ReCl}_{3}{ }^{2} \mathrm{An}(\mathrm{Cs})$

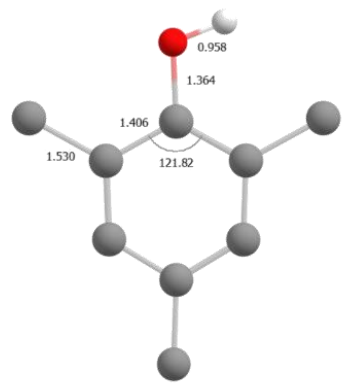

Mes*OH ${ }^{1} \mathrm{~A}^{\prime}(\mathrm{Cs})$

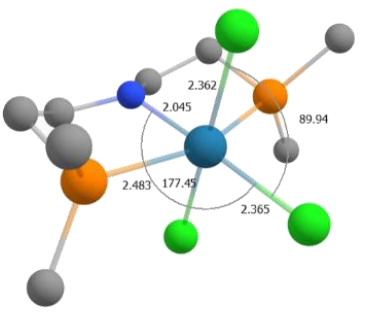

(PNP) $\operatorname{ReCl}_{3}{ }^{4} \mathrm{~A}^{\prime}(\mathrm{Cs})$

Figure 74: DFT-optimized structures (PBEO/def2TZVP-D3) of the rhenium amine complex 23, the rhenium amide complex 24 and the Mes*OH/Mes*O couple. (Top: left: Triplet ground state of 23; middle left: first singlet isomer of 23; middle right: Doublet ground state of 24; right: First quartet isomer of 24; Bottom: left: Mes*O doublet structure; right: $\mathrm{Mes}{ }^{*} \mathrm{OH}$ singlet structure). All ${ }^{\mathrm{B}} \mathrm{Bu}$ and ${ }^{\mathrm{P}} \mathrm{Pr}$ groups and carbon-bonded hydrogen atoms omitted for clarity. 


\subsubsection{State Averaged CASSCF Computations}

The following diagram shows the energies and occupation of the small active spaces of 23 and 24.
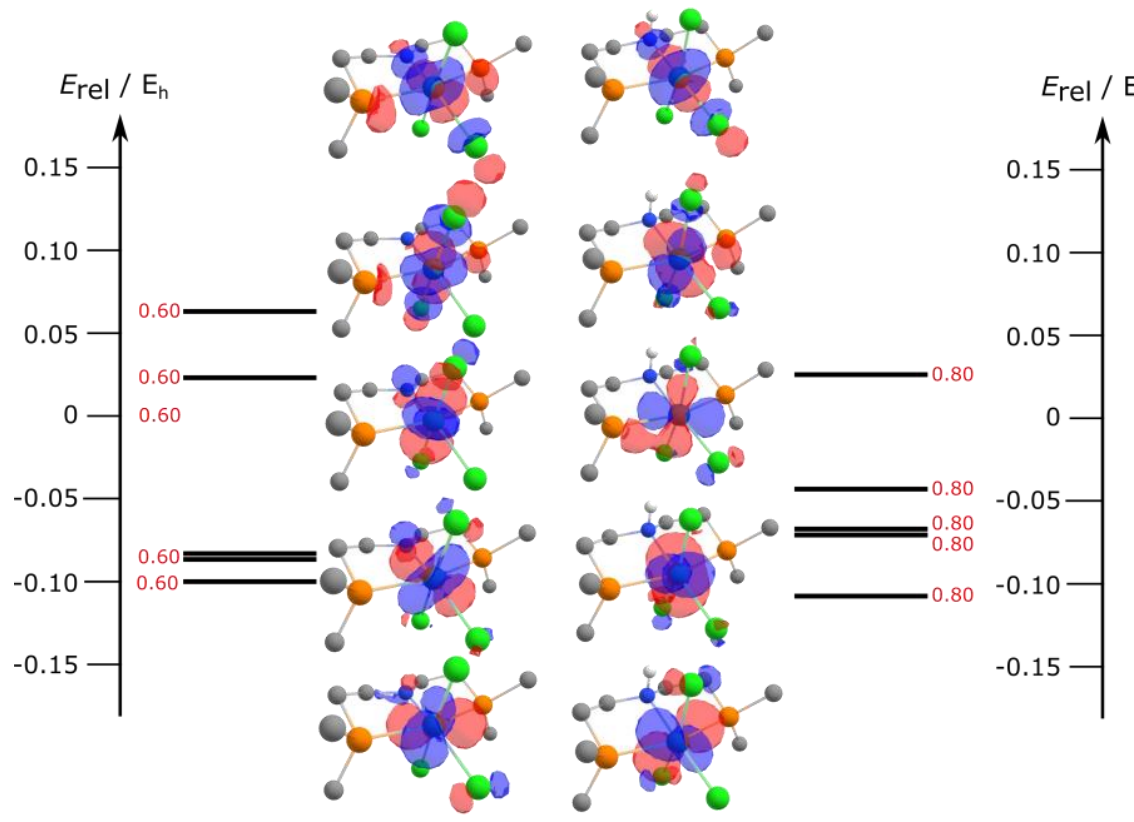

$\left(\mathrm{P}^{\mathrm{H} N P}\right) \mathrm{ReCl}_{3}$

CASSCF $(4 \times 5)$

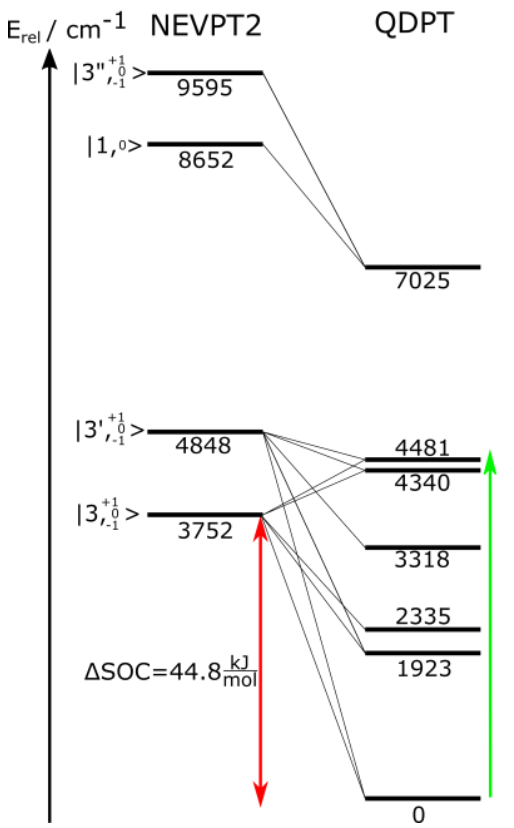

(PNP) $\mathrm{ReCl}_{3}$

$\operatorname{CASSCF}(3 \times 5)$

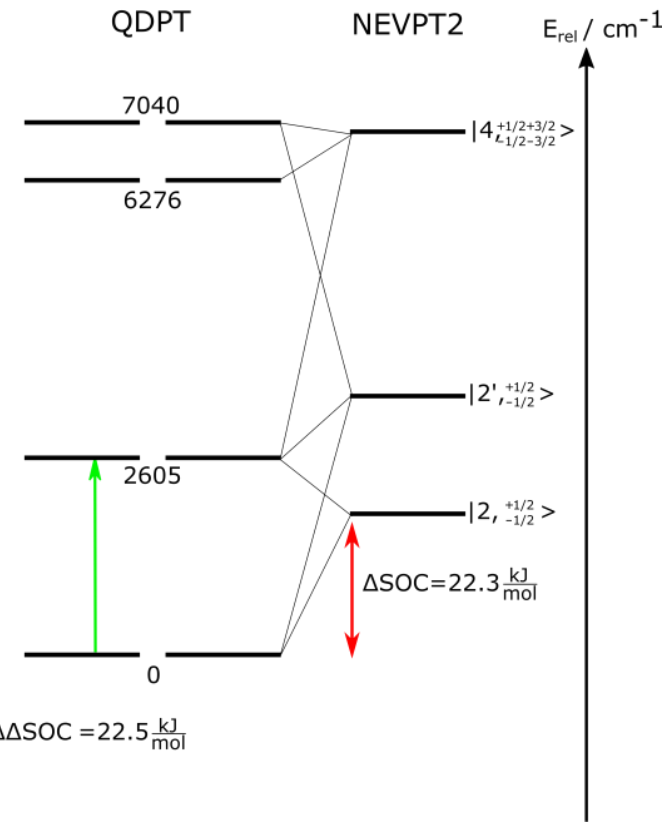

Figure 75: CASSCF wavefunctions state averaged over all excitations arising from the $5 \mathrm{~d}$ metal centre. MO's (top) are computed for the rhenium amine complex $23 \mathrm{CAS}(14 \times 10)$ averaged over 5 quintet, 45 triplet, and 50 singlet states (top left) and rhenium amide complex 24 CAS(15×11) averaged over 10 quartet and 40 doublet states (top right). Plotted at an isovalue of $0.05 \mathrm{a0}^{-3 / 2}$. Bottom: CASSCF state diagram of 1 (bottom left) and 2 (bottom right) with NEVPT2 and QDPT treatment. Red: Spin-orbit coupling stabilization. Green: Computed state transitions with non-negligible oscillator strength. 
The following diagram shows the energies and occupation of the big active spaces of 23 and 24 .
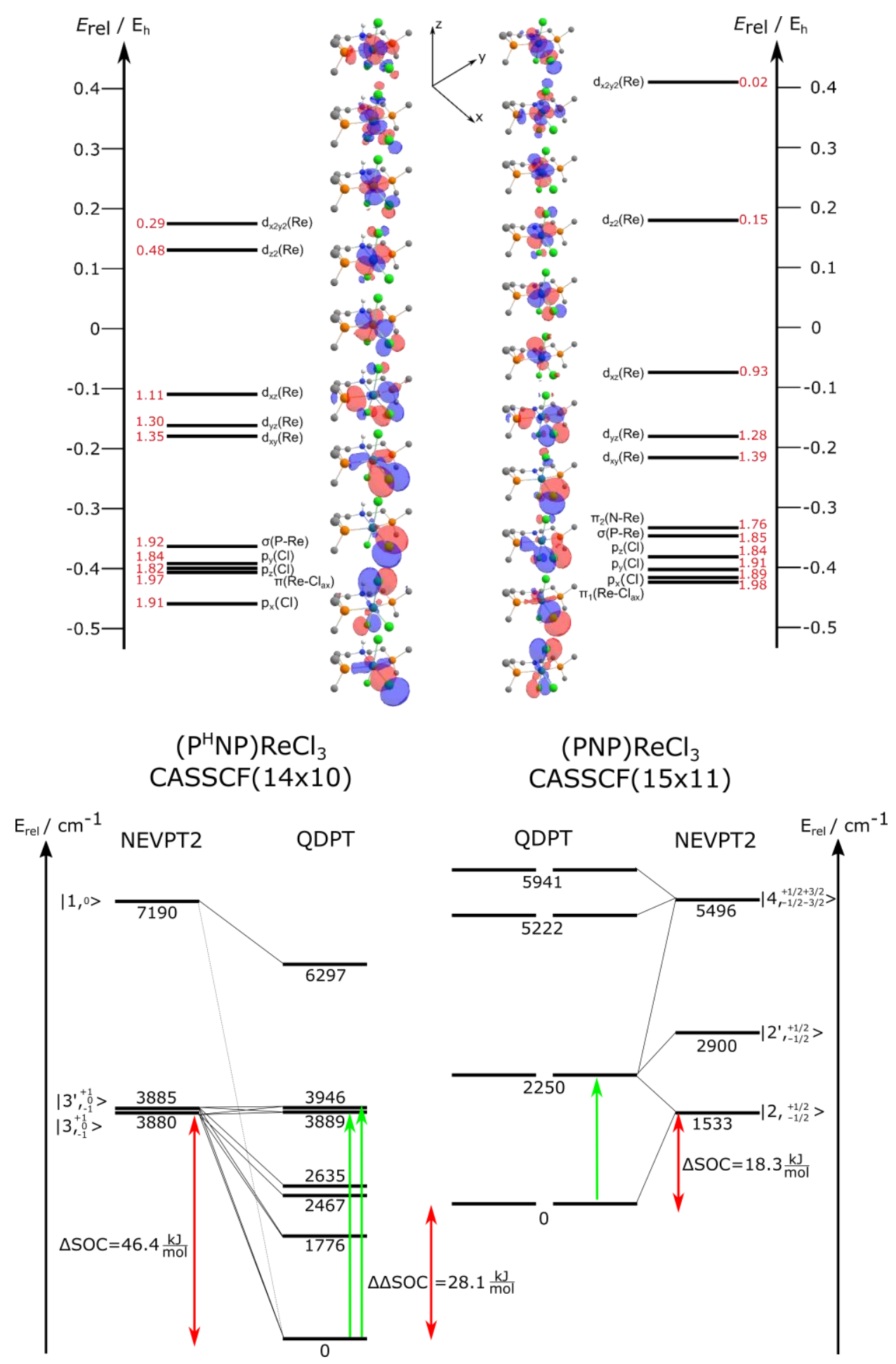

Figure 76: CASSCF wavefunctions state averaged over all excitations arising from the $5 \mathrm{~d}$ metal centre. MOs (top) are computed for $23 \mathrm{CAS}(14 \times 10)$ averaged over 5 quintet, 45 triplet, and 50 singlet states (top left) and 24 CAS(15x11) averaged over 10 quartet and 40 doublet states (top right). Plotted at an isovalue of 0.05 a $0^{-3 / 2}$. Bottom: CASSCF state diagram of 23 (bottom left) and 24 (bottom right) with NEVPT2 and QDPT treatment. Red: Spin-orbit coupling stabilization. Green: Computed state transitions with non-negligible oscillator strength. 


\subsubsection{CASSCF/NEVPT2 State Composition and QDPT Eigenvectors}

Table 26 and Table 27 collect the CASSCF/NEVPT2 state compositions and QDPT eigenvectors of 23 and 24.

Table 26: CASSCF/NEVPT2 state composition. Weight values rounded to 2 digits. Threshold for printing is Weight: 0.05 . States $<10000 \mathrm{~cm}^{-1}$

\begin{tabular}{|c|c|c|c|c|c|c|c|c|c|}
\hline \multicolumn{5}{|c|}{$23(14 \times 10)$} & \multicolumn{5}{|c|}{$24(15 \times 11)$} \\
\hline State & Mult & Energy $/ \mathrm{cm}^{-1}$ & Weight & Composition & State & Mult & Energy $/ \mathrm{cm}^{-1}$ & Weight & Composition \\
\hline \multirow{3}{*}{0} & \multirow{3}{*}{3} & \multirow{3}{*}{0} & 0.68 & 2222221100 & \multirow{3}{*}{0} & \multirow{3}{*}{2} & \multirow{3}{*}{0} & 0.82 & 22222221000 \\
\hline & & & & & & & & & \\
\hline & & & 0.28 & 2222212100 & & & & 0.05 & 22222021200 \\
\hline \multirow{3}{*}{1} & \multirow{3}{*}{3} & \multirow{3}{*}{5.3} & 0.68 & 2222212100 & \multirow{3}{*}{1} & \multirow{3}{*}{2} & \multirow{3}{*}{1368} & 0.83 & 22222212000 \\
\hline & & & & & & & & & \\
\hline & & & 0.28 & 2222221100 & & & & 0.05 & 22222012200 \\
\hline \multirow{3}{*}{2} & \multirow{3}{*}{1} & \multirow{3}{*}{3311} & 0.81 & 2222222000 & 3 & 4 & 3964 & 0.93 & 22222211100 \\
\hline & & & & & & & & & \\
\hline & & & 0.07 & 2222220200 & & & & & \\
\hline \multirow{3}{*}{3} & \multirow{3}{*}{1} & \multirow{3}{*}{6005} & 0.68 & 2222212100 & & & & & \\
\hline & & & & & & & & & \\
\hline & & & 0.25 & 2222221100 & & & & & \\
\hline \multirow[t]{2}{*}{4} & 3 & 6029 & 0.95 & 2222211200 & & & & & \\
\hline & \multirow{3}{*}{1} & \multirow{3}{*}{6335} & 0.68 & 2222221100 & & & & & \\
\hline \multirow[t]{2}{*}{5} & & & & & & & & & \\
\hline & & & 0.25 & 2222212100 & & & & & \\
\hline
\end{tabular}

Table 27: QDPT Eigenvectors, weight values rounded to 2 digits. Threshold for printing is 0.05 .

$23(14 \times 10) \quad 24(15 \times 11)$

\begin{tabular}{|c|c|c|c|c|c|c|c|c|c|c|c|c|c|}
\hline State & Energy $/ \mathrm{cm}^{-1}$ & Weight & Block & Root & Spin & $\mathrm{ms}$ & State & Energy $/ \mathrm{cm}^{-1}$ & Weight & Block & Root & Spin & $\mathrm{ms}$ \\
\hline \multirow{4}{*}{0} & \multirow{4}{*}{0} & 0.38 & 1 & 0 & 1 & 0 & \multirow{3}{*}{0} & \multirow{3}{*}{0} & 0.60 & 1 & 0 & $1 / 2$ & $-1 / 2$ \\
\hline & & 0.18 & 1 & 1 & 1 & 1 & & & 0.21 & 1 & 1 & $1 / 2$ & $-1 / 2$ \\
\hline & & 0.18 & 1 & 1 & 1 & -1 & & & 0.07 & 1 & 0 & $1 / 2$ & $-1 / 2$ \\
\hline & & 0.07 & 2 & 0 & 0 & 0 & \multirow{3}{*}{1} & \multirow{3}{*}{0} & 0.60 & 1 & 0 & $1 / 2$ & $1 / 2$ \\
\hline \multirow{3}{*}{1} & \multirow{3}{*}{1776} & 0.42 & 1 & 1 & 1 & 0 & & & 0.21 & 1 & 1 & $1 / 2$ & $1 / 2$ \\
\hline & & 0.24 & 1 & 0 & 1 & 1 & & & 0.07 & 1 & 0 & $1 / 2$ & $1 / 2$ \\
\hline & & 0.24 & 1 & 0 & 1 & -1 & \multirow{5}{*}{2} & \multirow{5}{*}{2250} & 0.32 & 1 & 1 & $1 / 2$ & $-1 / 2$ \\
\hline \multirow{2}{*}{2} & \multirow{2}{*}{2467} & 0.43 & 1 & 0 & 1 & 1 & & & 0.24 & 1 & 1 & $1 / 2$ & $1 / 2$ \\
\hline & & 0.43 & 1 & 0 & 1 & -1 & & & 0.15 & 1 & 0 & $1 / 2$ & $1 / 2$ \\
\hline \multirow{2}{*}{3} & \multirow{2}{*}{2635} & 0.44 & 1 & 1 & 1 & 1 & & & 0.08 & 0 & 0 & $3 / 2$ & $1 / 2$ \\
\hline & & 0.44 & 1 & 1 & 1 & -1 & & & 0.05 & 0 & 0 & $3 / 2$ & $-3 / 2$ \\
\hline \multirow{4}{*}{4} & \multirow{4}{*}{3889} & 0.34 & 1 & 1 & 1 & 0 & \multirow{5}{*}{3} & \multirow{5}{*}{2250} & 0.32 & 1 & 1 & $1 / 2$ & $1 / 2$ \\
\hline & & 0.16 & 1 & 0 & 1 & 0 & & & 0.24 & 1 & 1 & $1 / 2$ & $-1 / 2$ \\
\hline & & 0.14 & 1 & 0 & 1 & 1 & & & 0.15 & 1 & 0 & $1 / 2$ & $-1 / 2$ \\
\hline & & 0.14 & 1 & 0 & 1 & -1 & & & 0.08 & 0 & 0 & $3 / 2$ & $-1 / 2$ \\
\hline \multirow{5}{*}{5} & \multirow{5}{*}{3946} & 0.30 & 1 & 0 & 1 & 0 & & & 0.05 & 0 & 0 & $3 / 2$ & $3 / 2$ \\
\hline & & 0.16 & 1 & 1 & 1 & 0 & \multirow[t]{2}{*}{4} & \multirow[t]{2}{*}{5222} & 0.52 & 0 & 0 & $3 / 2$ & $-3 / 2$ \\
\hline & & 0.16 & 1 & 1 & 1 & 1 & & & 0.38 & 0 & 0 & $3 / 2$ & $1 / 2$ \\
\hline & & 0.16 & 1 & 1 & 1 & -1 & \multirow{2}{*}{5} & \multirow{2}{*}{5222} & 0.52 & 0 & 0 & $3 / 2$ & $3 / 2$ \\
\hline & & 0.07 & 1 & 0 & 1 & -1 & & & 0.38 & 0 & 0 & $3 / 2$ & $-1 / 2$ \\
\hline \multirow{8}{*}{6} & \multirow{3}{*}{6297} & 0.72 & 2 & 0 & 0 & 0 & \multirow{4}{*}{6} & \multirow{4}{*}{5941} & 0.34 & 0 & 0 & $3 / 2$ & $-1 / 2$ \\
\hline & & 0.10 & 1 & 2 & 1 & 1 & & & 0.32 & 0 & 0 & $3 / 2$ & $3 / 2$ \\
\hline & & 0.10 & 1 & 2 & 1 & -1 & & & 0.11 & 1 & 1 & $1 / 2$ & $1 / 2$ \\
\hline & & & & & & & & & 0.10 & 0 & 0 & $3 / 2$ & $1 / 2$ \\
\hline & & & & & & & & & 0.34 & 0 & 0 & $3 / 2$ & $1 / 2$ \\
\hline & & & & & & & 7 & 5941 & 0.32 & 0 & 0 & $3 / 2$ & $-3 / 2$ \\
\hline & & & & & & & & & 0.11 & 1 & 1 & $1 / 2$ & $-1 / 2$ \\
\hline & & & & & & & & & 0.10 & 0 & 0 & $3 / 2$ & $-1 / 2$ \\
\hline
\end{tabular}




\subsubsection{Comparison of Magnetic Properties Theory vs. Experiment}

Figure 77 and Figure 76 show the comparison of the obtained magnetic properties by CASSCF/NEVPT2-QDPT treatment with the experimentally derived magnetic data by SQUID magnetometry for 23 and 24.
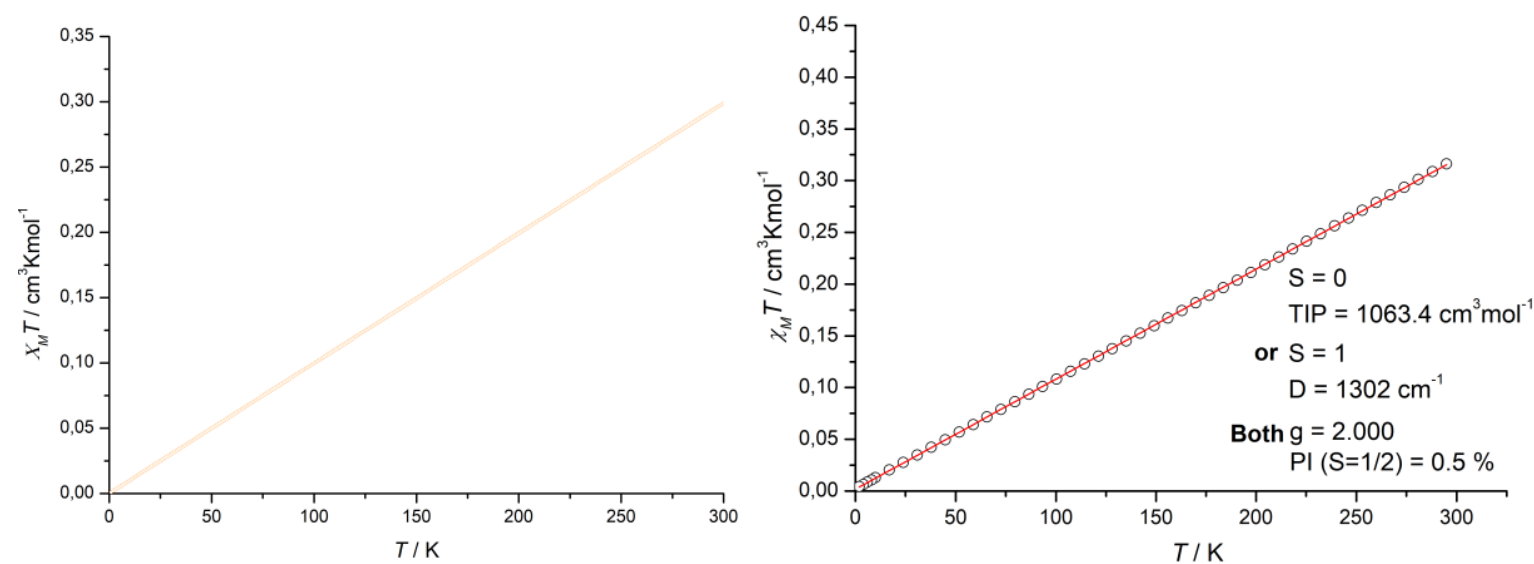

Figure 77: Computed magnetic susceptibility data on the CASSCF/NEVPT2/QDPT def2-TZVP(ZORA) level of theory at $0.5 \mathrm{~T}$ for reamin (left) and the experimental data obtained by SQUID magnetometry (right).
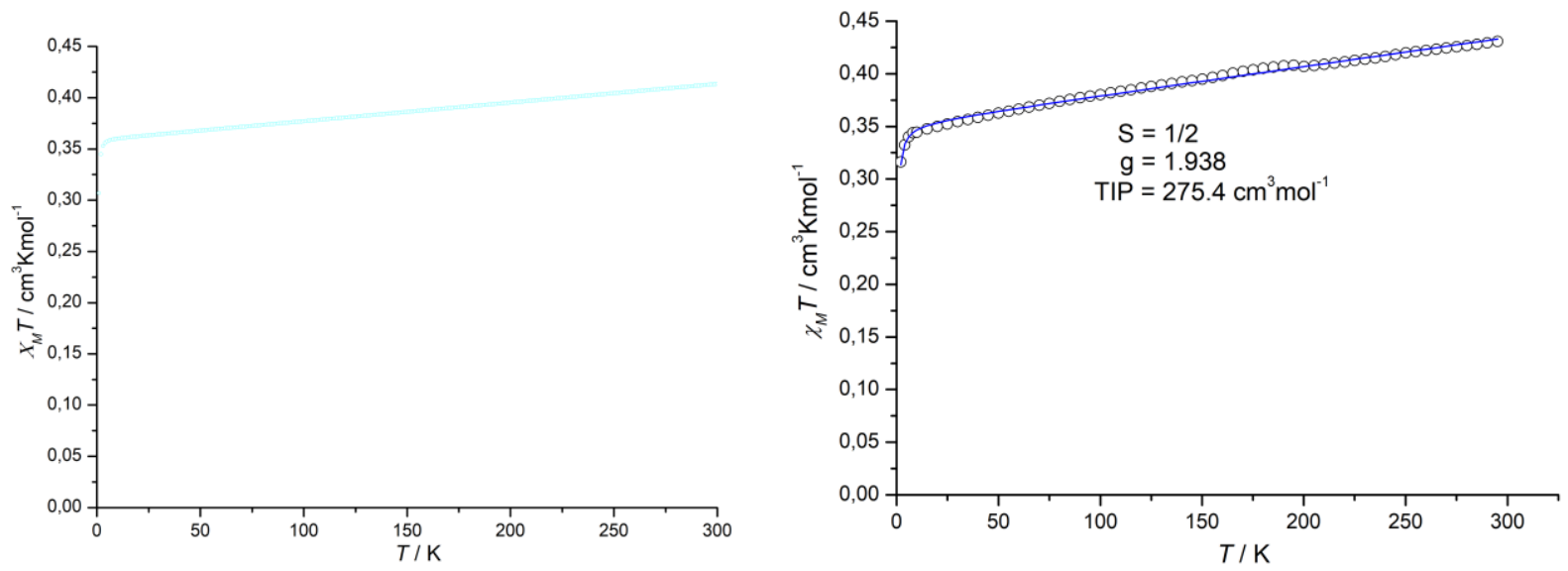

Figure 78: Computed magnetic susceptibility data on the CASSCF/NEVPT2/QDPT def2TZVP(ZORA) level of theory at $0.5 \mathrm{~T}$ for 24 (left) and the experimental obtained data by SQUID magnetometry (right). 


\subsubsection{Spin State and Reactions Energetics}

The relative spin-state and reaction energies were computed with DFT, coupled cluster methods and DLPNO. The results are summarized in following tables. Reaction 4.5 and 4.6 are used to compute the triplet/singlet gap $\Delta E(T / S)$ and the doublet/quartet, respectively.

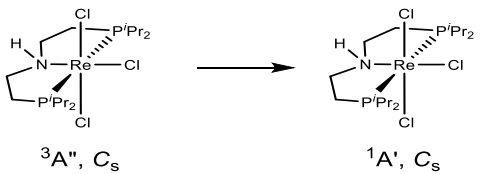

(4.5)

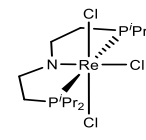

${ }^{2} \mathrm{~A} ", C_{\mathrm{s}}$

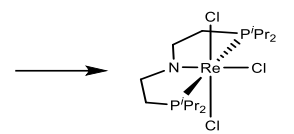

${ }^{4} \mathrm{~A}, C_{\mathrm{s}}$

The half reaction energetics were computed for reaction 4.7:

$$
\mathrm{X}-\mathrm{H} \rightarrow \mathrm{X}^{\cdot}+\mathrm{H}^{\cdot}
$$

The full reaction energetics were computed for the reaction 4.8:

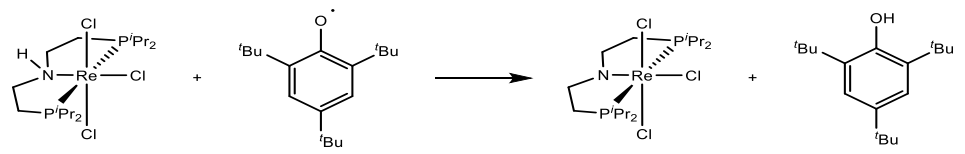

The ONIOM energies were computed with eq. 4.9:

$$
E^{\mathrm{ONIOM}}=E_{\mathrm{X}: \mathrm{iPr} / \mathrm{tBu}}^{\mathrm{CCSD}(\mathrm{T})-\mathrm{f} 12}-E_{\mathrm{X}: \mathrm{iPr} / \mathrm{tBu}}^{\mathrm{PBE0}-\mathrm{D} 3 / \mathrm{def2TZVP}}+E_{\mathrm{Fullsystem}}^{\mathrm{PBE} 0-\mathrm{D} 3 / \mathrm{def} 2 \mathrm{TZVP}}
$$


Table 28: Total energies in $E_{h}$ of 23 and 24 and the Mes* OH/Mes*O couple and the corresponding methyl and hydrogen truncated model systems at the level PBEO-D3/def2TZVP

\begin{tabular}{|c|c|c|c|c|c|c|c|}
\hline Method $^{[a]}$ & $\begin{array}{l}\Delta E(T / S)^{[b]} \\
\text { eq. }(4.5)\end{array}$ & $\begin{array}{l}\Delta E(\mathrm{D} / \mathrm{Q})^{[\mathrm{b}]} \\
\text { eq.(4.6) }\end{array}$ & $\begin{array}{l}\Delta E^{[c]} \\
\text { eq. (4.7) }\end{array}$ & Method $^{[a]}$ & $\begin{array}{l}\Delta E(T / S)^{[b]} \\
\text { eq. }(4.5)\end{array}$ & $\begin{array}{c}\Delta E(D / Q)^{[b]} \\
\text { eq. }(4.6)\end{array}$ & $\Delta E^{[c]}$ eq. (4.7) \\
\hline $\operatorname{DFT}^{[d]}(23 / 24)$ & 38.6 & 15.0 & 294 & & & & \\
\hline $\operatorname{DFT}\left(\mathrm{Mes}^{*} \mathrm{OH} / \mathrm{Mes}^{*} \mathrm{O}\right)^{[\mathrm{d}]}$ & & & 343 & $\begin{array}{c}\mathrm{ONIOM}\left(\mathrm{f}_{12}-\mathrm{VDZ}: \mathrm{PBEO}\right)\left(\mathrm{H} \mathrm{:}^{i} \mathrm{Pr}\right) \\
(23 / 24)^{[1]}\end{array}$ & 16.8 & 37.8 & 288 \\
\hline $\mathrm{DFT}^{\mathrm{H}}$ truncation $(23 / 24)^{[\mathrm{e}]}$ & 14.5 & 26.4 & 299 & $\begin{array}{l}\text { ONIOM }\left(\mathrm{f}_{12}-\mathrm{VDZ}: \mathrm{PBEO}\right)\left(\mathrm{H}:{ }^{t} \mathrm{Bu}\right) \\
\left(\text { Mes }^{*} \mathrm{OH} / \mathrm{Mes}^{*} \mathrm{O}\right)^{[1]}\end{array}$ & & & 357 \\
\hline $\begin{array}{c}\mathrm{DFT}^{\mathrm{H}} \text { truncation } \\
\left(\mathrm{Mes}^{*} \mathrm{OH} / \mathrm{Mes}^{*} \mathrm{O}\right)^{[\mathrm{e}]}\end{array}$ & & & 380 & $\begin{array}{c}\left.\text { ONIOM(f } f_{12}-\text { VDZ:PBEO)(Me: }{ }^{\mathrm{Pr}}\right) \\
(23 / 24)^{[\mathrm{m}]}\end{array}$ & 15.2 & 38.6 & 278 \\
\hline DFTMe truncation $(15 / 16)^{[f]}$ & 30.8 & 15.2 & 297 & $\begin{array}{l}\left.\text { ONIOM(f } f_{12}-\text { VDZ:PBEO)(Me: }{ }^{t} \mathrm{Bu}\right) \\
\left(\mathrm{Mes}^{*} \mathrm{OH} / \mathrm{Mes}^{*} \mathrm{O}\right)^{[\mathrm{m}]}\end{array}$ & & & 360 \\
\hline $\begin{array}{l}\text { DFT }^{\text {Me }} \text { truncation } \\
\left(\mathrm{Mes}^{*} \mathrm{OH} / \mathrm{Mes}^{*} \mathrm{O}\right)^{[\mathrm{ff}]}\end{array}$ & & & 353 & $\begin{array}{c}\left.\text { ONIOM(f } f_{12}-\mathrm{VTZ}: \mathrm{PBEO}\right)\left(\mathrm{H}:^{i} \mathrm{Pr}\right) \\
(23 / 24)^{[\mathrm{n}]}\end{array}$ & 18.5 & 36.3 & 286 \\
\hline $\begin{array}{c}\operatorname{CCSD}(\mathrm{T})-\mathrm{f}_{12} / \mathrm{VDZ}(\mathrm{H}: \mathrm{Pr}) \\
(23 / 24)^{[\mathrm{g}]}\end{array}$ & -7.3 & 49.2 & 284 & $\begin{array}{c}\text { ONIOM }\left(\mathrm{f}_{12}-\mathrm{VTZ}: \mathrm{PBE}\right)\left(\mathrm{Me} \mathrm{:}^{\mathrm{t} B \mathrm{Bu}}\right) \\
\left(\mathrm{Mes}^{*} \mathrm{OH} / \mathrm{Mes}^{*} \mathrm{O}\right)^{[\mathrm{o}]}\end{array}$ & & & 361 \\
\hline $\begin{array}{c}\operatorname{CCSD}(\mathrm{T})-\mathrm{f}_{12} / \operatorname{VDZ}\left(\mathrm{H}:{ }^{\mathrm{t}} \mathrm{Bu}\right) \\
\left(\mathrm{Mes}^{*} \mathrm{OH} / \mathrm{Mes}^{*} \mathrm{O}\right)^{[\mathrm{g}]}\end{array}$ & & & 394 & $\begin{array}{l}\text { DLPNO-CCSD (TO)/cc- } \\
\mathrm{pV}(\mathrm{T}, \mathrm{Q}) \mathrm{Z}(\mathrm{PP})(23 / 24)^{[\mathrm{p}]}\end{array}$ & 32.6 & 18.8 & 297 \\
\hline $\begin{array}{c}\operatorname{CCSD}(\mathrm{T})-\mathrm{f}_{12} / \mathrm{VDZ}\left(\mathrm{Me}:^{\mathrm{P} r} \mathrm{r}\right) \\
(23 / 24)^{[\mathrm{h}]}\end{array}$ & 7.4 & 38.8 & 281 & 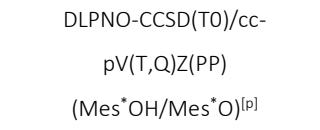 & & & 365 \\
\hline $\begin{array}{c}\operatorname{CCSD}(\mathrm{T})-\mathrm{f}_{12} / \mathrm{VDZ}\left(\mathrm{Me}:{ }^{\mathrm{t} B u}\right) \\
\left(\mathrm{Mes}^{*} \mathrm{OH} / \mathrm{Mes}^{*} \mathrm{O}\right)^{[\mathrm{h}]}\end{array}$ & & & 371 & $\begin{array}{l}\text { DLPNO-CCSD }(T 0) / \text { def2- } \\
(T, Q Z) Z V P P(23 / 24)^{[q]}\end{array}$ & 34.4 & 15.4 & 298 \\
\hline $\begin{array}{c}\operatorname{CCSD}(\mathrm{T})-\mathrm{f}_{12} / \operatorname{VDZ}\left({ }^{t} \mathrm{Bu}:{ }^{\mathrm{t}} \mathrm{Bu}\right) \\
\left(\mathrm{Mes}^{*} \mathrm{OH} / \mathrm{Mes}^{*} \mathrm{O}\right)^{[i]}\end{array}$ & & & 359 & $\begin{array}{c}\text { DLPNO-CCSD(TO)/def2- } \\
(\mathrm{T}, \mathrm{QZ}) Z \mathrm{ZVPP}\left(\mathrm{Mes}^{*} \mathrm{OH} / \mathrm{Mes}^{*} \mathrm{O}\right)^{[q]}\end{array}$ & & & 366 \\
\hline $\begin{array}{c}\operatorname{CCSD}(T)-f_{12} / V T Z(H: P r) \\
(23 / 24)^{[j]}\end{array}$ & -5.6 & 47.7 & 290 & $\begin{array}{c}\text { DLPNO-CCSD(T1)/def2- } \\
(T, Q) Z V P P(23 / 24)^{[r]}\end{array}$ & 15.9 & 18.8 & 296 \\
\hline $\begin{array}{c}\operatorname{CCSD}(\mathrm{T})-\mathrm{f}_{12} / \mathrm{NTZ}\left(\mathrm{H}:{ }^{t} \mathrm{Bu}\right) \\
\left(\mathrm{Mes}^{*} \mathrm{OH} / \mathrm{Mes}^{*} \mathrm{O}\right)^{[j]}\end{array}$ & & & 394 & $\begin{array}{c}\text { DLPNO-CCSD(T1)/def2- } \\
(\mathrm{T}, \mathrm{Q}) Z \mathrm{ZVPP}\left(\mathrm{Mes}^{*} \mathrm{OH} / \mathrm{Mes}^{*} \mathrm{O}\right)^{[r]}\end{array}$ & & & 369 \\
\hline $\begin{array}{c}\operatorname{CCSD}(\mathrm{T})-\mathrm{f}_{12} / \mathrm{VTZ}\left(\mathrm{Me}: \mathrm{t}^{\mathrm{Bu}}\right) \\
\left(\mathrm{Mes}^{*} \mathrm{OH} / \mathrm{Mes}^{*} \mathrm{O}\right)^{[k]}\end{array}$ & & & 372 & & & & \\
\hline
\end{tabular}

[a]All molecular geometries and thermal contributions to energies are at PBEO-D3/def2TZVP level. [b] Excitation energy at $0 \mathrm{~K}$ from the triplet(3A")/doublet(2A") ground state of 23 and 24 to the lowest energy singlet $\left(1 A^{\prime}\right) /$ quartet $\left(4 A^{\prime}\right)$ electromer. [c] Reaction energies for reaction (3) computed at $0 \mathrm{~K}$, without correction for thermal contributions or SOC effects. [d] PBEO-D3/def2TZVP level. [e] PBEO-D3/def2TZVP level for the hydrogen truncated model systems (H:iPr/tBu). [f] PBEOD3/def2TZVP level for the methyl truncated model systems (Me:iPr/tBu). [g] CCSD(T)-f12/VDZ level for hydrogen truncated model systems (H:iPr/tBu) [h] CCSD(T)f12/VDZ level for methyl truncated model systems (Me:iPr/tBu)). [i] CCSD(T)-f12/VDZ level for the untruncated Mes*OH/Mes*O couple. [j]CCSD(T)-f12/VTZ level for hydrogen truncated model systems (H:iPr/tBu)). [k]CCSD(T)-f12/NTZ level for the methyl truncated Mes*OH/Mes*O couple. [l] ONIOM results from eq. (5) on the CCSD(T)-f12/VDZ level with hydrogen truncated model systems. [m] ONIOM results from eq. (5) on the CCSD(T)-f12/VDZ level with methyl truncated model systems. [n] ONIOM results from eq. (5) on the CCSD(T)-f12/NTZ level with hydrogen truncated model systems. [o] ONIOM results from eq. (4.7) on the CCSD(T)f12/VTZ level with methyl truncated model systems.[p] DLPNO-CCSD(TO)/-cc-pV(T,Q)Z(PP) level. [q] DLPNO-CCSD(TO)/def2-pV(T,Q)ZVPP level. [r] DLPNO$\operatorname{CCSD}(T 1) /$ def2-pV(T,Q)ZVPP level. 
Table 29: Relative energies in $\mathrm{kJmol}^{-1}$ for the full reaction (4.6) energetics including enthalpic contribution and SOC effects.

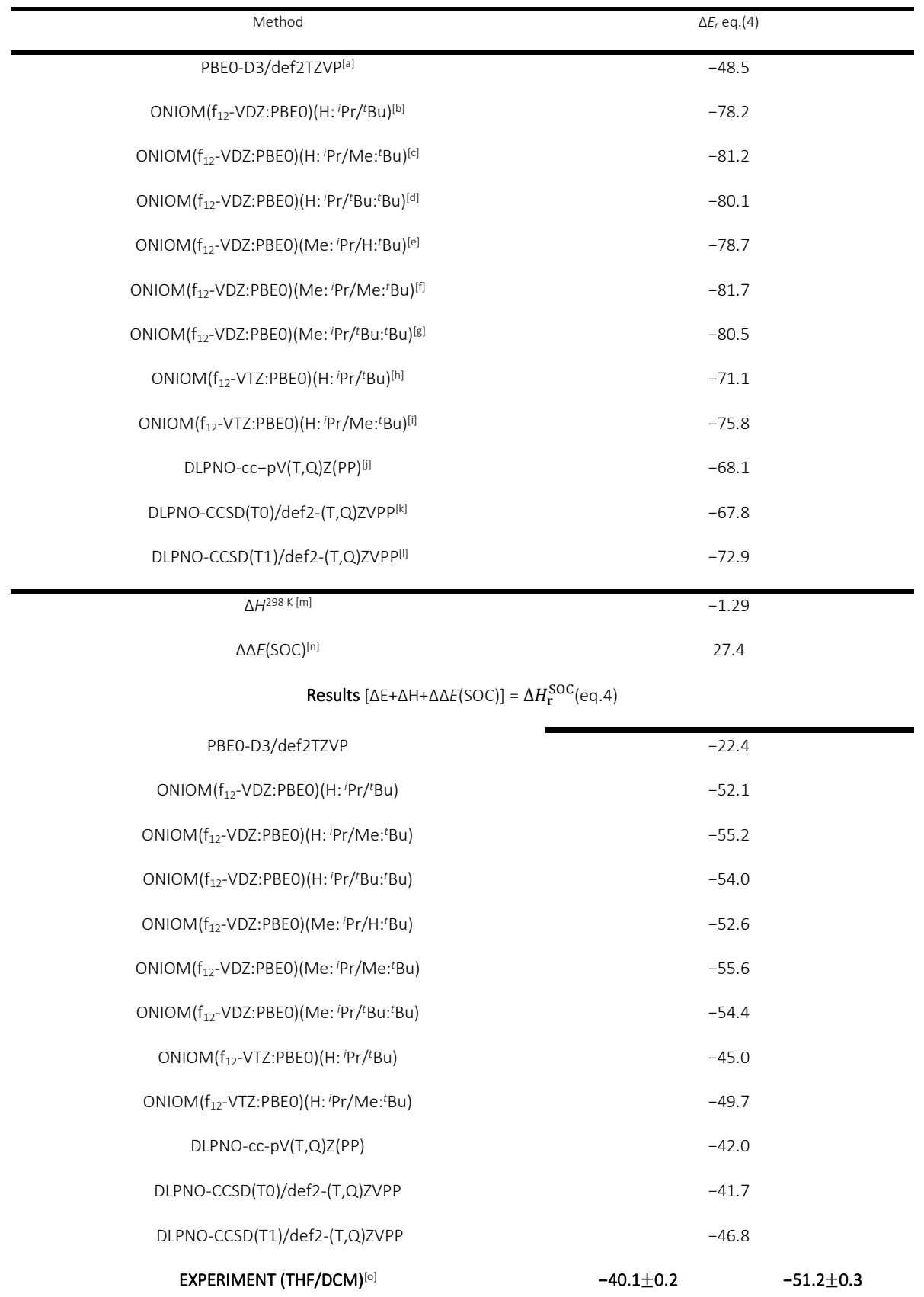

[a]PBEO-D3/def2TZVP level. [b] ONIOM energy for reaction (4.8) on the CCSD(T)-f12/VDZ level with H truncated model systems. [c] ONIOM energy for reaction (4.8) on the CCSD(T)-f12/VDZ level with $\mathrm{H}$ truncated model systems for 23 and 24 and Me truncated model systems for the Mes*OH/Mes*O couple. [d] ONIOM energy for reaction (4.8) on the CCSD(T)-f12/VDZ level with $\mathrm{H}$ truncated model systems for 23 and 24 and the untruncated Mes*OH/Mes*O couple. [e] ONIOM energy for reaction (4.8) on the CCSD(T)-f12/VDZ level with Me truncated model systems for 23 and 24 and the hydrogen truncated models systems for the Mes* OH/Mes*O couple. [f] ONIOM energy for reaction (4.8) on the CCSD(T)-f12/VDZ level with Me truncated model systems for 23 and 24 and the Me truncated models systems for the Mes*OH/Mes*O couple. [g] ONIOM energy for reaction (4.8) on the CCSD(T)-f12/VDZ level with Me truncated model systems for 23 and 24 and the untruncated the Mes* OH/Mes*O couple. [h] ONIOM energy for reaction (4.8) on the CCSD(T)-f12/VTZ level with $\mathrm{H}$ truncated model systems. [i] ONIOM energy for reaction (4.8) on the CCSD(T)-f12/VTZ level with $\mathrm{H}$ truncated model systems 23 and 24 and the Me truncated models systems for the Mes*OH/Mes*O couple. [i] DLPNO-CCSD(T0)/-cC-pV(T,Q)Z(PP) energy for reaction (4.8). [k] DLPNO-CCSD(TO)/def2-pV(T,Q)ZVPP energy for reaction (4.8). [I] DLPNO-CCSD(T1)/def2$\mathrm{pV}(\mathrm{T}, \mathrm{Q}) Z \mathrm{~V} P P$ energy for reaction (4.8) [m] Thermal contributions on the PBEO-D3/def2TZVP level. [n] Spin-Orbit-Coupling(SOC) contribution by CASSCF NEVPT2/QDPT treatment see Section 4.3.1. 


\subsubsection{Total Energies}

Table 30: Total energies in $E_{\mathrm{h}}$ of 23 and 24 as well as the Mes* OH/Mes*O couple and the corresponding methyl and hydrogen truncated model systems at the PBEO-D3/def2TZVP level.

\begin{tabular}{|c|c|c|c|c|c|}
\hline \multirow{3}{*}{ Species } & \multirow{3}{*}{ State } & \multicolumn{4}{|c|}{ PBE0-D3/def2TZVP } \\
\hline & & \multicolumn{2}{|c|}{ Fullsystem } & \multirow{2}{*}{$\begin{array}{c}\mathrm{Me} \\
\boldsymbol{E}_{\text {tot }}\end{array}$} & \multirow{2}{*}{$\begin{array}{c}\mathrm{H} \\
E_{\text {tot }}\end{array}$} \\
\hline & & $E_{t o t}$ & $\Delta H_{\text {corr }}^{298 K}$ & & \\
\hline Rhenium Amine $\mathbf{2 3}$ & ${ }^{3} A^{\prime \prime}$ & -2827.427856 & 0.554282 & -2513.175041 & -2355.988952 \\
\hline Rhenium Amine 23 & ${ }^{1} A^{\prime}$ & -2827.413110 & 0.554224 & -2513.163329 & -2355.983429 \\
\hline Rhenium Amide 24 & ${ }^{2} A^{\prime \prime}$ & -2826.841094 & 0.540469 & -2512.560877 & -2355.373971 \\
\hline Rhenium Amide 24 & ${ }^{4} A^{\prime}$ & -2826.834887 & 0.54034 & -2512.555096 & -2355.363929 \\
\hline Mes* $\mathrm{OH}$ & ${ }^{1} A^{\prime}$ & -778.6104991 & 0.465602 & -425.0888723 & -307.2358522 \\
\hline Mes*O & ${ }^{2} A^{\prime \prime}$ & -777.9789138 & 0.452282 & -424.4532747 & -306.5900897 \\
\hline
\end{tabular}

Table 31: Total energies at the $\operatorname{CCSD}\left(T^{*}\right)-f 12 / \operatorname{VnZ}(n=D, T)$ level in $E_{h}$ of the $(H / M e)$ truncated model systems as well as the un-truncated version of the Mes*OH/Mes*O couple.

\begin{tabular}{|c|c|c|c|c|c|c|}
\hline \multirow{2}{*}{ Species } & \multirow{2}{*}{ State } & \multicolumn{2}{|c|}{$\operatorname{CCSD}\left(T^{*}\right)-f 12 @ H$} & \multicolumn{2}{|c|}{$\operatorname{CCSD}\left(T^{*}\right)-f 12 @ \mathrm{Me}$} & \multirow{2}{*}{$\begin{array}{c}\operatorname{CCSD}\left(T^{*}\right)-f 12 \text { untruncated } \\
\text { VDZ }\end{array}$} \\
\hline & & VDZ & VTZ & VDZ & VTZ & \\
\hline Rhenium Amine 23 & ${ }^{3} A^{\prime \prime}$ & -2354.081467 & -2354.183069 & -2511.14589 & - & - \\
\hline Rhenium Amine 23 & ${ }^{1} \mathrm{~A}^{\prime}$ & -2354.084254 & -2354.185199 & -2511.143081 & - & - \\
\hline Rhenium Amide 24 & ${ }^{2} A^{\prime \prime}$ & -2353.473631 & -2353.572496 & -2510.539028 & - & - \\
\hline Rhenium Amide 24 & ${ }^{4} A^{\prime}$ & -2353.454891 & -2353.554311 & -2510.524251 & - & - \\
\hline Mes*OH & ${ }^{1} A^{\prime}$ & -307.0240698 & -307.0240698 & -424.7861547 & -424.8385756 & -778.044967 \\
\hline Mes*O & ${ }^{2} A^{\prime \prime}$ & -306.3741126 & -306.3741126 & -424.1452075 & -424.1969558 & -777.408469 \\
\hline
\end{tabular}

Table 32: Total energies at the ONIOM(CCSD $\left.\left(T^{*}\right)-f 12 / V n Z(n=D, T) / P B E 0\right)$ level in $E_{h}$ of the $(H / M e)$ truncated model systems as well as the untruncated version of the Mes* ${ }^{*}$ H/Mes* ${ }^{*}$ couple.

\begin{tabular}{|c|c|c|c|c|c|}
\hline \multirow{2}{*}{ Species } & \multirow{2}{*}{ State } & \multicolumn{2}{|c|}{ ONIOM (H: $\left.\mathrm{Pr} ; \mathrm{H}:{ }^{t} \mathrm{Bu}\right)$} & \multicolumn{2}{|c|}{ ONIOM (Me: $\left.{ }^{i} \mathrm{Pr} ; \mathrm{Me}:{ }^{t} \mathrm{Bu}\right)$} \\
\hline & & VDZ & VTZ & VDZ & VTZ \\
\hline Rhenium Amine 23 & ${ }^{2} A^{\prime \prime}$ & -2825.52037 & -2825.62197 & -2825.398705 & - \\
\hline Rhenium Amine 23 & ${ }^{4} A^{\prime}$ & -2825.51393 & -2825.61488 & -2825.392862 & - \\
\hline Rhenium Amide 24 & ${ }^{3} \mathrm{~A}^{\prime \prime}$ & -2824.91439 & -2825.01326 & -2824.792882 & - \\
\hline Rhenium Amide 24 & ${ }^{1} A^{\prime}$ & -2824.89987 & -2824.99929 & -2824.778062 & - \\
\hline Mes*OH & ${ }^{1} A^{\prime}$ & -777.762937 & -777.762937 & -777.6708466 & -777.7357124 \\
\hline Mes*O & ${ }^{2} \mathrm{~A}^{\prime \prime}$ & -778.398717 & -778.398717 & -778.3077814 & -778.3602023 \\
\hline
\end{tabular}


Table 33: Total energies in $E_{\mathrm{h}}$ computed at the DLPNO-cc-pV(T,Q)Z(PP) level of theory.

\begin{tabular}{|c|c|c|c|c|}
\hline \multirow{2}{*}{ Species } & \multirow{2}{*}{ State } & \multicolumn{3}{|c|}{ DLPNO-cc-pV(T,Q)Z(PP) } \\
\hline & & cc-pVTZ & cc-pVQZ & $\mathrm{CBS}(\mathrm{T}, \mathrm{Q})$ \\
\hline Rhenium Amine 23 & ${ }^{3} A^{\prime \prime}$ & -2825.107629 & -2825.490213 & -2825.736087 \\
\hline Rhenium Amine 23 & ${ }^{1} \mathrm{~A}^{\prime}$ & -2825.093800 & -2825.477307 & -2825.723677 \\
\hline Rhenium Amide 24 & ${ }^{2} A^{\prime \prime}$ & -2824.496800 & -2824.878193 & -2825.123150 \\
\hline Rhenium Amide 24 & ${ }^{4} A^{\prime}$ & -2824.491424 & -2824.871825 & -2825.115995 \\
\hline Mes*OH & ${ }^{1} \mathrm{~A}^{\prime}$ & -777.816691 & -778.044694 & -778.187228 \\
\hline Mes*O & ${ }^{2} A^{\prime \prime}$ & -777.180270 & -777.406721 & -777.548343 \\
\hline
\end{tabular}

Table 34: Total energies in $E_{\mathrm{h}}$ computed at the DLPNO-CCSD(TO)def2-(T,Q)ZVPP level of theory.

\begin{tabular}{|c|c|c|c|c|}
\hline \multirow{2}{*}{ Species } & \multirow{2}{*}{ State } & \multicolumn{3}{|c|}{ DLPNO-CCSD(TO)-def2-(T,Q)ZVPP } \\
\hline & & cc-pVTZ & cc-pVQZ & $\mathrm{CBS}(\mathrm{T}, \mathrm{Q})$ \\
\hline Rhenium Amine 23 & ${ }^{3} A^{\prime \prime}$ & -2824.765030 & -2825.164712 & -2825.406985 \\
\hline Rhenium Amine 23 & ${ }^{1} \mathrm{~A}^{\prime}$ & -2824.751862 & -2825.151617 & -2825.393868 \\
\hline Rhenium Amide 24 & ${ }^{2} A^{\prime \prime}$ & -2824.153006 & -2824.551895 & -2824.793466 \\
\hline Rhenium Amide 24 & ${ }^{4} A^{\prime}$ & -2824.147898 & -2824.546390 & -2824.787589 \\
\hline $\mathrm{Mes}^{*} \mathrm{OH}$ & ${ }^{1} \mathrm{~A}^{\prime}$ & -777.832086 & -778.047275 & -778.186284 \\
\hline Mes*O & ${ }^{2} A^{\prime \prime}$ & -777.195596 & -777.409059 & -777.546938 \\
\hline
\end{tabular}

Table 35: Total energies in $E_{\mathrm{h}}$ computed at the DLPNO-CCSD(T1)/def2-(T,Q)ZVPP level of theory.

\begin{tabular}{ccccc}
\hline \multirow{2}{*}{ Species } & \multirow{2}{*}{ State } & \multicolumn{3}{c}{ DLPNO-CCSD(T1)-def2-(T,Q)ZVPP } \\
\cline { 3 - 5 } & & cC-pVTZ & cc-pVQZ & CBS(T,Q) \\
\hline Rhenium Amine 23 & ${ }^{3} \mathrm{~A}^{\prime \prime}$ & -2824.775277 & -2825.173306 & -2825.414356 \\
Rhenium Amine 23 & ${ }^{1} \mathrm{~A}^{\prime}$ & -2824.764930 & -2825.165463 & -2825.408288 \\
Rhenium Amide 24 & ${ }^{2} \mathrm{~A}^{\prime \prime}$ & -2824.164967 & -2824.561681 & -2824.801641 \\
Rhenium Amide 24 & ${ }^{4} \mathrm{~A}^{\prime}$ & -2824.156341 & -2824.553934 & -2824.794467 \\
Mes*OH & ${ }^{1} \mathrm{~A}^{\prime}$ & -777.838427 & -778.053747 & -778.192853 \\
Mes*O & ${ }^{2} \mathrm{~A}^{\prime \prime}$ & -777.200845 & -777.414406 & -777.552359 \\
\hline
\end{tabular}




\subsection{Interconversion of Phosphinyl Radical and Phosphinidene Complexes by Proton Coupled Electron Transfer}

All molecules were optimized on the PBE-D3/def2SVP level of theory. The quartet isomer of the free phosphinyl radical was as well computed to ensure the discussion of the lowest energy structure. The singlet root for the PMes* molecule failed to converge. The BDE values were computed for the equation 4.10:

$$
\mathrm{X}-\mathrm{H} \rightarrow \mathrm{X}+\mathrm{H}
$$

Where $\mathrm{X}-\mathrm{H}$ is either 17 or HPMes* and $\mathrm{X}$ is 19 or PMes*

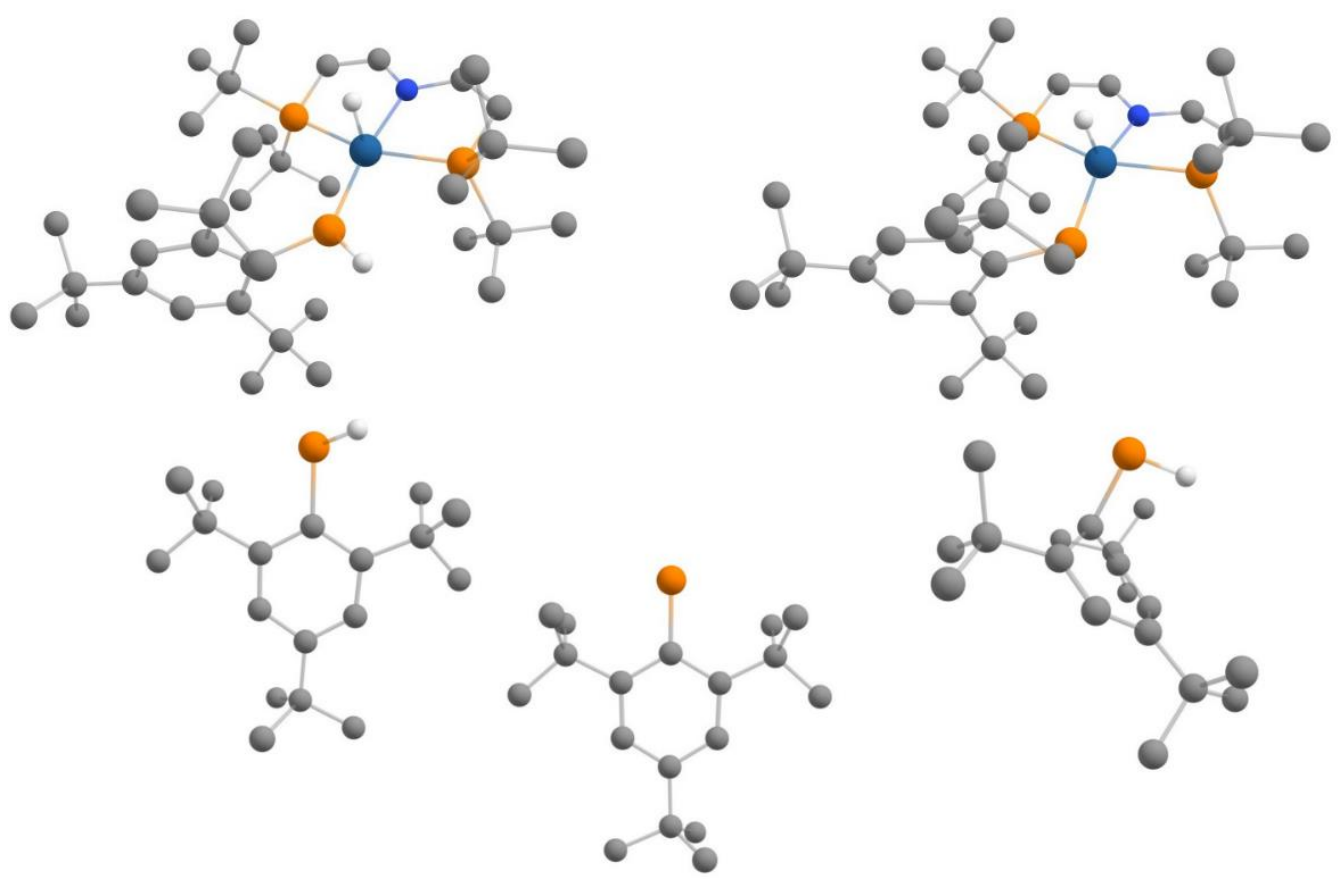

Figure 79: Computed minimum structures of $\mathrm{H}_{n} \mathrm{PMes}^{*}(\mathrm{n}=1,0)$ and the osmium complexes 17 and 19 on the PBE-D3/def2SVP level of theory. Top left: 17 doublet electromer. Top right: 19 singlet electromer. Bottom left: Doublet electromer of HPMes*. Bottom centre: Triplet electromer of PMes*. Bottom right: Quartet electromer of HPMes*.

The quartet isomer of HPMes* is significantly higher in energy with $\Delta(D / Q)=269 \mathrm{~kJ} \mathrm{~mol}^{-1}$. Coordination has a significant effect of $\triangle B D E=-23.4 \mathrm{~kJ} \mathrm{~mol}^{-1}$ on the $\mathrm{P}-\mathrm{H}$ bond strength compared to the free phosphinyl radical. The total energies of the computed structures can be found in Table 36.

Table 36: Total energies of 17 and 19 as well as the energies of the free phosphinyl radical, its quartet electromer and of PMes* at the PBE-D3/def2SVP level of theory.

\begin{tabular}{cccc}
\hline & \multicolumn{2}{c}{ PBE-D3/def2SVP } \\
Species & State & \multicolumn{2}{c}{ Fullsystem } \\
& & $\boldsymbol{E}_{\text {tot }}$ & $\Delta H_{\text {corr }}^{\mathbf{2 9}}$ \\
\hline Osmium Phosphinyl Radical 17 & ${ }^{2} \mathrm{~A}$ & -2656.56726 & 1.038222 \\
Osmium Phosphinidene 19 & ${ }^{\mathrm{A}} \mathrm{A}$ & -2655.96535 & 1.029094 \\
HPMes* & ${ }^{2} \mathrm{~A}$ & -1043.61561 & 0.444862 \\
HPMes* & ${ }^{4} \mathrm{~A}$ & -1043.51316 & 0.442785 \\
PMes* & ${ }^{3} \mathrm{~A}$ & -1043.00517 & 0.436193 \\
\hline
\end{tabular}




\section{Benchmarked Experimental Results}

Within this thesis several thermodynamic parameters have been benchmarked by isothermal titration calorimetry. Since BDE values might be object to changes of reference values only the pure reaction parameters are listed. If not otherwise noted, the experiments were performed at $298 \mathrm{~K}$.

Benchmarked values $\Delta \mathrm{H}_{\mathrm{r}}$ and $\mathrm{K}$ (see Section 3.1):

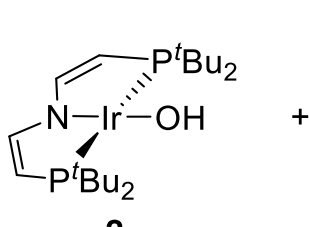

2<smiles>CC(C)(C)c1cc(Br)c([O])c(Br)c1</smiles><smiles>CCCC</smiles><smiles>CC(C)(C)c1cc(Br)c(O)c(C(C)(C)C)c1</smiles>

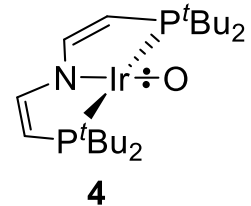

4

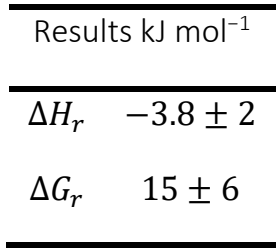

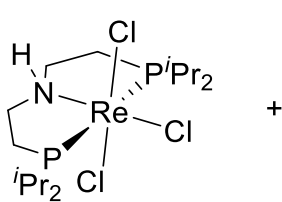

23<smiles>CC(C)(C)c1cc(C(C)(C)C)c(O)c(C(C)(C)C)c1</smiles><smiles>C[R9][Ba]F</smiles><smiles>PP1P2P3P1P(Cl)(Cl)(Cl)C23Cl</smiles>

24<smiles>CC(C)(C)c1cc(C(C)(C)C)c(O)c(C(C)(C)C)c1</smiles>

Benchmarked value $\Delta \mathrm{H}_{\mathrm{r}}$ (see Section 3.2):

\begin{tabular}{|c|c|c|c|c|}
\hline$\Delta H_{r} / \mathrm{kJ} \mathrm{mol}^{-1}$ & \multicolumn{2}{|c|}{$\begin{array}{l}\text { Error at } 95 \% \\
\text { confidence }\end{array}$} & $n$ value & Corrected Error \\
\hline-51.2 & \multicolumn{2}{|l|}{ \pm 0.28} & 0.943 & \pm 0.30 \\
\hline-51.3 & \multicolumn{2}{|l|}{ \pm 0.24} & 1.01 & \pm 0.24 \\
\hline-51.3 & \multicolumn{2}{|l|}{ \pm 0.36} & 1.05 & \pm 0.38 \\
\hline Weighted Average & \multicolumn{4}{|c|}{$\Delta H^{298 \mathrm{~K}}(\mathrm{DCM})=-51.2 \pm 0.3 \mathrm{~kJ} \mathrm{~mol}^{-1}$} \\
\hline$\Delta H_{r} / \mathrm{kJ} \mathrm{mol}^{-1}$ & \multicolumn{4}{|c|}{$\begin{array}{l}\text { Error at } 95 \% \\
\text { confidence }\end{array}$} \\
\hline-40.0 & \pm 0.25 & 1.02 & & \pm 0.26 \\
\hline-39.9 & \pm 0.20 & 1.04 & & \pm 0.21 \\
\hline-40.7 & \pm 0.29 & 1.05 & & \pm 0.30 \\
\hline Weighted Average & \multicolumn{4}{|c|}{$\Delta H^{298 \mathrm{~K}}(\mathrm{THF})=-40.1 \pm 0.2 \mathrm{~kJ} \mathrm{~mol}^{-1}$} \\
\hline
\end{tabular}




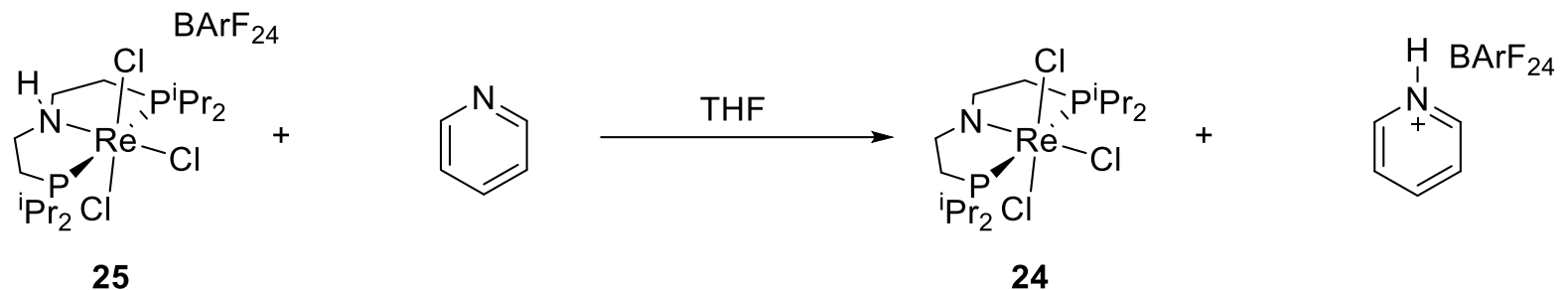

Benchmarked value $K$ (see Section 3.3)

\begin{tabular}{lll}
\hline Run & $K$ & $\begin{array}{l}95 \% \text { Confidence } \\
\text { Error }\end{array}$ \\
\hline 1 & & 0.12 \\
2 & 5.32 & 0.12 \\
\cline { 1 - 1 } & 5.32 & \\
\hline
\end{tabular}

Benchmarked value $K_{D}$ (see Section 3.4)<smiles>CC(C)Cc1cc(CC(C)C)c(O)c(C(C)(C)C)c1</smiles><smiles>CCN=P(N=P(N)(OC)N(C)C)(N(C)C)N(C)C</smiles>

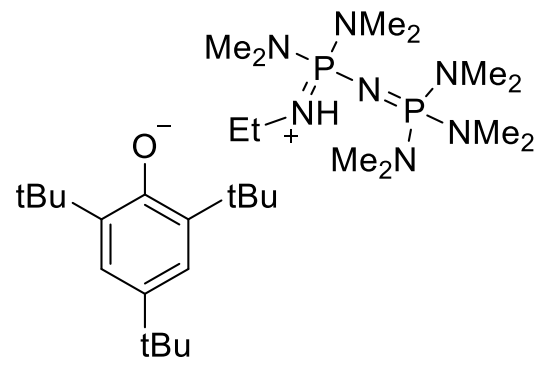

\begin{tabular}{cccc}
\hline$K_{\mathrm{D}} / \mathrm{mol} \mathrm{L}^{-1}$ & Error at 95\% confidence & $\mathrm{n}$ value & Corrected Error \\
\hline $1.32 \cdot 10^{-3}$ & $\pm 0.08 \cdot 10^{-3}$ & 1.00 & $\pm 0.08 \cdot 10^{-3}$ \\
$1.22 \cdot 10^{-3}$ & $\pm 0.04 \cdot 10^{-3}$ & 1.03 & $\pm 0.04 \cdot 10^{-3}$ \\
$1.20 \cdot 10^{-3}$ & $\pm 0.06 \cdot 10^{-3}$ & 1.00 & $\pm 0.06 \cdot 10^{-3}$ \\
\cline { 1 - 1 } Weighted Average & $K_{D}=1.24 \pm 0.06 \cdot 10^{-3} \mathrm{M}^{-1}$ & & \\
\hline
\end{tabular}

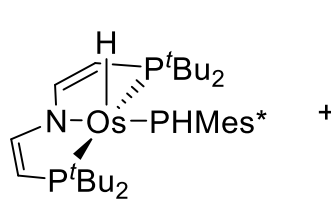

17<smiles>CC(C)(C)c1cc(C(C)(C)C)c([O])c(C(C)(C)C)c1</smiles>

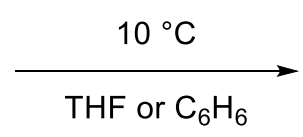

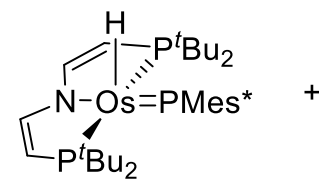

19<smiles>CC(C)(C)c1cc(C(C)(C)C)c(O)c(C(C)(C)C)c1</smiles> 


\section{Benchmarked value $\Delta \mathrm{H}_{\mathrm{r}}(283 \mathrm{~K})$ : (see Section 3.5)}

Benzene:

\begin{tabular}{|c|c|c|c|}
\hline Injection & $\begin{array}{l}\text { Heat of injection } \\
\text { of the } 1^{\text {st }} \text { run } / \mu \mathrm{J}\end{array}$ & $\begin{array}{l}\text { Heat of injection } \\
\text { of the } 2^{\text {nd }} \text { run } / \mu \text { J }\end{array}$ & $\begin{array}{c}\text { Added moles } \\
\text { per injection / nmol }\end{array}$ \\
\hline 3 & -4682 & -4678 & 68.2 \\
\hline 4 & -4708 & -4678 & 68.2 \\
\hline 5 & -4703 & -4709 & 68.2 \\
\hline 6 & -4716 & -4711 & 68.2 \\
\hline Mean & $-4702 \pm 20$ & $-4694 \pm 17$ & \\
\hline Weighted Mean & \multicolumn{2}{|c|}{$-4697 \pm 13$} & \\
\hline Reaction Enthalpy / $\mathrm{kJ} \mathrm{mol}^{-1}$ & \multicolumn{2}{|c|}{$-68.9 \pm 3.6$} & \\
\hline
\end{tabular}

THF:

\begin{tabular}{ccc}
\hline \multirow{2}{*}{ Injection } & $\begin{array}{c}\text { Heat of injection } \\
\text { of the } 1^{\text {st }} \text { run } / \mu \mathrm{J}\end{array}$ & Added moles \\
& & \\
\hline 2 & -4069 & 56.9 \\
3 & -4066 & 56.9 \\
4 & -4050 & 56.9 \\
5 & -4049 & 56.9 \\
Mean & $-4059 \pm 10$ & \\
\hline \hline Reaction Enthalpy / kJ mol & \multicolumn{2}{c|}{$-71 \pm 5 \mathrm{~kJ} \mathrm{~mol}^{-1}$} \\
\hline
\end{tabular}

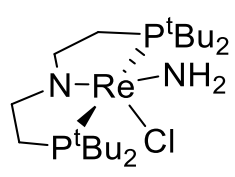

20

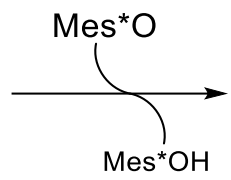

$\mathrm{Mes}^{*} \mathrm{OH}$

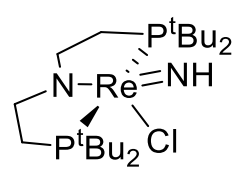

21

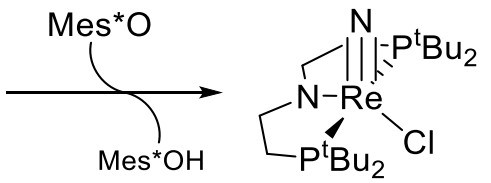

22

Benchmarked value $\Delta H_{r}$ (see Section 3.6):

\section{Results}

Mean Heat $[\mu \mathrm{J}] \quad$ Consumed moles per injection [nmol] Enthalpy $[\mathrm{kJ} / \mathrm{mol}]$

\begin{tabular}{|c|c|c|}
\hline$-4176 \pm 27$ & 19.62 & $-213 \pm 1$ \\
\hline$-3957 \pm 40$ & 18.76 & $-211 \pm 2$ \\
\hline \multirow[t]{2}{*}{$-4017 \pm 39^{*}$} & 18.76 & \multirow[t]{2}{*}{$-214 \pm 2$} \\
\hline & Weighted mean enthalpy $=-213 \pm 2 \mathrm{~kJ} \mathrm{~mol}^{-1}$ & \\
\hline
\end{tabular}


Chapter VII

6. Literature 
[1] I. G. Denisov, T. M. Makris, S. G. Sligar, I. Schlichting, Chem. Rev. 2005, 105, 2253-2277.

[2] H. Sitzmann, J. Hartmann-Schreier, "Sauerstoff," can be found under https://roempp.thieme.de/lexicon/RD-19-00520, 2011.

[3] T. W. Lyons, C. T. Reinhard, N. J. Planavsky, Nature 2014, 506, 307-315.

[4] D. Voet, J. G. Voet, C. W. Pratt, Fundamentals of Biochemistry: Life at the Molecular Level, John Wiley \& Sons, 2016.

[5] N. B. Terwilliger, J. Exp. Biol. 1998, 201, 1085-1098.

[6] L. Pauling, C. D. Coryell, Proc. N. A. S. 1936, 22, 210-216.

[7] G. Gilardi, G. Di Nardo, Rend. Fis. Acc. Lincei 2017, 28, 159-167.

[8] K. L. Bren, R. Eisenberg, H. B. Gray, Proc. Natl. Acad. Sci. 2015, 112, 13123-13127.

[9] T. Yamanaka, K. Okunuki, in Microb. Iron Metab. (Ed.: J. Neilands), Academic Press, 1974, pp. 349-400.

[10] J. Rittle, M. T. Green, Science 2010, 330, 933-937.

[11] D. L. Simmons, R. M. Botting, T. Hla, Pharmacol. Rev. 2004, 56, 387-437.

[12] J. N. Rodríguez-López, D. J. Lowe, J. Hernández-Ruiz, A. N. P. Hiner, F. García-Cánovas, R. N. F. Thorneley, J. Am. Chem. Soc. 2001, 123, 11838-11847.

[13] C. Krebs, D. G. Fujimori, C. T. Walsh, J. M. Bollinger, Acc. Chem. Res. 2007, 40, 484-492.

[14] N. Cox, M. Retegan, F. Neese, D. A. Pantazis, A. Boussac, W. Lubitz, Science 2014, 345, 804-808.

[15] R. Pal, C. F. A. Negre, L. Vogt, R. Pokhrel, M. Z. Ertem, G. W. Brudvig, V. S. Batista, Biochemistry 2013, 52, 7703-7706.

[16] K. Ray, F. Heims, M. Schwalbe, W. Nam, Curr. Opin. Chem. Biol. 2015, 25, 159-171.

[17] K. Gunay, A.; Theopold, Chem. Rev. 2010, 1060-1081.

[18] H. B. Gray, Chem. Int. 2019, 41, 16-19.

[19] C. J. Ballhausen, H. B. Gray, Inorg. Chem. 1962, 1, 111-122.

[20] J. R. et al. Winkler, in Mol. Electron. Struct. Transistion Met. Complexes I, 2012, pp. 17-28.

[21] U. Hintermair, S. W. Sheehan, A. R. Parent, D. H. Ess, D. T. Richens, P. H. Vaccaro, G. W. Brudvig, R. H. Crabtree, J. Am. Chem. Soc. 2013, 135, 10837-10851.

[22] M. Zhou, D. Balcells, A. R. Parent, R. H. Crabtree, O. Eisenstein, ACS Catal. 2012, 2, 208-218.

[23] B. A. Vaughan, M. S. Webster-Gardiner, T. R. Cundari, T. B. Gunnoe, Science 2015, 348, 421-424.

[24] J. L. Roizen, M. E. Harvey, J. Du Bois, Acc. Chem. Res. 2012, 45, 911-922.

[25] J. M. Mayer, Acc. Chem. Res. 2011, 44, 36-46.

[26] Y. Park, Y. Kim, S. Chang, Chem. Rev. 2017, 117, 9247-9301.

[27] D. M. Jenkins, T. A. Betley, J. C. Peters, J. Am. Chem. Soc. 2002, 124, 11238-11239.

[28] X. Hu, K. Meyer, J. Am. Chem. Soc. 2004, 126, 16322-16323.

[29] D. T. Shay, G. P. A. Yap, L. N. Zakharov, A. L. Rheingold, K. H. Theopold, Angew. Chemie - Int. Ed. 2005, 44, 1508-1510.

[30] R. E. Cowley, R. P. Bontchev, J. Sorrell, O. Sarracino, Y. Feng, H. Wang, J. M. Smith, J. Am. Chem. Soc. 2007, 129, 2424-2425.

[31] J. Du, L. Wang, M. Xie, L. Deng, Angew. Chemie - Int. Ed. 2015, 54, 12640-12644.

[32] E. R. King, G. T. Sazama, T. A. Betley, J. Am. Chem. Soc. 2012, 134, 17858-17861. 
[33] M. K. Goetz, E. A. Hill, A. S. Filatov, J. S. Anderson, J. Am. Chem. Soc. 2018, 140, 13176-13180.

[34] A. M. Geer, C. Tejel, J. A. López, M. A. Ciriano, 2014, 5720-5724.

[35] D. S. Glueck, J. Wu, F. J. Hollander, R. G. Bergman, J. Am. Chem. Soc. 1991, 113, 2041-2054.

[36] R. S. Hay-Motherwell, G. Wilkinson, B. Hussain-Bates, M. B. Hursthouse, Polyhedron 1993, 12, $2009-2012$.

[37] M. Kinauer, M. Diefenbach, H. Bamberger, S. Demeshko, E. J. Reijerse, C. Volkmann, C. Würtele, J. Van Slageren, B. De Bruin, M. C. Holthausen, et al., Chem. Sci. 2018, 9, 4325-4332.

[38] B. G. Jacobi, D. S. Laitar, L. Pu, M. F. Wargocki, A. G. DiPasquale, K. C. Fortner, S. M. Schuck, S. N. Brown, Inorg. Chem. 2002, 41, 4815-4823.

[39] Y. Shimoyama, T. Kojima, Inorg. Chem. 2019, 58, 9517-9542.

[40] P. F. Kuijpers, J. I. van der Vlugt, S. Schneider, B. de Bruin, I. Van Der Vlugt, S. Schneider, B. De Bruin, Chem. Eur. J. 2017, 23, 13819-13829.

[41] M. G. Scheibel, B. Askevold, F. W. Heinemann, E. J. Reijerse, B. De Bruin, S. Schneider, Nat. Chem. 2012, 4, 552-558.

[42] D. J. Mindiola, G. L. Hillhouse, J. Am. Chem. Soc. 2001, 123, 4623-4624.

[43] V. M. Iluc, G. L. Hillhouse, J. Am. Chem. Soc. 2010, 132, 15148-15150.

[44] C. A. Laskowski, A. J. M. Miller, G. L. Hillhouse, T. R. Cundari, J. Am. Chem. Soc. 2011, 133, 771-773.

[45] A. Grünwald, N. Orth, A. Scheurer, F. W. Heinemann, A. Pöthig, D. Munz, Angew. Chemie - Int. Ed. 2018, 57, 16228-16232.

[46] K. M. Carsch, I. M. DiMucci, D. A. Iovan, A. Li, S. L. Zheng, C. J. Titus, S. J. Lee, K. D. Irwin, D. Nordlund, K. M. Lancaster, et al., Science 2019, 365, 1138-1143.

[47] E. Poverenov, I. Efremenko, A. I. Frenkel, Y. Ben-David, L. J. W. Shimon, G. Leitus, L. Konstantinovski, J. M. L. Martin, D. Milstein, Nature 2008, 455, 1093-1096.

[48] R. L. S. James T. Hynes, Judith P. Klinman, Hans-Heinrich Limbach, Ed. , Hydrogen Transfer Reactions, WileyVCH, Weinheim, Germany, 2007.

[49] D. J. Vinyard, G. M. Ananyev, G. Charles Dismukes, Annu. Rev. Biochem. 2013, 82, 577-606.

[50] C. J. M. Van Der Ham, M. T. M. Koper, D. G. H. Hetterscheid, Chem. Soc. Rev. 2014, 43, 5183-5191.

[51] D. C. Miller, K. T. Tarantino, R. R. Knowles, Top. Curr. Chem. 2016, 374, 1-59.

[52] C. J. Gagliardi, B. C. Westlake, C. A. Kent, J. J. Paul, J. M. Papanikolas, T. J. Meyer, Coord. Chem. Rev. 2010, 254, 2459-2471.

[53] J. J. Warren, T. A. Tronic, J. M. Mayer, Chem Rev. 2010, 110, 6961-7001.

[54] M. H. V. Huynh, T. J. Meyer, Chem. Rev. 2007, 107, 5004-5064.

[55] R. A. Binstead, B. A. Moyer, G. J. Samuels, T. J. Meyer, J. Am. Chem. Soc. 1981, 103, 2897-2899.

[56] C. Costentin, D. H. Evans, M. Robert, J. M. Savéant, P. S. Singh, J. Am. Chem. Soc. 2005, 127, $12490-12491$.

[57] M. K. Goetz, J. S. Anderson, J. Am. Chem. Soc. 2019, 141, 4051.

[58] D. Bím, M. Maldonado-Domínguez, L. Rulísek, M. Srnec, Proc. Natl. Acad. Sci. 2018, 115, E10287-E10294.

[59] J. M. Mayer, Acc. Chem. Res. 1998, 31, 441-450.

[60] F. G. Bordwell, J.-P. Chen, J. A. Harrelson Jr., J. Am. Chem. Soc. 1988, 110, 1229-1231.

[61] W. S. Matthews, J. E. Bares, J. E. Bartmess, F. J. Cornforth, G. E. Drucker, R. J. McCallum, G. J. McCollum, N. R. Vanier, F. G. Bordwell, Z. Margolin, J. Am. Chem. Soc. 1975, 97, 7006-7014. 
[62] F. G. Bordwell, Acc. Chem. Res. 1988, 21, 456-463.

[63] M. Tilset, in Electron Transf. Chem. (Ed.: V. Balzani), WILEY-VCH Verlag GmbH, Weinheim, Germany, 2001, pp. 1-800.

[64] E. Roduner, Radiat. Phys. Chem. 2005, 72, 201-206.

[65] V. D. Parker, M. Tilset, J. Am. Chem. Soc. 1989, 111, 6711-6717.

[66] E. A. Mader, E. R. Davidson, J. M. Mayer, J. Am. Chem. Soc. 2007, 129, 5153-5166.

[67] K. B. WIBERG, Oxidation-Reduction Mechanisms in Organic Chemistry, ACADEMIC PRESS INC., 1963.

[68] K. B. Wiberg, R. Eisenthal, Tetrahedron 1964, 20, 1151-1161.

[69] T. R. Cundari, R. R. Conry, E. Spaltenstein, S. C. Critchlow, K. A. Hall, S. K. Tahmassebi, J. M. Mayer, Organometallics 1994, 13, 322-331.

[70] G. K. Cook, J. M. Mayer, J. Am. Chem. Soc. 1994, 116, 1855-1868.

[71] G. K. Cook, J. M. Mayer, J. Am. Chem. Soc. 1995, 117, 7139-7156.

[72] M. Szostak, N. J. Fazakerley, D. Parmar, D. J. Procter, Chem. Rev. 2014, 114, 5959-6039.

[73] T. V. Chciuk, W. R. Anderson, R. A. Flowers, J. Am. Chem. Soc. 2016, 138, 8738-8741.

[74] T. V. Chciuk, W. R. Anderson, R. A. Flowers, Angew. Chemie - Int. Ed. 2016, 55, 6033-6036.

[75] M. Pourbaix, Atlas of Electrochemical Equilibria in Aqueous Solutions, National Association Of Corrosion Engineers, Houston, 1974.

[76] W. A. Nugent, J. A. Mayer, Metal-Ligand Multiple Bonds, Wiley-Interscience, 1988.

[77] W. J. Mijs, C. R. H. I. de Jonge, Eds., Organic Synthesis by Oxidation with Metal Compounds, Plenum, New York, 1986.

[78] B. A. Moyer, T. J. Meyer, Inorg. Chem. 1981, 20, 436-444.

[79] E. L. Lebeau, R. A. Binstead, T. J. Meyer, J. Am. Chem. Soc. 2001, 123, 10535-10544.

[80] D. T. Sawyer, Oxygen Chemistry, Oxford University Press, New York, 1991.

[81] L. Duan, F. Bozoglian, S. Mandal, B. Stewart, T. Privalov, A. Llobet, L. Sun, Nat. Chem. 2012, 4, 418-423.

[82] H. S. Yu, S. L. Li, D. G. Truhlar, J. Chem. Phys. 2016, 145, DOI 10.1063/1.4963168.

[83] J. P. Perdew, K. Schmidt, AIP Conf. Proc. 2001, 577, 1-20.

[84] W. Koch, M. C. Holthausen, A Chemist's Guide to Density Functional Theory, Wiley-VCH Verlag GmbH, Weinheim, Germany, 2001.

[85] R. J. Bartlett, J. Chem. Phys. 2019, 151, DOI 10.1063/1.5116338.

[86] R. A. Friesner, Proc. Natl. Acad. Sci. U. S. A. 2005, 102, 6648-6653.

[87] R. J. Bartlett, J. F. Stanton, in Rev. Comput. Chem. (Eds.: K.B. Lipkowitz, D.B. Boyd), VCH Publishers, Inc., 1995, pp. 65-169.

[88] P.-O. Löwdin, in Adv. Chem. Phys. (Ed.: I. Prigogine), John Wiley \& Sons, Inc., 1958.

[89] M. Kodrycka, K. Patkowski, J. Chem. Phys. 2019, 151, 070901:1-17.

[90] H. J. Werner, F. R. Manby, J. Chem. Phys. 2006, 124, 054114:1-12.

[91] F. Neese, F. Wennmohs, A. Hansen, J. Chem. Phys. 2009, 130, 114108:1-18.

[92] F. Neese, A. Hansen, D. G. Liakos, J. Chem. Phys. 2009, 131, 064103:1-15.

[93] C. Riplinger, F. Neese, J. Chem. Phys. 2013, 138, 034106:1-18.

[94] Y. Guo, C. Riplinger, U. Becker, D. G. Liakos, Y. Minenkov, L. Cavallo, F. Neese, J. Chem. Phys. 2018, 148, 
011101.

[95] C. Riplinger, P. Pinski, U. Becker, E. F. Valeev, F. Neese, J. Chem. Phys. 2016, 144, 024109.

[96] L. W. Chung, W. M. C. Sameera, R. Ramozzi, A. J. Page, M. Hatanaka, G. P. Petrova, T. V. Harris, X. Li, Z. Ke, F. Liu, et al., Chem. Rev. 2015, 115, 5678-5796.

[97] M. Svensson, S. Humbel, K. Morokuma, J. Chem. Phys. 1996, 105, 3654-3661.

[98] M. Svensson, R. D. J. Froese, T. Matsubara, S. Sieber, K. Morokuma, J. Chem. Phys. 1996, 100, 1935719363.

[99] C. Edmiston, M. Krauss, J. Chem. Phys. 1965, 42, 1119-1120.

[100] C. Edmiston, M. Krauss, J. Chem. Phys. 1966, 45, 1833-1839.

[101] G. Czakó, E. Mátyus, A. G. Császár, J. Phys. Chem. A 2009, 113, 11665-11678.

[102] P. R. Bunker, P. Jensen, W. P. Krämer, R. Beardsworth, J. Chem. Phys. 1986, 85.

[103] G. Wedler, H.-J. Freund, Lehrbuch Der Physikalischen Chemie, WILEY-VCH Verlag GmbH, Weinheim, Germany, 2012.

[104] B. O. Roos, P. M. Siegbahn, J. Am. Chem. Soc. 1977, 99, 7716-7718.

[105] B. O. Roos, P. R. Taylor, P. E. M. Siegbahn, Chem. Phys. 1980, 48, 157-173.

[106] P. Siegbahn, A. Heiberg, B. Roos, B. Levy, Phys. Scr. 1980, 21, 323-327.

[107] P. E. M. Siegbahn, J. Almlöf, A. Heiberg, B. O. Roos, J. Chem. Phys. 1981, 74, 2384-2396.

[108] J. Olsen, Int. J. Quantum Chem. 2011, 111, 3267-3272.

[109] P. G. Szalay, T. Müller, G. Gidofalvi, H. Lischka, R. Shepard, Chem. Rev. 2012, 112, 108-181.

[110] C. Angeli, R. Cimiraglia, S. Evangelisti, T. Leininger, J. P. Malrieu, J. Chem. Phys. 2001, 114, 10252.

[111] C. Angeli, R. Cimiraglia, J. P. Malrieu, Chem. Phys. Lett. 2001, 350, 297-305.

[112] C. Angeli, R. Cimiraglia, J. P. Malrieu, J. Chem. Phys. 2002, 117, 9138-9153.

[113] K. Andersson, P. Å. Malmqvist, B. O. Roos, A. J. Sadlej, K. Wolinski, J. Phys. Chem. 1990, 94, 5483-5488.

[114] K. Andersson, P. Å. Malmqvist, B. O. Roos, J. Chem. Phys. 1991, 96, 1218-1226.

[115] J. M. Rintelman, I. Adamovic, S. Varganov, M. S. Gordon, J. Chem. Phys. 2005, 122, 044105:1-7.

[116] C. Camacho, H. A. Witek, S. Yamamoto, J. Comput. Chem. 2008, 30, 468-478.

[117] B. Nagy, F. Jensen, in Rev. Comput. Chem. (Eds.: A.L. Parrill, K.B. Lipkowitz.), John Wiley \& Sons, Inc., 2017, pp. 93-149.

[118] F. Weigend, R. Ahlrichs, Phys.Chem. Chem. Phys. 2005, 7, 3297-3305.

[119] T. H. Dunning, J. Chem. Phys. 1989, 90, 1007-1023.

[120] D. E. Woon, T. H. Dunning, J. Chem. Phys. 1993, 98, 1358-1371.

[121] K. A. Peterson, T. B. Adler, H. J. Werner, J. Chem. Phys. 2008, 128, 084102.

[122] L. Kong, F. A. Bischoff, E. F. Valeev, Chem. Rev. 2012, 112, 75-107.

[123] J. G. Hill, K. A. Peterson, G. Knizia, H. J. Werner, J. Chem. Phys. 2009, 131, 194105:1-13.

[124] G. Knizia, T. B. Adler, H. J. Werner, J. Chem. Phys. 2009, 130, 054104.

[125] W. Klopper, F. R. Manby, S. Ten-No, E. F. Valeev, Int. Rev. Phys. Chem. 2006, 25, 427-468.

[126] S. Ten-no, J. Noga, Wiley Interdiscip. Rev. Comput. Mol. Sci. 2012, 2, 114-125.

[127] S. Ten-No, Theor. Chem. Acc. 2012, 131, 1070:1-11.

[128] A. Einstein, Ann. Phys. 1905, 322, 891-921. 
[129] P. Pyykkö, in Adv. QUANTUM Chem., Academic Press, 1978, pp. 354-409.

[130] P. Pyykkö, Chem Rev. 1988, 12, 563-594.

[131] W. H. E. Schwarz, E. M. van Wezenbeek, E. J. Baerends, J. G. Snijders, J. Phys. B 1989, 22, 1515-1530.

[132] J. L. Dehmer, Phys. Rev. A 1973, 7, 4-9.

[133] B. Lepetit, J. M. Launay, M. LE Dourneuf, Chem. Phys. 1986, 106, 103-110.

[134] C. Heinemann, H. Schwarz, W. Koch, K. G. Dyall, J. Chem. Phys. 1996, 104, 4642.

[135] T. Fleig, Relativistic String-Based Electron Correlation Methods, Springer, Dordrecht, Heidelberg,London, New York, 2010.

[136] P. E. M. Siegbahn, M. Svensson, R. H. Crabtree, J. Am. Chem. Soc. 1995, 117, 6758-6765.

[137] J. J. Carroll, J. C. Weissharr, P. E. M. Siegbahn, C. A. M. Wittborn, M. R. A. Blomberg, J. Phys. Chem. 1995, 99, 14388-14396.

[138] K. Chen, G. Zhang, H. Chen, J. Yao, D. Danovich, S. Shaik, J. Chem. Theory Comput. 2012, 8, 1641-1645.

[139] P. Jerabek, L. Vondung, P. Schwerdtfeger, Chem. - A Eur. J. 2018, 24, 6047-6051.

[140] M. Srnec, J. Chalupsky, M. Fojta, L. Zendlova, L. Havran, M. Hocek, M. Kyvala, L. Rulisek, J. Am. Chem. Soc. 2008, 130, 10947-10954.

[141] P. Pyykkö, Annu. Rev. Phys. Chem. 2012, 63, 45-64.

[142] D. Peng, M. Reiher, Theor. Chem. Acc. 2012, 131, 1081:1-20.

[143] D. A. Pantazis, X. Y. Chen, C. R. Landis, F. Neese, J. Chem. Theory Comput. 2008, 4, 908-919.

[144] D. A. Pantazis, X. Y. Chen, C. R. Landis, F. Neese, J. Chem. Theory Comput. 2008, 4, 908-919.

[145] D. Figgen, K. A. Peterson, M. Dolg, H. Stoll, J. Chem. Phys. 2009, 130, 164108.

[146] M. Dolg, in Handb. Relativ. Quantum Chem., Springer-Verlag, Berlin Heidelberg, 2017, pp. 449-478.

[147] C. Chang, M. Pelissier, P. Durand, Phys. Scr. 1986, 34, 394-404.

[148] E. Van Lenthe, R. Van Leeuwen, E. J. Baerends, J. G. Snijders, Int. J. Quantum Chem. 1993, 99, 4597-4610.

[149] E. Van Lenthe, E. J. Baerends, J. G. Snijders, J. Chem. Phys. 1994, 101, 9783-9792.

[150] R. Van Leeuwen, E. Van Lenthe, E. J. Baerends, J. G. Snijders, J. Chem. Phys. 1994, 101, 1272-1281.

[151] E. Van Lenthe, R. Van Leeuwen, E. J. Baerends, J. G. Snijders, Int. J. Quant. Chem. 1996, 57, $281-293$.

[152] M. Barysz, in Theor. Chem. Phys. Heavy Super Heavy Elem. (Ed.: U.K. and S. Wilson), Kluwer Academic Publishers, 2003, pp. 349-397.

[153] "ADF Relativistic effects," can be found under https://www.scm.com/doc/ADF/Input/Relativistic_effects.html, 2020.

[154] "ORCA Input Libary Relativistic Approximations," can be found under https://sites.google.com/site/orcainputlibrary/relativistic-approximations, 2020.

[155] B. A. Heß, C. M. Marian, U. Wahlgren, O. Gropen, Chem. Phys. Lett. 1996, 251, 365-371.

[156] D. Ganyushin, F. Neese, J. Chem. Phys. 2013, 138, 1-18.

[157] D. Delony, M. Kinauer, M. Diefenbach, S. Demeshko, C. Würtele, M. C. Holthausen, S. Schneider, Angew. Chemie - Int. Ed. 2019, 58, 10971-10974.

[158] F. Schendzielorz, M. Finger, J. Abbenseth, C. Würtele, V. Krewald, S. Schneider, Angew. Chemie - Int. Ed. 2019, 131, 840-844.

[159] M. Kinauer, Transient and Stable Terminal Imido Complexes of Iridium, Georg-August Universität 
Göttingen, 2019.

[160] J. Meiners, M. G. Scheibel, M. H. Lemée-Cailleau, S. A. Mason, M. B. Boeddinghaus, T. F. Fässler, E. Herdtweck, M. M. Khusniyarov, S. Schneider, Angew. Chemie - Int. Ed. 2011, 50, 8184-8187.

[161] D. A. Ortmann, H. Werner, Zeitschrift fur Anorg. und Allg. Chemie 2002, 628, 1373-1376.

[162] K. Ilg, H. Werner, Organometallics 1999, 18, 5426-5428.

[163] R. J. Burford, W. E. Piers, D. H. Ess, M. Parvez, J. Am. Chem. Soc. 2014, 136, 3256-3263.

[164] D. Sieh, M. Schlimm, L. Andernach, F. Angersbach, S. Nückel, J. Schöffel, N. Šušnjar, P. Burger, Eur. J. Inorg. Chem. 2012, 444-462.

[165] L. M. Green, D. W. Meek, Organometallics 1989, 8, 659-666.

[166] D. Morales-Morales, D. W. Lee, Z. Wang, C. M. Jensen, Organometallics 2001, 20, 1144-1147.

[167] M. D. Millard, C. E. Moore, A. L. Rheingold, J. S. Figueroa, J. Am. Chem. Soc. 2010, 132, 8921-8923.

[168] P. Kläring, S. Pahl, T. Braun, A. Penner, Dalt. Trans. 2011, 40, 6785-6791.

[169] J. C. M. Ritter, R. G. Bergman, J. Am. Chem. Soc. 1997, 119, 2580-2581.

[170] C. A. Miller, T. S. Janik, C. H. Lake, L. M. Toomey, M. R. Churchill, J. D. Atwood, Organometallics 1994, 13, 5080-5087.

[171] F. T. Ladipo, M. Kooti, J. S. Merola, Inorg. Chem. 1993, 32, 1681-1688.

[172] B. J. Truscott, D. J. Nelson, C. Lujan, A. M. Z. Slawin, S. P. Nolan, Chem. - A Eur. J. 2013, 19, 7904-7916.

[173] V. Chandrasekhar, B. Mahanti, P. Bandipalli, K. Bhanuprakash, Inorg. Chem. 2012, 51, 10536-10547.

[174] W. J. Tenn, K. J. H. Young, J. Oxgaard, R. J. Nielsen, W. A. Goddard, R. A. Periana, Organometallics 2006, $25,5173-5175$.

[175] H. Jacobs, U. Metzner, Zeitschrift fur Anorg. und Allg. Chemie 1991, 597, 97-106.

[176] J. U. Rohde, W. T. Lee, J. Am. Chem. Soc. 2009, 131, 9162-9163.

[177] I. Efremenko, E. Poverenov, J. M. L. Martin, D. Milstein, J. Am. Chem. Soc. 2010, 132, 14886-14900.

[178] G. R. Fulmer, A. J. M. Miller, N. H. Sherden, H. E. Gottlieb, A. Nudelman, B. M. Stoltz, J. E. Bercaw, K. I. Goldberg, Organometallics 2010, 29, 2176-2179.

[179] M. Kinauer, M. G. Scheibel, J. Abbenseth, F. W. Heinemann, P. Stollberg, C. Würtele, S. Schneider, Dalt. Trans. 2014, 43, 4506-4513.

[180] H. Effenberger, K. Mereiter, J. Zemann, Zeitschrift fur Krist. - New Cryst. Struct. 1981, 156, $233-243$.

[181] J. J. Scepaniak, C. G. Margarit, J. N. Harvey, J. M. Smith, Inorg. Chem. 2011, 50, 9508-9517.

[182] F. S. Schendzielorz, M. Finger, C. Volkmann, C. Würtele, S. Schneider, Angew. Chemie - Int. Ed. 2016, 55, 11417-11420.

[183] Y. Y. Rusakov, I. L. Rusakova, L. B. Krivdin, Magn. Reson. Chem 2018, 56, 1061-1073.

[184] J. Abbenseth, D. Delony, M. C. Neben, W. Christian, B. De Bruin, S. Schneider, Angew. Chemie - Int. Ed. 2019, 6404-6407.

[185] M. H. Abraham, P. L. Grellier, D. V Prior, P. P. Duce, J. J. Morris, P. J. Taylor, J Chem. Soc. Perkin Trans 1989, II, 699-711.

[186] M. H. Abraham, P. L. Grellier, D. V Prior, J. J. Morris, P. J. Taylor, J Chem. Soc. Perkin Trans 1990, 2, 521529.

[187] E. P. Cappellani, S. D. Drouin, G. Jia, P. A. Maltby, R. H. Morris, C. T. Schweitzer, J. Am. Chem. Soc. 1994, 
116, 3375-3388.

[188] G. B. Shul'Pin, Catalysts 2016, 6, 1-40.

[189] F. Jia, Z. Li, Org. Chem. Front. 2014, 1, 194-214.

[190] P. C. Li, T. S. Wang, G. H. Lee, Y. H. Liu, Y. Wang, C. T. Chen, I. Chao, J. Org. Chem. 2002, 67, 8002-8009.

[191] Z. B. Alfassi, D. M. Golden, J. Am. Chem. Soc. 1973, 95, 319-323.

[192] C. Hansch, A. Leo, R. W. Taft, Chem. Rev. 1991, 91, 165-195.

[193] S. Marque, P. Tordo, in New Asp. Phosphorous Chem. V, Top. Curr. Chem. (Ed.: J. Majoral), Springer, Berlin, Heidelberg, 2005, p. 43.

[194] P. P. Power, Chem. Rev. 2003, 103, 789-809.

[195] A. Armstrong, T. Chivers, R. T. Boeré, The Diversity of Stable and Persistent Phosphorus-Containing Radicals, ACS Symposium Series, 2005.

[196] C. D. Martin, M. Soleilhavoup, G. Bertrand, Chem. Sci. 2013, 4, 3020-3030.

[197] V. Nesterov, D. Reiter, P. Bag, P. Frisch, R. Holzner, A. Porzelt, S. Inoue, Chem. Rev. 2018, 118, 9678-9842.

[198] J. K. Pagano, B. J. Ackley, R. Waterman, Chem. - A Eur. J. 2018, 24, 2554-2557.

[199] K. Pal, O. B. Hemming, B. M. Day, T. Pugh, D. J. Evans, R. A. Layfield, Angew. Chemie - Int. Ed. 2016, 55, 1690-1693.

[200] G. Dequirez, V. Pons, P. Dauban, Angew. Chemie - Int. Ed. 2012, 51, 7384-7395.

[201] M. J. Bezdek, S. Guo, P. J. Chirik, Science 2016, 354, 730.

[202] S. S. Kolmar, J. M. Mayer, J. Am. Chem. Soc. 2017, 139, 10687-10692.

[203] R. Melenkivitz, D. J. Mindiola, G. L. Hillhouse, J. Am. Chem. Soc. 2002, 124, 3846-3847.

[204] J. Abbenseth, M. Diefenbach, S. C. Bete, C. Würtele, C. Volkmann, S. Demeshko, M. C. Holthausen, S. Schneider, Chem. Commun. 2017, 53, 5511-5514.

[205] F. G. Bordwell, J. Cheng, J. Am. Chem. Soc. 1991, 113, 1736-1743.

[206] P. H. Pfromm, J. Renew. Sustain. Energy 2017, 9, 034702:1-11.

[207] R. Lan, S. Tao, Front. Energy Res. 2014, 2, 3-6.

[208] I. Klopsch, M. Kinauer, M. Finger, C. Würtele, S. Schneider, Angew. Chemie - Int. Ed. 2016, 55, 4786-4789.

[209] B. M. Lindley, R. S. van Alten, M. Finger, F. Schendzielorz, C. Würtele, A. J. M. Miller, I. Siewert, S. Schneider, J. Am. Chem. Soc. 2018, 140, 7922-7935.

[210] Coord. Chem. Rev. 1996, 150, 77-110.

[211] B. H. P. Gunz, G. J. Leigh, J.Chem. Soc. A. 1971, 2229-2233.

[212] B. Bleaney, J. Magn. Reson. 1972, 8, 91-100.

[213] B. Martin, J. Autschbach, J. Chem. Phys. 2015, 142, DOI 10.1063/1.4906318.

[214] J. Chatt, G. J. Leigh, D. M. P. Mingos, J. Chem. Soc. 1969, 1974-1980.

[215] I. P. Theor, A. Ligands, B. E. W. Randall, D. Shaw, M. College, M. E. Road, E. London, J. Chem. Soc. A 1969, 2867-2872.

[216] A. Soncini, W. Van Den Heuvel, J. Chem. Phys. 2013, 138, 021103.

[217] V. I. Belova, Y. L. Sykrin, E. G. Ippolitov, A. S. Kotelnikova, G. K. Babeshkina, R. A. Dovlyatshina, Zhurnal Strukt. Khimii 1964, 5, 252-257.

[218] A. Earnshaw, B. N. Figgis, J. Lewis, R. D. Peacock, J .Chem. Soc. 1961. 
[219] F. Cariati, A. Sgamellotti, F. Morazzoni, V. Valenti, Inorganica Chim. Acta 1971, 5, 531-535.

[220] G. Rouschias, G. Wilkinson, G. Wilkinson, J .Chem. Soc. A. 1967, 993-1000.

[221] N. S. Hush, J. R. Reimers, Chem. Rev. 2000, 100, 775-786.

[222] S. Tshepelevitsh, A. Kütt, M. Lõkov, I. Kaljurand, J. Saame, A. Heering, P. G. Plieger, R. Vianello, I. Leito, European J. Org. Chem. 2019, 2019, 6735-6748.

[223] R. M. Fuoss, J. Am. Chem. Soc. 1958, 80, 5059-5061.

[224] T. Wiseman, S. Williston, J. F. Brandts, L. N. Lin, Anal. Biochem. 1989, 179, 131-137.

[225] M. G. Scheibel, J. Abbenseth, M. Kinauer, F. W. Heinemann, C. Wu, B. De Bruin, S. Schneider, Inorg. Chem. 2015, 54, 9290-9302.

[226] V. W. Manner, T. F. Markle, J. H. Freudenthal, J. P. Roth, J. M. Mayer, Chem. Commun. 2008, 246, 256258.

[227] J. Meiners, A. Friedrich, E. Herdtweck, S. Schneider, Organometallics 2009, 28, 6331-6338.

[228] N. Elgrishi, K. J. Rountree, B. D. McCarthy, E. S. Rountree, T. T. Eisenhart, J. L. Dempsey, J. Chem. Educ. 2018, 95, 197-206.

[229] D. R. Lide, Ed. , CRC Handbook of Chemistry and Physics: A Ready-Reference Book of Chemical and Physical Data, CRC Press, Boca Raton, 2005.

[230] M. J. O. S. Budavari, A. Smith, P. E. Heckelman, The Merck Index, an Encyclopedia of Chemicals, Drugs, and Biologicals, Merck Co. Inc., Rahway, NJ, 1989.

[231] H. E. Gottlieb, V. Kotlyar, A. Nudelman, J. Org. Chem. 1997, 62, 7512-7515.

[232] E. Bill, JulX, Program for Simulation of Molecular Magnetic Data Matrix Diagonalization Is Done with the Routine ZHEEV from the LAPACK Numerical Package. Parameter Optimization Is Performed with the Simplex Routine AMOEBA from NUMERICAL RECIPES, Max-Planck Institute For Chemical Energy Conversion,, Mülheim/Ruhr, 2008.

[233] Bruker AXS Inc., APEX2 V2016.9-0 (SAINT/SADABS/SHELXT/SHELXL), Madison, WI, USA, 2016.

[234] G. M. Sheldrick, Acta Cryst. 2015, A71, 3-8.

[235] G. M. Sheldrick, Acta Cryst. 2015, C71, 3-8.

[236] G. M. Sheldrick, Acta Cryst. 2008, A64, 112-122.

[237] D. F. Evans, J. Chem. Soc. 1959, 2003-2005.

[238] S. K. Sur, J. Magn. Reson. 1989, 82, 169-173.

[239] M. J. Frisch, G. W. Trucks, H. B. Schlegel, G. E. Scuseria, M. A. Robb, J. R. Cheeseman, G. Scalmani, V. Barone, G. A. Petersson, H. Nakatsuji, et al., Gaussian, Inc., Wallingford, CT 2016.

[240] S. Grimme, J. Antony, S. Ehrlich, H. Krieg, J Chem Phys 2010, 154104, 1-9.

[241] A. D. Becke, J. Chem. Phys. 1993, 98, 5648-5652.

[242] C. Adamo, J Chem Phys 2008, 110, 6158-6170.

[243] D. Andrae, U. Häußermann, M. Dolg, H. Stoll, H. Preuß, Theor. Chim. Acta 1990, 123-141.

[244] A. V Marenich, C. J. Cramer, D. G. Truhlar, J. Phys. Chem. B 2009, 113, 6378-6396.

[245] D. Aravena, M. Atanasov, A. A. Auer, U. Becker, G. Bistoni, M. Brehm, D. Bykov, V. G. Chilkuri, D. Datta, A. K. Dutta, et al., Max-Planck-Institut f"ur Chem. Energiekonversion, Mülheim a. d. Ruhr, Ger. 2017.

[246] F. Neese, WIREs Comput Mol Sci 2012, 2, 73-78. 
[247] R. A. Kendall, T. H. Dunning, R. J. Harrison, J. Chem. Phys. 1992, 96, 6796-6806.

[248] F. Weigend, A. Köhn, C. Hättig, J. Chem. Phys. 2002, 116, 3175-3183.

[249] S. Ten-no, Chem. Phys. Lett. 2004, 398, 56-61.

[250] T. B. Adler, G. Knizia, H. J. Werner, J. Chem. Phys. 2007, 127, 221106.

[251] H.-J. Werner, P. J. Knowles, G. Knizia, M. F.R, M. Schütz, P. Celani, W. Györffy, D. Kats, T. Korona, R. Lindh, et al., "MOLPRO, version 2015.1 a package of ab initio programs," 2015.

[252] K. E. Yousaf, K. A. Peterson, J. Chem. Phys. 2008, 129, 184108.

[253] S. Kritikou, J. G. Hill, J. Chem. Theory Comput. 2015, 11, 5269-5276.

[254] F. Weigend, J. Comput. Chem. 2007, 29, 167-175.

[255] J. G. Hill, J. Chem. Phys. 2011, 135, 044105.

[256] C. Van Wüllen, J. Chem. Phys. 1998, 109, 392-399.

[257] F. Weigend, Phys. Chem. Chem. Phys. 2002, 4, 4285-4291.

[258] M. Atanasov, D. Aravena, E. Suturina, E. Bill, D. Maganas, F. Neese, Coord. Chem. Rev. 2015, 289-290, 177214.

[259] C. F. Wise, R. G. Agarwal, J. M. Mayer, J. Am. Chem. Soc. 2020, 142, 10681-10691.

[260] E. Brunner, J. Chem. Eng. Data 1985, 30, 269-273. 


\title{
Chapter VIII
}

\author{
7. Appendix
}




\section{A. Crystal Structures}

\section{A.1. X-Ray Single-Crystal Analysis of 1}

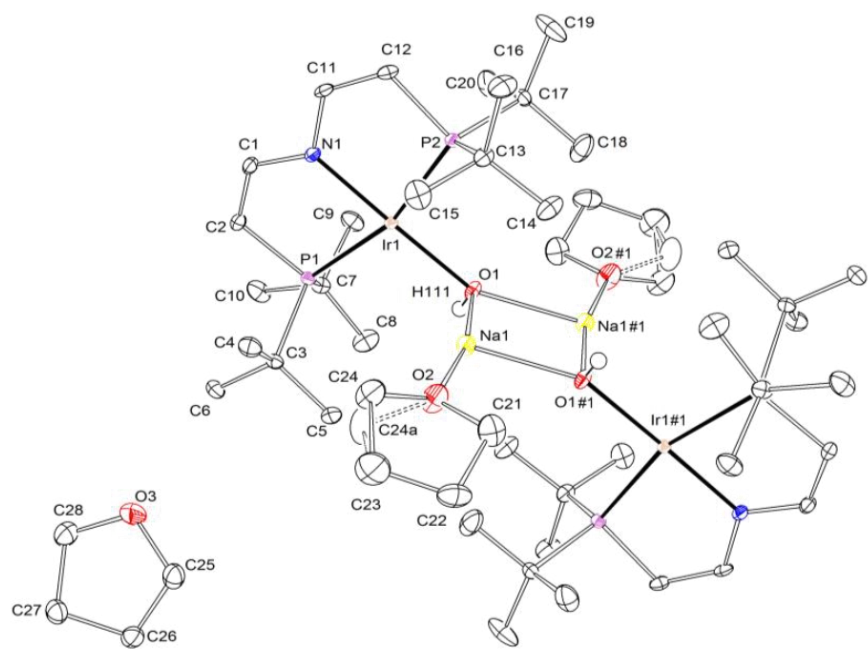

Figure A1: Thermal ellipsoid plot of 1 with the anisotropic displacement parameters drawn at the 50\% probability level. The asymmetric unit contains a half disordered complex molecule and one THF solvent molecule. The disordered complex molecule was refined with population of 0.64(3) on the main domain. The $\mathrm{O}-\mathrm{H}$ hydrogen atom was found from the residual density map and isotropically refined.

Table A1: Crystallographic data and structure refinement for 1.

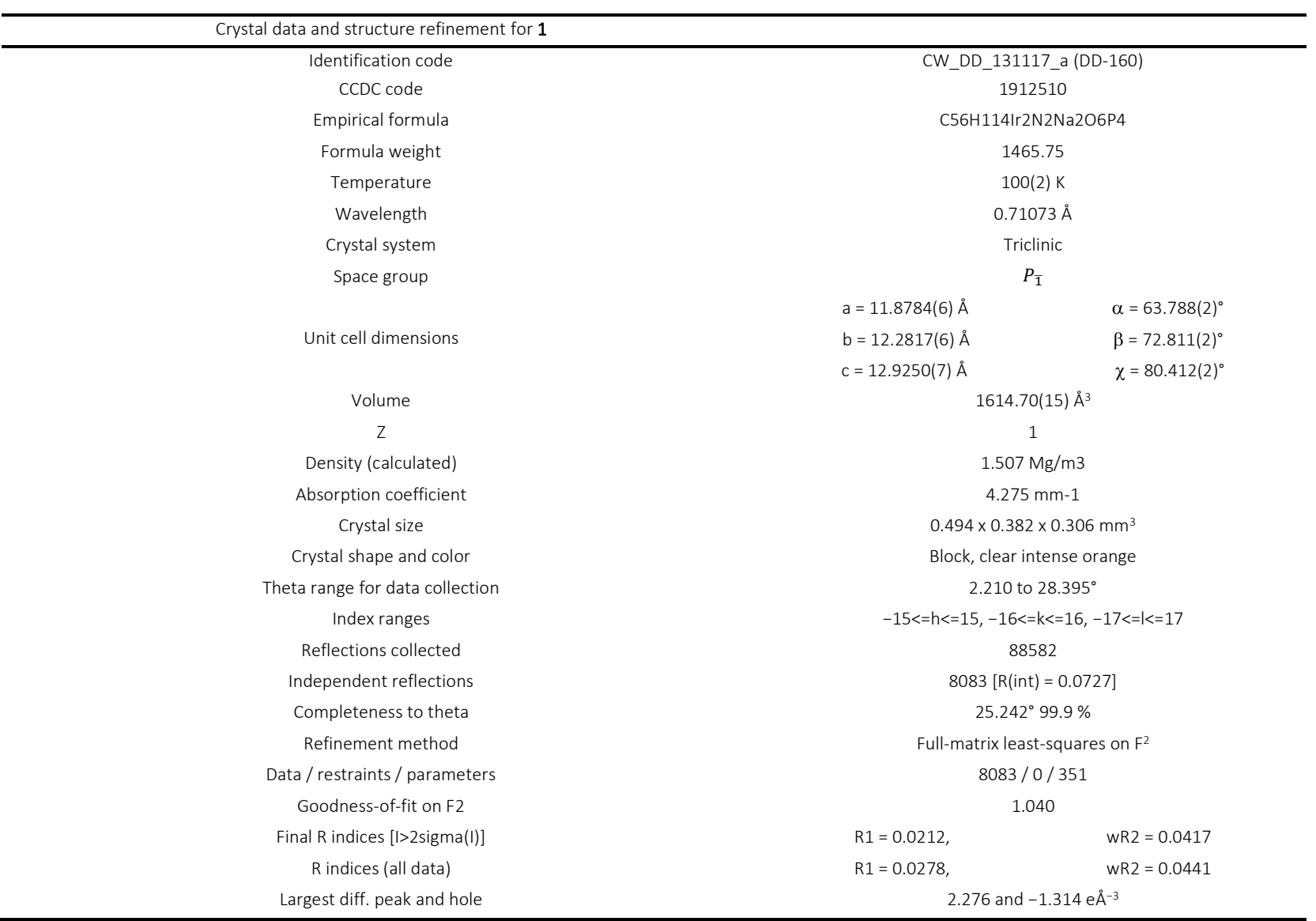


Table A2: Cartesian coordinates from X-Ray single-crystal analysis of 1

\begin{tabular}{|c|c|c|c|c|c|c|c|c|c|c|c|}
\hline Ir & 10.707566 & 9.649295 & 2.897519 & $\mathrm{H}$ & 8.44529 & 5.43972 & 0.057427 & $\mathrm{C}$ & 5.243511 & 8.538268 & 10.510628 \\
\hline$P$ & 11.142759 & 11.887684 & 3.095774 & $\mathrm{H}$ & 9.402813 & 4.834156 & 1.187689 & $\mathrm{H}$ & 4.584323 & 8.436769 & 11.187133 \\
\hline$P$ & 10.72123 & 7.463874 & 2.208846 & $\mathrm{H}$ & 10.027531 & 5.663 & -0.03042 & C & 5.83307 & 9.735739 & 10.34735 \\
\hline $\mathrm{Na}$ & 10.428574 & 8.762182 & 5.885073 & C & 8.745406 & 8.004715 & 0.425493 & $\mathrm{H}$ & 5.634105 & 10.487533 & 10.892983 \\
\hline 0 & 9.083787 & 9.540758 & 4.278042 & $\mathrm{H}$ & 9.431934 & 8.089645 & -0.268669 & C & 6.304429 & 11.139162 & 7.892532 \\
\hline 0 & 12.117705 & 8.206201 & 7.294478 & $\mathrm{H}$ & 8.678465 & 8.846138 & 0.923409 & C & 7.26713 & 11.511263 & 6.761045 \\
\hline$N$ & 12.176799 & 9.795448 & 1.496813 & $\mathrm{H}$ & 7.882076 & 7.802545 & 0.008104 & $\mathrm{H}$ & 7.959928 & 12.112435 & 7.106056 \\
\hline $\mathrm{C}$ & 12.750954 & 10.991658 & 1.204931 & $\mathrm{C}$ & 11.877878 & 7.232253 & 8.317239 & $\mathrm{H}$ & 7.685985 & 10.698649 & 6.408032 \\
\hline $\mathrm{H}$ & 13.416504 & 11.032161 & 0.528246 & $\mathrm{H}$ & 12.065004 & 6.319815 & 7.98177 & $\mathrm{H}$ & 6.771517 & 11.960809 & 6.04504 \\
\hline c & 12.401155 & 12.123741 & 1.850512 & $\mathrm{H}$ & 10.937609 & 7.272345 & 8.624477 & $\mathrm{H}$ & 8.91917 & 6.97393 & 6.902323 \\
\hline $\mathrm{H}$ & 12.789095 & 12.969725 & 1.659934 & $\mathrm{C}$ & 12.824064 & 7.592322 & 9.443315 & $\mathrm{C}$ & 5.037262 & 10.511556 & 7.292648 \\
\hline C & 11.971068 & 12.483777 & 4.688504 & $\mathrm{H}$ & 13.100813 & 6.787645 & 9.949279 & $\mathrm{H}$ & 4.402176 & 10.307969 & 8.010719 \\
\hline C & 13.235175 & 11.625091 & 4.829041 & $\mathrm{H}$ & 12.408875 & 8.240161 & 10.066232 & $\mathrm{H}$ & 4.627645 & 11.140928 & 6.662973 \\
\hline $\mathrm{H}$ & 13.846411 & 11.81988 & 4.088205 & C & 13.997936 & 8.210333 & 8.713042 & $\mathrm{H}$ & 5.273522 & 9.685337 & 6.821537 \\
\hline $\mathrm{H}$ & 12.989632 & 10.676559 & 4.809184 & $\mathrm{H}$ & 14.80076 & 7.636205 & 8.790202 & C & 5.886712 & 12.424641 & 8.616687 \\
\hline $\mathrm{H}$ & 13.676716 & 11.830493 & 5.679477 & $\mathrm{H}$ & 14.208276 & 9.105062 & 9.080872 & $\mathrm{H}$ & 6.685395 & 12.892614 & 8.938394 \\
\hline C & 11.082882 & 12.215667 & 5.914404 & $\mathrm{H}$ & 14.486331 & 8.843831 & 9.296346 & $\mathrm{H}$ & 5.396062 & 13.003976 & 7.99698 \\
\hline $\mathrm{H}$ & 10.329526 & 12.842437 & 5.915324 & $\mathrm{H}$ & 14.625929 & 7.514039 & 8.395387 & $\mathrm{H}$ & 5.311708 & 12.198878 & 9.37748 \\
\hline $\mathrm{H}$ & 11.609444 & 12.33661 & 6.732018 & C & 13.535893 & 8.318283 & 7.225758 & $\mathrm{C}$ & 8.618053 & 10.379863 & 9.842081 \\
\hline $\mathrm{H}$ & 10.74352 & 11.297059 & 5.877294 & $\mathrm{H}$ & 13.803575 & 9.187302 & 6.834288 & $\mathrm{C}$ & 9.731264 & 10.513804 & 8.805345 \\
\hline C & 12.376226 & 13.958389 & 4.672935 & $\mathrm{H}$ & 13.922594 & 7.58751 & 6.681202 & $\mathrm{H}$ & 10.59189 & 10.619719 & 9.261964 \\
\hline $\mathrm{H}$ & 12.877911 & 14.150672 & 3.853334 & $\mathrm{C}$ & 13.371415 & 8.907042 & 7.587651 & $\mathrm{H}$ & 9.755112 & 9.709559 & 8.245859 \\
\hline $\mathrm{H}$ & 12.937881 & 14.151092 & 5.452542 & $\mathrm{H}$ & 13.967783 & 8.889891 & 6.797616 & $\mathrm{H}$ & 9.561194 & 11.298099 & 8.242885 \\
\hline $\mathrm{H}$ & 11.572491 & 14.518315 & 4.702928 & $\mathrm{H}$ & 13.190252 & 9.850835 & 7.825384 & $\mathrm{C}$ & 8.481175 & 11.666602 & 10.647437 \\
\hline $\mathrm{C}$ & 9.774562 & 13.085254 & 2.582154 & Ir & 7.036157 & 7.605388 & 8.327431 & $\mathrm{H}$ & 9.298434 & 11.814962 & 11.167523 \\
\hline $\mathrm{C}$ & 8.739797 & 13.26281 & 3.697263 & $P$ & 6.600965 & 5.366998 & 8.129176 & $\mathrm{H}$ & 8.34091 & 12.420526 & 10.03726 \\
\hline $\mathrm{H}$ & 7.971186 & 13.763879 & 3.352915 & $P$ & 7.022494 & 9.790809 & 9.016104 & $\mathrm{H}$ & 7.716192 & 11.591683 & 11.25537 \\
\hline $\mathrm{H}$ & 9.143554 & 13.755175 & 4.442195 & $\mathrm{Na}$ & 7.315149 & 8.4925 & 5.339877 & $\mathrm{C}$ & 8.998317 & 9.249967 & 10.799457 \\
\hline $\mathrm{H}$ & 8.44253 & 12.382961 & 4.010169 & 0 & 8.659936 & 7.713925 & 6.946908 & $\mathrm{H}$ & 8.31179 & 9.165037 & 11.493619 \\
\hline $\mathrm{C}$ & 9.100728 & 12.418336 & 1.367872 & 0 & 5.626018 & 9.048482 & 3.930472 & $\mathrm{H}$ & 9.065258 & 8.408544 & 10.301541 \\
\hline $\mathrm{H}$ & 8.376541 & 12.99166 & 1.040396 & $N$ & 5.566924 & 7.459234 & 9.728137 & $\mathrm{H}$ & 9.861647 & 9.452137 & 11.216845 \\
\hline $\mathrm{H}$ & 8.734365 & 11.54924 & 1.634061 & $\mathrm{C}$ & 4.99277 & 6.263024 & 10.020019 & $\mathrm{C}$ & 5.865846 & 10.022429 & 2.907711 \\
\hline $\mathrm{H}$ & 9.762648 & 12.28983 & 0.656704 & $\mathrm{H}$ & 4.32722 & 6.222521 & 10.696704 & $\mathrm{H}$ & 5.678719 & 10.934867 & 3.24318 \\
\hline $\mathrm{C}$ & 10.264887 & 14.466258 & 2.126724 & C & 5.342569 & 5.130941 & 9.374438 & $\mathrm{H}$ & 6.806114 & 9.982337 & 2.600473 \\
\hline $\mathrm{H}$ & 10.955076 & 14.357586 & 1.439544 & $\mathrm{H}$ & 4.954628 & 4.284957 & 9.565016 & $\mathrm{C}$ & 4.919659 & 9.66236 & 1.781635 \\
\hline $\mathrm{H}$ & 10.639835 & 14.948789 & 2.892872 & $\mathrm{C}$ & 5.772656 & 4.770905 & 6.536445 & $\mathrm{H}$ & 4.64291 & 10.467037 & 1.275671 \\
\hline $\mathrm{H}$ & 9.512861 & 14.974314 & 1.756929 & C & 4.508548 & 5.629591 & 6.395909 & $\mathrm{H}$ & 5.334848 & 9.014521 & 1.158718 \\
\hline C & 12.500212 & 8.716414 & 0.714322 & $\mathrm{H}$ & 3.897313 & 5.434802 & 7.136745 & C & 3.745787 & 9.04435 & 2.511908 \\
\hline $\mathrm{H}$ & 13.1594 & 8.817913 & 0.037817 & $\mathrm{H}$ & 4.754092 & 6.578123 & 6.415766 & $\mathrm{H}$ & 2.942963 & 9.618477 & 2.434748 \\
\hline C & 11.910653 & 7.518943 & 0.8776 & $\mathrm{H}$ & 4.067007 & 5.424189 & 5.545473 & $\mathrm{H}$ & 3.535447 & 8.14962 & 2.144078 \\
\hline $\mathrm{H}$ & 12.109618 & 6.767149 & 0.331967 & $\mathrm{C}$ & 6.660842 & 5.039015 & 5.310546 & $\mathrm{H}$ & 3.257393 & 8.410851 & 1.928604 \\
\hline$c$ & 11.439294 & 6.11552 & 3.332418 & $\mathrm{H}$ & 7.414198 & 4.412245 & 5.309626 & $\mathrm{H}$ & 3.117794 & 9.740644 & 2.829563 \\
\hline C & 10.476593 & 5.743419 & 4.463904 & $\mathrm{H}$ & 6.13428 & 4.918072 & 4.492932 & C & 4.20783 & 8.936399 & 3.999191 \\
\hline $\mathrm{H}$ & 9.783796 & 5.142247 & 4.118894 & $\mathrm{H}$ & 7.000204 & 5.957623 & 5.347656 & $\mathrm{H}$ & 3.940148 & 8.06738 & 4.390662 \\
\hline $\mathrm{H}$ & 10.057739 & 6.556033 & 4.816918 & $\mathrm{C}$ & 5.367497 & 3.296293 & 6.552014 & $\mathrm{H}$ & 3.82113 & 9.667172 & 4.543747 \\
\hline $\mathrm{H}$ & 10.972207 & 5.293873 & 5.17991 & $\mathrm{H}$ & 4.865813 & 3.10401 & 7.371615 & $\mathrm{C}$ & 4.372308 & 8.34764 & 3.637299 \\
\hline $\mathrm{H}$ & 8.824554 & 10.280752 & 4.322627 & $\mathrm{H}$ & 4.805842 & 3.10359 & 5.772408 & $\mathrm{H}$ & 3.775941 & 8.364791 & 4.427334 \\
\hline C & 12.706462 & 6.743126 & 3.932302 & $\mathrm{H}$ & 6.171233 & 2.736368 & 6.522021 & $\mathrm{H}$ & 4.553471 & 7.403848 & 3.399566 \\
\hline $\mathrm{H}$ & 13.341548 & 6.946713 & 3.214231 & $C$ & 7.969162 & 4.169429 & 8.642796 & 0 & 14.74089 & 14.277018 & 7.237073 \\
\hline $\mathrm{H}$ & 13.116078 & 6.113754 & 4.561977 & $\mathrm{C}$ & 9.003927 & 3.991872 & 7.527687 & C & 14.24304 & 14.085715 & 8.567196 \\
\hline $\mathrm{H}$ & 12.470201 & 7.569345 & 4.403413 & $\mathrm{H}$ & 9.772537 & 3.490803 & 7.872035 & $\mathrm{H}$ & 14.70246 & 13.323049 & 9.000075 \\
\hline $\mathrm{C}$ & 11.857011 & 4.830042 & 2.608263 & $\mathrm{H}$ & 8.600169 & 3.499507 & 6.782755 & $\mathrm{H}$ & 13.27117 & 13.898133 & 8.54707 \\
\hline $\mathrm{H}$ & 11.058328 & 4.362068 & 2.286556 & $\mathrm{H}$ & 9.301193 & 4.871721 & 7.21478 & $\mathrm{C}$ & 14.51683 & 15.368612 & 9.330717 \\
\hline $\mathrm{H}$ & 12.347662 & 4.250706 & 3.22797 & C & 8.642996 & 4.836346 & 9.857078 & $\mathrm{H}$ & 14.68706 & 15.191533 & 10.289766 \\
\hline $\mathrm{H}$ & 12.432015 & 5.055804 & 1.84747 & $\mathrm{H}$ & 9.367183 & 4.263022 & 10.184554 & $\mathrm{H}$ & 13.76809 & 16.010378 & 9.243443 \\
\hline C & 9.125671 & 6.874819 & 1.382869 & $\mathrm{H}$ & 9.009358 & 5.705442 & 9.590889 & $\mathrm{C}$ & 15.76994 & 15.871199 & 8.623456 \\
\hline $\mathrm{C}$ & 8.01246 & 6.740878 & 2.419605 & $\mathrm{H}$ & 7.981076 & 4.964852 & 10.568245 & $\mathrm{H}$ & 15.88218 & 16.848335 & 8.736109 \\
\hline $\mathrm{H}$ & 7.151834 & 6.634963 & 1.962986 & $C$ & 7.478837 & 2.788424 & 9.098226 & $\mathrm{H}$ & 16.58133 & 15.408623 & 8.951752 \\
\hline $\mathrm{H}$ & 7.988611 & 7.545124 & 2.97909 & $\mathrm{H}$ & 6.788647 & 2.897096 & 9.785406 & C & 15.47026 & 15.509652 & 7.181195 \\
\hline $\mathrm{H}$ & 8.182529 & 5.956583 & 2.982065 & $\mathrm{H}$ & 7.103889 & 2.305893 & 8.332078 & $\mathrm{H}$ & 14.92776 & 16.216631 & 6.749944 \\
\hline C & 9.262548 & 5.58808 & 0.577512 & $\mathrm{H}$ & 8.230863 & 2.280368 & 9.468021 & $\mathrm{H}$ & 16.30953 & 15.395329 & 6.668709 \\
\hline
\end{tabular}




\section{A.2. X-Ray Single-Crystal Analysis of $\mathbf{3}$}
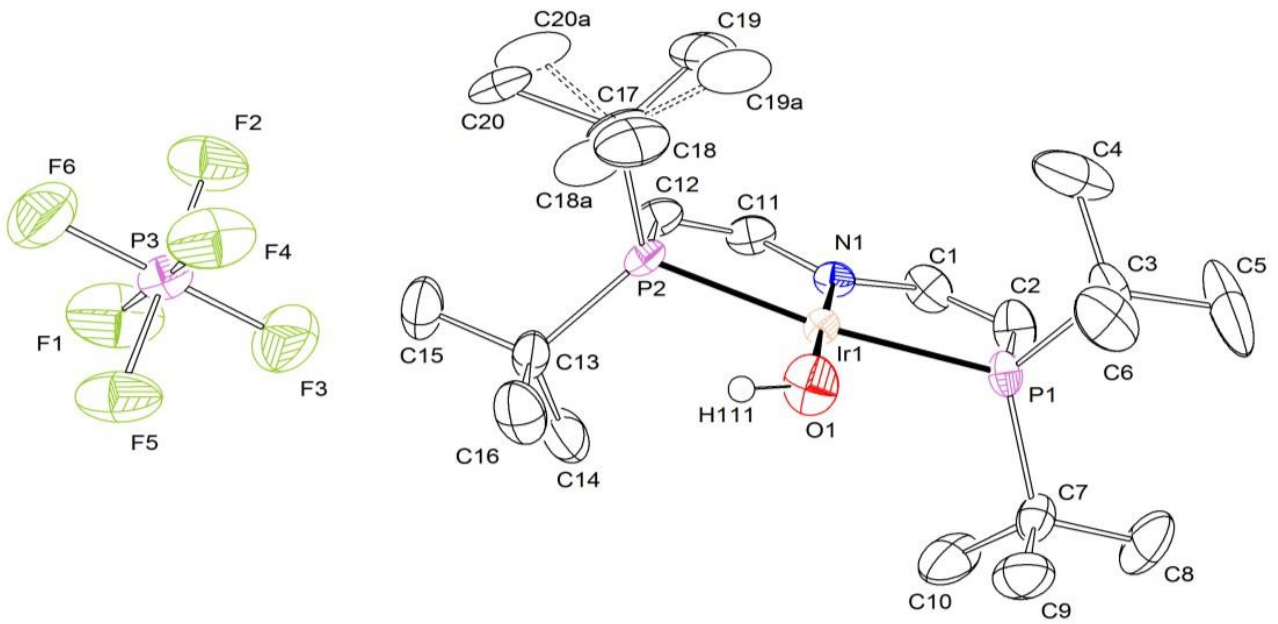

Figure A2: Thermal ellipsoid plot of 3 with the anisotropic displacement parameters drawn at the $50 \%$ probability level. The asymmetric unit contains one disordered complex molecule and one PF6 anion. The disordered complex molecule was refined with population of 0.59(3) on the main domain using some restraints (SADI, RIGU). The O-H hydrogen atom was calculated using AFIX 147 command.

Table A3: Crystal data and structure refinement for 3.

\begin{tabular}{|c|c|c|}
\hline \multicolumn{3}{|l|}{ Crystal data and structure refinement for 3} \\
\hline Identification code & \multicolumn{2}{|c|}{ mo_CW_DD_280717_0m_a (DD-91) } \\
\hline CCDC Identifier & \multicolumn{2}{|c|}{1912511} \\
\hline Empirical formula & \multicolumn{2}{|c|}{$\mathrm{C}_{20} \mathrm{H}_{41} \mathrm{~F}_{6} \mathrm{IrNOP} \mathrm{P}_{3}$} \\
\hline Formula weight & \multicolumn{2}{|c|}{710.65} \\
\hline Temperature & \multicolumn{2}{|c|}{$101(2) \mathrm{K}$} \\
\hline Wavelength & \multicolumn{2}{|c|}{$0.71073 \AA$} \\
\hline Crystal system & \multicolumn{2}{|c|}{ Monoclinic } \\
\hline Space group & \multicolumn{2}{|c|}{$\mathrm{P} 2_{1} / \mathrm{c}$} \\
\hline & $a=8.1470(4) \AA$ & $\alpha=90^{\circ}$ \\
\hline Unit cell dimensions & $b=12.7405(6) \AA$ & $\beta=97.255(2)^{\circ}$ \\
\hline & $c=26.4460(12) \AA$ & $\gamma=90^{\circ}$ \\
\hline Volume & \multicolumn{2}{|c|}{$163.74(13) \AA^{3}$} \\
\hline Z & \multicolumn{2}{|c|}{2} \\
\hline Density (calculated) & \multicolumn{2}{|c|}{$1.733 \mathrm{Mg} / \mathrm{m}^{3}$} \\
\hline Absorption coefficient & \multicolumn{2}{|c|}{$5.133 \mathrm{~mm}^{-1}$} \\
\hline$F(000)$ & \multicolumn{2}{|c|}{1408} \\
\hline Crystal size & \multicolumn{2}{|c|}{$0.283 \times 0.144 \times 0.058 \mathrm{~mm}^{3}$} \\
\hline Crystal shape and color & \multicolumn{2}{|c|}{ Plate, intense brown } \\
\hline Theta range for data collection & \multicolumn{2}{|c|}{2.228 to $26.461^{\circ}$} \\
\hline Index ranges & \multicolumn{2}{|c|}{$-10<=\mathrm{h}<=10,-15<=\mathrm{k}<=14,-33<=\mid<=33$} \\
\hline Reflections collected & \multicolumn{2}{|c|}{67143} \\
\hline Independent reflections & \multicolumn{2}{|c|}{$5588[R($ int $)=0.0500]$} \\
\hline Completeness to theta & \multicolumn{2}{|c|}{$100 \%$} \\
\hline Refinement method & \multicolumn{2}{|c|}{ Full-matrix least-squares on $\mathrm{F}^{2}$} \\
\hline Data / restraints / parameters & \multicolumn{2}{|c|}{$5588 / 72 / 333$} \\
\hline Goodness-of-fit on $F^{2}$ & \multicolumn{2}{|c|}{1.137} \\
\hline Final $R$ indices $[\mid>2 \operatorname{sigma}(I)]$ & $\mathrm{R} 1=0.0321$ & $w R 2=0.0656$ \\
\hline $\mathrm{R}$ indices (all data) & $\mathrm{R} 1=0.0390$ & $w R 2=0.0678$ \\
\hline Largest diff. peak and hole & \multicolumn{2}{|c|}{$\mathrm{d}-1.814 \mathrm{e} \AA^{-3}$} \\
\hline
\end{tabular}


Table A4: Cartesian coordinates from X-Ray single-crystal analysis of 3

\begin{tabular}{|c|c|c|c|c|c|c|c|}
\hline $\mathrm{Ir}$ & 3.932826 & 5.231249 & 10.178373 & $\overline{\mathrm{H}}$ & 7.699788 & 4.854131 & 12.76035 \\
\hline $\mathrm{P}$ & 3.174869 & 6.933253 & 8.771429 & H & 6.625512 & 5.40452 & 11.708355 \\
\hline$P$ & 4.872824 & 3.324506 & 11.151139 & C & 6.81133 & 2.336608 & 13.006952 \\
\hline 0 & 2.896504 & 5.836423 & 11.69655 & H & 7.211626 & 1.89069 & 12.230417 \\
\hline $\mathrm{H}$ & 3.166635 & 5.449112 & 12.390446 & H & 6.182822 & 1.726338 & 13.445064 \\
\hline$N$ & 4.978029 & 4.720355 & 8.676198 & H & 7.517028 & 2.587596 & 13.639198 \\
\hline c & 4.946648 & 5.438919 & 7.450795 & c & 5.302284 & 4.227298 & 13.712654 \\
\hline H & 5.49509 & 5.162451 & 6.726467 & H & 4.873868 & 5.05543 & 13.41096 \\
\hline c & 4.153935 & 6.492559 & 7.316476 & H & 5.92556 & 4.429872 & 14.441966 \\
\hline $\mathrm{H}$ & 4.103122 & 6.985616 & 6.506099 & H & 4.617111 & 3.604287 & 14.032712 \\
\hline c & 1.397423 & 6.767754 & 8.221821 & C & 3.667761 & 1.911075 & 11.35944 \\
\hline c & 1.225294 & 5.326803 & 7.820436 & C & 2.505357 & 2.304756 & 12.254028 \\
\hline H & 0.297385 & 5.173917 & 7.544976 & H & 2.062332 & 3.094667 & 11.881502 \\
\hline H & 1.439322 & 4.748384 & 8.583854 & H & 2.840852 & 2.508604 & 13.15124 \\
\hline H & 1.826071 & 5.121681 & 7.075383 & H & 1.867619 & 1.563259 & 12.303873 \\
\hline C & 1.01881 & 7.711825 & 7.093747 & C & 3.198792 & 1.590014 & 9.942789 \\
\hline H & 1.63118 & 7.583146 & 6.340823 & $\mathrm{H}$ & 2.964279 & 2.421969 & 9.481066 \\
\hline H & 1.080236 & 8.638059 & 7.411182 & $\mathrm{H}$ & 2.408892 & 1.009048 & 9.984764 \\
\hline H & 0.101533 & 7.524539 & 6.80517 & $\mathrm{H}$ & 3.913152 & 1.133905 & 9.457455 \\
\hline C & 0.487647 & 7.046771 & 9.410233 & C & 4.368692 & 0.649766 & 11.952334 \\
\hline H & 0.486893 & 8.008678 & 9.601743 & H & 5.180082 & 0.454836 & 11.440766 \\
\hline H & 0.814691 & 6.557535 & 10.194638 & H & 3.759605 & -0.115939 & 11.905112 \\
\hline H & -0.423836 & 6.755013 & 9.197736 & H & 4.607931 & 0.819214 & 12.888898 \\
\hline C & 3.726955 & 8.655896 & 9.179372 & $P$ & 5.124841 & 0.855525 & 16.992462 \\
\hline C & 3.512055 & 9.668765 & 8.064415 & $\mathrm{~F}$ & 6.689259 & 0.724934 & 16.87126 \\
\hline H & 3.896887 & 9.323498 & 7.230165 & $\mathrm{~F}$ & 4.949722 & -0.119761 & 15.758927 \\
\hline $\mathrm{H}$ & 3.95045 & 10.512187 & 8.300524 & $\mathrm{~F}$ & 5.126823 & 2.13276 & 16.070065 \\
\hline $\mathrm{H}$ & 2.552556 & 9.821651 & 7.941114 & $\mathrm{~F}$ & 3.555359 & 0.967004 & 17.202074 \\
\hline c & 3.043382 & 9.129842 & 10.459604 & $\mathrm{~F}$ & 5.325509 & 1.832084 & 18.246723 \\
\hline $\mathrm{H}$ & 2.088599 & 9.272536 & 10.286458 & $\mathrm{~F}$ & 5.071452 & -0.412792 & 17.951325 \\
\hline H & 3.449988 & 9.970715 & 10.753428 & C & 2.869756 & 2.229588 & 12.655413 \\
\hline $\mathrm{H}$ & 3.150887 & 8.452048 & 11.157436 & $\mathrm{H}$ & 2.471276 & 3.125245 & 12.579333 \\
\hline C & 5.229601 & 8.537409 & 9.439091 & $\mathrm{H}$ & 3.479876 & 2.214299 & 13.424077 \\
\hline H & 5.685458 & 8.282599 & 8.610088 & $\mathrm{H}$ & 2.165304 & 1.567082 & 12.781337 \\
\hline $\mathrm{H}$ & 5.389287 & 7.854518 & 10.123805 & C & 2.646324 & 2.076702 & 10.186768 \\
\hline $\mathrm{H}$ & 5.576026 & 9.399941 & 9.751279 & $\mathrm{H}$ & 3.125074 & 2.019369 & 9.33153 \\
\hline C & 5.848302 & 3.609384 & 8.694038 & H & 2.204701 & 2.94433 & 10.2576 \\
\hline $\mathrm{H}$ & 6.382982 & 3.420824 & 7.93062 & $\mathrm{H}$ & 1.979591 & 1.359411 & 10.233989 \\
\hline c & 5.931675 & 2.820747 & 9.767019 & C & 4.187606 & 0.486687 & 11.390921 \\
\hline $\mathrm{H}$ & 6.499253 & 2.060139 & 9.801124 & $\mathrm{H}$ & 3.435875 & -0.135049 & 11.472247 \\
\hline C & 6.068145 & 3.591547 & 12.54759 & $\mathrm{H}$ & 4.789098 & 0.375845 & 12.156961 \\
\hline c & 7.091991 & 4.605691 & 12.031037 & $\mathrm{H}$ & 4.678495 & 0.299402 & 10.561918 \\
\hline H & 7.605556 & 4.206913 & 11.299101 & & & & \\
\hline
\end{tabular}




\section{A.3. X-Ray Single-Crystal Analysis of 4}

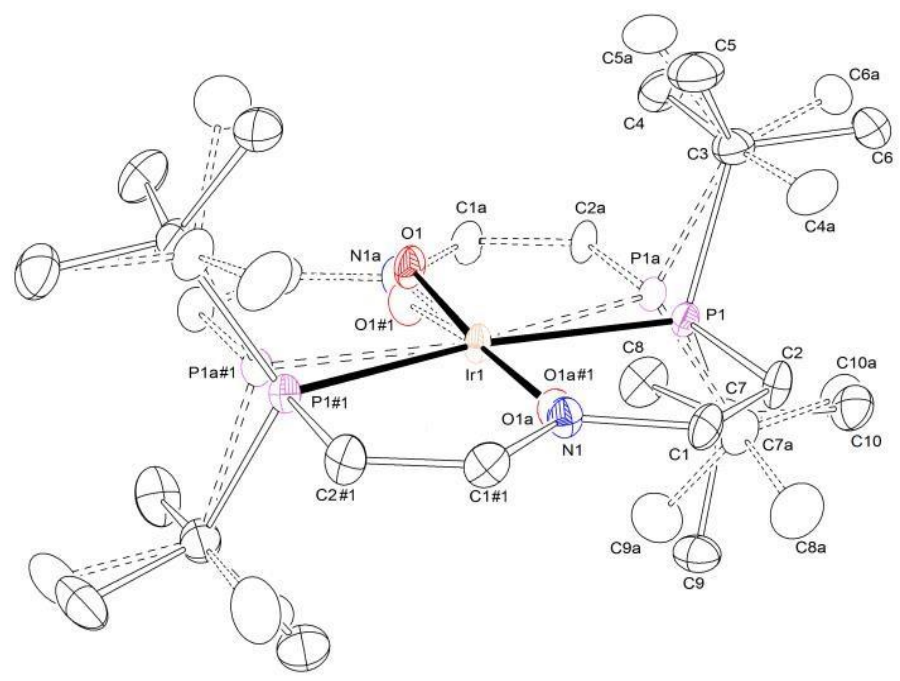

Figure A3: Thermal ellipsoid plot of 4 with the anisotropic displacement parameters drawn at the $50 \%$ probability level. The asymmetric unit contains a half disordered complex molecule. The disordered complex molecule was refined with population of 0.910(1) on the main domain using some restraints and constraints (RIGU, SADI, EADP).

Table A5: Crystal data and structure refinement for 4

\begin{tabular}{|c|c|c|}
\hline \multicolumn{3}{|l|}{ Crystal data and structure refinement for $\mathbf{4}$} \\
\hline Identification code & \multicolumn{2}{|c|}{ mo_CW_DD_050917_0m_a (DD-109) } \\
\hline CCDC Identifier & \multicolumn{2}{|c|}{1912512} \\
\hline Empirical formula & \multicolumn{2}{|c|}{$\mathrm{C}_{20} \mathrm{H}_{40} \mathrm{lrNOP} \mathrm{P}_{2}$} \\
\hline Formula weight & \multicolumn{2}{|c|}{564.67} \\
\hline Temperature & \multicolumn{2}{|c|}{$100(2) \mathrm{K}$} \\
\hline Wavelength & \multicolumn{2}{|c|}{$0.71073 \AA$} \\
\hline Crystal system & \multicolumn{2}{|c|}{ Monoclinic } \\
\hline Space group & \multicolumn{2}{|c|}{$\mathrm{C} 2 / \mathrm{c}$} \\
\hline \multirow{3}{*}{ Unit cell dimensions } & $a=22.0008(11) \AA$ & $\alpha=90^{\circ}$ \\
\hline & $b=7.6833(4) \AA$ & $\beta=103.429(2)^{\circ}$ \\
\hline & $c=13.9150(7)) \AA$ & $\gamma=90^{\circ}$ \\
\hline Volume & \multicolumn{2}{|c|}{$2287.9(2) \AA^{3}$} \\
\hline z & \multicolumn{2}{|c|}{4} \\
\hline Density (calculated) & \multicolumn{2}{|c|}{$1.639 \mathrm{Mg} / \mathrm{m}^{3}$} \\
\hline Absorption coefficient & \multicolumn{2}{|c|}{$5.984 \mathrm{~mm}^{-1}$} \\
\hline$F(000)$ & \multicolumn{2}{|c|}{1128} \\
\hline Crystal size & \multicolumn{2}{|c|}{$0.328 \times 0.238 \times 0.146 \mathrm{~mm}^{3}$} \\
\hline Crystal shape and color & \multicolumn{2}{|c|}{ Plate, clear dark orange } \\
\hline Theta range for data collection & \multicolumn{2}{|c|}{2.817 to $28.368^{\circ}$} \\
\hline Index ranges & \multicolumn{2}{|c|}{$-26<=h<=29,-10<=k<=10,-18<=\mid<=18$} \\
\hline Reflections collected & \multicolumn{2}{|c|}{32321} \\
\hline Independent reflections & \multicolumn{2}{|c|}{$2867[R($ int $)=0.0612]$} \\
\hline Completeness to theta & \multicolumn{2}{|c|}{$100 \%$} \\
\hline Refinement method & \multicolumn{2}{|c|}{ Full-matrix least-squares on $\mathrm{F}^{2}$} \\
\hline Data / restraints / parameters & \multicolumn{2}{|c|}{$2867 / 159 / 176$} \\
\hline Goodness-of-fit on $F^{2}$ & \multicolumn{2}{|c|}{1.066} \\
\hline Final $R$ indices $[\mid>2$ sigma $(I)]$ & $\mathrm{R} 1=0.0207$ & $w R 2=0.0360$ \\
\hline $\mathrm{R}$ indices (all data) & $\mathrm{R} 1=0.0346$ & $w R 2=0.0388$ \\
\hline Largest diff. peak and hole & \multicolumn{2}{|c|}{0.680 and $-0.613 e^{-3}$} \\
\hline
\end{tabular}


Table A6: Cartesian coordinates from X-Ray single-crystal analysis of $\mathbf{4}$

\begin{tabular}{|c|c|c|c|c|c|c|c|c|c|c|c|}
\hline Ir & 10.192494 & 5.722637 & 3.383636 & $\mathrm{H}$ & 6.695531 & 6.176528 & 0.419043 & C & 14.459516 & 5.665112 & 0.885416 \\
\hline C & 6.815715 & 5.848651 & 2.459294 & H & 8.122969 & 5.612228 & 0.871909 & $\mathrm{H}$ & 15.064713 & 6.232094 & 1.407592 \\
\hline$P$ & 7.940186 & 5.381422 & 3.871434 & H & 7.746386 & 7.144293 & 1.140299 & $\mathrm{H}$ & 14.616806 & 5.81297 & -0.070501 \\
\hline $\mathrm{N}$ & 10.192494 & 3.682829 & 3.383636 & C & 5.321754 & 6.199148 & 2.501576 & $\mathrm{H}$ & 14.625448 & 4.72387 & 1.102037 \\
\hline 0 & 10.253692 & 7.538378 & 3.577356 & $\mathrm{H}$ & 5.212478 & 7.129334 & 2.790065 & C & 11.350666 & 2.971632 & 3.099627 \\
\hline c & 7.874979 & 3.593226 & 3.942829 & H & 4.86467 & 5.605405 & 3.133192 & $\mathrm{H}$ & 11.322875 & 2.022037 & 3.09983 \\
\hline $\mathrm{H}$ & 7.080393 & 3.119489 & 4.159016 & H & 4.934377 & 6.086464 & 1.608472 & $P$ & 12.460056 & 6.145664 & 2.904472 \\
\hline C & 6.826241 & 7.382614 & 2.26732 & C & 7.643989 & 4.040878 & 5.708302 & C & 11.308529 & 8.459982 & 3.165716 \\
\hline H & 6.345266 & 7.612214 & 1.444921 & H & 8.273481 & 3.716535 & 5.030844 & $\mathrm{H}$ & 11.262917 & 9.408155 & 3.202963 \\
\hline H & 7.752076 & 7.697437 & 2.203017 & H & 6.789232 & 3.571405 & 5.611381 & C & 12.510098 & 7.888529 & 2.892995 \\
\hline H & 6.388063 & 7.810589 & 3.032333 & H & 8.009907 & 3.872145 & 6.601622 & $\mathrm{H}$ & 13.298166 & 8.387943 & 2.714055 \\
\hline c & 7.430986 & 5.211744 & 1.22045 & C & 7.421377 & 5.564522 & 5.519995 & C & 13.569048 & 4.235081 & 4.315557 \\
\hline $\mathrm{H}$ & 6.895607 & 5.444548 & 0.433322 & C & 8.28082 & 6.293714 & 6.56451 & $\mathrm{H}$ & 12.648566 & 3.908925 & 4.397616 \\
\hline H & 7.447518 & 4.237786 & 1.327847 & H & 9.22241 & 6.05315 & 6.438274 & $\mathrm{H}$ & 14.09897 & 3.911898 & 5.073938 \\
\hline $\mathrm{H}$ & 8.345788 & 5.543009 & 1.10293 & H & 7.994357 & 6.031106 & 7.464151 & $\mathrm{H}$ & 13.95948 & 3.90443 & 3.479704 \\
\hline c & 5.383525 & 5.362759 & 2.615862 & H & 8.173816 & 7.261932 & 6.457222 & C & 12.987861 & 6.229474 & 5.66606 \\
\hline H & 4.889165 & 5.530485 & 1.786492 & C & 5.940243 & 5.855889 & 5.815847 & $\mathrm{H}$ & 13.689458 & 6.176528 & 6.348228 \\
\hline H & 4.954576 & 5.84295 & 3.354658 & $\mathrm{H}$ & 5.751898 & 6.801449 & 5.640209 & $\mathrm{H}$ & 12.26202 & 5.612228 & 5.895362 \\
\hline $\mathrm{H}$ & 5.383738 & 4.401647 & 2.807362 & H & 5.748908 & 5.653756 & 6.755496 & $\mathrm{H}$ & 12.638602 & 7.144293 & 5.626972 \\
\hline c & 7.370902 & 6.017607 & 5.543789 & H & 5.376295 & 5.298089 & 5.240331 & C & 15.063235 & 6.199148 & 4.265695 \\
\hline C & 7.601306 & 7.530287 & 5.629246 & C & 13.569273 & 5.848651 & 4.307977 & $\mathrm{H}$ & 15.17251 & 7.129334 & 3.977206 \\
\hline $\mathrm{H}$ & 8.515551 & 7.738097 & 5.343965 & $\mathrm{P}$ & 12.444803 & 5.381422 & 2.895837 & $\mathrm{H}$ & 15.520318 & 5.605405 & 3.634079 \\
\hline H & 7.471777 & 7.828753 & 6.553669 & 0 & 10.131297 & 7.538378 & 3.189916 & $\mathrm{H}$ & 15.450612 & 6.086464 & 5.158799 \\
\hline H & 6.963969 & 7.991339 & 5.044771 & C & 12.510009 & 3.593226 & 2.824442 & C & 12.741 & 4.040878 & 1.05897 \\
\hline C & 8.296225 & 5.336482 & 6.564307 & $\mathrm{H}$ & 13.304595 & 3.119489 & 2.608255 & $\mathrm{H}$ & 12.111507 & 3.716535 & 1.736428 \\
\hline H & 8.124639 & 4.371621 & 6.567813 & C & 13.558747 & 7.382614 & 4.499951 & $\mathrm{H}$ & 13.595756 & 3.571405 & 1.155891 \\
\hline H & 8.123508 & 5.70223 & 7.456937 & H & 14.039723 & 7.612214 & 5.322351 & $\mathrm{H}$ & 12.375082 & 3.872145 & 0.165649 \\
\hline H & 9.23059 & 5.501227 & 6.318967 & $\mathrm{H}$ & 12.632913 & 7.697437 & 4.564254 & C & 12.963612 & 5.564522 & 1.247276 \\
\hline C & 5.925473 & 5.665112 & 5.881855 & H & 13.996926 & 7.810589 & 3.734938 & C & 12.104169 & 6.293714 & 0.202761 \\
\hline $\mathrm{H}$ & 5.320275 & 6.232094 & 5.359679 & C & 12.954003 & 5.211744 & 5.546821 & $\mathrm{H}$ & 11.162578 & 6.05315 & 0.328998 \\
\hline H & 5.768182 & 5.81297 & 6.837773 & H & 13.489382 & 5.444548 & 6.333949 & $\mathrm{H}$ & 12.390632 & 6.031106 & -0.69688 \\
\hline H & 5.759541 & 4.72387 & 5.665235 & $\mathrm{H}$ & 12.93747 & 4.237786 & 5.439424 & $\mathrm{H}$ & 12.211173 & 7.261932 & 0.310049 \\
\hline c & 9.034322 & 2.971632 & 3.667644 & $\mathrm{H}$ & 12.0392 & 5.543009 & 5.664341 & C & 14.444745 & 5.855889 & 0.951424 \\
\hline H & 9.062114 & 2.022037 & 3.667441 & C & 15.001464 & 5.362759 & 4.15141 & $\mathrm{H}$ & 14.633091 & 6.801449 & 1.127062 \\
\hline $\mathrm{P}$ & 7.924933 & 6.145664 & 3.862799 & $\mathrm{H}$ & 15.495823 & 5.530485 & 4.980779 & $\mathrm{H}$ & 14.636081 & 5.653756 & 0.011775 \\
\hline $\mathrm{N}$ & 10.192494 & 7.771289 & 3.383636 & H & 15.430412 & 5.84295 & 3.412613 & $\mathrm{H}$ & 15.008694 & 5.298089 & 1.52694 \\
\hline 0 & 10.192494 & 3.901826 & 3.383636 & $\mathrm{H}$ & 15.00125 & 4.401647 & 3.959909 & C & 9.034322 & 10.654932 & 3.667644 \\
\hline C & 9.076459 & 8.459982 & 3.601555 & C & 13.014087 & 6.017607 & 1.223482 & C & 11.350666 & 10.654932 & 3.099627 \\
\hline H & 9.122072 & 9.408155 & 3.564308 & C & 12.783682 & 7.530287 & 1.138025 & $\mathrm{H}$ & 9.062114 & 9.705337 & 3.667441 \\
\hline c & 7.874891 & 7.888529 & 3.874276 & $\mathrm{H}$ & 11.869437 & 7.738097 & 1.423306 & $\mathrm{H}$ & 11.322875 & 9.705337 & 3.09983 \\
\hline H & 7.086822 & 8.387943 & 4.053216 & $\mathrm{H}$ & 12.913212 & 7.828753 & 0.213602 & C & 9.076459 & 0.776682 & 3.601555 \\
\hline c & 6.815941 & 4.235081 & 2.451715 & $\mathrm{H}$ & 13.42102 & 7.991339 & 1.722501 & C & 11.308529 & 0.776682 & 3.165716 \\
\hline H & 7.736423 & 3.908925 & 2.369655 & C & 12.088764 & 5.336482 & 0.202964 & $\mathrm{H}$ & 9.122072 & 1.724855 & 3.564308 \\
\hline H & 6.286018 & 3.911898 & 1.693334 & $\mathrm{H}$ & 12.260349 & 4.371621 & 0.199459 & $\mathrm{H}$ & 11.262917 & 1.724855 & 3.202963 \\
\hline H & 6.425509 & 3.90443 & 3.287567 & $\mathrm{H}$ & 12.26148 & 5.70223 & -0.689666 & & & & \\
\hline c & 7.397128 & 6.229474 & 1.101211 & $\mathrm{H}$ & 11.154398 & 5.501227 & 0.448305 & & & & \\
\hline
\end{tabular}




\section{A.4. X-Ray Single-Crystal Analysis of 8}

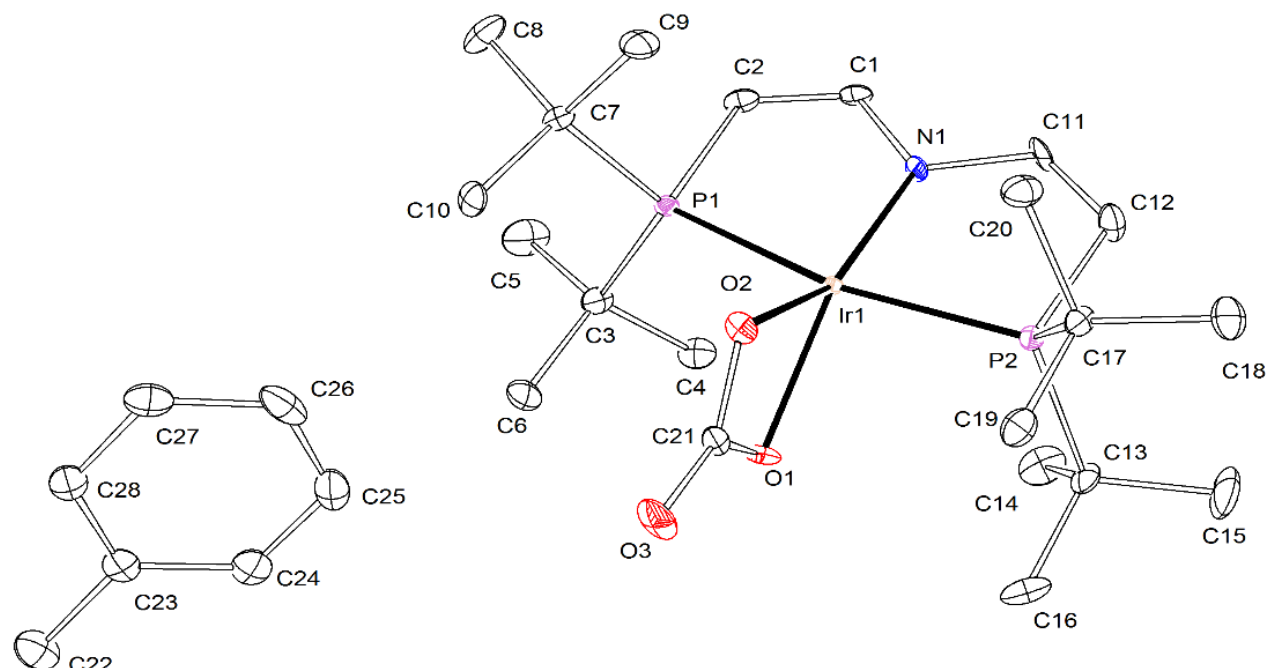

Figure A4: Thermal ellipsoid plot of 8 with the anisotropic displacement parameters drawn at the $50 \%$ probability level. The asymmetric unit contains one complex molecule and one toluene solvent molecule.

Table A7: Crystal data and structure refinement for 8 .

\begin{tabular}{|c|c|c|}
\hline \multicolumn{3}{|c|}{ Crystal data and structure refinement for 8} \\
\hline Identification code & \multicolumn{2}{|c|}{$\begin{array}{l}\text { SJKF_DD_081018_a (DD-266) } \\
\text { (DD_E }\end{array}$} \\
\hline CCDC Identifier & \multicolumn{2}{|c|}{1912514} \\
\hline Empirical formula & \multicolumn{2}{|c|}{$\mathrm{C}_{28} \mathrm{H}_{48} \mathrm{IrNO} \mathrm{P}_{3}$} \\
\hline Formula weight & \multicolumn{2}{|c|}{700.81} \\
\hline Temperature & \multicolumn{2}{|c|}{$100(2) \mathrm{K}$} \\
\hline Wavelength & \multicolumn{2}{|c|}{$0.71073 \AA$} \\
\hline Crystal system & \multicolumn{2}{|c|}{ Triclinic } \\
\hline Space group & \multicolumn{2}{|c|}{$P_{1}$} \\
\hline \multirow{3}{*}{ Unit cell dimensions } & $a=9.6329(3) \AA$ & $\alpha=112.299(2)^{\circ}$ \\
\hline & $\mathrm{b}=11.9945(3) \AA$ & $\beta=98.906(2)^{\circ}$ \\
\hline & $c=14.0860(4) \AA$ & $\gamma=91.614(2)^{\circ}$ \\
\hline Volume & \multicolumn{2}{|c|}{$1480.91(8) \AA^{3}$} \\
\hline z & \multicolumn{2}{|c|}{2} \\
\hline Density (calculated) & \multicolumn{2}{|c|}{$1.572 \mathrm{Mg} / \mathrm{m}^{3}$} \\
\hline Absorption coefficient & \multicolumn{2}{|c|}{$4.644 \mathrm{~mm}^{-1}$} \\
\hline$F(000)$ & \multicolumn{2}{|c|}{708} \\
\hline Crystal size & \multicolumn{2}{|c|}{$0.480 \times 0.373 \times 0.305 \mathrm{~mm}^{3}$} \\
\hline Crystal shape and color & \multicolumn{2}{|c|}{ Block, dark orange } \\
\hline Theta range for data collection & \multicolumn{2}{|c|}{2.434 to $30.639^{\circ}$} \\
\hline Index ranges & \multicolumn{2}{|c|}{$-13<=h<=13,-17<=k<=17,-20<=\mid<=20$} \\
\hline Reflections collected & \multicolumn{2}{|c|}{79020} \\
\hline Independent reflections & \multicolumn{2}{|c|}{$9015[R($ int $)=0.1147]$} \\
\hline Completeness to theta & \multicolumn{2}{|c|}{$99.9 \%$} \\
\hline Refinement method & \multicolumn{2}{|c|}{ Full-matrix least-squares on $\mathrm{F}^{2}$} \\
\hline Data / restraints / parameters & \multicolumn{2}{|c|}{$9015 / 0 / 329$} \\
\hline Goodness-of-fit on $\mathrm{F}^{2}$ & \multicolumn{2}{|c|}{1.063} \\
\hline Final R indices [I>2sigma(I)] & $\mathrm{R} 1=0.0371$ & $w R 2=0.0542$ \\
\hline $\mathrm{R}$ indices (all data) & $\mathrm{R} 1=0.0542$ & $w R 2=0.0580$ \\
\hline Largest diff. peak and hole & \multicolumn{2}{|c|}{1.834 and $-2.294 \mathrm{e}^{-3}$} \\
\hline
\end{tabular}


Table A8: Cartesian coordinates from X-Ray single-crystal analysis of 8 .

\begin{tabular}{|c|c|c|c|c|c|c|c|}
\hline Ir & 6.518685 & 4.345428 & 3.295313 & C & 6.340656 & 1.643647 & 1.041601 \\
\hline$P$ & 7.343656 & 6.479733 & 2.818108 & $\mathrm{H}$ & 6.070264 & 1.157195 & 0.23498 \\
\hline$P$ & 6.210528 & 2.066402 & 3.71801 & $\mathrm{H}$ & 7.299689 & 1.513418 & 1.195545 \\
\hline 0 & 4.708374 & 4.587738 & 2.326465 & $\mathrm{H}$ & 6.155346 & 2.598828 & 0.92456 \\
\hline o & 4.91276 & 5.001455 & 4.407782 & c & 5.761911 & -0.39367 & 2.362752 \\
\hline 0 & 2.880704 & 5.286188 & 3.467067 & $\mathrm{H}$ & 5.531826 & -0.823898 & 1.512831 \\
\hline$N$ & 8.387074 & 3.871134 & 3.299557 & $\mathrm{H}$ & 5.186534 & -0.745999 & 3.073527 \\
\hline c & 9.398462 & 4.828944 & 3.04996 & $\mathrm{H}$ & 6.699503 & -0.579042 & 2.579435 \\
\hline H & 10.309566 & 4.560393 & 3.066372 & c & 4.069379 & 1.409771 & 2.007679 \\
\hline C & 9.09712 & 6.094837 & 2.793553 & $\mathrm{H}$ & 3.916039 & 2.376506 & 2.05557 \\
\hline $\mathrm{H}$ & 9.760393 & 6.749818 & 2.610337 & $\mathrm{H}$ & 3.535199 & 0.959829 & 2.695156 \\
\hline C & 6.958158 & 7.138875 & 1.107648 & $\mathrm{H}$ & 3.806788 & 1.08081 & 1.122676 \\
\hline C & 7.20422 & 5.953724 & 0.163073 & C & 5.456902 & 1.574169 & 5.347857 \\
\hline H & 7.017983 & 6.227091 & -0.759422 & C & 5.704367 & 0.114075 & 5.724228 \\
\hline H & 6.613555 & 5.211551 & 0.409427 & $\mathrm{H}$ & 5.159605 & -0.467228 & 5.15351 \\
\hline H & 8.138187 & 5.666059 & 0.236314 & $\mathrm{H}$ & 5.459818 & -0.027338 & 6.662623 \\
\hline C & 7.862619 & 8.294823 & 0.680514 & $\mathrm{H}$ & 6.652404 & -0.099658 & 5.597942 \\
\hline H & 7.688984 & 8.515749 & -0.258329 & C & 3.957799 & 1.862729 & 5.355383 \\
\hline H & 8.800747 & 8.031796 & 0.786105 & $\mathrm{H}$ & 3.801044 & 2.784298 & 5.061203 \\
\hline H & 7.677995 & 9.077985 & 1.239948 & $\mathrm{H}$ & 3.607098 & 1.745407 & 6.262928 \\
\hline C & 5.484487 & 7.558806 & 0.999057 & $\mathrm{H}$ & 3.502921 & 1.243732 & 4.746828 \\
\hline H & 5.254452 & 7.703026 & 0.057418 & C & 6.14743 & 2.482981 & 6.392971 \\
\hline H & 5.343541 & 8.388855 & 1.500598 & $\mathrm{H}$ & 7.116944 & 2.344794 & 6.356287 \\
\hline H & 4.915363 & 6.852415 & 1.369876 & $\mathrm{H}$ & 5.81915 & 2.258644 & 7.288681 \\
\hline c & 7.162278 & 7.723185 & 4.208153 & $\mathrm{H}$ & 5.943467 & 3.421012 & 6.195752 \\
\hline c & 8.138857 & 8.897606 & 4.093651 & C & 4.065376 & 4.985217 & 3.404571 \\
\hline H & 9.057081 & 8.556837 & 4.060043 & C & 0.407821 & 11.436591 & 1.448117 \\
\hline H & 8.036537 & 9.484586 & 4.871716 & H & 0.988906 & 12.152532 & 1.116226 \\
\hline H & 7.947769 & 9.402811 & 3.275951 & $\mathrm{H}$ & -0.410707 & 11.826316 & 1.820295 \\
\hline C & 7.479242 & 6.933965 & 5.486093 & $\mathrm{H}$ & 0.176768 & 10.834783 & 0.70998 \\
\hline H & 6.787578 & 6.254935 & 5.630702 & C & 1.13022 & 10.659628 & 2.524171 \\
\hline H & 7.501082 & 7.546096 & 6.251093 & C & 1.673387 & 9.413063 & 2.257123 \\
\hline H & 8.351424 & 6.497039 & 5.392337 & $\mathrm{H}$ & 1.607409 & 9.049211 & 1.382044 \\
\hline C & 5.735831 & 8.252265 & 4.304409 & C & 2.312359 & 8.691111 & 3.254179 \\
\hline H & 5.532925 & 8.790749 & 3.511137 & $\mathrm{H}$ & 2.672904 & 7.833419 & 3.062179 \\
\hline H & 5.646654 & 8.806992 & 5.10735 & C & 2.424978 & 9.217861 & 4.525811 \\
\hline H & 5.110907 & 7.499166 & 4.356596 & $\mathrm{H}$ & 2.859855 & 8.722869 & 5.210172 \\
\hline c & 8.807047 & 2.558534 & 3.596802 & c & 1.901944 & 10.469476 & 4.799335 \\
\hline H & 9.737048 & 2.367687 & 3.631268 & $\mathrm{H}$ & 1.989586 & 10.841659 & 5.66899 \\
\hline c & 7.939504 & 1.583025 & 3.829577 & C & 1.249648 & 11.18013 & 3.802714 \\
\hline H & 8.210359 & 0.696901 & 4.039182 & $\mathrm{H}$ & 0.880638 & 12.033277 & 3.998894 \\
\hline c & 5.555843 & 1.115857 & 2.241813 & & & & \\
\hline
\end{tabular}




\section{A.5. X-Ray Single-Crystal Analysis of 10}

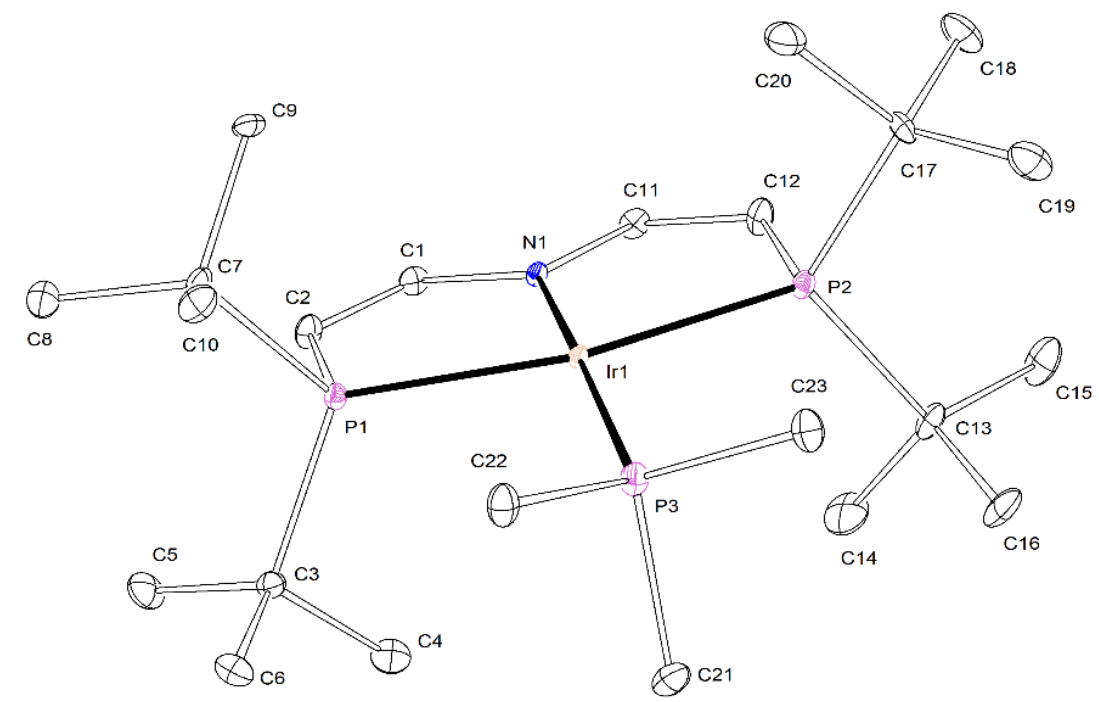

Figure A5: Thermal ellipsoid plot of 10 with the anisotropic displacement parameters drawn at the $50 \%$ probability level. The asymmetric unit contains one complex molecule.

Table A9: Crystal data and structure refinement for 10 .

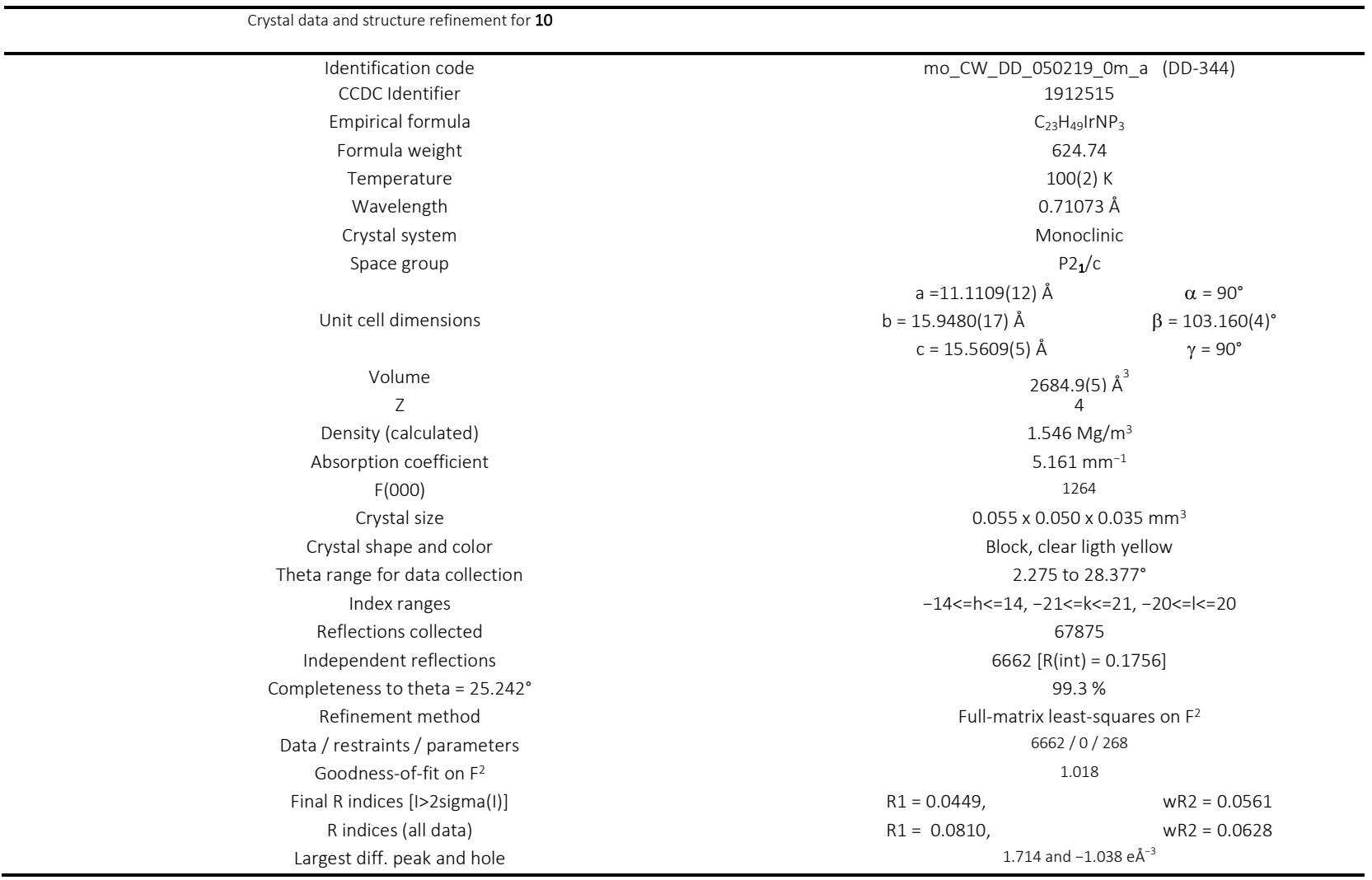


Table A10: Cartesian coordinates from X-Ray single-crystal analysis of 10.

\begin{tabular}{|c|c|c|c|c|c|c|c|}
\hline Ir & 1.752199 & 10.321083 & 4.464199 & C & 4.838035 & 10.878498 & 6.360729 \\
\hline$P$ & 0.203808 & 8.939045 & 3.391935 & c & 4.892618 & 9.373022 & 6.198418 \\
\hline $\mathrm{P}$ & 3.759011 & 11.474666 & 4.921039 & H & 3.984525 & 9.006872 & 6.239663 \\
\hline$P$ & 0.623737 & 10.798582 & 6.341486 & H & 5.433786 & 8.986283 & 6.918119 \\
\hline N & 2.891847 & 9.707723 & 2.821014 & H & 5.294858 & 9.15219 & 5.332483 \\
\hline c & 2.395774 & 8.807315 & 1.918471 & c & 6.277738 & 11.438719 & 6.289271 \\
\hline H & 2.951412 & 8.516998 & 1.204694 & H & 6.835256 & 10.993419 & 6.961046 \\
\hline c & 1.163116 & 8.327456 & 2.009536 & H & 6.262204 & 12.402776 & 6.464673 \\
\hline H & 0.813433 & 7.702836 & 1.384975 & H & 6.648064 & 11.274981 & 5.396834 \\
\hline c & -0.301495 & 7.312477 & 4.23643 & c & 4.28053 & 11.210758 & 7.746265 \\
\hline C & 0.934952 & 6.864035 & 5.029347 & H & 4.210452 & 12.183188 & 7.845588 \\
\hline H & 1.687968 & 6.735909 & 4.415393 & H & 4.880968 & 10.854576 & 8.43401 \\
\hline $\mathrm{H}$ & 0.740391 & 6.020258 & 5.488248 & $\mathrm{H}$ & 3.393079 & 10.807338 & 7.846633 \\
\hline H & 1.165481 & 7.549799 & 5.690409 & C & 3.851456 & 13.375986 & 4.793866 \\
\hline c & -0.627764 & 6.194921 & 3.227033 & C & 5.093458 & 13.865399 & 4.060225 \\
\hline H & -1.350831 & 6.49023 & 2.635126 & H & 5.103093 & 13.496665 & 3.152287 \\
\hline H & -0.909317 & 5.390201 & 3.710329 & H & 5.894594 & 13.570105 & 4.541248 \\
\hline H & 0.169347 & 5.99353 & 2.69369 & H & 5.081754 & 14.844287 & 4.015147 \\
\hline C & -1.479165 & 7.462978 & 5.19437 & C & 3.767465 & 14.155349 & 6.113914 \\
\hline H & -2.275222 & 7.737172 & 4.692861 & $\mathrm{H}$ & 3.736813 & 15.116376 & 5.924481 \\
\hline $\mathrm{H}$ & -1.267446 & 8.142427 & 5.868114 & $\mathrm{H}$ & 4.554865 & 13.955377 & 6.662001 \\
\hline H & -1.651368 & 6.606044 & 5.637528 & $\mathrm{H}$ & 2.957089 & 13.891792 & 6.597877 \\
\hline C & -1.271479 & 9.723145 & 2.48174 & $C$ & 2.619725 & 13.768817 & 3.938295 \\
\hline C & -2.060051 & 8.756887 & 1.604971 & $\mathrm{H}$ & 2.62467 & 13.255451 & 3.103543 \\
\hline H & -2.635403 & 9.264353 & 0.995169 & $\mathrm{H}$ & 2.656487 & 14.726399 & 3.733118 \\
\hline $\mathrm{H}$ & -2.613288 & 8.179203 & 2.171195 & $\mathrm{H}$ & 1.799418 & 13.572944 & 4.43741 \\
\hline $\mathrm{H}$ & -1.438384 & 8.205996 & 1.084946 & C & 0.861469 & 9.545022 & 7.672004 \\
\hline C & -0.623257 & 10.788136 & 1.573469 & $\mathrm{H}$ & 0.360473 & 8.732838 & 7.448917 \\
\hline H & -0.080224 & 10.346026 & 0.887846 & $\mathrm{H}$ & 0.536106 & 9.906148 & 8.522969 \\
\hline H & -0.053451 & 11.374879 & 2.113329 & $\mathrm{H}$ & 1.814206 & 9.32966 & 7.751462 \\
\hline H & -1.324241 & 11.320114 & 1.142161 & C & -1.204402 & 10.939244 & 6.465355 \\
\hline C & -2.252034 & 10.451011 & 3.393693 & $\mathrm{H}$ & -1.499195 & 11.76358 & 6.024925 \\
\hline $\mathrm{H}$ & -2.934177 & 10.89746 & 2.849864 & $\mathrm{H}$ & -1.467124 & 10.961252 & 7.409234 \\
\hline $\mathrm{H}$ & -1.769942 & 11.11826 & 3.925446 & $\mathrm{H}$ & -1.621764 & 10.16795 & 6.027986 \\
\hline $\mathrm{H}$ & -2.682483 & 9.805293 & 3.992176 & C & 0.968508 & 12.317837 & 7.283773 \\
\hline C & 4.177711 & 10.135066 & 2.660824 & $\mathrm{H}$ & 1.924776 & 12.362236 & 7.493495 \\
\hline H & 4.677894 & 9.823458 & 1.915683 & $\mathrm{H}$ & 0.451509 & 12.310293 & 8.116283 \\
\hline c & 4.754856 & 10.980708 & 3.523229 & $\mathrm{H}$ & 0.714818 & 13.09873 & 6.748763 \\
\hline H & 5.641277 & 11.299078 & 3.399133 & & & & \\
\hline
\end{tabular}




\section{A.6. X-Ray Single-Crystal Analysis of $14-\mathrm{H}$}

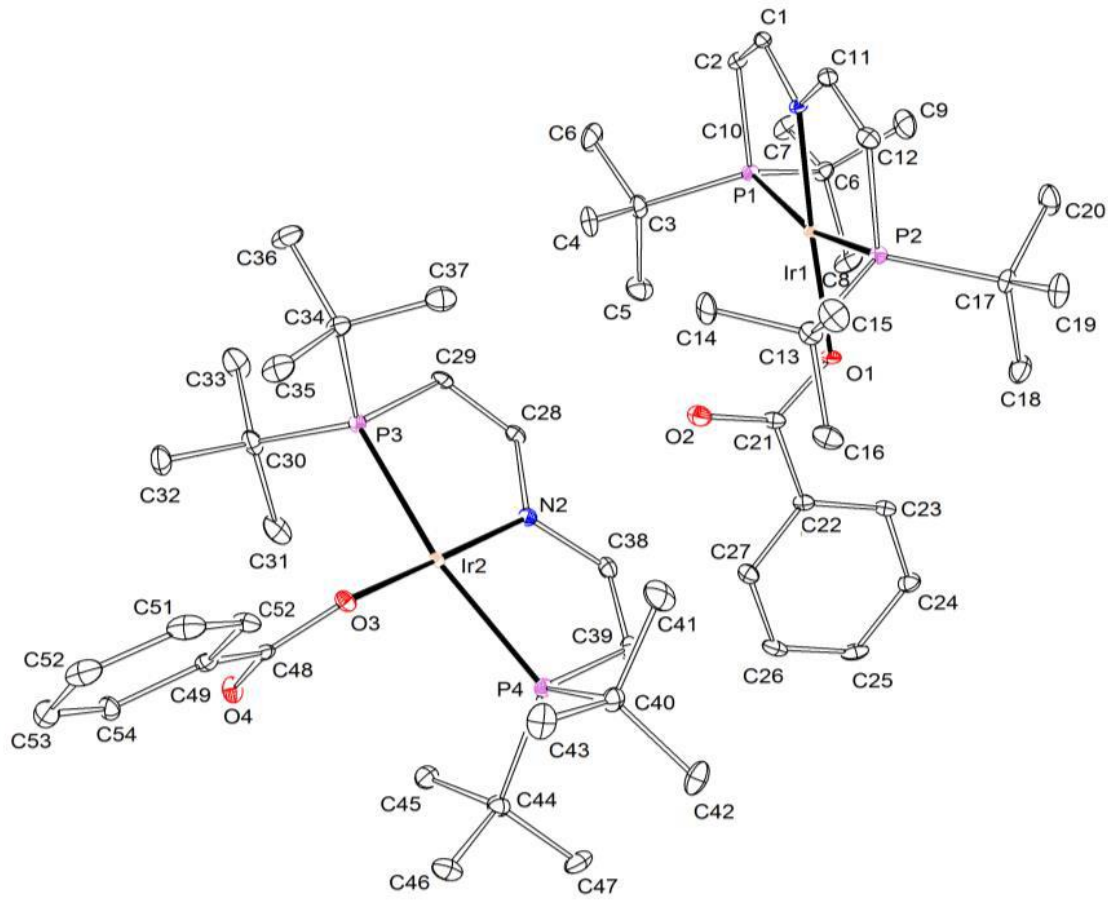

Figure A6: Thermal ellipsoid plot of $\mathbf{1 4}-\mathbf{H}$ with the anisotropic displacement parameters drawn at the $50 \%$ probability level. The asymmetric unit contains two complex molecules.

Table A11: Crystal data and structure refinement for $14-\mathrm{H}$.

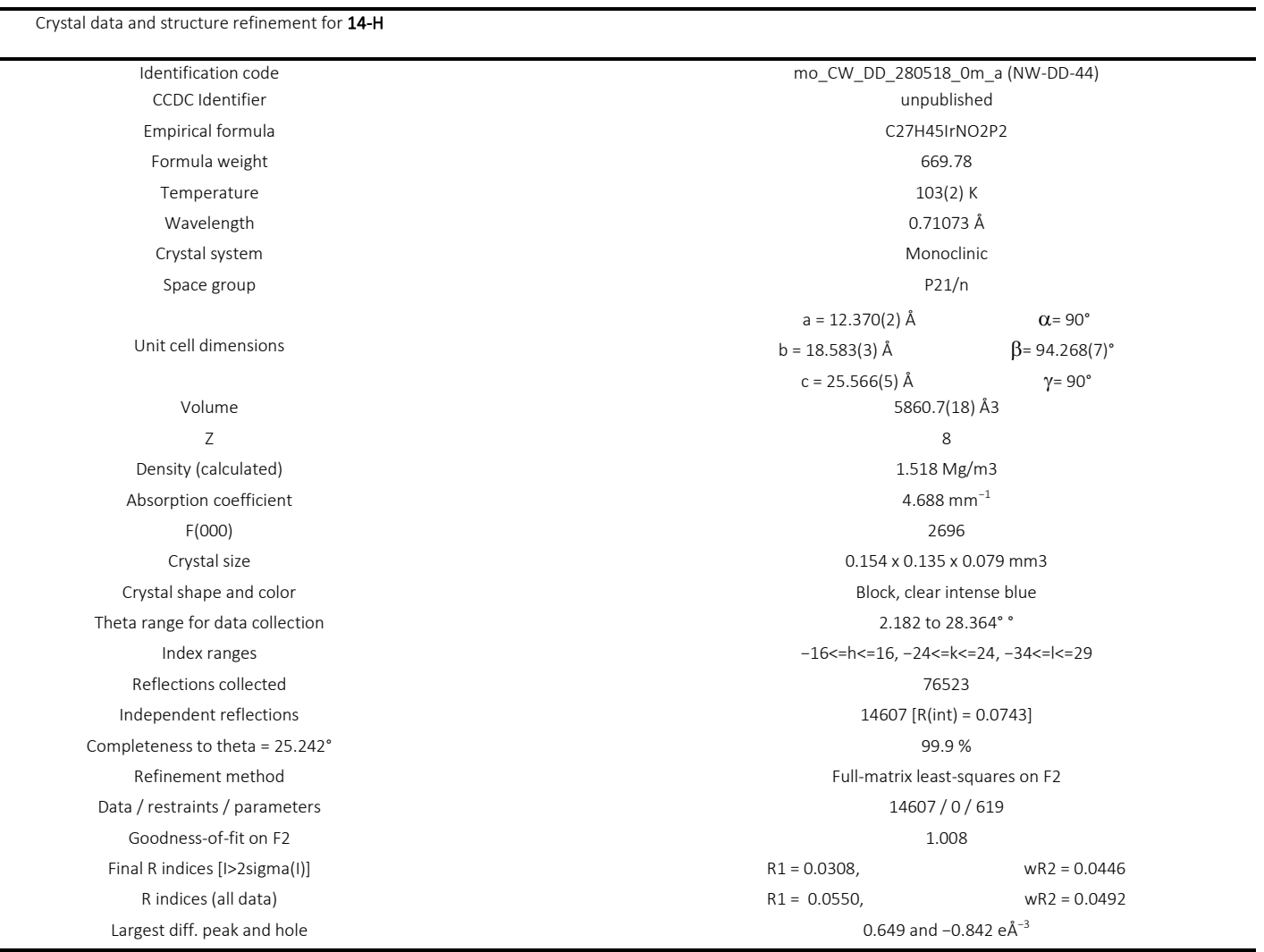


Table A12: Cartesian coordinates from X-Ray single-crystal analysis of 14-H

\begin{tabular}{|c|c|c|c|c|c|c|c|c|c|c|c|}
\hline Ir & 2.452817 & 11.13989 & 4.001664 & C & 0.931742 & 7.513328 & 6.765275 & $\mathrm{H}$ & 6.139481 & 10.07279 & 12.32370 \\
\hline$P$ & 0.612423 & 12.51779 & 4.280909 & $\mathrm{H}$ & 0.755394 & 7.558876 & 5.832905 & C & 9.236504 & 8.887131 & 12.74146 \\
\hline$P$ & 4.404435 & 10.12016 & 3.275164 & $c$ & 0.458567 & 6.443866 & 7.504803 & H & 9.784036 & 8.194896 & 12.31554 \\
\hline 0 & 1.892368 & 9.684995 & 5.348886 & $\mathrm{H}$ & -0.05074 & 5.764527 & 7.078657 & H & 9.126847 & 8.677695 & 13.69251 \\
\hline 0 & 2.857103 & 10.56603 & 7.164192 & $c$ & 0.722045 & 6.356486 & 8.86207 & $\mathrm{H}$ & 9.678177 & 9.756876 & 12.64733 \\
\hline$N$ & 2.938458 & 12.47447 & 2.650389 & $\mathrm{H}$ & 0.402901 & 5.61625 & 9.364751 & c & 7.179958 & 7.564209 & 12.15010 \\
\hline c & 2.130464 & 13.57580 & 2.386059 & $c$ & 1.447548 & 7.347228 & 9.475245 & H & 6.269004 & 7.630831 & 11.79499 \\
\hline $\mathrm{H}$ & 2.400503 & 14.20725 & 1.729669 & $\mathrm{H}$ & 1.626132 & 7.294674 & 10.40682 & H & 7.145609 & 7.271575 & 13.08477 \\
\hline c & 0.973428 & 13.76004 & 3.045252 & $c$ & 1.920704 & 8.423455 & 8.742448 & $\mathrm{H}$ & 7.689413 & 6.91401 & 11.62277 \\
\hline H & 0.393659 & 14.49167 & 2.868981 & $\mathrm{H}$ & 2.425898 & 9.101659 & 9.175248 & c & 10.01212 & 12.65514 & 11.86242 \\
\hline c & 0.488045 & 13.50165 & 5.872269 & 1 & 7.761746 & 11.85160 & 10.01073 & c & 11.47602 & 13.03844 & 11.94518 \\
\hline c & 1.939153 & 13.80837 & 6.257036 & $P$ & 7.053783 & 14.00300 & 9.520575 & C & 12.31533 & 12.89789 & 10.83824 \\
\hline H & 2.409064 & 12.97106 & 6.453193 & $P$ & 7.969004 & 9.562865 & 10.29505 & H & 11.97931 & 12.51976 & 10.03411 \\
\hline H & 1.952154 & 14.38383 & 7.05018 & 0 & 9.641185 & 12.30729 & 10.68884 & c & 13.6425 & 13.31004 & 10.90935 \\
\hline H & 2.384341 & 14.26642 & 5.513786 & 0 & 9.304564 & 12.71808 & 12.86098 & H & 14.21383 & 13.20558 & 10.15758 \\
\hline c & -0.170794 & 12.69940 & 6.99404 & $N$ & 6.051285 & 11.37053 & 9.184095 & c & 14.12531 & 13.86784 & 12.06725 \\
\hline H & -1.117994 & 12.56121 & 6.783989 & $c$ & 5.211448 & 12.32580 & 8.627237 & H & 15.02491 & 14.17043 & 12.10781 \\
\hline $\mathrm{H}$ & -0.091721 & 13.19175 & 7.837689 & $\mathrm{H}$ & 4.398262 & 12.05227 & 8.21932 & c & 13.30638 & 13.99053 & 13.17438 \\
\hline H & 0.274903 & 11.83083 & 7.079703 & $\mathrm{c}$ & 5.528685 & 13.62473 & 8.655842 & H & 13.64866 & 14.36671 & 13.97678 \\
\hline c & -0.244478 & 14.83836 & 5.689981 & $\mathrm{H}$ & 4.989353 & 14.29355 & 8.250501 & c & 11.98897 & 13.56712 & 13.11812 \\
\hline $\mathrm{H}$ & 0.2357 & 15.38741 & 5.035478 & $c$ & 6.473355 & 15.03817 & 10.97255 & H & 11.43521 & 13.63956 & 13.88663 \\
\hline H & -0.281289 & 15.31035 & 6.54806 & $c$ & 5.834332 & 14.01755 & 11.93123 & & & & \\
\hline $\mathrm{H}$ & -1.155486 & 14.67065 & 5.370072 & $\mathrm{H}$ & 5.103085 & 13.55272 & 11.47342 & & & & \\
\hline c & -1.013668 & 11.74951 & 3.776571 & $\mathrm{H}$ & 5.482881 & 14.48411 & 12.71813 & & & & \\
\hline c & -1.300129 & 10.52255 & 4.645382 & $\mathrm{H}$ & 6.510169 & 13.36601 & 12.21257 & & & & \\
\hline $\mathrm{H}$ & -2.076101 & 10.04282 & 4.287461 & $c$ & 7.646076 & 15.72327 & 11.67554 & & & & \\
\hline $\mathrm{H}$ & -1.488202 & 10.80911 & 5.563476 & $\mathrm{H}$ & 8.352945 & 15.06653 & 11.84705 & & & & \\
\hline H & -0.519497 & 9.930093 & 4.641507 & $\mathrm{H}$ & 7.340408 & 16.10364 & 12.52541 & & & & \\
\hline c & -0.817187 & 11.26613 & 2.335069 & $\mathrm{H}$ & 7.996362 & 16.43868 & 11.10466 & & & & \\
\hline H & -1.614378 & 10.77655 & 2.043179 & $c$ & 5.415643 & 16.09509 & 10.58676 & & & & \\
\hline H & -0.03682 & 10.67486 & 2.292314 & $\mathrm{H}$ & 5.806692 & 16.73249 & 9.953349 & & & & \\
\hline H & -0.674767 & 12.03798 & 1.748255 & $\mathrm{H}$ & 5.122033 & 16.57207 & 11.39092 & & & & \\
\hline C & -2.199287 & 12.71793 & 3.824399 & $\mathrm{H}$ & 4.646983 & 15.65141 & 10.17115 & & & & \\
\hline $\mathrm{H}$ & -1.982513 & 13.52523 & 3.312871 & $c$ & 8.140334 & 14.94756 & 8.325991 & & & & \\
\hline H & -2.385045 & 12.96117 & 4.755393 & $c$ & 9.565027 & 15.02905 & 8.903269 & & & & \\
\hline $\mathrm{H}$ & -2.988936 & 12.28703 & 3.435578 & $\mathrm{H}$ & 9.896597 & 14.12468 & 9.083747 & & & & \\
\hline c & 4.097421 & 12.34914 & 1.882332 & $\mathrm{H}$ & 9.550752 & 15.54415 & 9.736848 & & & & \\
\hline H & 4.308852 & 13.01987 & 1.243662 & $\mathrm{H}$ & 10.15473 & 15.47022 & 8.256721 & & & & \\
\hline c & 4.916657 & 11.29958 & 2.030177 & $c$ & 7.641863 & 16.35370 & 8.00185 & & & & \\
\hline H & 5.707167 & 11.18994 & 1.51485 & $\mathrm{H}$ & 8.211321 & 16.75079 & 7.31015 & & & & \\
\hline c & 5.831877 & 10.07322 & 4.48808 & $\mathrm{H}$ & 7.675862 & 16.90676 & 8.810162 & & & & \\
\hline c & 5.751459 & 11.41190 & 5.234134 & $\mathrm{H}$ & 6.718446 & 16.30618 & 7.677148 & & & & \\
\hline H & 5.834817 & 12.14857 & 4.59322 & $c$ & 8.196932 & 14.10950 & 7.054259 & & & & \\
\hline H & 6.478413 & 11.46497 & 5.889197 & $\mathrm{H}$ & 7.308538 & 14.08179 & 6.641498 & & & & \\
\hline H & 4.889786 & 11.47700 & 5.69638 & $\mathrm{H}$ & 8.482791 & 13.19867 & 7.275732 & & & & \\
\hline c & 7.208076 & 9.969657 & 3.815272 & $\mathrm{H}$ & 8.835875 & 14.50917 & 6.427826 & & & & \\
\hline H & 7.278924 & 9.111806 & 3.346779 & $c$ & 5.667488 & 10.03503 & 9.068425 & & & & \\
\hline H & 7.910038 & 10.02873 & 4.496569 & $\mathrm{H}$ & 4.838209 & 9.826193 & 8.654695 & & & & \\
\hline H & 7.313685 & 10.70231 & 3.173058 & $c$ & 6.431093 & 9.047228 & 9.52162 & & & & \\
\hline c & 5.650829 & 8.922793 & 5.481434 & $\mathrm{H}$ & 6.182132 & 8.133849 & 9.442561 & & & & \\
\hline H & 4.734548 & 8.932382 & 5.828902 & $c$ & 9.329363 & 8.744726 & 9.305399 & & & & \\
\hline $\mathrm{H}$ & 6.282568 & 9.029091 & 6.223052 & $c$ & 9.108088 & 9.207399 & 7.862317 & & & & \\
\hline H & 5.819456 & 8.070368 & 5.028289 & $\mathrm{H}$ & 9.843458 & 8.886054 & 7.29985 & & & & \\
\hline c & 4.140434 & 8.496265 & 2.36913 & $\mathrm{H}$ & 9.078978 & 10.18656 & 7.834017 & & & & \\
\hline c & 3.458854 & 7.492477 & 3.310118 & $\mathrm{H}$ & 8.260216 & 8.845653 & 7.52966 & & & & \\
\hline H & 3.207781 & 6.690562 & 2.805856 & $c$ & 9.292344 & 7.214579 & 9.356898 & & & & \\
\hline H & 2.655779 & 7.900218 & 3.69644 & $\mathrm{H}$ & 8.38351 & 6.90312 & 9.163495 & & & & \\
\hline H & 4.077988 & 7.246004 & 4.028689 & $\mathrm{H}$ & 9.558474 & 6.911259 & 10.24998 & & & & \\
\hline H & 5.196128 & 7.113336 & 1.236625 & $\mathrm{H}$ & 9.911343 & 6.850213 & 8.690235 & & & & \\
\hline H & 6.007382 & 7.602231 & 2.52656 & $c$ & 10.70166 & 9.263856 & 9.737663 & & & & \\
\hline H & 5.886537 & 8.557033 & 1.247945 & $\mathrm{H}$ & 11.38023 & 8.966892 & 9.095985 & & & & \\
\hline c & 3.185434 & 8.812537 & 1.21651 & $\mathrm{H}$ & 10.91792 & 8.913985 & 10.62717 & & & & \\
\hline H & 3.632497 & 9.402693 & 0.574449 & $\mathrm{H}$ & 10.68607 & 10.2433 & 9.766804 & & & & \\
\hline H & 2.38594 & 9.257556 & 1.567446 & $c$ & 7.862851 & 8.93701 & 12.06837 & & & & \\
\hline $\mathrm{H}$ & 2.926132 & 7.979959 & 0.769306 & $c$ & 6.991359 & 9.971386 & 12.79744 & & & & \\
\hline c & 2.191708 & 9.693228 & 6.606366 & $\mathrm{H}$ & 7.45723 & 10.83327 & 12.81998 & & & & \\
\hline c & 1.663472 & 8.520367 & 7.380873 & $\mathrm{H}$ & 6.821503 & 9.667787 & 13.71360 & & & & \\
\hline
\end{tabular}




\section{A.7. X-Ray Single-Crystal Analysis of 14-F}

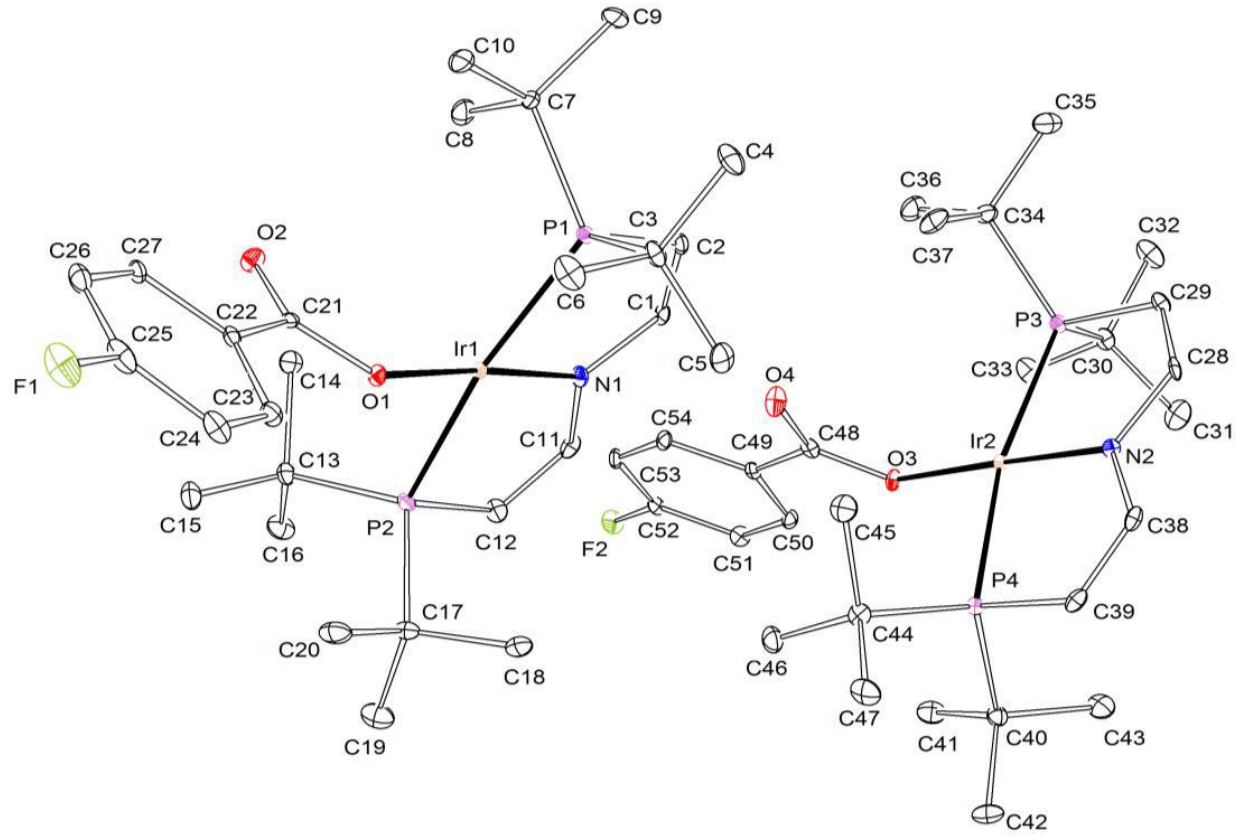

Figure A7: Thermal ellipsoid plot of 14-F with the anisotropic displacement parameters drawn at the 50\% probability level. The asymmetric unit contains two complex molecules.

Table A13: Crystal data and structure refinement for914-F.

\begin{tabular}{|c|c|c|}
\hline \multicolumn{3}{|c|}{ Crystal data and structure refinement for $14-\mathrm{F}$} \\
\hline Identification code & \multicolumn{2}{|c|}{ mo_CW_DD_250618_0m_a (NW-DD-61) } \\
\hline CCDC Identifier & \multicolumn{2}{|c|}{ unpublished } \\
\hline Empirical formula & \multicolumn{2}{|c|}{$\mathrm{C} 27 \mathrm{H} 44 \mathrm{FIrNO} 2 \mathrm{P} 2$} \\
\hline Formula weight & \multicolumn{2}{|c|}{687.77} \\
\hline Temperature & \multicolumn{2}{|c|}{$100(2) \mathrm{K}$} \\
\hline Wavelength & \multicolumn{2}{|c|}{$0.71073 \AA$} \\
\hline Crystal system & \multicolumn{2}{|c|}{ Monoclinic } \\
\hline Space group & \multicolumn{2}{|c|}{$\mathrm{P} 21 / \mathrm{n}$} \\
\hline \multirow{3}{*}{ Unit cell dimensions } & $a=12.4837(5) \AA$ & $\alpha=90^{\circ}$ \\
\hline & $b=18.583(3) \AA$ & $\beta=94.480(2)$ \\
\hline & $c=25.5619(10) \AA$ & $\gamma=90^{\circ}$ \\
\hline Volume & \multicolumn{2}{|c|}{$5911.4(4) \AA 3$} \\
\hline z & \multicolumn{2}{|c|}{8} \\
\hline Density (calculated) & \multicolumn{2}{|c|}{$1.546 \mathrm{Mg} / \mathrm{m} 3$} \\
\hline Absorption coefficient & \multicolumn{2}{|c|}{$4.655 \mathrm{~mm}^{-1}$} \\
\hline$F(000)$ & \multicolumn{2}{|c|}{2760} \\
\hline Crystal size & \multicolumn{2}{|c|}{$0.200 \times 0.105 \times 0.089 \mathrm{~mm} 3$} \\
\hline Crystal shape and color & \multicolumn{2}{|c|}{ Block, clear intense blue } \\
\hline Theta range for data collection & \multicolumn{2}{|c|}{2.192 to $28.343^{\circ} \circ$} \\
\hline Index ranges & \multicolumn{2}{|c|}{$-16<=h<=16,-24<=k<=24,-34<=\mid<=34$} \\
\hline Reflections collected & \multicolumn{2}{|c|}{219961} \\
\hline Independent reflections & \multicolumn{2}{|c|}{$14732[R(\mathrm{int})=0.1746]$} \\
\hline Completeness to theta $=25.242^{\circ}$ & \multicolumn{2}{|c|}{$99.9 \%$} \\
\hline Refinement method & \multicolumn{2}{|c|}{ Full-matrix least-squares on F2 } \\
\hline Data / restraints / parameters & \multicolumn{2}{|c|}{14732 / 0 / 637} \\
\hline Goodness-of-fit on F2 & \multicolumn{2}{|c|}{1.016} \\
\hline Final R indices [l>2sigma $(\mathrm{I})]$ & $\mathrm{R} 1=0.0365$ & $w R 2=0.0478$ \\
\hline$R$ indices (all data) & $\mathrm{R} 1=0.0667$ & $w R 2=0.0529$ \\
\hline Largest diff. peak and hole & \multicolumn{2}{|c|}{0.904 and $-1.093 \mathrm{e}^{-3}$} \\
\hline
\end{tabular}


Table A14: Cartesian coordinates from X-Ray single-crystal analysis of 14-F.

\begin{tabular}{|c|c|c|c|c|c|c|c|c|c|c|c|}
\hline Ir & 2.661991 & 11.816838 & 15.51165 & C & 0.442023 & 12.60319 & 13.62937 & $\mathrm{H}$ & 5.509987 & 11.34964 & 19.79686 \\
\hline$P$ & 3.325785 & 13.971356 & 16.03715 & C & -1.01419 & 12.98378 & 13.51946 & $\mathrm{H}$ & 4.603406 & 12.04695 & 20.91618 \\
\hline$P$ & 2.477499 & 9.532305 & 15.21477 & c & -1.88451 & 12.88645 & 14.60672 & $\mathrm{H}$ & 3.916161 & 11.34516 & 19.65275 \\
\hline$F$ & -4.89300 & 14.285311 & 13.21197 & H & -1.57081 & 12.52955 & 15.42934 & C & 4.770636 & 8.801621 & 20.08179 \\
\hline o & 0.785569 & 12.265528 & 14.82346 & c & -3.19331 & 13.30241 & 14.49928 & $\mathrm{H}$ & 4.135232 & 8.899323 & 19.34212 \\
\hline 0 & 1.175261 & 12.642326 & 12.64715 & H & -3.79325 & 13.22162 & 15.23141 & $\mathrm{H}$ & 4.603198 & 7.955285 & 20.54664 \\
\hline$N$ & 4.376557 & 11.348043 & 16.34553 & c & -3.60358 & 13.83392 & 13.31103 & $\mathrm{H}$ & 5.685126 & 8.805504 & 19.72955 \\
\hline c & 5.201982 & 12.313153 & 16.89965 & c & -2.80169 & 13.93371 & 12.21186 & $c$ & 3.21825 & 9.87337 & 21.73900 \\
\hline $\mathrm{H}$ & 6.021278 & 12.052119 & 17.30352 & $\mathrm{H}$ & -3.13190 & 14.29430 & 11.39737 & $\mathrm{H}$ & 2.516811 & 9.885634 & 21.05474 \\
\hline c & 4.862168 & 13.609257 & 16.87458 & c & -1.49274 & 13.49436 & 12.31723 & $\mathrm{H}$ & 3.098837 & 10.63586 & 22.34294 \\
\hline $\mathrm{H}$ & 5.398195 & 14.2852 & 17.27241 & H & -0.91728 & 13.54201 & 11.56289 & $\mathrm{H}$ & 3.16107 & 9.03941 & 22.25051 \\
\hline c & 2.230464 & 14.852124 & 17.26147 & Ir & 7.95995 & 11.07210 & 21.53628 & C & 8.235924 & 9.637756 & 18.91914 \\
\hline c & 2.691907 & 16.260201 & 17.61763 & $P$ & 9.791645 & 12.45913 & 21.2554 & $C$ & 8.806677 & 8.46154 & 18.16569 \\
\hline $\mathrm{H}$ & 2.644063 & 16.830637 & 16.82218 & $P$ & 6.026397 & 10.03032 & 22.27067 & c & 9.579124 & 7.509252 & 18.82047 \\
\hline $\mathrm{H}$ & 3.616141 & 16.228835 & 17.94199 & $\mathrm{~F}$ & 10.43427 & 5.32426 & 16.09526 & $\mathrm{H}$ & 9.735063 & 7.588447 & 19.75425 \\
\hline $\mathrm{H}$ & 2.112253 & 16.62663 & 18.31773 & 0 & 8.519097 & 9.643553 & 20.17269 & $c$ & 10.12409 & 6.44304 & 18.11770 \\
\hline c & 2.211838 & 13.984196 & 18.52641 & 0 & 7.557341 & 10.47760 & 18.32922 & $\mathrm{H}$ & 10.65158 & 5.786087 & 18.55664 \\
\hline $\mathrm{H}$ & 1.91668 & 13.078362 & 18.29673 & N & 7.470629 & 12.39156 & 22.89120 & $c$ & 9.877746 & 6.366911 & 16.77004 \\
\hline $\mathrm{H}$ & 1.594118 & 14.373053 & 19.18033 & c & 8.261255 & 13.50780 & 23.13743 & c & 9.130718 & 7.276833 & 16.08320 \\
\hline $\mathrm{H}$ & 3.11279 & 13.946736 & 18.91020 & $\mathrm{H}$ & 7.981559 & 14.14371 & 23.78540 & $\mathrm{H}$ & 8.987277 & 7.189834 & 15.14813 \\
\hline c & 0.797794 & 14.906828 & 16.70832 & c & 9.408588 & 13.70285 & 22.47867 & $c$ & 8.586592 & 8.335724 & 16.79858 \\
\hline $\mathrm{H}$ & 0.779747 & 15.464499 & 15.90268 & H & 9.976079 & 14.44561 & 22.64826 & $\mathrm{H}$ & 8.056891 & 8.982773 & 16.34775 \\
\hline H & 0.200989 & 15.290297 & 17.38448 & c & 11.42466 & 11.70105 & 21.77705 & $c$ & 5.507203 & 11.20516 & 23.51023 \\
\hline $\mathrm{H}$ & 0.498985 & 14.000585 & 16.48508 & c & 11.22805 & 11.24761 & 23.23166 & & & & \\
\hline c & 3.843354 & 15.057247 & 14.60165 & $\mathrm{H}$ & 10.45658 & 10.64558 & 23.28472 & & & & \\
\hline c & 4.460973 & 14.079315 & 13.59657 & $\mathrm{H}$ & 11.07100 & 12.03069 & 23.79955 & & & & \\
\hline H & 3.783144 & 13.430836 & 13.31299 & $\mathrm{H}$ & 12.03116 & 10.77727 & 23.53859 & & & & \\
\hline $\mathrm{H}$ & 5.209012 & 13.606804 & 14.01797 & c & 12.61344 & 12.67543 & 21.72820 & & & & \\
\hline $\mathrm{H}$ & 4.785777 & 14.57485 & 12.81598 & H & 12.77514 & 12.94721 & 20.80061 & & & & \\
\hline c & 4.903057 & 16.108426 & 14.98628 & H & 13.41286 & 12.23351 & 22.08319 & & & & \\
\hline H & 5.147978 & 16.630458 & 14.19389 & $\mathrm{H}$ & 12.40988 & 13.46653 & 22.26963 & & & & \\
\hline $\mathrm{H}$ & 5.698269 & 15.656856 & 15.33857 & c & 11.71892 & 10.46781 & 20.92472 & & & & \\
\hline H & 4.536663 & 16.706289 & 15.67090 & $\mathrm{H}$ & 11.91081 & 10.74492 & 20.00450 & & & & \\
\hline c & 2.649678 & 15.761173 & 13.95520 & H & 10.94015 & 9.872924 & 20.93106 & & & & \\
\hline $\mathrm{H}$ & 2.931489 & 16.175041 & 13.11276 & H & 12.49454 & 9.994485 & 21.29181 & & & & \\
\hline $\mathrm{H}$ & 2.310587 & 16.452502 & 14.56141 & c & 9.918849 & 13.42528 & 19.65369 & & & & \\
\hline $\mathrm{H}$ & 1.941935 & 15.10712 & 13.77712 & C & 10.64069 & 14.77174 & 19.82598 & & & & \\
\hline c & 4.767473 & 10.018901 & 16.46518 & H & 10.64961 & 15.24945 & 18.97034 & & & & \\
\hline $\mathrm{H}$ & 5.592971 & 9.814337 & 16.88852 & H & 11.56161 & 14.61325 & 20.12124 & & & & \\
\hline c & 4.008344 & 9.022091 & 15.99811 & H & 10.17096 & 15.31081 & 20.49618 & & & & \\
\hline H & 4.261461 & 8.109716 & 16.07558 & c & 10.58144 & 12.61118 & 18.53826 & & & & \\
\hline c & 2.596767 & 8.897112 & 13.45226 & H & 10.46427 & 13.07392 & 17.68239 & & & & \\
\hline c & 3.475638 & 9.928874 & 12.72085 & H & 10.16617 & 11.72482 & 18.48994 & & & & \\
\hline H & 3.671487 & 9.608062 & 11.81579 & H & 11.53780 & 12.51390 & 18.72880 & & & & \\
\hline $\mathrm{H}$ & 4.314384 & 10.050825 & 13.21281 & c & 8.457053 & 13.71825 & 19.25239 & & & & \\
\hline $\mathrm{H}$ & 3.000305 & 10.784278 & 12.66848 & H & 8.44507 & 14.30944 & 18.47086 & & & & \\
\hline c & 1.23624 & 8.837966 & 12.75953 & H & 7.994483 & 14.15377 & 19.99853 & & & & \\
\hline $\mathrm{H}$ & 0.772327 & 9.693147 & 12.87719 & H & 8.003757 & 12.87755 & 19.03300 & & & & \\
\hline $\mathrm{H}$ & 0.700025 & 8.118524 & 13.15359 & c & 6.321867 & 12.25536 & 23.66061 & & & & \\
\hline H & 1.363726 & 8.664303 & 11.80351 & $\mathrm{H}$ & 6.11104 & 12.92038 & 24.30545 & & & & \\
\hline c & 3.294631 & 7.528001 & 13.37451 & c & 5.507203 & 11.20516 & 23.51023 & & & & \\
\hline $\mathrm{H}$ & 3.334687 & 7.234337 & 12.44040 & $\mathrm{H}$ & 4.715032 & 11.09340 & 24.02255 & & & & \\
\hline $\mathrm{H}$ & 2.789877 & 6.873668 & 13.90126 & c & 6.305395 & 8.414102 & 23.16102 & & & & \\
\hline H & 4.203797 & 7.603962 & 13.73232 & c & 7.023207 & 7.429481 & 22.23945 & & & & \\
\hline c & 1.103919 & 8.726886 & 16.20499 & H & 6.406382 & 7.127103 & 21.54053 & & & & \\
\hline c & 1.296984 & 9.237285 & 17.63955 & $\mathrm{H}$ & 7.329718 & 6.658252 & 22.76065 & & & & \\
\hline H & 1.343103 & 10.216201 & 17.63456 & H & 7.793985 & 7.8715 & 21.82603 & & & & \\
\hline $\mathrm{H}$ & 2.129356 & 8.872918 & 18.0067 & c & 5.035853 & 7.780841 & 23.73031 & & & & \\
\hline H & 0.541758 & 8.948676 & 18.19339 & $\mathrm{H}$ & 4.440204 & 7.525511 & 22.99517 & & & & \\
\hline c & 1.16592 & 7.193049 & 16.20267 & H & 4.580648 & 8.426347 & 24.31042 & & & & \\
\hline $\mathrm{H}$ & 0.982116 & 6.860067 & 15.29950 & H & 5.272798 & 6.984693 & 24.25030 & & & & \\
\hline $\mathrm{H}$ & 0.496509 & 6.838122 & 16.82420 & c & 7.261754 & 8.736771 & 24.33121 & & & & \\
\hline H & 2.058712 & 6.901782 & 16.48284 & $\mathrm{H}$ & 8.042254 & 9.221974 & 23.99095 & & & & \\
\hline c & -0.26464 & 9.205473 & 15.72370 & H & 7.554454 & 7.902271 & 24.75351 & & & & \\
\hline $\mathrm{H}$ & -0.25225 & 10.179967 & 15.62067 & H & 6.795016 & 9.290726 & 24.9913 & & & & \\
\hline $\mathrm{H}$ & -0.94775 & 8.955068 & 16.38027 & c & 4.596019 & 9.96626 & 21.06241 & & & & \\
\hline $\mathrm{H}$ & -0.47188 & 8.788428 & 14.86143 & c & 4.662451 & 11.29859 & 20.28622 & & & & \\
\hline
\end{tabular}




\section{A.8. X-Ray Single-Crystal Analysis of 14-OMe}

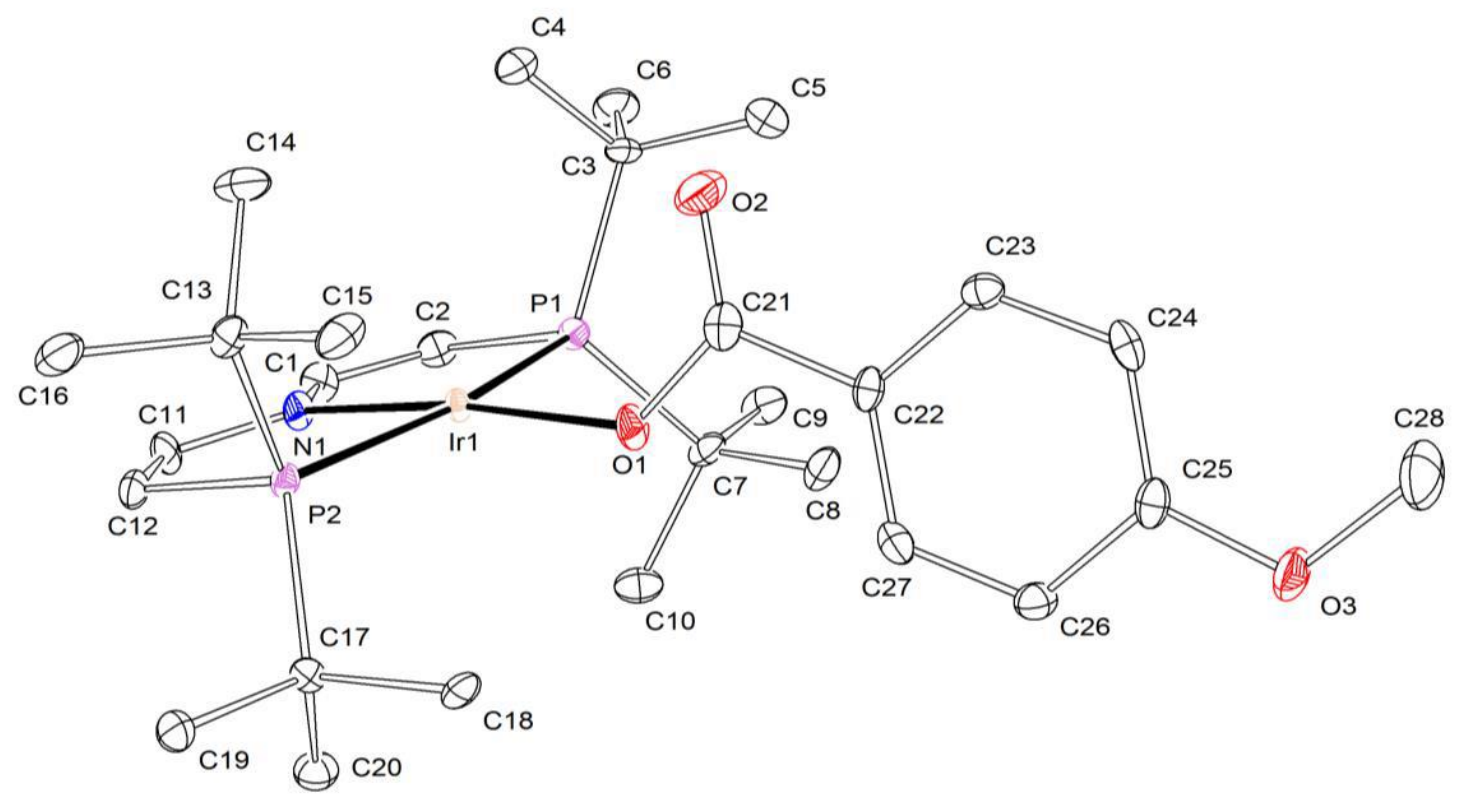

Figure A8: Thermal ellipsoid plot of 14-OMe with the anisotropic displacement parameters drawn at the 50\% probability level. The asymmetric unit contains one complex molecule.

Table A15: Crystal data and structure refinement for 14-OMe.

\begin{tabular}{|c|c|c|}
\hline \multicolumn{3}{|c|}{ Crystal data and structure refinement for $14-\mathrm{OMe}$} \\
\hline Identification code & \multicolumn{2}{|c|}{ MO_DD_021219_Om_a (MH-DD-018) } \\
\hline CCDC Identifier & \multicolumn{2}{|c|}{ unpublished } \\
\hline Empirical formula & \multicolumn{2}{|c|}{$\mathrm{C} 28 \mathrm{H} 47 \mathrm{IrNO} 3 \mathrm{P} 2$} \\
\hline Formula weight & \multicolumn{2}{|c|}{699.80} \\
\hline Temperature & \multicolumn{2}{|c|}{$100(2) \mathrm{K}$} \\
\hline Wavelength & \multicolumn{2}{|c|}{$0.71073 \AA$} \\
\hline Crystal system & \multicolumn{2}{|c|}{ Monoclinic } \\
\hline Space group & \multicolumn{2}{|c|}{$\mathrm{P} 21 / \mathrm{c}$} \\
\hline \multirow{3}{*}{ Unit cell dimensions } & $a=8.4979(2) \AA$ & $\alpha=90^{\circ}$ \\
\hline & $b=11.9821(3) \AA$ & $\beta=94.2010(10)^{\circ}$ \\
\hline & $c=28.9933(7) \AA$ & $\gamma=90^{\circ}$ \\
\hline Volume & \multicolumn{2}{|c|}{$2944.24(12) \AA 3$} \\
\hline z & \multicolumn{2}{|c|}{4} \\
\hline Density (calculated) & \multicolumn{2}{|c|}{$1.579 \mathrm{Mg} / \mathrm{m} 3$} \\
\hline Absorption coefficient & \multicolumn{2}{|c|}{$4.672 \mathrm{~mm}^{-1}$} \\
\hline$F(000)$ & \multicolumn{2}{|c|}{1412} \\
\hline Crystal size & \multicolumn{2}{|c|}{$0.174 \times 0.107 \times 0.095 \mathrm{~mm} 3$} \\
\hline Crystal shape and color & \multicolumn{2}{|c|}{ Block, clear intense blue } \\
\hline Theta range for data collection & \multicolumn{2}{|c|}{2.208 to $28.354^{\circ}$} \\
\hline Index ranges & \multicolumn{2}{|c|}{$-11<=h<=11,-15<=k<=15,-38<=\mid<=38$} \\
\hline Reflections collected & \multicolumn{2}{|c|}{70890} \\
\hline Independent reflections & \multicolumn{2}{|c|}{$7330[\mathrm{R}$ (int) $=0.1037]$} \\
\hline Completeness to theta $=25.242^{\circ}$ & \multicolumn{2}{|c|}{$100 \%$} \\
\hline Refinement method & \multicolumn{2}{|c|}{ Full-matrix least-squares on F2 } \\
\hline Data / restraints / parameters & \multicolumn{2}{|c|}{$7330 / 0 / 329$} \\
\hline Goodness-of-fit on F2 & \multicolumn{2}{|c|}{1.029} \\
\hline Final R indices [ $[>2 \operatorname{sigma}(I)]$ & $\mathrm{R} 1=0.0290$ & $w R 2=0.0414$ \\
\hline $\mathrm{R}$ indices (all data) & $R 1=0.0503$ & $w R 2=0.0453$ \\
\hline Largest diff. peak and hole & \multicolumn{2}{|c|}{0.648 and $-0.831 e^{-3}$} \\
\hline
\end{tabular}


Table A16: Cartesian coordinates from X-Ray single-crystal analysis of 14-OMe.

\begin{tabular}{|c|c|c|c|c|c|c|c|}
\hline Ir & 4.742295 & 3.126549 & 11.380726 & C & 4.530399 & 3.493333 & 14.895335 \\
\hline$P$ & 3.799737 & 3.071168 & 9.255299 & c & 3.073131 & 3.554071 & 14.403484 \\
\hline $\mathrm{P}$ & 5.492279 & 2.674441 & 13.524225 & H & 3.02189 & 4.116259 & 13.602412 \\
\hline 0 & 5.548542 & 4.98985 & 11.307136 & $\mathrm{H}$ & 2.506645 & 3.934946 & 15.106649 \\
\hline 0 & 3.759925 & 6.336494 & 11.415482 & H & 2.763037 & 2.649686 & 14.188267 \\
\hline 0 & 8.448441 & 10.322028 & 9.694756 & c & 5.027116 & 4.923697 & 15.140624 \\
\hline $\mathrm{N}$ & 4.188991 & 1.234576 & 11.423723 & H & 5.102183 & 5.394749 & 14.284555 \\
\hline C & 3.610908 & 0.64109 & 10.310943 & H & 5.904837 & 4.894616 & 15.575541 \\
\hline H & 3.378511 & -0.279291 & 10.348157 & H & 4.391669 & 5.395576 & 15.718499 \\
\hline C & 3.371979 & 1.322812 & 9.192235 & C & 4.559202 & 2.702587 & 16.212431 \\
\hline $\mathrm{H}$ & 3.002656 & 0.916367 & 8.417042 & H & 4.206434 & 1.801077 & 16.059989 \\
\hline C & 2.145231 & 3.915774 & 8.981673 & H & 4.007586 & 3.160578 & 16.880551 \\
\hline C & 1.420242 & 3.828017 & 10.344861 & $\mathrm{H}$ & 5.481955 & 2.641765 & 16.536833 \\
\hline $\mathrm{H}$ & 0.501897 & 4.156171 & 10.248167 & C & 7.347066 & 2.743853 & 13.789841 \\
\hline $\mathrm{H}$ & 1.89547 & 4.37645 & 11.003496 & C & 7.870411 & 4.148371 & 13.459049 \\
\hline $\mathrm{H}$ & 1.403953 & 2.895834 & 10.646795 & H & 7.520676 & 4.430573 & 12.588175 \\
\hline C & 2.289163 & 5.392269 & 8.595103 & H & 8.849817 & 4.133213 & 13.428428 \\
\hline H & 2.687823 & 5.458853 & 7.70234 & H & 7.57488 & 4.778066 & 14.149375 \\
\hline $\mathrm{H}$ & 2.865943 & 5.845384 & 9.245034 & C & 7.785714 & 2.34927 & 15.198571 \\
\hline $\mathrm{H}$ & 1.405721 & 5.816435 & 8.590823 & H & 7.377894 & 1.491328 & 15.439407 \\
\hline c & 1.275007 & 3.206386 & 7.927678 & H & 7.498909 & 3.037331 & 15.834767 \\
\hline $\mathrm{H}$ & 0.437322 & 3.701007 & 7.809153 & H & 8.761796 & 2.265995 & 15.225462 \\
\hline $\mathrm{H}$ & 1.076476 & 2.29486 & 8.227819 & C & 7.942556 & 1.765071 & 12.765976 \\
\hline $\mathrm{H}$ & 1.757401 & 3.171878 & 7.075338 & H & 7.640989 & 2.009937 & 11.866273 \\
\hline c & 4.985787 & 3.376124 & 7.8455 & H & 7.64559 & 0.854491 & 12.973502 \\
\hline c & 5.633192 & 4.763376 & 7.994848 & H & 8.920896 & 1.806505 & 12.805156 \\
\hline $\mathrm{H}$ & 6.27884 & 4.902225 & 7.270806 & C & 4.951767 & 6.137375 & 11.215879 \\
\hline $\mathrm{H}$ & 6.09382 & 4.817667 & 8.858146 & c & 5.882424 & 7.265682 & 10.833271 \\
\hline $\mathrm{H}$ & 4.939389 & 5.454108 & 7.950781 & C & 5.378349 & 8.546185 & 10.678429 \\
\hline c & 4.359616 & 3.232327 & 6.451113 & H & 4.456219 & 8.707069 & 10.840586 \\
\hline $\mathrm{H}$ & 5.055082 & 3.335829 & 5.768449 & c & 6.196674 & 9.60013 & 10.290239 \\
\hline $\mathrm{H}$ & 3.675246 & 3.922808 & 6.327557 & H & 5.837934 & 10.473494 & 10.185219 \\
\hline $\mathrm{H}$ & 3.948835 & 2.346539 & 6.367258 & C & 7.539971 & 9.362849 & 10.058396 \\
\hline C & 6.098412 & 2.329488 & 8.004419 & C & 8.055518 & 8.079662 & 10.194732 \\
\hline H & 5.716077 & 1.431022 & 7.920854 & H & 8.974034 & 7.914992 & 10.016642 \\
\hline $\mathrm{H}$ & 6.514739 & 2.426627 & 8.886252 & c & 7.231431 & 7.043761 & 10.589398 \\
\hline $\mathrm{H}$ & 6.774407 & 2.462945 & 7.307558 & H & 7.591062 & 6.170829 & 10.695026 \\
\hline c & 4.456579 & 0.445986 & 12.536012 & c & 7.962214 & 11.65554 & 9.542892 \\
\hline H & 4.210673 & -0.47152 & 12.521467 & H & 8.709069 & 12.253315 & 9.330132 \\
\hline C & 5.048549 & 0.940715 & 13.622739 & H & 7.538943 & 11.945962 & 10.377708 \\
\hline H & 5.222603 & 0.413011 & 14.393248 & H & 7.304821 & 11.682823 & 8.816624 \\
\hline
\end{tabular}




\section{A.9. X-Ray Single-Crystal Analysis of 11}

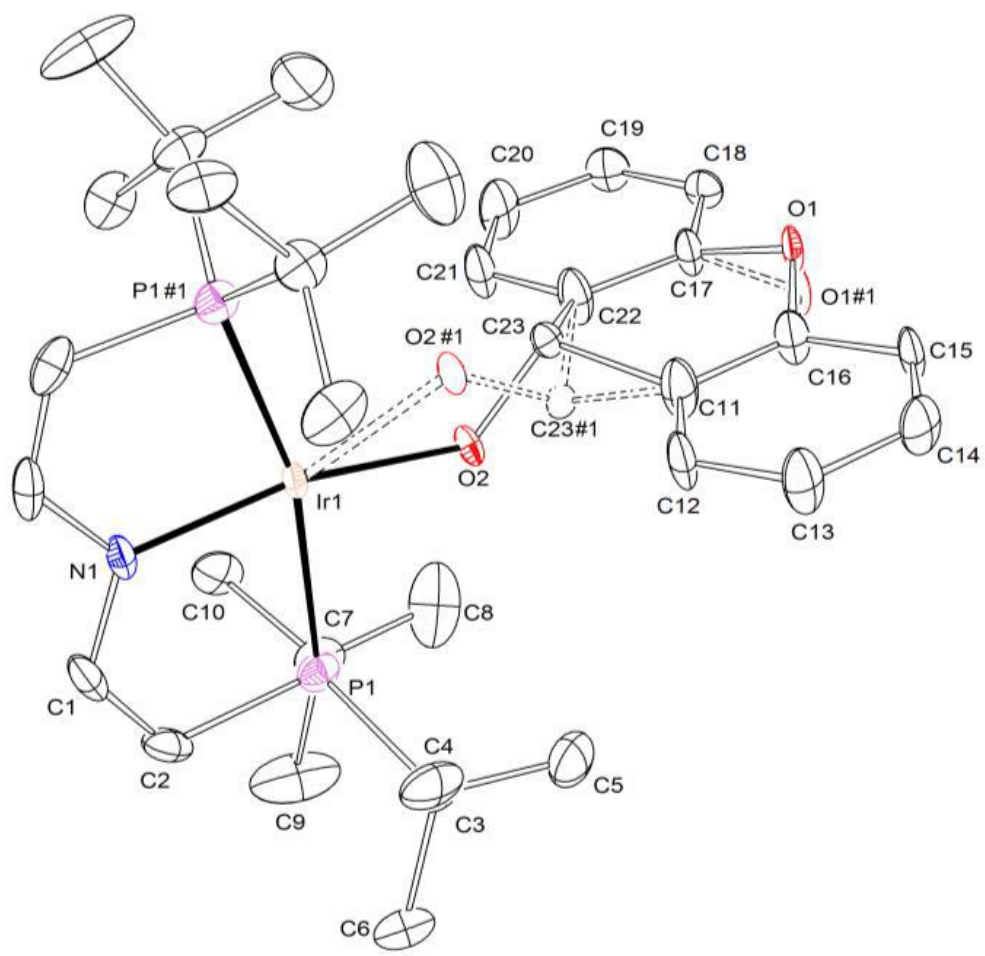

Figure A9: Thermal ellipsoid plot of 11 with the anisotropic displacement parameters drawn at the 50\% probability level. The asymmetric unit contains a half complex molecule. The disorder was refined using PART -1 command.

Table A17: Crystal data and structure refinement for 11.

\begin{tabular}{|c|c|c|}
\hline \multicolumn{3}{|c|}{ Crystal data and structure refinement for 11} \\
\hline Identification code & \multicolumn{2}{|c|}{ MO_DD_171219_2_Om_a (DD-MH-22) } \\
\hline CCDC Identifier & \multicolumn{2}{|c|}{ unpublished } \\
\hline Empirical formula & \multicolumn{2}{|c|}{ C33H491rNO2P2 } \\
\hline Formula weight & \multicolumn{2}{|c|}{745.87} \\
\hline Temperature & \multicolumn{2}{|c|}{$100(2) \mathrm{K}$} \\
\hline Wavelength & \multicolumn{2}{|c|}{$0.71073 \AA$} \\
\hline Crystal system & \multicolumn{2}{|c|}{ Orthorhombic } \\
\hline Space group & \multicolumn{2}{|c|}{ Pnma } \\
\hline \multirow{3}{*}{ Unit cell dimensions } & $a=13.0432(6) \AA$ & $\alpha=90^{\circ}$ \\
\hline & $b=15.6336(7) \AA$ & $\beta=90^{\circ}$ \\
\hline & $c=15.8611(7) \AA$ & $\gamma=90^{\circ}$ \\
\hline Volume & \multicolumn{2}{|c|}{$3234.3(3) \AA ̊ 3$} \\
\hline z & \multicolumn{2}{|c|}{4} \\
\hline Density (calculated) & \multicolumn{2}{|c|}{$1.532 \mathrm{Mg} / \mathrm{m} 3$} \\
\hline Absorption coefficient & \multicolumn{2}{|c|}{$4.256 \mathrm{~mm}^{-1}$} \\
\hline$F(000)$ & \multicolumn{2}{|c|}{1508} \\
\hline Crystal size & \multicolumn{2}{|c|}{$0.190 \times 0.118 \times 0.098 \mathrm{~mm} 3$} \\
\hline Crystal shape and color & \multicolumn{2}{|c|}{ Block, clear intense blue } \\
\hline Theta range for data collection & \multicolumn{2}{|c|}{2.405 to $30.564^{\circ}$} \\
\hline Index ranges & \multicolumn{2}{|c|}{$-18<=h<=18,-22<=k<=22,-22<=\mid<=22$} \\
\hline Reflections collected & \multicolumn{2}{|c|}{47456} \\
\hline Independent reflections & \multicolumn{2}{|c|}{$5121[R($ int $)=0.1422]$} \\
\hline Completeness to theta $=25.242^{\circ}$ & \multicolumn{2}{|c|}{$99.9 \%$} \\
\hline Refinement method & \multicolumn{2}{|c|}{ Full-matrix least-squares on F2 } \\
\hline Data / restraints / parameters & \multicolumn{2}{|c|}{$5121 / 0 / 217$} \\
\hline Goodness-of-fit on F2 & \multicolumn{2}{|c|}{1.012} \\
\hline Final R indices [l>2sigma(I)] & $\mathrm{R} 1=0.0393$ & $w R 2=0.0534$ \\
\hline $\mathrm{R}$ indices (all data) & $\mathrm{R} 1=0.0797$ & $w R 2=0.0622$ \\
\hline Largest diff. peak and hole & \multicolumn{2}{|c|}{0.984 and $-1.722 \mathrm{e}^{-3}$} \\
\hline
\end{tabular}


Table A18: Cartesian coordinates from X-Ray single-crystal analysis of 11

\begin{tabular}{|c|c|c|c|c|c|c|c|}
\hline Ir & 3.350185 & 11.7252 & 6.751975 & $\mathrm{C}$ & 7.221629 & 11.7252 & 10.506377 \\
\hline$P$ & 3.077126 & 9.412834 & 6.611557 & $c$ & 8.466941 & 11.7252 & 8.49281 \\
\hline $\mathrm{N}$ & 1.620057 & 11.7252 & 5.77206 & $\mathrm{C}$ & 9.728206 & 11.7252 & 7.931565 \\
\hline c & 1.008422 & 10.54552 & 5.422038 & $\mathrm{H}$ & 10.499711 & 11.7252 & 8.485911 \\
\hline $\mathrm{H}$ & 0.184457 & 10.581086 & 4.950519 & $c$ & 9.857672 & 11.7252 & 6.558263 \\
\hline 0 & 8.43719 & 11.990752 & 9.86911 & $\mathrm{H}$ & 10.721184 & 11.7252 & 6.162228 \\
\hline 0 & 5.009893 & 11.272639 & 7.738218 & $c$ & 8.744931 & 11.7252 & 5.769269 \\
\hline c & 5.944673 & 12.112241 & 8.320194 & $\mathrm{H}$ & 8.835085 & 11.7252 & 4.823567 \\
\hline $\mathrm{H}$ & 5.726969 & 13.078663 & 8.183709 & $c$ & 7.49941 & 11.7252 & 6.335542 \\
\hline c & 1.523485 & 9.358492 & 5.716769 & $\mathrm{H}$ & 6.735495 & 11.7252 & 5.770792 \\
\hline H & 1.101876 & 8.542918 & 5.472682 & $\mathrm{C}$ & 7.319713 & 11.7252 & 7.719597 \\
\hline c & 2.686077 & 8.507836 & 8.214083 & $P$ & 3.077126 & 14.037566 & 6.611557 \\
\hline c & 3.945072 & 8.206639 & 9.025918 & $c$ & 1.008422 & 12.90488 & 5.422038 \\
\hline $\mathrm{H}$ & 4.459914 & 7.501533 & 8.580744 & $H$ & 0.184457 & 12.869314 & 4.950519 \\
\hline $\mathrm{H}$ & 3.690965 & 7.908069 & 9.924068 & $\mathrm{C}$ & 1.523485 & 14.091908 & 5.716769 \\
\hline $\mathrm{H}$ & 4.491804 & 9.017132 & 9.093597 & $\mathrm{H}$ & 1.101876 & 14.907482 & 5.472682 \\
\hline c & 1.791066 & 9.484452 & 8.994227 & $\mathrm{C}$ & 2.686077 & 14.942564 & 8.214083 \\
\hline $\mathrm{H}$ & 2.295199 & 10.300588 & 9.194664 & $\mathrm{C}$ & 3.945072 & 15.243761 & 9.025918 \\
\hline $\mathrm{H}$ & 1.50212 & 9.065049 & 9.831503 & $\mathrm{H}$ & 4.459914 & 15.948867 & 8.580744 \\
\hline $\mathrm{H}$ & 1.005331 & 9.710279 & 8.453839 & $\mathrm{H}$ & 3.690965 & 15.542331 & 9.924068 \\
\hline c & 1.895933 & 7.216016 & 7.995866 & $\mathrm{H}$ & 4.491804 & 14.433268 & 9.093597 \\
\hline $\mathrm{H}$ & 1.080264 & 7.413531 & 7.489833 & $C$ & 1.791066 & 13.965948 & 8.994227 \\
\hline $\mathrm{H}$ & 1.656265 & 6.829116 & 8.863785 & $\mathrm{H}$ & 2.295199 & 13.149812 & 9.194664 \\
\hline $\mathrm{H}$ & 2.445117 & 6.577947 & 7.494227 & $\mathrm{H}$ & 1.50212 & 14.385351 & 9.831503 \\
\hline c & 4.2669 & 8.461389 & 5.506895 & $\mathrm{H}$ & 1.005331 & 13.740121 & 8.453839 \\
\hline c & 5.668705 & 8.36754 & 6.106539 & c & 1.895933 & 16.234384 & 7.995866 \\
\hline $\mathrm{H}$ & 6.296044 & 8.052336 & 5.422815 & $\mathrm{H}$ & 1.080264 & 16.036869 & 7.489833 \\
\hline H & 5.661153 & 7.738366 & 6.85788 & $\mathrm{H}$ & 1.656265 & 16.621284 & 8.863785 \\
\hline $\mathrm{H}$ & 5.948221 & 9.251683 & 6.42365 & $\mathrm{H}$ & 2.445117 & 16.872453 & 7.494227 \\
\hline c & 3.766667 & 7.06448 & 5.13289 & c & 4.2669 & 14.989011 & 5.506895 \\
\hline H & 2.860087 & 7.129422 & 4.766419 & c & 5.668705 & 15.08286 & 6.106539 \\
\hline $\mathrm{H}$ & 3.756285 & 6.496558 & 5.93148 & $\mathrm{H}$ & 6.296044 & 15.398064 & 5.422815 \\
\hline $\mathrm{H}$ & 4.363237 & 6.672843 & 4.461236 & $\mathrm{H}$ & 5.661153 & 15.712034 & 6.85788 \\
\hline c & 4.354094 & 9.304165 & 4.22987 & $\mathrm{H}$ & 5.948221 & 14.198717 & 6.42365 \\
\hline $\mathrm{H}$ & 4.944729 & 8.86089 & 3.585624 & $\mathrm{C}$ & 3.766667 & 16.38592 & 5.13289 \\
\hline H & 4.713369 & 10.189746 & 4.446818 & $\mathrm{H}$ & 2.860087 & 16.320978 & 4.766419 \\
\hline $\mathrm{H}$ & 3.459944 & 9.400796 & 3.840559 & $\mathrm{H}$ & 3.756285 & 16.953842 & 5.93148 \\
\hline c & 6.024354 & 11.7252 & 9.837974 & $\mathrm{H}$ & 4.363237 & 16.777557 & 4.461236 \\
\hline c & 4.860692 & 11.7252 & 10.57063 & c & 4.354094 & 14.146235 & 4.22987 \\
\hline $\mathrm{H}$ & 4.023097 & 11.7252 & 10.122348 & $\mathrm{H}$ & 4.944729 & 14.58951 & 3.585624 \\
\hline c & 4.896652 & 11.7252 & 11.949832 & $H$ & 4.713369 & 13.260654 & 4.446818 \\
\hline $\mathrm{H}$ & 4.087335 & 11.7252 & 12.447347 & $\mathrm{H}$ & 3.459944 & 14.049604 & 3.840559 \\
\hline c & 6.113674 & 11.7252 & 12.606069 & 0 & 8.43719 & 11.459648 & 9.86911 \\
\hline H & 6.141717 & 11.7252 & 13.555657 & 0 & 5.009893 & 12.177761 & 7.738218 \\
\hline c & 7.27821 & 11.7252 & 11.886372 & c & 5.944673 & 11.338159 & 8.320194 \\
\hline $\mathrm{H}$ & 8.117266 & 11.7252 & 12.331894 & $\mathrm{H}$ & 5.726969 & 10.371737 & 8.183709 \\
\hline
\end{tabular}




\section{A.10. X-Ray Single-Crystal Analysis of 12}

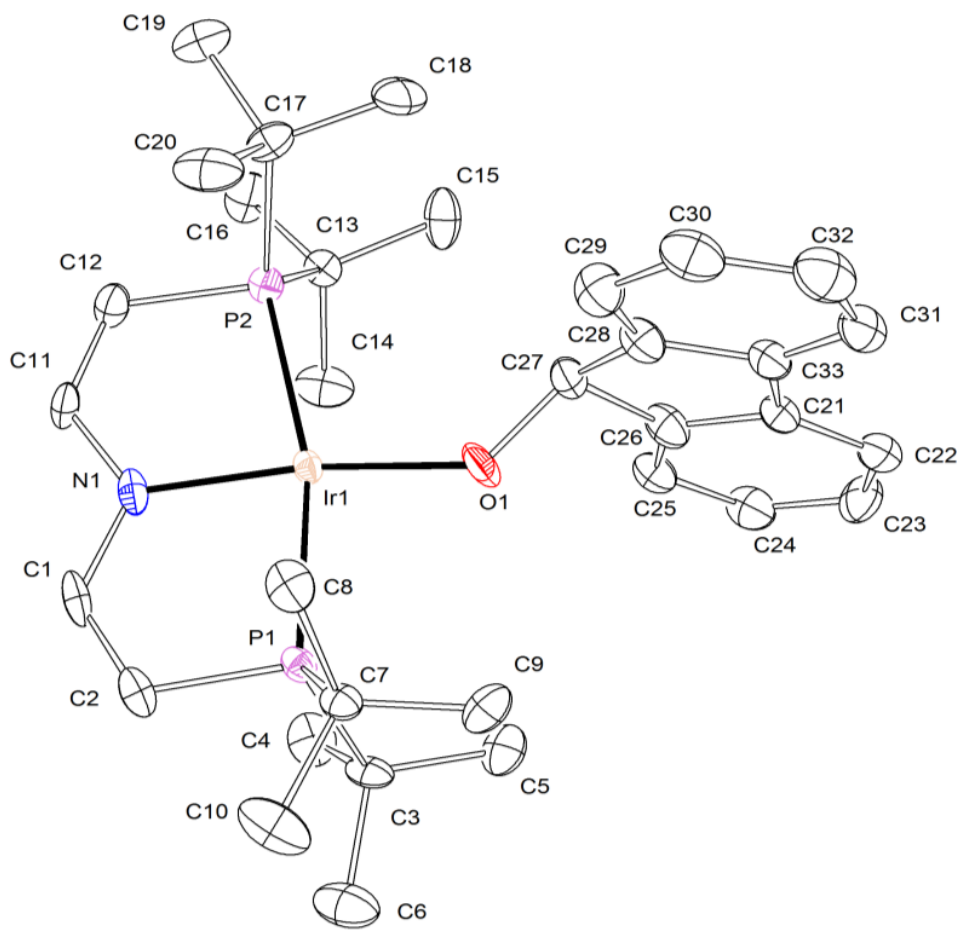

Figure A10: Thermal ellipsoid plot of 12 with the anisotropic displacement parameters drawn at the 50\% probability level. The asymmetric unit one complex molecule.

Table A19: Crystal data and structure refinement for 12 .

\begin{tabular}{|c|c|c|}
\hline \multicolumn{3}{|c|}{ Crystal data and structure refinement for 12} \\
\hline Identification code & \multicolumn{2}{|c|}{ MO_DD_181219_Om_a (MH-DD-21) } \\
\hline CCDC Identifier & \multicolumn{2}{|c|}{ unpublished } \\
\hline Empirical formula & \multicolumn{2}{|c|}{ C33H49IrNOP2 } \\
\hline Formula weight & \multicolumn{2}{|c|}{729.87} \\
\hline Temperature & \multicolumn{2}{|c|}{$100(2) \mathrm{K}$} \\
\hline Wavelength & \multicolumn{2}{|c|}{$0.71073 \AA$} \\
\hline Crystal system & \multicolumn{2}{|c|}{ Triclinic } \\
\hline Space group & \multicolumn{2}{|c|}{ P1 } \\
\hline \multirow{3}{*}{ Unit cell dimensions } & $a=8.7365(16) \AA$ & $\alpha=98.628(16)^{\circ}$ \\
\hline & $b=12.087(3) \AA$ & $\beta=98.206(12)^{\circ}$ \\
\hline & $c=15.565(3) \AA$ & $\gamma=91.124(14)^{\circ}$ \\
\hline Volume & \multicolumn{2}{|c|}{ 1606.9(6) Å3 } \\
\hline z & \multicolumn{2}{|c|}{2} \\
\hline Density (calculated) & \multicolumn{2}{|c|}{$1.508 \mathrm{Mg} / \mathrm{m} 3$} \\
\hline Absorption coefficient & \multicolumn{2}{|c|}{$4.279 \mathrm{~mm}^{-1}$} \\
\hline$F(000)$ & \multicolumn{2}{|c|}{738} \\
\hline Crystal size & \multicolumn{2}{|c|}{$0.249 \times 0.118 \times 0.092 \mathrm{~mm} 3$} \\
\hline Crystal shape and color & \multicolumn{2}{|c|}{ Plate, clear intense blue } \\
\hline Theta range for data collection & \multicolumn{2}{|c|}{2.325 to $28.484^{\circ}$} \\
\hline Index ranges & \multicolumn{2}{|c|}{$-11<=h<=11,-16<=k<=16,-20<=\mid<=20$} \\
\hline Reflections collected & \multicolumn{2}{|c|}{25068} \\
\hline Independent reflections & \multicolumn{2}{|c|}{$7991[R($ int $)=0.0978]$} \\
\hline Completeness to theta $=25.242^{\circ}$ & \multicolumn{2}{|c|}{$99.8 \%$} \\
\hline Refinement method & \multicolumn{2}{|c|}{ Full-matrix least-squares on F2 } \\
\hline Data / restraints / parameters & \multicolumn{2}{|c|}{$7991 / 0 / 355$} \\
\hline Goodness-of-fit on F2 & \multicolumn{2}{|c|}{1.027} \\
\hline Final $R$ indices $[1>2 \operatorname{sigma}(\mid)]$ & $R 1=0.0472$ & $w R 2=0.0746$ \\
\hline$R$ indices (all data) & $\mathrm{R} 1=0.0800$ & $w R 2=0.0846$ \\
\hline Largest diff. peak and hole & \multicolumn{2}{|c|}{1.742 and $-1.988 \mathrm{e}^{-3}$} \\
\hline
\end{tabular}


Table A20: Cartesian coordinates from X-Ray single-crystal analysis of 12.

\begin{tabular}{|c|c|c|c|c|c|c|c|}
\hline Ir & 4.26921 & 2.642109 & 12.249178 & C & 1.315497 & 4.984311 & 10.074406 \\
\hline$P$ & 4.955528 & 0.663459 & 13.221627 & H & 1.673634 & 5.744378 & 9.570001 \\
\hline $\mathrm{P}$ & 3.565832 & 4.8506 & 11.842245 & H & 0.338465 & 4.973412 & 9.998943 \\
\hline 0 & 4.160095 & 1.692399 & 10.537625 & H & 1.680479 & 4.150331 & 9.711536 \\
\hline N & 4.559171 & 3.349301 & 14.084169 & C & 1.219116 & 6.473865 & 12.056518 \\
\hline c & 5.080344 & 2.52314 & 15.074365 & H & 1.628351 & 7.187947 & 11.524533 \\
\hline H & 5.245789 & 2.892027 & 15.934046 & H & 1.467329 & 6.584761 & 12.998069 \\
\hline C & 5.35886 & 1.229383 & 14.867124 & H & 0.24412 & 6.518569 & 11.968285 \\
\hline $\mathrm{H}$ & 5.735489 & 0.665798 & 15.532729 & C & 4.648375 & 5.96257 & 10.784638 \\
\hline c & 3.572034 & -0.5973 & 13.454561 & c & 4.544146 & 5.670444 & 9.283948 \\
\hline C & 2.458595 & 0.18465 & 14.177136 & H & 3.633292 & 5.860879 & 8.976572 \\
\hline $\mathrm{H}$ & 2.139009 & 0.908017 & 13.598348 & H & 4.753998 & 4.727428 & 9.11937 \\
\hline $\mathrm{H}$ & 2.812498 & 0.563762 & 15.008659 & H & 5.178493 & 6.236214 & 8.796179 \\
\hline $\mathrm{H}$ & 1.715984 & -0.420427 & 14.384073 & C & 4.372847 & 7.46234 & 10.979764 \\
\hline c & 3.016752 & -1.069224 & 12.102499 & H & 5.073394 & 7.983739 & 10.535038 \\
\hline H & 2.830565 & -0.291274 & 11.536329 & H & 4.367799 & 7.671693 & 11.937129 \\
\hline H & 2.188992 & -1.573429 & 12.247337 & H & 3.501704 & 7.687653 & 10.591505 \\
\hline $\mathrm{H}$ & 3.67641 & -1.644059 & 11.661122 & c & 6.081068 & 5.650871 & 11.230688 \\
\hline c & 3.975467 & -1.807494 & 14.298093 & H & 6.260595 & 4.695214 & 11.108635 \\
\hline $\mathrm{H}$ & 4.638295 & -2.339451 & 13.810156 & H & 6.187002 & 5.884983 & 12.176394 \\
\hline $\mathrm{H}$ & 3.184826 & -2.357347 & 14.479611 & H & 6.711754 & 6.173105 & 10.69225 \\
\hline $\mathrm{H}$ & 4.361793 & -1.501186 & 15.145049 & c & 2.922965 & 0.701091 & 7.531163 \\
\hline c & 6.54088 & -0.131212 & 12.580436 & c & 2.012341 & -0.124361 & 6.869881 \\
\hline C & 7.453578 & 1.048601 & 12.241994 & H & 2.237332 & -0.525078 & 6.038419 \\
\hline $\mathrm{H}$ & 8.329952 & 0.713102 & 11.959473 & c & 0.784269 & -0.346264 & 7.444528 \\
\hline $\mathrm{H}$ & 7.560798 & 1.616255 & 13.033624 & H & 0.15929 & -0.910149 & 7.00414 \\
\hline $\mathrm{H}$ & 7.054769 & 1.572365 & 11.516025 & C & 0.43522 & 0.242492 & 8.667171 \\
\hline C & 6.287639 & -0.918494 & 11.3023 & H & -0.423825 & 0.093791 & 9.044546 \\
\hline $\mathrm{H}$ & 5.715688 & -0.394225 & 10.703604 & C & 1.363464 & 1.048727 & 9.322669 \\
\hline $\mathrm{H}$ & 5.842783 & -1.763641 & 11.521915 & H & 1.147872 & 1.443332 & 10.159519 \\
\hline $\mathrm{H}$ & 7.14142 & -1.104409 & 10.858563 & c & 2.592517 & 1.268883 & 8.750228 \\
\hline C & 7.250963 & -0.998587 & 13.637084 & C & 3.760262 & 2.112651 & 9.27786 \\
\hline $\mathrm{H}$ & 6.693645 & -1.776187 & 13.849546 & $\mathrm{H}$ & 3.504157 & 3.079009 & 9.301908 \\
\hline $\mathrm{H}$ & 7.397174 & -0.469809 & 14.449125 & c & 4.797237 & 1.877288 & 8.214134 \\
\hline H & 8.113797 & -1.301559 & 13.284777 & c & 6.098087 & 2.339884 & 8.169508 \\
\hline c & 4.279082 & 4.672852 & 14.397299 & H & 6.447021 & 2.872809 & 8.874305 \\
\hline $\mathrm{H}$ & 4.434276 & 4.981408 & 15.282292 & C & 6.897764 & 2.005664 & 7.057579 \\
\hline c & 3.799914 & 5.519002 & 13.492292 & $\mathrm{H}$ & 7.793776 & 2.317905 & 7.011096 \\
\hline H & 3.593879 & 6.421707 & 13.704861 & c & 5.071117 & 0.754184 & 6.087748 \\
\hline c & 1.714378 & 5.117521 & 11.555416 & H & 4.714115 & 0.226034 & 5.383407 \\
\hline C & 1.03918 & 4.014075 & 12.369922 & C & 6.391621 & 1.230979 & 6.038875 \\
\hline $\mathrm{H}$ & 0.0662 & 4.119987 & 12.319953 & H & 6.941323 & 1.016287 & 5.294413 \\
\hline $\mathrm{H}$ & 1.326874 & 4.075487 & 13.304731 & C & 4.318652 & 1.073804 & 7.178277 \\
\hline H & 1.291611 & 3.139339 & 12.00725 & & & & \\
\hline
\end{tabular}




\section{A.11. X-Ray Single-Crystal Analysis of 15}

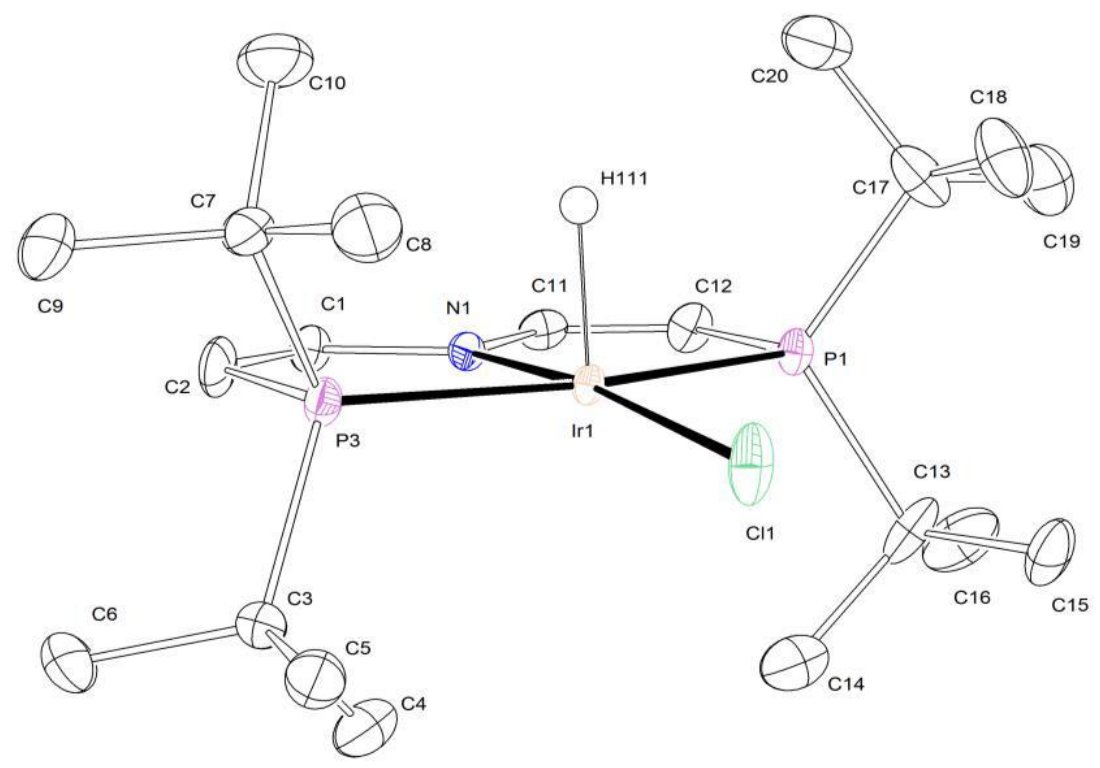

Figure A11: Thermal ellipsoid plot of 15 with the anisotropic displacement parameters drawn at the 50\% probability level. The asymmetric unit contains one complex molecule. The structure was refined as twin using the twin law -100 0-10 001 (BASF: 0.0898(4)). The Ir-H hydrogen atom was found from the residual density map and isotropically refined.

Table A21: Crystal data and structure refinement for 15

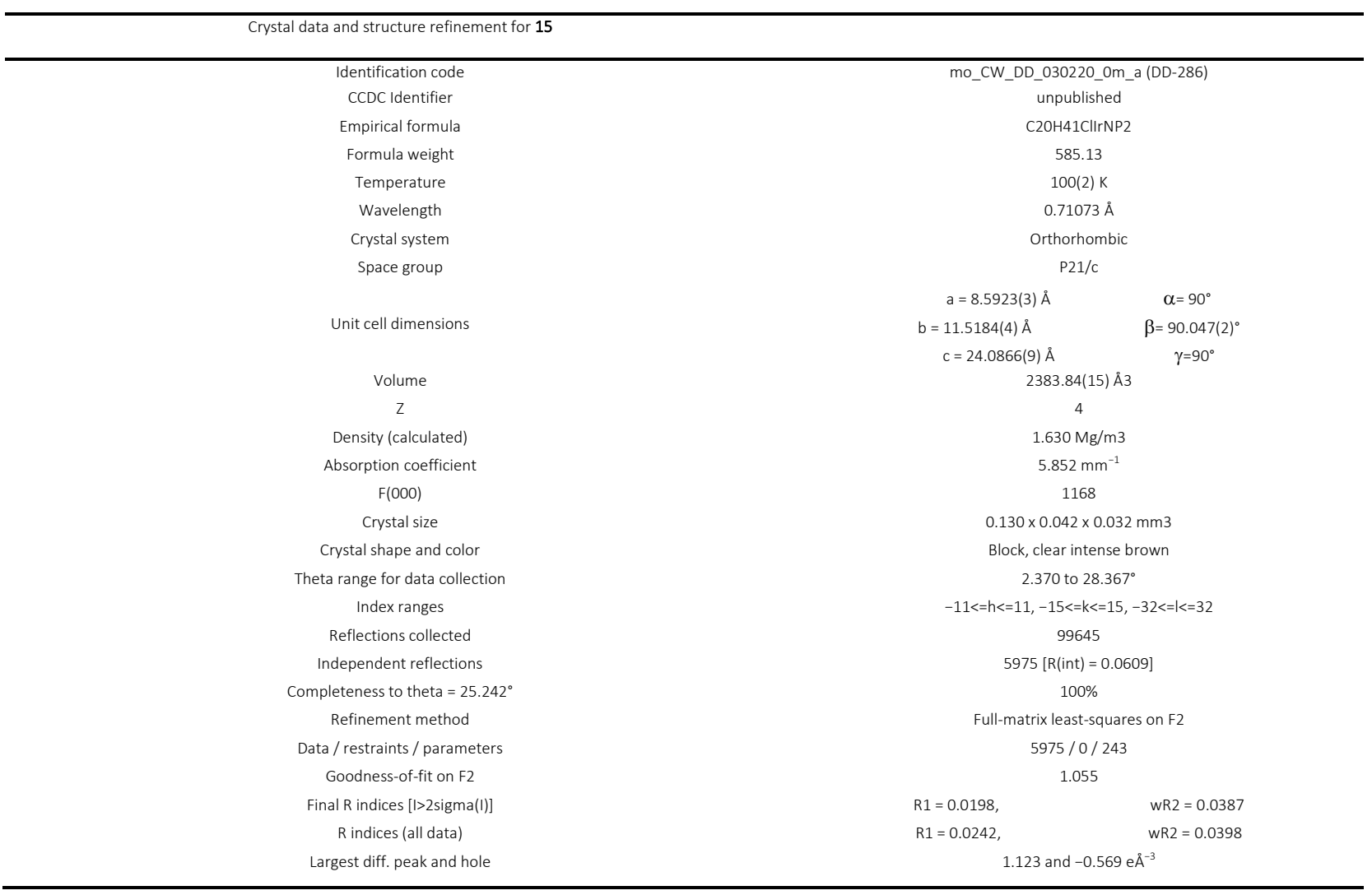


Table A22: Cartesian coordinates from X-Ray single-crystal analysis of 15.

\begin{tabular}{|c|c|c|c|c|c|c|c|}
\hline Ir & 2.251553 & 4.509361 & 15.133991 & $\mathrm{H}$ & 2.440326 & 5.527254 & 12.022822 \\
\hline $\mathrm{Cl}$ & -0.104047 & 4.414623 & 15.328033 & $\mathrm{H}$ & 2.337575 & 5.164206 & 10.467623 \\
\hline $\mathrm{P}$ & 2.625706 & 6.02797 & 16.84055 & C & 4.991518 & 5.16621 & 16.013055 \\
\hline$P$ & 2.464391 & 3.012833 & 13.377452 & $\mathrm{H}$ & 5.939839 & 5.112419 & 15.995689 \\
\hline $\mathrm{N}$ & 4.274138 & 4.416132 & 15.123658 & C & 4.405223 & 5.978637 & 16.913123 \\
\hline c & 4.912726 & 3.628146 & 14.202563 & H & 4.904067 & 6.488165 & 17.540844 \\
\hline H & 5.86191 & 3.589755 & 14.210824 & C & 2.031276 & 5.413199 & 18.524251 \\
\hline c & 4.240983 & 2.906783 & 13.286598 & c & 2.174008 & 3.870528 & 18.423136 \\
\hline H & 4.684236 & 2.376338 & 12.634959 & $\mathrm{H}$ & 1.507176 & 3.521209 & 17.795656 \\
\hline c & 1.885312 & 1.278646 & 13.81867 & H & 2.032223 & 3.470736 & 19.30656 \\
\hline c & 2.212219 & 1.15806 & 15.32059 & $\mathrm{H}$ & 3.072707 & 3.645885 & 18.103314 \\
\hline H & 2.028329 & 0.244063 & 15.622564 & H & 2.387103 & 5.601329 & 14.131555 \\
\hline $\mathrm{H}$ & 1.657446 & 1.787322 & 15.827203 & C & 0.557629 & 5.735691 & 18.742066 \\
\hline H & 3.15867 & 1.366151 & 15.466627 & $\mathrm{H}$ & 0.041504 & 5.449562 & 17.959686 \\
\hline c & 0.378828 & 1.117803 & 13.635058 & $\mathrm{H}$ & 0.449898 & 6.701428 & 18.869147 \\
\hline $\mathrm{H}$ & 0.165862 & 1.104914 & 12.678556 & $\mathrm{H}$ & 0.233983 & 5.263448 & 19.537454 \\
\hline $\mathrm{H}$ & -0.08433 & 1.8681 & 14.062764 & C & 2.883162 & 5.881595 & 19.683804 \\
\hline $\mathrm{H}$ & 0.087582 & 0.277121 & 14.045927 & $\mathrm{H}$ & 3.807243 & 5.583222 & 19.551737 \\
\hline c & 2.635454 & 0.180793 & 13.05409 & $\mathrm{H}$ & 2.534377 & 5.504805 & 20.518549 \\
\hline H & 2.474484 & 0.283802 & 12.092914 & $\mathrm{H}$ & 2.859588 & 6.860117 & 19.732146 \\
\hline $\mathrm{H}$ & 2.315305 & -0.698038 & 13.346597 & C & 2.158637 & 7.802553 & 16.526509 \\
\hline $\mathrm{H}$ & 3.595873 & 0.255167 & 13.234282 & C & 0.691035 & 7.913325 & 16.120602 \\
\hline c & 1.858517 & 3.558368 & 11.697509 & $\mathrm{H}$ & 0.493697 & 8.836456 & 15.85736 \\
\hline C & 0.358806 & 3.898195 & 11.752354 & $\mathrm{H}$ & 0.122669 & 7.661015 & 16.878029 \\
\hline $\mathrm{H}$ & 0.10543 & 4.38972 & 10.943285 & $\mathrm{H}$ & 0.514696 & 7.31362 & 15.365849 \\
\hline $\mathrm{H}$ & 0.177824 & 4.451378 & 12.540805 & C & 2.406737 & 8.696853 & 17.775784 \\
\hline $\mathrm{H}$ & -0.16201 & 3.069895 & 11.807922 & $\mathrm{H}$ & 2.257356 & 9.636236 & 17.539928 \\
\hline c & 2.12034 & 2.525847 & 10.599546 & $\mathrm{H}$ & 3.329968 & 8.58001 & 18.083009 \\
\hline H & 1.91662 & 2.919603 & 9.725564 & $\mathrm{H}$ & 1.78912 & 8.437435 & 18.491084 \\
\hline H & 1.550447 & 1.742077 & 10.745559 & C & 3.039493 & 8.287696 & 15.417442 \\
\hline H & 3.061683 & 2.254416 & 10.624138 & $\mathrm{H}$ & 2.858895 & 7.768677 & 14.606013 \\
\hline c & 2.625048 & 4.841771 & 11.347266 & $\mathrm{H}$ & 3.97879 & 8.176659 & 15.67394 \\
\hline H & 3.586499 & 4.653019 & 11.327804 & $\mathrm{H}$ & 2.857399 & 9.235315 & 15.246379 \\
\hline
\end{tabular}




\section{A.12. X-Ray Single-Crystal Analysis of 25}

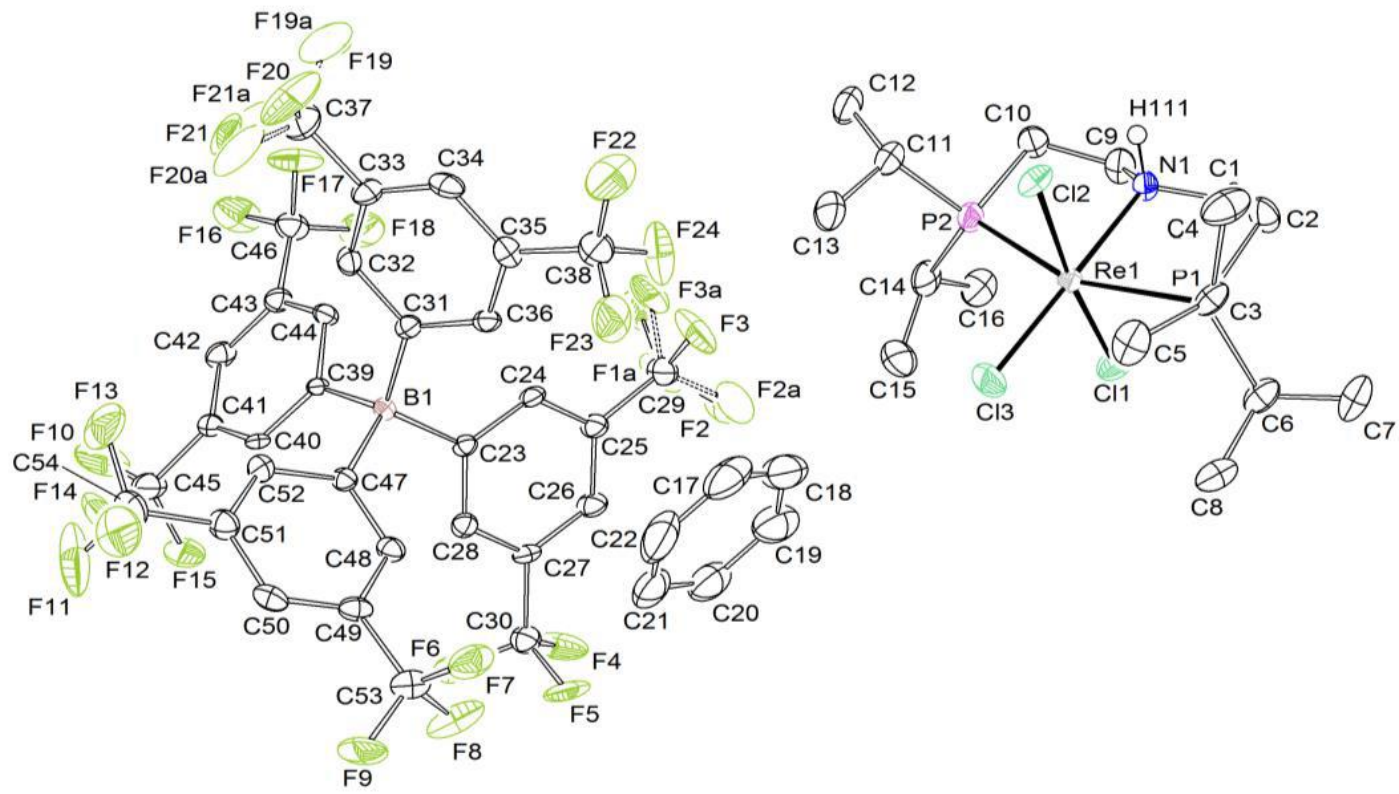

Figure A12: Thermal ellipsoid plot of 25 with the anisotropic displacement parameters drawn at the 50\% probability level. The asymmetric unit contains one complex molecule, one benzene solvent molecule and one $\mathrm{BArF}_{24}$ anion with two disordered $\mathrm{CF} 3$ groups. The disordered $\mathrm{CF} 3$ groups were refined in two different positions with population of $0.829(5)$ on their main domains using PART commands and some restraints and constrains (SADI, RIGU, SIMU, EADP). The N-H hydrogen atom was refined isotropically on a calculated position using AFIX 13 command.

Table A23: Crystal data and structure refinement for 25.

\begin{tabular}{|c|c|c|}
\hline \multicolumn{3}{|c|}{ Crystal data and structure refinement for 25} \\
\hline Identification code & \multicolumn{2}{|c|}{ mo_CW_DD_22719_0m_a (DD-380) } \\
\hline CCDC Identifier & \multicolumn{2}{|c|}{ unpublished } \\
\hline Empirical formula & \multicolumn{2}{|c|}{ C54H55BCl3F24NP2Re } \\
\hline Formula weight & \multicolumn{2}{|c|}{1539.29} \\
\hline Temperature & \multicolumn{2}{|c|}{$100(2) \mathrm{K}$} \\
\hline Wavelength & \multicolumn{2}{|c|}{$0.71073 \AA$} \\
\hline Crystal system & \multicolumn{2}{|c|}{ Orthorhombic } \\
\hline Space group & \multicolumn{2}{|c|}{$\mathrm{P} 21 / \mathrm{c}$} \\
\hline & $a=14.0923(10) \AA$ & $\alpha=90^{\circ}$ \\
\hline Unit cell dimensions & $b=11.5184(4) \AA$ & $\beta=103.942(4)^{\circ}$ \\
\hline & $c=29.199(2) \AA$ & $\gamma=90^{\circ}$ \\
\hline Volume & \multicolumn{2}{|c|}{$6200.2(8) \AA 3$} \\
\hline z & \multicolumn{2}{|c|}{4} \\
\hline Density (calculated) & \multicolumn{2}{|c|}{$1.649 \mathrm{Mg} / \mathrm{m} 3$} \\
\hline Absorption coefficient & \multicolumn{2}{|c|}{$2.250 \mathrm{~mm}^{-1}$} \\
\hline$F(000)$ & \multicolumn{2}{|c|}{3052} \\
\hline Crystal size & \multicolumn{2}{|c|}{$0.297 \times 0.206 \times 0.118 \mathrm{~mm} 3$} \\
\hline Crystal shape and color & \multicolumn{2}{|c|}{ Block, clear intense orange } \\
\hline Theta range for data collection & \multicolumn{2}{|c|}{2.229 to $26.585^{\circ}$} \\
\hline Index ranges & \multicolumn{2}{|c|}{$-17<=h<=17,-19<=k<=19,-36<=\mid<=36$} \\
\hline Reflections collected & \multicolumn{2}{|c|}{65141} \\
\hline Independent reflections & \multicolumn{2}{|c|}{$12767[R$ (int) $=0.1000]$} \\
\hline Completeness to theta $=25.242^{\circ}$ & \multicolumn{2}{|c|}{$99.4 \%$} \\
\hline Refinement method & \multicolumn{2}{|c|}{ Full-matrix least-squares on $\mathrm{F} 2$} \\
\hline Data / restraints / parameters & \multicolumn{2}{|c|}{$12767 / 120 / 834$} \\
\hline Goodness-of-fit on F2 & \multicolumn{2}{|c|}{1.023} \\
\hline Final R indices $[\mid>2 \operatorname{sigma}(I)]$ & $\mathrm{R} 1=0.0422$ & $w R 2=0.0946$ \\
\hline $\mathrm{R}$ indices (all data) & $\mathrm{R} 1=0.0693$ & $w R 2=0.1059$ \\
\hline Largest diff. peak and hole & \multicolumn{2}{|c|}{1.206 and $-1.072 e^{-3}$} \\
\hline
\end{tabular}


Table A24: Cartesian coordinates from X-Ray single-crystal analysis of 25 .

\begin{tabular}{|c|c|c|c|c|c|c|c|c|c|c|c|}
\hline$R$ & 13.977905 & 3.432378 & 4.700529 & $F$ & 3.66329 & 3.125454 & 17.761542 & $\mathrm{H}$ & 10.911576 & 2.241975 & 11.84683 \\
\hline C & 13.693873 & 1.899033 & 2.983707 & $\mathrm{~F}$ & -1.197985 & 4.436256 & 10.921916 & $\mathrm{C}$ & 10.680521 & 1.565545 & 9.974294 \\
\hline C & 14.436847 & 5.095423 & 6.263187 & $\mathrm{~F}$ & -0.288329 & 3.523854 & 12.609406 & $\mathrm{H}$ & 11.441757 & 2.011965 & 9.622582 \\
\hline C & 12.798105 & 2.169394 & 6.195259 & $\mathrm{~F}$ & -0.14853 & 2.579329 & 10.741341 & C & 9.900325 & 0.778868 & 9.144053 \\
\hline$P$ & 16.277448 & 2.557764 & 5.091916 & $\mathrm{~F}$ & 1.189865 & 7.948109 & 8.756605 & $\mathrm{H}$ & 10.124637 & 0.677766 & 8.226471 \\
\hline$P$ & 12.161827 & 4.882894 & 3.799893 & $\mathrm{~F}$ & 3.090425 & 8.468679 & 9.580612 & C & 8.780748 & 0.136826 & 9.672656 \\
\hline$N$ & 15.103317 & 4.664745 & 3.261513 & $\mathrm{~F}$ & 2.985966 & 7.185667 & 7.887142 & $\mathrm{H}$ & 8.240078 & -0.409547 & 9.114382 \\
\hline $\mathrm{H}$ & 15.360081 & 5.493015 & 3.759567 & $\mathrm{~F}$ & 10.619353 & 6.512342 & 13.143961 & C & 8.455015 & 0.294193 & 11.01121 \\
\hline C & 16.393179 & 4.04933 & 2.810132 & $\mathrm{~F}$ & 10.169536 & 4.455787 & 13.351004 & $\mathrm{H}$ & 7.683466 & -0.128831 & 11.36932 \\
\hline $\mathrm{H}$ & 16.885927 & 4.686776 & 2.234826 & $\mathrm{~F}$ & 10.223827 & 5.304799 & 11.437398 & $C$ & 9.25839 & 1.067984 & 11.81631 \\
\hline $\mathrm{H}$ & 16.208584 & 3.233046 & 2.281245 & C & 5.516414 & 3.174701 & 10.54671 & $\mathrm{H}$ & 9.051801 & 1.165872 & 12.73840 \\
\hline C & 17.231661 & 3.697398 & 4.022183 & $C$ & 6.408359 & 3.684807 & 9.600307 & & & & \\
\hline $\mathrm{H}$ & 17.459988 & 4.519411 & 4.524431 & $\mathrm{H}$ & 6.786914 & 4.543646 & 9.747244 & & & & \\
\hline $\mathrm{H}$ & 18.07422 & 3.264143 & 3.735026 & C & 6.763901 & 2.987634 & 8.453946 & & & & \\
\hline C & 17.024584 & 2.590569 & 6.798847 & $c$ & 6.222156 & 1.734586 & 8.194221 & & & & \\
\hline H & 17.724545 & 1.876955 & 6.827639 & $\mathrm{H}$ & 6.461268 & 1.249663 & 7.41309 & & & & \\
\hline C & 17.719857 & 3.916385 & 7.113379 & C & 5.321055 & 1.214203 & 9.111775 & & & & \\
\hline $\mathrm{H}$ & 18.469625 & 4.046597 & 6.495877 & C & 4.972097 & 1.924106 & 10.247792 & & & & \\
\hline $\mathrm{H}$ & 18.054349 & 3.8994 & 8.034362 & $\mathrm{H}$ & 4.338562 & 1.546262 & 10.846449 & & & & \\
\hline $\mathrm{H}$ & 17.081028 & 4.652511 & 7.011359 & $C$ & 7.736151 & 3.596327 & 7.508875 & & & & \\
\hline C & 15.968991 & 2.230129 & 7.850528 & C & 4.774995 & -0.164477 & 8.880955 & & & & \\
\hline H & 15.2951 & 2.940359 & 7.893716 & $C$ & 6.076914 & 5.179773 & 12.312784 & & & & \\
\hline $\mathrm{H}$ & 16.399257 & 2.134415 & 8.7258 & $C$ & 5.679094 & 6.386135 & 12.911413 & & & & \\
\hline H & 15.537791 & 1.385216 & 7.604349 & $\mathrm{H}$ & 4.751959 & 6.582983 & 12.975969 & & & & \\
\hline C & 16.580949 & 0.876352 & 4.404898 & C & 6.594286 & 7.299065 & 13.411196 & & & & \\
\hline $\mathrm{H}$ & 16.229083 & 0.88056 & 3.468868 & $c$ & 7.954624 & 7.061603 & 13.3393 & & & & \\
\hline C & 18.060169 & 0.496785 & 4.316821 & $\mathrm{H}$ & 8.577624 & 7.69863 & 13.668796 & & & & \\
\hline $\mathrm{H}$ & 18.145415 & -0.395528 & 3.920701 & $c$ & 8.389222 & 5.867226 & 12.772751 & & & & \\
\hline H & 18.450747 & 0.493882 & 5.215615 & C & 7.466426 & 4.950787 & 12.286231 & & & & \\
\hline H & 18.531989 & 1.14884 & 3.757697 & $\mathrm{H}$ & 7.78589 & 4.13458 & 11.919838 & & & & \\
\hline C & 15.79134 & -0.176137 & 5.163245 & $c$ & 6.132775 & 8.569222 & 14.04318 & & & & \\
\hline $\mathrm{H}$ & 14.853367 & 0.100916 & 5.225307 & $C$ & 9.846614 & 5.562414 & 12.683597 & & & & \\
\hline $\mathrm{H}$ & 16.162605 & -0.276571 & 6.064617 & $c$ & 3.571914 & 4.67271 & 11.342237 & & & & \\
\hline $\mathrm{H}$ & 15.848491 & -1.031933 & 4.689165 & $C$ & 2.340087 & 4.053848 & 11.565121 & & & & \\
\hline C & 14.311826 & 5.132451 & 2.084971 & $\mathrm{H}$ & 2.313271 & 3.2806 & 12.116368 & & & & \\
\hline $\mathrm{H}$ & 14.02886 & 4.356424 & 1.53925 & $C$ & 1.150752 & 4.520249 & 11.015235 & & & & \\
\hline $\mathrm{H}$ & 14.86764 & 5.724609 & 1.518846 & $\mathrm{C}$ & 1.139982 & 5.6397 & 10.203952 & & & & \\
\hline C & 13.091983 & 5.887673 & 2.580701 & $\mathrm{H}$ & 0.332689 & 5.9601 & 9.819083 & & & & \\
\hline $\mathrm{H}$ & 12.504144 & 6.105977 & 1.814618 & $C$ & 2.349948 & 6.279242 & 9.971376 & & & & \\
\hline H & 13.377226 & 6.737384 & 3.001107 & $\mathrm{C}$ & 3.526055 & 5.806723 & 10.514432 & & & & \\
\hline C & 11.499647 & 6.13116 & 4.972609 & $\mathrm{H}$ & 4.335456 & 6.26516 & 10.321558 & & & & \\
\hline H & 12.293018 & 6.583417 & 5.380065 & $C$ & -0.131676 & 3.792585 & 11.305595 & & & & \\
\hline C & 10.692647 & 7.219699 & 4.286045 & $C$ & 2.392229 & 7.467812 & 9.070258 & & & & \\
\hline $\mathrm{H}$ & 11.203087 & 7.576817 & 3.529513 & 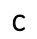 & 4.793719 & 3.099557 & 13.169863 & & & & \\
\hline $\mathrm{H}$ & 10.506833 & 7.940129 & 4.923895 & $\mathrm{C}$ & 5.433686 & 1.869503 & 13.353867 & & & & \\
\hline H & 9.847427 & 6.844432 & 3.961764 & $\mathrm{H}$ & 5.980056 & 1.526948 & 12.656279 & & & & \\
\hline C & 10.735238 & 5.491804 & 6.120416 & $\mathrm{C}$ & 5.295613 & 1.128021 & 14.530267 & & & & \\
\hline H & 9.915025 & 5.079664 & 5.777205 & $\mathrm{C}$ & 4.528358 & 1.611547 & 15.568402 & & & & \\
\hline H & 10.504401 & 6.177612 & 6.781305 & $\mathrm{H}$ & 4.413073 & 1.104111 & 16.36322 & & & & \\
\hline $\mathrm{H}$ & 11.29347 & 4.805919 & 6.542721 & $C$ & 3.925188 & 2.86017 & 15.42804 & & & & \\
\hline C & 10.724121 & 4.09305 & 2.93658 & $C$ & 4.043264 & 3.567667 & 14.250903 & & & & \\
\hline $\mathrm{H}$ & 9.982511 & 4.763829 & 2.929382 & $\mathrm{H}$ & 3.598417 & 4.403373 & 14.172093 & & & & \\
\hline C & 10.216832 & 2.870432 & 3.714877 & $C$ & 6.003733 & -0.170377 & 14.647901 & & & & \\
\hline $\mathrm{H}$ & 9.372482 & 2.562018 & 3.324538 & $C$ & 3.11896 & 3.406916 & 16.573834 & & & & \\
\hline H & 10.072579 & 3.116806 & 4.652381 & B & 5.000389 & 4.038183 & 11.839243 & & & & \\
\hline $\mathrm{H}$ & 10.881659 & 2.152191 & 3.664434 & $\mathrm{~F}$ & 7.274588 & 4.675489 & 6.912712 & & & & \\
\hline C & 11.015357 & 3.736988 & 1.489714 & $\mathrm{~F}$ & 8.218206 & 2.789839 & 6.614701 & & & & \\
\hline $\mathrm{H}$ & 10.259584 & 3.235871 & 1.118136 & $\mathrm{~F}$ & 8.849772 & 4.079574 & 8.178465 & & & & \\
\hline H & 11.825358 & 3.187152 & 1.445307 & $\mathrm{~F}$ & 7.075188 & 3.594355 & 6.308359 & & & & \\
\hline $\mathrm{H}$ & 11.149118 & 4.558132 & 0.971794 & $\mathrm{~F}$ & 8.705975 & 2.810209 & 7.140726 & & & & \\
\hline $\mathrm{F}$ & 4.625077 & -0.459384 & 7.596074 & $\mathrm{~F}$ & 7.897015 & 4.876544 & 7.599588 & & & & \\
\hline $\mathrm{F}$ & 5.588651 & -1.107822 & 9.377904 & $\mathrm{~F}$ & 6.902363 & 9.607769 & 14.061146 & & & & \\
\hline $\mathrm{F}$ & 3.579885 & -0.358329 & 9.450763 & $\mathrm{~F}$ & 5.724441 & 8.278926 & 15.311823 & & & & \\
\hline $\mathrm{F}$ & 7.30549 & -0.017761 & 14.924658 & $\mathrm{~F}$ & 4.887428 & 8.782139 & 13.596532 & & & & \\
\hline $\mathrm{F}$ & 5.952602 & -0.903336 & 13.542915 & $\mathrm{~F}$ & 6.4901 & 9.646925 & 13.278315 & & & & \\
\hline $\mathrm{F}$ & 5.519832 & -0.936669 & 15.631144 & $\mathrm{~F}$ & 6.758523 & 8.816388 & 15.192771 & & & & \\
\hline $\mathrm{F}$ & 3.009584 & 4.729223 & 16.560005 & $\mathrm{~F}$ & 4.857795 & 8.694559 & 14.204484 & & & & \\
\hline $\mathrm{F}$ & 1.917837 & 2.888348 & 16.609173 & C & 10.365544 & 1.703272 & 11.286324 & & & & \\
\hline
\end{tabular}




\section{B. List of Chemical Compounds}
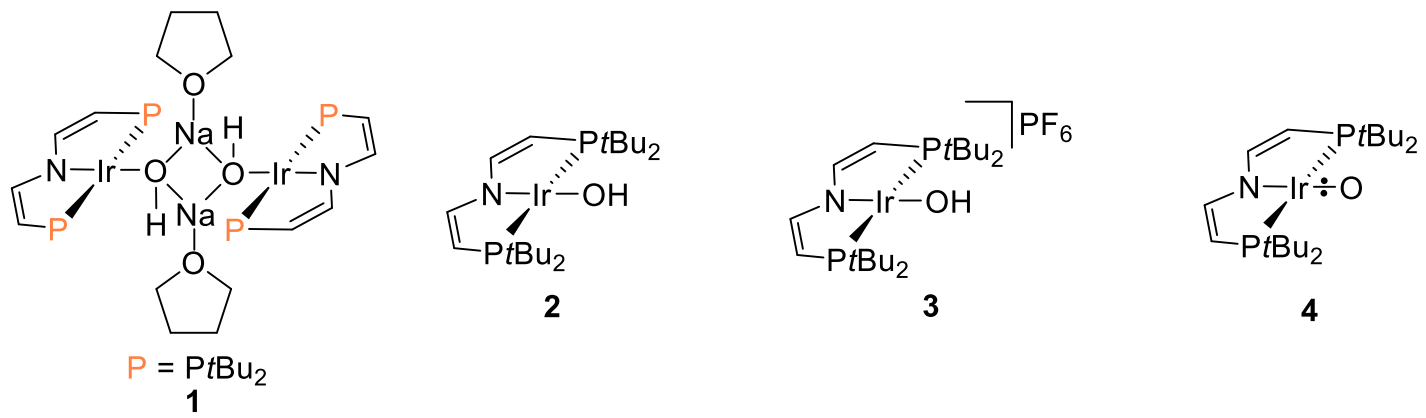

4

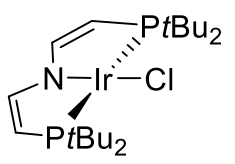

5

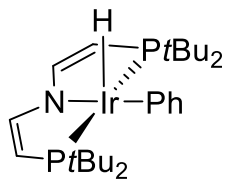

9

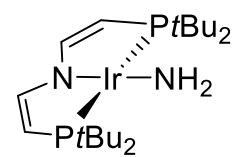

13

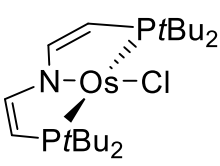

16

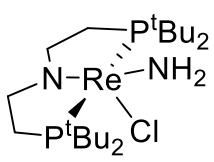

20

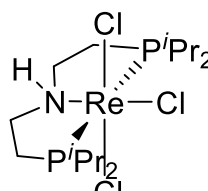

23

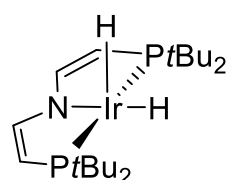

6

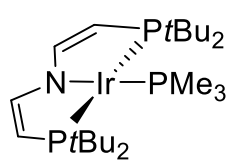

10

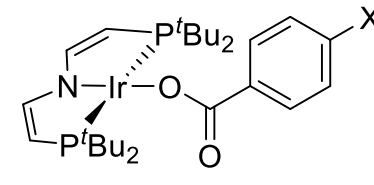

$\mathrm{X}=\mathrm{H}, \mathrm{F}, \mathrm{Me}, \mathrm{OMe}$

14

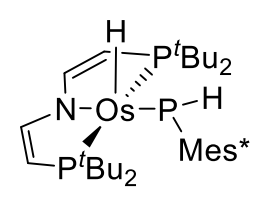

17
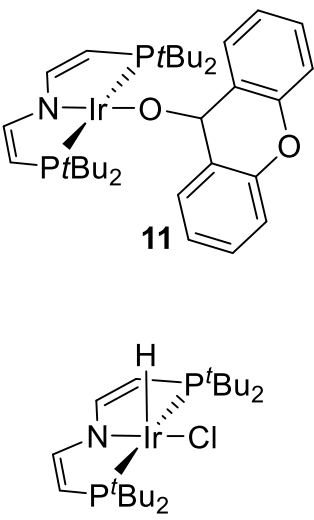

15

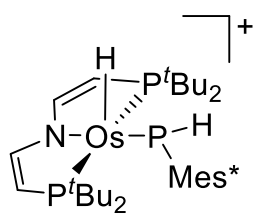

18

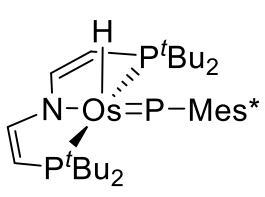

19

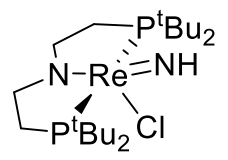

21

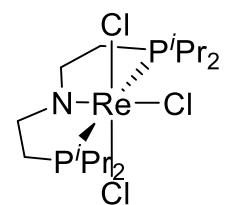

24

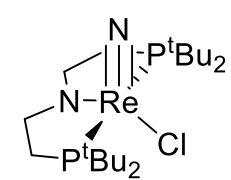

22<smiles>[Y17]C(C)(C)CC</smiles>

25 


\section{Independent PCET Model}

\section{C.1. Derivation of the fitting function}

For a general HAT reaction (Scheme C1) the equilibrium constant is derived via eq. $\mathrm{c} 1$.

$$
\begin{gathered}
\text { A-H }+ \text { B } \stackrel{K}{=} \text { A }+ \text { B } \quad \text { C } \quad \text { D } \\
\text { Scheme C1: General HAT reaction } \\
K=\frac{[C][D]}{[A][B]}
\end{gathered}
$$

For the calorimetric titration reactant $\mathbf{A}$ will be the sample cell concentration and $\mathbf{B}$ the syringe concentration. The products $\mathrm{C}$ and $\mathrm{D}$ are always simultaneously formed, therefore eq. $\mathrm{C} 2$ holds true.

$$
[\mathrm{C}]=[\mathrm{D}]
$$

Only the starting concentrations of $\mathrm{A}$ and $\mathrm{B}$ are known, but not the concentrations within the experiment. Therefore, the total concentrations must be defined following eq. C3.

$$
\left[\mathrm{A}_{\mathrm{T}}\right]=[\mathrm{A}]+[\mathrm{C}] \text { and }\left[\mathrm{B}_{\mathrm{T}}\right]=[\mathrm{B}]+[\mathrm{C}]
$$

Inserting eq. C2 and C3 in eq. C1 leads to eq. 4.

$$
\mathrm{K}=\frac{[\mathrm{C}]^{2}}{\left[\mathrm{~A}_{\mathrm{T}}-[\mathrm{C}]\right]\left[\mathrm{B}_{\mathrm{T}}-[\mathrm{C}]\right]}
$$

To analyse the calorimetric process eq. C4 has to be solved for the product formation. Therefore follows:

$$
[C]=-\frac{\sqrt{K} \sqrt{A_{T}^{2} K-2 A_{T} B_{T}(K-2)+B_{T}^{2} K}+K\left(A_{T}+B_{T}\right)}{2(K-1)}
$$

Which can be simplified to eq. C6.

$$
[C]=\frac{K\left(A_{T}+B_{T}\right)-\sqrt{K\left[K\left(A_{T}-B_{T}\right)^{2}+4 A_{T} B_{T}\right]}}{2(K-1)}
$$

The heat is proportional to the formed products and for integration plotted as a function of the added titrant B. It follows:

$$
\frac{d[C]}{d\left[B_{T}\right]}=\frac{\frac{\sqrt{K}\left(A_{T}(K-2)-B_{T} K\right)}{\sqrt{K\left(A_{T}-B_{T}\right)^{2}+4 A_{T} B_{T}}}+K}{2(K-1)}
$$

The observable in calorimetry is the heat of reaction $d Q$, which is directly connected to the reaction enthalpy via eq. C8 with the reaction volume for normalisation.

$$
d[\mathrm{C}]=\frac{1}{\Delta H^{0} V_{0}} \frac{d Q}{d \mathrm{~B}_{\mathrm{T}}}
$$

This yield the final product function for the calorimetric fitting process in Isothermal Titration Calorimetry with only $\Delta H^{0}$ and $K$ as unknown variables. 


$$
\frac{1}{V_{0}} \frac{\mathrm{d}[\mathrm{Q}]}{\mathrm{d}\left[\mathrm{B}_{\mathrm{T}}\right]}=\frac{\Delta H^{0}}{2(\mathrm{~K}-1)}\left(\frac{\sqrt{\mathrm{K}}\left(\mathrm{A}_{\mathrm{T}}(\mathrm{K}-2)-\mathrm{B}_{\mathrm{T}} \mathrm{K}\right)}{\sqrt{\mathrm{K}\left(\mathrm{A}_{\mathrm{T}}-\mathrm{B}_{\mathrm{T}}\right)^{2}+4 \mathrm{~A}_{\mathrm{T}} \mathrm{B}_{\mathrm{T}}}}+\mathrm{K}\right)
$$

\section{C.2. Incooperation into Visual Basics}

The following figure shows the translation of the results in Section C.1. into visual basic directly within the NANOANALYZE Program by TAINSTRUMENTS.

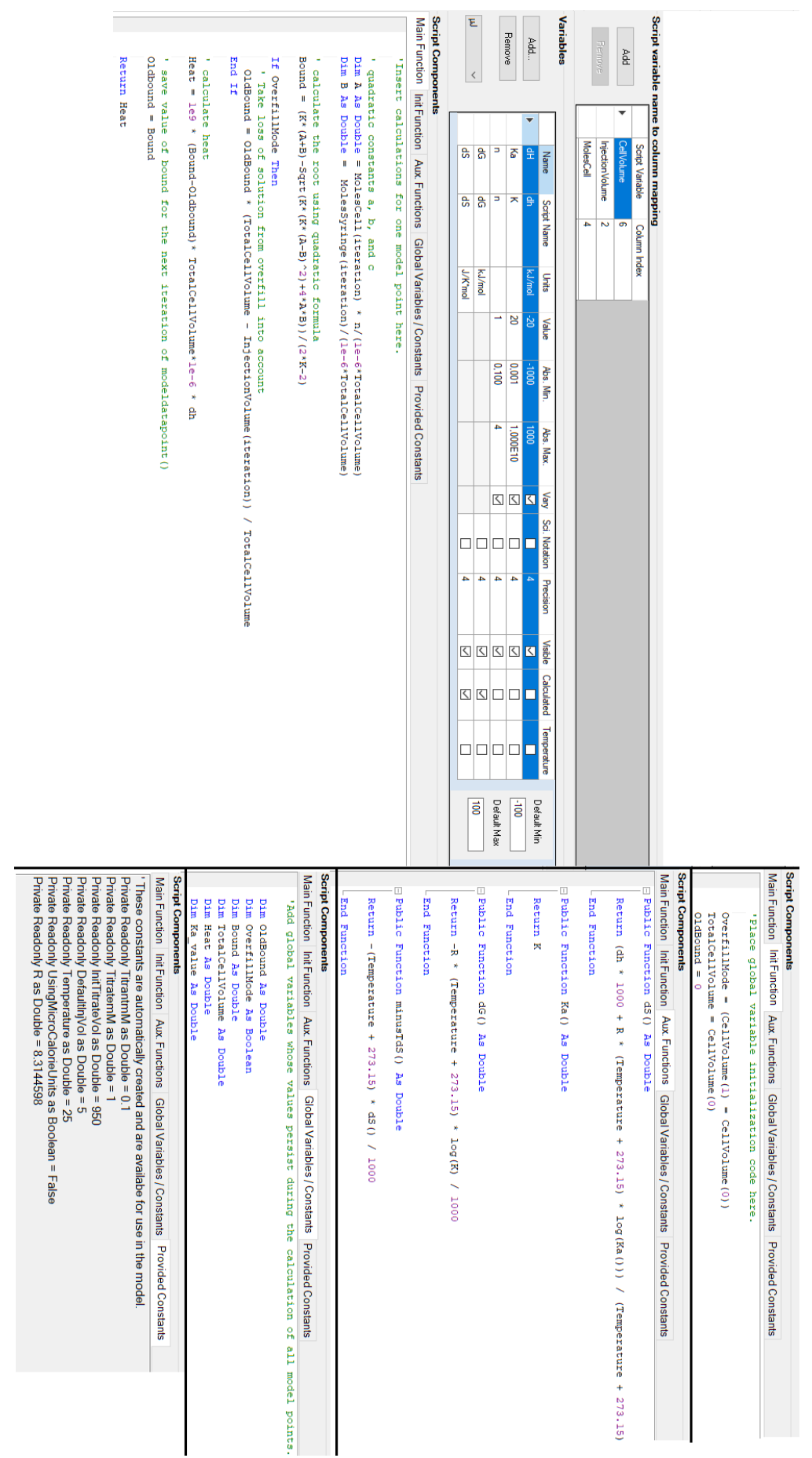

Scheme C2: Main function of the Independend PCET model (left) and the intial function, auxilliary functions, global variables and provied constants (Right top to bottom) of the independend PCET model. 


\section{Cartesian Coordinates of}

\section{Computed Structures}

\begin{tabular}{|c|c|c|c|}
\hline \multicolumn{4}{|c|}{$\begin{array}{l}\text { able D1: xyz data of } 4{ }^{3} \mathrm{~A}^{\prime \prime}(\mathrm{Cs}) \\
65\end{array}$} \\
\hline Ir & 0.02322186 & -0.14047041 & 0.00000000 \\
\hline$P$ & 0.00831253 & 0.22776427 & 2.33116093 \\
\hline$P$ & 0.00831253 & 0.22776427 & -2.33116093 \\
\hline $\mathrm{N}$ & 0.06831915 & 1.95655412 & 0.00000000 \\
\hline 0 & 0.01677776 & -1.94355902 & 0.00000000 \\
\hline c & 0.08491872 & 2.63988700 & 1.18561360 \\
\hline H & 0.11502875 & 3.72764850 & 1.12704192 \\
\hline c & 0.08491872 & 2.63988700 & -1.18561360 \\
\hline $\mathrm{H}$ & 0.11502875 & 3.72764850 & -1.12704192 \\
\hline c & 0.06473572 & 2.01724657 & 2.38754281 \\
\hline H & 0.07067232 & 2.58819506 & 3.30486097 \\
\hline c & 0.06473572 & 2.01724657 & -2.38754281 \\
\hline H & 0.07067232 & 2.58819506 & -3.30486097 \\
\hline C & 1.55115456 & -0.37586696 & -3.24622368 \\
\hline c & -1.60754443 & -0.31352409 & -3.16069497 \\
\hline c & 1.55115456 & -0.37586696 & 3.24622368 \\
\hline c & -1.60754443 & -0.31352409 & 3.16069497 \\
\hline C & 2.73935234 & -0.02277196 & -2.32978671 \\
\hline H & 3.67065113 & -0.28685244 & -2.83979431 \\
\hline $\mathrm{H}$ & 2.69350150 & -0.56995160 & -1.38849788 \\
\hline H & 2.76805124 & 1.04407131 & -2.10133173 \\
\hline c & 1.76181177 & 0.32150679 & -4.59895065 \\
\hline H & 1.83811081 & 1.40373568 & -4.48376980 \\
\hline $\mathrm{H}$ & 0.97095436 & 0.10684366 & -5.31447724 \\
\hline H & 2.70200660 & -0.02908994 & -5.03542956 \\
\hline C & 1.50056862 & -1.89918863 & -3.42649950 \\
\hline $\mathrm{H}$ & 2.48305928 & -2.25444701 & -3.75105169 \\
\hline $\mathrm{H}$ & 0.78002115 & -2.19539341 & -4.18923339 \\
\hline H & 1.24913897 & -2.40474650 & -2.49196280 \\
\hline c & -2.71861641 & 0.50802275 & -2.47855872 \\
\hline H & -2.61431685 & 1.57554014 & -2.67389061 \\
\hline H & -2.71583584 & 0.35811169 & -1.39767321 \\
\hline H & -3.68753471 & 0.18083312 & -2.86639107 \\
\hline C & -1.86810104 & -1.80324814 & -2.87903314 \\
\hline H & -1.17183906 & -2.45468549 & -3.40381503 \\
\hline $\mathrm{H}$ & -2.87585298 & -2.05592556 & -3.22250273 \\
\hline $\mathrm{H}$ & -1.79832369 & -2.02722502 & -1.81447919 \\
\hline c & -1.64121314 & -0.04550641 & -4.67044254 \\
\hline H & -1.39498552 & 0.99107582 & -4.90971416 \\
\hline $\mathrm{H}$ & -2.64968063 & -0.24068721 & -5.04766802 \\
\hline $\mathrm{H}$ & -0.96018475 & -0.69844906 & -5.21587235 \\
\hline C & 1.76181177 & 0.32150679 & 4.59895065 \\
\hline $\mathrm{H}$ & 2.70200660 & -0.02908994 & 5.03542956 \\
\hline $\mathrm{H}$ & 0.97095436 & 0.10684366 & 5.31447724 \\
\hline H & 1.83811081 & 1.40373568 & 4.48376980 \\
\hline C & 2.73935234 & -0.02277196 & 2.32978671 \\
\hline H & 2.76805124 & 1.04407131 & 2.10133173 \\
\hline $\mathrm{H}$ & 2.69350150 & -0.56995160 & 1.38849788 \\
\hline $\mathrm{H}$ & 3.67065113 & -0.28685244 & 2.83979431 \\
\hline C & 1.50056862 & -1.89918863 & 3.42649950 \\
\hline $\mathrm{H}$ & 1.24913897 & -2.40474650 & 2.49196280 \\
\hline $\mathrm{H}$ & 0.78002115 & -2.19539341 & 4.18923339 \\
\hline $\mathrm{H}$ & 2.48305928 & -2.25444701 & 3.75105169 \\
\hline c & -2.71861641 & 0.50802275 & 2.47855872 \\
\hline $\mathrm{H}$ & -2.61431685 & 1.57554014 & 2.67389061 \\
\hline $\mathrm{H}$ & -3.68753471 & 0.18083312 & 2.86639107 \\
\hline H & -2.71583584 & 0.35811169 & 1.39767321 \\
\hline c & -1.64121314 & -0.04550641 & 4.67044254 \\
\hline $\mathrm{H}$ & -0.96018475 & -0.69844906 & 5.21587235 \\
\hline $\mathrm{H}$ & -2.64968063 & -0.24068721 & 5.04766802 \\
\hline $\mathrm{H}$ & -1.39498552 & 0.99107582 & 4.90971416 \\
\hline C & -1.86810104 & -1.80324814 & 2.87903314 \\
\hline H & -1.79832369 & -2.02722502 & 1.81447919 \\
\hline H & -2.87585298 & -2.05592556 & 3.22250273 \\
\hline H & -1.17183906 & -2.45468549 & 3.40381503 \\
\hline
\end{tabular}

\begin{tabular}{|c|c|c|c|}
\hline \multicolumn{4}{|c|}{$\begin{array}{l}\text { Table D2: :xyz data of } 4 m e^{3} A^{\prime \prime}(C s){ }^{t} B u: M e \\
29 \\
E(B 3 L Y P(V)-D 3 / \text { def2TZVP) }=-1231.40496563\end{array}$} \\
\hline Ir & 807232718 & -014047041 & P \\
\hline P & 0.00831253 & 0.22776427 & 2.33116093 \\
\hline $\mathrm{P}$ & 0.00831253 & 0.22776427 & -2.33116093 \\
\hline $\mathrm{N}$ & 0.06831915 & 1.95655412 & 0.00000000 \\
\hline 0 & 0.01677776 & -1.94355902 & 0.000000000 \\
\hline c & 0.08491872 & 2.63988700 & 1.18561360 \\
\hline H & 0.11502875 & 3.72764850 & 1.12704192 \\
\hline C & 0.08491872 & 2.63988700 & -1.18561360 \\
\hline H & 0.11502875 & 3.72764850 & -1.12704192 \\
\hline C & 0.06473572 & 2.01724657 & 2.38754281 \\
\hline $\mathrm{H}$ & 0.07067232 & 2.58819506 & 3.30486097 \\
\hline c & 0.06473572 & 2.01724657 & -2.38754281 \\
\hline $\mathrm{H}$ & 0.07067232 & 2.58819506 & -3.30486097 \\
\hline c & 1.55115456 & -0.37586696 & -3.24622368 \\
\hline c & -1.60754443 & -0.31352409 & -3.16069497 \\
\hline c & 1.55115456 & -0.37586696 & 3.24622368 \\
\hline c & -1.60754443 & -0.31352409 & 3.16069497 \\
\hline H & 2.39053148 & -0.12643055 & -2.59882638 \\
\hline H & 1.70073618 & 0.11931809 & -4.20675621 \\
\hline $\mathrm{H}$ & 1.51522810 & -1.45773994 & -3.37425674 \\
\hline H & -2.39291823 & 0.26719559 & -2.67851924 \\
\hline H & -1.79207542 & -1.36857409 & -2.96121688 \\
\hline H & -1.63150404 & -0.12279506 & -4.23507442 \\
\hline H & 1.70073618 & 0.11931809 & 4.20675621 \\
\hline H & 2.39053148 & -0.12643055 & 2.59882638 \\
\hline $\mathrm{H}$ & 1.51522810 & -1.45773994 & 3.37425674 \\
\hline $\mathrm{H}$ & -2.39291823 & 0.26719559 & 2.67851924 \\
\hline $\mathrm{H}$ & -1.63150404 & -0.12279506 & 4.23507442 \\
\hline H & -1.79207542 & -1.36857409 & 2.96121688 \\
\hline
\end{tabular}

Table D3: :xyz data of $4 \mathrm{~h}^{3} \mathrm{~A}^{\prime \prime}(\mathrm{Cs}){ }^{\mathrm{t}} \mathrm{Bu}: \mathrm{H}$

17

$E(B 3 L Y P(V)-D 3 /$ def2TZVP $)=-1074.14708225$

\begin{tabular}{cccc}
\hline Ir & 0.0232218606 & -0.1404704069 & 0.0000000000 \\
P & 0.0083125323 & 0.2277642692 & 2.3311609277 \\
P & 0.0083125323 & 0.2277642692 & -2.3311609277 \\
N & 0.0683191483 & 1.9565541211 & 0.0000000000 \\
O & 0.0167777607 & -1.9435590241 & 0.0000000000 \\
C & 0.0849187197 & 2.6398869982 & 1.1856135957 \\
H & 0.1150287507 & 3.7276484966 & 1.1270419209 \\
C & 0.0849187197 & 2.6398869982 & -1.1856135957 \\
H & 0.1150287507 & 3.7276484966 & -1.1270419209 \\
C & 0.0647357246 & 2.0172465734 & 2.3875428070 \\
H & 0.0706723246 & 2.5881950609 & 3.3048609693 \\
C & 0.0647357246 & 2.0172465734 & -2.3875428070 \\
H & 0.0706723246 & 2.5881950609 & -3.3048609693 \\
H & 1.1548704977 & -0.2208222876 & 3.0111867958 \\
H & -1.1911142254 & -0.1740260841 & 2.9469117971 \\
H & 1.1548704977 & -0.2208222876 & -3.0111867958 \\
H & -1.1911142254 & -0.1740260841 & -2.9469117971 \\
\hline
\end{tabular}




\begin{tabular}{|c|c|c|c|}
\hline \multicolumn{4}{|c|}{$\begin{array}{l}\text { Table D4: xyz data of } 4{ }_{1}{ }^{1} A^{\prime}(\mathrm{Cs}) \\
65\end{array}$} \\
\hline Ir & -0.0298281579 & -0.0973829171 & 0.0000000000 \\
\hline$P$ & -0.0186695120 & 0.1846628807 & 2.3325446875 \\
\hline$P$ & -0.0186695120 & 0.1846628807 & -2.3325446875 \\
\hline $\mathrm{N}$ & -0.2471987979 & 1.9030873015 & -0.0000000000 \\
\hline 0 & 0.8567711343 & -1.6835949596 & -0.00000000000 \\
\hline c & -0.2273192968 & 2.6027725485 & 1.1992341983 \\
\hline $\mathrm{H}$ & -0.2978954486 & 3.6871576584 & 1.1309505548 \\
\hline c & -0.2273192968 & 2.6027725485 & -1.1992341983 \\
\hline $\mathrm{H}$ & -0.2978954486 & 3.6871576584 & -1.1309505548 \\
\hline c & -0.1277468141 & 1.9881392673 & 2.3934301135 \\
\hline H & -0.1297821396 & 2.5663808244 & 3.3067711449 \\
\hline c & -0.1277468141 & 1.9881392673 & -2.3934301135 \\
\hline $\mathrm{H}$ & -0.1297821396 & 2.5663808244 & -3.3067711449 \\
\hline c & 1.5924389747 & -0.2622678441 & -3.2117418542 \\
\hline c & -1.5624645946 & -0.4670333280 & -3.2157995472 \\
\hline c & 1.5924389747 & -0.2622678441 & 3.2117418542 \\
\hline c & -1.5624645946 & -0.4670333280 & 3.2157995472 \\
\hline c & 2.7180892418 & 0.1921319386 & -2.2598704591 \\
\hline H & 3.6818353101 & 0.0226371493 & -2.7495032255 \\
\hline H & 2.6983547965 & -0.3746845813 & -1.3303722001 \\
\hline $\mathrm{H}$ & 2.6437800918 & 1.2552045367 & -2.0228378268 \\
\hline c & 1.7790918059 & 0.4456089533 & -4.5613892425 \\
\hline H & 1.7610186226 & 1.5314647830 & -4.4526969233 \\
\hline H & 1.0298879803 & 0.1619267018 & -5.2975246025 \\
\hline $\mathrm{H}$ & 2.7576536724 & 0.1742994820 & -4.9695797005 \\
\hline c & 1.6784076346 & -1.7870805036 & -3.3743636870 \\
\hline $\mathrm{H}$ & 2.6919082134 & -2.0542987077 & -3.6889901767 \\
\hline $\mathrm{H}$ & 0.9941440339 & -2.1556656602 & -4.1395326832 \\
\hline H & 1.4697629140 & -2.2894200183 & -2.4273274461 \\
\hline c & -2.7553779613 & 0.2798676378 & -2.5880522719 \\
\hline H & -2.7351956980 & 1.3461065157 & -2.8140366308 \\
\hline $\mathrm{H}$ & -2.7750312504 & 0.1604807860 & -1.5029709590 \\
\hline $\mathrm{H}$ & -3.6843425089 & -0.1362979749 & -2.9882955693 \\
\hline c & -1.7197402767 & -1.9662028139 & -2.9058876729 \\
\hline H & -0.9396701401 & -2.5714806121 & -3.3633083042 \\
\hline $\mathrm{H}$ & -2.6808743867 & -2.3118891910 & -3.2987947359 \\
\hline H & -1.6982992421 & -2.1506675404 & -1.8314401561 \\
\hline c & -1.5685525621 & -0.2356854903 & -4.7313238094 \\
\hline $\mathrm{H}$ & -1.3926034510 & 0.8109718440 & -4.9877669084 \\
\hline H & -2.5467222719 & -0.5147701138 & -5.1353558248 \\
\hline $\mathrm{H}$ & -0.8223479912 & -0.8460846932 & -5.2392636422 \\
\hline c & 1.7790918059 & 0.4456089533 & 4.5613892425 \\
\hline $\mathrm{H}$ & 2.7576536724 & 0.1742994820 & 4.9695797005 \\
\hline H & 1.0298879803 & 0.1619267018 & 5.2975246025 \\
\hline $\mathrm{H}$ & 1.7610186226 & 1.5314647830 & 4.4526969233 \\
\hline c & 2.7180892418 & 0.1921319386 & 2.2598704591 \\
\hline H & 2.6437800918 & 1.2552045367 & 2.0228378268 \\
\hline H & 2.6983547965 & -0.3746845813 & 1.3303722001 \\
\hline $\mathrm{H}$ & 3.6818353101 & 0.0226371493 & 2.7495032255 \\
\hline c & 1.6784076346 & -1.7870805036 & 3.3743636870 \\
\hline $\mathrm{H}$ & 1.4697629140 & -2.2894200183 & 2.4273274461 \\
\hline $\mathrm{H}$ & 0.9941440339 & -2.1556656602 & 4.1395326832 \\
\hline $\mathrm{H}$ & 2.6919082134 & -2.0542987077 & 3.6889901767 \\
\hline c & -2.7553779613 & 0.2798676378 & 2.5880522719 \\
\hline H & -2.7351956980 & 1.3461065157 & 2.8140366308 \\
\hline $\mathrm{H}$ & -3.6843425089 & -0.1362979749 & 2.9882955693 \\
\hline $\mathrm{H}$ & -2.7750312504 & 0.1604807860 & 1.5029709590 \\
\hline c & -1.5685525621 & -0.2356854903 & 4.7313238094 \\
\hline $\mathrm{H}$ & -0.8223479912 & -0.8460846932 & 5.2392636422 \\
\hline H & -2.5467222719 & -0.5147701138 & 5.1353558248 \\
\hline $\mathrm{H}$ & -1.3926034510 & 0.8109718440 & 4.9877669084 \\
\hline c & -1.7197402767 & -1.9662028139 & 2.9058876729 \\
\hline $\mathrm{H}$ & -1.6982992421 & -2.1506675404 & 1.8314401561 \\
\hline $\mathrm{H}$ & -2.6808743867 & -2.3118891910 & 3.2987947359 \\
\hline $\mathrm{H}$ & -0.9396701401 & -2.5714806121 & 3.3633083042 \\
\hline
\end{tabular}

\begin{tabular}{|c|c|c|c|}
\hline \multicolumn{4}{|c|}{$\begin{array}{l}\text { Table D5: :xyz data of } 4 \mathrm{me}_{1}{ }^{1} \mathrm{~A}^{\mathrm{A}}(\mathrm{Cs}){ }^{\mathrm{t}} \mathrm{Bu}: \mathrm{Me} \\
29 \\
\mathrm{E}(\mathrm{B} 3 \mathrm{LYP}(\mathrm{V})-\mathrm{D} 3 / \text { def2TZVP) }=-1231.40496563\end{array}$} \\
\hline 1 & -0.02982816 & -0.09738292 & 0.00000000 \\
\hline$P$ & -0.01866951 & 0.18466288 & 2.33254469 \\
\hline$P$ & -0.01866951 & 0.18466288 & -2.33254469 \\
\hline $\mathrm{N}$ & -0.24719880 & 1.90308730 & 0.00000000 \\
\hline 0 & 0.85677113 & -1.68359496 & 0.00000000 \\
\hline c & -0.22731930 & 2.60277255 & 1.19923420 \\
\hline $\mathrm{H}$ & -0.29789545 & 3.68715766 & 1.13095056 \\
\hline c & -0.22731930 & 2.60277255 & -1.19923420 \\
\hline H & -0.29789545 & 3.68715766 & -1.13095056 \\
\hline c & -0.12774681 & 1.98813927 & 2.39343011 \\
\hline H & -0.12978214 & 2.56638082 & 3.30677115 \\
\hline c & -0.12774681 & 1.98813927 & -2.39343011 \\
\hline $\mathrm{H}$ & -0.12978214 & 2.56638082 & -3.30677115 \\
\hline c & 1.59243898 & -0.26226784 & -3.21174185 \\
\hline c & -1.56246460 & -0.46703333 & -3.21579955 \\
\hline c & 1.59243898 & -0.26226784 & 3.21174185 \\
\hline c & -1.56246460 & -0.46703333 & 3.21579955 \\
\hline H & 2.38724762 & 0.05857859 & -2.53963646 \\
\hline H & 1.72499478 & 0.24044717 & -4.17022503 \\
\hline H & 1.65342771 & -1.34401540 & -3.32711063 \\
\hline H & -2.40608766 & 0.06117174 & -2.77185945 \\
\hline H & -1.67379460 & -1.52824343 & -2.99642368 \\
\hline H & -1.56679785 & -0.30236591 & -4.29451059 \\
\hline H & 1.72499478 & 0.24044717 & 4.17022503 \\
\hline H & 2.38724762 & 0.05857859 & 2.53963646 \\
\hline H & 1.65342771 & -1.34401540 & 3.32711063 \\
\hline H & -2.40608766 & 0.06117174 & 2.77185945 \\
\hline H & -1.56679785 & -0.30236591 & 4.29451059 \\
\hline H & -1.67379460 & -1.52824343 & 2.99642368 \\
\hline
\end{tabular}

\begin{tabular}{|c|c|c|c|}
\hline \multicolumn{4}{|c|}{$\begin{array}{l}\text { Table D6: :xyz data of } 4 \mathrm{~h}{ }_{1}{ }^{1} \mathrm{~A}^{\prime}(\mathrm{CS})^{\mathrm{t}} \mathrm{Bu}: \mathrm{H} \\
17\end{array}$} \\
\hline \multicolumn{4}{|c|}{$E(B 3 L Y P(V)-D 3 /$ def2TZVP) $=-1074.11808336$} \\
\hline Ir & -0.02982816 & -0.09738292 & 0.00000000 \\
\hline$P$ & -0.01866951 & 0.18466288 & 2.33254469 \\
\hline$P$ & -0.01866951 & 0.18466288 & -2.33254469 \\
\hline $\mathrm{N}$ & -0.24719880 & 1.90308730 & 0.00000000 \\
\hline 0 & 0.85677113 & -1.68359496 & 0.00000000 \\
\hline c & -0.22731930 & 2.60277255 & 1.19923420 \\
\hline H & -0.29789545 & 3.68715766 & 1.13095056 \\
\hline c & -0.22731930 & 2.60277255 & -1.19923420 \\
\hline H & -0.29789545 & 3.68715766 & -1.13095055 \\
\hline c & -0.12774681 & 1.98813927 & 2.39343011 \\
\hline H & -0.12978214 & 2.56638082 & 3.30677115 \\
\hline c & -0.12774681 & 1.98813927 & -2.39343011 \\
\hline H & -0.12978214 & 2.56638083 & -3.30677115 \\
\hline H & 1.18152063 & -0.14827674 & -2.98749982 \\
\hline $\mathrm{H}$ & -1.16602787 & -0.29968190 & -2.98898534 \\
\hline H & 1.18152063 & -0.14827674 & 2.98749982 \\
\hline H & -1.16602787 & -0.29968190 & 2.98898534 \\
\hline
\end{tabular}




\begin{tabular}{|c|c|c|c|}
\hline \multicolumn{4}{|c|}{$\begin{array}{l}\text { Table D7: xyz data of } 2{ }^{2} A(C 1) \\
66\end{array}$} \\
\hline Ir & 0.0050958848 & -0.0156777177 & -0.0882343990 \\
\hline$P$ & -2.3124919190 & -0.0023251148 & 0.2028011184 \\
\hline $\mathrm{P}$ & 2.3243994711 & -0.0141122055 & 0.2134758945 \\
\hline $\mathrm{N}$ & -0.0017481669 & -0.0543978270 & 1.9312039329 \\
\hline 0 & 0.1472304536 & -0.0255323898 & -2.0622208719 \\
\hline H & -0.7026424592 & -0.0245540780 & -2.5115971839 \\
\hline C & -1.1919566468 & -0.0603323501 & 2.6234292271 \\
\hline H & -1.1254519701 & -0.0822505932 & 3.7099385235 \\
\hline c & 1.1887359932 & -0.0858461070 & 2.6276294720 \\
\hline H & 1.1156417377 & -0.1165700739 & 3.7135721441 \\
\hline C & -2.3898016333 & -0.0390265573 & 2.0008413680 \\
\hline H & -3.3073033307 & -0.0328197877 & 2.5717051577 \\
\hline c & 2.3898295740 & -0.0786063806 & 2.0120729664 \\
\hline H & 3.3029031461 & -0.0952036685 & 2.5902553202 \\
\hline c & 3.2386485545 & -1.5593207391 & -0.3854775126 \\
\hline c & 3.1876083130 & 1.5944795914 & -0.2980978610 \\
\hline C & -3.2705549530 & -1.5465776493 & -0.3506172359 \\
\hline C & -3.1723670696 & 1.6033523041 & -0.3339373118 \\
\hline c & 2.3159075649 & -2.7430408202 & -0.0325160000 \\
\hline H & 2.8164750092 & -3.6772505567 & -0.3051141328 \\
\hline H & 1.3714679948 & -2.6865338340 & -0.5732738996 \\
\hline H & 2.0936289730 & -2.7762841036 & 1.0356788813 \\
\hline C & 4.5933796531 & -1.7843379437 & 0.3016100866 \\
\hline H & 4.4860017573 & -1.8597340832 & 1.3849638763 \\
\hline H & 5.3148182757 & -1.0002188357 & 0.0824421749 \\
\hline H & 5.0192351836 & -2.7286018496 & -0.0520153182 \\
\hline C & 3.4057483122 & -1.5038369124 & -1.9111699929 \\
\hline $\mathrm{H}$ & 3.7125355610 & -2.4892855774 & -2.2749953239 \\
\hline H & 4.1779266579 & -0.7937343776 & -2.2086731951 \\
\hline H & 2.4699766884 & -1.2312864958 & -2.4037958700 \\
\hline C & 2.5026906513 & 2.7075452708 & 0.5191456688 \\
\hline H & 2.6787212312 & 2.5949720193 & 1.5893851581 \\
\hline H & 1.4243931595 & 2.7159568926 & 0.3515082189 \\
\hline H & 2.9052539409 & 3.6752011507 & 0.2057254678 \\
\hline C & 2.9262117827 & 1.8650484407 & -1.7903304587 \\
\hline H & 3.4759456499 & 1.1837625167 & -2.4372479707 \\
\hline H & 3.2558965740 & 2.8812054378 & -2.0285424526 \\
\hline H & 1.8680426358 & 1.7722226746 & -2.0348995321 \\
\hline c & 4.6933022486 & 1.6190667060 & -0.0103772707 \\
\hline H & 4.9185561140 & 1.3640646378 & 1.0273374740 \\
\hline H & 5.07946866606 & 2.6266404914 & -0.1940588016 \\
\hline H & 5.2430632153 & 0.9392481916 & -0.6608863413 \\
\hline C & -4.5999716637 & -1.7492486789 & 0.3930052742 \\
\hline H & -5.0505385628 & -2.6915366111 & 0.0658407031 \\
\hline H & -5.3213368183 & -0.9588605053 & 0.1976195371 \\
\hline H & -4.4481317211 & -1.8179839925 & 1.4707398486 \\
\hline C & -2.3475813728 & -2.7359953764 & -0.0195464197 \\
\hline H & -2.0921499057 & -2.7602006624 & 1.0411988426 \\
\hline H & -1.4179955912 & -2.6924365813 & -0.5870272595 \\
\hline H & -2.8632577394 & -3.6690958443 & -0.2667560156 \\
\hline c & -3.5160748362 & -1.5222594973 & -1.8652107891 \\
\hline H & -2.5914833962 & -1.3536239307 & -2.4210265166 \\
\hline H & -4.2410079698 & -0.7616549938 & -2.1551880122 \\
\hline H & -3.9141678833 & -2.4907313012 & -2.1824041044 \\
\hline c & -2.5384287577 & 2.7225515551 & 0.5143564023 \\
\hline H & -2.7924333425 & 2.6244865844 & 1.5698952992 \\
\hline H & -2.9083958822 & 3.6895861455 & 0.1608598466 \\
\hline H & -1.4506010617 & 2.7200858593 & 0.4258675514 \\
\hline C & -4.6901234262 & 1.6143332817 & -0.1197319133 \\
\hline H & -5.2052304428 & 0.9266394911 & -0.7903479188 \\
\hline H & -5.0768283636 & 2.6176527063 & -0.3241203157 \\
\hline H & -4.9591160014 & 1.3619051994 & 0.9077514410 \\
\hline c & -2.8430187354 & 1.8845474615 & -1.8103614247 \\
\hline H & -1.7648524327 & 1.9356820940 & -1.9679293234 \\
\hline $\mathrm{H}$ & -3.2711176045 & 2.8500733175 & -2.0965550073 \\
\hline H & -3.2556138754 & 1.1348813063 & -2.4848956458 \\
\hline
\end{tabular}

\begin{tabular}{|c|c|c|c|}
\hline \multicolumn{4}{|c|}{$\begin{array}{l}\text { Table D8: :xyz data of } 2 \mathrm{me}^{2} \mathrm{~A}(\mathrm{C} 1)^{\mathrm{t}} \mathrm{Bu}: \mathrm{Me} \\
30\end{array}$} \\
\hline \multicolumn{4}{|c|}{$E(B 3 L Y P(V)-D 3 / d e f 2 T Z V P)=-1232.03745741$} \\
\hline Ir & 0.0050958848 & -0.0156777177 & -0.0882343990 \\
\hline$P$ & -2.3124919190 & -0.0023251148 & 0.2028011184 \\
\hline$P$ & 2.3243994711 & -0.0141122055 & 0.2134758945 \\
\hline $\mathrm{N}$ & -0.0017481669 & -0.0543978270 & 1.9312039329 \\
\hline 0 & 0.1472304536 & -0.0255323898 & -2.0622208719 \\
\hline H & -0.7026424592 & -0.0245540780 & -2.5115971839 \\
\hline c & -1.1919566468 & -0.0603323501 & 2.6234292271 \\
\hline $\mathrm{H}$ & -1.1254519701 & -0.0822505932 & 3.7099385235 \\
\hline c & 1.1887359932 & -0.0858461070 & 2.6276294720 \\
\hline $\mathrm{H}$ & 1.1156417377 & -0.1165700739 & 3.7135721441 \\
\hline c & -2.3898016333 & -0.0390265573 & 2.0008413680 \\
\hline $\mathrm{H}$ & -3.3073033307 & -0.0328197877 & 2.5717051577 \\
\hline c & 2.3898295740 & -0.0786063806 & 2.0120729664 \\
\hline $\mathrm{H}$ & 3.3029031461 & -0.0952036685 & 2.5902553202 \\
\hline c & 3.2386485545 & -1.5593207391 & -0.3854775126 \\
\hline c & 3.1876083130 & 1.5944795914 & -0.2980978610 \\
\hline c & -3.2705549530 & -1.5465776493 & -0.3506172359 \\
\hline c & -3.1723670696 & 1.6033523041 & -0.3339373118 \\
\hline $\mathrm{H}$ & 2.5867727657 & -2.3955667391 & -0.1361257801 \\
\hline $\mathrm{H}$ & 4.2011395354 & -1.7191879114 & 0.1026751680 \\
\hline $\mathrm{H}$ & 3.3572504733 & -1.5199401416 & -1.4683639161 \\
\hline $\mathrm{H}$ & 2.7034809351 & 2.3812392253 & 0.2795627679 \\
\hline $\mathrm{H}$ & 3.0025009675 & 1.7860822933 & -1.3548189076 \\
\hline $\mathrm{H}$ & 4.2594987768 & 1.6119829452 & -0.0932720663 \\
\hline $\mathrm{H}$ & -4.2143361794 & -1.6904581281 & 0.1772962158 \\
\hline $\mathrm{H}$ & -2.6183288502 & -2.3870884780 & -0.1166635965 \\
\hline $\mathrm{H}$ & -3.4450898908 & -1.5292903848 & -1.4273100404 \\
\hline $\mathrm{H}$ & -2.7241461093 & 2.3946730899 & 0.2658419034 \\
\hline $\mathrm{H}$ & -4.2529692398 & 1.6111704682 & -0.1814287636 \\
\hline $\mathrm{H}$ & -2.9390142620 & 1.8025871951 & -1.3800264405 \\
\hline \multicolumn{4}{|c|}{$\begin{array}{l}\text { Table D9: :xyz data of } 2 \mathrm{~h}^{2} \mathrm{~A} \text { ( (C1) }{ }^{\mathrm{t} B u}: \mathrm{H} \\
18\end{array}$} \\
\hline \multicolumn{4}{|c|}{$E(B 3 L Y P(V)-D 3 /$ def2TZVP $)=-1074.78244850$} \\
\hline $\mathrm{Ir}$ & 0.0050958848 & -0.0156777177 & -0.0882343990 \\
\hline$P$ & -2.3124919190 & -0.0023251148 & 0.2028011184 \\
\hline$P$ & 2.3243994711 & -0.0141122055 & 0.2134758945 \\
\hline $\mathrm{N}$ & -0.0017481669 & -0.0543978270 & 1.9312039329 \\
\hline 0 & 0.1472304536 & -0.0255323898 & -2.0622208719 \\
\hline $\mathrm{H}$ & -0.7026424592 & -0.0245540780 & -2.5115971839 \\
\hline C & -1.1919566468 & -0.0603323501 & 2.6234292271 \\
\hline $\mathrm{H}$ & -1.1254519701 & -0.0822505932 & 3.7099385235 \\
\hline C & 1.1887359932 & -0.0858461070 & 2.6276294720 \\
\hline $\mathrm{H}$ & 1.1156417377 & -0.1165700739 & 3.7135721441 \\
\hline C & -2.3898016333 & -0.0390265573 & 2.0008413680 \\
\hline $\mathrm{H}$ & -3.3073033307 & -0.0328197877 & 2.5717051577 \\
\hline C & 2.3898295740 & -0.0786063806 & 2.0120729664 \\
\hline $\mathrm{H}$ & 3.3029031461 & -0.0952036685 & 2.5902553202 \\
\hline $\mathrm{H}$ & -3.0234805337 & -1.1483310991 & -0.2078964403 \\
\hline $\mathrm{H}$ & -2.9509967012 & 1.1899790353 & -0.1957568051 \\
\hline $\mathrm{H}$ & 3.0043142266 & -1.1632630238 & -0.2319577042 \\
\hline $\mathrm{H}$ & 2.9654541046 & 1.1804946785 & -0.1664399618 \\
\hline
\end{tabular}




\begin{tabular}{|c|c|c|c|}
\hline \multicolumn{4}{|c|}{$\begin{array}{l}\text { Table D10: xyz data of } 4+{ }^{2} A^{\prime \prime}(\mathrm{Cs}) \\
65\end{array}$} \\
\hline \multicolumn{4}{|c|}{ E(UPBE1PBE-D3(BJ)/def2TZVP) $=-1702.18117658<S 2\rangle=0.9298$} \\
\hline $\bar{N}$ & -0.04402339 & 0.00000000 & 1.85406003 \\
\hline c & -0.04499775 & 1.18409063 & 2.59510905 \\
\hline c & -0.04499775 & -1.18409063 & 2.59510905 \\
\hline c & -0.02671447 & -2.37904680 & 1.99645369 \\
\hline $\mathrm{P}$ & 0.01446540 & -2.34282627 & 0.20194402 \\
\hline c & -1.57704960 & -3.11286682 & -0.38800661 \\
\hline c & 1.57550258 & -3.23673873 & -0.28659437 \\
\hline c & -0.02671447 & 2.37904680 & 1.99645369 \\
\hline $\mathrm{P}$ & 0.01446540 & 2.34282627 & 0.20194402 \\
\hline C & 1.57550258 & 3.23673873 & -0.28659437 \\
\hline c & -1.57704960 & 3.11286682 & -0.38800661 \\
\hline c & 1.73309837 & -4.56042978 & 0.45777010 \\
\hline c & 2.72861344 & -2.30982109 & 0.10403497 \\
\hline c & 1.59051143 & -3.45661222 & -1.79594879 \\
\hline C & 2.72861344 & 2.30982109 & 0.10403497 \\
\hline c & 1.73309837 & 4.56042978 & 0.45777010 \\
\hline C & 1.59051143 & 3.45661222 & -1.79594879 \\
\hline C & -1.61854121 & -4.62248667 & -0.18277398 \\
\hline c & -1.77714236 & -2.76574472 & -1.86280256 \\
\hline c & -2.69016715 & -2.45204841 & 0.42717433 \\
\hline c & -2.69016715 & 2.45204841 & 0.42717433 \\
\hline c & -1.77714236 & 2.76574472 & -1.86280256 \\
\hline c & -1.61854121 & 4.62248667 & -0.18277398 \\
\hline Ir & 0.08237422 & 0.00000000 & -0.10305373 \\
\hline 0 & 0.50626869 & 0.00000000 & -1.79877184 \\
\hline H & 2.70427796 & 2.05090805 & 1.16523660 \\
\hline H & 3.67129414 & 2.82870026 & -0.08975388 \\
\hline H & 2.72151780 & 1.38900242 & -0.48034963 \\
\hline H & 1.39676236 & 2.53246414 & -2.34523130 \\
\hline H & 2.58275910 & 3.81353159 & -2.08477991 \\
\hline H & 0.86907893 & 4.21236716 & -2.10849421 \\
\hline H & -1.42218064 & 4.90849233 & 0.85268265 \\
\hline H & -2.62050419 & 4.98006281 & -0.43588800 \\
\hline H & -0.91486175 & 5.14509492 & -0.83097387 \\
\hline H & -2.65786415 & 1.36256493 & 0.34474564 \\
\hline H & -3.65335471 & 2.78725447 & 0.03393327 \\
\hline H & -2.64501243 & 2.72231069 & 1.48297183 \\
\hline H & -1.77274893 & 1.68655195 & -2.02390651 \\
\hline $\mathrm{H}$ & -1.01946210 & 3.20882170 & -2.50738303 \\
\hline H & -2.74946632 & 3.15267758 & -2.17946196 \\
\hline H & -2.64501243 & -2.72231069 & 1.48297183 \\
\hline H & -3.65335471 & -2.78725447 & 0.03393327 \\
\hline H & -2.65786415 & -1.36256493 & 0.34474564 \\
\hline $\mathrm{H}$ & -0.91486175 & -5.14509492 & -0.83097387 \\
\hline H & -2.62050419 & -4.98006281 & -0.43588800 \\
\hline H & -1.42218064 & -4.90849233 & 0.85268265 \\
\hline H & -1.01946210 & -3.20882170 & -2.50738303 \\
\hline H & -1.77274893 & -1.68655195 & -2.02390651 \\
\hline H & -2.74946632 & -3.15267758 & -2.17946196 \\
\hline H & 2.70427796 & -2.05090805 & 1.16523660 \\
\hline H & 2.72151780 & -1.38900242 & -0.48034963 \\
\hline H & 3.67129414 & -2.82870026 & -0.08975388 \\
\hline $\mathrm{H}$ & 0.86907893 & -4.21236716 & -2.10849421 \\
\hline H & 2.58275910 & -3.81353159 & -2.08477991 \\
\hline H & 1.39676236 & -2.53246414 & -2.34523130 \\
\hline H & 1.80526492 & -4.41290601 & 1.53674209 \\
\hline H & 2.66620496 & -5.02782950 & 0.13152856 \\
\hline H & 0.92716844 & -5.26339419 & 0.25485995 \\
\hline H & 1.80526492 & 4.41290601 & 1.53674209 \\
\hline H & 0.92716844 & 5.26339419 & 0.25485995 \\
\hline H & 2.66620496 & 5.02782950 & 0.13152856 \\
\hline H & -0.06831680 & -1.07899195 & 3.67646059 \\
\hline H & -0.03791943 & -3.28983941 & 2.58035035 \\
\hline H & -0.06831680 & 1.07899195 & 3.67646059 \\
\hline H & -0.03791943 & 3.28983941 & 2.58035035 \\
\hline
\end{tabular}

\begin{tabular}{|c|c|c|c|}
\hline \multicolumn{4}{|c|}{$\begin{array}{l}\text { Table D11: xyz data of } 4+{ }^{4} A^{\prime \prime}(\mathrm{Cs}) \\
65\end{array}$} \\
\hline $\mathrm{N}$ & 0.03800444 & 0.00000000 & 1.92148364 \\
\hline c & 0.01810707 & 1.17597021 & 2.62125454 \\
\hline C & 0.01810707 & -1.17597021 & 2.62125454 \\
\hline c & -0.04067448 & -2.36830445 & 1.99096263 \\
\hline$P$ & -0.01054829 & -2.33221609 & 0.19674380 \\
\hline c & -1.58524123 & -3.12983278 & -0.39351135 \\
\hline C & 1.56865296 & -3.19782748 & -0.28615254 \\
\hline c & -0.04067448 & 2.36830445 & 1.99096263 \\
\hline$P$ & -0.01054829 & 2.33221609 & 0.19674380 \\
\hline c & 1.56865296 & 3.19782748 & -0.28615254 \\
\hline c & -1.58524123 & 3.12983278 & -0.39351135 \\
\hline C & 1.75208905 & -4.50964277 & 0.47235267 \\
\hline c & 2.70067964 & -2.24059685 & 0.09361274 \\
\hline c & 1.58918535 & -3.43312422 & -1.79275092 \\
\hline c & 2.70067964 & 2.24059685 & 0.09361274 \\
\hline c & 1.75208905 & 4.50964277 & 0.47235267 \\
\hline c & 1.58918535 & 3.43312422 & -1.79275092 \\
\hline c & -1.63737194 & -4.63011106 & -0.13367850 \\
\hline C & -1.76291709 & -2.83147008 & -1.88143051 \\
\hline C & -2.70227534 & -2.42942764 & 0.38222351 \\
\hline C & -2.70227534 & 2.42942764 & 0.38222351 \\
\hline C & -1.76291709 & 2.83147008 & -1.88143051 \\
\hline c & -1.63737194 & 4.63011106 & -0.13367850 \\
\hline Ir & 0.01549097 & 0.00000000 & -0.12969436 \\
\hline 0 & 0.51623709 & 0.00000000 & -1.84638212 \\
\hline H & 2.65985227 & 1.95428959 & 1.14753498 \\
\hline $\mathrm{H}$ & 3.65538783 & 2.74629548 & -0.07480305 \\
\hline H & 2.68405698 & 1.33787190 & -0.51840058 \\
\hline H & 1.36524731 & 2.52151720 & -2.35157074 \\
\hline $\mathrm{H}$ & 2.59240019 & 3.76063375 & -2.07879220 \\
\hline H & 0.89295128 & 4.21627157 & -2.09480051 \\
\hline H & -1.45631623 & 4.87791217 & 0.91446622 \\
\hline H & -2.63566283 & 4.99674195 & -0.38832388 \\
\hline $\mathrm{H}$ & -0.92425492 & 5.17685340 & -0.75100609 \\
\hline H & -2.67182419 & 1.34396993 & 0.24211705 \\
\hline $\mathrm{H}$ & -3.66573772 & 2.78354638 & 0.00690946 \\
\hline $\mathrm{H}$ & -2.65619445 & 2.63556576 & 1.45289667 \\
\hline $\mathrm{H}$ & -1.69391862 & 1.76148332 & -2.09026125 \\
\hline $\mathrm{H}$ & -1.03066102 & 3.34421584 & -2.50270868 \\
\hline H & -2.75562193 & 3.17152373 & -2.18849808 \\
\hline $\mathrm{H}$ & -2.65619445 & -2.63556576 & 1.45289667 \\
\hline H & -3.66573772 & -2.78354638 & 0.00690946 \\
\hline $\mathrm{H}$ & -2.67182419 & -1.34396993 & 0.24211705 \\
\hline H & -0.92425492 & -5.17685340 & -0.75100609 \\
\hline H & -2.63566283 & -4.99674195 & -0.38832388 \\
\hline $\mathrm{H}$ & -1.45631623 & -4.87791217 & 0.91446622 \\
\hline $\mathrm{H}$ & -1.03066102 & -3.34421584 & -2.50270868 \\
\hline H & -1.69391862 & -1.76148332 & -2.09026125 \\
\hline $\mathrm{H}$ & -2.75562193 & -3.17152373 & -2.18849808 \\
\hline $\mathrm{H}$ & 2.65985227 & -1.95428959 & 1.14753498 \\
\hline $\mathrm{H}$ & 2.68405698 & -1.33787190 & -0.51840058 \\
\hline $\mathrm{H}$ & 3.65538783 & -2.74629548 & -0.07480305 \\
\hline H & 0.89295128 & -4.21627157 & -2.09480051 \\
\hline $\mathrm{H}$ & 2.59240019 & -3.76063375 & -2.07879220 \\
\hline $\mathrm{H}$ & 1.36524731 & -2.52151720 & -2.35157074 \\
\hline $\mathrm{H}$ & 1.82909613 & -4.34763795 & 1.54896380 \\
\hline H & 2.68888155 & -4.96847657 & 0.14454470 \\
\hline H & 0.95399106 & -5.22607428 & 0.28414954 \\
\hline $\mathrm{H}$ & 1.82909613 & 4.34763795 & 1.54896380 \\
\hline H & 0.95399106 & 5.22607428 & 0.28414954 \\
\hline $\mathrm{H}$ & 2.68888155 & 4.96847657 & 0.14454470 \\
\hline H & 0.04061579 & -1.10617031 & 3.70668937 \\
\hline H & -0.08448584 & -3.28512176 & 2.56470279 \\
\hline $\mathrm{H}$ & 0.04061579 & 1.10617031 & 3.70668937 \\
\hline $\mathrm{H}$ & -0.08448584 & 3.28512176 & 2.56470279 \\
\hline
\end{tabular}




\begin{tabular}{|c|c|c|c|}
\hline \multicolumn{4}{|c|}{$\begin{array}{l}\text { Table D12: xyz data of Mes* }{ }^{*}{ }^{1} A^{\prime}(C s) \\
49\end{array}$} \\
\hline C & 0.40442879 & 1.18230327 & 0.00000000 \\
\hline c & -0.47890621 & -1.47666010 & 0.00000000 \\
\hline c & 1.35508336 & 0.14577914 & 0.00000000 \\
\hline c & -0.98246635 & 0.92342527 & 0.00000000 \\
\hline c & -1.37669709 & -0.40951321 & 0.00000000 \\
\hline c & 0.87360170 & -1.16720701 & 0.00000000 \\
\hline $\mathrm{H}$ & -2.43148325 & -0.63306780 & 0.00000000 \\
\hline $\mathrm{H}$ & 1.58715691 & -1.97232425 & 0.00000000 \\
\hline 0 & 0.77372584 & 2.50675302 & 0.00000000 \\
\hline $\mathrm{H}$ & 1.72930373 & 2.58561535 & 0.00000000 \\
\hline c & -1.00820434 & -2.91797490 & 0.00000000 \\
\hline c & -2.02525536 & 2.05980519 & 0.00000000 \\
\hline c & 2.87613810 & 0.41884766 & 0.00000000 \\
\hline c & 0.12216822 & -3.95775724 & 0.00000000 \\
\hline $\mathrm{H}$ & 0.75516695 & -3.86733241 & 0.88544228 \\
\hline $\mathrm{H}$ & 0.75516695 & -3.86733241 & -0.88544228 \\
\hline $\mathrm{H}$ & -0.30337380 & -4.96346252 & 0.00000000 \\
\hline c & -1.87083642 & -3.15003432 & 1.25794262 \\
\hline $\mathrm{H}$ & -1.28153274 & -2.99484739 & 2.16429581 \\
\hline $\mathrm{H}$ & -2.25862597 & -4.17213696 & 1.27243201 \\
\hline $\mathrm{H}$ & -2.72310404 & -2.46995548 & 1.29371219 \\
\hline c & -1.87083642 & -3.15003432 & -1.25794262 \\
\hline $\mathrm{H}$ & -2.72310404 & -2.46995548 & -1.29371219 \\
\hline $\mathrm{H}$ & -2.25862597 & -4.17213696 & -1.27243201 \\
\hline $\mathrm{H}$ & -1.28153274 & -2.99484739 & -2.16429581 \\
\hline c & -1.87083642 & 2.92946601 & -1.26725216 \\
\hline H & -0.89319689 & 3.40121976 & -1.31932464 \\
\hline $\mathrm{H}$ & -2.63115302 & 3.71503916 & -1.27323219 \\
\hline $\mathrm{H}$ & -2.00807119 & 2.32141395 & -2.16468428 \\
\hline c & -1.87083642 & 2.92946601 & 1.26725216 \\
\hline $\mathrm{H}$ & -0.89319689 & 3.40121976 & 1.31932464 \\
\hline $\mathrm{H}$ & -2.00807119 & 2.32141395 & 2.16468428 \\
\hline H & -2.63115302 & 3.71503916 & 1.27323219 \\
\hline c & -3.46567748 & 1.51724646 & 0.00000000 \\
\hline H & -3.67770527 & 0.91548282 & 0.88630282 \\
\hline H & -3.67770527 & 0.91548282 & -0.88630282 \\
\hline $\mathrm{H}$ & -4.16265727 & 2.35753922 & 0.00000000 \\
\hline c & 3.29481238 & 1.18890276 & 1.27595121 \\
\hline $\mathrm{H}$ & 3.05517724 & 0.59914695 & 2.16250509 \\
\hline H & 2.80221415 & 2.15410638 & 1.40282409 \\
\hline $\mathrm{H}$ & 4.37177883 & 1.37286730 & 1.26840033 \\
\hline c & 3.29481238 & 1.18890276 & -1.27595121 \\
\hline H & 2.80221415 & 2.15410638 & -1.40282409 \\
\hline $\mathrm{H}$ & 3.05517724 & 0.59914695 & -2.16250509 \\
\hline $\mathrm{H}$ & 4.37177883 & 1.37286730 & -1.26840033 \\
\hline c & 3.69962789 & -0.88253905 & 0.00000000 \\
\hline $\mathrm{H}$ & 3.50285395 & -1.48922200 & 0.88535989 \\
\hline H & 4.76292099 & -0.63443460 & 0.00000000 \\
\hline $\mathrm{H}$ & 3.50285395 & -1.48922200 & -0.88535989 \\
\hline
\end{tabular}

\begin{tabular}{|c|c|c|c|}
\hline \multirow{2}{*}{\multicolumn{4}{|c|}{$\begin{array}{l}\text { Table D13: xyz data of Mes* }{ }^{*} \mathrm{OH}^{\mathrm{Me}}{ }^{1} \mathrm{~A}^{\prime}(\mathrm{Cs}){ }^{\mathrm{t}} \mathrm{Bu}: \mathrm{Me} \\
\mathrm{E}(\mathrm{B} 3 \mathrm{LYP}(\mathrm{V})-\mathrm{D} 3 / \text { def2TZVP) }=-425.325964546\end{array}$}} \\
\hline & & & \\
\hline C & 0.404428790 & 1.182303275 & 0.000000000 \\
\hline c & -0.478906207 & -1.476660103 & 0.000000000 \\
\hline c & 1.355083361 & 0.145779137 & 0.000000000 \\
\hline C & -0.982466347 & 0.923425269 & 0.000000000 \\
\hline c & -1.376697092 & -0.409513205 & 0.000000000 \\
\hline c & 0.873601700 & -1.167207011 & 0.0000000000 \\
\hline $\mathrm{H}$ & -2.431483251 & -0.633067800 & 0.000000000 \\
\hline H & 1.587156912 & -1.972324247 & 0.0000000000 \\
\hline 0 & 0.773725839 & 2.506753017 & 0.000000000 \\
\hline H & 1.729303734 & 2.585615350 & 0.000000000 \\
\hline C & -1.008204339 & -2.917974904 & 0.000000000 \\
\hline $\mathrm{H}$ & -0.205682842 & -3.656180789 & 0.000000000 \\
\hline H & -1.618831584 & -3.082241690 & 0.890453829 \\
\hline H & -1.618831584 & -3.082241690 & -0.890453829 \\
\hline c & -2.025255356 & 2.059805187 & 0.000000000 \\
\hline H & -1.916151730 & 2.674257980 & -0.895368178 \\
\hline $\mathrm{H}$ & -1.916151730 & 2.674257980 & 0.895368178 \\
\hline H & -3.045862512 & 1.675376681 & 0.000000000 \\
\hline c & 2.876138096 & 0.418847662 & 0.000000000 \\
\hline H & 3.172084764 & 0.963173525 & 0.901926686 \\
\hline $\mathrm{H}$ & 3.172084764 & 0.963173525 & -0.901926686 \\
\hline H & 3.458820774 & -0.501984031 & 0.000000000 \\
\hline
\end{tabular}

\begin{tabular}{|c|c|c|c|}
\hline \multicolumn{4}{|c|}{ 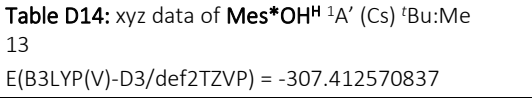 } \\
\hline C & 0.40442879 & 1.18230328 & 0.00000000 \\
\hline c & -0.47890621 & -1.47666010 & 0.00000000 \\
\hline c & 1.35508336 & 0.14577914 & 0.00000000 \\
\hline c & -0.98246635 & 0.92342527 & 0.00000000 \\
\hline c & -1.37669709 & -0.40951321 & 0.00000000 \\
\hline c & 0.87360170 & -1.16720701 & 0.00000000 \\
\hline H & -2.43148325 & -0.63306780 & 0.00000000 \\
\hline $\mathrm{H}$ & 1.58715691 & -1.97232425 & 0.00000000 \\
\hline 0 & 0.77372584 & 2.50675302 & 0.00000000 \\
\hline H & 1.72930373 & 2.58561535 & 0.00000000 \\
\hline H & -0.85141752 & -2.49103374 & 0.00000000 \\
\hline $\mathrm{H}$ & -1.71263515 & 1.71912714 & 0.00000000 \\
\hline H & 2.42057903 & 0.33706307 & 0.00000000 \\
\hline
\end{tabular}




\begin{tabular}{|c|c|c|c|}
\hline \multicolumn{4}{|c|}{$\begin{array}{l}\text { Table D15: xyz data of Mes*0 2A" (Cs) } \\
48 \\
E(B 3 L Y P(V)-D 3 / \text { def2TZVP) }=-778.40576486694818\end{array}$} \\
\hline $\mathrm{C}$ & 0.42887515 & 1.23904273 & 0.00000000 \\
\hline c & -0.45989312 & -1.45264666 & 0.00000000 \\
\hline c & 1.38723894 & 0.13308698 & 0.00000000 \\
\hline c & -1.00283896 & 0.92563434 & 0.00000000 \\
\hline c & -1.38078327 & -0.38865632 & 0.00000000 \\
\hline c & 0.90719205 & -1.15475784 & 0.00000000 \\
\hline $\mathrm{H}$ & -2.43455432 & -0.63128204 & 0.00000000 \\
\hline $\mathrm{H}$ & 1.61060641 & -1.97338915 & 0.00000000 \\
\hline 0 & 0.82057433 & 2.41930657 & 0.00000000 \\
\hline c & -0.98219231 & -2.87873524 & 0.00000000 \\
\hline c & -2.02047147 & 2.05465003 & 0.00000000 \\
\hline c & 2.87779205 & 0.42917507 & 0.00000000 \\
\hline c & 0.13751951 & -3.91404963 & 0.00000000 \\
\hline H & 0.77073551 & -3.82750843 & 0.88613283 \\
\hline $\mathrm{H}$ & 0.77073551 & -3.82750843 & -0.88613283 \\
\hline H & -0.29581615 & -4.91663524 & 0.00000000 \\
\hline c & -1.84188218 & -3.09717364 & 1.25117212 \\
\hline H & -1.25291409 & -2.94710932 & 2.15892549 \\
\hline H & -2.23204013 & -4.11835951 & 1.26138579 \\
\hline $\mathrm{H}$ & -2.69175459 & -2.41338445 & 1.28453084 \\
\hline c & -1.84188218 & -3.09717364 & -1.25117212 \\
\hline $\mathrm{H}$ & -2.69175459 & -2.41338445 & -1.28453084 \\
\hline $\mathrm{H}$ & -2.23204013 & -4.11835951 & -1.26138579 \\
\hline $\mathrm{H}$ & -1.25291409 & -2.94710932 & -2.15892549 \\
\hline c & -1.84033589 & 2.91811772 & -1.25613531 \\
\hline H & -0.85254335 & 3.37331535 & -1.28222294 \\
\hline H & -2.59308423 & 3.71125015 & -1.26426270 \\
\hline $\mathrm{H}$ & -1.97485302 & 2.31732067 & -2.15963970 \\
\hline c & -1.84033589 & 2.91811772 & 1.25613531 \\
\hline $\mathrm{H}$ & -0.85254335 & 3.37331535 & 1.28222294 \\
\hline $\mathrm{H}$ & -1.97485302 & 2.31732067 & 2.15963970 \\
\hline $\mathrm{H}$ & -2.59308423 & 3.71125015 & 1.26426270 \\
\hline c & -3.45308061 & 1.52711245 & 0.00000000 \\
\hline $\mathrm{H}$ & -3.66944472 & 0.92674794 & 0.88763635 \\
\hline $\mathrm{H}$ & -3.66944472 & 0.92674794 & -0.88763635 \\
\hline H & -4.14268216 & 2.37392620 & 0.00000000 \\
\hline c & 3.24995152 & 1.22928437 & 1.25620079 \\
\hline $\mathrm{H}$ & 2.99829626 & 0.66759814 & 2.15982530 \\
\hline H & 2.73106366 & 2.18510199 & 1.28197617 \\
\hline $\mathrm{H}$ & 4.32774642 & 1.41380803 & 1.26406519 \\
\hline c & 3.24995152 & 1.22928437 & -1.25620079 \\
\hline $\mathrm{H}$ & 2.73106366 & 2.18510199 & -1.28197617 \\
\hline $\mathrm{H}$ & 2.99829626 & 0.66759814 & -2.15982530 \\
\hline $\mathrm{H}$ & 4.32774642 & 1.41380803 & -1.26406519 \\
\hline c & 3.71214012 & -0.84958819 & 0.00000000 \\
\hline $\mathrm{H}$ & 3.52761195 & -1.46071242 & 0.88738275 \\
\hline $\mathrm{H}$ & 4.77074592 & -0.58122904 & 0.00000000 \\
\hline $\mathrm{H}$ & 3.52761195 & -1.46071242 & -0.88738275 \\
\hline
\end{tabular}

\begin{tabular}{|c|c|c|c|}
\hline \multicolumn{4}{|c|}{$\begin{array}{l}\text { Table D16: xyz data of Mes*OMe }{ }^{2} A^{\prime \prime}(C s){ }^{t} \text { Bu:Me } \\
21 \\
E(B 3 L Y P(V)-D 3 / \text { def2TZVP) }=-424.694213273\end{array}$} \\
\hline c & 0.4308158593 & 1.2440794891 & 0.0000000000 \\
\hline c & -0.4611465907 & -1.4568441096 & 0.0000000000 \\
\hline c & 1.3945046177 & 0.1333174589 & 0.0000000000 \\
\hline c & -1.0080569431 & 0.9302754976 & 0.0000000000 \\
\hline c & -1.3834579905 & -0.3881976167 & 0.0000000000 \\
\hline c & 0.9098863314 & -1.1564480544 & 0.0000000000 \\
\hline $\mathrm{H}$ & -2.4338848979 & -0.6342056593 & 0.0000000000 \\
\hline $\mathrm{H}$ & 1.6104913531 & -1.9739497447 & 0.0000000000 \\
\hline 0 & 0.8240289343 & 2.4286391599 & 0.0000000000 \\
\hline c & -0.9879795502 & -2.8928557501 & 0.0000000000 \\
\hline $\mathrm{H}$ & -0.1905108562 & -3.6353892886 & 0.0000000000 \\
\hline $\mathrm{H}$ & -1.5999065480 & -3.0507260807 & 0.8914051236 \\
\hline H & -1.5999065480 & -3.0507260807 & -0.8914051236 \\
\hline c & -2.0332965572 & 2.0676503195 & 0.0000000000 \\
\hline H & -1.9061594053 & 2.6816106033 & -0.8930034687 \\
\hline H & -1.9061594053 & 2.6816106033 & 0.8930034687 \\
\hline $\mathrm{H}$ & -3.0579685857 & 1.6938669500 & 0.0000000000 \\
\hline c & 2.8961436521 & 0.4316053298 & 0.0000000000 \\
\hline $\mathrm{H}$ & 3.1614554855 & 0.9998915439 & 0.8929773445 \\
\hline H & 3.1614554855 & 0.9998915439 & -0.8929773445 \\
\hline H & 3.4950225405 & -0.4799722393 & 0.0000000000 \\
\hline
\end{tabular}

\begin{tabular}{|c|c|c|c|}
\hline \multicolumn{4}{|c|}{$\begin{array}{l}\text { Table D17: xyz data of Mes* }{ }^{*} \mathrm{H}^{2} \mathrm{~A}^{\prime \prime}(\mathrm{Cs}){ }^{\mathrm{t}} \mathrm{Bu}: \mathrm{Me} \\
12 \\
\mathrm{E}(\mathrm{B} 3 \mathrm{LYP}(\mathrm{V})-\mathrm{D} 3 / \text { def2TZVP) }=-306.770251191\end{array}$} \\
\hline $\mathrm{c}$ & 0.4308158593 & 1.2440794891 & 0.0000000000 \\
\hline c & -0.4611465907 & -1.4568441096 & 0.0000000000 \\
\hline c & 1.3945046177 & 0.1333174589 & 0.0000000000 \\
\hline c & -1.0080569431 & 0.9302754976 & 0.0000000000 \\
\hline c & -1.3834579905 & -0.3881976167 & 0.0000000000 \\
\hline c & 0.9098863314 & -1.1564480544 & 0.0000000000 \\
\hline $\mathrm{H}$ & -2.4338848979 & -0.6342056593 & 0.0000000000 \\
\hline H & 1.6104913531 & -1.9739497447 & 0.0000000000 \\
\hline 0 & 0.8240289343 & 2.4286391599 & 0.0000000000 \\
\hline H & -0.8335078280 & -2.4718054205 & 0.0000000000 \\
\hline $\mathrm{H}$ & -1.7315181965 & 1.7328650610 & 0.0000000000 \\
\hline H & 2.4542896221 & 0.3438347706 & 0.0000000000 \\
\hline
\end{tabular}




\begin{tabular}{|c|c|c|c|}
\hline \multicolumn{4}{|c|}{$\begin{array}{l}\text { Table D18: xyz data of } 23^{3} \mathrm{~A}^{\prime \prime}(\mathrm{Cs}) \\
60\end{array}$} \\
\hline $\mathrm{Re}$ & 0.1518433799 & 0.0661960579 & 0.0000000000 \\
\hline $\mathrm{N}$ & -0.2532309345 & -2.0907674786 & 0.0000000000 \\
\hline $\mathrm{H}$ & 0.7085356660 & -2.4348286738 & 0.0000000000 \\
\hline$P$ & 0.0204876528 & -0.2595350252 & 2.3954180254 \\
\hline$P$ & 0.0204876528 & -0.2595350252 & -2.3954180254 \\
\hline $\mathrm{Cl}$ & 2.4397617653 & -0.6259757032 & 0.0000000000 \\
\hline $\mathrm{Cl}$ & 0.6905150586 & 2.3648247163 & 0.0000000000 \\
\hline $\mathrm{Cl}$ & -2.2291542895 & 0.3138010227 & 0.0000000000 \\
\hline C & -0.9248724832 & -2.5726185276 & 1.2216829378 \\
\hline $\mathrm{H}$ & -0.9828237827 & -3.6676813150 & 1.2039378150 \\
\hline $\mathrm{H}$ & -1.9435002468 & -2.1793545500 & 1.1968305700 \\
\hline C & -0.1899552105 & -2.0932903392 & 2.4584788139 \\
\hline $\mathrm{H}$ & 0.8169720808 & -2.5224182575 & 2.4809344625 \\
\hline $\mathrm{H}$ & -0.7079515426 & -2.4284321927 & 3.3594013698 \\
\hline C & -0.9248724832 & -2.5726185276 & -1.2216829378 \\
\hline $\mathrm{H}$ & -1.9435002468 & -2.1793545500 & -1.1968305700 \\
\hline $\mathrm{H}$ & -0.9828237827 & -3.6676813150 & -1.2039378150 \\
\hline C & -0.1899552105 & -2.0932903392 & -2.4584788139 \\
\hline $\mathrm{H}$ & -0.7079515426 & -2.4284321927 & -3.3594013698 \\
\hline $\mathrm{H}$ & 0.8169720808 & -2.5224182575 & -2.4809344625 \\
\hline $\mathrm{C}$ & -1.4482542269 & 0.4069807733 & -3.2883055553 \\
\hline $\mathrm{H}$ & -2.2610375332 & -0.0605231178 & -2.7185909534 \\
\hline $\mathrm{C}$ & -1.5759072085 & 1.9130043245 & -3.0878461140 \\
\hline $\mathrm{H}$ & -1.4413977511 & 2.1915310957 & -2.0426970291 \\
\hline $\mathrm{H}$ & -2.5690863183 & 2.2405942764 & -3.4066025132 \\
\hline $\mathrm{H}$ & -0.8407788999 & 2.4576066869 & -3.6826372038 \\
\hline $\mathrm{C}$ & -1.5890014223 & 0.0210563375 & -4.7528214392 \\
\hline $\mathrm{H}$ & -0.8865003936 & 0.5763265203 & -5.3768427360 \\
\hline $\mathrm{H}$ & -2.5957089540 & 0.2695171600 & -5.1009344122 \\
\hline $\mathrm{H}$ & -1.4325422026 & -1.0444155374 & -4.9344608128 \\
\hline $\mathrm{C}$ & 1.5855088940 & 0.0278416648 & -3.3272489450 \\
\hline $\mathrm{H}$ & 2.3104545402 & -0.3879225797 & -2.6170226434 \\
\hline $\mathrm{C}$ & 1.7290957429 & -0.7089269108 & -4.6517209361 \\
\hline $\mathrm{H}$ & 2.7620381179 & -0.6187705541 & -5.0001609820 \\
\hline $\mathrm{H}$ & 1.0869667761 & -0.2879346602 & -5.4255857573 \\
\hline $\mathrm{H}$ & 1.5058182079 & -1.7745781906 & -4.5701938028 \\
\hline$C$ & 1.8842094489 & 1.5146133116 & -3.4666836826 \\
\hline $\mathrm{H}$ & 1.7432500128 & 2.0460123832 & -2.5233800269 \\
\hline $\mathrm{H}$ & 1.2482604834 & 1.9774398294 & -4.2247157124 \\
\hline $\mathrm{H}$ & 2.9218645108 & 1.6496443580 & -3.7829981438 \\
\hline C & 1.5855088940 & 0.0278416648 & 3.3272489450 \\
\hline $\mathrm{H}$ & 2.3104545402 & -0.3879225797 & 2.6170226434 \\
\hline C & 1.7290957429 & -0.7089269108 & 4.6517209361 \\
\hline $\mathrm{H}$ & 2.7620381179 & -0.6187705541 & 5.0001609820 \\
\hline $\mathrm{H}$ & 1.5058182080 & -1.7745781906 & 4.5701938028 \\
\hline $\mathrm{H}$ & 1.0869667761 & -0.2879346602 & 5.4255857573 \\
\hline $\mathrm{C}$ & 1.8842094489 & 1.5146133116 & 3.4666836826 \\
\hline $\mathrm{H}$ & 1.2482604834 & 1.9774398294 & 4.2247157124 \\
\hline $\mathrm{H}$ & 1.7432500128 & 2.0460123832 & 2.5233800269 \\
\hline $\mathrm{H}$ & 2.9218645109 & 1.6496443580 & 3.7829981437 \\
\hline C & -1.4482542269 & 0.4069807733 & 3.2883055553 \\
\hline $\mathrm{H}$ & -2.2610375332 & -0.0605231178 & 2.7185909534 \\
\hline C & -1.5759072085 & 1.9130043245 & 3.0878461140 \\
\hline $\mathrm{H}$ & -1.4413977510 & 2.1915310957 & 2.0426970291 \\
\hline $\mathrm{H}$ & -0.8407788999 & 2.4576066869 & 3.6826372038 \\
\hline $\mathrm{H}$ & -2.5690863183 & 2.2405942764 & 3.4066025132 \\
\hline C & -1.5890014223 & 0.0210563375 & 4.7528214392 \\
\hline $\mathrm{H}$ & -1.4325422026 & -1.0444155374 & 4.9344608128 \\
\hline $\mathrm{H}$ & -2.5957089540 & 0.2695171600 & 5.1009344122 \\
\hline $\mathrm{H}$ & -0.8865003936 & 0.5763265203 & 5.3768427360 \\
\hline
\end{tabular}

\begin{tabular}{|c|c|c|c|}
\hline \multirow{2}{*}{\multicolumn{4}{|c|}{$\begin{array}{l}\text { Table D20: :xyz data of } 23 \mathrm{me}^{3} \mathrm{~A}^{\prime \prime}(\mathrm{Cs})^{i} \mathrm{Pr}: \mathrm{Me} \\
36 \\
\text { E(UPBE1PBE-D3/def2TZVP) }=-2513.1750413\end{array}$}} \\
\hline & & & \\
\hline Ree & -0.1061115559 & \multicolumn{2}{|c|}{$\begin{array}{lll}\text { Ree } & -0.1061115559 & -0.0700213799\end{array}$} \\
\hline $\mathrm{N}$ & 0.0000000000 & 1.9908532315 & 0.0000000000 \\
\hline H & -0.2435638982 & 2.4885207565 & 0.0000000000 \\
\hline$P$ & 0.0770653000 & 0.2296472689 & 2.3954180254 \\
\hline$P$ & 0.0770653162 & 0.2296472689 & -2.3954180250 \\
\hline $\mathrm{Cl}$ & -2.2489011998 & 0.9892825555 & 0.0000000000 \\
\hline $\mathrm{Cl}$ & -1.0157716943 & -2.2486400521 & 0.0000000000 \\
\hline $\mathrm{Cl}$ & 2.2016598989 & -0.7061416772 & 0.0000000000 \\
\hline c & 1.3902465602 & 2.3555861430 & 1.2216829370 \\
\hline $\mathrm{H}$ & 1.6276456457 & 3.4261759503 & 1.2039378150 \\
\hline H & 2.3302541294 & 1.8000280029 & 1.1968305700 \\
\hline c & 0.5864588408 & 2.0037563375 & 2.4584788139 \\
\hline H & -0.3361049259 & 2.0000000000 & 2.4809344625 \\
\hline $\mathrm{H}$ & 1.1525521707 & 2.2490696146 & 3.3594013698 \\
\hline c & 1.3000000000 & 2.3555861430 & -1.2216829378 \\
\hline H & 2.3302541294 & 1.8000280029 & -1.1960000000 \\
\hline $\mathrm{H}$ & 1.6276456457 & 3.4261759503 & -1.2039378150 \\
\hline $\mathrm{C}$ & 0.5864588408 & 2.0037560000 & -2.4584788139 \\
\hline $\mathrm{H}$ & 1.1525521707 & 2.2490696146 & -3.3594013698 \\
\hline $\mathrm{H}$ & -0.3361000000 & 2.5927634992 & -2.4809344625 \\
\hline c & 1.4160733015 & -0.6695210325 & -3.2883000000 \\
\hline $\mathrm{H}$ & 2.2947189221 & -0.3421703575 & -2.7185909534 \\
\hline $\mathrm{H}$ & 1.3289711378 & -1.7400000000 & -3.1451481490 \\
\hline $\mathrm{H}$ & 1.5611144884 & -0.4132711410 & -4.3380592718 \\
\hline c & -1.5000000000 & 0.2037792305 & -3.3272489450 \\
\hline $\mathrm{H}$ & -2.1605390369 & 0.7331931644 & -2.6100000000 \\
\hline H & -1.5284934840 & 0.7411124032 & -4.2757121312 \\
\hline $\mathrm{H}$ & -1.8995829235 & 0.0000000000 & -3.4269565103 \\
\hline c & -1.5139116452 & 0.2037792305 & 3.3272489450 \\
\hline $\mathrm{H}$ & -2.0000000000 & 0.7331931644 & 2.6170226434 \\
\hline $\mathrm{H}$ & -1.5284934840 & 0.7411124032 & 4.2700000000 \\
\hline H & -1.8995829235 & -0.8097317603 & 3.4269565103 \\
\hline c & 1.4160733015 & 0.0000000000 & 3.2883055553 \\
\hline $\mathrm{H}$ & 2.2947189221 & -0.3421703575 & 2.7185909534 \\
\hline $\mathrm{H}$ & 1.0000000000 & -1.7453789437 & 3.1451481490 \\
\hline $\mathrm{H}$ & 1.5611144884 & -0.4132711410 & 4.3380000000 \\
\hline \multicolumn{4}{|c|}{$\begin{array}{l}\text { Table D21: xyz data of } 23 \mathrm{H} 3 \mathrm{~A}^{\prime \prime}(\mathrm{Cs})^{\mathrm{i}} \mathrm{Pr}: \mathrm{H} \\
24\end{array}$} \\
\hline \multicolumn{4}{|c|}{ E(UPBE1PBE-D3/def2TZVP) $=-2355.98895176$} \\
\hline $\operatorname{Re}$ & -0.10611156 & -0.07002138 & 0.00000000 \\
\hline $\mathrm{N}$ & 0.64845636 & 1.99085323 & 0.00000000 \\
\hline H & -0.24356390 & 2.48852076 & 0.00000000 \\
\hline $\mathrm{P}$ & 0.07706532 & 0.22964727 & 2.39541803 \\
\hline$P$ & 0.07706532 & 0.22964727 & -2.39541803 \\
\hline $\mathrm{Cl}$ & -2.24890120 & 0.98928256 & 0.00000000 \\
\hline $\mathrm{Cl}$ & -1.01577169 & -2.24864005 & 0.00000000 \\
\hline $\mathrm{Cl}$ & 2.20165990 & -0.70614168 & 0.00000000 \\
\hline c & 1.39024656 & 2.35558614 & 1.22168294 \\
\hline H & 1.62764565 & 3.42617595 & 1.20393782 \\
\hline H & 2.33025413 & 1.80002800 & 1.19683057 \\
\hline c & 0.58645884 & 2.00375634 & 2.45847881 \\
\hline H & -0.33610493 & 2.59276350 & 2.48093446 \\
\hline H & 1.15255217 & 2.24906961 & 3.35940137 \\
\hline c & 1.39024656 & 2.35558614 & -1.22168294 \\
\hline H & 2.33025413 & 1.80002800 & -1.19683057 \\
\hline H & 1.62764565 & 3.42617595 & -1.20393782 \\
\hline c & 0.58645884 & 2.00375634 & -2.45847881 \\
\hline $\mathrm{H}$ & 1.15255217 & 2.24906961 & -3.35940137 \\
\hline H & -0.33610493 & 2.59276350 & -2.48093446 \\
\hline H & 1.10095661 & -0.45791442 & -3.07817703 \\
\hline H & -1.13560430 & 0.20993021 & -3.10567535 \\
\hline H & -1.13560430 & 0.20993021 & 3.10567535 \\
\hline H & 1.10095661 & -0.45791442 & 3.07817703 \\
\hline
\end{tabular}




\begin{tabular}{|c|c|c|c|}
\hline \multicolumn{4}{|c|}{$\begin{array}{l}\text { Table D22: xyz data of } 2{ }^{2} \mathrm{~A}(\mathrm{C} 1) \\
66\end{array}$} \\
\hline \multicolumn{4}{|c|}{$\mathrm{E}(\mathrm{UPBE} 1 \mathrm{PBE}-\mathrm{D} 3(\mathrm{BJ}) / \mathrm{def} 2 \mathrm{TZVP})=-1703.03656783<\mathrm{S} 2>=0.7561$} \\
\hline Ir & 0.0048245 & -0.01354098 & -0.08555563 \\
\hline$P$ & -2.28958551 & 0.00101498 & 0.19570832 \\
\hline$P$ & 2.30190885 & -0.01265728 & 0.20200033 \\
\hline $\mathrm{N}$ & -0.00077528 & -0.04869755 & 1.90868597 \\
\hline 0 & 0.1383322 & -0.04012764 & -2.04115916 \\
\hline H & -0.71786906 & -0.03420379 & -2.47173432 \\
\hline c & -1.18192243 & -0.05448759 & 2.60266814 \\
\hline H & -1.10789281 & -0.07600755 & 3.68984564 \\
\hline c & 1.1817513 & -0.08181722 & 2.60467578 \\
\hline H & 1.10313426 & -0.11197948 & 3.69140481 \\
\hline c & -2.37816961 & -0.03373065 & 1.9832946 \\
\hline H & -3.29605906 & -0.02749024 & 2.55566329 \\
\hline c & 2.38015028 & -0.07645888 & 1.99033533 \\
\hline H & 3.2942501 & -0.09389551 & 2.56891265 \\
\hline c & 3.20187613 & -1.54227214 & -0.38913126 \\
\hline c & 3.15526334 & 1.57651306 & -0.3029798 \\
\hline C & -3.2310043 & -1.52839625 & -0.34899129 \\
\hline C & -3.14151498 & 1.58564246 & -0.33548809 \\
\hline C & 2.26314167 & -2.703042 & -0.05296301 \\
\hline H & 2.748788 & -3.64494312 & -0.32902176 \\
\hline H & 1.32240456 & -2.61840565 & -0.59919631 \\
\hline H & 2.03360242 & -2.73638387 & 1.01478285 \\
\hline C & 4.5328471 & -1.77980685 & 0.31545218 \\
\hline H & 4.40115604 & -1.86352802 & 1.39613958 \\
\hline H & 5.26315152 & -0.99650835 & 0.11724235 \\
\hline H & 4.95821864 & -2.72483625 & -0.03843447 \\
\hline C & 3.38852945 & -1.4838125 & -1.90195031 \\
\hline H & 3.68821981 & -2.47283159 & -2.26379808 \\
\hline H & 4.17316619 & -0.78098064 & -2.18716025 \\
\hline H & 2.4607094 & -1.20010509 & -2.4053355 \\
\hline C & 2.46208716 & 2.67028072 & 0.51202016 \\
\hline H & 2.64260967 & 2.55576558 & 1.5820399 \\
\hline H & 1.38189852 & 2.65518021 & 0.34625908 \\
\hline H & 2.84780992 & 3.64475961 & 0.19646889 \\
\hline c & 2.89314383 & 1.84766855 & -1.78383225 \\
\hline H & 3.45648378 & 1.17847009 & -2.4332839 \\
\hline H & 3.20276092 & 2.87211757 & -2.01559734 \\
\hline H & 1.83513956 & 1.73418307 & -2.02711898 \\
\hline c & 4.64967913 & 1.60586699 & -0.01225923 \\
\hline H & 4.87250064 & 1.34821182 & 1.02599563 \\
\hline H & 5.03031185 & 2.61720116 & -0.19047022 \\
\hline H & 5.20507573 & 0.93113496 & -0.66515283 \\
\hline C & -4.54507268 & -1.73431252 & 0.39780705 \\
\hline H & -4.9911963 & -2.68132722 & 0.07641443 \\
\hline H & -5.27189583 & -0.9465812 & 0.20512349 \\
\hline H & -4.38181594 & -1.79819696 & 1.47492839 \\
\hline C & -2.300821 & -2.69683384 & -0.016499 \\
\hline H & -2.05107379 & -2.71497387 & 1.04680144 \\
\hline H & -1.36752567 & -2.63385085 & -0.57856035 \\
\hline H & -2.80282745 & -3.63728467 & -0.26703091 \\
\hline C & -3.47771456 & -1.51021356 & -1.85288135 \\
\hline H & -2.55311202 & -1.34034532 & -2.41017822 \\
\hline H & -4.20731175 & -0.75328264 & -2.1445847 \\
\hline H & -3.87107837 & -2.48302031 & -2.16481985 \\
\hline c & -2.50255151 & 2.68766549 & 0.51101759 \\
\hline H & -2.76205019 & 2.58879199 & 1.56584332 \\
\hline H & -2.85648719 & 3.66043003 & 0.15498919 \\
\hline H & -1.41301848 & 2.66384636 & 0.42591109 \\
\hline c & -4.6487102 & 1.5986994 & -0.12078616 \\
\hline H & -5.16691135 & 0.91705366 & -0.79702967 \\
\hline H & -5.03104839 & 2.60585765 & -0.31768584 \\
\hline H & -4.91734035 & 1.34005834 & 0.90594921 \\
\hline c & -2.81004266 & 1.86764623 & -1.79980911 \\
\hline H & -1.72926769 & 1.91631752 & -1.94920308 \\
\hline H & -3.23452528 & 2.83596579 & -2.08410865 \\
\hline H & -3.22176964 & 1.11909104 & -2.4779125 \\
\hline
\end{tabular}

\begin{tabular}{|c|c|c|c|}
\hline \multicolumn{4}{|c|}{$\begin{array}{l}\text { Table D23: xyz data of } 4^{3} \mathrm{~A}^{\prime \prime}(\mathrm{CS}) \\
65\end{array}$} \\
\hline $\mathrm{Ir}$ & 0.02633205 & -0.13117178 & 0.00000000 \\
\hline$P$ & 0.00711979 & 0.21834134 & 2.30776158 \\
\hline$P$ & 0.00711978 & 0.21834134 & -2.30776158 \\
\hline$N$ & 0.06152214 & 1.93385832 & 0.00000000 \\
\hline 0 & 0.04840650 & -1.92176986 & 0.00000000 \\
\hline c & 0.07869579 & 2.61848419 & 1.17753100 \\
\hline H & 0.10714185 & 3.70688614 & 1.11154177 \\
\hline c & 0.07869579 & 2.61848419 & -1.17753100 \\
\hline H & 0.10714185 & 3.70688614 & -1.11154177 \\
\hline c & 0.06088827 & 1.99839362 & 2.37724022 \\
\hline $\mathrm{H}$ & 0.06645750 & 2.57056079 & 3.29510959 \\
\hline c & 0.06088827 & 1.99839362 & -2.37724022 \\
\hline H & 0.06645750 & 2.57056079 & -3.29510959 \\
\hline C & 1.53382645 & -0.37630266 & -3.20971778 \\
\hline c & -1.58964223 & -0.31661762 & -3.12621707 \\
\hline c & 1.53382646 & -0.37630266 & 3.20971778 \\
\hline c & -1.58964223 & -0.31661762 & 3.12621707 \\
\hline c & 2.70111592 & -0.03099016 & -2.28275917 \\
\hline $\mathrm{H}$ & 3.63950392 & -0.29788287 & -2.77971093 \\
\hline $\mathrm{H}$ & 2.63288571 & -0.57846451 & -1.34149980 \\
\hline H & 2.72689414 & 1.03680522 & -2.05325920 \\
\hline C & 1.75140731 & 0.33226195 & -4.54286929 \\
\hline H & 1.82939099 & 1.41297188 & -4.40947496 \\
\hline $\mathrm{H}$ & 0.96182569 & 0.12948958 & -5.26511887 \\
\hline H & 2.69416610 & -0.01494437 & -4.97794622 \\
\hline c & 1.48425612 & -1.88790418 & -3.40111353 \\
\hline H & 2.47070968 & -2.23989327 & -3.71923596 \\
\hline $\mathrm{H}$ & 0.76966282 & -2.17806838 & -4.17307850 \\
\hline H & 1.22527863 & -2.40009520 & -2.47113324 \\
\hline c & -2.68125844 & 0.50579025 & -2.43925859 \\
\hline H & -2.57603566 & 1.57205919 & -2.64461094 \\
\hline H & -2.65403032 & 0.36336402 & -1.35604568 \\
\hline $\mathrm{H}$ & -3.65700724 & 0.17417979 & -2.80744396 \\
\hline C & -1.85229122 & -1.79361766 & -2.83931720 \\
\hline $\mathrm{H}$ & -1.16001080 & -2.45034421 & -3.36480584 \\
\hline H & -2.86430781 & -2.04286699 & -3.17433747 \\
\hline $\mathrm{H}$ & -1.77527790 & -2.00904384 & -1.77219247 \\
\hline C & -1.62758370 & -0.05053581 & -4.62564705 \\
\hline $\mathrm{H}$ & -1.37583513 & 0.98512601 & -4.86613419 \\
\hline H & -2.64039143 & -0.23875863 & -4.99657525 \\
\hline $\mathrm{H}$ & -0.95349928 & -0.70961327 & -5.17452696 \\
\hline c & 1.75140731 & 0.33226195 & 4.54286928 \\
\hline H & 2.69416610 & -0.01494437 & 4.97794622 \\
\hline $\mathrm{H}$ & 0.96182570 & 0.12948958 & 5.26511887 \\
\hline H & 1.82939099 & 1.41297188 & 4.40947496 \\
\hline C & 2.70111592 & -0.03099016 & 2.28275917 \\
\hline H & 2.72689414 & 1.03680522 & 2.05325919 \\
\hline H & 2.63288571 & -0.57846451 & 1.34149980 \\
\hline H & 3.63950392 & -0.29788287 & 2.77971092 \\
\hline c & 1.48425612 & -1.88790418 & 3.40111352 \\
\hline $\mathrm{H}$ & 1.22527863 & -2.40009520 & 2.47113324 \\
\hline H & 0.76966283 & -2.17806838 & 4.17307850 \\
\hline $\mathrm{H}$ & 2.47070968 & -2.23989327 & 3.71923596 \\
\hline C & -2.68125844 & 0.50579025 & 2.43925859 \\
\hline $\mathrm{H}$ & -2.57603566 & 1.57205919 & 2.64461095 \\
\hline $\mathrm{H}$ & -3.65700724 & 0.17417979 & 2.80744397 \\
\hline $\mathrm{H}$ & -2.65403032 & 0.36336402 & 1.35604568 \\
\hline c & -1.62758370 & -0.05053581 & 4.62564705 \\
\hline $\mathrm{H}$ & -0.95349927 & -0.70961327 & 5.17452697 \\
\hline $\mathrm{H}$ & -2.64039142 & -0.23875863 & 4.99657525 \\
\hline $\mathrm{H}$ & -1.37583513 & 0.98512601 & 4.86613419 \\
\hline C & -1.85229122 & -1.79361766 & 2.83931720 \\
\hline H & -1.77527790 & -2.00904384 & 1.77219248 \\
\hline $\mathrm{H}$ & -2.86430781 & -2.042866999 & 3.17433747 \\
\hline $\mathrm{H}$ & -1.16001080 & -2.45034421 & 3.36480584 \\
\hline
\end{tabular}




\begin{tabular}{|c|c|c|c|}
\hline \multicolumn{4}{|c|}{$\begin{array}{l}\text { Table D24: xyz data of } 2^{\text {Rh }{ }^{2}} \mathrm{~A}(\mathrm{C} 1) \\
66\end{array}$} \\
\hline \multicolumn{4}{|c|}{$\mathrm{E}(\mathrm{UPBE} 1 \mathrm{PBE}-\mathrm{D} 3(\mathrm{BJ}) / \mathrm{def} 2 \mathrm{TZVP})=-1709.22482819<\mathrm{S} 2\rangle=0.7565$} \\
\hline $\mathrm{Rh}$ & 0.01066531 & -0.00734290 & -0.08429858 \\
\hline$P$ & -2.29792350 & 0.00264047 & 0.19142356 \\
\hline $\mathrm{P}$ & 2.31295934 & -0.00827354 & 0.20588853 \\
\hline $\mathrm{N}$ & 0.00274389 & -0.04154497 & 1.91346215 \\
\hline 0 & 0.14781297 & -0.05855629 & -2.02644840 \\
\hline $\mathrm{H}$ & -0.71033575 & -0.05556378 & -2.45368214 \\
\hline C & -1.17829159 & -0.05218371 & 2.59816098 \\
\hline H & -1.11267499 & -0.07415581 & 3.68723849 \\
\hline c & 1.18274642 & -0.07467451 & 2.60555075 \\
\hline H & 1.10802327 & -0.10509022 & 3.69384974 \\
\hline c & -2.37617918 & -0.03630517 & 1.97784526 \\
\hline H & -3.29309611 & -0.03449620 & 2.55251100 \\
\hline c & 2.38397129 & -0.07004200 & 1.99346935 \\
\hline H & 3.29630148 & -0.08905556 & 2.57546442 \\
\hline c & 3.20110137 & -1.54317406 & -0.38458149 \\
\hline c & 3.16434072 & 1.57842224 & -0.30385442 \\
\hline c & -3.23131895 & -1.52863888 & -0.35677434 \\
\hline c & -3.14933796 & 1.58761022 & -0.33418096 \\
\hline c & 2.24299466 & -2.69087891 & -0.05779703 \\
\hline $\mathrm{H}$ & 2.71754221 & -3.64039056 & -0.32677095 \\
\hline $\mathrm{H}$ & 1.31159608 & -2.59419820 & -0.61845649 \\
\hline H & 1.99992610 & -2.71964268 & 1.00714802 \\
\hline c & 4.52431168 & -1.79599013 & 0.32879029 \\
\hline H & 4.38376188 & -1.88291944 & 1.40810466 \\
\hline $\mathrm{H}$ & 5.26141018 & -1.01641173 & 0.13946895 \\
\hline H & 4.94565851 & -2.74238290 & -0.02643772 \\
\hline c & 3.39793452 & -1.48450548 & -1.89600319 \\
\hline H & 3.68808957 & -2.47663920 & -2.25719492 \\
\hline H & 4.19307116 & -0.79098194 & -2.17478764 \\
\hline $\mathrm{H}$ & 2.47627444 & -1.18895386 & -2.40380774 \\
\hline c & 2.45405816 & 2.67370013 & 0.49410915 \\
\hline $\mathrm{H}$ & 2.61235795 & 2.56219200 & 1.56794385 \\
\hline H & 1.37725053 & 2.65901092 & 0.30648235 \\
\hline H & 2.84392330 & 3.64810010 & 0.18365669 \\
\hline c & 2.91630378 & 1.83499157 & -1.79000317 \\
\hline $\mathrm{H}$ & 3.50529863 & 1.17576524 & -2.42668123 \\
\hline H & 3.20459431 & 2.86505052 & -2.02438400 \\
\hline $\mathrm{H}$ & 1.86466140 & 1.69337357 & -2.04759999 \\
\hline C & 4.65480428 & 1.61682073 & 0.00530796 \\
\hline H & 4.86377874 & 1.37586219 & 1.05044239 \\
\hline H & 5.03682525 & 2.62560786 & -0.18443178 \\
\hline H & 5.21913165 & 0.93177876 & -0.62900648 \\
\hline c & -4.53073384 & -1.75471646 & 0.40923646 \\
\hline H & -4.97806403 & -2.69886624 & 0.08106562 \\
\hline $\mathrm{H}$ & -5.26398584 & -0.96690190 & 0.24085888 \\
\hline H & -4.34821371 & -1.83317123 & 1.48224241 \\
\hline c & -2.27910723 & -2.68648756 & -0.05011588 \\
\hline $\mathrm{H}$ & -2.00549340 & -2.70600836 & 1.00736870 \\
\hline H & -1.36050196 & -2.60942956 & -0.63480288 \\
\hline $\mathrm{H}$ & -2.77258095 & -3.63315484 & -0.29376894 \\
\hline c & -3.50322716 & -1.50034745 & -1.85606959 \\
\hline H & -2.59152057 & -1.31127616 & -2.42794665 \\
\hline H & -4.24881522 & -0.75166292 & -2.12789190 \\
\hline H & -3.88809844 & -2.47597413 & -2.16976589 \\
\hline C & -2.49379893 & 2.68719823 & 0.50263331 \\
\hline $\mathrm{H}$ & -2.73519372 & 2.58758396 & 1.56159665 \\
\hline H & -2.85016118 & 3.66145022 & 0.15343086 \\
\hline H & -1.40554823 & 2.66315614 & 0.40011320 \\
\hline c & -4.65349366 & 1.60544206 & -0.09890098 \\
\hline H & -5.18135790 & 0.91883515 & -0.76260238 \\
\hline $\mathrm{H}$ & -5.03817123 & 2.61137343 & -0.29776361 \\
\hline H & -4.90743022 & 1.35395565 & 0.93336630 \\
\hline c & -2.83483251 & 1.86533270 & -1.80312325 \\
\hline H & -1.75572238 & 1.89619133 & -1.96978839 \\
\hline H & -3.24816442 & 2.84033095 & -2.08095856 \\
\hline H & -3.26788516 & 1.12347782 & -2.47487209 \\
\hline
\end{tabular}

\begin{tabular}{|c|c|c|c|}
\hline \multicolumn{4}{|c|}{$\begin{array}{l}\text { Table D25: } x y z \text { data of } 4^{\text {Rh }}{ }^{1} A^{\prime}(C s) \\
65 \\
\text { E(RPBE1PBE-D3(BJ)/def2TZVP) }=-1708.54535146\end{array}$} \\
\hline $\mathrm{Rh}$ & -0.01703227 & -0.08947148 & 0.00000000 \\
\hline$P$ & -0.00764073 & 0.17240218 & 2.32490995 \\
\hline$P$ & -0.00764073 & 0.17240218 & -2.32490995 \\
\hline$N$ & -0.21012262 & 1.87961986 & 0.00000000 \\
\hline 0 & 1.05652181 & -1.50891653 & 0.00000000 \\
\hline$c$ & -0.19252410 & 2.57070464 & 1.18716642 \\
\hline $\mathrm{H}$ & -0.25417345 & 3.65726099 & 1.11332862 \\
\hline$c$ & -0.19252410 & 2.57070464 & -1.18716642 \\
\hline $\mathrm{H}$ & -0.25417345 & 3.65726099 & -1.11332862 \\
\hline$c$ & -0.10967381 & 1.96172322 & 2.38499758 \\
\hline $\mathrm{H}$ & -0.10917465 & 2.54859652 & 3.29454071 \\
\hline C & -0.10967381 & 1.96172322 & -2.38499758 \\
\hline $\mathrm{H}$ & -0.10917465 & 2.54859652 & -3.29454071 \\
\hline$c$ & 1.57170542 & -0.26931554 & -3.21411563 \\
\hline$c$ & -1.55153083 & -0.468855813 & -3.16683529 \\
\hline$c$ & 1.57170542 & -0.26931554 & 3.21411563 \\
\hline C & -1.55153083 & -0.468858813 & 3.16683529 \\
\hline$c$ & 2.68678747 & 0.19398984 & -2.27221037 \\
\hline $\mathrm{H}$ & 3.65230391 & 0.01857166 & -2.75789608 \\
\hline $\mathrm{H}$ & 2.65518504 & -0.36514775 & -1.33644133 \\
\hline $\mathrm{H}$ & 2.60819702 & 1.26135336 & -2.05183159 \\
\hline$c$ & 1.73629535 & 0.43601767 & -4.55538743 \\
\hline $\mathrm{H}$ & 1.70609531 & 1.52184792 & -4.44303077 \\
\hline $\mathrm{H}$ & 0.98233479 & 0.14358271 & -5.28527000 \\
\hline $\mathrm{H}$ & 2.71590697 & 0.17683191 & -4.97052001 \\
\hline$c$ & 1.66626628 & -1.78300951 & -3.37407109 \\
\hline $\mathrm{H}$ & 2.67899735 & -2.04244054 & -3.69933391 \\
\hline $\mathrm{H}$ & 0.97485760 & -2.15919035 & -4.13047242 \\
\hline $\mathrm{H}$ & 1.47526447 & -2.28218415 & -2.42090168 \\
\hline$c$ & -2.70973663 & 0.27029426 & -2.49459025 \\
\hline $\mathrm{H}$ & -2.69037350 & 1.33948189 & -2.70925418 \\
\hline $\mathrm{H}$ & -2.68560768 & 0.13987223 & -1.40872741 \\
\hline $\mathrm{H}$ & -3.65538673 & -0.14095316 & -2.86107764 \\
\hline$c$ & -1.70075161 & -1.95908118 & -2.86432203 \\
\hline $\mathrm{H}$ & -0.93430704 & -2.56243806 & -3.34891869 \\
\hline $\mathrm{H}$ & -2.67545767 & -2.30162625 & -3.22691931 \\
\hline $\mathrm{H}$ & -1.64460634 & -2.14721047 & -1.79010839 \\
\hline$c$ & -1.60261155 & -0.22344015 & -4.66883297 \\
\hline $\mathrm{H}$ & -1.42830152 & 0.82567813 & -4.91871344 \\
\hline $\mathrm{H}$ & -2.59497829 & -0.49324943 & -5.04557425 \\
\hline $\mathrm{H}$ & -0.87443449 & -0.83334133 & -5.20505122 \\
\hline$c$ & 1.73629535 & 0.43601767 & 4.55538743 \\
\hline $\mathrm{H}$ & 2.71590697 & 0.17683191 & 4.97052001 \\
\hline $\mathrm{H}$ & 0.98233479 & 0.14358271 & 5.28527000 \\
\hline $\mathrm{H}$ & 1.70609531 & 1.52184792 & 4.44303077 \\
\hline C & 2.68678747 & 0.19398984 & 2.27221037 \\
\hline $\mathrm{H}$ & 2.60819702 & 1.26135336 & 2.05183159 \\
\hline $\mathrm{H}$ & 2.65518504 & -0.36514775 & 1.33644133 \\
\hline $\mathrm{H}$ & 3.65230391 & 0.01857166 & 2.75789608 \\
\hline$c$ & 1.66626628 & -1.78300951 & 3.37407109 \\
\hline $\mathrm{H}$ & 1.47526447 & -2.28218415 & 2.42090168 \\
\hline $\mathrm{H}$ & 0.97485760 & -2.15919035 & 4.13047242 \\
\hline $\mathrm{H}$ & 2.67899735 & -2.04244054 & 3.69933391 \\
\hline$c$ & -2.70973663 & 0.27029426 & 2.49459025 \\
\hline $\mathrm{H}$ & -2.69037350 & 1.33948189 & 2.70925418 \\
\hline $\mathrm{H}$ & -3.65538673 & -0.14095316 & 2.86107764 \\
\hline $\mathrm{H}$ & -2.68560768 & 0.13987223 & 1.40872741 \\
\hline$c$ & -1.60261155 & -0.22344015 & 4.66883297 \\
\hline $\mathrm{H}$ & -0.87443449 & -0.83334133 & 5.20505122 \\
\hline $\mathrm{H}$ & -2.59497829 & -0.49324943 & 5.04557425 \\
\hline $\mathrm{H}$ & -1.42830152 & 0.82567813 & 4.91871344 \\
\hline C & -1.70075161 & -1.95908118 & 2.86432203 \\
\hline $\mathrm{H}$ & -1.64460634 & -2.14721047 & 1.79010839 \\
\hline $\mathrm{H}$ & -2.67545767 & -2.30162625 & 3.22691931 \\
\hline $\mathrm{H}$ & -0.93430704 & -2.56243806 & 3.34891869 \\
\hline
\end{tabular}




\begin{tabular}{|c|c|c|c|}
\hline \multicolumn{4}{|c|}{$\begin{array}{l}\text { Table D26: xyz data of } 4^{\text {Rh }}{ }^{3} A^{\prime \prime}(C s) \\
65\end{array}$} \\
\hline $\mathrm{Rh}$ & 0.02314609 & -0.12941801 & 0.00000000 \\
\hline$P$ & 0.00437260 & 0.21457979 & 2.31442203 \\
\hline $\mathrm{P}$ & 0.00437260 & 0.21457979 & -2.31442203 \\
\hline $\mathrm{N}$ & 0.06065934 & 1.93299156 & 0.00000000 \\
\hline 0 & 0.04395996 & -1.90111941 & 0.00000000 \\
\hline c & 0.07777974 & 2.61381779 & 1.17687560 \\
\hline $\mathrm{H}$ & 0.10717228 & 3.70359891 & 1.11504975 \\
\hline C & 0.07777974 & 2.61381779 & -1.17687560 \\
\hline H & 0.10717228 & 3.70359891 & -1.11504975 \\
\hline c & 0.05912497 & 1.99524269 & 2.37870189 \\
\hline H & 0.06572700 & 2.57113249 & 3.29474268 \\
\hline C & 0.05912497 & 1.99524269 & -2.37870189 \\
\hline H & 0.06572700 & 2.57113249 & -3.29474268 \\
\hline c & 1.53424085 & -0.37661951 & -3.21126819 \\
\hline C & -1.59070167 & -0.31697914 & -3.13552024 \\
\hline c & 1.53424085 & -0.37661951 & 3.21126819 \\
\hline C & -1.59070167 & -0.31697914 & 3.13552024 \\
\hline c & 2.69080898 & -0.04594221 & -2.26553939 \\
\hline H & 3.63603316 & -0.30590837 & -2.75306033 \\
\hline $\mathrm{H}$ & 2.61122273 & -0.60962678 & -1.33434077 \\
\hline H & 2.71417926 & 1.01828587 & -2.01951572 \\
\hline C & 1.76734164 & 0.34529213 & -4.53434997 \\
\hline $\mathrm{H}$ & 1.85051707 & 1.42386707 & -4.38788570 \\
\hline H & 0.98032351 & 0.15578621 & -5.26339852 \\
\hline H & 2.71052751 & -0.00303788 & -4.96781100 \\
\hline C & 1.48272887 & -1.88569858 & -3.41972135 \\
\hline H & 2.47090535 & -2.23699993 & -3.73338773 \\
\hline H & 0.77386360 & -2.16549674 & -4.20074371 \\
\hline H & 1.21457903 & -2.40709937 & -2.49754354 \\
\hline C & -2.68155670 & 0.49464491 & -2.43481364 \\
\hline H & -2.57713423 & 1.56384786 & -2.62441587 \\
\hline H & -2.65361365 & 0.33717657 & -1.35344699 \\
\hline H & -3.65841337 & 0.16785391 & -2.80429729 \\
\hline C & -1.84749774 & -1.79751742 & -2.86147639 \\
\hline H & -1.15905458 & -2.44697339 & -3.40080295 \\
\hline H & -2.86224618 & -2.04660255 & -3.18834225 \\
\hline H & -1.75860808 & -2.02361839 & -1.79719612 \\
\hline C & -1.63407740 & -0.03500669 & -4.63183424 \\
\hline H & -1.39187321 & 1.00568284 & -4.86001668 \\
\hline H & -2.64509453 & -0.22876961 & -5.00507686 \\
\hline H & -0.95327594 & -0.68093065 & -5.18819293 \\
\hline C & 1.76734164 & 0.34529213 & 4.53434997 \\
\hline $\mathrm{H}$ & 2.71052751 & -0.00303788 & 4.96781100 \\
\hline H & 0.98032351 & 0.15578621 & 5.26339852 \\
\hline H & 1.85051707 & 1.42386707 & 4.38788570 \\
\hline C & 2.69080898 & -0.04594221 & 2.26553939 \\
\hline H & 2.71417926 & 1.01828587 & 2.01951572 \\
\hline H & 2.61122273 & -0.60962678 & 1.33434077 \\
\hline H & 3.63603316 & -0.30590837 & 2.75306033 \\
\hline c & 1.48272887 & -1.88569858 & 3.41972135 \\
\hline $\mathrm{H}$ & 1.21457903 & -2.40709937 & 2.49754354 \\
\hline H & 0.77386360 & -2.16549674 & 4.20074371 \\
\hline H & 2.47090535 & -2.23699993 & 3.73338773 \\
\hline C & -2.68155670 & 0.49464491 & 2.43481364 \\
\hline H & -2.57713423 & 1.56384786 & 2.62441587 \\
\hline $\mathrm{H}$ & -3.65841337 & 0.16785391 & 2.80429729 \\
\hline H & -2.65361365 & 0.33717657 & 1.35344699 \\
\hline C & -1.63407740 & -0.03500669 & 4.63183424 \\
\hline H & -0.95327594 & -0.68093065 & 5.18819293 \\
\hline H & -2.64509453 & -0.22876961 & 5.00507686 \\
\hline H & -1.39187321 & 1.00568284 & 4.86001668 \\
\hline C & -1.84749774 & -1.79751742 & 2.86147639 \\
\hline H & -1.75860808 & -2.02361839 & 1.79719612 \\
\hline H & -2.86224618 & -2.04660255 & 3.18834225 \\
\hline H & -1.15905458 & -2.44697339 & 3.40080295 \\
\hline
\end{tabular}

\begin{tabular}{|c|c|c|c|}
\hline \multirow{2}{*}{\multicolumn{4}{|c|}{$\begin{array}{l}\text { Table D27: xyz data of } 23^{1} A^{\prime}(\mathrm{Cs}) \\
60 \\
\text { E(RPBE1PBE-D3/def2TZVP) }=-2827.41310991\end{array}$}} \\
\hline & & & \\
\hline$\overline{R e}$ & 0.0712099339 & 0.0993327626 & 0.0000000000 \\
\hline N & -0.4835944919 & -2.0345140444 & 0.0000000000 \\
\hline H & 0.4546104888 & -2.4324177636 & 0.0000000000 \\
\hline$P$ & -0.0081740653 & -0.2040317720 & 2.3556383962 \\
\hline$P$ & -0.0081740650 & -0.2040317720 & -2.3556383962 \\
\hline $\mathrm{Cl}$ & 2.2472752190 & -0.8835734151 & 0.0000000001 \\
\hline $\mathrm{Cl}$ & 0.7157954063 & 2.3274540658 & 0.0000000000 \\
\hline $\mathrm{Cl}$ & -2.27368883148 & 0.3526098012 & -0.0000000001 \\
\hline c & -1.1538318070 & -2.4730308099 & 1.2372963843 \\
\hline H & -1.2686422175 & -3.5636545064 & 1.2272087523 \\
\hline H & -2.1504860613 & -2.0280046091 & 1.2391510049 \\
\hline c & -0.3517171037 & -2.0249634660 & 2.4458991827 \\
\hline $\mathrm{H}$ & 0.6210762168 & -2.5274699021 & 2.4478958381 \\
\hline H & -0.8650809148 & -2.3106702014 & 3.3662790600 \\
\hline c & -1.1538318069 & -2.4730308099 & -1.2372963845 \\
\hline H & -2.1504860612 & -2.0280046091 & -1.2391510051 \\
\hline H & -1.2686422174 & -3.5636545064 & -1.2272087524 \\
\hline c & -0.3517171034 & -2.0249634660 & -2.4458991827 \\
\hline H & -0.8650809144 & -2.3106702014 & -3.3662790601 \\
\hline H & 0.6210762171 & -2.5274699021 & -2.4478958380 \\
\hline c & -1.4298311667 & 0.5394540725 & -3.2751480389 \\
\hline H & -2.2764915156 & 0.0948826268 & -2.7371846125 \\
\hline c & -1.5101049312 & 2.0457140323 & -3.0552043706 \\
\hline H & -1.4261742824 & 2.3020615089 & -1.9995762144 \\
\hline H & -2.46885534184 & 2.4202841494 & -3.4247798361 \\
\hline $\mathrm{H}$ & -0.7197850388 & 2.5719579563 & -3.5932025939 \\
\hline c & -1.5512588003 & 0.1884680125 & -4.7499554095 \\
\hline $\mathrm{H}$ & -0.8059985905 & 0.7201987101 & -5.3445347296 \\
\hline H & -2.5350916257 & 0.4914220083 & -5.1204229591 \\
\hline H & -1.4403531298 & -0.8796693612 & -4.9478239943 \\
\hline c & 1.5562623364 & -0.0331336541 & -3.3217599514 \\
\hline H & 2.2692276374 & -0.4590531637 & -2.6050067895 \\
\hline c & 1.6719934493 & -0.8137357654 & -4.6235401875 \\
\hline $\mathrm{H}$ & 2.7079443785 & -0.7792645060 & -4.9741706011 \\
\hline H & 1.0483476546 & -0.3921853598 & -5.4120999845 \\
\hline H & 1.4039538465 & -1.8658203207 & -4.5088209481 \\
\hline c & 1.9190287146 & 1.4350368530 & -3.5068796048 \\
\hline H & 1.8053252365 & 1.9992625987 & -2.5790058803 \\
\hline H & 1.2966494284 & 1.9036044627 & -4.2730235420 \\
\hline H & 2.9589525450 & 1.5215994387 & -3.8334961317 \\
\hline c & 1.5562623361 & -0.0331336541 & 3.3217599516 \\
\hline H & 2.2692276371 & -0.4590531637 & 2.6050067897 \\
\hline c & 1.6719934488 & -0.8137357654 & 4.6235401877 \\
\hline $\mathrm{H}$ & 2.7079443780 & -0.7792645060 & 4.9741706014 \\
\hline H & 1.4039538461 & -1.8658203207 & 4.5088209482 \\
\hline H & 1.0483476540 & -0.3921853598 & 5.4120999846 \\
\hline c & 1.9190287142 & 1.4350368530 & 3.5068796050 \\
\hline H & 1.2966494280 & 1.9036044627 & 4.2730235421 \\
\hline H & 1.8053252362 & 1.9992625987 & 2.5790058805 \\
\hline H & 2.9589525446 & 1.5215994387 & 3.8334961320 \\
\hline c & -1.4298311671 & 0.5394540725 & 3.2751480387 \\
\hline H & -2.2764915159 & 0.0948826268 & 2.7371846123 \\
\hline c & -1.5101049315 & 2.0457140323 & 3.0552043705 \\
\hline H & -1.4261742826 & 2.3020615089 & 1.9995762143 \\
\hline H & -0.7197850392 & 2.5719579563 & 3.5932025938 \\
\hline H & -2.46885534187 & 2.4202841494 & 3.4247798359 \\
\hline c & -1.5512588008 & 0.1884680125 & 4.7499554094 \\
\hline H & -1.4403531303 & -0.8796693612 & 4.9478239941 \\
\hline H & -2.5350916262 & 0.4914220083 & 5.1204229589 \\
\hline H & -0.8059985911 & 0.7201987101 & 5.3445347295 \\
\hline
\end{tabular}




\begin{tabular}{|c|c|c|c|}
\hline \multirow{2}{*}{\multicolumn{4}{|c|}{$\begin{array}{l}\text { Table D28: } x y z \text { data of } 23 m e{ }^{1}{ }^{\prime}(\mathrm{Cs})^{i} \mathrm{Pr}: \mathrm{Me} \\
36 \\
\text { E(RPBE1PBE-D3/def2TZVP) }=-2513.16332935\end{array}$}} \\
\hline & & & \\
\hline$\overline{R e}$ & -0.0712099339 & -0.0993327626 & 0.0000000000 \\
\hline $\mathrm{N}$ & 0.4835000000 & 2.0345140444 & 0.0000000000 \\
\hline H & -0.4546104889 & 2.4324177636 & 0.0000000000 \\
\hline$P$ & 0.0081740651 & 0.2040317720 & 2.3556383962 \\
\hline $\mathrm{P}$ & 0.0081740651 & 0.2040317720 & -2.3556383962 \\
\hline $\mathrm{Cl}$ & 0.0000000000 & 0.8835734150 & 0.0000000000 \\
\hline $\mathrm{Cl}$ & -0.7157954062 & -2.3274540659 & 0.0000000000 \\
\hline $\mathrm{Cl}$ & 2.2730000000 & -0.3526098011 & 0.0000000000 \\
\hline c & 1.1538318068 & 2.4730308099 & 1.2372963844 \\
\hline $\mathrm{H}$ & 1.0000000000 & 3.5636545064 & 1.2272087524 \\
\hline H & 2.1504860612 & 2.0280046093 & 1.2390000000 \\
\hline c & 0.3517171035 & 2.0249634660 & 2.4458991827 \\
\hline H & -0.6210762171 & 2.5274600000 & 2.4478958381 \\
\hline $\mathrm{H}$ & 0.8650809145 & 2.3106702014 & 3.3662790601 \\
\hline c & 1.1538310000 & 2.4730308099 & -1.2372963844 \\
\hline H & 2.1504860612 & 2.0280046093 & -1.2391510000 \\
\hline $\mathrm{H}$ & 1.2686422173 & 3.5636545064 & -1.2272087524 \\
\hline c & 0.3517171035 & 2.0249634600 & -2.4458991827 \\
\hline H & 0.8650809145 & 2.3106702014 & -3.3662790601 \\
\hline H & -0.6210762000 & 2.5274699021 & -2.4478958381 \\
\hline c & 1.4298311669 & -0.5394540724 & -3.2751480000 \\
\hline H & 2.2764915158 & -0.0948826266 & -2.7371846124 \\
\hline H & 1.4871795380 & -1.6155410000 & -3.1180181075 \\
\hline $\mathrm{H}$ & 1.5168955349 & -0.2877948861 & -4.3325940890 \\
\hline c & -1.5562000000 & 0.0331336541 & -3.3217599515 \\
\hline H & -2.2692276373 & 0.4590531636 & -2.6050000000 \\
\hline H & -1.6391550982 & 0.5922422929 & -4.2541640090 \\
\hline $\mathrm{H}$ & -1.8156575466 & -1.0100000000 & -3.4541293063 \\
\hline c & -1.5562623363 & 0.0331336541 & 3.3217599515 \\
\hline H & -2.0000000000 & 0.4590531636 & 2.6050067896 \\
\hline H & -1.6391550982 & 0.5922422929 & 4.2500000000 \\
\hline H & -1.8156575466 & -1.0166780719 & 3.4541293063 \\
\hline c & 1.4298311669 & -0.5000000000 & 3.2751480388 \\
\hline H & 2.2764915158 & -0.0948826266 & 2.7371846124 \\
\hline H & 1.4000000000 & -1.6155410809 & 3.1180181075 \\
\hline H & 1.5168955349 & -0.2877948861 & 4.3320000000 \\
\hline
\end{tabular}

\begin{tabular}{|c|c|c|c|}
\hline \multirow{2}{*}{\multicolumn{4}{|c|}{$\begin{array}{l}\text { Table D29: xyz data of } 23 \mathrm{~h}^{1} \mathrm{~A}^{\prime}(\mathrm{CS})^{i} \mathrm{Pr}: \mathrm{H} \\
24 \\
\text { E(RPBE1PBE-D3/def2TZVP) }=-2355.98342861\end{array}$}} \\
\hline & & & \\
\hline $\operatorname{Re}$ & -0.0712099339 & -0.0993327626 & 0.0000000000 \\
\hline $\mathrm{N}$ & 0.4835944918 & 2.0345140444 & 0.0000000000 \\
\hline $\mathrm{H}$ & -0.4546104889 & 2.4324177636 & 0.0000000000 \\
\hline $\mathrm{P}$ & 0.0081740651 & 0.2040317720 & 2.3556383962 \\
\hline $\mathrm{P}$ & 0.0081740651 & 0.2040317720 & -2.3556383962 \\
\hline $\mathrm{Cl}$ & -2.2472752191 & 0.8835734150 & 0.0000000000 \\
\hline $\mathrm{Cl}$ & -0.7157954062 & -2.3274540659 & 0.0000000000 \\
\hline $\mathrm{Cl}$ & 2.2736883148 & -0.3526098011 & 0.0000000000 \\
\hline$C$ & 1.1538318068 & 2.4730308099 & 1.2372963844 \\
\hline $\mathrm{H}$ & 1.2686422173 & 3.5636545064 & 1.2272087524 \\
\hline $\mathrm{H}$ & 2.1504860612 & 2.0280046093 & 1.2391510050 \\
\hline C & 0.3517171035 & 2.0249634660 & 2.4458991827 \\
\hline $\mathrm{H}$ & -0.6210762171 & 2.5274699021 & 2.4478958381 \\
\hline $\mathrm{H}$ & 0.8650809145 & 2.3106702014 & 3.3662790601 \\
\hline C & 1.1538318068 & 2.4730308099 & -1.2372963844 \\
\hline $\mathrm{H}$ & 2.1504860612 & 2.0280046093 & -1.2391510050 \\
\hline $\mathrm{H}$ & 1.2686422173 & 3.5636545064 & -1.2272087524 \\
\hline$C$ & 0.3517171035 & 2.0249634660 & -2.4458991827 \\
\hline $\mathrm{H}$ & 0.8650809145 & 2.3106702014 & -3.3662790601 \\
\hline $\mathrm{H}$ & -0.6210762171 & 2.5274699021 & -2.4478958381 \\
\hline $\mathrm{H}$ & 1.0935208897 & -0.3635734539 & -3.0576268500 \\
\hline $\mathrm{H}$ & -1.1867332670 & 0.0735007936 & -3.0935563641 \\
\hline $\mathrm{H}$ & -1.1867332670 & 0.0735007936 & 3.0935563641 \\
\hline H & 1.0935208897 & -0.3635734539 & 3.0576268500 \\
\hline
\end{tabular}

\begin{tabular}{|c|c|c|c|}
\hline \multicolumn{4}{|c|}{$\begin{array}{l}\text { Table D30: xyz data of } 24^{2} \mathrm{~A}^{\prime \prime}(\mathrm{CS}) \\
59 \\
\text { E(UPBE1PBE-D3/def2TZVP) }=-2826.81473043\end{array}$} \\
\hline $\operatorname{Re}$ & 0.0693461503 & -0.0293170956 & 0.0000000000 \\
\hline $\mathrm{N}$ & -0.9160798892 & -1.6770430334 & 0.0000000000 \\
\hline$P$ & -0.0710102583 & -0.2674440395 & 2.4128075904 \\
\hline$P$ & -0.0710102584 & -0.2674440395 & -2.4128075904 \\
\hline $\mathrm{Cl}$ & 2.2164075338 & -0.9132762085 & 0.0000000000 \\
\hline $\mathrm{Cl}$ & 1.0388343672 & 2.2007696546 & 0.0000000000 \\
\hline $\mathrm{Cl}$ & -2.0480505281 & 1.0418173021 & 0.0000000000 \\
\hline c & -1.5439642112 & -2.2197861965 & 1.1991777525 \\
\hline $\mathrm{H}$ & -1.7260928306 & -3.2925256097 & 1.0644324505 \\
\hline $\mathrm{H}$ & -2.5317840998 & -1.7442934728 & 1.3103698529 \\
\hline c & -0.7017541916 & -1.9897007298 & 2.4398115614 \\
\hline H & 0.1801029203 & -2.6358410937 & 2.4035102925 \\
\hline H & -1.2628921822 & -2.2348950823 & 3.3433484474 \\
\hline c & -1.5439642113 & -2.2197861965 & -1.1991777524 \\
\hline H & -2.5317840998 & -1.7442934728 & -1.3103698528 \\
\hline H & -1.7260928307 & -3.2925256097 & -1.0644324505 \\
\hline c & -0.7017541917 & -1.9897007298 & -2.4398115614 \\
\hline $\mathrm{H}$ & -1.2628921823 & -2.2348950823 & -3.3433484474 \\
\hline $\mathrm{H}$ & 0.1801029202 & -2.6358410937 & -2.4035102925 \\
\hline c & -1.3412185901 & 0.7443975252 & -3.2806278059 \\
\hline H & -2.2181438057 & 0.5421052407 & -2.6532903474 \\
\hline c & -1.0400967016 & 2.2345107666 & -3.1635607742 \\
\hline H & -0.7461220966 & 2.5118339062 & -2.1508119586 \\
\hline H & -1.9309668413 & 2.8078654293 & -3.4329281140 \\
\hline H & -0.2397160945 & 2.5296654302 & -3.8443041681 \\
\hline c & -1.6642129340 & 0.3467482308 & -4.7131513772 \\
\hline H & -0.8767814895 & 0.6600208925 & -5.4001326686 \\
\hline H & -2.5847270318 & 0.8465292864 & -5.0278977057 \\
\hline H & -1.8153318590 & -0.7272428820 & -4.8381067459 \\
\hline c & 1.5197945631 & -0.3518173764 & -3.3352178649 \\
\hline $\mathrm{H}$ & 2.0964668163 & -0.9962458705 & -2.6608967759 \\
\hline C & 1.4604793953 & -1.0194740551 & -4.7015230365 \\
\hline H & 2.4782080555 & -1.2167444884 & -5.0503241070 \\
\hline $\mathrm{H}$ & 0.9808200096 & -0.3813923998 & -5.4441372179 \\
\hline H & 0.9300931023 & -1.9738274297 & -4.6810065302 \\
\hline c & 2.2201599054 & 0.9996357684 & -3.3800948037 \\
\hline H & 2.2104615327 & 1.4927867634 & -2.4059664185 \\
\hline H & 1.7507290972 & 1.6655789683 & -4.1069149080 \\
\hline H & 3.2603795665 & 0.8610997469 & -3.6863452472 \\
\hline c & 1.5197945632 & -0.3518173764 & 3.3352178649 \\
\hline H & 2.0964668164 & -0.9962458705 & 2.6608967758 \\
\hline c & 1.4604793955 & -1.0194740551 & 4.7015230365 \\
\hline H & 2.4782080556 & -1.2167444884 & 5.0503241069 \\
\hline H & 0.9300931024 & -1.9738274297 & 4.6810065302 \\
\hline H & 0.9808200097 & -0.3813923998 & 5.4441372178 \\
\hline c & 2.2201599055 & 0.9996357684 & 3.3800948036 \\
\hline $\mathrm{H}$ & 1.7507290973 & 1.6655789683 & 4.1069149079 \\
\hline H & 2.2104615328 & 1.4927867634 & 2.4059664184 \\
\hline $\mathrm{H}$ & 3.2603795666 & 0.8610997469 & 3.6863452471 \\
\hline c & -1.3412185900 & 0.7443975252 & 3.2806278059 \\
\hline $\mathrm{H}$ & -2.2181438057 & 0.5421052407 & 2.6532903474 \\
\hline c & -1.0400967015 & 2.2345107666 & 3.1635607742 \\
\hline $\mathrm{H}$ & -0.7461220965 & 2.5118339062 & 2.1508119586 \\
\hline $\mathrm{H}$ & -0.2397160944 & 2.5296654302 & 3.8443041682 \\
\hline H & -1.9309668412 & 2.8078654293 & 3.4329281140 \\
\hline c & -1.6642129339 & 0.3467482308 & 4.7131513772 \\
\hline $\mathrm{H}$ & -1.8153318589 & -0.7272428820 & 4.8381067459 \\
\hline $\mathrm{H}$ & -2.5847270316 & 0.8465292864 & 5.0278977058 \\
\hline $\mathrm{H}$ & -0.8767814893 & 0.6600208925 & 5.4001326687 \\
\hline
\end{tabular}




\begin{tabular}{|c|c|c|c|}
\hline \multicolumn{4}{|c|}{$\begin{array}{l}\text { Table D31: xyz data of } 24 \mathrm{me}^{2} \mathrm{~A}^{\prime \prime}(\mathrm{Cs})^{\mathrm{i}} \mathrm{Pr}: \mathrm{Me} \\
35 \\
\text { E(UPBE1PBE-D3/def2TZVP) }=-2512.56087673\end{array}$} \\
\hline $\operatorname{Re}$ & 0.0693461503 & -0.0293170956 & 0.0000000000 \\
\hline $\mathrm{N}$ & -0.9000000000 & -1.6770430334 & 0.0000000000 \\
\hline$P$ & -0.0710102583 & -0.2674440395 & 2.4128075904 \\
\hline $\mathrm{P}$ & -0.0710102583 & -0.2674440395 & -2.4128075904 \\
\hline $\mathrm{Cl}$ & 2.2164075338 & -0.9132762000 & 0.0000000000 \\
\hline $\mathrm{Cl}$ & 1.0388343672 & 2.2007696546 & 0.0000000000 \\
\hline $\mathrm{Cl}$ & -2.0480505282 & 1.0418173021 & 0.0000000000 \\
\hline c & -1.5439642112 & -2.2197861965 & 1.1991777524 \\
\hline H & -1.7260928306 & -3.2925250000 & 1.0644324505 \\
\hline $\mathrm{H}$ & -2.5317840998 & -1.7442934728 & 1.3103698528 \\
\hline c & -0.7017000000 & -1.9897007298 & 2.4398115614 \\
\hline H & 0.1801029203 & -2.6358410936 & 2.4035100000 \\
\hline H & -1.2628921822 & -2.2348950824 & 3.3433484474 \\
\hline c & -1.5439642112 & -2.2100000000 & -1.1991777524 \\
\hline H & -2.5317840998 & -1.7442934728 & -1.3103698528 \\
\hline $\mathrm{H}$ & 0.0000000000 & -3.2925256097 & -1.0644324505 \\
\hline c & -0.7017541916 & -1.9897007298 & -2.4398115614 \\
\hline H & -1.2628921822 & -2.2348950824 & -3.3433484474 \\
\hline H & 0.1801029000 & -2.6358410936 & -2.4035102925 \\
\hline c & -1.3412185901 & 0.7443975252 & -3.2806270000 \\
\hline H & -2.2181438057 & 0.5421052407 & -2.6532903474 \\
\hline H & -1.1261767633 & 1.8085000000 & -3.1970260841 \\
\hline $\mathrm{H}$ & -1.5726407636 & 0.4594858364 & -4.3070164245 \\
\hline c & 1.5100000000 & -0.3518173764 & -3.3352178649 \\
\hline H & 2.0964668163 & -0.9962458705 & -2.6000000000 \\
\hline H & 1.4773117532 & -0.8300075737 & -4.3137952693 \\
\hline H & 2.0205297026 & 0.0000000000 & -3.3673032050 \\
\hline c & 1.5197945631 & -0.3518173764 & 3.3352178649 \\
\hline $\mathrm{H}$ & 2.0000000000 & -0.9962458705 & 2.6608967758 \\
\hline H & 1.4773117532 & -0.8300075737 & 4.3000000000 \\
\hline $\mathrm{H}$ & 2.0205297026 & 0.6144212967 & 3.3673032050 \\
\hline c & -1.3412185901 & 0.7400000000 & 3.2806278059 \\
\hline $\mathrm{H}$ & -2.2181438057 & 0.5421052407 & 2.6532903474 \\
\hline $\mathrm{H}$ & -1.1000000000 & 1.8085402722 & 3.1970260841 \\
\hline $\mathrm{H}$ & -1.5726407636 & 0.4594858364 & 4.3070000000 \\
\hline
\end{tabular}

\begin{tabular}{|c|c|c|c|}
\hline \multicolumn{4}{|c|}{$\begin{array}{l}\text { Table D32: xyz data of } 24 \mathrm{~h}^{2} \mathrm{~A}^{\prime \prime}(\mathrm{Cs})^{i} \mathrm{Pr}: \mathrm{H} \\
23 \\
\text { E(UPBE1PBE-D3/def2TZVP) }=-2355.37397073\end{array}$} \\
\hline $\operatorname{Re}$ & 0.06934615 & -0.029317096 & 0.0000000001 \\
\hline $\mathrm{N}$ & -0.916079889 & -1.677043033 & -0.0000000007 \\
\hline $\mathrm{P}$ & -0.071010258 & -0.26744404 & 2.4128075904 \\
\hline $\mathrm{P}$ & -0.071010257 & -0.267444038 & -2.4128075903 \\
\hline $\mathrm{Cl}$ & 2.216407534 & -0.913276208 & 0.0000000003 \\
\hline $\mathrm{Cl}$ & 1.038834367 & 2.200769655 & 0.0000000012 \\
\hline $\mathrm{Cl}$ & -2.048050528 & 1.041817302 & 0.0000000001 \\
\hline c & -1.543964211 & -2.219786197 & 1.1991777524 \\
\hline $\mathrm{H}$ & -1.726092831 & -3.29252561 & 1.0644324505 \\
\hline $\mathrm{H}$ & -2.5317841 & -1.744293473 & 1.3103698528 \\
\hline c & -0.701754192 & -1.98970073 & 2.4398115614 \\
\hline $\mathrm{H}$ & 0.18010292 & -2.635841094 & 2.4035102925 \\
\hline H & -1.262892182 & -2.234895082 & 3.3433484474 \\
\hline c & -1.543964211 & -2.219786196 & -1.1991777546 \\
\hline $\mathrm{H}$ & -2.531784099 & -1.744293472 & -1.3103698551 \\
\hline $\mathrm{H}$ & -1.72609283 & -3.292525609 & -1.0644324536 \\
\hline c & -0.701754191 & -1.989700728 & -2.4398115630 \\
\hline H & -1.262892181 & -2.23489508 & -3.3433484495 \\
\hline $\mathrm{H}$ & 0.180102921 & -2.635841092 & -2.4035102942 \\
\hline $\mathrm{H}$ & -1.0415911 & 0.505715772 & -3.0759190296 \\
\hline H & 1.143053293 & -0.331835717 & -3.1167686783 \\
\hline H & 1.143053292 & -0.331835719 & 3.1167686789 \\
\hline $\mathrm{H}$ & -1.041591102 & 0.50571577 & 3.0759190298 \\
\hline
\end{tabular}

\begin{tabular}{|c|c|c|c|}
\hline \multicolumn{4}{|c|}{$\begin{array}{l}\text { Table D33: xyz data of } 24{ }^{4} A^{\prime}(C s) \\
59 \\
2 \text { E(UPBE1PBE-D3/def2TZVP) }=-2826.80890737\end{array}$} \\
\hline $\operatorname{Re}$ & -0.053732088 & -0.0712042708 & 0.000000 \\
\hline N & 0.814242484 & 1.7804058554 & 0.000000 \\
\hline$P$ & 0.048660198 & 0.3187911341 & 2.449927392 \\
\hline$P$ & 0.048660198 & 0.3187911341 & -2.449927392 \\
\hline $\mathrm{Cl}$ & -2.235360674 & 0.8328209074 & 0.000000 \\
\hline $\mathrm{Cl}$ & -0.961389261 & -2.2549093001 & 0.000000 \\
\hline $\mathrm{Cl}$ & 2.130707878 & -0.9734141439 & 0.000000 \\
\hline c & 1.488489849 & 2.2393779783 & 1.189813455 \\
\hline H & 1.733086415 & 3.3060103638 & 1.085863501 \\
\hline $\mathrm{H}$ & 2.451378687 & 1.7168451069 & 1.340331503 \\
\hline c & 0.606556101 & 2.0539349347 & 2.41338002 \\
\hline H & -0.29390501 & 2.6650461208 & 2.305295213 \\
\hline $\mathrm{H}$ & 1.118168811 & 2.3460261308 & 3.332463499 \\
\hline c & 1.488489849 & 2.2393779783 & -1.189813455 \\
\hline H & 2.451378687 & 1.7168451069 & -1.340331503 \\
\hline H & 1.733086415 & 3.3060103638 & -1.085863501 \\
\hline c & 0.606556101 & 2.0539349347 & -2.41338002 \\
\hline H & 1.118168811 & 2.3460261308 & -3.332463499 \\
\hline H & -0.29390501 & 2.6650461208 & -2.305295213 \\
\hline c & 1.362443061 & -0.6307107397 & -3.320344343 \\
\hline H & 2.233304655 & -0.3728456877 & -2.704843431 \\
\hline c & 1.155069953 & -2.1361815815 & -3.204676914 \\
\hline H & 0.94197959 & -2.4406659916 & -2.180089127 \\
\hline H & 2.06282785 & -2.6506673058 & -3.530205112 \\
\hline H & 0.336887842 & -2.4751514185 & -3.842139791 \\
\hline c & 1.632427492 & -0.2106859711 & -4.757957224 \\
\hline H & 0.848539827 & -0.5652392070 & -5.429037635 \\
\hline H & 2.572160987 & -0.6582524752 & -5.092924837 \\
\hline H & 1.721463566 & 0.8703759897 & -4.879277524 \\
\hline c & -1.559859727 & 0.3299917651 & -3.337270122 \\
\hline H & -2.160581822 & 0.9135765633 & -2.629361175 \\
\hline c & -1.552559783 & 1.0533720093 & -4.676902059 \\
\hline H & -2.583806199 & 1.1983403824 & -5.010552042 \\
\hline H & -1.038618198 & 0.4782189877 & -5.447648584 \\
\hline H & -1.085203449 & 2.0383068306 & -4.618597231 \\
\hline c & -2.18283122 & -1.0556983530 & -3.429755076 \\
\hline H & -2.159580715 & -1.5745629736 & -2.469947024 \\
\hline H & -1.673432071 & -1.6740356286 & -4.171614252 \\
\hline H & -3.226525982 & -0.9630818314 & -3.740736355 \\
\hline c & -1.559859727 & 0.3299917651 & 3.337270122 \\
\hline H & -2.160581822 & 0.9135765633 & 2.629361175 \\
\hline c & -1.552559783 & 1.0533720093 & 4.676902059 \\
\hline H & -2.583806199 & 1.1983403824 & 5.010552042 \\
\hline H & -1.085203449 & 2.0383068306 & 4.618597231 \\
\hline H & -1.038618198 & 0.4782189877 & 5.447648584 \\
\hline c & -2.18283122 & -1.0556983530 & 3.429755076 \\
\hline H & -1.673432071 & -1.6740356286 & 4.171614252 \\
\hline H & -2.159580715 & -1.5745629736 & 2.469947024 \\
\hline H & -3.226525982 & -0.9630818314 & 3.740736355 \\
\hline c & 1.362443061 & -0.6307107397 & 3.320344343 \\
\hline $\mathrm{H}$ & 2.233304655 & -0.3728456877 & 2.704843431 \\
\hline c & 1.155069953 & -2.1361815815 & 3.204676914 \\
\hline $\mathrm{H}$ & 0.94197959 & -2.4406659916 & 2.180089127 \\
\hline H & 0.336887842 & -2.4751514185 & 3.842139791 \\
\hline H & 2.06282785 & -2.6506673058 & 3.530205112 \\
\hline c & 1.632427492 & -0.2106859711 & 4.757957224 \\
\hline H & 1.721463566 & 0.8703759897 & 4.879277524 \\
\hline H & 2.572160987 & -0.6582524752 & 5.092924837 \\
\hline H & 0.848539827 & -0.5652392070 & 5.429037635 \\
\hline
\end{tabular}




\begin{tabular}{|c|c|c|c|}
\hline \multicolumn{4}{|c|}{$\begin{array}{l}\text { Table D34: xyz data of } 24 \mathrm{me}^{4} \mathrm{~A}^{\prime}(\mathrm{Cs}){ }^{\mathrm{i}} \mathrm{Pr}: \mathrm{Me} \\
35 \\
\text { E(UPBE1PBE-D3(BJ)/def2TZVP) }=-2512.55509575\end{array}$} \\
\hline $\mathrm{Re}$ & -0.053732088 & -0.071204271 & 0.0000000000 \\
\hline $\mathrm{N}$ & 0.80000000 & 1.780405855 & 0.0000000000 \\
\hline$P$ & 0.048660198 & 0.318791134 & 2.4499273922 \\
\hline$P$ & 0.00000000 & 0.318791134 & -2.4499273922 \\
\hline $\mathrm{Cl}$ & -2.235360674 & 0.832820907 & 0.0000000000 \\
\hline $\mathrm{Cl}$ & -0.961389261 & -2.2549093 & 0.0000000000 \\
\hline $\mathrm{Cl}$ & 2.130707878 & -0.973414144 & 0.00000000000 \\
\hline C & 1.488489849 & 2.239377978 & 1.1898134551 \\
\hline H & 1.733086415 & 3.306010364 & 1.0000000000 \\
\hline $\mathrm{H}$ & 2.451378687 & 1.716845107 & 1.3403315033 \\
\hline c & 0.606556101 & 2.00000000 & 2.4133800202 \\
\hline H & -0.29390501 & 2.665046121 & 2.3052952126 \\
\hline H & 1.100000000 & 2.346026131 & 3.3324634993 \\
\hline c & 1.488489849 & 2.239377978 & -1.1890000000 \\
\hline H & 2.451378687 & 1.716845107 & -1.3403315033 \\
\hline H & 1.733086415 & 3.30600000 & -1.0858635009 \\
\hline C & 0.606556101 & 2.053934935 & -2.4133800202 \\
\hline H & 1.11000000 & 2.346026131 & -3.3324634993 \\
\hline H & -0.29390501 & 2.665046121 & -2.3000000000 \\
\hline C & 1.362443061 & -0.63071074 & -3.3203443431 \\
\hline H & 2.233304655 & 0.00000000 & -2.7048434313 \\
\hline H & 1.214332674 & -1.705950778 & -3.2377321477 \\
\hline H & 1.555822219 & -0.329863629 & -4.3500495272 \\
\hline c & -1.559859727 & 0.329991765 & -3.3372701223 \\
\hline $\mathrm{H}$ & -2.160581822 & 0.913576563 & -2.6293611747 \\
\hline H & -1.554633973 & 0.847832323 & -4.2962619589 \\
\hline H & -2.005389918 & -0.661011499 & -3.4034125300 \\
\hline C & -1.559859727 & 0.329991765 & 3.3372701223 \\
\hline H & -2.160581822 & 0.91357656 & 2.6293611747 \\
\hline $\mathrm{H}$ & -1.554633973 & 0.847832323 & 4.2962619589 \\
\hline H & -2.0053899 & -0.661011499 & 3.4034125374 \\
\hline c & 1.362443061 & -0.63071074 & 3.3203443400 \\
\hline H & 2.233304655 & -0.372845688 & 2.7048434313 \\
\hline $\mathrm{H}$ & 1.214332674 & -1.7059507 & 3.2377321477 \\
\hline H & 1.555822219 & -0.329863629 & 4.3500495272 \\
\hline
\end{tabular}

\begin{tabular}{|c|c|c|c|}
\hline \multicolumn{4}{|c|}{$\begin{array}{l}\text { Table D35: xyz data of } 24 h^{4} A^{\prime}(C S)^{i} P r: H \\
23 \\
\text { E(UPBE1PBE-D3/def2TZVP) }=-2355.36392902\end{array}$} \\
\hline $\operatorname{Re}$ & -0.053732088 & -0.071204271 & 0.0000000000 \\
\hline $\mathrm{N}$ & 0.814242484 & 1.780405855 & 0.0000000000 \\
\hline$P$ & 0.048660198 & 0.318791134 & 2.4499273922 \\
\hline $\mathrm{P}$ & 0.048660198 & 0.318791134 & -2.4499273922 \\
\hline $\mathrm{Cl}$ & -2.235360674 & 0.832820907 & 0.0000000000 \\
\hline $\mathrm{Cl}$ & -0.961389261 & -2.2549093 & 0.0000000000 \\
\hline $\mathrm{Cl}$ & 2.130707878 & -0.973414144 & 0.0000000000 \\
\hline c & 1.488489849 & 2.239377978 & 1.1898134551 \\
\hline $\mathrm{H}$ & 1.733086415 & 3.306010364 & 1.0858635009 \\
\hline $\mathrm{H}$ & 2.451378687 & 1.716845107 & 1.3403315033 \\
\hline c & 0.606556101 & 2.053934935 & 2.4133800202 \\
\hline H & -0.29390501 & 2.665046121 & 2.3052952126 \\
\hline H & 1.118168811 & 2.346026131 & 3.3324634993 \\
\hline c & 1.488489849 & 2.239377978 & -1.1898134551 \\
\hline $\mathrm{H}$ & 2.451378687 & 1.716845107 & -1.3403315033 \\
\hline $\mathrm{H}$ & 1.733086415 & 3.306010364 & -1.0858635009 \\
\hline c & 0.606556101 & 2.053934935 & -2.4133800202 \\
\hline H & 1.118168811 & 2.346026131 & -3.3324634993 \\
\hline $\mathrm{H}$ & -0.29390501 & 2.665046121 & -2.3052952126 \\
\hline $\mathrm{H}$ & 1.051853461 & -0.406240307 & -3.1145702682 \\
\hline H & -1.180001271 & 0.327346691 & -3.1277205643 \\
\hline H & -1.180001271 & 0.327346691 & 3.1277205643 \\
\hline $\mathrm{H}$ & 1.051853461 & -0.406240307 & 3.1145702682 \\
\hline
\end{tabular}

\begin{tabular}{|c|c|c|c|}
\hline \multicolumn{4}{|c|}{$\begin{array}{l}\text { Table D36: xyz data of } 25^{2} A^{\prime \prime}(\mathrm{Cs}) \\
60\end{array}$} \\
\hline $\operatorname{Re}$ & 0.18584665 & -0.08508213 & 0.00000000 \\
\hline $\mathrm{Cl}$ & -1.51019687 & 1.47589730 & 0.00000000 \\
\hline $\mathrm{Cl}$ & 1.30483117 & -2.12602689 & 0.00000000 \\
\hline $\mathrm{Cl}$ & 2.05692248 & 1.15245699 & 0.00000000 \\
\hline $\mathrm{P}$ & -0.17700374 & -0.24907186 & 2.42350039 \\
\hline$P$ & -0.17700374 & -0.24907186 & -2.42350039 \\
\hline $\mathrm{N}$ & -1.57453606 & -1.46201071 & 0.00000000 \\
\hline H & -1.09462209 & -2.36044101 & 0.00000000 \\
\hline c & -2.39477084 & -1.40365121 & 1.22965459 \\
\hline H & -3.13103535 & -2.21324922 & 1.20824442 \\
\hline H & -2.93945928 & -0.45850273 & 1.21534955 \\
\hline c & -1.51185065 & -1.51567008 & 2.45594203 \\
\hline H & -1.02018490 & -2.49301576 & 2.47350518 \\
\hline H & -2.11331500 & -1.44254516 & 3.36367692 \\
\hline c & 1.21537321 & -1.01692782 & 3.35793478 \\
\hline H & 1.56365444 & -1.76703256 & 2.63881215 \\
\hline c & 2.35046337 & -0.02942960 & 3.59621834 \\
\hline H & 2.62376691 & 0.51825631 & 2.69326570 \\
\hline H & 3.23367828 & -0.57368756 & 3.93702833 \\
\hline $\mathrm{H}$ & 2.09285445 & 0.69120753 & 4.37415673 \\
\hline c & 0.81666090 & -1.73605395 & 4.64129782 \\
\hline H & 0.50876761 & -1.04208490 & 5.42231446 \\
\hline H & 1.68378045 & -2.28574276 & 5.01559168 \\
\hline H & 0.01539391 & -2.46136471 & 4.49334622 \\
\hline c & -0.87449375 & 1.22177698 & 3.27934666 \\
\hline $\mathrm{H}$ & -1.82048098 & 1.36190864 & 2.74218586 \\
\hline c & -1.18431168 & 1.02057949 & 4.75675111 \\
\hline H & -1.78580276 & 1.85979701 & 5.11365032 \\
\hline $\mathrm{H}$ & -0.27159017 & 1.00037962 & 5.35365563 \\
\hline H & -1.74536215 & 0.10673271 & 4.95990202 \\
\hline c & -0.02797250 & 2.46952339 & 3.05036531 \\
\hline H & 0.19410048 & 2.62804610 & 1.99543121 \\
\hline $\mathrm{H}$ & 0.91596538 & 2.41979285 & 3.59340445 \\
\hline $\mathrm{H}$ & -0.57121452 & 3.34420397 & 3.41451650 \\
\hline c & -2.39477084 & -1.40365121 & -1.22965459 \\
\hline H & -2.93945928 & -0.45850273 & -1.21534955 \\
\hline H & -3.13103535 & -2.21324922 & -1.20824442 \\
\hline c & -1.51185065 & -1.51567008 & -2.45594203 \\
\hline H & -2.11331500 & -1.44254516 & -3.36367692 \\
\hline H & -1.02018490 & -2.49301576 & -2.47350518 \\
\hline c & 1.21537321 & -1.01692782 & -3.35793478 \\
\hline H & 1.56365444 & -1.76703256 & -2.63881215 \\
\hline c & 0.81666090 & -1.73605395 & -4.64129782 \\
\hline H & 0.01539391 & -2.46136471 & -4.49334622 \\
\hline H & 1.68378045 & -2.28574276 & -5.01559168 \\
\hline H & 0.50876761 & -1.04208490 & -5.42231446 \\
\hline c & 2.35046337 & -0.02942960 & -3.59621834 \\
\hline H & 2.09285445 & 0.69120753 & -4.37415673 \\
\hline H & 3.23367828 & -0.57368756 & -3.93702833 \\
\hline H & 2.62376691 & 0.51825631 & -2.69326570 \\
\hline c & -0.87449375 & 1.22177698 & -3.27934666 \\
\hline H & -1.82048098 & 1.36190864 & -2.74218586 \\
\hline c & -1.18431168 & 1.02057949 & -4.75675111 \\
\hline H & -1.78580276 & 1.85979701 & -5.11365032 \\
\hline H & -1.74536215 & 0.10673271 & -4.95990202 \\
\hline H & -0.27159017 & 1.00037962 & -5.35365563 \\
\hline c & -0.02797250 & 2.46952339 & -3.05036531 \\
\hline H & -0.57121452 & 3.34420397 & -3.41451650 \\
\hline H & 0.91596538 & 2.41979285 & -3.59340445 \\
\hline $\mathrm{H}$ & 0.19410048 & 2.62804610 & -1.99543121 \\
\hline
\end{tabular}




\begin{tabular}{|c|c|c|c|}
\hline \multicolumn{4}{|c|}{$\begin{array}{l}\text { Table D37: xyz data of } 25^{4} \mathrm{~A}^{\prime}(\mathrm{Cs}) \\
60\end{array}$} \\
\hline $\operatorname{Re}$ & 0.12657891 & 0.02482185 & 0.00000000 \\
\hline $\mathrm{Cl}$ & -1.49596587 & 1.69286923 & 0.00000000 \\
\hline $\mathrm{Cl}$ & 1.45985395 & -1.89645793 & 0.00000000 \\
\hline $\mathrm{Cl}$ & 1.88759281 & 1.46641479 & 0.00000000 \\
\hline P & -0.18461113 & -0.26361071 & 2.46657692 \\
\hline $\mathrm{P}$ & -0.18461113 & -0.26361071 & -2.46657692 \\
\hline $\mathrm{N}$ & -1.56159607 & -1.41565764 & 0.00000000 \\
\hline H & -1.06105706 & -2.30589646 & 0.00000000 \\
\hline c & -2.38756079 & -1.36651916 & 1.22514238 \\
\hline $\mathrm{H}$ & -3.14409235 & -2.15651420 & 1.18317985 \\
\hline $\mathrm{H}$ & -2.90501811 & -0.40564854 & 1.22772344 \\
\hline c & -1.51475934 & -1.52867208 & 2.45271914 \\
\hline H & -1.02845401 & -2.50913472 & 2.44547749 \\
\hline $\mathrm{H}$ & -2.12051287 & -1.47686532 & 3.35889307 \\
\hline c & 1.21901696 & -1.04330184 & 3.35933543 \\
\hline $\mathrm{H}$ & 1.51231979 & -1.81909842 & 2.64241011 \\
\hline c & 2.39364311 & -0.08665797 & 3.51832966 \\
\hline $\mathrm{H}$ & 2.64630849 & 0.41833339 & 2.58478234 \\
\hline $\mathrm{H}$ & 3.27100296 & -0.64980702 & 3.84280501 \\
\hline $\mathrm{H}$ & 2.19177159 & 0.66881513 & 4.27930813 \\
\hline c & 0.84601481 & -1.71898102 & 4.67331597 \\
\hline $\mathrm{H}$ & 0.61673322 & -0.99587639 & 5.45496349 \\
\hline $\mathrm{H}$ & 1.69893522 & -2.31013686 & 5.01496373 \\
\hline $\mathrm{H}$ & -0.00149226 & -2.39992632 & 4.57793841 \\
\hline c & -0.8905514 & 1.20360815 & 3.32192310 \\
\hline H & -1.81501521 & 1.35827577 & 2.75153163 \\
\hline c & -1.24579721 & 0.97184717 & 4.78471378 \\
\hline $\mathrm{H}$ & -1.86499952 & 1.80156085 & 5.13326136 \\
\hline $\mathrm{H}$ & -0.35254014 & 0.95079510 & 5.40985287 \\
\hline H & -1.80614627 & 0.05129103 & 4.95712895 \\
\hline c & -0.02770836 & 2.44829827 & 3.14712264 \\
\hline $\mathrm{H}$ & 0.22013214 & 2.63770135 & 2.10358091 \\
\hline $\mathrm{H}$ & 0.90110268 & 2.37476910 & 3.71312524 \\
\hline $\mathrm{H}$ & -0.57569156 & 3.31397984 & 3.52513801 \\
\hline c & -2.38756079 & -1.36651916 & -1.22514238 \\
\hline $\mathrm{H}$ & -2.90501811 & -0.40564854 & -1.22772344 \\
\hline $\mathrm{H}$ & -3.14409235 & -2.15651420 & -1.18317985 \\
\hline c & -1.51475934 & -1.52867208 & -2.45271914 \\
\hline H & -2.12051287 & -1.47686532 & -3.35889307 \\
\hline $\mathrm{H}$ & -1.02845401 & -2.50913472 & -2.44547749 \\
\hline c & 1.21901696 & -1.04330184 & -3.35933543 \\
\hline $\mathrm{H}$ & 1.51231979 & -1.81909842 & -2.64241011 \\
\hline c & 0.84601481 & -1.71898102 & -4.67331597 \\
\hline $\mathrm{H}$ & -0.00149226 & -2.39992632 & -4.57793841 \\
\hline $\mathrm{H}$ & 1.69893522 & -2.31013686 & -5.01496373 \\
\hline $\mathrm{H}$ & 0.61673322 & -0.99587639 & -5.45496349 \\
\hline c & 2.39364311 & -0.08665797 & -3.51832966 \\
\hline H & 2.19177159 & 0.66881513 & -4.27930813 \\
\hline $\mathrm{H}$ & 3.27100296 & -0.64980702 & -3.84280501 \\
\hline H & 2.64630849 & 0.41833339 & -2.58478234 \\
\hline c & -0.8905514 & 1.20360815 & -3.3219231 \\
\hline H & -1.81501521 & 1.35827577 & -2.75153163 \\
\hline C & -1.24579721 & 0.97184717 & -4.78471378 \\
\hline H & -1.86499952 & 1.80156085 & -5.13326136 \\
\hline $\mathrm{H}$ & -1.80614627 & 0.05129103 & -4.95712895 \\
\hline $\mathrm{H}$ & -0.35254014 & 0.9507951 & -5.40985287 \\
\hline c & -0.02770836 & 2.44829827 & -3.14712264 \\
\hline $\mathrm{H}$ & -0.57569156 & 3.31397984 & -3.52513801 \\
\hline $\mathrm{H}$ & 0.90110268 & 2.3747691 & -3.71312524 \\
\hline $\mathrm{H}$ & 0.22013214 & 2.63770135 & -2.10358091 \\
\hline
\end{tabular}

\begin{tabular}{|c|c|c|c|}
\hline \multicolumn{4}{|c|}{ Table D38: xyz data of Mes* $\mathrm{OH}^{1} \mathrm{~A}^{\prime}$} \\
\hline \multicolumn{4}{|l|}{49} \\
\hline \multicolumn{4}{|c|}{$\mathrm{E}(\mathrm{RPBE} 1 \mathrm{PBE}-\mathrm{D} 3(\mathrm{BJ}) / \mathrm{def} 2 \mathrm{TZVP})=-778.610534501$} \\
\hline C & 0.400141343 & 1.179146088 & 0.0000000000 \\
\hline c & -0.479041371 & -1.472189579 & 0.0000000000 \\
\hline c & 1.346321971 & 0.145607972 & 0.0000000000 \\
\hline c & -0.981242207 & 0.919160338 & 0.0000000000 \\
\hline c & -1.375696921 & -0.409493935 & 0.0000000000 \\
\hline c & 0.869921685 & -1.164360287 & 0.0000000000 \\
\hline H & -2.43379699 & -0.630371379 & 0.0000000000 \\
\hline H & 1.585876745 & -1.971129708 & 0.0000000000 \\
\hline 0 & 0.776456973 & 2.489925836 & 0.0000000000 \\
\hline $\mathrm{H}$ & 1.733502267 & 2.540362506 & 0.0000000000 \\
\hline c & -1.002531314 & -2.903740563 & 0.0000000000 \\
\hline c & -2.014268089 & 2.047660817 & 0.0000000000 \\
\hline c & 2.854840941 & 0.422291827 & 0.0000000000 \\
\hline c & 0.12471686 & -3.930794234 & 0.0000000000 \\
\hline H & 0.757464507 & -3.836922237 & 0.8861648427 \\
\hline H & 0.757464507 & -3.836922237 & -0.8861648427 \\
\hline H & -0.297866518 & -4.938418234 & 0.0000000000 \\
\hline c & -1.859431541 & -3.132883953 & 1.2489793930 \\
\hline H & -1.269740553 & -2.980780461 & 2.1561873738 \\
\hline H & -2.251085034 & -4.154124349 & 1.2611462515 \\
\hline H & -2.708862505 & -2.447912926 & 1.2841632459 \\
\hline c & -1.859431541 & -3.132883953 & -1.2489793930 \\
\hline H & -2.708862505 & -2.447912926 & -1.2841632459 \\
\hline H & -2.251085034 & -4.154124349 & -1.2611462515 \\
\hline H & -1.269740553 & -2.980780461 & -2.1561873738 \\
\hline c & -1.859380187 & 2.910952052 & -1.2583540554 \\
\hline H & -0.87877795 & 3.379292939 & -1.3110643798 \\
\hline $\mathrm{H}$ & -2.617977528 & 3.698941357 & -1.2610180659 \\
\hline H & -2.000947028 & 2.303837997 & -2.1562557636 \\
\hline c & -1.859380187 & 2.910952052 & 1.2583540554 \\
\hline H & -0.87877795 & 3.379292939 & 1.3110643798 \\
\hline H & -2.000947028 & 2.303837997 & 2.1562557636 \\
\hline $\mathrm{H}$ & -2.617977528 & 3.698941357 & 1.2610180659 \\
\hline c & -3.443025872 & 1.50669775 & 0.0000000000 \\
\hline H & -3.653812098 & 0.90438773 & 0.8871960443 \\
\hline $\mathrm{H}$ & -3.653812098 & 0.90438773 & -0.8871960443 \\
\hline H & -4.139387254 & 2.348373926 & 0.0000000000 \\
\hline c & 3.273883959 & 1.183435262 & 1.2688256594 \\
\hline H & 3.037225744 & 0.58804765 & 2.1531052569 \\
\hline H & 2.785871714 & 2.150611525 & 1.4079785828 \\
\hline $\mathrm{H}$ & 4.352175496 & 1.363020039 & 1.2567342207 \\
\hline c & 3.273883959 & 1.183435262 & -1.2688256594 \\
\hline H & 2.785871714 & 2.150611525 & -1.4079785828 \\
\hline H & 3.037225744 & 0.58804765 & -2.1531052569 \\
\hline H & 4.352175496 & 1.363020039 & -1.2567342207 \\
\hline c & 3.669766159 & -0.871239181 & 0.0000000000 \\
\hline H & 3.470964543 & -1.477246193 & 0.8863288491 \\
\hline H & 4.733485993 & -0.622195841 & 0.0000000000 \\
\hline $\mathrm{H}$ & 3.470964543 & -1.477246193 & -0.8863288491 \\
\hline
\end{tabular}




\begin{tabular}{|c|c|c|c|}
\hline $\begin{array}{l}\text { Tak } \\
22\end{array}$ & 9: xyz data of & $\mathrm{u}: \mathrm{Me}{ }^{1} \mathrm{~A}^{\prime}$ & \\
\hline \multicolumn{4}{|c|}{$E(R P B E 1 P B E-D 3(B J) /$ def2TZVP) $=-425.088872333$} \\
\hline $\mathrm{C}$ & 0.40068800 & 1.179294 & 0.0000000000 \\
\hline $\mathrm{C}$ & -0.47847200 & -1.472049 & 0.0000000000 \\
\hline C & 1.34687700 & 0.145764 & 0.0000000000 \\
\hline C & -0.98069400 & 0.919297 & 0.0000000000 \\
\hline c & -1.37513700 & -0.409361 & 0.0000000000 \\
\hline C & 0.87048800 & -1.164208 & 0.0000000000 \\
\hline $\mathrm{H}$ & -2.43323500 & -0.630247 & 0.0000000000 \\
\hline $\mathrm{H}$ & 1.5864500 & -1.970971 & 0.0000000000 \\
\hline 0 & 0.77699200 & 2.490077 & 0.0000000000 \\
\hline $\mathrm{H}$ & 1.73403700 & 2.540522 & 0.0000000000 \\
\hline C & -1.00195000 & -2.903604 & 0.0000000000 \\
\hline $\mathrm{H}$ & -0.195829105 & -3.638060853 & 0.0000000000 \\
\hline $\mathrm{H}$ & -1.613024011 & -3.067016939 & 0.8906761453 \\
\hline $\mathrm{H}$ & -1.613024011 & -3.067016939 & -0.8906761453 \\
\hline C & -2.013729 & 2.047789 & 0.0000000000 \\
\hline $\mathrm{H}$ & -1.903469516 & 2.662364901 & -0.8958197733 \\
\hline $\mathrm{H}$ & -1.903469516 & 2.662364901 & 0.8958197732 \\
\hline $\mathrm{H}$ & -3.033853625 & 1.661534036 & 0.0000000000 \\
\hline C & 2.855394 & 0.422461 & 0.0000000000 \\
\hline $\mathrm{H}$ & 3.153737598 & 0.964379437 & 0.9033737279 \\
\hline $\mathrm{H}$ & 3.153737598 & 0.964379437 & -0.9033737280 \\
\hline $\mathrm{H}$ & 3.436482823 & -0.499884237 & 0.0000000000 \\
\hline
\end{tabular}

\begin{tabular}{|c|c|c|c|}
\hline \multicolumn{4}{|c|}{ Table D42: xyz data of Mes*OMe tBu:Me 2A" } \\
\hline \multicolumn{4}{|l|}{21} \\
\hline \multicolumn{4}{|c|}{ E(UPBE1PBE-D3(BJ)/def2TZVP) $=-424.453274740$} \\
\hline c & 0.428747 & 1.239799 & 0.0000000000 \\
\hline c & -0.459329 & -1.452119 & 0.0000000000 \\
\hline c & 1.387395 & 0.13409 & 0.0000000000 \\
\hline c & -1.002886 & 0.926023 & 0.0000000000 \\
\hline c & -1.380493 & -0.388365 & 0.0000000000 \\
\hline c & 0.90768 & -1.153879 & 0.0000000000 \\
\hline $\mathrm{H}$ & -2.434201 & -0.631262 & 0.0000000000 \\
\hline $\mathrm{H}$ & 1.611304 & -1.972329 & 0.0000000000 \\
\hline 0 & 0.820143 & 2.420163 & 0.0000000000 \\
\hline c & -0.981262 & -2.878342 & 0.0000000000 \\
\hline $\mathrm{H}$ & -0.18089098 & -3.618003686 & 0.0000000000 \\
\hline $\mathrm{H}$ & -1.593880144 & -3.034169511 & 0.8916487249 \\
\hline H & -1.593880144 & -3.034169511 & -0.8916487249 \\
\hline c & -2.020809 & 2.054777 & 0.0000000000 \\
\hline H & -1.892848701 & 2.668934372 & -0.8934013470 \\
\hline $\mathrm{H}$ & -1.892848701 & 2.668934372 & 0.8934013469 \\
\hline $\mathrm{H}$ & -3.044373549 & 1.677565097 & 0.0000000000 \\
\hline c & 2.877872 & 0.430561 & 0.0000000000 \\
\hline $\mathrm{H}$ & 3.142390406 & 0.999633924 & 0.8933585474 \\
\hline $\mathrm{H}$ & 3.142390406 & 0.999633924 & -0.8933585475 \\
\hline $\mathrm{H}$ & 3.474183245 & -0.482861973 & 0.0000000000 \\
\hline
\end{tabular}

\begin{tabular}{|c|c|c|c|}
\hline \multicolumn{4}{|c|}{$\begin{array}{l}13 \\
! 1-A^{\prime}(C S): E(R P B E 1 P B E-D 3(B J) / d e f 2 T Z V P)=-307.235852150\end{array}$} \\
\hline $\bar{c}$ & 0.400688 & 1.179294 & 0.0000000000 \\
\hline C & -0.478472 & -1.472049 & 0.0000000000 \\
\hline H & -0.849882414 & -2.487744854 & 0.0000000000 \\
\hline C & 1.346877 & 0.145764 & 0.0000000000 \\
\hline $\mathrm{H}$ & 2.412955759 & 0.341307567 & 0.0000000000 \\
\hline c & -0.980694 & 0.919297 & 0.0000000000 \\
\hline $\mathrm{H}$ & -1.710673789 & 1.716730149 & 0.0000000000 \\
\hline C & -1.375137 & -0.409361 & 0.0000000000 \\
\hline c & 0.870488 & -1.164208 & 0.0000000000 \\
\hline H & -2.433235 & -0.630247 & 0.0000000000 \\
\hline $\mathrm{H}$ & 1.58645 & -1.970971 & 0.0000000000 \\
\hline 0 & 0.776992 & 2.490077 & 0.0000000000 \\
\hline H & 1.734037 & 2.540522 & 0.0000000000 \\
\hline
\end{tabular}

\begin{tabular}{|c|c|c|c|}
\hline \multicolumn{4}{|c|}{$\begin{array}{l}\text { Table D41: xyz data of pyridinium } 1 \mathrm{~A}^{\prime} \\
12 \\
\text { E(RPBE1PBE-D3(BJ)/def2TZVP) }=-248.454161612\end{array}$} \\
\hline $\mathrm{C}$ & 2.11612442 & -2.46169864 & 0.00000000 \\
\hline c & 3.30150603 & -1.73726782 & 0.00000000 \\
\hline$c$ & 0.89510164 & -1.79910311 & 0.00000000 \\
\hline c & 0.88348091 & -0.42452649 & 0.00000000 \\
\hline$c$ & 3.24251545 & -0.36390844 & 0.00000000 \\
\hline$N$ & 2.04664589 & 0.24215332 & 0.00000000 \\
\hline $\mathrm{H}$ & -0.02022982 & 0.1702531 & 0.00000000 \\
\hline $\mathrm{H}$ & 4.26506435 & -2.22886627 & 0.00000000 \\
\hline $\mathrm{H}$ & 4.11448661 & 0.27649934 & 0.00000000 \\
\hline $\mathrm{H}$ & 2.14396135 & -3.54500974 & 0.00000000 \\
\hline $\mathrm{H}$ & -0.0419374 & -2.33953953 & 0.00000000 \\
\hline $\mathrm{H}$ & 2.02061057 & 1.25535427 & 0.00000000 \\
\hline
\end{tabular}




\begin{tabular}{|c|c|c|c|}
\hline \multicolumn{4}{|c|}{ Table D43: xyz data of Mes*O ${ }^{2} \mathrm{~A}^{\prime \prime}$} \\
\hline \multicolumn{4}{|l|}{48} \\
\hline \multicolumn{4}{|c|}{ E(UPBE1PBE-D3(BJ)/def2TZVP) $=-777.978971168$} \\
\hline C & 0.42887515 & 1.23904273 & 0.00000000 \\
\hline c & -0.45989312 & -1.45264666 & 0.00000000 \\
\hline c & 1.38723894 & 0.13308698 & 0.00000000 \\
\hline c & -1.00283896 & 0.92563434 & 0.00000000 \\
\hline c & -1.38078327 & -0.38865632 & 0.00000000 \\
\hline c & 0.90719205 & -1.15475784 & 0.00000000 \\
\hline $\mathrm{H}$ & -2.43455432 & -0.63128204 & 0.00000000 \\
\hline $\mathrm{H}$ & 1.61060641 & -1.97338915 & 0.00000000 \\
\hline 0 & 0.82057433 & 2.41930657 & 0.00000000 \\
\hline c & -0.98219231 & -2.87873524 & 0.00000000 \\
\hline c & -2.02047147 & 2.05465003 & 0.00000000 \\
\hline C & 2.87779205 & 0.42917507 & 0.00000000 \\
\hline c & 0.13751951 & -3.91404963 & 0.00000000 \\
\hline H & 0.77073551 & -3.82750843 & 0.88613283 \\
\hline H & 0.77073551 & -3.82750843 & -0.88613283 \\
\hline H & -0.29581615 & -4.91663524 & 0.00000000 \\
\hline c & -1.84188218 & -3.09717364 & 1.25117212 \\
\hline H & -1.25291409 & -2.94710932 & 2.15892549 \\
\hline H & -2.23204013 & -4.11835950 & 1.26138579 \\
\hline $\mathrm{H}$ & -2.69175459 & -2.41338445 & 1.28453084 \\
\hline C & -1.84188218 & -3.09717364 & -1.25117212 \\
\hline H & -2.69175459 & -2.41338445 & -1.28453084 \\
\hline H & -2.23204013 & -4.11835950 & -1.26138579 \\
\hline $\mathrm{H}$ & -1.25291409 & -2.94710932 & -2.15892549 \\
\hline C & -1.84033589 & 2.91811772 & -1.25613531 \\
\hline H & -0.85254335 & 3.37331535 & -1.28222294 \\
\hline $\mathrm{H}$ & -2.59308423 & 3.71125015 & -1.26426270 \\
\hline $\mathrm{H}$ & -1.97485302 & 2.31732067 & -2.15963970 \\
\hline C & -1.84033589 & 2.91811772 & 1.25613531 \\
\hline H & -0.85254335 & 3.37331535 & 1.28222294 \\
\hline H & -1.97485302 & 2.31732067 & 2.15963970 \\
\hline $\mathrm{H}$ & -2.59308423 & 3.71125015 & 1.26426270 \\
\hline C & -3.45308061 & 1.52711245 & 0.00000000 \\
\hline H & -3.669444472 & 0.92674794 & 0.88763635 \\
\hline H & -3.66944472 & 0.92674794 & -0.88763635 \\
\hline H & -4.14268216 & 2.37392620 & 0.00000000 \\
\hline C & 3.24995152 & 1.22928436 & 1.25620079 \\
\hline $\mathrm{H}$ & 2.99829626 & 0.66759814 & 2.15982530 \\
\hline H & 2.73106366 & 2.18510199 & 1.28197617 \\
\hline $\mathrm{H}$ & 4.32774642 & 1.41380803 & 1.26406519 \\
\hline c & 3.24995152 & 1.22928436 & -1.25620079 \\
\hline $\mathrm{H}$ & 2.73106366 & 2.18510199 & -1.28197617 \\
\hline $\mathrm{H}$ & 2.99829626 & 0.66759814 & -2.15982530 \\
\hline H & 4.32774642 & 1.41380803 & -1.26406519 \\
\hline C & 3.71214012 & -0.84958819 & 0.00000000 \\
\hline $\mathrm{H}$ & 3.52761195 & -1.46071242 & 0.88738275 \\
\hline $\mathrm{H}$ & 4.77074592 & -0.58122904 & 0.00000000 \\
\hline H & 3.52761195 & -1.46071242 & -0.88738275 \\
\hline
\end{tabular}

\begin{tabular}{|c|c|c|c|}
\hline \multicolumn{4}{|c|}{ Table D44: xyz data of Mes*0Me tBu:Me 2A" } \\
\hline \multicolumn{4}{|l|}{21} \\
\hline \multicolumn{4}{|c|}{ E(UPBE1PBE-D3(BJ)/def2TZVP) $=-424.453274740$} \\
\hline C & 0.428747 & 1.239799 & 0.0000000000 \\
\hline c & -0.459329 & -1.452119 & 0.0000000000 \\
\hline c & 1.387395 & 0.13409 & 0.0000000000 \\
\hline c & -1.002886 & 0.926023 & 0.0000000000 \\
\hline c & -1.380493 & -0.388365 & 0.0000000000 \\
\hline c & 0.90768 & -1.153879 & 0.0000000000 \\
\hline H & -2.434201 & -0.631262 & 0.0000000000 \\
\hline H & 1.611304 & -1.972329 & 0.0000000000 \\
\hline 0 & 0.820143 & 2.420163 & 0.0000000000 \\
\hline c & -0.981262 & -2.878342 & 0.0000000000 \\
\hline H & -0.18089098 & -3.618003686 & 0.0000000000 \\
\hline H & -1.593880144 & -3.034169511 & 0.8916487249 \\
\hline H & -1.593880144 & -3.034169511 & -0.8916487249 \\
\hline c & -2.020809 & 2.054777 & 0.0000000000 \\
\hline H & -1.892848701 & 2.668934372 & -0.8934013470 \\
\hline H & -1.892848701 & 2.668934372 & 0.8934013469 \\
\hline $\mathrm{H}$ & -3.044373549 & 1.677565097 & 0.0000000000 \\
\hline c & 2.877872 & 0.430561 & 0.0000000000 \\
\hline H & 3.142390406 & 0.999633924 & 0.8933585474 \\
\hline H & 3.142390406 & 0.999633924 & -0.8933585475 \\
\hline H & 3.474183245 & -0.482861973 & 0.0000000000 \\
\hline \multicolumn{4}{|c|}{ Table D45: xyz data of Mes* ${ }^{*}{ }^{H}$ tBu:H 2A" } \\
\hline \multicolumn{4}{|l|}{12} \\
\hline \multicolumn{4}{|c|}{ E(UPBE1PBE-D3(BJ)/def2TZVP) $=-306.590089742$} \\
\hline C & 0.428747 & 1.239799 & 0.0000000000 \\
\hline C & 0.90768 & -1.153879 & 0.0000000000 \\
\hline c & -0.459329 & -1.452119 & 0.0000000000 \\
\hline H & -0.80304663 & -2.47806005 & 0.0000000000 \\
\hline c & 1.387395 & 0.13409 & 0.0000000000 \\
\hline $\mathrm{H}$ & 2.4417846 & 0.37632374 & 0.0000000000 \\
\hline C & -1.002886 & 0.926023 & 0.0000000000 \\
\hline $\mathrm{H}$ & -1.7042911 & 1.74962255 & 0.0000000000 \\
\hline C & -1.380493 & -0.388365 & 0.0000000000 \\
\hline $\mathrm{H}$ & -2.434201 & -0.631262 & 0.0000000000 \\
\hline $\mathrm{H}$ & 1.611304 & -1.972329 & 0.0000000000 \\
\hline 0 & 0.820143 & 2.420163 & 0.0000000000 \\
\hline
\end{tabular}




\begin{tabular}{|c|c|c|c|}
\hline \multicolumn{4}{|c|}{ Table D46: $x y z$ data of $17^{2} \mathrm{~A}$} \\
\hline \multicolumn{4}{|l|}{114} \\
\hline \multicolumn{4}{|c|}{$E(U P B E-P B E-D 3(B J) /$ def2SVP) $=-2656.56726064<S 2>=0.7544$} \\
\hline $\mathrm{N}$ & 5.2453802 & 9.37972619 & 14.4794318 \\
\hline Os & 5.13619555 & 7.70643968 & 15.7588866 \\
\hline$P$ & 5.17038953 & 9.43646522 & 17.3892599 \\
\hline$P$ & 5.17560649 & 6.59293147 & 13.6300561 \\
\hline $\mathrm{P}$ & 5.24064514 & 6.06867156 & 17.3319205 \\
\hline c & 5.19774122 & 9.23424838 & 13.1087582 \\
\hline H & 5.20690079 & 10.1679962 & 12.5135237 \\
\hline c & 5.13857679 & 8.01264491 & 12.5052471 \\
\hline H & 5.09963627 & 7.92763444 & 11.4105892 \\
\hline $\mathrm{c}$ & 6.8653321 & 5.7838459 & 13.1972003 \\
\hline $\mathrm{C}$ & 7.19396879 & 5.79526719 & 11.6965411 \\
\hline $\mathrm{H}$ & 6.49828516 & 5.17697311 & 11.0998867 \\
\hline $\mathrm{H}$ & 8.21530357 & 5.38242559 & 11.5471451 \\
\hline $\mathrm{H}$ & 7.1863691 & 6.82375227 & 11.2861685 \\
\hline$C$ & 7.87915672 & 6.69174257 & 13.9202007 \\
\hline $\mathrm{H}$ & 8.91275463 & 6.32051328 & 13.7492462 \\
\hline $\mathrm{H}$ & 7.70129206 & 6.70460445 & 15.0139729 \\
\hline $\mathrm{H}$ & 7.82021023 & 7.73411398 & 13.5471042 \\
\hline$c$ & 6.93509556 & 4.35333715 & 13.7457931 \\
\hline $\mathrm{H}$ & 6.34013547 & 3.64935706 & 13.131517 \\
\hline $\mathrm{H}$ & 6.57395302 & 4.28390615 & 14.789471 \\
\hline $\mathrm{H}$ & 7.98696453 & 3.99427467 & 13.7252668 \\
\hline$c$ & 3.71218434 & 5.51480427 & 13.0298621 \\
\hline$c$ & 3.83861092 & 5.08669337 & 11.5608635 \\
\hline $\mathrm{H}$ & 3.99874143 & 5.94602781 & 10.8797372 \\
\hline $\mathrm{H}$ & 2.8965458 & 4.58739229 & 11.2466111 \\
\hline $\mathrm{H}$ & 4.65820243 & 4.35828497 & 11.4079521 \\
\hline$c$ & 2.47038794 & 6.41146809 & 13.1958215 \\
\hline $\mathrm{H}$ & 2.52993893 & 7.3155997 & 12.5580879 \\
\hline $\mathrm{H}$ & 2.35496761 & 6.74482903 & 14.2454309 \\
\hline $\mathrm{H}$ & 1.56302451 & 5.83765782 & 12.9095941 \\
\hline$c$ & 3.57215285 & 4.28509189 & 13.9327891 \\
\hline $\mathrm{H}$ & 2.6002511 & 3.78177261 & 13.7393882 \\
\hline $\mathrm{H}$ & 3.60230528 & 4.56778631 & 14.9984172 \\
\hline $\mathrm{H}$ & 4.36829032 & 3.53698667 & 13.7679558 \\
\hline$c$ & 5.26135937 & 10.656809 & 14.995783 \\
\hline $\mathrm{H}$ & 5.28942209 & 11.4851358 & 14.2614563 \\
\hline$c$ & 5.23910314 & 10.9067847 & 16.3378689 \\
\hline $\mathrm{H}$ & 5.24023185 & 11.9371017 & 16.7191619 \\
\hline$c$ & 3.62230322 & 9.7527502 & 18.4864219 \\
\hline$c$ & 6.79828815 & 9.57585593 & 18.4169477 \\
\hline$c$ & 7.88332024 & 9.04427307 & 17.459996 \\
\hline $\mathrm{H}$ & 7.73526111 & 7.96760147 & 17.2436043 \\
\hline $\mathrm{H}$ & 8.88587766 & 9.16539348 & 17.9240336 \\
\hline $\mathrm{H}$ & 7.88198766 & 9.59752691 & 16.4983917 \\
\hline $\mathrm{H}$ & 4.99921664 & 6.37628012 & 18.7077226 \\
\hline$c$ & 7.14769564 & 11.0269111 & 18.7867263 \\
\hline $\mathrm{H}$ & 8.12377991 & 11.0384916 & 19.3183599 \\
\hline $\mathrm{H}$ & 6.40196361 & 11.4932893 & 19.4561973 \\
\hline $\mathrm{H}$ & 7.25135088 & 11.6605221 & 17.8847426 \\
\hline$c$ & 6.75731351 & 8.70581871 & 19.6807512 \\
\hline $\mathrm{H}$ & 6.49228319 & 7.65613267 & 19.4486856 \\
\hline $\mathrm{H}$ & 6.04162636 & 9.09150467 & 20.4330944 \\
\hline $\mathrm{H}$ & 7.76207804 & 8.69766913 & 20.1557945 \\
\hline$c$ & 5.08523808 & 4.20793669 & 17.3414017 \\
\hline$c$ & 6.27270476 & 3.40687652 & 17.2579645 \\
\hline$c$ & 6.15823443 & 2.09814394 & 16.7438818 \\
\hline$H$ & 7.06961581 & 1.50713984 & 16.6115406 \\
\hline$c$ & 4.92856704 & 1.52304186 & 16.3994981 \\
\hline$c$ & 3.77364959 & 2.24059472 & 16.7529776 \\
\hline $\mathrm{H}$ & 2.80059482 & 1.75306682 & 16.6194325 \\
\hline$c$ & 3.80576853 & 3.5552205 & 17.2508428 \\
\hline$c$ & 2.48135193 & 4.15902892 & 17.8106573 \\
\hline$c$ & 1.93910778 & 5.35890143 & 17.0045922 \\
\hline $\mathrm{H}$ & 1.60034361 & 5.03780649 & 16.0004281 \\
\hline $\mathrm{H}$ & 2.69572406 & 6.1517896 & 16.8642017 \\
\hline $\mathrm{H}$ & 1.06651227 & 5.79817679 & 17.5311145 \\
\hline$C$ & 2.73674922 & 4.56981854 & 19.2810355 \\
\hline
\end{tabular}

\begin{tabular}{|c|c|c|c|}
\hline $\mathrm{H}$ & 3.50715248 & 5.35783386 & 19.366194 \\
\hline H & 3.0776952 & 3.69835317 & 19.8758137 \\
\hline H & 1.80331947 & 4.95922648 & 19.7378163 \\
\hline c & 1.34718582 & 3.11183232 & 17.8456906 \\
\hline $\mathrm{H}$ & 1.04188392 & 2.78756589 & 16.8305163 \\
\hline $\mathrm{H}$ & 0.45595834 & 3.56625417 & 18.3231519 \\
\hline H & 1.62145646 & 2.21284508 & 18.4333282 \\
\hline c & 7.64879094 & 3.83660471 & 17.8558871 \\
\hline c & 8.393078 & 4.89821842 & 17.0234447 \\
\hline $\mathrm{H}$ & 8.65053769 & 4.51219907 & 16.0191474 \\
\hline H & 9.33577645 & 5.18685081 & 17.5332508 \\
\hline H & 7.77630954 & 5.80775192 & 16.8991777 \\
\hline c & 8.60516465 & 2.63149523 & 17.9934644 \\
\hline $\mathrm{H}$ & 8.91850638 & 2.22563955 & 17.0107526 \\
\hline H & 8.1585891 & 1.80780059 & 18.5856153 \\
\hline H & 9.52560287 & 2.96233309 & 18.5151436 \\
\hline c & 7.4090645 & 4.37360588 & 19.2867699 \\
\hline $\mathrm{H}$ & 6.80301381 & 5.29925002 & 19.2807734 \\
\hline $\mathrm{H}$ & 8.37802129 & 4.60901558 & 19.7738545 \\
\hline H & 6.87920427 & 3.62330192 & 19.9075005 \\
\hline c & 4.80609957 & 0.14734474 & 15.7239236 \\
\hline C & 4.07655432 & 0.32645028 & 14.3725721 \\
\hline $\mathrm{H}$ & 4.65112017 & 0.99506476 & 13.6997255 \\
\hline H & 3.07098765 & 0.77233877 & 14.5066943 \\
\hline H & 3.95184559 & -0.65178643 & 13.8631763 \\
\hline c & 6.17960712 & -0.49011692 & 15.4527433 \\
\hline H & 6.04835379 & -1.46565155 & 14.9424891 \\
\hline H & 6.74078123 & -0.67747189 & 16.3904252 \\
\hline H & 6.80617036 & 0.14907674 & 14.7983842 \\
\hline c & 3.99326053 & -0.80794752 & 16.6264479 \\
\hline $\mathrm{H}$ & 2.9709452 & -0.42572731 & 16.8173142 \\
\hline $\mathrm{H}$ & 4.49166834 & -0.9437722 & 17.6075563 \\
\hline H & 3.89483429 & -1.80445975 & 16.1478698 \\
\hline H & 3.55129876 & 7.79706536 & 15.6977041 \\
\hline c & 3.26435772 & 8.50273306 & 19.3034064 \\
\hline $\mathrm{H}$ & 3.06710368 & 7.63999579 & 18.6405575 \\
\hline $\mathrm{H}$ & 2.34016719 & 8.69999324 & 19.8882461 \\
\hline H & 4.05555329 & 8.21444527 & 20.0214492 \\
\hline H & 4.09098055 & 11.8773822 & 18.8645148 \\
\hline H & 2.82832494 & 11.1784099 & 19.9185045 \\
\hline c & 2.47374172 & 10.0306637 & 17.4984347 \\
\hline $\mathrm{H}$ & 2.64830007 & 10.9536017 & 16.9121902 \\
\hline H & 1.5250938 & 10.146914 & 18.0648538 \\
\hline H & 2.35065613 & 9.19313868 & 16.78315 \\
\hline c & 3.79616844 & 10.9625835 & 19.4161986 \\
\hline
\end{tabular}




\begin{tabular}{|c|c|c|c|}
\hline \multicolumn{4}{|c|}{ Table D47: xyz data of $19^{1} \mathrm{~A}$} \\
\hline \multicolumn{4}{|l|}{113} \\
\hline \multicolumn{4}{|c|}{ E(RPBE-PBE-D3(BJ)/def2SVP) $=-2655.96534768$} \\
\hline $\mathrm{N}$ & 5.53174285 & 9.33924349 & 14.5435534 \\
\hline Os & 5.19295584 & 7.63525275 & 15.8094306 \\
\hline $\mathrm{P}$ & 5.03900605 & 9.43148113 & 17.4277058 \\
\hline$P$ & 5.29447078 & 6.58128408 & 13.6571548 \\
\hline $\mathrm{P}$ & 5.70563667 & 6.07987033 & 17.3056903 \\
\hline c & 5.49563831 & 9.21929774 & 13.1690901 \\
\hline H & 5.57822913 & 10.1571161 & 12.5849332 \\
\hline c & 5.35303939 & 8.01512561 & 12.5498844 \\
\hline H & 5.33021817 & 7.94130467 & 11.454218 \\
\hline c & 6.92783879 & 5.68414535 & 13.1654138 \\
\hline c & 7.21634488 & 5.70498905 & 11.6551222 \\
\hline H & 6.47650419 & 5.13536556 & 11.0640517 \\
\hline H & 8.20992015 & 5.23932385 & 11.4776979 \\
\hline H & 7.26049822 & 6.73831046 & 11.260219 \\
\hline c & 8.01294643 & 6.52592232 & 13.8643746 \\
\hline H & 9.01579304 & 6.09167859 & 13.6632555 \\
\hline H & 7.86345487 & 6.55641576 & 14.960039 \\
\hline H & 8.00835625 & 7.56879277 & 13.4878638 \\
\hline c & 6.93432683 & 4.24083003 & 13.6794176 \\
\hline H & 6.26503618 & 3.59005228 & 13.0826302 \\
\hline H & 6.62291849 & 4.166676 & 14.7374382 \\
\hline H & 7.95844838 & 3.8165152 & 13.5966953 \\
\hline c & 3.76100996 & 5.60165169 & 13.0694708 \\
\hline c & 3.87726976 & 5.11649885 & 11.6172757 \\
\hline H & 4.09883523 & 5.94022616 & 10.9097225 \\
\hline H & 2.90773644 & 4.66843072 & 11.3097835 \\
\hline H & 4.64911129 & 4.3320721 & 11.499012 \\
\hline c & 2.57299358 & 6.5763089 & 13.1798037 \\
\hline $\mathrm{H}$ & 2.70056829 & 7.45428954 & 12.516184 \\
\hline H & 2.45071579 & 6.94896703 & 14.2153686 \\
\hline H & 1.64015867 & 6.04773157 & 12.8886826 \\
\hline c & 3.53981122 & 4.4148564 & 14.0134856 \\
\hline $\mathrm{H}$ & 2.55837567 & 3.93883336 & 13.8010669 \\
\hline H & 3.54581856 & 4.74382359 & 15.0657113 \\
\hline H & 4.31588959 & 3.63484251 & 13.911047 \\
\hline c & 5.58558164 & 10.6111654 & 15.073697 \\
\hline H & 5.76049122 & 11.4368332 & 14.3552997 \\
\hline c & 5.39196109 & 10.8741054 & 16.3985089 \\
\hline H & 5.40944442 & 11.9052343 & 16.7767969 \\
\hline c & 3.31533749 & 9.81457372 & 18.1767009 \\
\hline c & 6.44165425 & 9.50000191 & 18.7430897 \\
\hline c & 7.70825851 & 9.04245398 & 17.9905394 \\
\hline $\mathrm{H}$ & 7.64954538 & 7.9728515 & 17.7117502 \\
\hline H & 8.59345556 & 9.18039675 & 18.6477963 \\
\hline H & 7.86931065 & 9.64173811 & 17.07073 \\
\hline c & 6.68179576 & 10.9261472 & 19.2669093 \\
\hline $\mathrm{H}$ & 7.5342955 & 10.9021997 & 19.9793308 \\
\hline H & 5.81296858 & 11.3429121 & 19.8073038 \\
\hline $\mathrm{H}$ & 6.9529837 & 11.620541 & 18.4481688 \\
\hline c & 6.14962482 & 8.54662286 & 19.9078967 \\
\hline H & 5.91567526 & 7.5235899 & 19.5436148 \\
\hline $\mathrm{H}$ & 5.31724537 & 8.90744235 & 20.5437224 \\
\hline H & 7.05118804 & 8.46712404 & 20.552385 \\
\hline c & 5.32768179 & 4.24212566 & 17.2698002 \\
\hline c & 6.44270406 & 3.34354552 & 17.1010038 \\
\hline c & 6.18038054 & 2.0655048 & 16.5633653 \\
\hline H & 7.02744734 & 1.40899684 & 16.3407955 \\
\hline c & 4.88336319 & 1.5904659 & 16.321248 \\
\hline c & 3.82523209 & 2.37323793 & 16.8195446 \\
\hline H & 2.80995871 & 1.95439994 & 16.8070802 \\
\hline c & 4.01222793 & 3.6657859 & 17.3304793 \\
\hline c & 2.82742796 & 4.34945346 & 18.0680091 \\
\hline c & 2.00627304 & 5.27432802 & 17.1472817 \\
\hline H & 1.547136 & 4.69897866 & 16.3193531 \\
\hline H & 2.6400066 & 6.06552866 & 16.7052185 \\
\hline H & 1.18929834 & 5.76056496 & 17.7198729 \\
\hline c & 3.37365707 & 5.13319807 & 19.2790898 \\
\hline H & 4.05694685 & 5.9550227 & 18.9738947 \\
\hline
\end{tabular}

\begin{tabular}{|c|c|c|c|}
\hline H & 3.94456881 & 4.46616649 & 19.9559012 \\
\hline $\mathrm{H}$ & 2.54398513 & 5.59604417 & 19.8514756 \\
\hline c & 1.86038135 & 3.28778262 & 18.6409469 \\
\hline H & 1.29730975 & 2.75436392 & 17.8496935 \\
\hline H & 1.11000814 & 3.78904582 & 19.2855577 \\
\hline $\mathrm{H}$ & 2.39393636 & 2.53416034 & 19.2545275 \\
\hline c & 7.87755695 & 3.63458237 & 17.6411324 \\
\hline c & 8.6595369 & 4.70777901 & 16.8589637 \\
\hline H & 8.80781202 & 4.41217328 & 15.8036017 \\
\hline H & 9.65940556 & 4.8548909 & 17.3181494 \\
\hline H & 8.13233012 & 5.682202 & 16.880058 \\
\hline c & 8.75070393 & 2.36092142 & 17.629002 \\
\hline H & 8.97082129 & 2.00942209 & 16.6006462 \\
\hline H & 8.28423967 & 1.52737251 & 18.1910264 \\
\hline H & 9.72295662 & 2.58854949 & 18.1101705 \\
\hline c & 7.74178087 & 4.07549001 & 19.1198392 \\
\hline H & 7.19091808 & 5.03365225 & 19.2002951 \\
\hline $\mathrm{H}$ & 8.74702127 & 4.21565336 & 19.5691061 \\
\hline H & 7.19979138 & 3.30977965 & 19.7108082 \\
\hline c & 4.59689856 & 0.24179024 & 15.6425069 \\
\hline c & 3.69959865 & 0.48784597 & 14.4079678 \\
\hline H & 4.21132759 & 1.13933098 & 13.6709082 \\
\hline H & 2.74767141 & 0.9825618 & 14.6856162 \\
\hline $\mathrm{H}$ & 3.45238776 & -0.47172652 & 13.9079075 \\
\hline c & 5.88517734 & -0.45593298 & 15.1731937 \\
\hline H & 5.63389491 & -1.40353343 & 14.6552592 \\
\hline H & 6.55231381 & -0.70822174 & 16.0219522 \\
\hline H & 6.45626218 & 0.17535132 & 14.4626561 \\
\hline c & 3.86572178 & -0.69346629 & 16.6323236 \\
\hline H & 2.90225107 & -0.26308636 & 16.9703754 \\
\hline H & 4.48697401 & -0.87806645 & 17.5319895 \\
\hline H & 3.64852381 & -1.67178555 & 16.1550939 \\
\hline H & 3.63959273 & 7.30606258 & 15.8256535 \\
\hline c & 2.7911638 & 8.57739567 & 18.9157422 \\
\hline H & 2.78498746 & 7.69853359 & 18.2473053 \\
\hline H & 1.7487752 & 8.76105616 & 19.2543166 \\
\hline H & 3.3939553 & 8.32530558 & 19.8081505 \\
\hline H & 3.74091298 & 11.9360003 & 18.619619 \\
\hline H & 2.24839976 & 11.2874995 & 19.3593852 \\
\hline c & 2.41325259 & 10.0814953 & 16.9559454 \\
\hline H & 2.71312716 & 11.0010383 & 16.4169711 \\
\hline $\mathrm{H}$ & 1.36268522 & 10.2003015 & 17.296812 \\
\hline $\mathrm{H}$ & 2.45142615 & 9.23746545 & 16.2373102 \\
\hline c & 3.3006618 & 11.0398156 & 19.1006706 \\
\hline $\mathrm{H}$ & 3.83414612 & 10.8520689 & 20.0525323 \\
\hline
\end{tabular}




\begin{tabular}{|c|c|c|c|}
\hline \multicolumn{4}{|c|}{ Table D48: xyz data of Mes*P ${ }^{3} \mathrm{~A}$} \\
\hline \multicolumn{4}{|l|}{48} \\
\hline \multicolumn{4}{|c|}{ E(UPBE-PBE-D3(BJ)/def2SVP) $=-1043.00517060<S 2>=2.0095$} \\
\hline C & -4.29229334 & 1.42606636 & 0.22200909 \\
\hline C & -2.99198846 & 0.81085245 & 0.07847518 \\
\hline C & -5.47486304 & 0.60068952 & 0.07929523 \\
\hline c & -5.31104726 & -0.76354046 & -0.19266727 \\
\hline C & -4.05454603 & -1.37931613 & -0.3355638 \\
\hline c & -2.92108818 & -0.56598655 & -0.19439347 \\
\hline c & -1.6637529 & 1.60118599 & 0.21179058 \\
\hline c & -1.58864046 & 2.70372974 & -0.869759 \\
\hline c & -0.42301689 & 0.70510659 & 0.01385345 \\
\hline c & -1.54609878 & 2.21091953 & 1.62790395 \\
\hline c & -6.91587345 & 1.15855329 & 0.21390892 \\
\hline C & -7.17429031 & 2.23307866 & -0.86755954 \\
\hline c & -3.97032955 & -2.88478971 & -0.63403464 \\
\hline C & -2.51714459 & -3.3749683 & -0.75551203 \\
\hline C & -4.69760363 & -3.17816175 & -1.96658947 \\
\hline C & -4.65539365 & -3.66687531 & 0.51039908 \\
\hline H & -6.47431541 & 3.09071684 & -0.77555828 \\
\hline H & -8.20590536 & 2.63149422 & -0.7771844 \\
\hline H & -7.05189899 & 1.80478922 & -1.88291956 \\
\hline C & -7.13174992 & 1.74023986 & 1.6302411 \\
\hline C & -7.98949851 & 0.06798657 & 0.01701514 \\
\hline H & -7.94186088 & -0.39087581 & -0.99090978 \\
\hline $\mathrm{H}$ & -8.9930087 & 0.52586208 & 0.12444555 \\
\hline $\mathrm{H}$ & -7.91182279 & -0.73891945 & 0.77306131 \\
\hline $\mathrm{H}$ & -6.42963527 & 2.57308369 & 1.8479073 \\
\hline H & -6.97891535 & 0.95927322 & 2.40231633 \\
\hline $\mathrm{H}$ & -8.163175 & 2.13645006 & 1.73178939 \\
\hline $\mathrm{H}$ & -1.63974143 & 2.26118863 & -1.88509648 \\
\hline $\mathrm{H}$ & -0.63721113 & 3.26764747 & -0.78073231 \\
\hline H & -2.42105941 & 3.43334091 & -0.77649744 \\
\hline H & -0.39393565 & 0.24462296 & -0.99397529 \\
\hline H & -0.36389552 & -0.10338734 & 0.76979788 \\
\hline $\mathrm{H}$ & 0.48973182 & 1.3246013 & 0.12054038 \\
\hline $\mathrm{H}$ & -2.376391 & 2.91589633 & 1.84601841 \\
\hline H & -0.59447127 & 2.77254546 & 1.72854243 \\
\hline $\mathrm{H}$ & -1.5667517 & 1.41565603 & 2.40023392 \\
\hline $\mathrm{H}$ & -4.15212568 & -3.47238716 & 1.47898985 \\
\hline $\mathrm{H}$ & -4.61429199 & -4.7580558 & 0.31233085 \\
\hline H & -5.72118735 & -3.38481323 & 0.62138712 \\
\hline $\mathrm{H}$ & -1.97733823 & -2.86621758 & -1.57946068 \\
\hline $\mathrm{H}$ & -2.50451964 & -4.46245384 & -0.97029298 \\
\hline $\mathrm{H}$ & -1.94732624 & -3.21370857 & 0.18172425 \\
\hline $\mathrm{H}$ & -6.20442734 & -1.38941083 & -0.30094042 \\
\hline P & -4.44205099 & 3.19864575 & 0.57430733 \\
\hline $\mathrm{H}$ & -1.93509525 & -1.02676825 & -0.30210388 \\
\hline $\mathrm{H}$ & -4.2251241 & -2.62720371 & -2.8047307 \\
\hline $\mathrm{H}$ & -5.76461699 & -2.88198573 & -1.92714665 \\
\hline H & -4.65708945 & -4.26253652 & -2.19915142 \\
\hline
\end{tabular}

\begin{tabular}{|c|c|c|c|}
\hline $\begin{array}{l}\text { Table } \\
49 \\
\text { E(UPE }\end{array}$ & $z$ data of Me & 43.61560710 & 0.7541 \\
\hline c & -4.30275837 & 1.44510214 & 0.23520462 \\
\hline c & -3.00461847 & 0.83793583 & 0.10132009 \\
\hline c & -5.47982558 & 0.62842654 & 0.05528223 \\
\hline c & -5.31375994 & -0.74329807 & -0.1919054 \\
\hline c & -4.05925832 & -1.36522783 & -0.28568588 \\
\hline c & -2.93247959 & -0.54736221 & -0.14543651 \\
\hline c & -1.65205738 & 1.60143083 & 0.2054077 \\
\hline c & -1.65611572 & 2.88710663 & -0.64926016 \\
\hline c & -0.46597086 & 0.76451307 & -0.32982155 \\
\hline c & -1.33287767 & 1.90258412 & 1.68955888 \\
\hline c & -6.93418814 & 1.17357867 & 0.14291345 \\
\hline c & -7.18179940 & 2.2206555 & -0.96931411 \\
\hline c & -3.96887993 & -2.87822072 & -0.54519874 \\
\hline c & -2.51321537 & -3.37057261 & -0.61830655 \\
\hline c & -4.66311253 & -3.20599493 & -1.88693217 \\
\hline c & -4.68168386 & -3.63085849 & 0.60169619 \\
\hline H & -6.51521925 & 3.10088666 & -0.88820039 \\
\hline H & -8.22631414 & 2.59185895 & -0.91705588 \\
\hline H & -7.02656077 & 1.76555275 & -1.96833192 \\
\hline c & -7.20692615 & 1.76832976 & 1.5441546 \\
\hline c & -7.99039991 & 0.06627449 & -0.06460375 \\
\hline $\mathrm{H}$ & -7.91124622 & -0.40951784 & -1.06253895 \\
\hline H & -9.00032749 & 0.51711377 & 0.00586057 \\
\hline H & -7.92800398 & -0.72619407 & 0.70783013 \\
\hline H & -6.53262516 & 2.62271719 & 1.76891839 \\
\hline H & -7.05785843 & 1.00170836 & 2.33131609 \\
\hline H & -8.25013622 & 2.1408825 & 1.60951758 \\
\hline H & -1.80502216 & 2.64353538 & -1.72071042 \\
\hline H & -0.69112290 & 3.42370539 & -0.54265855 \\
\hline $\mathrm{H}$ & -2.46192078 & 3.60037992 & -0.36737044 \\
\hline H & -0.62797555 & 0.4363945 & -1.37610036 \\
\hline H & -0.25919521 & -0.1304451 & 0.28959103 \\
\hline H & 0.45061584 & 1.38736928 & -0.30945486 \\
\hline H & -2.10595172 & 2.52421456 & 2.17868544 \\
\hline H & -0.36517686 & 2.43949486 & 1.77478808 \\
\hline H & -1.25409304 & 0.95676046 & 2.2621461 \\
\hline H & -4.20159495 & -3.41189926 & 1.57685028 \\
\hline $\mathrm{H}$ & -4.63765631 & -4.72688832 & 0.43294747 \\
\hline H & -5.74932682 & -3.34467525 & 0.67989842 \\
\hline H & -1.95390605 & -2.88482815 & -1.443183 \\
\hline H & -2.49496395 & -4.46373784 & -0.80179366 \\
\hline H & -1.96606450 & -3.18199894 & 0.32738165 \\
\hline H & -6.20586573 & -1.36777829 & -0.31293872 \\
\hline$P$ & -4.53733615 & 3.2520471 & 0.56803194 \\
\hline H & -1.94532794 & -1.00822932 & -0.23771776 \\
\hline H & -4.17067016 & -2.67559438 & -2.7268799 \\
\hline H & -5.73079431 & -2.90956218 & -1.88081716 \\
\hline $\mathrm{H}$ & -4.61710714 & -4.29581471 & -2.09157962 \\
\hline H & -3.61604477 & 3.3502193 & 1.67223545 \\
\hline
\end{tabular}




\begin{tabular}{|c|c|c|c|}
\hline \multicolumn{4}{|c|}{ Table D50: xyz data of Mes*PH ${ }^{4} \mathrm{~A}$} \\
\hline \multicolumn{4}{|l|}{49} \\
\hline \multicolumn{4}{|c|}{ E(UPBE-PBE-D3(BJ)/def2SVP) $=-1043.51315469<S 2>=3.7640$} \\
\hline C & -4.27144164 & 1.18478336 & 0.75666526 \\
\hline C & -2.96422795 & 0.75254911 & 0.19232865 \\
\hline C & -5.48580925 & 0.53280405 & 0.19630713 \\
\hline c & -5.32897116 & -0.82320305 & -0.02384777 \\
\hline c & -4.03615846 & -1.45836202 & -0.0044699 \\
\hline c & -2.87909333 & -0.61562378 & -0.02737433 \\
\hline c & -1.83635498 & 1.7527852 & -0.05705776 \\
\hline c & -2.4297574 & 3.00406147 & -0.74055906 \\
\hline c & -0.74328404 & 1.15577466 & -0.96159978 \\
\hline c & -1.19009598 & 2.16967173 & 1.28625123 \\
\hline c & -6.74264972 & 1.33822501 & -0.12906373 \\
\hline C & -6.35935945 & 2.36938482 & -1.21791533 \\
\hline c & -3.94957981 & -2.98212195 & -0.13126548 \\
\hline C & -2.49560951 & -3.48620685 & -0.13642461 \\
\hline C & -4.63095345 & -3.45081727 & -1.43961833 \\
\hline c & -4.67978378 & -3.61243329 & 1.0792886 \\
\hline H & -5.53916293 & 3.02701121 & -0.86670507 \\
\hline H & -7.23020615 & 3.00848723 & -1.47351752 \\
\hline H & -6.01605143 & 1.86121873 & -2.14148792 \\
\hline C & -7.25657543 & 2.09574899 & 1.11489447 \\
\hline C & -7.8721381 & 0.44049952 & -0.66033752 \\
\hline H & -7.56985656 & -0.09777315 & -1.58091618 \\
\hline $\mathrm{H}$ & -8.75987769 & 1.05704181 & -0.9076579 \\
\hline $\mathrm{H}$ & -8.18429563 & -0.31054148 & 0.0934087 \\
\hline $\mathrm{H}$ & -6.49525829 & 2.799762 & 1.50714277 \\
\hline H & -7.52367331 & 1.39458044 & 1.93141381 \\
\hline $\mathrm{H}$ & -8.16015467 & 2.68657751 & 0.85643184 \\
\hline $\mathrm{H}$ & -2.89249308 & 2.74516873 & -1.71425942 \\
\hline $\mathrm{H}$ & -1.63944968 & 3.76165429 & -0.92020484 \\
\hline H & -3.21335294 & 3.46600171 & -0.10615817 \\
\hline H & -1.16205846 & 0.80815355 & -1.92727693 \\
\hline H & -0.2306669 & 0.29920364 & -0.47966977 \\
\hline $\mathrm{H}$ & 0.02931377 & 1.92182663 & -1.17658909 \\
\hline $\mathrm{H}$ & -1.93508819 & 2.62312642 & 1.97315662 \\
\hline H & -0.38723339 & 2.91736673 & 1.11398001 \\
\hline H & -0.74718085 & 1.29318702 & 1.80054539 \\
\hline H & -4.20193919 & -3.30506852 & 2.03160659 \\
\hline $\mathrm{H}$ & -4.65069338 & -4.72076686 & 1.01910398 \\
\hline H & -5.74227584 & -3.30012185 & 1.12049797 \\
\hline $\mathrm{H}$ & -1.93447366 & -3.12339299 & -1.02105606 \\
\hline $\mathrm{H}$ & -2.48060757 & -4.59444207 & -0.16748417 \\
\hline $\mathrm{H}$ & -1.94911653 & -3.1657976 & 0.77335136 \\
\hline $\mathrm{H}$ & -6.19783417 & -1.4265074 & -0.32866574 \\
\hline P & -4.3008938 & 1.473989866 & 2.5826926 \\
\hline $\mathrm{H}$ & -1.91597137 & -1.05302599 & -0.32542257 \\
\hline $\mathrm{H}$ & -4.13280265 & -3.00613684 & -2.32469494 \\
\hline $\mathrm{H}$ & -5.70175438 & -3.16834903 & -1.47557585 \\
\hline H & -4.57516963 & -4.55566453 & -1.52996295 \\
\hline $\mathrm{H}$ & -4.142598 & 0.08779111 & 2.9955117 \\
\hline
\end{tabular}




\section{E. List of abbreviations}

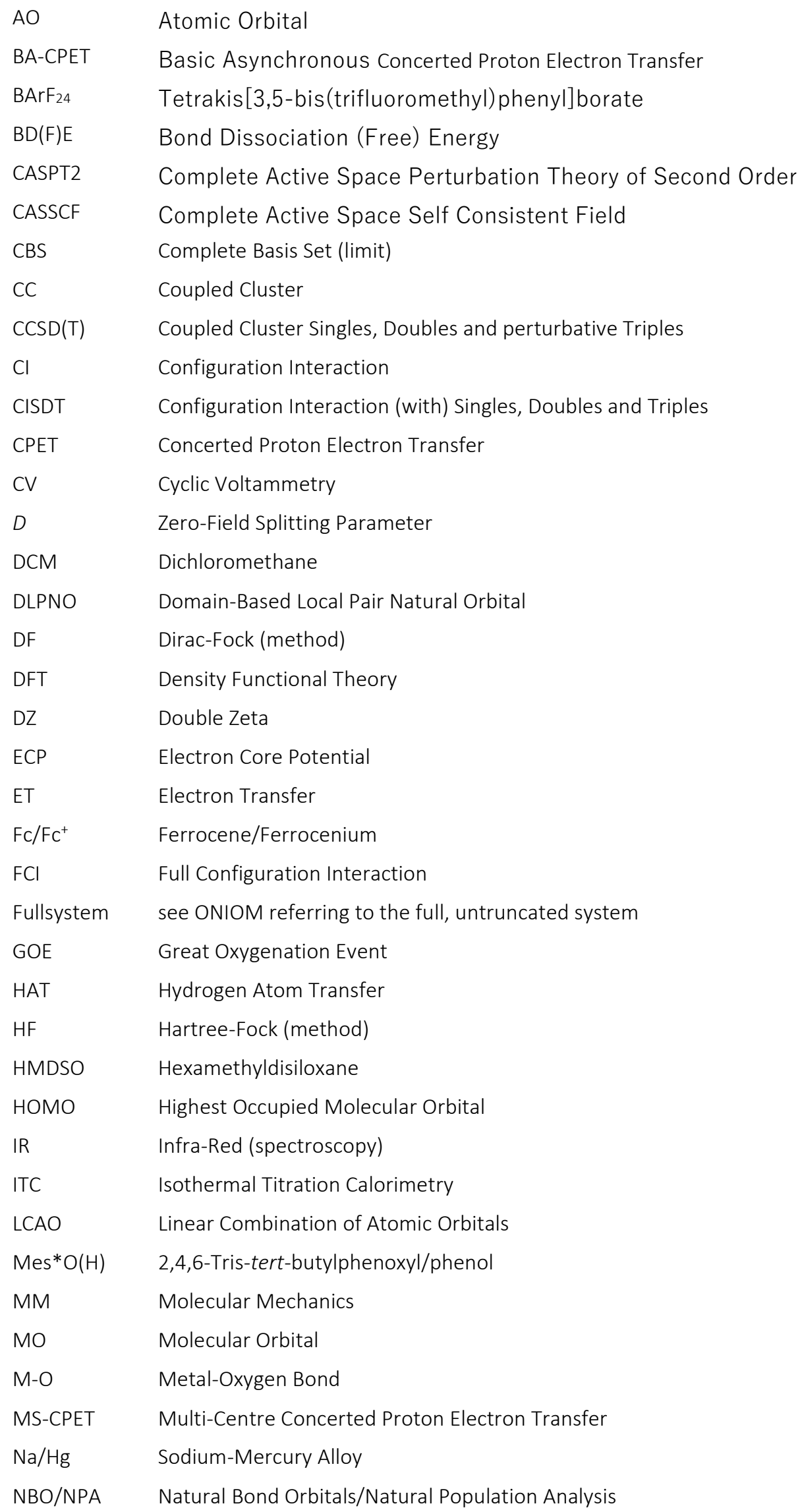




\begin{tabular}{|c|c|}
\hline NEVPT2 & n-Electron Valence Perturbation Theory of Second Order \\
\hline NIR & Near-Infra-Red (spectroscopy) \\
\hline NMR & Nuclear Magnetic Resonance (technique) \\
\hline OA-CPET & Oxidative Asynchronous Concerted Proton Electron Transfer \\
\hline OAT & Oxygen Atom Transfer \\
\hline OEC & Oxygen Evolving Reaction \\
\hline ONIOM & Our Own n-layered integrated molecular orbital and molecular mechanics \\
\hline PAO & Projected Atomic Orbitals \\
\hline PCET & Proton Coupled Electron Transfer \\
\hline PNO & Pair of Natural Orbitals \\
\hline PNP & Bis(di-tert-butylphosphinoethylene)amide (Chapter II-III) \\
\hline $\mathrm{PH}^{\mathrm{H}} \mathrm{NP}$ & Bis(di-isopropylphosphinoethyl)amine \\
\hline PSII & Photosystem II \\
\hline PT & Proton Transfer \\
\hline QDPT & Quasi-Degenerate Pertubation Theory \\
\hline SARC & All-Electron Relativistic Contracted (basis set) \\
\hline SMD & Solvation Model Bases Density \\
\hline SOC & Spin-Orbit Coupling \\
\hline SOMF & Spin-Orbit Mean Field (operator) \\
\hline SOMO & Singly Occupied Molecular Orbital \\
\hline SORCl & Spectroscopy Oriented Configuration Interaction \\
\hline SQUID & Superconducting Quantum Interference Device (technique) \\
\hline SZ & Single Zeta \\
\hline TD-DFT & Time-Dependent Density Functional Theory \\
\hline THF & Tetrahydrofurane \\
\hline TIP & Temperature Independent Paramagnetism \\
\hline Truncation & See ONIOM Referring to truncated model system \\
\hline UV-VIS & Ultra-Violet-Visual (spectroscopy) \\
\hline WFT & Wavefunction Theory \\
\hline $\mathrm{XRD}$ & X-Ray Diffraction \\
\hline ZORA & Zeorth Order Regular Approximation \\
\hline
\end{tabular}




\title{
F. Curriculum Vitae
}

\author{
Daniel Delony
}

* 02. August 1992 in Celle

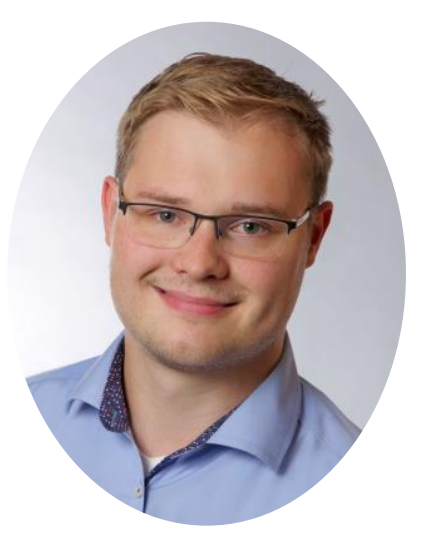

\section{7-2020 Dissertation}

"Proton Coupled Electron Transfer at Heavy Metal Sites"

Georg-August Universität Göttingen, Working Group Prof. S. Schneider

\section{4 - 2016 Master of Science (Chemie)}

„Amin Aktivierung durch PNP-Komplexe der Gruppe 9“

Georg-August Universität Göttingen, Working Group Prof. S. Schneider

\section{1 - 2014 Bachelor of Science (Chemie)}

„Mechanistische Untersuchung zur selenkatalysierten C-H Acyloxylierung Georg-August Universität Göttingen, Working Group Dr. A. Breder

\section{6-2011 Abitur}

Christian-Gymnasium Hermannsburg 